\title{
Ionic liquid crystals: versatile materials
}

Karel Goossens, ${ }^{\left[{ }^{[a][b]}\right.}$ Kathleen Lava, ${ }^{[\mathrm{c}][\mathrm{b}]}$ Christopher W. Bielawski, ${ }^{[\mathrm{a}][\mathrm{d}]}$ and Koen Binnemans ${ }^{[\mathrm{b}]}$

${ }^{[a]}$ Center for Multidimensional Carbon Materials, Institute for Basic Science (IBS), Ulsan 689798, Republic of Korea;

${ }^{[b]}$ Department of Chemistry, KU Leuven, Celestijnenlaan 200F PO box 2404, B-3001 Heverlee, Belgium;

${ }^{[c]}$ Department of Organic and Macromolecular Chemistry, Ghent University, Krijgslaan 281 S4, B-9000 Ghent, Belgium;

${ }^{[d]}$ Department of Chemistry and Department of Energy Engineering, Ulsan National Institute of Science and Technology (UNIST), Ulsan 689-798, Republic of Korea.

* Contact details of the corresponding author:

E-mail: karel.cw.goossens@gmail.com

Fax: +82-52-217-5759

Tel.: +82-52-217-5753

\section{Supporting Information}

\section{Table of contents}

1. List of abbreviations ....................................................................................... S3

2. Additional tables referred to in the main text ........................................................... 99

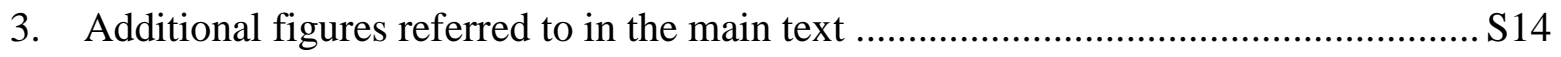

4. Thermal transitions of all compounds discussed in the main text (in the order

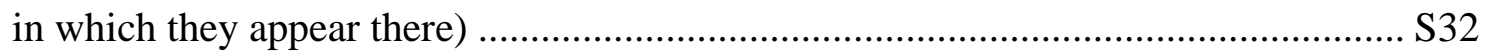

4.1. Imidazolium-based ionic liquid crystals .................................................. S33

4.2. Ammonium-based ionic liquid crystals .................................................... S86

4.3. Phosphonium-based ionic liquid crystals ...................................................S112 
4.4. Pyridinium-based ionic liquid crystals .......................................................S119

4.5. 4,4'-Bipyridinium-based ionic liquid crystals ............................................... S136

4.6. New cationic cores for ionic liquid crystals ..................................................S143

4.7. Ionic liquid crystals formed by ‘ionic self-assembly’ (ISA) ............................S171

4.8. Structures mentioned in section about applications of ionic liquid

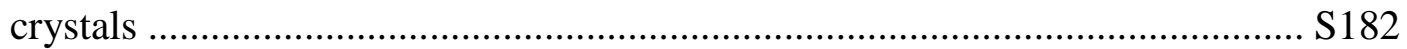

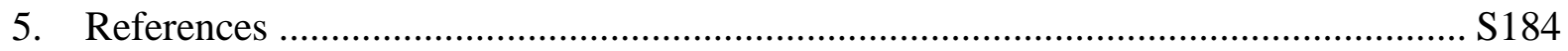




\section{List of abbreviations}

\begin{tabular}{|c|c|}
\hline 1D & one-dimensional(ly) \\
\hline 2D & two-dimensional(ly) \\
\hline $3 \mathrm{D}$ & three-dimensional(ly) \\
\hline 5CB & 4'-pentyl-4-cyanobiphenyl \\
\hline 5OCB & 4'-(n-pentyloxy)-4-cyanobiphenyl \\
\hline$\lambda$ & wavelength \\
\hline$\sigma_{\|}$ & ionic conductivity parallel to smectic layers (in $\mathrm{S} \mathrm{cm}^{-1}$ ) \\
\hline$\sigma_{\perp}$ & ionic conductivity perpendicular to smectic layers (in $\mathrm{S} \mathrm{cm}^{-1}$ ) \\
\hline$\left[\mathrm{C}_{n} \mathrm{C}_{m} \mathrm{Bim}\right]^{+}$ & $\begin{array}{l}\text { 1,3-bis( } n \text {-alkyl)benzimidazolium ( } n \text { and } m \text { indicate the number of carbon atoms in } \\
\text { both alkyl chains, respectively) }\end{array}$ \\
\hline$\left[\mathrm{C}_{n} \mathrm{C}_{m} \mathrm{im}\right]^{+}$ & $\begin{array}{l}\text { 1,3-bis( } n \text {-alkyl)imidazolium ( } n \text { and } m \text { indicate the number of carbon atoms in } \\
\text { both alkyl chains, respectively) }\end{array}$ \\
\hline$\left[\mathrm{C}_{n} \mathrm{C}_{m} \text { mmpiperaz }\right]^{+}$ & $\begin{array}{l}N, N^{\prime} \text {-bis(n-alkyl)- } N, N^{\prime} \text {-dimethylpiperazinium ( } n \text { and } m \text { indicate the number of } \\
\text { carbon atoms in both alkyl chains, respectively) }\end{array}$ \\
\hline$\left[\mathrm{C}_{n} \mathrm{C}_{m} \text { piperid }\right]^{+}$ & $\begin{array}{l}N, N \text {-bis( } n \text {-alkyl)piperidinium ( } n \text { and } m \text { indicate the number of carbon atoms in } \\
\text { both alkyl chains, respectively) }\end{array}$ \\
\hline$\left[\mathrm{C}_{n} \mathrm{C}_{m} \mathrm{pyrr}\right]^{+}$ & $\begin{array}{l}N, N \text {-bis( } n \text {-alkyl)pyrrolidinium ( } n \text { and } m \text { indicate the number of carbon atoms in } \\
\text { both alkyl chains, respectively) }\end{array}$ \\
\hline$\left[\mathrm{C}_{n} \mathrm{dmim}\right]^{+}$ & $\begin{array}{l}\text { 1,2-dimethyl-3-( } n \text {-alkyl)imidazolium ( } n \text { indicates the number of carbon atoms in } \\
\text { the alkyl chain) }\end{array}$ \\
\hline$\left[\mathrm{C}_{n} \mathrm{Him}\right]^{+}$ & $\begin{array}{l}\text { 1-( } n \text {-alkyl)imidazolium ( } n \text { indicates the number of carbon atoms in the alkyl } \\
\text { chain) }\end{array}$ \\
\hline$\left[\mathrm{C}_{n} \mathrm{mim}\right]^{+}$ & $\begin{array}{l}\text { 1-methyl-3-( } n \text {-alkyl)imidazolium ( } n \text { indicates the number of carbon atoms in the } \\
\text { alkyl chain) }\end{array}$ \\
\hline$\left[\mathrm{C}_{n} \text { mpiperid }\right]^{+}$ & $\begin{array}{l}N \text {-( } n \text {-alkyl)- } N \text {-methylpiperidinium ( } n \text { indicates the number of carbon atoms in the } \\
\text { alkyl chain) }\end{array}$ \\
\hline$\left[\mathrm{C}_{n} \mathrm{mpyrr}\right]^{+}$ & $\begin{array}{l}N \text {-( } n \text {-alkyl)- } N \text {-methylpyrrolidinium ( } n \text { indicates the number of carbon atoms in } \\
\text { the alkyl chain) }\end{array}$ \\
\hline$\left[\mathrm{C}_{n} \mathrm{pyr}\right]^{+}$ & $N$-( $n$-alkyl)pyridinium ( $n$ indicates the number of carbon atoms in the alkyl chain) \\
\hline$\left[\mathrm{C}_{n} \mathrm{vim}\right]^{+}$ & $\begin{array}{l}\text { 1-vinyl-3-( } n \text {-alkyl)imidazolium ( } n \text { indicates the number of carbon atoms in the } \\
\text { alkyl chain) }\end{array}$ \\
\hline [DcHSS $^{-}$ & dicyclohexylsulfosuccinate \\
\hline [DDSS $^{-}$ & bis(2-butyloctyl)sulfosuccinate (didodecylsulfosuccinate) \\
\hline [DHSS $^{-}$ & bis(n-hexyl)sulfosuccinate \\
\hline 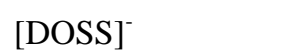 & bis(2-ethylhexyl)sulfosuccinate (dioctylsulfosuccinate) \\
\hline
\end{tabular}




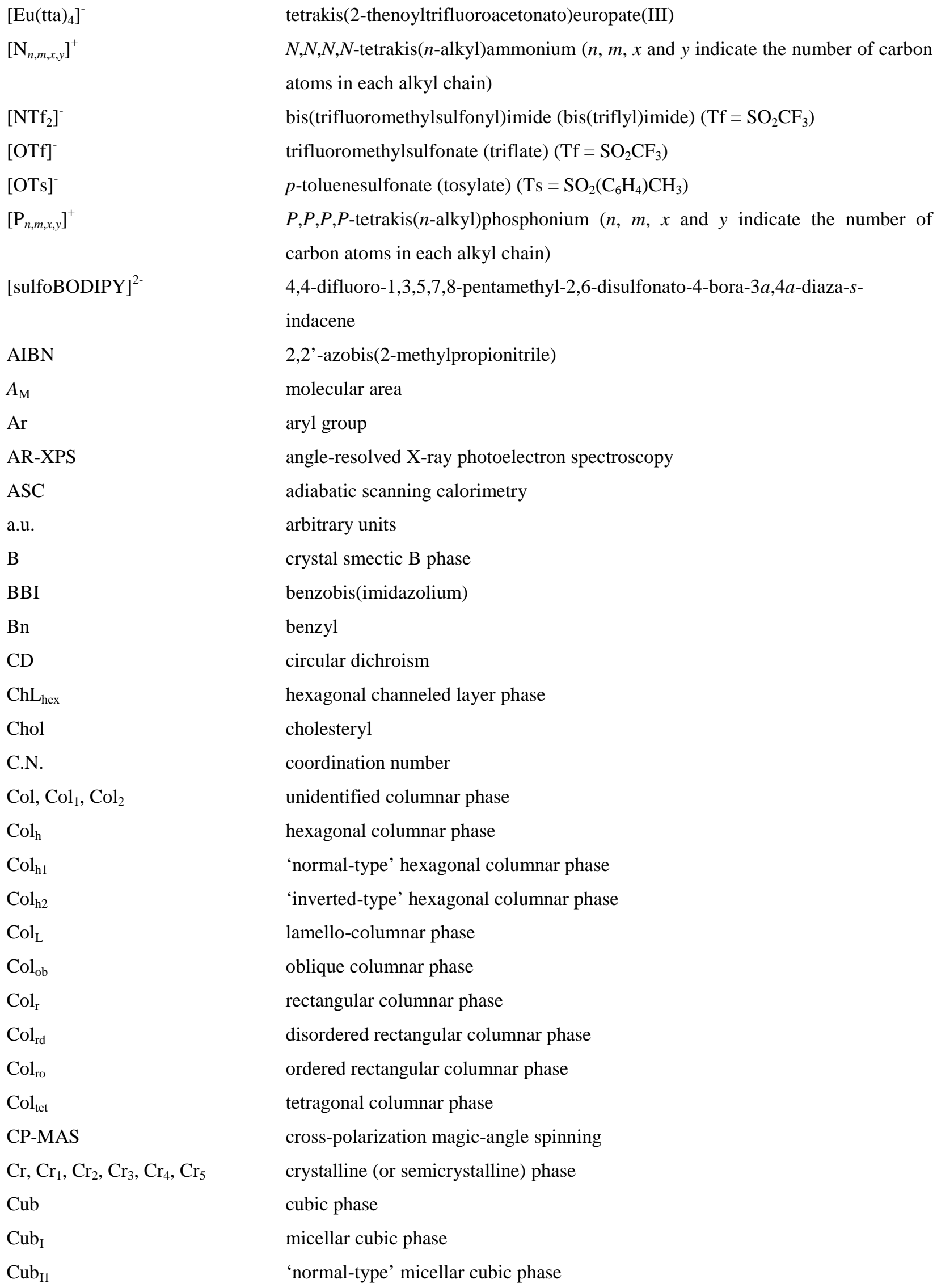




\begin{tabular}{|c|c|}
\hline $\mathrm{Cub}_{\mathrm{I} 2}$ & ‘inverted-type’ micellar cubic phase \\
\hline $\mathrm{Cub}_{\mathrm{V}}$ & bicontinuous cubic phase \\
\hline $\mathrm{Cub}_{\mathrm{V} 1}$ & 'normal-type' bicontinuous cubic phase \\
\hline $\mathrm{Cub}_{\mathrm{V} 2}$ & ‘inverted-type’ bicontinuous cubic phase \\
\hline$d$ & smectic layer thickness \\
\hline $\mathrm{DAB}$ & 1,4-diaminobutane \\
\hline DABCO & 1,4-diazabicyclo[2.2.2]octane \\
\hline DAE & 1,2-diaminoethane \\
\hline dcnm $^{-}$ & dicyanonitrosomethanide \\
\hline dec. & (thermal) decomposition \\
\hline DFT & density functional theory \\
\hline DMSO & dimethyl sulfoxide \\
\hline DOSY & diffusion ordered spectroscopy \\
\hline DRIFTS & diffuse reflectance infrared Fourier-transform spectroscopy \\
\hline DSC & differential scanning calorimetry \\
\hline DSSC & dye-sensitized solar cell \\
\hline $\mathrm{E}$ & crystal smectic E phase \\
\hline EPR & electron paramagnetic resonance \\
\hline FP-TRMC & flash-photolysis time-resolved microwave conductivity \\
\hline FT-IR & Fourier-transform infrared \\
\hline g & glass \\
\hline G & crystal smectic $G$ phase \\
\hline $\mathrm{G}^{*}$ & chiral crystal smectic G phase \\
\hline $\mathrm{g}_{\text {Iso }}$ & vitrified isotropic liquid phase \\
\hline $\mathrm{g}_{\mathrm{SmA}}$ & vitrified SmA phase \\
\hline $\mathrm{H}$ & crystal smectic H phase \\
\hline $\mathrm{H}^{*}$ & chiral crystal smectic $\mathrm{H}$ phase \\
\hline $\mathrm{H}_{2} \mathrm{dpa}$ & 2,6-pyridinedicarboxylic acid ( dpa $^{2-}=$ pyridine-2,6-dicarboxylate or dipicolinate) \\
\hline $\mathrm{H}_{\mathrm{II}}^{\mathrm{C}}$ & ‘inverted-type’ hexagonal columnar phase \\
\hline HOMO & highest occupied molecular orbital \\
\hline HOP & heterogeneity order parameter \\
\hline HOPG & highly oriented pyrolytic graphite \\
\hline Htta & 2-thenoyltrifluoroacetone (tta ${ }^{-}=$2-thenoyltrifluoroacetonate) \\
\hline I & isotropic liquid phase \\
\hline$i-\mathrm{Bu}$ & isobutyl \\
\hline IL & ionic liquid \\
\hline ILC & ionic liquid crystal \\
\hline$i-\operatorname{Pr}$ & isopropyl \\
\hline
\end{tabular}


IR

IRAS

ISA

ITO

J

J*

K

$\mathrm{K}^{*}$

$\mathrm{L}_{\alpha}{ }^{\mathrm{C}}$

LC

$\mathrm{L}_{\mathrm{Col}}$

LED

Ln

LUMO

$\mathrm{M}, \mathrm{M}_{1}, \mathrm{M}_{2}, \mathrm{M}_{3}$

MD

$\mathrm{MeOH}$

MMAB

n

N

N*

NBO

$\mathrm{N}_{\mathrm{C}}$

$\mathrm{N}_{\mathrm{C}}$ *

$\mathrm{N}_{\mathrm{D}}$

$\mathrm{N}_{\mathrm{D}}$ *

NHC

NIR

NMR

NPA

PAH

PAMAM

PC

PEDOT-PSS

PEI

PEIMe

$\mathrm{Ph}$

POM infrared

infrared reflection absorption spectroscopy

ionic self-assembly

indium tin oxide

crystal smectic J phase

chiral crystal smectic J phase

crystal smectic K phase

chiral crystal smectic K phase

lamello-columnar phase

liquid crystal / liquid-crystalline

lamello-columnar phase

light-emitting diode

lanthanide(III) ion

lowest unoccupied molecular orbital

unidentified mesophase

molecular dynamics

methanol

4-methoxy-4'-methylazobenzene

director of the liquid crystal

nematic phase

chiral nematic phase

natural bond orbital (analysis)

columnar nematic phase

chiral columnar nematic phase

discotic nematic phase

chiral discotic nematic phase

$\mathrm{N}$-heterocyclic carbene

near-infrared

nuclear magnetic resonance

natural population analysis

polycyclic aromatic hydrocarbon

poly(amidoamine)

propylene carbonate

poly(3,4-ethylenedioxythiophene)-poly(4-styrenesulfonate)

poly(ethylene imine)

fully methylated poly(ethylene imine)

phenyl group

polarizing optical microscopy 


\begin{tabular}{|c|c|}
\hline PPI & poly(propylene imine) \\
\hline PR-TRMC & pulse-radiolysis time-resolved microwave conductivity \\
\hline PTFE & poly(tetrafluoroethylene) \\
\hline PXRD & powder X-ray diffraction \\
\hline$r_{\mathrm{i}}$ & ionic radius \\
\hline r.t. & room temperature \\
\hline SAXS & small-angle X-ray scattering \\
\hline SEP & surfactant-encapsulated polyoxometalate (cluster/complex) \\
\hline SILP & supported ionic liquid phase \\
\hline SILCP & supported ionic liquid crystal phase \\
\hline SmA & smectic A phase \\
\hline SmA* & chiral smectic A phase \\
\hline $\operatorname{Sm} \tilde{A}$ & modulated (ribbon-like) SmA phase \\
\hline $\mathrm{SmB}$ & smectic B phase \\
\hline $\mathrm{SmC}$ & smectic C phase \\
\hline $\mathrm{SmC}^{*}$ & chiral smectic $C$ phase \\
\hline $\mathrm{SmCP}$ & polar SmC phase or $\mathrm{B}_{2}$ 'banana' phase \\
\hline $\mathrm{SmF}$ & smectic F phase \\
\hline $\mathrm{SmF}^{*}$ & chiral smectic F phase \\
\hline SmI & smectic I phase \\
\hline $\mathrm{SmI}^{*}$ & chiral smectic I phase \\
\hline $\mathrm{SmX}, \mathrm{SmX}_{1}, \mathrm{SmX}_{2}, \mathrm{SmX}_{3}$ & unidentified smectic phase \\
\hline SWCNT & single-walled carbon nanotube \\
\hline $\mathrm{T}, \mathrm{T}_{1}, \mathrm{~T}_{2}$ & crystal smectic $\mathrm{T}$ phase \\
\hline$T_{\mathrm{C}}$ & clearing temperature \\
\hline$T_{\text {dec. }}$ & decomposition temperature \\
\hline TEM & transmission electron microscopy \\
\hline Tet & $P 4_{2} / m n m$ tetragonal phase \\
\hline$T_{\mathrm{g}}$ & glass transition temperature \\
\hline TGA & thermogravimetric analysis \\
\hline THF & tetrahydrofuran \\
\hline$T_{\mathrm{m}}$ & melting temperature \\
\hline TOF & time-of-flight \\
\hline $\mathrm{TTF}$ & tetrathiafulvalene \\
\hline UV & ultraviolet \\
\hline Vis & visible region of the electromagnetic spectrum \\
\hline$V_{\text {mol }}$ & molecular volume \\
\hline WAXD & wide-angle X-ray diffraction \\
\hline
\end{tabular}


wt.\%

$\mathrm{X}, \mathrm{X}_{1}, \mathrm{X}_{2}, \mathrm{X}_{3}, \mathrm{X}_{4}, \mathrm{X}_{5}$

$\mathrm{X}_{\mathrm{L}}$

XPS

XRD weight percent

unidentified crystal smectic phase or soft crystal phase unidentified soft crystal phase with a simple lamellar structure X-ray photoelectron spectroscopy

$\mathrm{X}$-ray diffraction 


\section{Additional tables referred to in the main text}

Table S1. Important non-covalent intermolecular interactions. ${ }^{1-4}$

\begin{tabular}{|c|c|c|}
\hline Type of interaction & $\begin{array}{l}\text { Typical dissociation energy } \\
\qquad\left(\mathrm{kJ} \mathrm{mol}^{-1}\right)^{a}\end{array}$ & Interacting species (+ comments) \\
\hline Covalent & usually $>$ or $>>200-350$ & two atoms (short range, irreversible) \\
\hline $\begin{array}{l}\text { ion-ion } \\
\text { (Coulombic force) }\end{array}$ & $200-300^{b}$ & $\begin{array}{l}\text { ions only (long range, non-directional, non- } \\
\text { selective; distance dependence of potential } \\
\text { energy: } 1 / r \text { ) }\end{array}$ \\
\hline ion-dipole ${ }^{c}$ & $15-150$ & $\begin{array}{l}\text { ions and polar molecules (short range; } \\
\text { distance dependence of potential energy: } 1 / r^{2} \text { ) }\end{array}$ \\
\hline ion - induced dipole & 5 & ions and non-polar molecules (short range) \\
\hline dipole-dipole & $2-40$ & stationary polar molecules (short range) \\
\hline 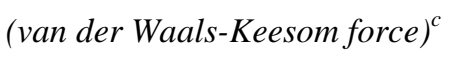 & $0.3-0.6$ & rotating polar molecules (short range) \\
\hline $\begin{array}{l}\text { dipole-induced dipole } \\
\text { (van der Waals-Debye force) }^{c}\end{array}$ & 2 & $\begin{array}{l}\text { at least one molecule must be polar (short } \\
\text { range) }\end{array}$ \\
\hline $\begin{array}{l}\text { induced dipole - induced dipole } \\
\text { (van der Waals-London } \\
\text { dispersion force) }\end{array}$ & $1-2$ & all types of molecules (short range) \\
\hline hydrogen bonding & $2-150$ & $\begin{array}{l}\text { in the strictest sense: } \mathrm{N}, \mathrm{O} \text { and } \mathrm{F} \text {, with the link } \\
\text { being a shared } \mathrm{H} \text { atom (short range, } \\
\text { directional, selective) }^{d}\end{array}$ \\
\hline halogen bonding & $5-180$ & $\begin{array}{l}\text { halogen atoms and Lewis bases (short range, } \\
\text { directional, selective) }\end{array}$ \\
\hline$\pi-\pi$ (stacking) & & $\pi$-electron clouds (short range) \\
\hline $\mathrm{C}-\mathrm{H} \cdots \pi$ & & $\begin{array}{l}\mathrm{C}-\mathrm{H} \text { groups and } \pi \text {-electron clouds (short } \\
\text { range) }\end{array}$ \\
\hline cation- $\pi$ & & cations and $\pi$-electron clouds (short range) $^{e}$ \\
\hline arene-perfluoroarene & & $\begin{array}{l}\text { face-to-face interaction between } \\
\text { perfluorinated and non-fluorinated aromatic } \\
\text { rings }\end{array}$ \\
\hline
\end{tabular}


Table S1. Continued.

\begin{tabular}{|c|c|c|}
\hline $\begin{array}{l}\text { metal coordination } \\
\text { (complexation) }\end{array}$ & & $\begin{array}{l}\text { metals/metal ions and suitable ligands (short } \\
\text { range, directional, selective) }\end{array}$ \\
\hline charge transfer & & $\begin{array}{l}\text { electron donors and electron acceptors (short } \\
\text { range) }\end{array}$ \\
\hline hydrophobic & (entropy-driven) & (short range) \\
\hline
\end{tabular}

${ }^{a}$ These are only indicative values, which can strongly vary according to the system under consideration.

${ }^{b}$ Interaction energies in solution strongly dependent on the type of solvent, etc.

${ }^{c}$ Multipoles can also be involved in this kind of interaction.

${ }^{d}$ A special class of hydrogen bonds are 'ionic' or 'charge-assisted' hydrogen bonds between ions and molecules, with bond strengths of $20-160 \mathrm{~kJ} \mathrm{~mol}^{-1} .^{5-7}$ 'Non-classical' hydrogen bonds such as the C-H $\cdots \mathrm{X}^{-}$and C-H $\cdots \mathrm{X}$ interactions found in many ionic liquids can be considered to belong to this class. ${ }^{8-39}$

${ }^{e}$ Anion- $\pi$ interactions between anions and electron-deficient aromatic ring systems can also occur. 
Table S2. Thermal phase behavior of $\left[\mathrm{C}_{n} \operatorname{mim}\right][\mathrm{X}],\left[\mathrm{C}_{n} \mathrm{C}_{m} \mathrm{im}\right][\mathrm{X}]$ and $\left[\mathrm{C}_{n} \mathrm{Him}\right][\mathrm{X}]$ salts that are not included in Figure 16 and Figure 17.

\begin{tabular}{|c|c|c|c|}
\hline $\begin{array}{l}{\left[\mathrm{C}_{12} \mathrm{mim}\right][\mathrm{Br}]} \\
\text { (anhydrous) }\end{array}$ & $\begin{array}{c}\mathrm{Cr} \cdot 42 \cdot \mathrm{SmA} \cdot 100 \cdot \\
\mathrm{I}\left({ }^{\circ} \mathrm{C}\right)^{40, a}\end{array}$ & $\begin{array}{c}{\left[\mathrm{C}_{7} \mathrm{C}_{6} \mathrm{im}\right][\mathrm{Br}]} \\
\text { (anhydrous) }\end{array}$ & $\mathrm{g} \cdot-57 \cdot \mathrm{I}\left({ }^{\circ} \mathrm{C}\right)^{41}$ \\
\hline$\left[\mathrm{C}_{12} \mathrm{mim}\right][\mathrm{Br}] \cdot \mathrm{H}_{2} \mathrm{O}$ & $\mathrm{Cr} \cdot 44 \cdot \mathrm{SmA} \cdot 129 \cdot \mathrm{I}\left({ }^{\circ} \mathrm{C}\right)^{40}$ & $\begin{array}{c}{\left[\mathrm{C}_{8} \mathrm{C}_{5} \mathrm{im}\right][\mathrm{Br}]} \\
\text { (anhydrous) }\end{array}$ & $\mathrm{g} \cdot$ ca. $-60 \cdot \mathrm{I}\left({ }^{\circ} \mathrm{C}\right)^{41}$ \\
\hline $\begin{array}{l}{\left[\mathrm{C}_{14} \mathrm{mim}\right][\mathrm{Br}]} \\
\text { (anhydrous) }\end{array}$ & $\mathrm{Cr} \cdot 53 \cdot \mathrm{SmA} \cdot 173 \cdot \mathrm{I}\left({ }^{\circ} \mathrm{C}\right)^{40}$ & $\begin{array}{c}{\left[\mathrm{C}_{9} \mathrm{C}_{4} \mathrm{im}\right][\mathrm{Br}]} \\
\text { (anhydrous) }\end{array}$ & $\mathrm{g} \cdot$ ca. $-60 \cdot \mathrm{I}\left({ }^{\circ} \mathrm{C}\right)^{41}$ \\
\hline$\left[\mathrm{C}_{14} \mathrm{mim}\right][\mathrm{Br}] \cdot \mathrm{H}_{2} \mathrm{O}$ & $\mathrm{Cr} \cdot 53 \cdot \mathrm{SmA} \cdot 188 \cdot \mathrm{I}\left({ }^{\circ} \mathrm{C}\right)^{40}$ & $\begin{array}{c}{\left[\mathrm{C}_{10} \mathrm{C}_{3} \mathrm{im}\right][\mathrm{Br}]} \\
\text { (anhydrous) }\end{array}$ & $\mathrm{g} \cdot$ ca. $-60 \cdot \mathrm{I}\left({ }^{\circ} \mathrm{C}\right)^{41}$ \\
\hline$\left[\mathrm{C}_{11} \mathrm{mim}\right][\mathrm{I}]$ & $\mathrm{Cr} \cdot 50 \cdot \mathrm{I}\left({ }^{\circ} \mathrm{C}\right)^{42,43}$ & $\begin{array}{c}{\left[\mathrm{C}_{11} \mathrm{C}_{2} \mathrm{im}\right][\mathrm{Br}]} \\
\text { (anhydrous) }\end{array}$ & $\mathrm{Cr} \cdot 38 \cdot \mathrm{I}\left({ }^{\circ} \mathrm{C}\right)^{41}$ \\
\hline$\left[\mathrm{C}_{12} \operatorname{mim}\right][\mathrm{I}]$ & $\begin{array}{c}\mathrm{Cr} \cdot 27 \cdot \mathrm{SmA} \cdot 80 \cdot \\
\left.\mathrm{I}\left({ }^{\circ} \mathrm{C}\right)^{42,43} \text { (see also ref. } 44\right)\end{array}$ & $\begin{array}{c}{\left[\mathrm{C}_{12} \mathrm{C}_{2} \mathrm{im}\right][\mathrm{Br}]} \\
\text { (anhydrous) }\end{array}$ & $\begin{array}{c}\mathrm{Cr}_{1} \cdot-1 \cdot \mathrm{Cr}_{2} \cdot 1 \cdot \mathrm{Cr}_{3} \cdot 35 \cdot \\
\mathrm{I}\left({ }^{\circ} \mathrm{C}\right)^{45}\end{array}$ \\
\hline$\left[\mathrm{C}_{16} \mathrm{mim}\right][\mathrm{I}]$ & see ref. 44 & $\begin{array}{c}{\left[\mathrm{C}_{12} \mathrm{C}_{3} \mathrm{im}\right][\mathrm{Br}]} \\
\text { (anhydrous) }\end{array}$ & $\begin{array}{l}\mathrm{Cr} \cdot-24 \cdot \operatorname{SmA}(?) \cdot-4 \cdot \\
\operatorname{SmX}(?) \cdot 21 \cdot \mathrm{I}\left({ }^{\circ} \mathrm{C}\right)^{45}\end{array}$ \\
\hline$\left[\mathrm{C}_{18} \operatorname{mim}\right][\mathrm{I}]$ & see ref. 44 & $\begin{array}{l}{\left[\mathrm{C}_{12} \mathrm{C}_{4} \mathrm{im}\right][\mathrm{Br}]} \\
\text { (anhydrous) }\end{array}$ & $\begin{array}{c}\mathrm{Cr}_{1} \cdot-40 \cdot \mathrm{Cr}_{2} \cdot-29 \cdot \operatorname{SmA}(?) \cdot \\
-19 \cdot \operatorname{SmX}(?) \cdot 4 \cdot \mathrm{I}\left({ }^{\circ} \mathrm{C}\right)^{45}\end{array}$ \\
\hline$\left[\mathrm{C}_{12} \mathrm{mim}\right]\left[\mathrm{NTf}_{2}\right]$ & $\mathrm{Cr} \cdot 17 \cdot \mathrm{I}\left({ }^{\circ} \mathrm{C}\right)^{46,47}$ & $\begin{array}{c}{\left[\mathrm{C}_{12} \mathrm{C}_{5} \mathrm{im}\right][\mathrm{Br}]} \\
\text { (anhydrous) }\end{array}$ & $\begin{array}{c}\mathrm{Cr} \cdot-39 \cdot \operatorname{SmA}(?) \cdot 8 \cdot \operatorname{SmX} \\
(?) \cdot 18 \cdot \mathrm{I}\left({ }^{\circ} \mathrm{C}\right)^{45}\end{array}$ \\
\hline$\left[\mathrm{C}_{14} \mathrm{mim}\right]\left[\mathrm{NTf}_{2}\right]$ & $\mathrm{Cr} \cdot 34 \cdot \mathrm{I}\left({ }^{\circ} \mathrm{C}\right)^{46}$ & $\begin{array}{c}{\left[\mathrm{C}_{12} \mathrm{C}_{6} \mathrm{im}\right][\mathrm{Br}]} \\
\text { (anhydrous) }\end{array}$ & $\mathrm{g} \cdot-57 \cdot \mathrm{I}\left({ }^{\circ} \mathrm{C}\right)^{45}$ \\
\hline$\left[\mathrm{C}_{16} \mathrm{mim}\right]\left[\mathrm{NTf}_{2}\right]$ & $\mathrm{Cr} \cdot 42 \cdot \mathrm{I}\left({ }^{\circ} \mathrm{C}\right)^{46}$ & $\begin{array}{c}{\left[\mathrm{C}_{12} \mathrm{C}_{7} \mathrm{im}\right][\mathrm{Br}]} \\
\text { (anhydrous) }\end{array}$ & $\mathrm{Cr} \cdot(\mathrm{SmC}(?) \cdot 8 \cdot) 9 \cdot \mathrm{I}\left({ }^{\circ} \mathrm{C}\right)^{45}$ \\
\hline$\left[\mathrm{C}_{18} \mathrm{mim}\right]\left[\mathrm{NTf}_{2}\right]$ & $\begin{array}{c}\mathrm{Cr} \cdot 45 \cdot \mathrm{I}\left({ }^{\circ} \mathrm{C}\right)^{46,47} \\
\mathrm{Cr} \cdot 52 \cdot \mathrm{I}\left({ }^{\circ} \mathrm{C}\right)^{48}\end{array}$ & $\begin{array}{c}{\left[\mathrm{C}_{12} \mathrm{C}_{8} \mathrm{im}\right][\mathrm{Br}]} \\
\text { (anhydrous) }\end{array}$ & $\begin{array}{c}\mathrm{Cr} \cdot 28 \cdot \mathrm{SmC}(?)^{b} \cdot 34 \cdot \\
\mathrm{I}\left({ }^{\circ} \mathrm{C}\right)^{45}\end{array}$ \\
\hline$\left[\mathrm{C}_{22} \mathrm{mim}\right]\left[\mathrm{NTf}_{2}\right]$ & $\begin{array}{c}\mathrm{Cr} \cdot(\mathrm{SmA} \cdot 65 \cdot) 66 \cdot \\
\mathrm{I}\left({ }^{\circ} \mathrm{C}\right)^{48}\end{array}$ & $\begin{array}{c}{\left[\mathrm{C}_{12} \mathrm{C}_{9} \mathrm{im}\right][\mathrm{Br}]} \\
\text { (anhydrous) }\end{array}$ & $\mathrm{Cr} \cdot 25 \cdot \mathrm{SmC}(?) \cdot 56 \cdot \mathrm{I}\left({ }^{\circ} \mathrm{C}\right)^{45}$ \\
\hline$\left[\mathrm{C}_{18} \mathrm{mim}\right]\left[\mathrm{N}\left(\mathrm{SO}_{2} \mathrm{~F}\right)_{2}\right]$ & $\begin{array}{c}\mathrm{Cr}_{1} \cdot 40 \cdot \mathrm{Cr}_{2} \cdot 66 \cdot \mathrm{SmA} \cdot 70 \\
\cdot \mathrm{I}\left({ }^{\circ} \mathrm{C}\right)^{48}\end{array}$ & $\begin{array}{c}{\left[\mathrm{C}_{12} \mathrm{C}_{10} \mathrm{im}\right][\mathrm{Br}]} \\
\text { (anhydrous) }\end{array}$ & $\mathrm{Cr} \cdot 39 \cdot \mathrm{SmC}(?) \cdot 72 \cdot \mathrm{I}\left({ }^{\circ} \mathrm{C}\right)^{45}$ \\
\hline$\left[\mathrm{C}_{22} \mathrm{mim}\right]\left[\mathrm{N}\left(\mathrm{SO}_{2} \mathrm{~F}\right)_{2}\right]$ & $\begin{array}{c}\mathrm{Cr}_{1} \cdot 67 \cdot \mathrm{Cr}_{2} \cdot 78 \cdot \mathrm{SmA} \cdot 129 \\
\cdot \mathrm{I}\left({ }^{\circ} \mathrm{C}\right)^{48}\end{array}$ & $\begin{array}{c}{\left[\mathrm{C}_{12} \mathrm{C}_{11} \mathrm{im}\right][\mathrm{Br}]} \\
\text { (anhydrous) }\end{array}$ & $\begin{array}{c}\mathrm{Cr}_{1} \cdot-22 \cdot \mathrm{Cr}_{2} \cdot 41 \cdot \mathrm{SmC}(?) \cdot \\
85 \cdot \mathrm{I}\left({ }^{\circ} \mathrm{C}\right)^{45}\end{array}$ \\
\hline
\end{tabular}


Table S2. Continued.

\begin{tabular}{|c|c|c|c|}
\hline$\left[\mathrm{C}_{16} \mathrm{mim}\right]\left[\mathrm{CH}_{3} \mathrm{SO}_{3}\right]$ & $\mathrm{Cr} \cdot 75 \cdot \mathrm{SmA} \cdot 153 \cdot \mathrm{I}\left({ }^{\circ} \mathrm{C}\right){ }^{49}$ & $\begin{array}{c}{\left[\mathrm{C}_{12} \mathrm{C}_{12} \mathrm{im}\right][\mathrm{Br}]} \\
\text { (anhydrous) }\end{array}$ & $\begin{array}{c}\mathrm{Cr}_{1} \cdot-9 \cdot \mathrm{Cr}_{2} \cdot 31 \cdot \mathrm{Cr}_{3} \cdot 60 \cdot \\
\mathrm{SmA} \cdot 103 \cdot \mathrm{I}\left({ }^{\circ} \mathrm{C}\right){ }^{45}\end{array}$ \\
\hline$\left[\mathrm{C}_{8} \mathrm{mim}\right][\mathrm{OTs}]$ & $\begin{array}{c}\mathrm{Cr} \cdot 80 \cdot \mathrm{SmX} \cdot 90 \cdot \mathrm{I}\left({ }^{\circ} \mathrm{C}\right) \\
\text { (non-sheared sample) }{ }^{50}\end{array}$ & $\begin{array}{c}{\left[\mathrm{C}_{12} \mathrm{C}_{13} \mathrm{im}\right][\mathrm{Br}]} \\
\text { (anhydrous) }\end{array}$ & $\begin{array}{c}\mathrm{Cr}_{1} \cdot-13 \cdot \mathrm{Cr}_{2} \cdot 33 \cdot \mathrm{Cr}_{3} \cdot 59 \cdot \\
\mathrm{SmA} \cdot 111 \cdot \mathrm{I}\left({ }^{\circ} \mathrm{C}\right)^{45}\end{array}$ \\
\hline$\left[\mathrm{C}_{10} \mathrm{mim}\right][\mathrm{OTs}]$ & $\begin{array}{c}\mathrm{Cr} \cdot 49 \cdot \mathrm{SmA} \cdot 79 \cdot \mathrm{I}\left({ }^{\circ} \mathrm{C}\right) \\
\text { (non-sheared sample) }{ }^{50}\end{array}$ & {$\left[\mathrm{C}_{16} \mathrm{C}_{2} \mathrm{im}\right][\mathrm{Br}]$} & $\mathrm{Cr} \cdot 56 \cdot \mathrm{SmA} \cdot 117 \cdot \mathrm{I}\left({ }^{\circ} \mathrm{C}\right)^{51}$ \\
\hline$\left[\mathrm{C}_{12} \mathrm{mim}\right][$ salicylate $]$ & $\begin{array}{c}\mathrm{Cr} \cdot(\mathrm{SmC}(?) \cdot 48 \cdot) 48 \cdot \mathrm{I} \\
\left({ }^{\circ} \mathrm{C}\right)^{52, c}\end{array}$ & {$\left[\mathrm{C}_{12} \mathrm{C}_{2} \mathrm{im}\right][\mathrm{I}]$} & see ref. 44 \\
\hline $\begin{array}{c}{\left[\mathrm{C}_{10} \mathrm{C}_{10} \mathrm{im}\right][\mathrm{Br}]} \\
\text { (anhydrous) }\end{array}$ & not LC ${ }^{53}$ & {$\left[\mathrm{C}_{16} \mathrm{C}_{2} \mathrm{im}\right][\mathrm{I}]$} & $\begin{array}{c}\mathrm{Cr} \cdot 60 \cdot \mathrm{SmA} \cdot 100 \cdot \mathrm{I}\left({ }^{\circ} \mathrm{C}\right){ }^{51} \text { (see } \\
\text { also ref. } 44)\end{array}$ \\
\hline$\left[\mathrm{C}_{12} \mathrm{C}_{12} \mathrm{im}\right][\mathrm{OTf}]$ & $\mathrm{Cr} \cdot 48 \cdot \mathrm{I}\left({ }^{\circ} \mathrm{C}\right)^{54}$ & {$\left[\mathrm{C}_{18} \mathrm{C}_{2} \mathrm{im}\right][\mathrm{I}]$} & see ref. 44 \\
\hline$\left[\mathrm{C}_{12} \mathrm{C}_{12} \mathrm{im}\right]\left[\mathrm{NTf}_{2}\right]$ & $\mathrm{Cr} \cdot 42 \cdot \mathrm{I}\left({ }^{\circ} \mathrm{C}\right)^{54, d}$ & $\begin{array}{l}{\left[\mathrm{C}_{12} \mathrm{Him}\right][\mathrm{Br}]} \\
\text { (anhydrous) }\end{array}$ & $\begin{array}{c}\mathrm{Cr}_{1} \cdot-1 \cdot \mathrm{Cr}_{2} \cdot 6 \cdot \mathrm{Cr}_{3} \cdot 42 \cdot \\
\mathrm{SmA} \cdot 86 \cdot \mathrm{I}\left({ }^{\circ} \mathrm{C}\right){ }^{45}\end{array}$ \\
\hline$\left[\mathrm{C}_{12} \mathrm{C}_{12} \mathrm{im}\right]\left[\mathrm{I}_{3}\right]$ & $\mathrm{Cr} \cdot 46 \cdot \mathrm{I}\left({ }^{\circ} \mathrm{C}\right)^{55}$ & $\begin{array}{l}{\left[\mathrm{C}_{13} \mathrm{Him}\right][\mathrm{Br}]} \\
\text { (anhydrous) }\end{array}$ & $\mathrm{Cr} \cdot 71 \cdot \mathrm{SmA} \cdot 142 \cdot \mathrm{I}\left({ }^{\circ} \mathrm{C}\right){ }^{41}$ \\
\hline$\left[\mathrm{C}_{12} \mathrm{C}_{12} \mathrm{im}\right]\left[\mathrm{I}_{5}\right]$ & $\mathrm{Cr} \cdot 36 \cdot \mathrm{I}\left({ }^{\circ} \mathrm{C}\right)^{55}$ & {$\left[\mathrm{C}_{12} \mathrm{C}_{2} \mathrm{im}\right]\left[\mathrm{BF}_{4}\right]$} & see ref. 44 \\
\hline$\left[\mathrm{C}_{12} \mathrm{C}_{12} \mathrm{im}\right]\left[\mathrm{B}(\mathrm{CN})_{4}\right]$ & $\mathrm{Cr} \cdot 48 \cdot \mathrm{I}\left({ }^{\circ} \mathrm{C}\right)^{55}$ & {$\left[\mathrm{C}_{14} \mathrm{C}_{2} \mathrm{im}\right]\left[\mathrm{BF}_{4}\right]$} & see ref. 44 \\
\hline$\left[\mathrm{C}_{12} \mathrm{C}_{12} \mathrm{im}\right]\left[\mathrm{C}(\mathrm{CN})_{3}\right]$ & $\mathrm{Cr} \cdot 44 \cdot \mathrm{I}\left({ }^{\circ} \mathrm{C}\right)^{55}$ & {$\left[\mathrm{C}_{16} \mathrm{C}_{2} \mathrm{im}\right]\left[\mathrm{BF}_{4}\right]$} & see ref. 44 \\
\hline$\left[\mathrm{C}_{12} \mathrm{C}_{12} \mathrm{im}\right]\left[\mathrm{N}(\mathrm{CN})_{2}\right]$ & $\mathrm{Cr} \cdot 44 \cdot \mathrm{I}\left({ }^{\circ} \mathrm{C}\right)^{55}$ & {$\left[\mathrm{C}_{18} \mathrm{C}_{2} \mathrm{im}\right]\left[\mathrm{BF}_{4}\right]$} & see ref. 44 \\
\hline$\left[\mathrm{C}_{12} \mathrm{C}_{12} \mathrm{im}\right]\left[\mathrm{SbF}_{6}\right]$ & $\mathrm{Cr} \cdot 41 \cdot \mathrm{I}\left({ }^{\circ} \mathrm{C}\right)^{55}$ & & \\
\hline
\end{tabular}

${ }^{a}$ Or: $1^{\text {st }}$ DSC heating run: $\mathrm{Cr} \cdot 43 \cdot \mathrm{SmA} \cdot 101 \cdot \mathrm{I}\left({ }^{\circ} \mathrm{C}\right), 2^{\text {nd }} \mathrm{DSC}$ heating run: $\mathrm{Cr}_{1} \cdot-1 \cdot \mathrm{Cr}_{2} \cdot 1 \cdot \mathrm{Cr}_{3} \cdot 6 \cdot \mathrm{Cr}_{4} \cdot 39 \cdot$ $\mathrm{SmA} \cdot 100 \cdot \mathrm{I}\left({ }^{\circ} \mathrm{C}\right){ }^{41,45}$

${ }^{b}$ This phase does not appear during the $1^{\text {st }}$ heating run and is probably monotropic.

${ }^{c}$ [salicylate $]^{-}=$2-hydroxybenzoate.

${ }^{d}$ Or: $1^{\text {st }}$ DSC heating run: $\mathrm{Cr} \cdot 34 \cdot \mathrm{I}\left({ }^{\circ} \mathrm{C}\right), 2^{\text {nd }}$ DSC heating run: $\mathrm{Cr} \cdot 17 \cdot \mathrm{M} \cdot 28$ [broad transition] $\cdot \mathrm{I}\left({ }^{\circ} \mathrm{C}\right){ }^{47}$ 
Table S3. Number of primary amino groups $\left(-\mathrm{NH}_{2}\right)$ and tertiary amino groups $\left(\mathrm{NR}_{3}\right)$ in poly(propylene imine) (PPI) and poly(amidoamine) (PAMAM) dendrimers of different generations.

\begin{tabular}{ccc|ccc}
\hline & $\begin{array}{c}\text { \# of }-\mathbf{N H}_{\mathbf{2}} \\
\text { groups }\end{array}$ & $\begin{array}{c}\text { \# of } \mathbf{N R}_{\mathbf{3}} \\
\text { groups }\end{array}$ & & $\begin{array}{c}\text { \# of }-\mathrm{NH}_{\mathbf{2}} \\
\text { groups }\end{array}$ & $\begin{array}{c}\text { \# of } \mathbf{N R}_{\mathbf{3}} \\
\text { groups }\end{array}$ \\
\hline PPI-G1 & 4 & 2 & PAMAM-G0 & 4 & 2 \\
PPI-G2 & 8 & 6 & PAMAM-G1 & 8 & 6 \\
PPI-G3 & 16 & 14 & PAMAM-G2 & 16 & 14 \\
PPI-G4 & 32 & 30 & PAMAM-G3 & 32 & 30 \\
PPI-G5 & 64 & 62 & PAMAM-G4 & 64 & 62 \\
& & & PAMAM-G5 & 128 & 126 \\
\hline
\end{tabular}




\section{Additional figures referred to in the main text}

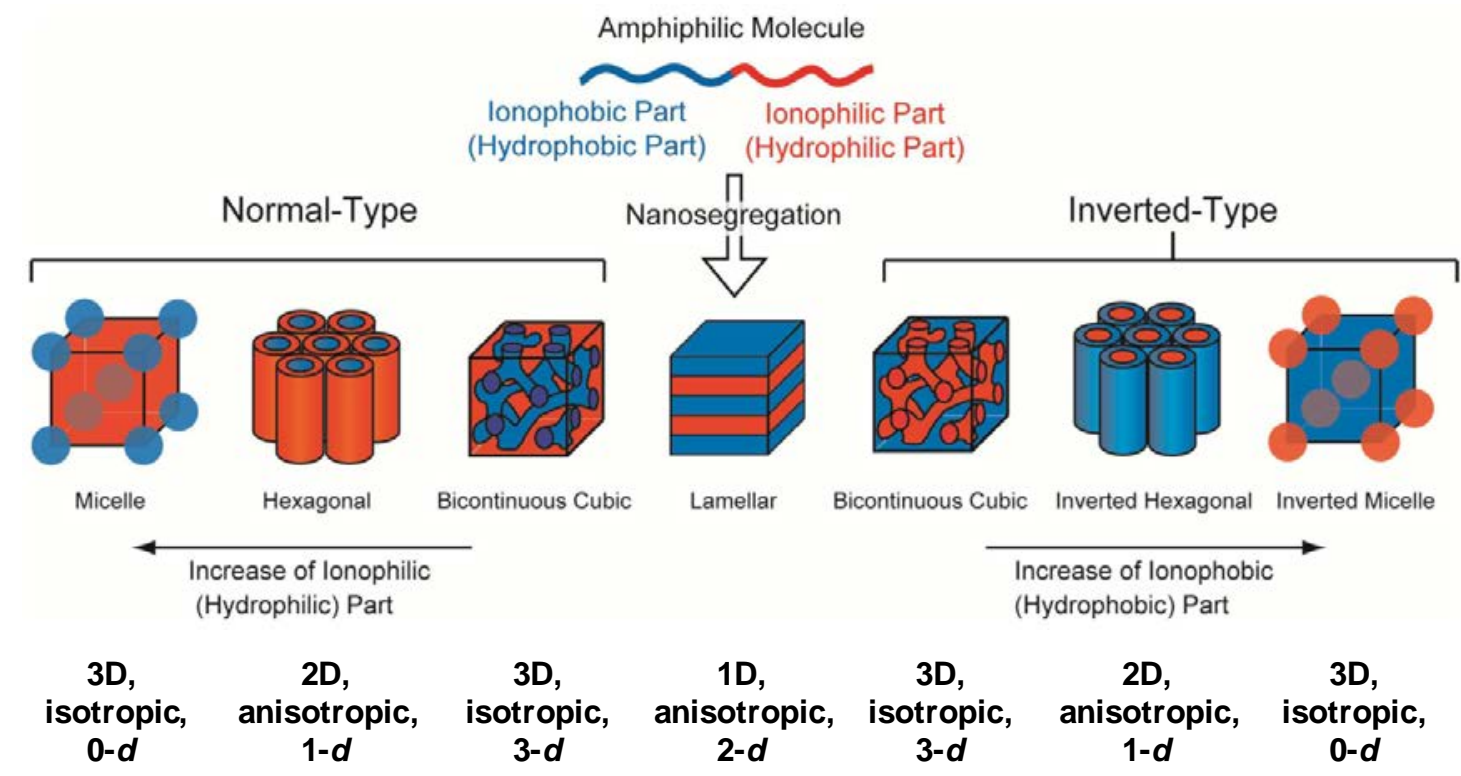

Figure S1. Phase-segregated mesophase morphologies of thermotropic LC 'block molecules' that consist, for example, of an ionophobic/hydrophobic and an ionophilic/hydrophilic part (amphiphilic molecules). The lamellar morphology corresponds to smectic mesophases; the hexagonal ordering of columns corresponds to (hexagonal) columnar mesophases; furthermore, there exist two kinds of cubic mesophases: bicontinuous cubic phases (Cub ${ }_{\mathrm{V}}$ ) and micellar cubic phases ( $\left.\mathrm{Cub}_{\mathrm{I}}\right)$. 'Normal-type' bicontinuous cubic, hexagonal columnar and micellar cubic phases are abbreviated as $\mathrm{Cub}_{\mathrm{V} 1}, \mathrm{Col}_{\mathrm{h} 1}$ and $\mathrm{Cub}_{\mathrm{I} 1}$, respectively; the 'inverted-type' variants are abbreviated as $\mathrm{Cub}_{\mathrm{V} 2}, \mathrm{Col}_{\mathrm{h} 2}$ and $\mathrm{Cub}_{\mathrm{I} 2}$, respectively. 1D, 2D and 3D indicate the dimensionality of the crystallographic lattice; $0-d, 1-d, 2-d$ and

$3-d$ indicate the dimensionality of the connectivity of the aggregates, which is for example expressed by the directionality of conducting properties. ${ }^{56,57}$ Adapted with permission from ref. 58. Copyright 2012 American Chemical Society. 


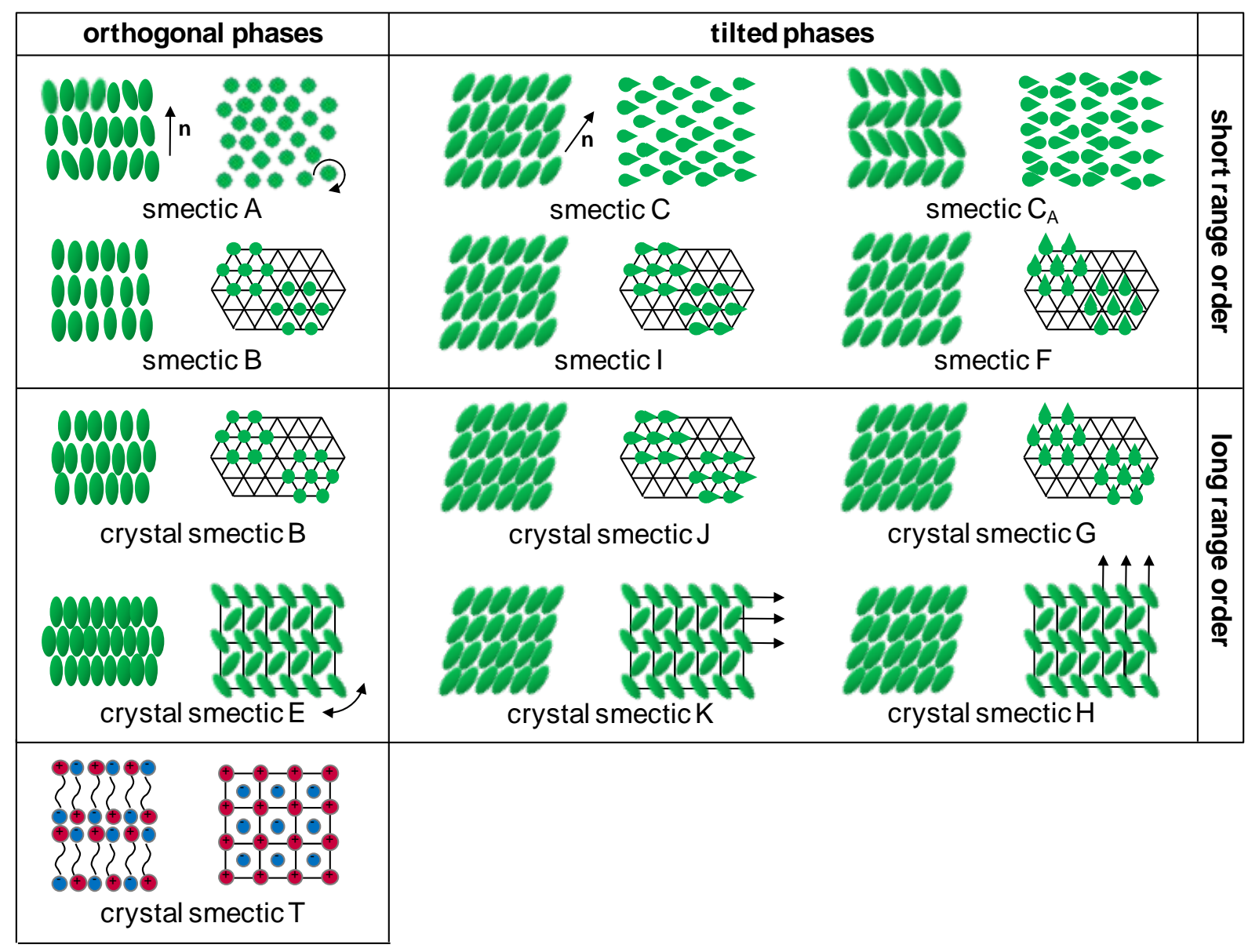

Figure S2. Classification of smectic and crystal smectic phases (side views and top views). The rod-like molecules are depicted as green ellipsoids of revolution. 

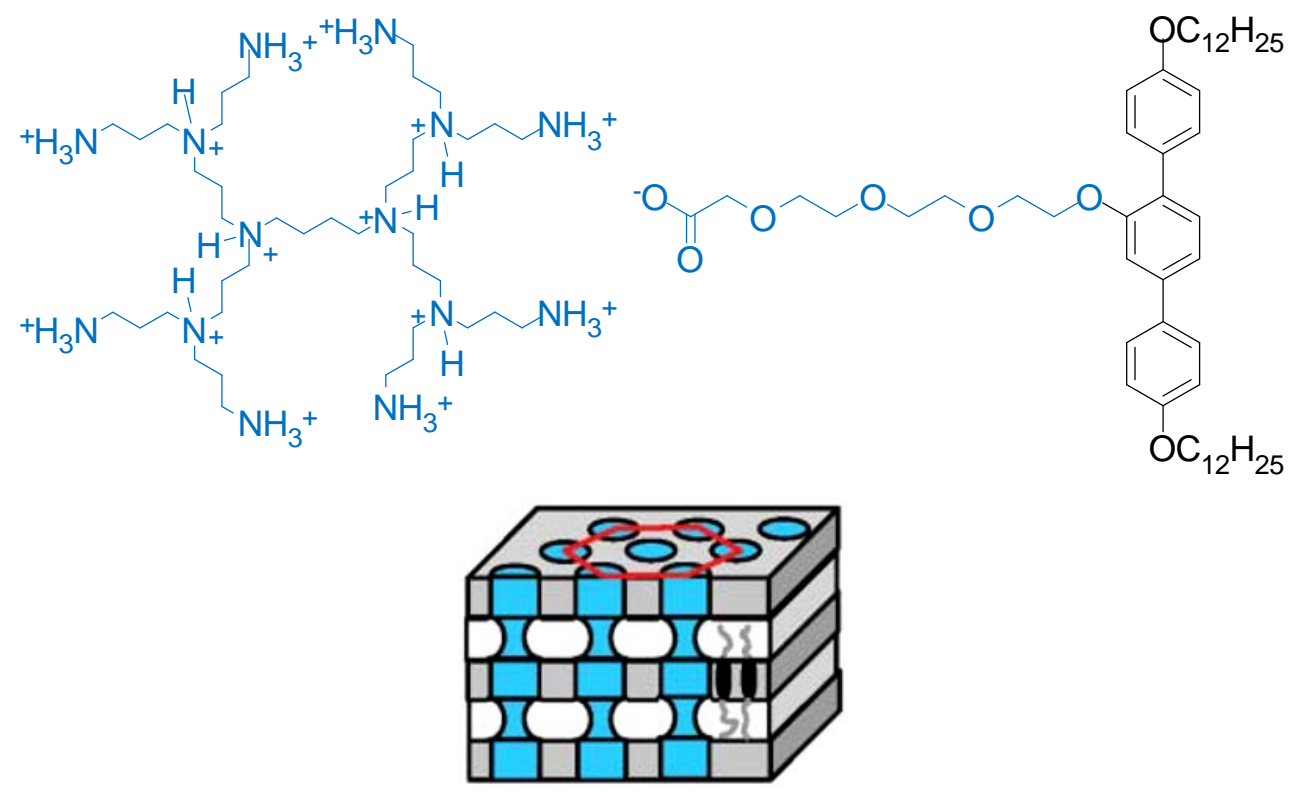

Figure S3. Proposed structure of the $\mathrm{ChL}_{\text {hex }}$ phase formed by an ionic dendritic complex of a T-shaped facial amphiphilic carboxylate (top right) with a fully protonated second-generation DAB PPI dendrimer (top left) (14:1 molar ratio). ${ }^{59,60}$ Color code for the model: blue = polar regions; white = alkyl chains; black = terphenyl cores. Adapted from ref. 59 (http://dx.doi.org/10.1039/b415892j) with permission of The Royal Society of Chemistry. 
(a)
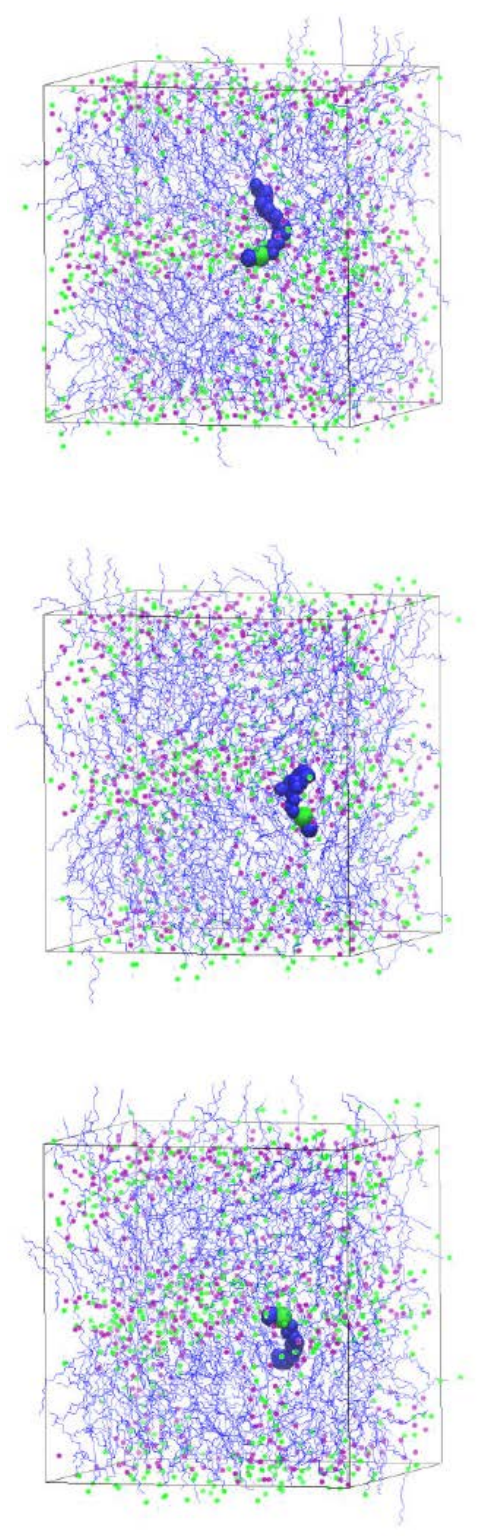
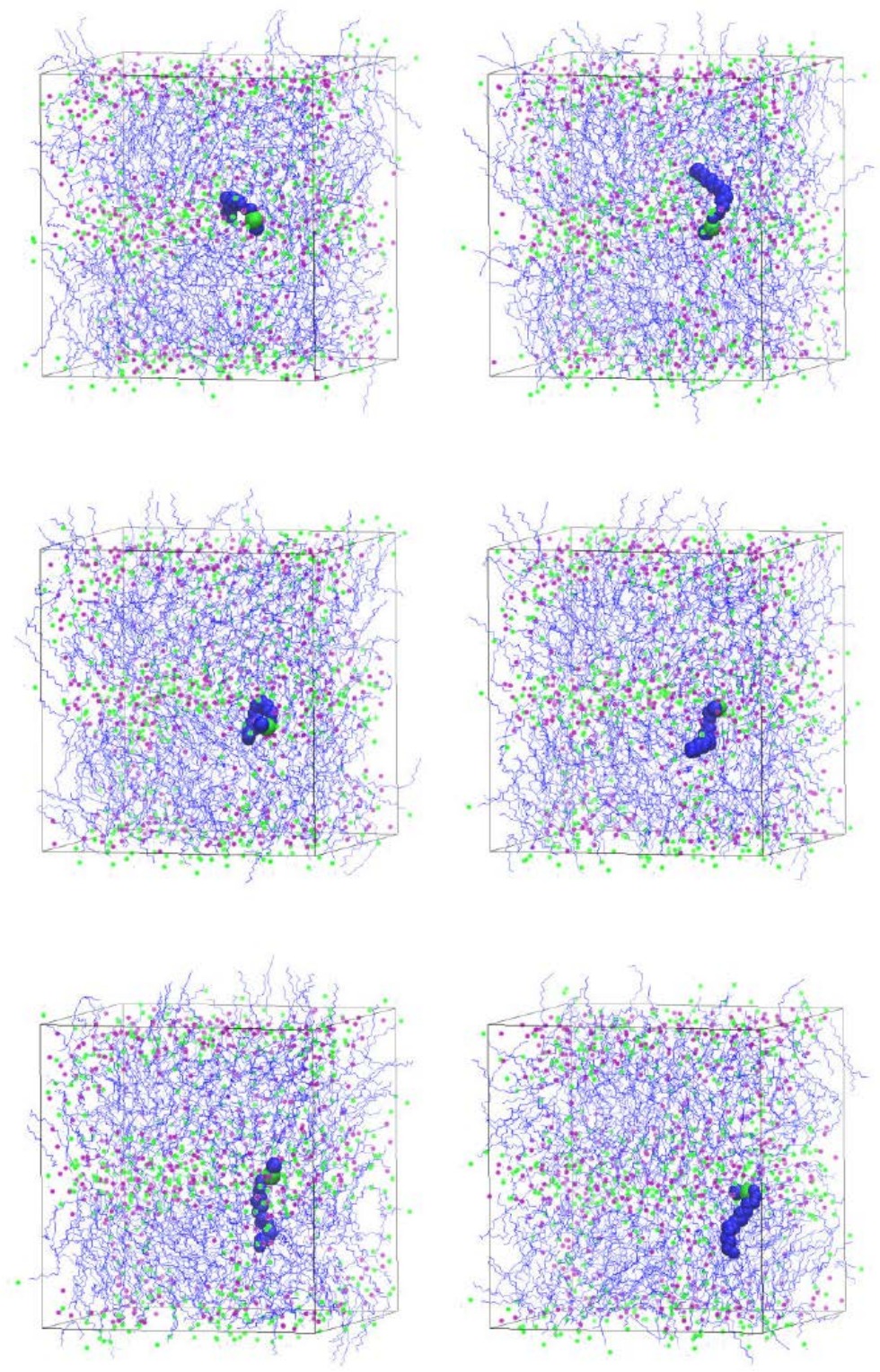
(b)
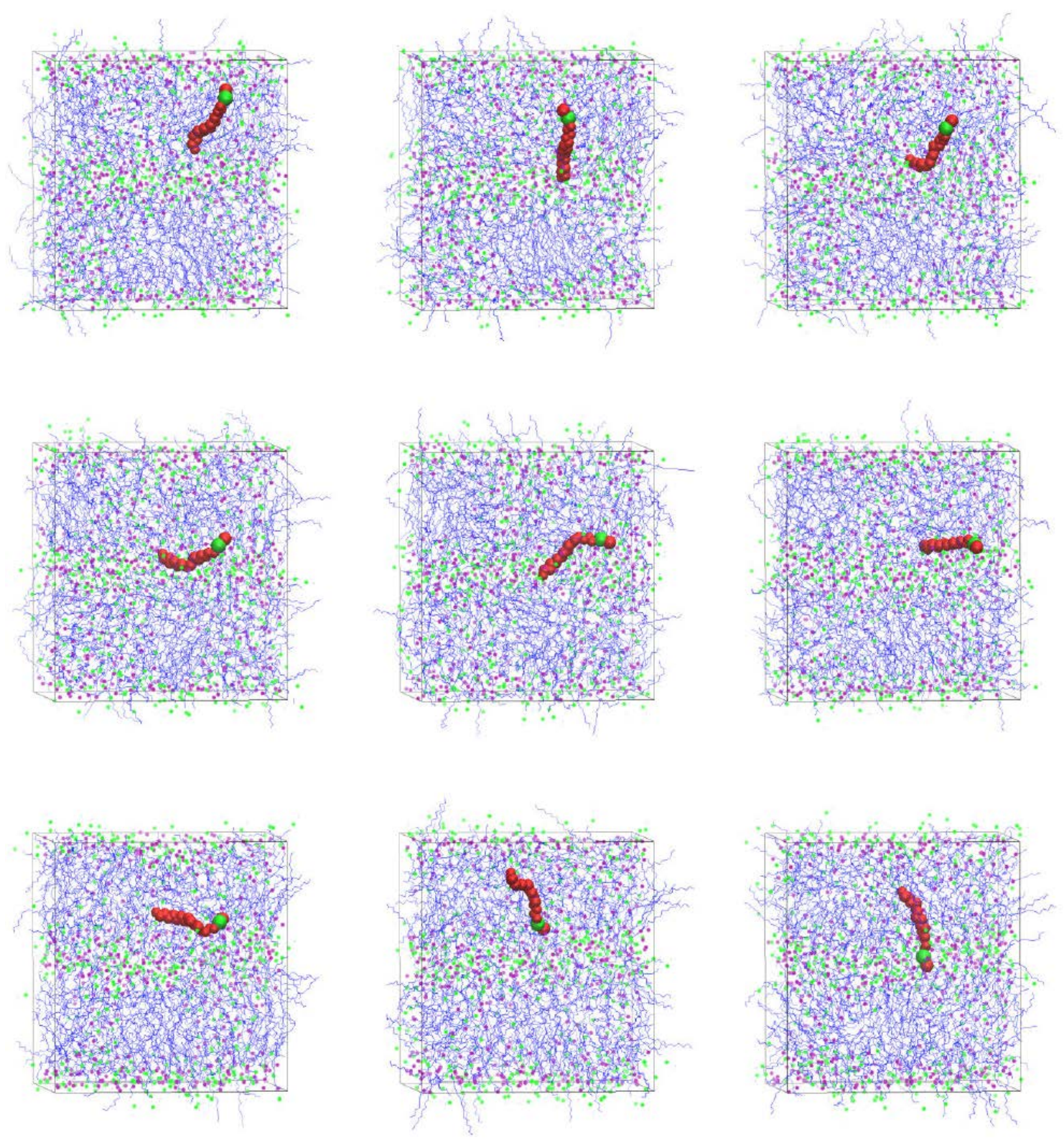

Figure S4. Investigation of the self-diffusion mechanisms in smectogenic ILCs, via coarse-grained MD simulations on $\left[\mathrm{C}_{16} \mathrm{mim}\right]\left[\mathrm{NO}_{3}\right]$. During simulation of the SmA phase at $505 \mathrm{~K}$, the trajectories of two molecules were monitored as a function of time. (a) Snapshots of one of the trajectories (blue line in Figure 6 in ref. 61), taken at times - from top left to bottom right - $6.75 \mathrm{~ns}, 8.37 \mathrm{~ns}, 8.56 \mathrm{~ns}, 8.69 \mathrm{~ns}, 8.72 \mathrm{~ns}, 8.75 \mathrm{~ns}, 8.80 \mathrm{~ns}, 8.86 \mathrm{~ns}$ and $10.80 \mathrm{~ns}$. The cationic headgroup acts as a pivotal site to displace its alkyl chain between two adjacent hydrophobic sublayers, but itself remains in the same ionic sublayer. (b) Snapshots of the other trajectory (red line in Figure 6 in ref. 61), taken at times - from top left to bottom right - $13.50 \mathrm{~ns}, 13.77 \mathrm{~ns}, 14.04 \mathrm{~ns}, 14.31 \mathrm{~ns}, 14.58 \mathrm{~ns}, 14.85 \mathrm{~ns}, 15.12 \mathrm{~ns}, 15.39$

ns and 15.66 ns. The diffusion mechanism that is highlighted is direct 'permeation' through the hydrophobic sublayer followed by a reorientation. This mechanism cannot be observed in the lower-temperature crystalline phase. 
Reproduced from ref. 61 (http://dx.doi.org/10.1039/c3sm50375e) with permission of The Royal Society of Chemistry.
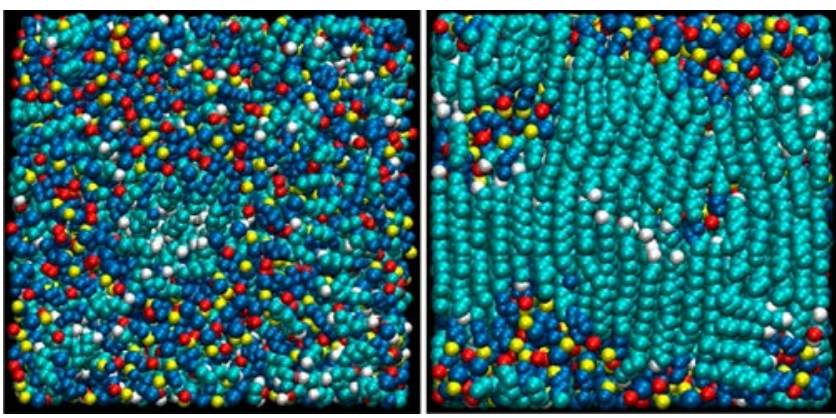

Figure S5. Snapshots of MD simulations of 512 molecules of $\left[\mathrm{C}_{10} \mathrm{mim}\right]\left[\mathrm{NO}_{3}\right]$ (left) and $\left[\mathrm{C}_{22} \mathrm{mim}\right]\left[\mathrm{NO}_{3}\right]$ (right) equilibrated at $400 \mathrm{~K}$. Yellow: A sites; red: D sites; blue: $\mathrm{B}, \mathrm{M}_{1}, \mathrm{M}_{2}, \mathrm{M}_{3}$ and $\mathrm{M}_{4}$ sites; cyan: $\mathrm{C}$ sites; white: E sites (see Figure 9). Reprinted in part with permission from ref. 62. Copyright 2013 American Chemical Society.

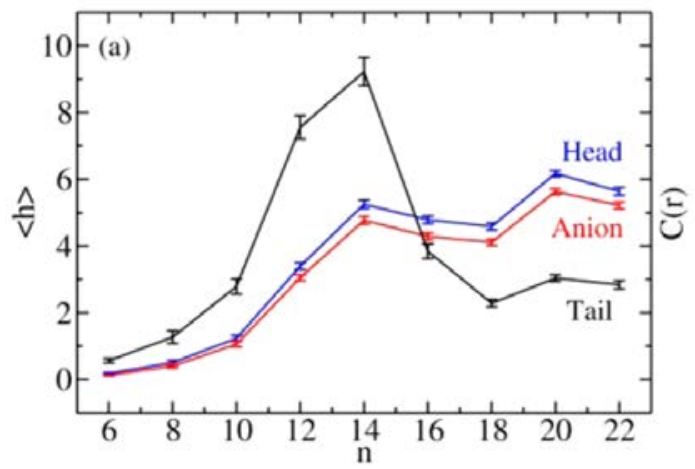

Figure S6. Heterogeneity order parameter (HOP) for the coarse-grained aliphatic tail sites E (see Figure 9), and for the cationic headgroups (A sites) and anions (D sites) in the series $\left[\mathrm{C}_{n} \mathrm{mim}\right]\left[\mathrm{NO}_{3}\right]$, at $400 \mathrm{~K}$. Reprinted in part with permission from ref. 62. Copyright 2013 American Chemical Society. 
(a)

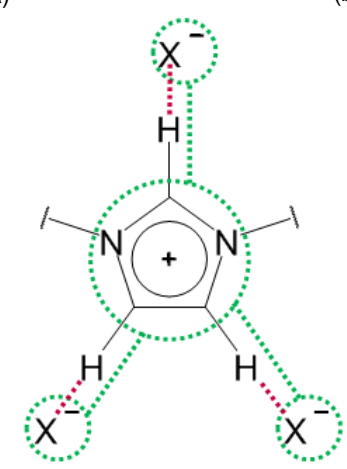

(b)

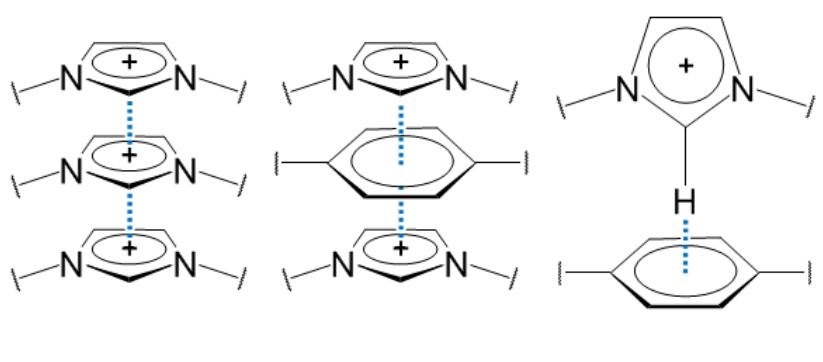

(c)

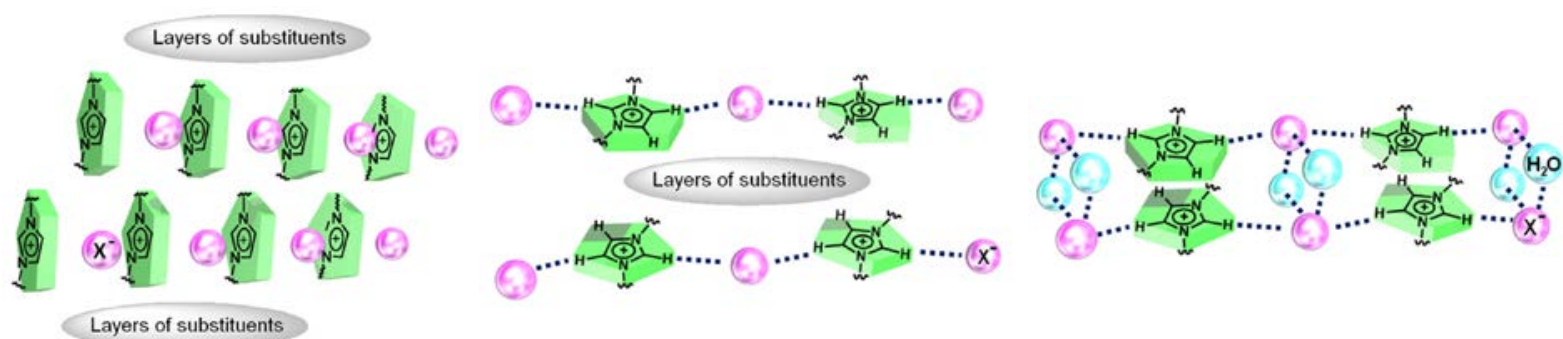

Figure S7. (a, b) Some of the possible interactions with imidazolium cations that have been found in single-crystal structures: (a) hydrogen-bonding interactions with strongly coordinating anions (red lines) and electrostatic interactions (green lines); (b) aromatic stacking interactions (blue lines; these are less important than the interactions in (a)): $\pi$-stacking between imidazolium rings (left) (this interaction is weak since the imidazolium cations are electron-poor rings so that the interaction between the $\pi$-electrons is weak), $\pi$-stacking between two different aromatic rings (middle), and T-stacking (right) (the latter two types of stacking are more stable because the interactions take place between an electron-rich aromatic system and an electron-poor moiety). ${ }^{27,34,63}$ (c) Three types of network structures formed by imidazolium halide salts in their crystalline solid state. Halide anions and water molecules are depicted by pink and blue spheres, respectively. Reprinted from ref. 64 (http://dx.doi.org/10.1016/j.molstruc.2011.05.032), copyright 2011, with permission from Elsevier. 
(a)

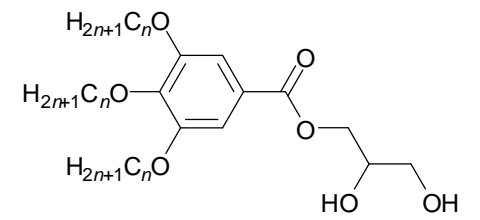

NDA1- $n(n=8,12)$

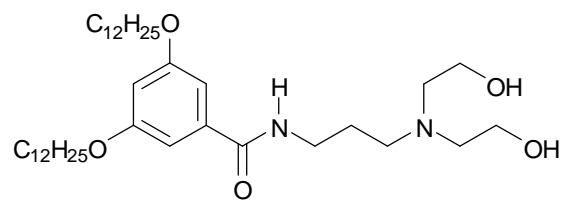

NDA3

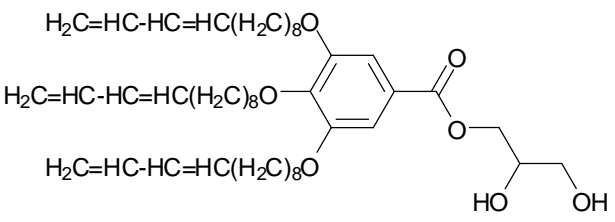

NDA5

(b)

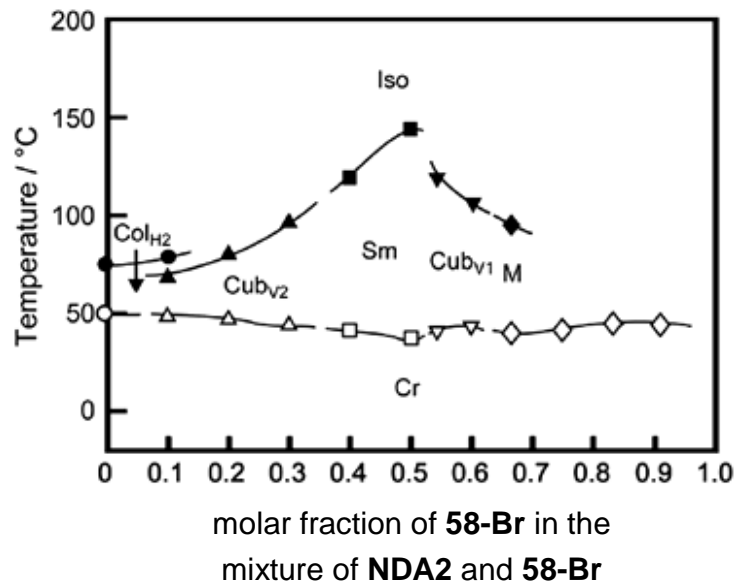

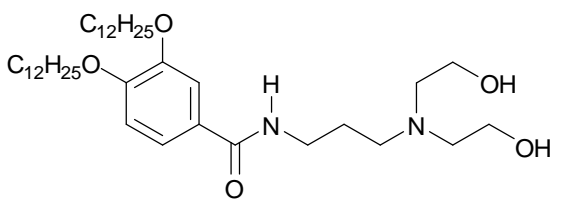

NDA2

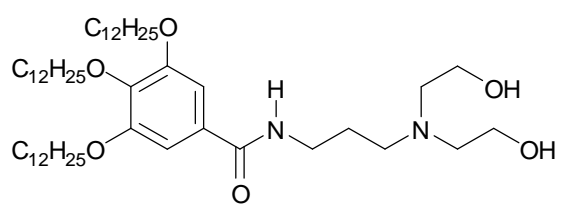

NDA4

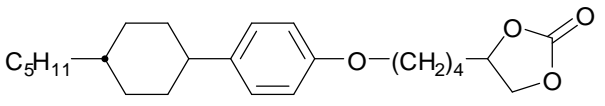

NCM

(c)

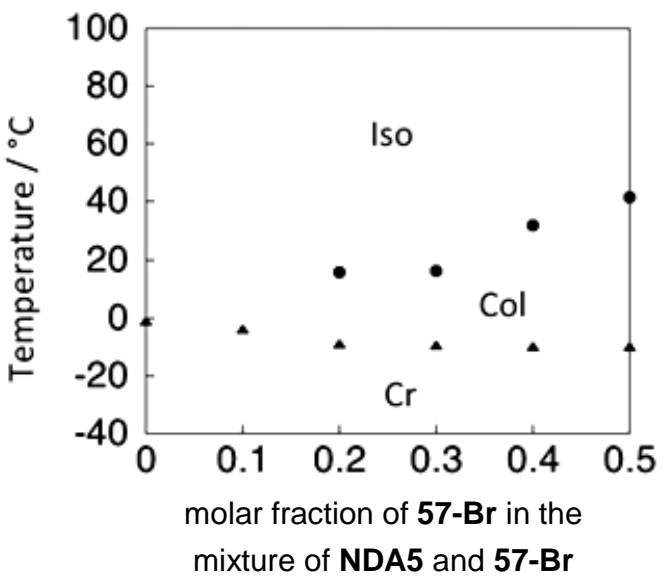

Figure S8. (a) (NDA1-n)-NDA5: neutral dihydroxy amphiphiles (consisting of an ionophilic and an ionophobic part) that were mixed with imidazolium ILs 57-X and 58-X. NCM: neutral carbonate-based mesogen that was mixed with the IL $\left[\mathrm{C}_{3} \mathrm{mim}\right][\mathrm{I}]$ (in combination with $\mathrm{LiNTf}_{2}$ it was also used as the electrolyte in lithium-ion batteries, see section 12.1). (b) Phase diagram for NDA2/58-Br mixtures. The phase sequence as a function of the molar fraction of the polar solvent (i.e. the IL 58-Br) resembles that of typical amphiphilic lyotropic LCs, and is related to the gradual change of the volume ratio between the ionophilic and ionophobic components. Abbreviations: $\mathrm{Cr}=$ crystalline phase; $\mathrm{Col}_{\mathrm{h} 2}=$ 'inverted-type' hexagonal columnar phase; $\mathrm{Cub}_{\mathrm{V} 2}=$ 'inverted-type' bicontinuous cubic phase (Ia $\overline{3} d$ symmetry); $\mathrm{Sm}=$ smectic phase; $\mathrm{Cub}_{\mathrm{V} 1}=$ 'normal-type' bicontinuous cubic phase (Ia $\overline{3} d$ symmetry); $\mathrm{M}=$ 
unidentified mesophase; Iso = isotropic liquid phase. Adapted from ref. 65 (http://dx.doi.org/10.1039/c2sc00981a) with permission of The Royal Society of Chemistry. (c) Phase diagram for NDA5/57-Br mixtures. Adapted with permission from ref. 66. Copyright 2015 Wiley.

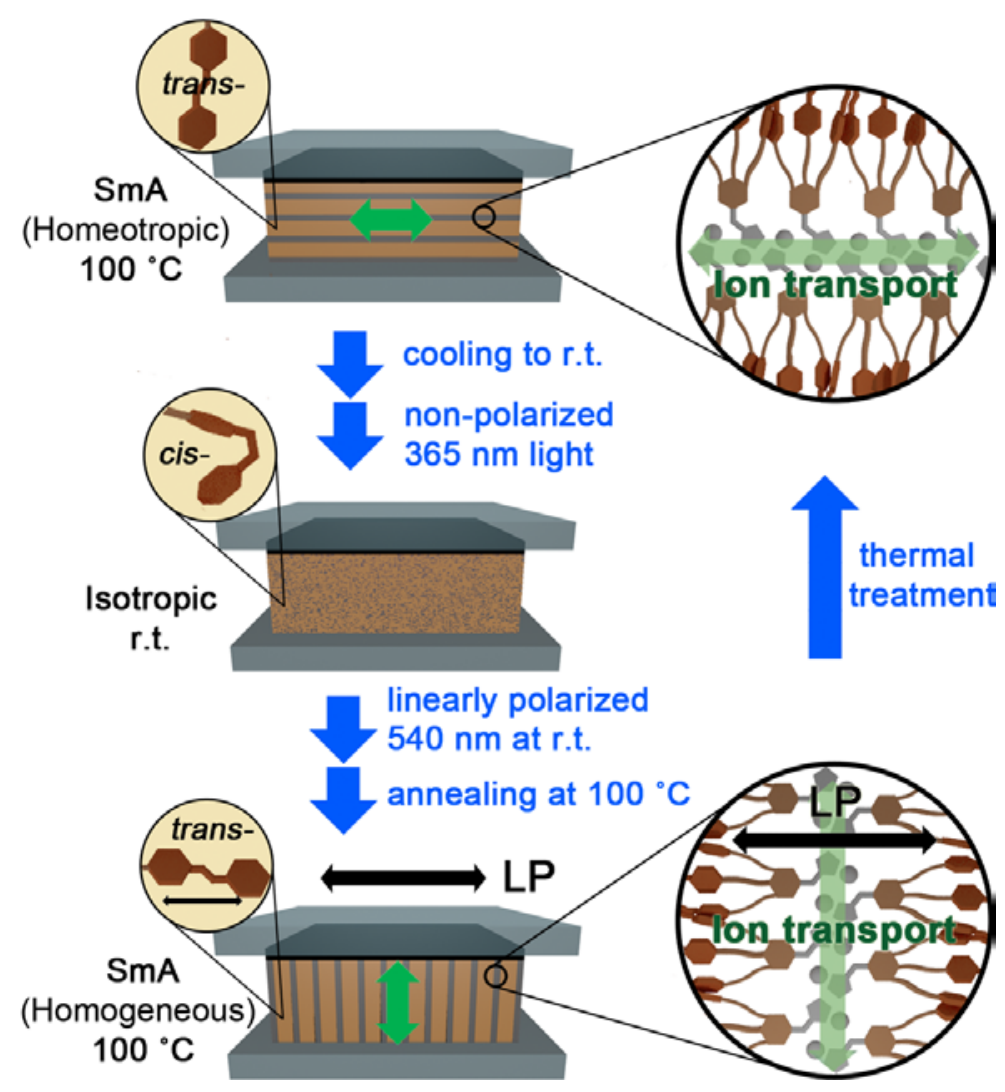

Figure S9. Macroscopic photocontrol over ion-transporting pathways formed by salt 60, based on the trans-to-cis photoisomerization and subsequent photoinduced reorientation of the azobenzene moieties in the ILC. The direction of the linearly polarized (LP) light is marked with black arrows. Reprinted with permission from ref. 67. Copyright 2014 American Chemical Society. 


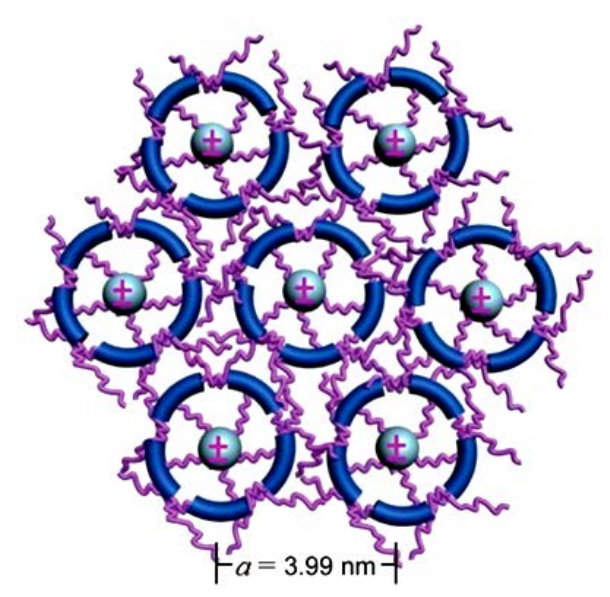

Figure S10. Schematic representation of the structure of the $\mathrm{Col}_{h}$ phase formed by 98-8. The bent-shaped, $\pi$ conjugated mesogenic groups are displayed in blue, aliphatic chains are depicted in purple, and the imidazolium cations are shown in green. Reprinted in part with permission from ref. 68. Copyright 2013 Wiley.

(a)

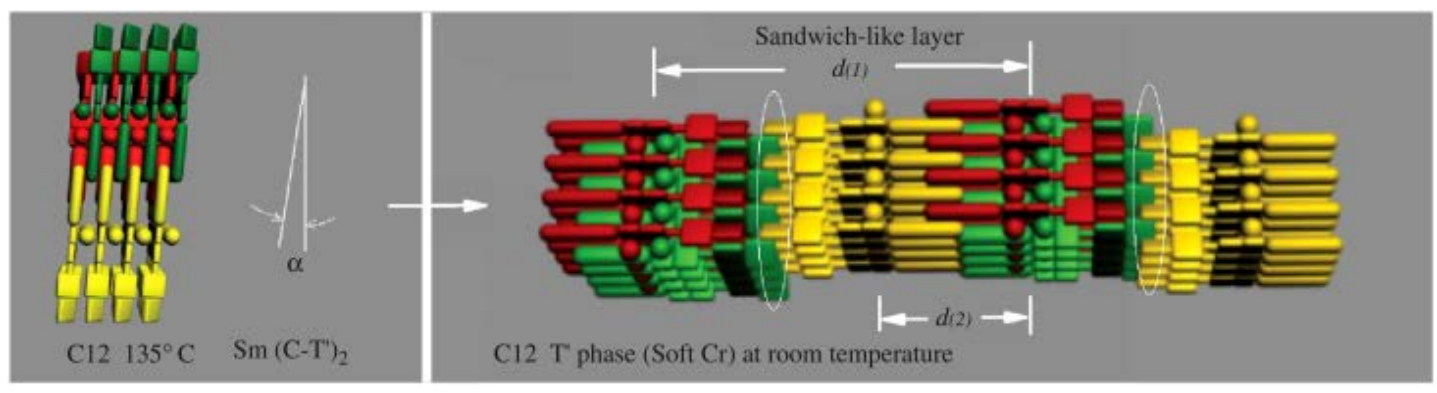

(b)
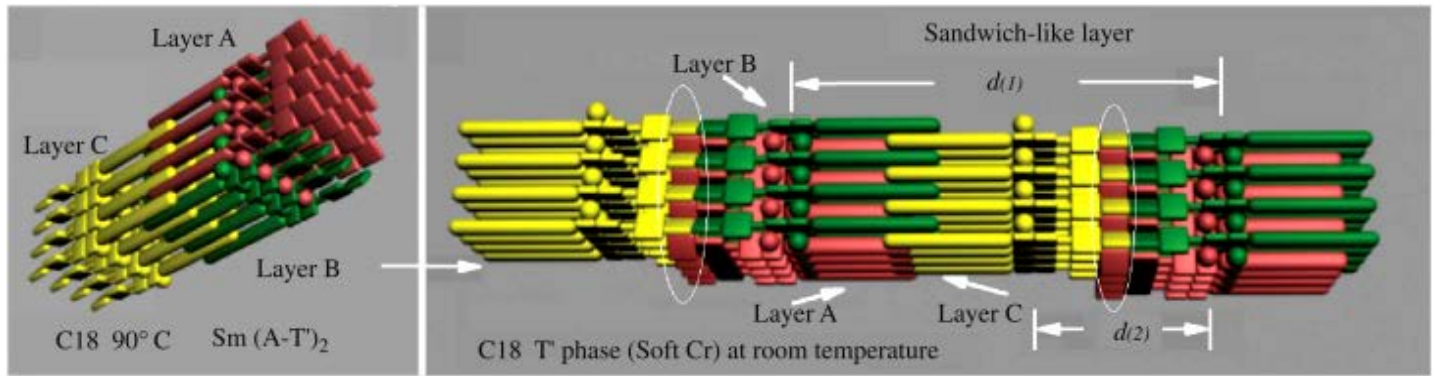

Figure S11. Schematic representation of the structures of (a) the transitional Sm(C-T') $)_{2}$ phase and the roomtemperature tilted T’ phase formed by 127-12 on cooling; and (b) the transitional Sm(A-T') 2 phase and the roomtemperature orthogonal T' phase formed by 127-18 on cooling. Each smectic layer is composed of an ionic monolayer (in yellow; the bromide anions are depicted as little spheres) and an ionic bilayer (in red and green; the bromide anions are again depicted as little spheres). Reprinted in part with permission from ref. 69. Copyright 2012 Taylor \& Francis Ltd (http://www.tandfonline.com). 


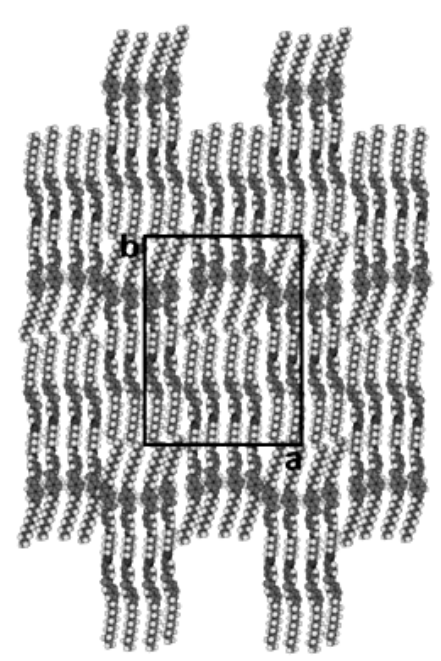

Figure S12. Proposed molecular packing in a SmÃ phase formed by LC dimers. Reproduced from ref. 70 (http://dx.doi.org/10.1039/b612517d) with permission of The Royal Society of Chemistry.
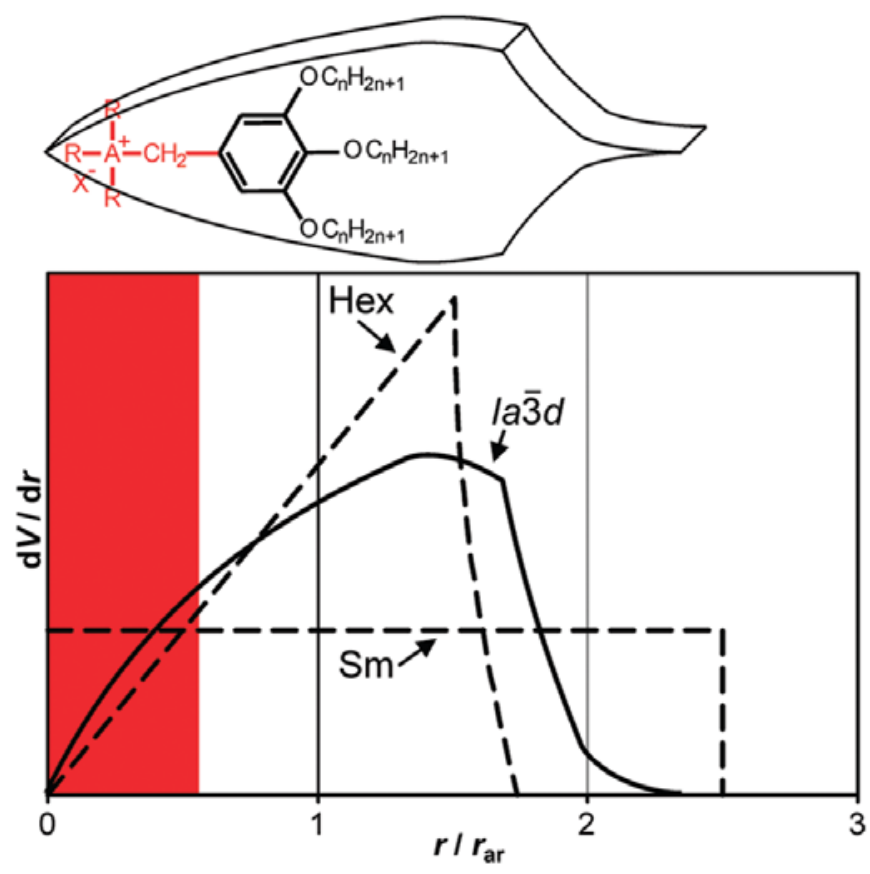

Figure S13. Top: schematic drawing of the molecular cross-sectional area profile, $A(r)$, for one of the taper-shaped ionic mesogens in the series $161-X-m / n$ and $162-m / n$. Bottom: the solid line depicts the calculated rate of increase in volume of the branched cylinder network in a bicontinuous cubic phase ( $\left.\mathrm{Cub}_{\mathrm{v}}(I a \overline{3} d)\right), \mathrm{d} V / \mathrm{d} r$, as a function of increasing radius $r$ of the cylinders (starting from their central axis); the dashed lines are the calculated results for a hexagonal columnar phase and a smectic phase, respectively. The curves are scaled horizontally to the same radius $r_{\text {ar }}$ which delimits the 'aromatic' volume fraction (including all but the peripheral $n$-alkyloxy chains) from the aliphatic volume fraction. The ionic moieties of the taper-shaped mesogens are located near the apex (narrow end) of 
the molecules, and near the central axis of the cylinders in the network. Reprinted with permission from ref. 71.

Copyright 2012 American Chemical Society.
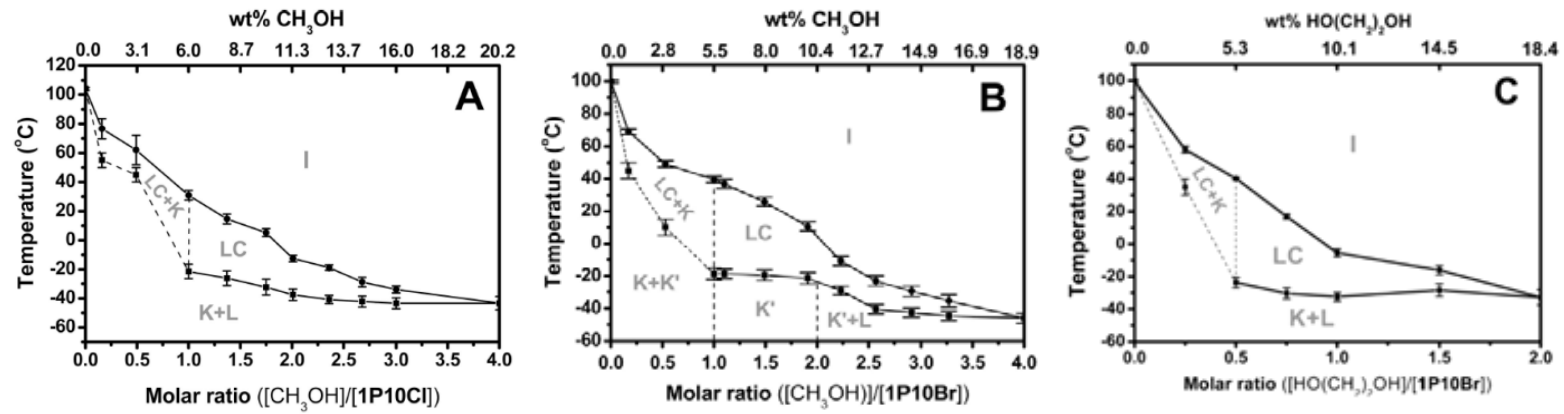

Figure S14. Phase diagrams for mixtures of (a) 227-Cl-10 and methanol; (b) 227-Br-10 and methanol; and (c) 227-

Br-10 and ethylene glycol. ${ }^{72}$ Abbreviations: $\mathrm{L}=$ (phase-separated) liquid of the alcohol; $\mathrm{K}=$ solid of 227-Cl-10 or 227-Br-10; $\mathrm{K}^{\prime}=$ solid of 227-Br-10 $\cdot \mathrm{CH}_{3} \mathrm{OH}$; $\mathrm{LC}=\mathrm{LC}$ mesophase $\left(\mathrm{SmA}_{2}\right)$; I = isotropic liquid state. Lines between data points are drawn as a visual aid and are not based on a physical model. Data points for the solid lines were determined by POM; data points for dashed lines were determined (less precisely) using PXRD and ${ }^{2} \mathrm{H}$ NMR spectroscopy. Reprinted with permission from ref. 72. Copyright 2008 American Chemical Society.
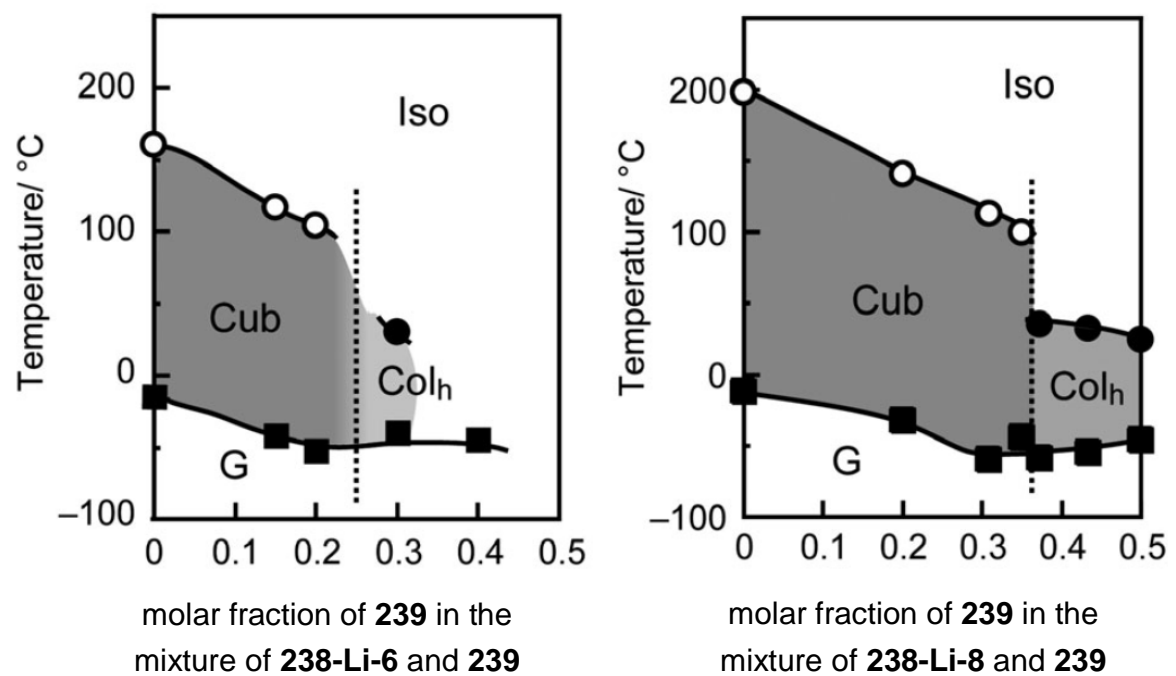

Figure S15. Phase diagrams for binary mixtures of 238-Li-n $\left(\mathrm{A}^{+}=\mathrm{Li}^{+}\right)$and 239. Abbreviations: $\mathrm{g}=$ glass; $\mathrm{Cub}=$ micellar cubic phase $(P m \overline{3} n) ; \mathrm{Col}_{\mathrm{h}}=$ hexagonal columnar phase; Iso = isotropic liquid. Adapted from ref. 73 (http://dx.doi.org/10.1039/c2cc31074k) with permission of The Royal Society of Chemistry. 
(a)

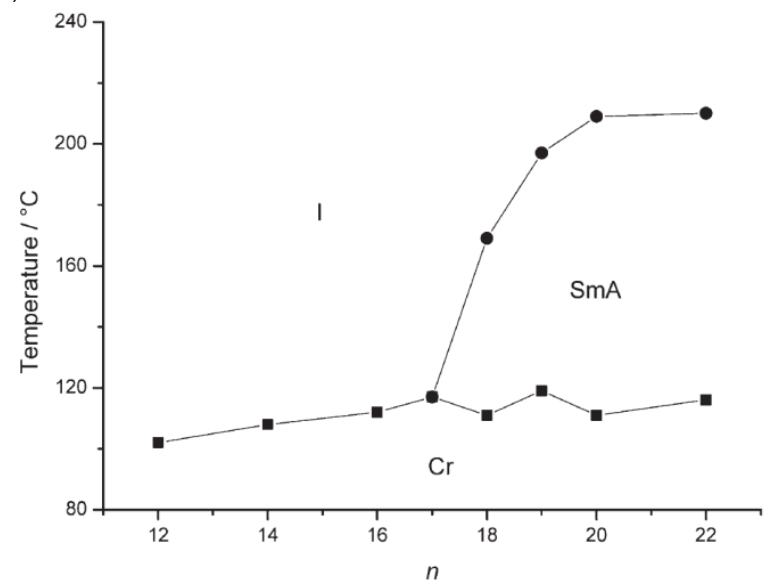

(b)

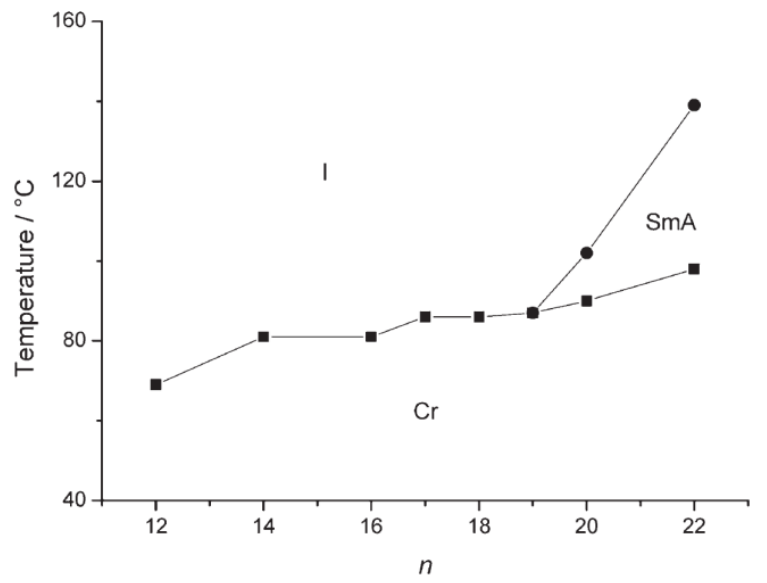

(c)

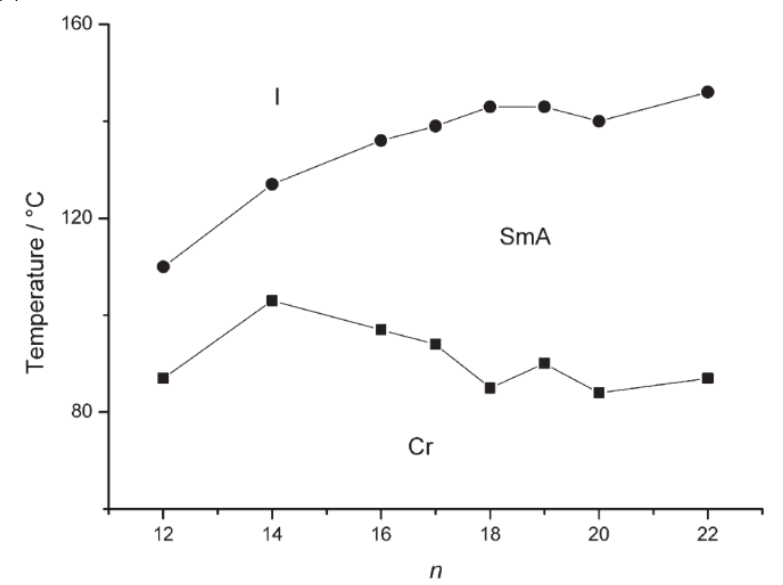

Figure S16. Phase diagram for compounds (a) 255-Br-n, (b) 255-PF $-\mathbf{n}$ and (c) $255-\mathbf{C}_{\mathbf{1 2}} \mathbf{H}_{25} \mathbf{O S O}_{3}-\boldsymbol{n}$. Abbreviations: $\mathrm{Cr}$ = crystalline phase; $\mathrm{SmA}$ = smectic A phase; $\mathrm{I}$ = isotropic liquid phase. Reproduced from ref. 74 (http://dx.doi.org/10.1039/c2ra21208k) with permission of The Royal Society of Chemistry. 


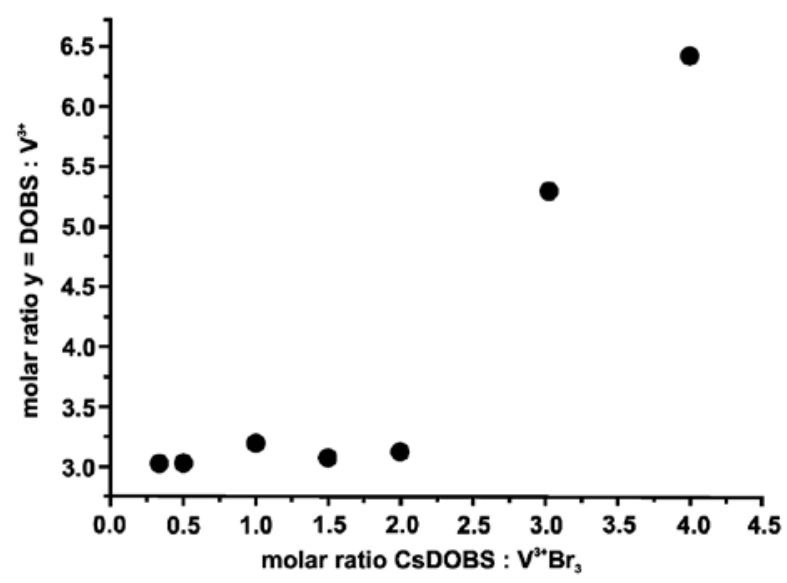

Figure S17. Resulting ionic complex stoechiometry as a function of the molar ratio of the reagents $\mathrm{Cs}\left[\left(\mathrm{C}_{12} \mathrm{H}_{25} \mathrm{O}\right)_{3} \mathrm{PhSO}_{3}\right]$ (= CsDOBS) and 291- $\mathbf{Y}_{\mathbf{1}}-\mathrm{Br}_{3}$ (equal volumes of organic and aqueous phases; [291- $\left.\mathbf{Y}_{1}-\mathrm{Br}_{3}\right]=$ $0.01 \mathrm{M}$ ). The organic ions show a strong affinity for each other, and the release of small $\mathrm{Cs}^{+}$ions into the aqueous phase ensures an entropic gain in free energy. Reproduced from ref. 75 (http://dx.doi.org/10.1039/c2jm31830j) with permission of The Royal Society of Chemistry.

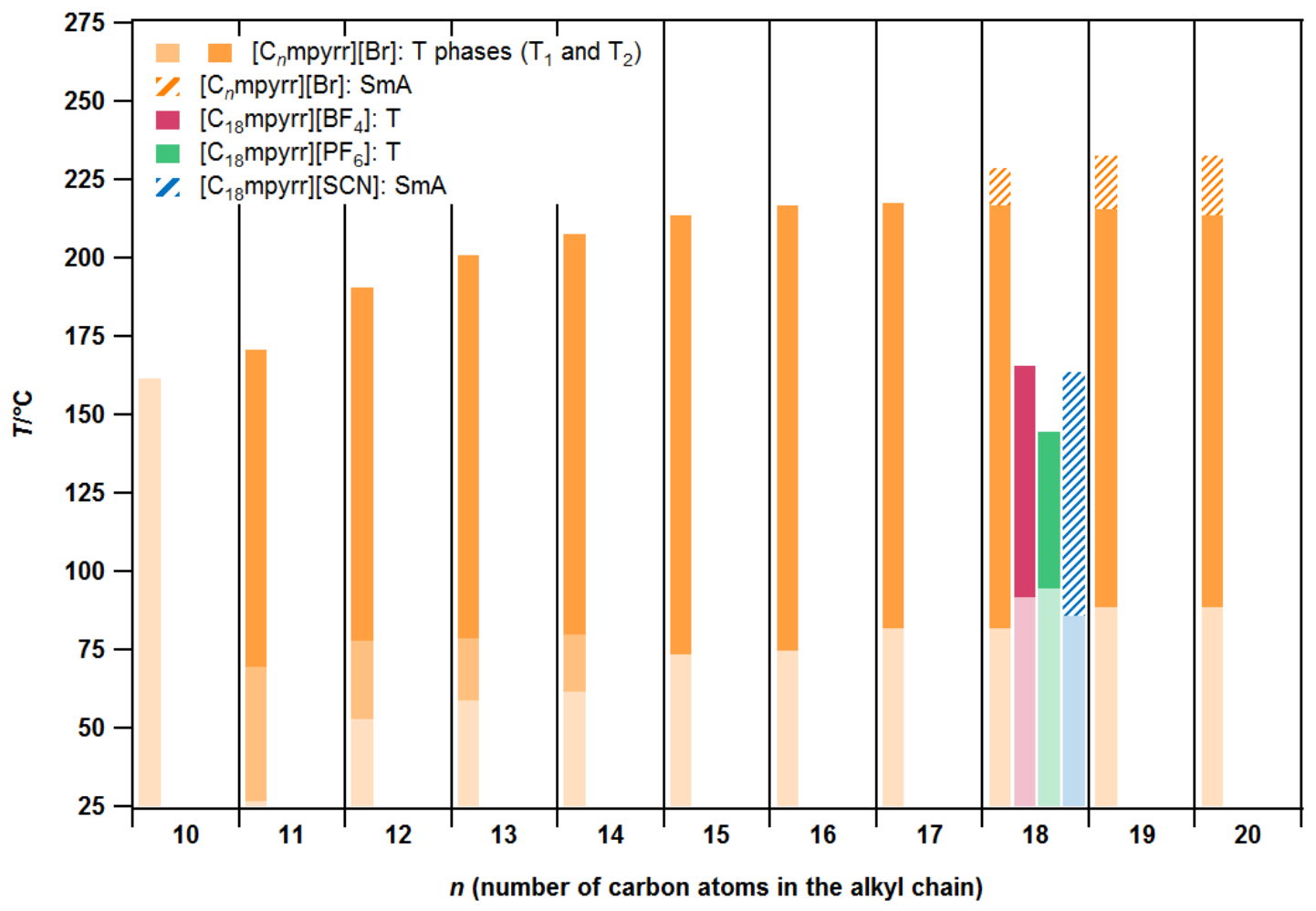

Figure S18. Phase diagrams for $\left[\mathrm{C}_{n} \mathrm{mpyrr}\right][\mathrm{Br}],\left[\mathrm{C}_{18} \mathrm{mpyrr}\right]\left[\mathrm{BF}_{4}\right],\left[\mathrm{C}_{18} \mathrm{mpyrr}\right]\left[\mathrm{PF}_{6}\right]$ and $\left[\mathrm{C}_{18} \mathrm{mpyrr}\right][\mathrm{SCN}]{ }^{76}$ Salts [ $\left.\mathrm{C}_{n} \mathrm{mpyrr}\right][\mathrm{Br}]$ with $n=11-16$ show two types of $\mathrm{T}$ phases $\left(\mathrm{T}_{1}\right.$ and $\mathrm{T}_{2}$; the $\mathrm{T}_{1}$ phase is monotropic in the case of $\left[\mathrm{C}_{15} \mathrm{mpyrr}\right][\mathrm{Br}]$ and $\left.\left[\mathrm{C}_{16} \mathrm{mpyrr}\right][\mathrm{Br}]\right)$, but there are only subtle structural differences between these two phases. 


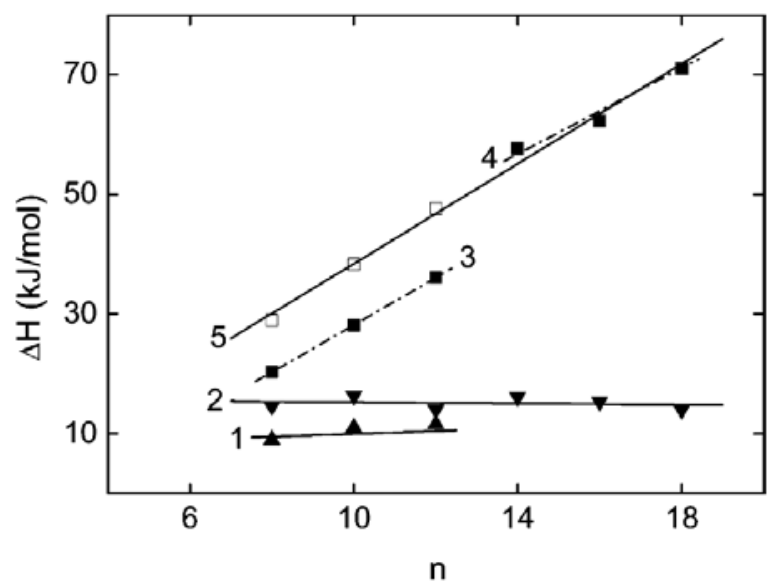

Figure S19. Change of transition enthalpy values as a function of the alkyl chain length in the series 317-0/n: $1=$ $\mathrm{SmX} \rightarrow \mathrm{SmY} ; 2=\mathrm{SmY} \rightarrow \mathrm{SmA} ; 3=\mathrm{Cr} \rightarrow \mathrm{SmX} ; 4=\mathrm{Cr} \rightarrow \mathrm{SmY} ; 5=\mathrm{Cr} \rightarrow \mathrm{SmX} \rightarrow \mathrm{SmY}$. Reprinted with permission from ref. 77. Copyright 2005 American Chemical Society.

(a)

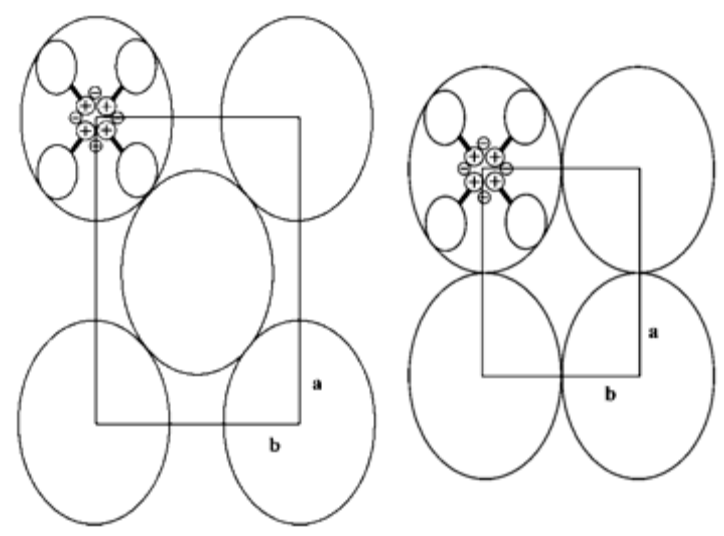

(b)

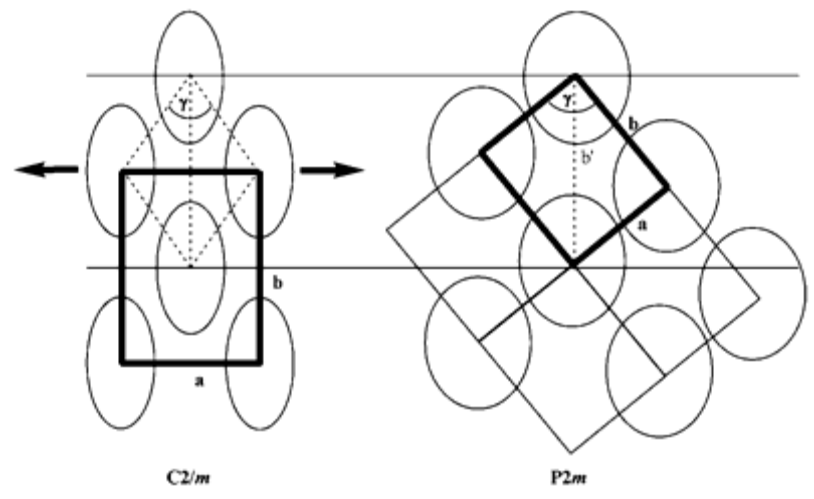

Figure S20. (a) $\mathrm{Col}_{\mathrm{r}}$ phases of $\mathrm{C2} / \mathrm{m}\left(\mathrm{Col}_{\mathrm{r}, 2}\right)$ and $\mathrm{P} 2 \mathrm{~m}\left(\mathrm{Col}_{\mathrm{r}, 3}\right)$ symmetry, formed by salts 338-n. (b) Possible mechanism for the symmetry change of 338-8 from $C 2 / m$ to $P 2 m$. Reprinted with permission from ref. 78. Copyright 2008 American Chemical Society. 


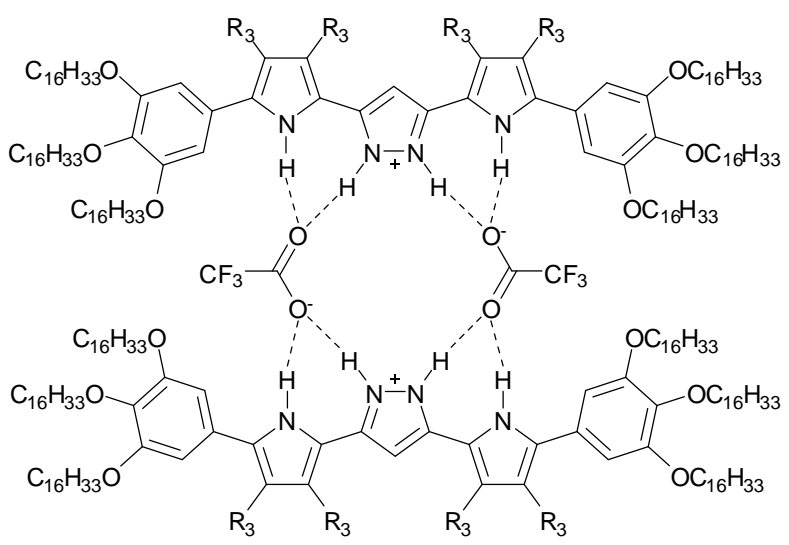

Figure S21. Formation of [2+2]-type ion-pair complexes by compounds $\left[\mathbf{3 5 4}-\mathbf{R}_{3}\right]\left[\mathrm{CF}_{3} \mathrm{COO}\right]\left(\mathrm{R}_{3}=\mathrm{H}\right.$ or F). ${ }^{79}$ The $\left[\mathrm{CF}_{3} \mathrm{COO}\right]^{-}$anions act as bridging units through hydrogen bonding.

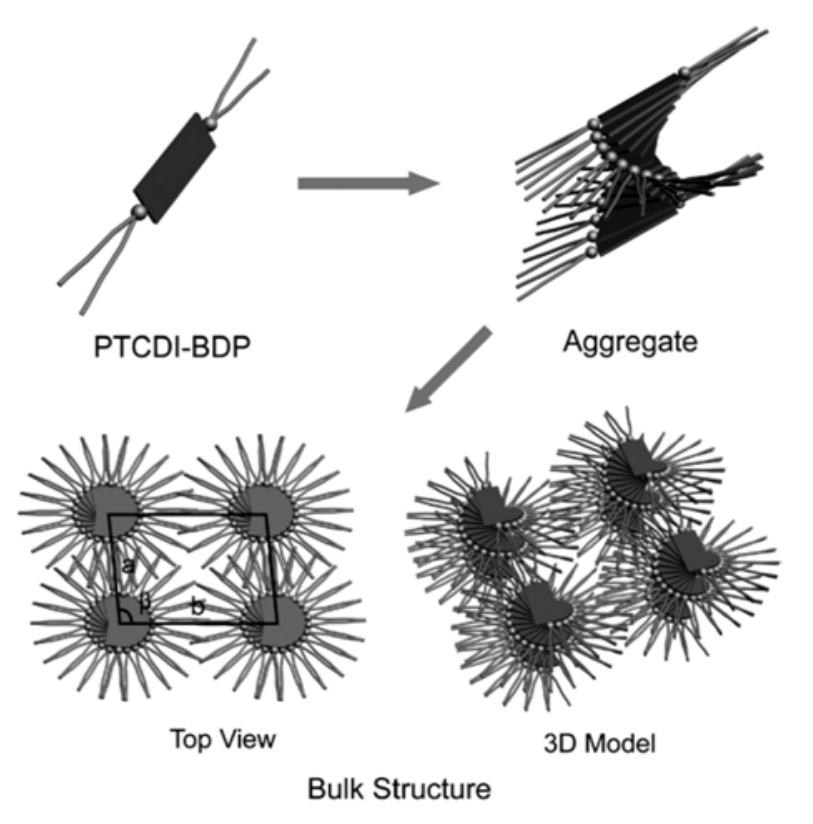

Figure S22. Schematic representation of the helical ordering in the $\mathrm{Col}_{\mathrm{ob}}$ phase formed by $\mathbf{4 1 1}$ (Maeda and coworkers coined the term 'charge-segregated assemblies' for similar phase structures ${ }^{80}$ ). Reproduced from ref. 81 (http://dx.doi.org/10.1039/b817838k) with permission of The Royal Society of Chemistry. 


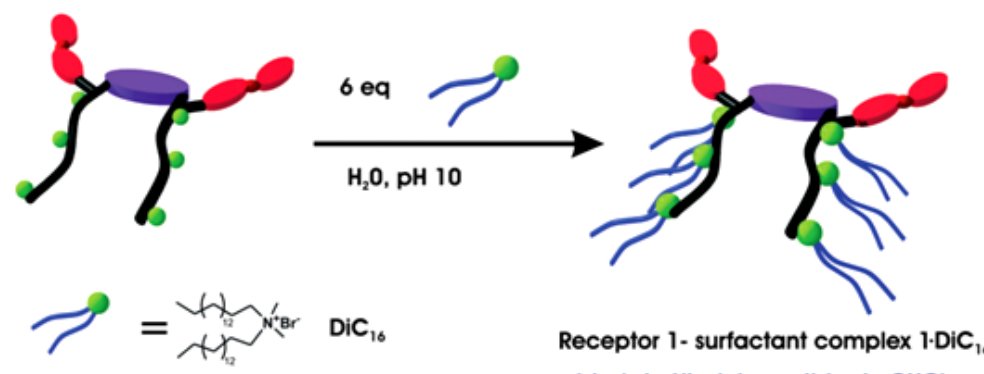

binds to His-rich peptides in $\mathrm{CHCl}_{3}$

Figure S23. Formation of the receptor-surfactant complex 388-(399-16) 6 . Ionic moieties (carboxylate groups and ammonium headgroups) are represented as green spheres, azobenzene chromophores are depicted in red. Reproduced from ref. 82 (http://dx.doi.org/10.1039/b802690d) with permission of The Royal Society of Chemistry.

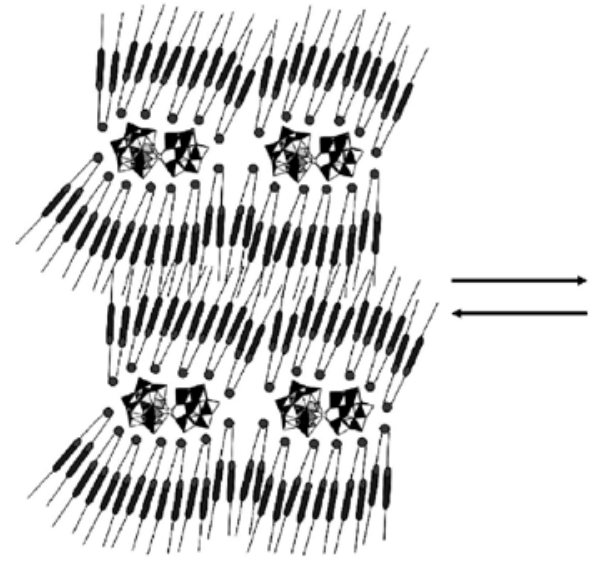

Solid state

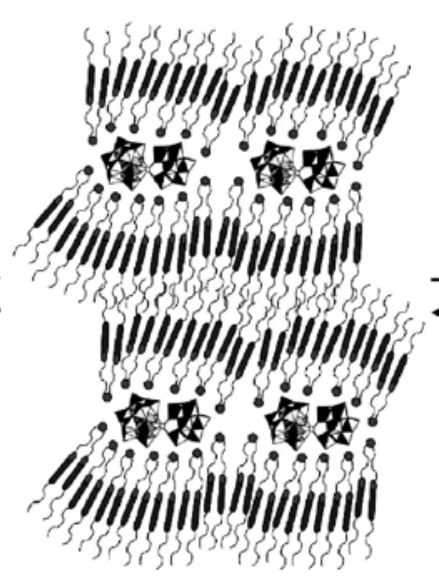

Smectic C

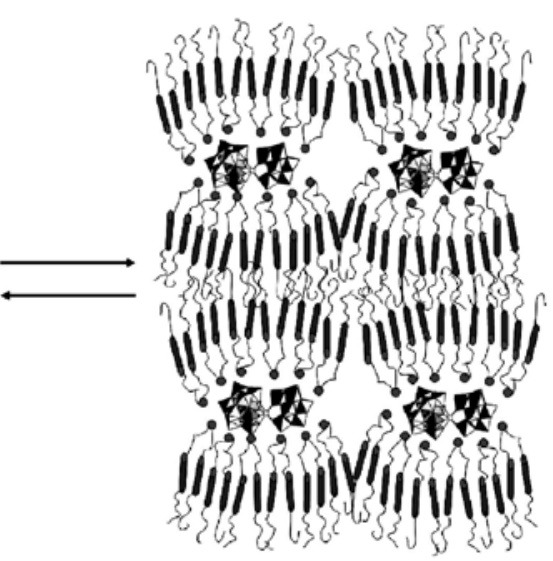

Smectic A

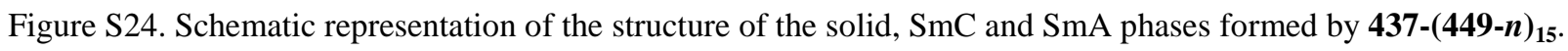
Reprinted with permission from ref. 83. Copyright 2008 American Chemical Society. 

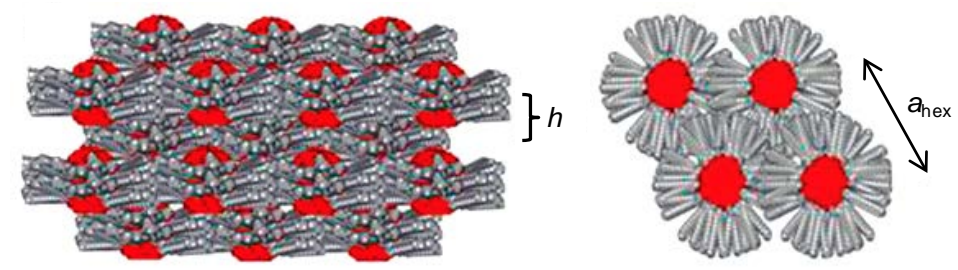

Figure S25. Schematic representation of the molecular organization of 440-(399-18) $)_{36}\left(\mathbf{N H}_{4}\right)_{6}$ in its lamellar mesophase. Left: view parallel to the layers. Right: view perpendicular to the layers illustrating the local hexagonal packing of the polyoxometalate clusters. Adapted from ref. 84 (http://dx.doi.org/10.1039/c2nj20923c) with permission from the Centre National de la Recherche Scientifique (CNRS) and The Royal Society of Chemistry.

(a)

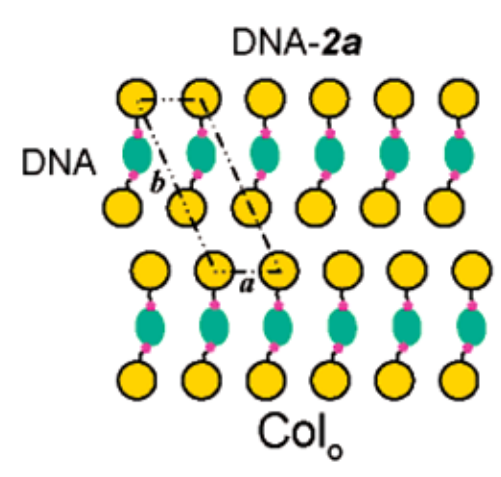

(b)

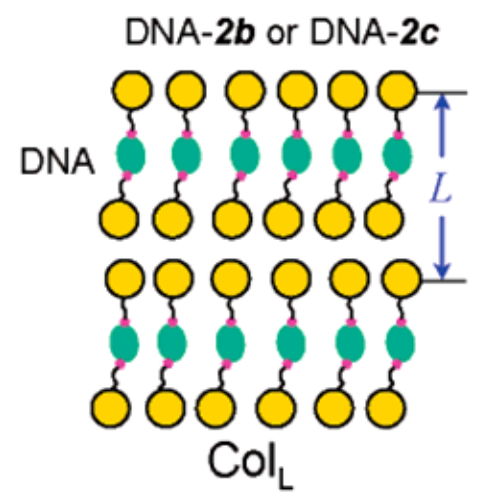

Figure S26. Schematic representations of the proposed structures of (a) the $\mathrm{Col}_{\mathrm{ob}}$ phase formed by $\mathbf{4 5 6 - 4}$ and (b) the $\mathrm{Col}_{\mathrm{L}}\left(\mathrm{L}_{\alpha}{ }^{\mathrm{C}}\right)$ phases formed by 456-7 and 456-10. Reprinted with permission from ref. 85. Copyright 2006 American Chemical Society.

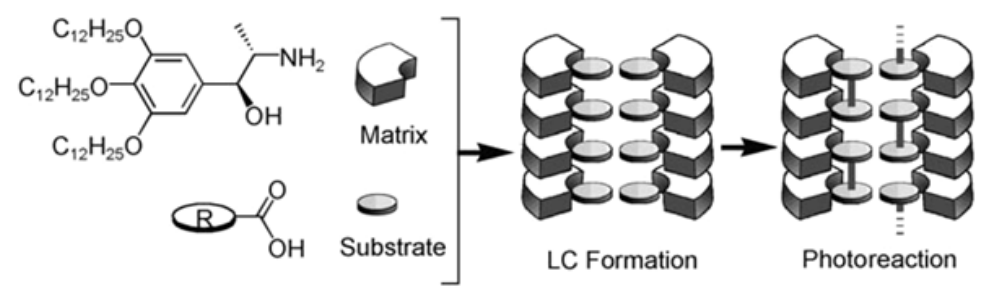

Figure S27. Photoreaction (photodimerization) in a two-component LC ( $\mathrm{R}=$ anthracene). Reprinted with permission from ref. 86. Copyright 2008 Wiley. 


\section{Thermal transitions of all compounds discussed in the main text (in the order in which they appear there)}

Comment on the reproduction of phase transition temperatures: One should bear in mind that these data have not always been obtained in the same way. Some research groups report transition temperatures from the first DSC heating run (DSC = differential scanning calorimetry) of a virgin sample, while others report transitions from the second DSC heating run or even from a cooling run, or they have determined the values via POM (= polarizing optical microscopy) or PXRD (= powder X-ray diffraction) or do not specify how the data were obtained. Furthermore, DSC experiments are not always performed at the same heating/cooling rate. In principle, one should always report the onset temperatures of thermal transitions that are observed during a heating run, and preferably data are provided for both the first heating run (during which the sample is heated up to its isotropic liquid state) and second heating run - if possible. Onset temperatures are in principle not dependent on the rate of heating. In a cooling run, supercooling effects can be in play, especially for transitions to crystalline phases and cubic mesophases. Differences between the first and second heating run can be due to the loss of water during the first heating of a virgin sample, which may be particularly important in the case of amphiphilic charged compounds. It is therefore recommended to mention the maximum temperature to which a specific sample has been heated during its first heating run and whether or not it has been kept for some time at that temperature with a hole pierced in the lid of the DSC pan. Of course, it should be avoided that the sample is already heated to a temperature at which (partial) decomposition occurs. This can lead to erroneous results and conclusions. ${ }^{46,87}$ Thermal decomposition usually results in color changes of the sample (which can be observed during POM experiments, besides a possible bad smell) and in an alteration of the baseline of a DSC thermogram, and must be checked by thermogravimetric analysis (TGA). ${ }^{76}$

Thermal phase transitions are represented here as follows: $\mathrm{Cr}_{1} \cdot 50 \cdot \mathrm{Cr}_{2} \cdot\left(\mathrm{M}_{1} \cdot 55 \cdot\right) 60 \cdot \mathrm{M}_{2}$. $90 \cdot \mathrm{M}_{3} \cdot 120 \cdot \mathrm{I}\left({ }^{\circ} \mathrm{C}\right)$, for a material that exhibits a crystalline phase between room temperature and $50{ }^{\circ} \mathrm{C}$, another (distinct) crystalline phase between $50{ }^{\circ} \mathrm{C}$ and $60{ }^{\circ} \mathrm{C}$, an enantiotropic LC mesophase $\left(\mathrm{M}_{2}\right)$ between $60{ }^{\circ} \mathrm{C}$ and $90{ }^{\circ} \mathrm{C}$ and another enantiotropic LC mesophase $\left(\mathrm{M}_{3}\right)$ between $90^{\circ} \mathrm{C}$ and $120^{\circ} \mathrm{C}$, and that clears out into an isotropic liquid at $120^{\circ} \mathrm{C}$; the monotropic LC mesophase $\mathrm{M}_{1}$ is only formed upon quickly cooling the material below its melting point. 


\subsection{Imidazolium-based ionic liquid crystals}

Remark: The phase transition temperatures of basic $\left[\mathrm{C}_{n} \operatorname{mim}\right][\mathrm{X}],\left[\mathrm{C}_{n} \mathrm{C}_{m} \mathrm{im}\right][\mathrm{X}]$ and $\left[\mathrm{C}_{n} \mathrm{Him}\right][\mathrm{X}]$ salts are summarized in Figure 16 and Figure 17 of the review paper and in Table S2.

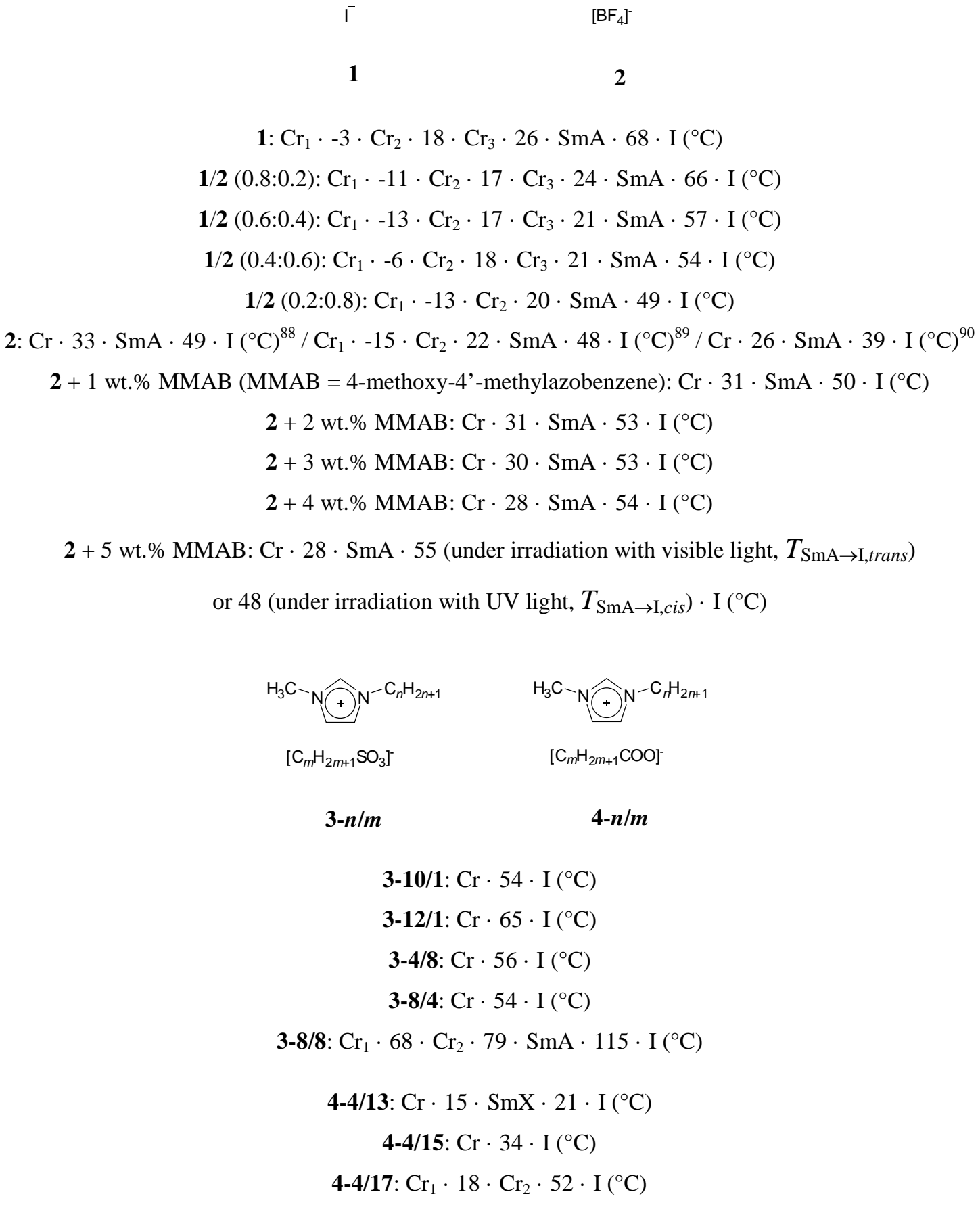

I

1

$\left[\mathrm{BF}_{4}\right]^{-}$

2

$$
\begin{aligned}
& 2+2 \text { wt.\% MMAB: } \mathrm{Cr} \cdot 31 \cdot \mathrm{SmA} \cdot 53 \cdot \mathrm{I}\left({ }^{\circ} \mathrm{C}\right) \\
& 2+3 \text { wt.\% MMAB: } \mathrm{Cr} \cdot 30 \cdot \mathrm{SmA} \cdot 53 \cdot \mathrm{I}\left({ }^{\circ} \mathrm{C}\right) \\
& 2+4 \text { wt.\% MMAB: } \mathrm{Cr} \cdot 28 \cdot \mathrm{SmA} \cdot 54 \cdot \mathrm{I}\left({ }^{\circ} \mathrm{C}\right)
\end{aligned}
$$$$
\text { 1: } \mathrm{Cr}_{1} \cdot-3 \cdot \mathrm{Cr}_{2} \cdot 18 \cdot \mathrm{Cr}_{3} \cdot 26 \cdot \mathrm{SmA} \cdot 68 \cdot \mathrm{I}\left({ }^{\circ} \mathrm{C}\right)
$$$$
\text { 1/2 (0.8:0.2): } \mathrm{Cr}_{1} \cdot-11 \cdot \mathrm{Cr}_{2} \cdot 17 \cdot \mathrm{Cr}_{3} \cdot 24 \cdot \mathrm{SmA} \cdot 66 \cdot \mathrm{I}\left({ }^{\circ} \mathrm{C}\right)
$$$$
\text { 1/2 (0.6:0.4): } \mathrm{Cr}_{1} \cdot-13 \cdot \mathrm{Cr}_{2} \cdot 17 \cdot \mathrm{Cr}_{3} \cdot 21 \cdot \mathrm{SmA} \cdot 57 \cdot \mathrm{I}\left({ }^{\circ} \mathrm{C}\right)
$$$$
\text { 1/2 (0.4:0.6): } \mathrm{Cr}_{1} \cdot-6 \cdot \mathrm{Cr}_{2} \cdot 18 \cdot \mathrm{Cr}_{3} \cdot 21 \cdot \mathrm{SmA} \cdot 54 \cdot \mathrm{I}\left({ }^{\circ} \mathrm{C}\right)
$$$$
\text { 1/2 (0.2:0.8): } \mathrm{Cr}_{1} \cdot-13 \cdot \mathrm{Cr}_{2} \cdot 20 \cdot \mathrm{SmA} \cdot 49 \cdot \mathrm{I}\left({ }^{\circ} \mathrm{C}\right)
$$$$
\text { 2: } \mathrm{Cr} \cdot 33 \cdot \mathrm{SmA} \cdot 49 \cdot \mathrm{I}\left({ }^{\circ} \mathrm{C}\right)^{88} / \mathrm{Cr}_{1} \cdot-15 \cdot \mathrm{Cr}_{2} \cdot 22 \cdot \mathrm{SmA} \cdot 48 \cdot \mathrm{I}\left({ }^{\circ} \mathrm{C}\right)^{89} / \mathrm{Cr} \cdot 26 \cdot \mathrm{SmA} \cdot 39 \cdot \mathrm{I}\left({ }^{\circ} \mathrm{C}\right)^{90}
$$$$
2+1 \text { wt.\% MMAB (MMAB = 4-methoxy-4'-methylazobenzene): } \mathrm{Cr} \cdot 31 \cdot \mathrm{SmA} \cdot 50 \cdot \mathrm{I}\left({ }^{\circ} \mathrm{C}\right)
$$

$2+5$ wt.\% MMAB: $\mathrm{Cr} \cdot 28 \cdot \mathrm{SmA} \cdot 55$ (under irradiation with visible light, $T_{\mathrm{SmA} \rightarrow \mathrm{I}, \text { trans }}$ ) or 48 (under irradiation with UV light, $\left.T_{\mathrm{SmA} \rightarrow \mathrm{I}, c i s}\right) \cdot \mathrm{I}\left({ }^{\circ} \mathrm{C}\right)$ 


\section{4-10/13: $\mathrm{Cr}_{1} \cdot 30 \cdot \mathrm{Cr}_{2} \cdot$ ca. $32 \cdot \mathrm{Cr}_{3} \cdot 55 \cdot \mathrm{I}\left({ }^{\circ} \mathrm{C}\right)$ \\ 4-10/15: $\mathrm{Cr} \cdot 61 \cdot \mathrm{I}\left({ }^{\circ} \mathrm{C}\right)$}

[remark: See ref. 91 for $\left[\mathrm{C}_{4} \mathrm{mim}\right]^{+}$salts with a palmitoyl ascorbate or palmitoyl-L-tryptophanate anion, respectively; these salts are ILs, but not LC.]
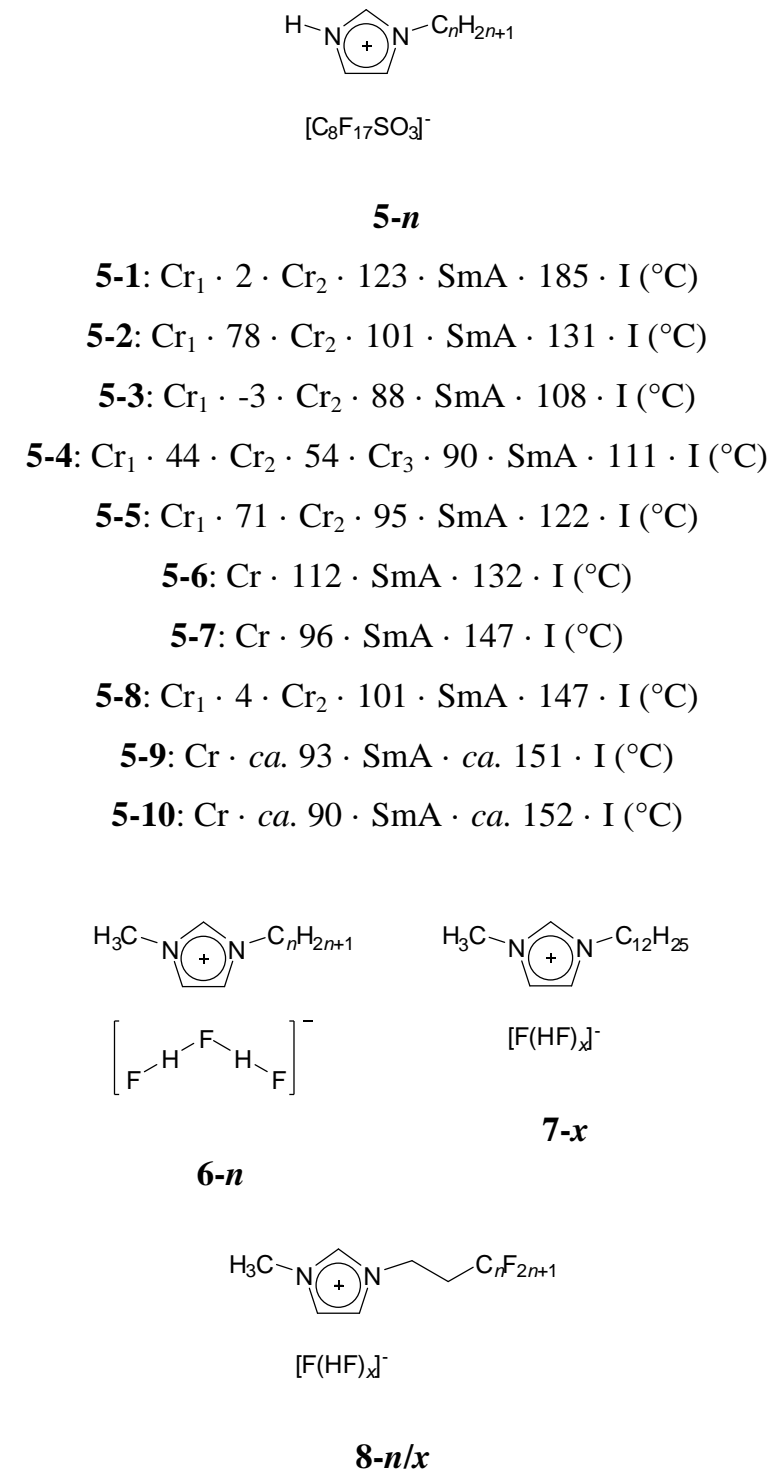

6-8: $\mathrm{Cr} \cdot-28 \cdot \mathrm{I}\left({ }^{\circ} \mathrm{C}\right)$

6-10: $\mathrm{Cr} \cdot 0 \cdot \mathrm{SmA} \cdot 10 \cdot \mathrm{I}\left({ }^{\circ} \mathrm{C}\right)$

6-12: $\mathrm{Cr} \cdot 20 \cdot \mathrm{SmA} \cdot 62 \cdot \mathrm{I}\left({ }^{\circ} \mathrm{C}\right)$

6-14: $\mathrm{Cr} \cdot 29 \cdot \mathrm{SmA} \cdot 109 \cdot \mathrm{I}\left({ }^{\circ} \mathrm{C}\right)$

6-16: $\mathrm{Cr} \cdot 46 \cdot \mathrm{SmA} \cdot 153 \cdot \mathrm{I}\left({ }^{\circ} \mathrm{C}\right)$

6-18: $\mathrm{Cr} \cdot 62 \cdot \mathrm{SmA} \cdot 189 \cdot \mathrm{I}\left({ }^{\circ} \mathrm{C}\right)$

7-1.0 $(x=1.0): \mathrm{Cr}_{1} \cdot-1 \cdot \mathrm{Cr}_{2} \cdot 41 \cdot \mathrm{SmA} \cdot 103 \cdot \mathrm{I}\left({ }^{\circ} \mathrm{C}\right)$ 
7-1.1 $\left(x=1.1\right.$; anionic species: $[\mathrm{FHF}]^{-}$and $\left.\left[\mathrm{F}(\mathrm{HF})_{2}\right]\right): \mathrm{Cr}_{1} \cdot 2 \cdot \mathrm{Cr}_{2} \cdot 39 \cdot \mathrm{SmA} \cdot 99 \cdot \mathrm{I}\left({ }^{\circ} \mathrm{C}\right)$

7-1.2 $\left(x=1.2\right.$; anionic species: $[\mathrm{FHF}]^{-}$and $\left.\left[\mathrm{F}(\mathrm{HF})_{2}\right]^{-}\right): \mathrm{Cr}_{1} \cdot 2 \cdot \mathrm{Cr}_{2} \cdot 36 \cdot \mathrm{SmA} \cdot 95 \cdot \mathrm{I}\left({ }^{\circ} \mathrm{C}\right)$

7-1.3 ( $x=1.3$; anionic species: $[\mathrm{FHF}]^{-}$and $\left.\left[\mathrm{F}(\mathrm{HF})_{2}\right]^{-}\right): \mathrm{Cr}_{1} \cdot 3 \cdot \mathrm{Cr}_{2} \cdot 36 \cdot \mathrm{SmA} \cdot 90 \cdot \mathrm{I}\left({ }^{\circ} \mathrm{C}\right)$

7-1.4 $\left(x=1.4\right.$; anionic species: $[\mathrm{FHF}]^{-}$and $\left[\mathrm{F}(\mathrm{HF})_{2}{ }^{\top}\right): \mathrm{Cr}_{1} \cdot 3 \cdot \mathrm{Cr}_{2} \cdot 35 \cdot \mathrm{SmA} \cdot 87 \cdot \mathrm{I}\left({ }^{\circ} \mathrm{C}\right)$

7-1.5 ( $x=1.5$; anionic species: $[\mathrm{FHF}]^{-}$and $\left.\left[\mathrm{F}(\mathrm{HF})_{2}\right]^{\top}\right): \mathrm{Cr}_{1} \cdot 1 \cdot \mathrm{Cr}_{2} \cdot 34 \cdot \mathrm{SmA} \cdot 82 \cdot \mathrm{I}\left({ }^{\circ} \mathrm{C}\right)$

7-1.6 ( $x=1.6$; anionic species: $[\mathrm{FHF}]^{-}$and $\left.\left[\mathrm{F}(\mathrm{HF})_{2}\right]^{-}\right): \mathrm{Cr}_{1} \cdot 1 \cdot \mathrm{Cr}_{2} \cdot 34 \cdot \mathrm{SmA} \cdot 79 \cdot \mathrm{I}\left({ }^{\circ} \mathrm{C}\right)$

7-1.7 ( $x=1.7$; anionic species: $[\mathrm{FHF}]^{-}$and $\left.\left[\mathrm{F}(\mathrm{HF})_{2}\right]\right): \mathrm{Cr}_{1} \cdot 0 \cdot \mathrm{Cr}_{2} \cdot 32 \cdot \mathrm{SmA} \cdot 74 \cdot \mathrm{I}\left({ }^{\circ} \mathrm{C}\right)$

7-1.8 $\left(x=1.8\right.$; anionic species: $[\mathrm{FHF}]^{-}$and $\left.\left[\mathrm{F}(\mathrm{HF})_{2}\right]\right): \mathrm{Cr}_{1} \cdot-3 \cdot \mathrm{Cr}_{2} \cdot 29 \cdot \mathrm{SmA} \cdot 70 \cdot \mathrm{I}\left({ }^{\circ} \mathrm{C}\right)$

7-1.9 $\left(x=1.9\right.$; anionic species: $[\mathrm{FHF}]^{-}$and $\left.\left[\mathrm{F}(\mathrm{HF})_{2}\right]^{-}\right): \mathrm{Cr}_{1} \cdot-4 \cdot \mathrm{Cr}_{2} \cdot 23 \cdot \mathrm{SmA} \cdot 66 \cdot \mathrm{I}\left({ }^{\circ} \mathrm{C}\right)$

$$
\text { 7-2.0 ( } x=2.0): \mathrm{Cr}_{1} \cdot 3 \cdot \mathrm{Cr}_{2} \cdot 20 \cdot \mathrm{SmA} \cdot 62 \cdot \mathrm{I}\left({ }^{\circ} \mathrm{C}\right)
$$

7-2.1 $\left(x=2.1\right.$; anionic species: $\left[\mathrm{F}(\mathrm{HF})_{2}\right]^{-}$and $\left.\left[\mathrm{F}(\mathrm{HF})_{3}\right]^{-}\right): \mathrm{Cr}_{1} \cdot 3 \cdot \mathrm{Cr}_{2} \cdot 18 \cdot \mathrm{SmA} \cdot 57 \cdot \mathrm{I}\left({ }^{\circ} \mathrm{C}\right)$

7-2.2 $\left(x=2.2\right.$; anionic species: $\left[\mathrm{F}(\mathrm{HF})_{2}\right]^{-}$and $\left.\left[\mathrm{F}(\mathrm{HF})_{3}\right]^{-}\right): \mathrm{Cr}_{1} \cdot-8 \cdot \mathrm{Cr}_{2} \cdot 1 \cdot \mathrm{Cr}_{3} \cdot 15 \cdot \mathrm{SmA} \cdot 53 \cdot \mathrm{I}\left({ }^{\circ} \mathrm{C}\right)$

7-2.3 $\left(x=2.3\right.$; anionic species: $\left[\mathrm{F}(\mathrm{HF})_{2}\right]^{-}$and $\left.\left[\mathrm{F}(\mathrm{HF})_{3}\right]^{-}\right): \mathrm{Cr}_{1} \cdot-7 \cdot \mathrm{Cr}_{2} \cdot 2 \cdot \mathrm{Cr}_{3} \cdot 12 \cdot \mathrm{SmA} \cdot 49 \cdot \mathrm{I}\left({ }^{\circ} \mathrm{C}\right)$

8-1/1.7 ( $x=1.7$; anionic species: $[\mathrm{FHF}]^{-}$and $\left.\left[\mathrm{F}(\mathrm{HF})_{2}\right]^{-}\right): \mathrm{Cr} \cdot 1 \cdot \mathrm{I}\left({ }^{\circ} \mathrm{C}\right)$

8-2/1.7 ( $x=1.7$; anionic species: $[\mathrm{FHF}]^{-}$and $\left.\left[\mathrm{F}(\mathrm{HF})_{2}\right]^{-}\right)$: $\mathrm{g} \cdot-87 \cdot \mathrm{I}\left({ }^{\circ} \mathrm{C}\right)$

8-4/1.8 $\left(x=1.8\right.$; anionic species: $[\mathrm{FHF}]^{-}$and $\left.\left[\mathrm{F}(\mathrm{HF})_{2}\right]^{-}\right): \mathrm{Cr} \cdot 3 \cdot \mathrm{I}\left({ }^{\circ} \mathrm{C}\right)$

8-6/2.0 $(x=2.0): \mathrm{Cr} \cdot 53 \cdot \mathrm{SmX} \cdot 109 \cdot \mathrm{I}\left({ }^{\circ} \mathrm{C}\right)$

8-8/2.0 $(x=2.0): \mathrm{Cr} \cdot 76 \cdot \mathrm{SmX} \cdot\left[\right.$ not reported] $\cdot \operatorname{dec} .\left({ }^{\circ} \mathrm{C}\right)$

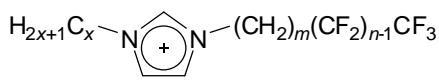

$1^{-}$

\section{$9-x / m / n$}

9-1/2/4: $\mathrm{Cr} \cdot 65 \cdot \mathrm{I}\left({ }^{\circ} \mathrm{C}\right)$

9-1/2/6: $\mathrm{Cr} \cdot 94 \cdot \mathrm{SmA} \cdot 224 \cdot \mathrm{I}\left({ }^{\circ} \mathrm{C}\right)$

9-1/3/6: $\mathrm{Cr} \cdot 86 \cdot \mathrm{SmA} \cdot 198 \cdot \mathrm{I}\left({ }^{\circ} \mathrm{C}\right)$

9-2/3/6: $\mathrm{Cr} \cdot 10 \cdot \mathrm{SmA} \cdot 68 \cdot \mathrm{I}\left({ }^{\circ} \mathrm{C}\right)$

9-3/3/6: $\mathrm{Cr} \cdot-7 \cdot \mathrm{I}\left({ }^{\circ} \mathrm{C}\right)$

9-4/2/4: $\mathrm{Cr} \cdot 71 \cdot \mathrm{I}\left({ }^{\circ} \mathrm{C}\right)$

9-6/2/8: $\mathrm{Cr} \cdot 92 \cdot \mathrm{SmA} \cdot 197 \cdot \mathrm{I}\left({ }^{\circ} \mathrm{C}\right)$

9-6/3/6: $\mathrm{Cr} \cdot-29 \cdot \mathrm{SmA} \cdot 37 \cdot \mathrm{I}\left({ }^{\circ} \mathrm{C}\right)$

9-8/3/6: $\mathrm{Cr} \cdot 48 \cdot \mathrm{SmA} \cdot 119 \cdot \mathrm{I}\left({ }^{\circ} \mathrm{C}\right)$

$(\mathbf{9 - 8} / \mathbf{3} / \mathbf{6}) / \mathrm{I}_{2}(4: 1): \mathrm{Cr} \cdot 27 \cdot \mathrm{SmA} \cdot 119 \cdot \mathrm{I}\left({ }^{\circ} \mathrm{C}\right)$

9-10/3/6: $\mathrm{Cr} \cdot 74 \cdot \mathrm{SmA} \cdot 154 \cdot \mathrm{I}\left({ }^{\circ} \mathrm{C}\right)$

9-12/3/6: $\mathrm{Cr} \cdot 77 \cdot \mathrm{SmA} \cdot 160 \cdot \mathrm{I}\left({ }^{\circ} \mathrm{C}\right)$ 


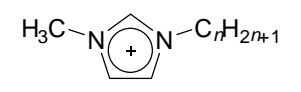

$\left[\mathrm{ZF}_{6}\right]^{-}$

10-P-n (Z = P: $\left.\left[\mathrm{PF}_{6}\right]^{-}\right)$

10-P-14: $\mathrm{Cr} \cdot 67^{92} / 74^{93} \cdot \mathrm{SmA} \cdot 73^{92} / 77^{93} \cdot \mathrm{I}\left({ }^{\circ} \mathrm{C}\right)$

10-P-16: $\mathrm{Cr} \cdot 75 \cdot \mathrm{SmA} \cdot 125 \cdot \mathrm{I}\left({ }^{\circ} \mathrm{C}\right)$

10-P-18: $\mathrm{Cr} \cdot 80 \cdot \mathrm{SmA} \cdot 164^{92} / 165^{93} \cdot \mathrm{I}\left({ }^{\circ} \mathrm{C}\right)$

10-As-n (Z = As: $\left.\left[\mathrm{AsF}_{6}\right]^{-}\right)$

10-As-14: $\mathrm{Cr} \cdot 65 \cdot \mathrm{I}\left({ }^{\circ} \mathrm{C}\right)$

10-As-16: $\mathrm{Cr} \cdot 75 \cdot \mathrm{SmA} \cdot 104 \cdot \mathrm{I}\left({ }^{\circ} \mathrm{C}\right)$

10-As-18: $\mathrm{Cr} \cdot 78 \cdot \mathrm{SmA} \cdot 143 \cdot \mathrm{I}\left({ }^{\circ} \mathrm{C}\right)$

10-Sb-n (Z = Sb: $\left.\left[\mathrm{SbF}_{6}\right]^{-}\right)$

10-Sb-14: $\mathrm{Cr} \cdot 57 \cdot \mathrm{I}\left({ }^{\circ} \mathrm{C}\right)$

10-Sb-16: $\mathrm{Cr} \cdot 68 \cdot \mathrm{SmA} \cdot 77 \cdot \mathrm{I}\left({ }^{\circ} \mathrm{C}\right)$

10-Sb-18: $\mathrm{Cr} \cdot 75 \cdot \mathrm{SmA} \cdot 118 \cdot \mathrm{I}\left({ }^{\circ} \mathrm{C}\right)$

10-Nb-n (Z = Nb: $\left.\left[\mathrm{NbF}_{6}\right]^{-}\right)$

10-Nb-14: $\mathrm{Cr} \cdot 56 \cdot \mathrm{I}\left({ }^{\circ} \mathrm{C}\right)$

10-Nb-16: $\mathrm{Cr} \cdot 67 \cdot \mathrm{SmA} \cdot 77 \cdot \mathrm{I}\left({ }^{\circ} \mathrm{C}\right)$

10-Nb-18: $\mathrm{Cr} \cdot 74 \cdot \mathrm{SmA} \cdot 117 \cdot \mathrm{I}\left({ }^{\circ} \mathrm{C}\right)$

10-Ta-n (Z = Ta: $\left.\left[\mathrm{TaF}_{6}\right]^{-}\right)$

10-Ta-14: $\mathrm{Cr} \cdot 56 \cdot \mathrm{I}\left({ }^{\circ} \mathrm{C}\right)$

10-Ta-16: $\mathrm{Cr} \cdot 66 \cdot \mathrm{SmA} \cdot 76 \cdot \mathrm{I}\left({ }^{\circ} \mathrm{C}\right)$

10-Ta-18: $\mathrm{Cr} \cdot 74 \cdot \mathrm{SmA} \cdot 115 \cdot \mathrm{I}\left({ }^{\circ} \mathrm{C}\right)$

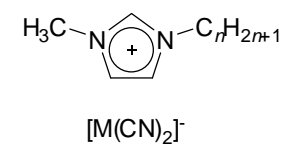

11-Ag(CN $)_{2}-\boldsymbol{n}\left(\mathrm{M}=\mathrm{Ag}^{+}\right)$

11-Ag(CN) $)_{2}-12: \mathrm{Cr} \cdot-17 \cdot \mathrm{SmA} \cdot\left[\right.$ not reported] $\cdot \mathrm{I}\left({ }^{\circ} \mathrm{C}\right)$

11-Ag(CN $)_{2}-14: \mathrm{Cr} \cdot 24 \cdot \mathrm{SmA} \cdot 38 \cdot \mathrm{I}\left({ }^{\circ} \mathrm{C}\right)$

11-Ag(CN $)_{2}-\mathbf{1 8}: \mathrm{Cr} \cdot 48 \cdot \mathrm{SmA} \cdot 129 \cdot \mathrm{I}\left({ }^{\circ} \mathrm{C}\right)$

11-Au(CN) $)_{2}$-n $\left(\mathrm{M}=\mathrm{Au}^{+}\right)$

11-Au(CN) $)_{2}$-12: $\mathrm{Cr} \cdot 18 \cdot \mathrm{SmA} \cdot\left[\right.$ not reported] $\cdot \mathrm{I}\left({ }^{\circ} \mathrm{C}\right)$

11-Au(CN) $)_{2}$-14: Cr $\cdot 23 \cdot \mathrm{SmA} \cdot\left[\right.$ not reported] $\cdot \mathrm{I}\left({ }^{\circ} \mathrm{C}\right)$

11-Au(CN) $)_{2} \mathbf{- 1 8}: \mathrm{Cr} \cdot 47 \cdot \mathrm{SmA} \cdot 106 \cdot \mathrm{I}\left({ }^{\circ} \mathrm{C}\right)$ 


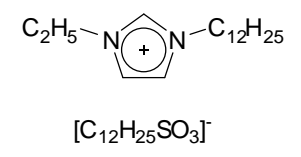

12: $\mathrm{Cr} \cdot 67 \cdot \mathrm{SmA} \cdot 121 \cdot \mathrm{I}\left({ }^{\circ} \mathrm{C}\right)$

12/LiBF 4 (95:5): $\mathrm{Cr}_{1} \cdot 30 \cdot \mathrm{SmX} \cdot 50 \cdot \mathrm{Cr}_{2} \cdot 65 \cdot \mathrm{SmA} \cdot 125 \cdot \mathrm{I}\left({ }^{\circ} \mathrm{C}\right)$

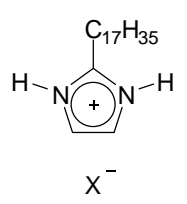

13-X $\left(\mathrm{X}^{-}=\left[\mathrm{BF}_{4}\right]^{-}\right.$,

$\left.\left[\mathrm{CH}_{3} \mathrm{SO}_{3}\right]^{-},[\mathrm{OTf}]^{-}\right)$

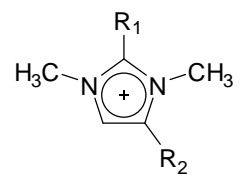

$\left[\mathrm{C}_{12} \mathrm{H}_{25} \mathrm{SO}_{3}\right]^{-}$

16- $\mathbf{R}_{\mathbf{1}} / \mathbf{R}_{\mathbf{2}}\left(\mathrm{R}_{1}, \mathrm{R}_{2}=\right.$

$\mathrm{H}$ or $\mathrm{CH}_{3}$ )

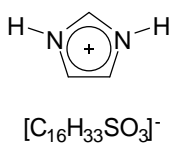

15

14

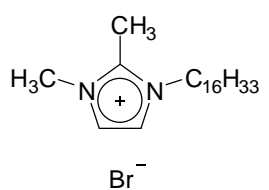

17

$$
\begin{aligned}
& \text { 13-BF } 4\left(\mathrm{X}^{-}=\left[\mathrm{BF}_{4}\right]^{-}\right): \mathrm{Cr} \cdot 91 \cdot \mathrm{SmA} \cdot 117 \cdot \mathrm{I}\left({ }^{\circ} \mathrm{C}\right) \\
& \text { 13- } \mathrm{CH}_{3} \mathrm{SO}_{3}\left(\mathrm{X}^{-}=\left[\mathrm{CH}_{3} \mathrm{SO}_{3}\right]^{-}\right): \mathrm{Cr} \cdot 80 \cdot \mathrm{I}\left({ }^{\circ} \mathrm{C}\right) \\
& \text { 13-OTf }\left(\mathrm{X}^{-}=[\mathrm{OTf}]^{-}\right): \mathrm{Cr} \cdot 82 \cdot \mathrm{I}\left({ }^{\circ} \mathrm{C}\right)
\end{aligned}
$$

14: $\mathrm{Cr} \cdot 92 \cdot \mathrm{I}\left({ }^{\circ} \mathrm{C}\right)$

15: $\mathrm{Cr} \cdot 100 \cdot \mathrm{SmA} \cdot 230 \cdot \mathrm{I}\left({ }^{\circ} \mathrm{C}\right)$

16-H/H $\left(\mathrm{R}_{1}=\mathrm{R}_{2}=\mathrm{H}\right): \mathrm{Cr} \cdot 90 \cdot \mathrm{SmA} \cdot 177 \cdot \mathrm{I}\left({ }^{\circ} \mathrm{C}\right)$

16- $\mathrm{CH}_{3} / \mathrm{H}\left(\mathrm{R}_{1}=\mathrm{CH}_{3}, \mathrm{R}_{2}=\mathrm{H}\right): \mathrm{Cr} \cdot 202 \cdot \mathrm{I}\left({ }^{\circ} \mathrm{C}\right)$

16-H/CH $\mathbf{C H}_{3}\left(\mathrm{R}_{1}=\mathrm{H}, \mathrm{R}_{2}=\mathrm{CH}_{3}\right): \mathrm{Cr} \cdot(41 \cdot \mathrm{SmA} \cdot) 95 \cdot \mathrm{I}\left({ }^{\circ} \mathrm{C}\right)$

17: $\mathrm{Cr} \cdot 90 \cdot \mathrm{SmA} \cdot 232 \cdot \mathrm{I}\left({ }^{\circ} \mathrm{C}\right)$ 


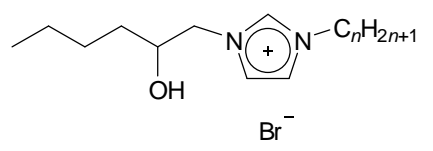

18-n

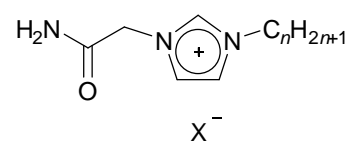

20-X-n $\left(\mathrm{X}^{-}=\mathrm{Br}^{-}\right.$, $\left.\left[\mathrm{BF}_{4}\right]^{-},\left[\mathrm{PF}_{6}\right]^{-}\right)$

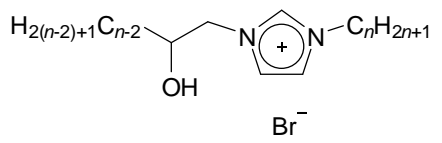

19-n

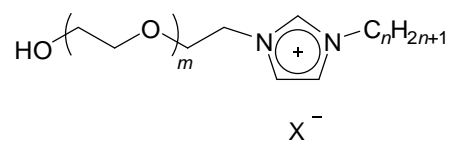

21-X-m/n $\left(\mathrm{X}^{-}=\mathrm{Cl}^{-},\left[\mathrm{NO}_{3}\right]^{-}\right.$,

$\left.\left[\mathrm{ClO}_{4}\right]^{-},\left[\mathrm{BF}_{4}\right]^{-},\left[\mathrm{PF}_{6}\right]^{-}\right)$

18-10: $\mathrm{Cr} \cdot<-20 \cdot \mathrm{SmA} \cdot 15 \cdot \mathrm{I}\left({ }^{\circ} \mathrm{C}\right)$

18-12: $\mathrm{Cr} \cdot-14 \cdot \mathrm{SmA} \cdot 56 \cdot \mathrm{I}\left({ }^{\circ} \mathrm{C}\right)$

18-14: $\mathrm{Cr} \cdot 9 \cdot \mathrm{SmA} \cdot 81 \cdot \mathrm{I}\left({ }^{\circ} \mathrm{C}\right)$

18-16: $\mathrm{Cr} \cdot 31 \cdot \mathrm{SmA} \cdot 118 \cdot \mathrm{I}\left({ }^{\circ} \mathrm{C}\right)$

18-18: $\mathrm{Cr} \cdot 45 \cdot \mathrm{SmA} \cdot 142 \cdot \mathrm{I}\left({ }^{\circ} \mathrm{C}\right)$

19-10: $\mathrm{Cr} \cdot<-20 \cdot \mathrm{SmA} \cdot 144 \cdot \mathrm{I}\left({ }^{\circ} \mathrm{C}\right)$

19-12: $\mathrm{Cr} \cdot-4 \cdot \mathrm{SmA} \cdot 185 \cdot \mathrm{I}\left({ }^{\circ} \mathrm{C}\right)$

19-14: $\mathrm{Cr} \cdot 68 \cdot \mathrm{SmA} \cdot 207 \cdot \mathrm{I}\left({ }^{\circ} \mathrm{C}\right)$

19-16: $\mathrm{Cr} \cdot 75 \cdot \mathrm{SmA} \cdot 207 \cdot \mathrm{I}\left({ }^{\circ} \mathrm{C}\right)$

19-18: $\mathrm{Cr} \cdot 75 \cdot \mathrm{SmA} \cdot 209 \cdot \mathrm{I}\left({ }^{\circ} \mathrm{C}\right)$

$$
\text { 20-Br-n }\left(\mathrm{X}^{-}=\mathrm{Br}^{-}\right)
$$

20-Br-10: $\mathrm{Cr} \cdot 123 \cdot \mathrm{SmA} \cdot 197 \cdot \mathrm{I}\left({ }^{\circ} \mathrm{C}\right)$

20-Br-12: $\mathrm{Cr} \cdot 125 \cdot \mathrm{SmA} \cdot 250 \cdot \mathrm{I} / \mathrm{dec} .\left({ }^{\circ} \mathrm{C}\right)$

20-Br-14: $\mathrm{Cr} \cdot 124 \cdot \mathrm{SmA} \cdot 287 \cdot \mathrm{I} /$ dec. $\left({ }^{\circ} \mathrm{C}\right)$

20-Br-16: $\mathrm{Cr} \cdot 126 \cdot \mathrm{SmA} \cdot 287 \cdot \mathrm{I} / \mathrm{dec} .\left({ }^{\circ} \mathrm{C}\right)$

20-Br-18: $\mathrm{Cr} \cdot 129 \cdot \mathrm{SmA} \cdot 287 \cdot \mathrm{I} / \mathrm{dec} .\left({ }^{\circ} \mathrm{C}\right)$

$$
\text { 20-BF } \mathbf{H}_{4} \boldsymbol{n}\left(\mathrm{X}^{-}=\left[\mathrm{BF}_{4}\right]^{-}\right)
$$

20-BF ${ }_{4}-10: \mathrm{Cr} \cdot 90 \cdot \mathrm{SmA} \cdot 120 \cdot \mathrm{I}\left({ }^{\circ} \mathrm{C}\right)$

20- $\mathbf{B F}_{4}-\mathbf{1 2}: \mathrm{Cr} \cdot 94 \cdot \mathrm{SmA} \cdot 206 \cdot \mathrm{I} / \mathrm{dec} .\left({ }^{\circ} \mathrm{C}\right)$

20- $\mathrm{BF}_{4}-14: \mathrm{Cr} \cdot 100 \cdot \mathrm{SmA} \cdot 233 \cdot \mathrm{I} /$ dec. $\left({ }^{\circ} \mathrm{C}\right)$

20- $\mathbf{B F}_{4}-16: \mathrm{Cr} \cdot 104 \cdot \mathrm{SmA} \cdot 230 \cdot \mathrm{I} /$ dec. $\left({ }^{\circ} \mathrm{C}\right)$

20- $\mathbf{B F}_{4}-\mathbf{1 8}: \mathrm{Cr} \cdot 107 \cdot \mathrm{SmA} \cdot 243 \cdot \mathrm{I} /$ dec. $\left({ }^{\circ} \mathrm{C}\right)$

20-PF ${ }_{6}-\boldsymbol{n}\left(\mathrm{X}^{-}=\left[\mathrm{PF}_{6}\right]^{-}\right)$

20- $\mathbf{P F}_{\mathbf{6}}-\mathbf{1 0}: \mathrm{Cr} \cdot 113 \cdot \mathrm{I}\left({ }^{\circ} \mathrm{C}\right)$

20- $\mathrm{PF}_{6}$-12: $\mathrm{Cr} \cdot 115 \cdot \mathrm{SmA} \cdot 162 \cdot \mathrm{I}\left({ }^{\circ} \mathrm{C}\right)$

20- $\mathbf{P F}_{6} \mathbf{- 1 4}: \mathrm{Cr} \cdot 116 \cdot \mathrm{SmA} \cdot 200 \cdot \mathrm{I} / \mathrm{dec} .\left({ }^{\circ} \mathrm{C}\right)$ 
20- $\mathbf{P F}_{\mathbf{6}} \mathbf{- 1 6}: \mathrm{Cr} \cdot 119 \cdot \mathrm{SmA} \cdot 220 \cdot \mathrm{I} /$ dec. $\left({ }^{\circ} \mathrm{C}\right)$

20- $\mathbf{P F}_{\mathbf{6}}$-18: $\mathrm{Cr} \cdot 119 \cdot \mathrm{SmA} \cdot 240 \cdot \mathrm{I} /$ dec. $\left({ }^{\circ} \mathrm{C}\right)$

21-Cl-m/n $\left(\mathrm{X}^{-}=\mathrm{Cl}^{-}\right)$

21-Cl-0/10: $\mathrm{SmA} \cdot 27 \cdot \mathrm{I}\left({ }^{\circ} \mathrm{C}\right)$

21-Cl-0/12: $\mathrm{SmA} \cdot 140 \cdot \mathrm{I}\left({ }^{\circ} \mathrm{C}\right)$

21-Cl-0/14: $\mathrm{Cr} \cdot 25 \cdot \mathrm{SmA} \cdot 223 \cdot \mathrm{I} /$ dec. $\left({ }^{\circ} \mathrm{C}\right)$

21-Cl-0/16: $\mathrm{Cr} \cdot 44 \cdot \mathrm{SmA} \cdot 260 \cdot \mathrm{I} /$ dec. $\left({ }^{\circ} \mathrm{C}\right)$

21-Cl-0/18: $\mathrm{Cr} \cdot 57 \cdot \mathrm{SmA} \cdot 273 \cdot \mathrm{I} /$ dec. $\left({ }^{\circ} \mathrm{C}\right)$

21-Cl-1/10: liquid at r.t. (no LC mesophase detected)

21-Cl-1/12: $\mathrm{SmA} \cdot 25 \cdot \mathrm{I}\left({ }^{\circ} \mathrm{C}\right)$

21-Cl-1/14: SmA $\cdot 85 \cdot \mathrm{I}\left({ }^{\circ} \mathrm{C}\right)$

21-Cl-1/16: $\mathrm{Cr} \cdot 40 \cdot \mathrm{SmA} \cdot 169 \cdot \mathrm{I}\left({ }^{\circ} \mathrm{C}\right)$

21-Cl-1/18: $\mathrm{Cr} \cdot 54 \cdot \mathrm{SmA} \cdot 228 \cdot \mathrm{I} /$ dec. $\left({ }^{\circ} \mathrm{C}\right)$

21-Cl-2/14: $\mathrm{Cr} \cdot 10 \cdot \mathrm{I}\left({ }^{\circ} \mathrm{C}\right)$

21-Cl-2/16: $\mathrm{Cr} \cdot 33 \cdot \mathrm{SmA} \cdot 74 \cdot \mathrm{I}\left({ }^{\circ} \mathrm{C}\right)$

21-Cl-2/18: $\mathrm{Cr} \cdot 49 \cdot \mathrm{SmA} \cdot 142 \cdot \mathrm{I}\left({ }^{\circ} \mathrm{C}\right)$

21- $\mathrm{NO}_{3}-\mathbf{- 0} / \boldsymbol{n}\left(\mathrm{X}^{-}=\left[\mathrm{NO}_{3}\right]^{-}, m=0\right)$

21- $\mathrm{NO}_{3}-\mathbf{0} / 12: \mathrm{SmA} \cdot 99 \cdot \mathrm{I}\left({ }^{\circ} \mathrm{C}\right)$

21-NO $\mathbf{3}_{3} \mathbf{- 0 / 1 4}: \mathrm{Cr} \cdot 25 \cdot \mathrm{SmA} \cdot 194 \cdot \mathrm{I}\left({ }^{\circ} \mathrm{C}\right)$

21-NO $\mathbf{H}_{3} \mathbf{- 0 / 1 6}: \mathrm{Cr} \cdot 43 \cdot \mathrm{SmA} \cdot 229 \cdot \mathrm{I}\left({ }^{\circ} \mathrm{C}\right)$

21-NO $\mathbf{N}_{3} \mathbf{- 0 / 1 8} \mathrm{Cr} \cdot 57 \cdot \mathrm{SmA} \cdot 267 \cdot \mathrm{I}\left({ }^{\circ} \mathrm{C}\right)$

21- $\mathrm{ClO}_{4}-\mathbf{0} / \boldsymbol{n}\left(\mathrm{X}^{-}=\left[\mathrm{ClO}_{4}\right]^{-}, m=0\right)$

21-ClO ${ }_{4}^{-0 / 12: ~} \mathrm{SmA} \cdot 72 \cdot \mathrm{I}\left({ }^{\circ} \mathrm{C}\right)$

21-ClO $-\mathbf{- 0} / 14: \mathrm{Cr} \cdot 29 \cdot \mathrm{SmA} \cdot 142 \cdot \mathrm{I}\left({ }^{\circ} \mathrm{C}\right)$

21-ClO $-\mathbf{- 0 / 1 6}: \mathrm{Cr} \cdot 47 \cdot \mathrm{SmA} \cdot 199 \cdot \mathrm{I}\left({ }^{\circ} \mathrm{C}\right)$

21-ClO $\mathbf{4}^{-\mathbf{0}} / \mathbf{1 8}: \mathrm{Cr} \cdot 60 \cdot \mathrm{SmA} \cdot 238 \cdot \mathrm{I}\left({ }^{\circ} \mathrm{C}\right)$

21-BF ${ }_{4}^{-0 / n}\left(\mathrm{X}^{-}=\left[\mathrm{BF}_{4}\right]^{-}, m=0\right)$

21-BF $-\mathbf{- 0} / \mathbf{1 2}: \mathrm{SmA} \cdot 69 \cdot \mathrm{I}\left({ }^{\circ} \mathrm{C}\right)$

21-BF $\mathbf{4}_{\mathbf{4}} \mathbf{0 / 1 4}: \mathrm{Cr} \cdot 31 \cdot \mathrm{SmA} \cdot 146 \cdot \mathrm{I}\left({ }^{\circ} \mathrm{C}\right)$

21-BF $\mathbf{4}_{\mathbf{4}} \mathbf{- 0 / 1 6}: \mathrm{Cr} \cdot 47 \cdot \mathrm{SmA} \cdot 220 \cdot \mathrm{I}\left({ }^{\circ} \mathrm{C}\right)$

21-BF $\mathbf{4}_{\mathbf{- 1}} \mathbf{0 / 1 8}: \mathrm{Cr} \cdot 60 \cdot \mathrm{SmA} \cdot 248 \cdot \mathrm{I}\left({ }^{\circ} \mathrm{C}\right)$

21- $\mathbf{P F}_{\mathbf{6}} \mathbf{- 0 / n}\left(\mathrm{X}^{-}=\left[\mathrm{PF}_{6}\right]^{-}, m=0\right)$

21-PF $-\mathbf{6}-\mathbf{0}$ 12: $\mathrm{SmA} \cdot 45 \cdot \mathrm{I}\left({ }^{\circ} \mathrm{C}\right)$

21-PF $\mathbf{6}_{\mathbf{6}} \mathbf{- 0 / 1 4} \mathrm{Cr} \cdot 43 \cdot \mathrm{SmA} \cdot 106 \cdot \mathrm{I}\left({ }^{\circ} \mathrm{C}\right)$

21-PF $\mathbf{6}_{\mathbf{6}} \mathbf{- 0 / 1 6}: \mathrm{Cr} \cdot 48 \cdot \mathrm{SmA} \cdot 165 \cdot \mathrm{I}\left({ }^{\circ} \mathrm{C}\right)$

21-PF $\mathbf{6}_{\mathbf{6}} \mathbf{- 0 / 1 8} \mathrm{Cr} \cdot 61 \cdot \mathrm{SmA} \cdot 199 \cdot \mathrm{I}\left({ }^{\circ} \mathrm{C}\right)$

21-Cl-0/16 / $\mathrm{LiCl}(1: 0.5): \mathrm{Cr} \cdot 47 \cdot \mathrm{SmA} \cdot 300 \cdot \mathrm{I} /$ dec. $\left({ }^{\circ} \mathrm{C}\right)$ 
21-Cl-1/16 / $\mathrm{LiCl}$ (1:0.5): $\mathrm{Cr} \cdot 40 \cdot \mathrm{SmA} \cdot 240 \cdot \mathrm{I} /$ dec. $\left({ }^{\circ} \mathrm{C}\right)$

21-Cl-2/16 / $\mathrm{LiCl}(1: 0.5): \mathrm{Cr} \cdot 31 \cdot \mathrm{SmA} \cdot 128 \cdot \mathrm{I}\left({ }^{\circ} \mathrm{C}\right)$

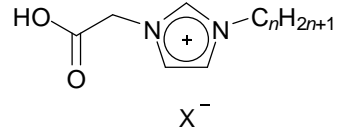

$x^{-}$

22-X-n $\left(\mathrm{X}^{-}=\mathrm{Cl}^{-}, \mathrm{Br}^{-},\left[\mathrm{BF}_{4}\right]^{-},\left[\mathrm{PF}_{6}\right]^{-}\right)$

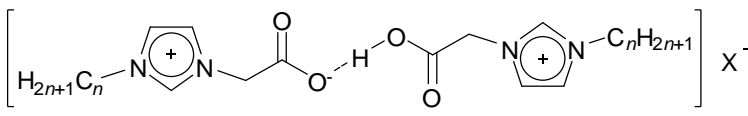

24-X-n $\left(\mathrm{X}^{-}=\mathrm{Br}^{-},\left[\mathrm{NO}_{3}\right]^{-}\right)$

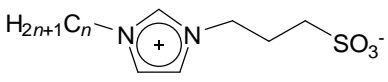

26-n

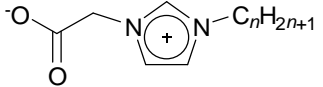

23-n

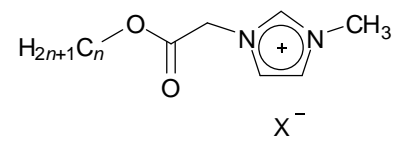

25-X-n $\left(\mathrm{X}^{-}=\mathrm{Br}^{-},\left[\mathrm{BF}_{4}\right]^{-},\left[\mathrm{PF}_{6}\right]^{-}\right)$

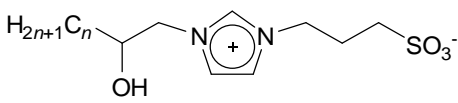

27-n

22-Cl-n $\left(\mathrm{X}^{-}=\mathrm{Cl}^{-}\right)$

22-Cl-10: $\mathrm{Cr}_{1} \cdot 79 \cdot \mathrm{Cr}_{2} \cdot 130 \cdot \mathrm{I}\left({ }^{\circ} \mathrm{C}\right)$

22-Cl-12: $\mathrm{Cr} \cdot 136 \cdot \mathrm{SmA} \cdot 238 \cdot \mathrm{I}\left({ }^{\circ} \mathrm{C}\right)$

22-Cl-14: $\mathrm{Cr} \cdot 134 \cdot \mathrm{SmA} \cdot 245 \cdot \mathrm{I} / \mathrm{dec} .\left({ }^{\circ} \mathrm{C}\right)$

22-Cl-16: $\mathrm{Cr} \cdot 135 \cdot \mathrm{SmA} \cdot 259 \cdot \mathrm{I} / \mathrm{dec} .\left({ }^{\circ} \mathrm{C}\right)$

22-Cl-18: $\mathrm{Cr} \cdot 134 \cdot \mathrm{SmA} \cdot 265 \cdot \mathrm{I} / \mathrm{dec} .\left({ }^{\circ} \mathrm{C}\right)$

$$
\text { 22-Br-n }\left(\mathrm{X}^{-}=\mathrm{Br}^{-}\right)
$$

22-Br-10: $\mathrm{Cr}_{1} \cdot 102 \cdot \mathrm{Cr}_{2} \cdot 130 \cdot \mathrm{I}\left({ }^{\circ} \mathrm{C}\right)$

22-Br-12: $\mathrm{Cr}_{1} \cdot 123 \cdot \mathrm{Cr}_{2} \cdot 135 \cdot \mathrm{SmA} \cdot 224 \cdot \mathrm{I}\left({ }^{\circ} \mathrm{C}\right)$

22-Br-14: $\mathrm{Cr}_{1} \cdot 126 \cdot \mathrm{Cr}_{2} \cdot 136 \cdot \mathrm{SmA} \cdot 231 \cdot \mathrm{I} /$ dec. $\left({ }^{\circ} \mathrm{C}\right)$

22-Br-16: $\mathrm{Cr}_{1} \cdot 128 \cdot \mathrm{Cr}_{2} \cdot 135 \cdot \mathrm{SmA} \cdot 240 \cdot \mathrm{I} /$ dec. $\left({ }^{\circ} \mathrm{C}\right)$

22-Br-18: $\mathrm{Cr}_{1} \cdot 129 \cdot \mathrm{Cr}_{2} \cdot 135 \cdot \mathrm{SmA} \cdot 252 \cdot \mathrm{I} /$ dec. $\left({ }^{\circ} \mathrm{C}\right)$

22-BF $-\boldsymbol{n}\left(\mathrm{X}^{-}=\left[\mathrm{BF}_{4}\right]^{-}\right)$

22-BF 4 -10: $\mathrm{SmA} \cdot 85 \cdot \mathrm{I}\left({ }^{\circ} \mathrm{C}\right)$

22-BF ${ }_{4}-12: \mathrm{Cr} \cdot 61 \cdot \mathrm{SmA} \cdot 188 \cdot \mathrm{I} / \mathrm{dec} .\left({ }^{\circ} \mathrm{C}\right)$

22-BF ${ }_{4}-14: \mathrm{Cr} \cdot 66 \cdot \mathrm{SmA} \cdot 210 \cdot \mathrm{I} /$ dec. $\left({ }^{\circ} \mathrm{C}\right)$

22-BF ${ }_{4}-16: \mathrm{Cr} \cdot 70 \cdot \mathrm{SmA} \cdot 210 \cdot \mathrm{I} / \mathrm{dec} .\left({ }^{\circ} \mathrm{C}\right)$

22-BF 4 -18: $\mathrm{Cr} \cdot 63 \cdot \mathrm{SmA} \cdot 210 \cdot \mathrm{I} /$ dec. $\left({ }^{\circ} \mathrm{C}\right)$

22-PF $-\boldsymbol{n}\left(\mathrm{X}^{-}=\left[\mathrm{PF}_{6}\right]^{-}\right)$

22-PF -10 : not LC

22-PF -12 : not LC 
22-PF $-14:$ not LC

22-PF $\mathbf{6}_{\mathbf{6}}$ 16: not LC

22-PF $\mathbf{6}-18$ : not LC

23-10: $\mathrm{Cr}_{1} \cdot 29 \cdot \mathrm{Cr}_{2} \cdot 39 \cdot \mathrm{SmA} \cdot 96 \cdot \mathrm{I}\left({ }^{\circ} \mathrm{C}\right)$

23-12: $\mathrm{Cr}_{1} \cdot 43 \cdot \mathrm{Cr}_{2} \cdot 104 \cdot \mathrm{SmA} \cdot 187 \cdot \mathrm{I} /$ dec. $\left({ }^{\circ} \mathrm{C}\right)$

23-14: $\mathrm{Cr}_{1} \cdot 54 \cdot \mathrm{Cr}_{2} \cdot 111 \cdot \mathrm{SmA} \cdot 214 \cdot \mathrm{I} /$ dec. $\left({ }^{\circ} \mathrm{C}\right)$

23-16: $\mathrm{Cr}_{1} \cdot 66 \cdot \mathrm{Cr}_{2} \cdot 120 \cdot \mathrm{SmA} \cdot 234 \cdot \mathrm{I} /$ dec. $\left({ }^{\circ} \mathrm{C}\right)$

23-18: $\mathrm{Cr}_{1} \cdot 70 \cdot \mathrm{Cr}_{2} \cdot 122 \cdot \mathrm{SmA} \cdot 231 \cdot \mathrm{I} /$ dec. $\left({ }^{\circ} \mathrm{C}\right)$

23-10/LiBF 4 (3:1): soft phase $\cdot 32 \cdot \mathrm{SmA} \cdot 105 \cdot \mathrm{I}\left({ }^{\circ} \mathrm{C}\right)$

23-10/LiBF 4 (4:1): soft phase $\cdot 30 \cdot \mathrm{SmA} \cdot 95 \cdot \mathrm{I}\left({ }^{\circ} \mathrm{C}\right)$

23-10/LiBF 4 (6:1): soft phase $\cdot 24 \cdot \mathrm{SmA} \cdot 94 \cdot \mathrm{I}\left({ }^{\circ} \mathrm{C}\right)$

23-10/ $\mathrm{LiClO}_{4}$ (3:1): soft phase $\cdot 32 \cdot \mathrm{SmA} \cdot 136 \cdot \mathrm{I}\left({ }^{\circ} \mathrm{C}\right)$

23-10/ $\mathrm{LiClO}_{4}$ (4:1): soft phase $\cdot 15 \cdot \mathrm{SmA} \cdot 104 \cdot \mathrm{I}\left({ }^{\circ} \mathrm{C}\right)$

23-10/ $\mathrm{LiClO}_{4}(6: 1): \mathrm{SmA} \cdot 98 \cdot \mathrm{I}\left({ }^{\circ} \mathrm{C}\right)$

23-10/LiNTf 2 (3:1): soft phase $\cdot 19 \cdot \mathrm{SmX} \cdot 52 \cdot \mathrm{SmA} \cdot 88 \cdot \mathrm{I}\left({ }^{\circ} \mathrm{C}\right)$

23-10/ $\mathrm{CH}_{3} \mathrm{COOLi}(3: 1)$ : soft phase $\cdot 40 \cdot \mathrm{SmA} \cdot 143 \cdot \mathrm{I}\left({ }^{\circ} \mathrm{C}\right)$

24-Br-n $\left(\mathrm{X}^{-}=\mathrm{Br}^{-}\right)$

24-Br-10: $x$

24-Br-12: $\mathrm{Cr} \cdot 38 \cdot \mathrm{SmA} \cdot 234 \cdot \mathrm{I} /$ dec. $\left({ }^{\circ} \mathrm{C}\right)$

24-Br-14: $\mathrm{Cr} \cdot 67 \cdot \mathrm{SmA} \cdot 256 \cdot \mathrm{I} /$ dec. $\left({ }^{\circ} \mathrm{C}\right)$

24-Br-16: $\mathrm{Cr} \cdot 89 \cdot \mathrm{SmA} \cdot 261 \cdot \mathrm{I} /$ dec. $\left({ }^{\circ} \mathrm{C}\right)$

24-Br-18: $\mathrm{Cr} \cdot 96 \cdot \mathrm{SmA} \cdot 265 \cdot \mathrm{I} /$ dec. $\left({ }^{\circ} \mathrm{C}\right)$

$$
\text { 24- } \mathrm{NO}_{3}-\boldsymbol{n}\left(\mathrm{X}^{-}=\left[\mathrm{NO}_{3}\right]^{-}\right)
$$

$$
\text { 24-NO } \mathbf{N}_{3}-10 \text { : } \mathrm{x}
$$

24-NO $\mathbf{N}_{3} \mathbf{- 1 2}: \mathrm{Cr} \cdot 39 \cdot \mathrm{SmA} \cdot 173 \cdot \mathrm{I} /$ dec. $\left({ }^{\circ} \mathrm{C}\right)$

24-NO $\mathbf{N}_{3}$-14: $\mathrm{Cr} \cdot 47 \cdot \mathrm{SmA} \cdot 180 \cdot \mathrm{I} /$ dec. $\left({ }^{\circ} \mathrm{C}\right)$

24-NO $\mathbf{N}_{3}$-16: $\mathrm{Cr} \cdot 61 \cdot \mathrm{SmA} \cdot 188 \cdot \mathrm{I} /$ dec. $\left({ }^{\circ} \mathrm{C}\right)$

24-NO $\mathbf{N}_{3}$-18: $\mathrm{Cr} \cdot 68 \cdot \mathrm{SmA} \cdot 193 \cdot \mathrm{I} /$ dec. $\left({ }^{\circ} \mathrm{C}\right)$

$$
\begin{gathered}
\text { 25-Br-n }\left(\mathrm{X}^{-}=\mathrm{Br}^{-}\right) \\
\text {25-Br-8: } \mathrm{SmA} \cdot 76 \cdot \mathrm{I}\left({ }^{\circ} \mathrm{C}\right) \\
\text { 25-Br-10: } \mathrm{SmA} \cdot 137 \cdot \mathrm{I}\left({ }^{\circ} \mathrm{C}\right) \\
\text { 25-Br-12: } \mathrm{SmA} \cdot 162 \cdot \mathrm{I}\left({ }^{\circ} \mathrm{C}\right)
\end{gathered}
$$

25-Br-14: $\mathrm{Cr} \cdot 82 \cdot \mathrm{SmA} \cdot 228 \cdot \mathrm{I} /$ dec. $\left({ }^{\circ} \mathrm{C}\right)$

25-Br-16: $\mathrm{Cr} \cdot 89 \cdot \mathrm{SmA} \cdot 230 \cdot \mathrm{I} /$ dec. $\left({ }^{\circ} \mathrm{C}\right)$

25-Br-18: $\mathrm{Cr}_{1} \cdot 66 \cdot \mathrm{Cr}_{2} \cdot 91 \cdot \mathrm{SmA} \cdot 229 \cdot \mathrm{I} /$ dec. $\left({ }^{\circ} \mathrm{C}\right)$

25-BF $\mathbf{H}^{-} \boldsymbol{n}\left(\mathrm{X}^{-}=\left[\mathrm{BF}_{4}\right]^{-}\right)$ 
25-BF ${ }_{4}-12: \mathrm{Cr} \cdot 52 \cdot \mathrm{SmA} \cdot 164 \cdot \mathrm{I}\left({ }^{\circ} \mathrm{C}\right)$

25-BF ${ }_{4}-14: \mathrm{Cr} \cdot 63 \cdot \mathrm{SmA} \cdot 212 \cdot \mathrm{I}\left({ }^{\circ} \mathrm{C}\right)$

25-BF 4 -16: $\mathrm{Cr} \cdot 73 \cdot \mathrm{SmA} \cdot 246 \cdot \mathrm{I}\left({ }^{\circ} \mathrm{C}\right)$

25-PF $\mathbf{6}_{6}-\boldsymbol{n}\left(\mathrm{X}^{-}=\left[\mathrm{PF}_{6}\right]^{-}\right)$

25-PF $\mathbf{P}_{\mathbf{6}} \mathbf{- 1 2 :} \mathrm{Cr} \cdot 53 \cdot \mathrm{SmA} \cdot 121 \cdot \mathrm{I}\left({ }^{\circ} \mathrm{C}\right)$

25-PF $\mathbf{P}_{\mathbf{6}}-\mathbf{1 4 :} \mathrm{Cr} \cdot 62 \cdot \mathrm{SmA} \cdot 169 \cdot \mathrm{I}\left({ }^{\circ} \mathrm{C}\right)$

25-PF ${ }_{6}-16: \mathrm{Cr} \cdot 70 \cdot \mathrm{SmA} \cdot 205 \cdot \mathrm{I}\left({ }^{\circ} \mathrm{C}\right)$

26-12: $\mathrm{Cr} \cdot 85 \cdot \mathrm{SmA} \cdot 139 \cdot \mathrm{I}\left({ }^{\circ} \mathrm{C}\right)$

26-14: $\mathrm{M}_{1} \cdot 11 \cdot \mathrm{M}_{2} \cdot 74 \cdot \mathrm{SmA} \cdot 220 \cdot \mathrm{I} / \mathrm{dec} .\left({ }^{\circ} \mathrm{C}\right)$

26-16: $\mathrm{Cr} \cdot 40 \cdot \mathrm{M} \cdot 82 \cdot \mathrm{SmA} \cdot 250 \cdot \mathrm{I} / \mathrm{dec} .\left({ }^{\circ} \mathrm{C}\right)$

26-18: $\mathrm{Cr} \cdot 53 \cdot \mathrm{M} \cdot 89 \cdot \mathrm{SmA} \cdot 270 \cdot \mathrm{I} / \mathrm{dec} .\left({ }^{\circ} \mathrm{C}\right)$

26-12/ $\mathrm{LiClO}_{4}(6: 1): \mathrm{SmA} \cdot 147 \cdot \mathrm{I}\left({ }^{\circ} \mathrm{C}\right)$

26-14/ $\mathrm{LiClO}_{4}(6: 1): \mathrm{Cr} \cdot 20 \cdot \mathrm{SmA} \cdot 258 \cdot \mathrm{I}\left({ }^{\circ} \mathrm{C}\right)$

26-16/ $\mathrm{LiClO}_{4}(6: 1): \mathrm{Cr} \cdot 43 \cdot \mathrm{SmA} \cdot 275 \cdot \mathrm{I} /$ dec. $\left({ }^{\circ} \mathrm{C}\right)$

26-18/ $\mathrm{LiClO}_{4}(6: 1): \mathrm{Cr} \cdot 59 \cdot \mathrm{SmA} \cdot 295 \cdot \mathrm{I} /$ dec. $\left({ }^{\circ} \mathrm{C}\right)$

27-10: $\mathrm{Cr} \cdot(\mathrm{SmA} \cdot 122 \cdot) 150 \cdot \mathrm{I}\left({ }^{\circ} \mathrm{C}\right)$

27-12: $\mathrm{Cr} \cdot 154 \cdot \mathrm{SmA} \cdot 206 \cdot \mathrm{I}\left({ }^{\circ} \mathrm{C}\right)$

27-14: $\mathrm{Cr} \cdot 155 \cdot \mathrm{SmA} \cdot 250 \cdot \mathrm{I} /$ dec. $\left({ }^{\circ} \mathrm{C}\right)$

27-16: $\mathrm{Cr} \cdot 155 \cdot \mathrm{SmA} \cdot 260 \cdot \mathrm{I} / \mathrm{dec} .\left({ }^{\circ} \mathrm{C}\right)$

27-18: $\mathrm{Cr} \cdot 156 \cdot \mathrm{SmA} \cdot 270 \cdot \mathrm{I} / \mathrm{dec} .\left({ }^{\circ} \mathrm{C}\right)$

27-10/ $\mathrm{LiClO}_{4}(6: 1): \mathrm{M} \cdot(\mathrm{SmA} \cdot 81 \cdot) 135 \cdot \mathrm{I}\left({ }^{\circ} \mathrm{C}\right)$

27-12/ $/ \mathrm{LClO}_{4}(6: 1): \mathrm{M} \cdot 143 \cdot \mathrm{SmA} \cdot 213 \cdot \mathrm{I}\left({ }^{\circ} \mathrm{C}\right)$

27-14/ $\mathrm{LiClO}_{4}(6: 1): \mathrm{Cr} \cdot 25 \cdot \mathrm{M} \cdot 138 \cdot \mathrm{SmA} \cdot 255 \cdot \mathrm{I} / \mathrm{dec} .\left({ }^{\circ} \mathrm{C}\right)$

27-16/ $\mathrm{LiClO}_{4}(6: 1): \mathrm{Cr} \cdot 39 \cdot \mathrm{M} \cdot 148 \cdot \mathrm{SmA} \cdot 275 \cdot \mathrm{I} / \mathrm{dec} .\left({ }^{\circ} \mathrm{C}\right)$

27-18/ $\mathrm{LiClO}_{4}$ (6:1): $\mathrm{Cr} \cdot 57 \cdot \mathrm{M} \cdot 156 \cdot \mathrm{SmA} \cdot 295 \cdot \mathrm{I} / \mathrm{dec} .\left({ }^{\circ} \mathrm{C}\right)$

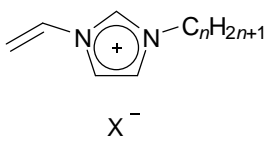

28-Br-n $\left(\mathrm{X}^{-}=\mathrm{Br}^{-}\right)$

28-Br-12· $\mathrm{H}_{2} \mathrm{O}: \mathrm{Cr} \cdot 42 \cdot \mathrm{I}\left({ }^{\circ} \mathrm{C}\right)$

28-Br-14 $\cdot \mathrm{H}_{2} \mathrm{O}: \mathrm{Cr} \cdot 60 \cdot \mathrm{SmA} \cdot 99 \cdot \mathrm{I}\left({ }^{\circ} \mathrm{C}\right)$

28-Br-16· $\mathrm{H}_{2} \mathrm{O}: \mathrm{Cr} \cdot 67 \cdot \mathrm{SmA} \cdot 158 \cdot \mathrm{I}\left({ }^{\circ} \mathrm{C}\right)$

28-Br-18 $\cdot \mathrm{H}_{2} \mathrm{O}: \mathrm{Cr} \cdot 71 \cdot \mathrm{SmA} \cdot 198 \cdot \mathrm{I} / \mathrm{dec} .\left({ }^{\circ} \mathrm{C}\right)$

28-I-n $\left(\mathrm{X}^{-}=\mathrm{I}^{-}\right)$

28-I-12: $\mathrm{Cr}_{1} \cdot 35 \cdot \mathrm{Cr}_{2} \cdot 51 \cdot \mathrm{I}\left({ }^{\circ} \mathrm{C}\right)$ 
28-I-14: $\mathrm{Cr}_{1} \cdot 35 \cdot \mathrm{Cr}_{2} \cdot 61 \cdot \mathrm{SmA} \cdot 91 \cdot \mathrm{I}\left({ }^{\circ} \mathrm{C}\right)$

28-I-16: $\mathrm{Cr}_{1} \cdot 41 \cdot \mathrm{Cr}_{2} \cdot 64 \cdot \mathrm{SmA} \cdot 144 \cdot \mathrm{I}\left({ }^{\circ} \mathrm{C}\right)$

28-I-18: $\mathrm{Cr} \cdot 74 \cdot \mathrm{SmA} \cdot 186 \cdot \mathrm{I}\left({ }^{\circ} \mathrm{C}\right)$

$$
\text { 28-BF } \mathbf{H}_{4} \boldsymbol{n}\left(\mathrm{X}^{-}=\left[\mathrm{BF}_{4}\right]^{-}\right)
$$

28- $\mathrm{BF}_{4}-\mathbf{1 2}: \mathrm{Cr} \cdot 36 \cdot \mathrm{I}\left({ }^{\circ} \mathrm{C}\right)$

28-BF ${ }_{4}-14: \mathrm{Cr} \cdot 53 \cdot \mathrm{SmA} \cdot 62 \cdot \mathrm{I}\left({ }^{\circ} \mathrm{C}\right)$

28- $\mathrm{BF}_{4}-\mathbf{1 4} / \mathrm{LiBF}_{4}(1: 0.1): \mathrm{Cr} \cdot 42 \cdot \mathrm{SmA} \cdot 60 \cdot \mathrm{I}\left({ }^{\circ} \mathrm{C}\right)$

28- $\mathrm{BF}_{4}-14 / \mathrm{LiBF}_{4}(1: 0.3): \mathrm{Cr} \cdot 40 \cdot \mathrm{SmA} \cdot 54 \cdot \mathrm{I}\left({ }^{\circ} \mathrm{C}\right)$

28-BF 4 -16: $\mathrm{Cr}_{1} \cdot 48 \cdot \mathrm{Cr}_{2} \cdot 60 \cdot \mathrm{SmA} \cdot 119 \cdot \mathrm{I}\left({ }^{\circ} \mathrm{C}\right)$

28- $\mathrm{BF}_{4}-\mathbf{1 6} / \mathrm{LiBF}_{4}(1: 0.1): \mathrm{Cr} \cdot 52 \cdot \mathrm{SmA} \cdot 121 \cdot \mathrm{I}\left({ }^{\circ} \mathrm{C}\right)$

28-BF $-16 / \mathrm{LiBF}_{4}$ (1:0.3): $\mathrm{Cr} \cdot 67 \cdot \mathrm{SmA} \cdot 123 \cdot \mathrm{I}\left({ }^{\circ} \mathrm{C}\right)$

28-BF ${ }_{4}-18: \mathrm{Cr}_{1} \cdot 60 \cdot \mathrm{Cr}_{2} \cdot 65 \cdot \mathrm{SmA} \cdot 161 \cdot \mathrm{I}\left({ }^{\circ} \mathrm{C}\right)$

28-PF $-\mathbf{n}\left(\mathrm{X}^{-}=\left[\mathrm{PF}_{6}\right]^{-}\right)$

28-PF ${ }_{6}-12: \mathrm{Cr} \cdot 69 \cdot \mathrm{I}\left({ }^{\circ} \mathrm{C}\right)$

28- $\mathrm{PF}_{\mathbf{6}}-\mathbf{1 4}: \mathrm{Cr} \cdot 79 \cdot \mathrm{I}\left({ }^{\circ} \mathrm{C}\right)$

28-PF $-16: \mathrm{Cr} \cdot 87 \cdot \mathrm{SmA} \cdot 90 \cdot \mathrm{I}\left({ }^{\circ} \mathrm{C}\right)$

28-PF $\mathbf{6}-\mathbf{1 8}: \mathrm{Cr} \cdot 91 \cdot \mathrm{SmA} \cdot 128 \cdot \mathrm{I}\left({ }^{\circ} \mathrm{C}\right)$

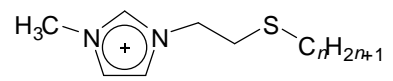

$x^{-}$

$$
\text { 29-Br-n }\left(\mathrm{X}^{-}=\mathrm{Br}^{-}\right)
$$

29-Br-8: $\mathrm{Cr} \cdot 22 \cdot \mathrm{SmA} \cdot 51 \cdot \mathrm{I}\left({ }^{\circ} \mathrm{C}\right)$

29-Br-10: $\mathrm{Cr} \cdot 35 \cdot \mathrm{SmA} \cdot 148 \cdot \mathrm{I}\left({ }^{\circ} \mathrm{C}\right)$

29-Br-12: $\mathrm{Cr} \cdot 46 \cdot \mathrm{SmA} \cdot>200 \cdot \operatorname{dec} .\left({ }^{\circ} \mathrm{C}\right)$

29-Br-14: $\mathrm{Cr} \cdot 55 \cdot \mathrm{SmA} \cdot>200 \cdot \operatorname{dec}\left({ }^{\circ} \mathrm{C}\right)$

29-Br-16: $\mathrm{Cr} \cdot 62 \cdot \mathrm{SmA} \cdot>200 \cdot \operatorname{dec} .\left({ }^{\circ} \mathrm{C}\right)$

29-Br-18: $\mathrm{Cr} \cdot 41 \cdot \mathrm{SmA} \cdot>200 \cdot \operatorname{dec} .\left({ }^{\circ} \mathrm{C}\right)$

29-BF ${ }_{4}-18\left(\mathrm{X}^{-}=\left[\mathrm{BF}_{4}\right]^{-}\right): \mathrm{Cr} \cdot 64 \cdot \mathrm{SmA} \cdot>200 \cdot \operatorname{dec} .\left({ }^{\circ} \mathrm{C}\right)$

29-OTf-n $\left(\mathrm{X}^{-}=[\mathrm{OTf}]^{-}\right)$

29-OTf-8: liquid at r.t.

29-OTf-10: liquid at r.t.

29-OTf-12: $\mathrm{Cr} \cdot 6 \cdot \mathrm{SmA}$ (monotropic?) $\cdot 36 \cdot \mathrm{I}\left({ }^{\circ} \mathrm{C}\right)$

29-OTf-14: $\mathrm{Cr} \cdot 28 \cdot \mathrm{SmA} \cdot 71 \cdot \mathrm{I}\left({ }^{\circ} \mathrm{C}\right)$

29-OTf-16: $\mathrm{Cr} \cdot 43 \cdot \mathrm{SmA} \cdot 124 \cdot \mathrm{I}\left({ }^{\circ} \mathrm{C}\right)$

29-OTf-18: $\mathrm{Cr} \cdot 54 \cdot \mathrm{SmA} \cdot 149 \cdot \mathrm{I}\left({ }^{\circ} \mathrm{C}\right)$ 


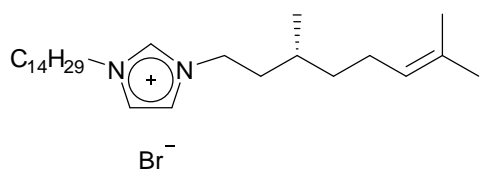

30

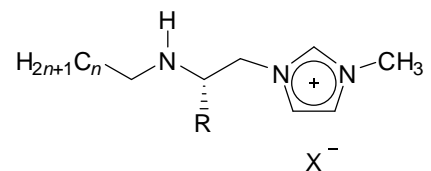

32-X-n-R (X' = $\left.\mathrm{Cl}^{-},[\mathrm{OTf}]^{-}\right)$

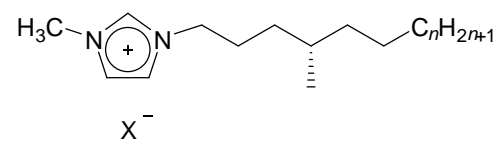

31-X-n $\left(\mathrm{X}^{-}=\mathrm{Br}^{-}, \mathrm{I}^{-},\left[\mathrm{BF}_{4}\right]^{-}\right.$,

$\left.\left[\mathrm{PF}_{6}\right]^{-},[\mathrm{SCN}]^{-},\left[\mathrm{CH}_{3} \mathrm{COO}\right]^{-}\right)$

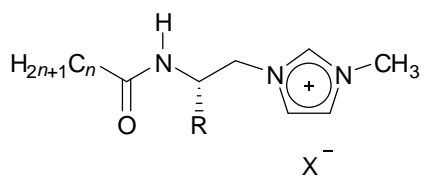

33-X-n-R $\left(\mathrm{X}^{-}=\mathrm{Cl}^{-},[\mathrm{OTf}]^{-}\right)$

30: $\mathrm{Cr} \cdot(\mathrm{SmA} \cdot 9 \cdot) 45 \cdot \mathrm{I}\left({ }^{\circ} \mathrm{C}\right)$

$$
\text { 31-Br-n }\left(\mathrm{X}^{-}=\mathrm{Br}^{-}\right)
$$

31-Br-6: $\mathrm{Cr} \cdot 7 \cdot \mathrm{SmA} \cdot 102 \cdot \mathrm{I}\left({ }^{\circ} \mathrm{C}\right)$

31-Br-7: $\mathrm{Cr} \cdot 17 \cdot \mathrm{SmA} \cdot 153 \cdot \mathrm{I}\left({ }^{\circ} \mathrm{C}\right)$

31-Br-8: $\mathrm{Cr} \cdot 1 \cdot \mathrm{SmA} \cdot 144 \cdot \mathrm{I}\left({ }^{\circ} \mathrm{C}\right)$

31-Br-9: $\mathrm{Cr} \cdot 9 \cdot \mathrm{SmA} \cdot 167 \cdot \mathrm{I}\left({ }^{\circ} \mathrm{C}\right)$

31-Br-10: $\mathrm{Cr} \cdot 20 \cdot \mathrm{SmA} \cdot 159 \cdot \mathrm{I}\left({ }^{\circ} \mathrm{C}\right)$

31-Br-12: $\mathrm{Cr} \cdot 30 \cdot \mathrm{SmA} \cdot 177 \cdot \mathrm{I}\left({ }^{\circ} \mathrm{C}\right)$

31-I-9 $\left(\mathrm{X}^{-}=\mathrm{I}^{-}\right): \mathrm{Cr} \cdot 6 \cdot \mathrm{SmA} \cdot 147 \cdot \mathrm{I}\left({ }^{\circ} \mathrm{C}\right)$

$$
\text { 31-BF } \mathbf{H}_{\mathbf{-}} \mathbf{9}\left(\mathrm{X}^{-}=\left[\mathrm{BF}_{4}\right]^{-}\right): \mathrm{Cr} \cdot 5 \cdot \mathrm{SmA} \cdot 127 \cdot \mathrm{I}\left({ }^{\circ} \mathrm{C}\right)
$$

31-PF $\mathbf{6}^{-\mathbf{9}}\left(\mathrm{X}^{-}=\left[\mathrm{PF}_{6}\right]^{-}\right): \mathrm{Cr} \cdot 53 \cdot \mathrm{SmA} \cdot 93 \cdot \mathrm{I}\left({ }^{\circ} \mathrm{C}\right)$

31-SCN-9 $\left(\mathrm{X}^{-}=[\mathrm{SCN}]^{-}\right): \mathrm{Cr} \cdot 8 \cdot \mathrm{SmA} \cdot 116 \cdot \mathrm{I}\left({ }^{\circ} \mathrm{C}\right)$

31- $\mathbf{C H}_{3}$ COO-9 $\left(\mathrm{X}^{-}=\left[\mathrm{CH}_{3} \mathrm{COO}^{-}\right): \mathrm{Cr} \cdot 4 \cdot \mathrm{SmA} \cdot 153 \cdot \mathrm{I}\left({ }^{\circ} \mathrm{C}\right)\right.$

$$
\text { 32-Cl-n-R }\left(\mathrm{X}^{-}=\mathrm{Cl}^{-}\right)
$$

32-Cl-17-H: $\mathrm{Cr} \cdot 62 \cdot \mathrm{I}\left({ }^{\circ} \mathrm{C}\right)$

32-Cl-15- $\mathrm{CH}_{3}: \mathrm{Cr} \cdot 52 \cdot \mathrm{I}\left({ }^{\circ} \mathrm{C}\right)$

32-Cl-15-(i-Pr): $\mathrm{Cr} \cdot 38 \cdot \mathrm{I}\left({ }^{\circ} \mathrm{C}\right)$

32-Cl-15-(i-Bu): $\mathrm{Cr} \cdot 27 \cdot \mathrm{I}\left({ }^{\circ} \mathrm{C}\right)$

32-Cl-8-Bn: $\mathrm{Cr} \cdot 34 \cdot \mathrm{SmA}$ (monotropic) $\cdot 55 \cdot \mathrm{I}\left({ }^{\circ} \mathrm{C}\right)\left(1^{\text {st }} \mathrm{DSC}\right.$ cooling run)

32-Cl-9-Bn: $\mathrm{Cr} \cdot 56 \cdot \mathrm{SmA} \cdot 87 \cdot \mathrm{I}\left({ }^{\circ} \mathrm{C}\right)$

32-Cl-11-Bn: $\mathrm{Cr} \cdot 53 \cdot \mathrm{SmA} \cdot 113 \cdot \mathrm{I}\left({ }^{\circ} \mathrm{C}\right)$

32-Cl-13-Bn: $\mathrm{Cr} \cdot 57 \cdot \mathrm{I}\left({ }^{\circ} \mathrm{C}\right)$

$$
\text { 32-OTf-n-R ( } \left.\mathrm{X}^{-}=[\mathrm{OTf}]^{-}\right)
$$

32-OTf-13-H: $\mathrm{Cr} \cdot 31 \cdot \mathrm{SmA} \cdot 76 \cdot \mathrm{I}\left({ }^{\circ} \mathrm{C}\right)$

32-OTf-15-H: $\mathrm{Cr} \cdot 44 \cdot \mathrm{SmA} \cdot 115 \cdot \mathrm{I}\left({ }^{\circ} \mathrm{C}\right)$ 
32-OTf-17-H: $\mathrm{Cr} \cdot 51 \cdot \mathrm{SmA} \cdot 116 \cdot \mathrm{I}\left({ }^{\circ} \mathrm{C}\right)$

32-OTf-15- $\mathbf{C H}_{3}: \mathrm{Cr} \cdot 35 \cdot \mathrm{SmA} \cdot 58 \cdot \mathrm{I}\left({ }^{\circ} \mathrm{C}\right)$

33-Cl-n-R $\left(\mathrm{X}^{-}=\mathrm{Cl}^{-}\right)$

33-Cl-13-H: $\mathrm{Cr} \cdot 37 \cdot \mathrm{I}\left({ }^{\circ} \mathrm{C}\right)$

33-Cl-15-H: $\mathrm{Cr} \cdot 39 \cdot \mathrm{I}\left({ }^{\circ} \mathrm{C}\right)$

33-Cl-17-H: $\mathrm{Cr} \cdot 67 \cdot \mathrm{I}\left({ }^{\circ} \mathrm{C}\right)$

33-Cl-15- $\mathrm{CH}_{3}: \mathrm{Cr} \cdot 40 \cdot \mathrm{I}\left({ }^{\circ} \mathrm{C}\right)$

33-Cl-17- $\mathrm{CH}_{3}: \mathrm{Cr} \cdot 54 \cdot \mathrm{I}\left({ }^{\circ} \mathrm{C}\right)$

33-OTf-n-R ( $\left.\mathrm{X}^{-}=[\mathrm{OTf}]^{-}\right)$

33-OTf-15-H: $\mathrm{Cr} \cdot 37 \cdot \mathrm{SmA} \cdot 84 \cdot \mathrm{I}\left({ }^{\circ} \mathrm{C}\right)$

33-OTf-17-H: $\mathrm{Cr} \cdot 43 \cdot \mathrm{SmA} \cdot 106 \cdot \mathrm{I}\left({ }^{\circ} \mathrm{C}\right)$

33-OTf-15- $\mathrm{CH}_{3}: \mathrm{Cr} \cdot 55 \cdot \mathrm{I}\left({ }^{\circ} \mathrm{C}\right)$

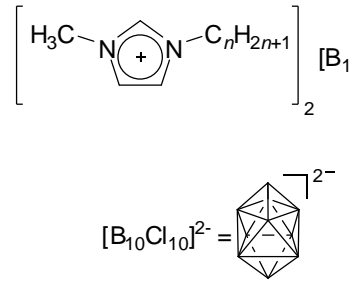

34-n
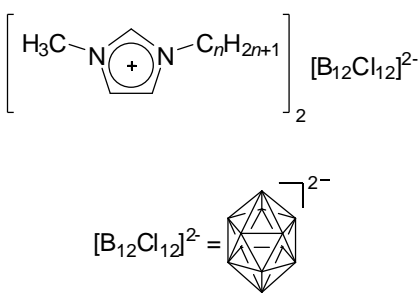

35-n

34-16: $\mathrm{Cr} \cdot 71 \cdot \mathrm{SmA} \cdot 125 \cdot \mathrm{I}\left({ }^{\circ} \mathrm{C}\right)$

34-18: $\mathrm{Cr} \cdot 81 \cdot \mathrm{SmA} \cdot 139 \cdot \mathrm{I}\left({ }^{\circ} \mathrm{C}\right)$

35-16: $\mathrm{Cr} \cdot 105 \cdot \mathrm{SmA} \cdot 150 \cdot \mathrm{I}\left({ }^{\circ} \mathrm{C}\right)$

35-18: $\mathrm{Cr} \cdot 110 \cdot \mathrm{SmA} \cdot 157 \cdot \mathrm{I}\left({ }^{\circ} \mathrm{C}\right)$

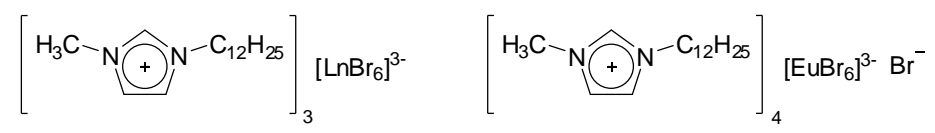

36- $\operatorname{LnBr}_{6}\left(\operatorname{Ln}=\mathrm{Dy}^{3+}, \mathrm{Tb}^{3+}\right)$

37

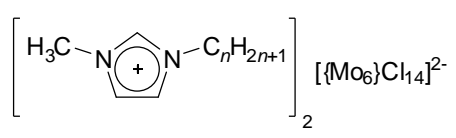

38-n

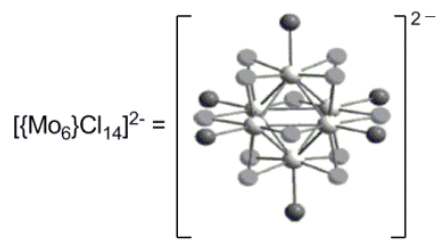

36- $\mathrm{DyBr}_{6}\left(\mathrm{Ln}=\mathrm{Dy}^{3+}\right): \mathrm{g} \cdot \mathrm{ca} \cdot-20 \cdot \mathrm{SmA} \cdot 28 \cdot \mathrm{SmA}^{\prime} \cdot 50 \cdot \mathrm{SmA}^{\prime \prime} \cdot 87 \cdot \mathrm{SmA}^{\prime \prime \prime} \cdot 112 \cdot \mathrm{SmA}^{\prime,}, \cdot 116 \cdot \mathrm{I}\left({ }^{\circ} \mathrm{C}\right)$

36- $\operatorname{TbBr}_{6}\left(\mathrm{Ln}=\mathrm{Tb}^{3+}\right): \mathrm{g} \cdot-6 \cdot \mathrm{SmA} \cdot 101 \cdot \mathrm{I}\left({ }^{\circ} \mathrm{C}\right)$

36- $\mathbf{T b B r}_{6} /\left[\mathrm{C}_{12} \mathrm{mim}\right][\mathrm{Br}](1: 6): \mathrm{Cr} \cdot-4 \cdot \mathrm{SmA} \cdot 102 \cdot \mathrm{I}\left({ }^{\circ} \mathrm{C}\right)$ 
37: $\mathrm{Cr} \cdot-3 \cdot \mathrm{SmA} \cdot 98 \cdot \mathrm{I}\left({ }^{\circ} \mathrm{C}\right)$

38-4: $\mathrm{Cr} \cdot 227 \cdot \mathrm{I}\left({ }^{\circ} \mathrm{C}\right)$

38-6: $\mathrm{Cr} \cdot 226 \cdot \mathrm{I}\left({ }^{\circ} \mathrm{C}\right)$

38-12: $\mathrm{Cr} \cdot 167 \cdot \mathrm{I}\left({ }^{\circ} \mathrm{C}\right)$

38-16: $\mathrm{g} \cdot\left[\right.$ not reported] $\cdot \mathrm{M}_{1}\left(\right.$ ?) $\cdot 133 \cdot \mathrm{M}_{2} \cdot 160 \cdot \mathrm{I}\left({ }^{\circ} \mathrm{C}\right)$

38-18: g $\cdot\left[\right.$ not reported] $\cdot \mathrm{M}_{1}\left(\right.$ ?) $\cdot 133 \cdot \mathrm{M}_{2} \cdot 148 \cdot \mathrm{I}\left({ }^{\circ} \mathrm{C}\right)$

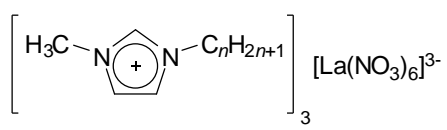

39-n

39-8: $\mathrm{Cr} \cdot 57 \cdot \mathrm{I}\left({ }^{\circ} \mathrm{C}\right)$

39-12: $\mathrm{SmA} \cdot 54 \cdot \mathrm{I}\left({ }^{\circ} \mathrm{C}\right)$

39-14: $\mathrm{Cr}_{1} \cdot 8 \cdot \mathrm{Cr}_{2} \cdot 36 \cdot \mathrm{SmA} \cdot 111 \cdot \mathrm{I}\left({ }^{\circ} \mathrm{C}\right)$

39-16: $\mathrm{Cr} \cdot 51 \cdot \mathrm{SmA} \cdot 118 \cdot \mathrm{I}\left({ }^{\circ} \mathrm{C}\right)$

39-18: $\mathrm{Cr} \cdot 63 \cdot \mathrm{SmA} \cdot 220 \cdot \mathrm{I}\left({ }^{\circ} \mathrm{C}\right)$

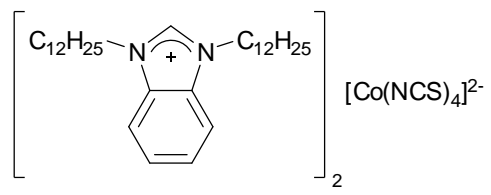

40: $\mathrm{Cr} \cdot 49 \cdot \mathrm{SmA} \cdot 92 \cdot \mathrm{I}\left({ }^{\circ} \mathrm{C}\right)$

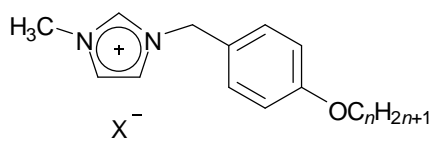

41-Br-n $\left(\mathrm{X}^{-}=\mathrm{Br}^{-}\right)$

41-Br-8: g $\cdot-16 \cdot \mathrm{SmA} \cdot 155 \cdot \mathrm{I}\left({ }^{\circ} \mathrm{C}\right)$

41-Br-10: g $\cdot-15 \cdot \mathrm{SmA} \cdot 227 \cdot \mathrm{I}\left({ }^{\circ} \mathrm{C}\right)$

41-Br-12: $\mathrm{Cr}_{1} \cdot-18 \cdot \mathrm{Cr}_{2} \cdot 30 \cdot \mathrm{SmA} \cdot 250 \cdot \mathrm{I}\left({ }^{\circ} \mathrm{C}\right)\left(\mathrm{Cr} \cdot 81 \cdot \mathrm{SmA} \cdot 249 \cdot \mathrm{I}\left({ }^{\circ} \mathrm{C}\right)\right)$

41-Br-14: $\mathrm{Cr} \cdot 33 \cdot \mathrm{SmA} \cdot 250 \cdot$ dec. $\left({ }^{\circ} \mathrm{C}\right)$

41-Br-16: $\mathrm{Cr} \cdot 48 \cdot \mathrm{SmA} \cdot 250 \cdot$ dec. $\left({ }^{\circ} \mathrm{C}\right)$

41-BF $\mathbf{B}_{4} \boldsymbol{n}\left(\mathrm{X}^{-}=\left[\mathrm{BF}_{4}\right]^{-}\right)$

41-BF ${ }_{4}-8: \mathrm{g} \cdot-29 \cdot \mathrm{SmA} \cdot 71 \cdot \mathrm{I}\left({ }^{\circ} \mathrm{C}\right)$

41-BF ${ }_{4}-10: \mathrm{g} \cdot-29 \cdot \mathrm{Cr}_{1} \cdot 0 \cdot \mathrm{Cr}_{2} \cdot 30 \cdot \mathrm{SmA} \cdot 143 \cdot \mathrm{I}\left({ }^{\circ} \mathrm{C}\right)$

41- $\mathrm{BF}_{4}-\mathbf{- 1 2}: \mathrm{Cr}_{1} \cdot 14 \cdot \mathrm{Cr}_{2} \cdot 62 \cdot \mathrm{Cr}_{3} \cdot 65 \cdot \mathrm{SmA} \cdot 203 \cdot \mathrm{I}\left({ }^{\circ} \mathrm{C}\right)$ 
41-BF $-14: \mathrm{Cr}_{1} \cdot 35 \cdot \mathrm{Cr}_{2} \cdot 49 \cdot \mathrm{Cr}_{3} \cdot 56 \cdot \mathrm{Cr}_{4} \cdot 72 \cdot \mathrm{SmA} \cdot 250 \cdot$ dec. $\left({ }^{\circ} \mathrm{C}\right)$

41-BF $\mathbf{B}_{4}-16: \mathrm{Cr}_{1} \cdot 51 \cdot \mathrm{Cr}_{2} \cdot 59 \cdot \mathrm{Cr}_{3} \cdot 78 \cdot \mathrm{SmA} \cdot 250 \cdot$ dec. $\left({ }^{\circ} \mathrm{C}\right)$

41-PF $\mathbf{6}_{\mathbf{6}} \boldsymbol{n}\left(\mathrm{X}^{-}=\left[\mathrm{PF}_{6}\right]^{-}\right)$

41- $\mathbf{P F}_{\mathbf{6}} \mathbf{- 8}: \mathrm{g} \cdot-26 \cdot \mathrm{Cr} \cdot 35 \cdot \mathrm{I}\left({ }^{\circ} \mathrm{C}\right)$

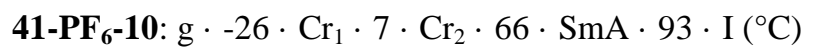

41-PF $-12: \mathrm{Cr}_{1} \cdot 17 \cdot \mathrm{Cr}_{2} \cdot 75 \cdot \mathrm{SmA} \cdot 148 \cdot \mathrm{I}\left({ }^{\circ} \mathrm{C}\right)$

41- $\mathrm{PF}_{6}-\mathbf{- 1 4}: \mathrm{Cr}_{1} \cdot 36 \cdot \mathrm{Cr}_{2} \cdot 81 \cdot \mathrm{SmA} \cdot 195 \cdot \mathrm{I}\left({ }^{\circ} \mathrm{C}\right)$

41-PF $\mathbf{6}-\mathbf{1 6}: \mathrm{Cr}_{1} \cdot 45 \cdot \mathrm{Cr}_{2} \cdot 87 \cdot \mathrm{SmA} \cdot 250 \cdot$ dec. $\left({ }^{\circ} \mathrm{C}\right)$

41-SCN-n $\left(\mathrm{X}^{-}=[\mathrm{SCN}]^{-}\right)$

41-SCN-8: $\mathrm{Cr} \cdot 34 \cdot \mathrm{SmA} \cdot 51 \cdot \mathrm{I}\left({ }^{\circ} \mathrm{C}\right)$

41-SCN-10: $\mathrm{Cr} \cdot 32 \cdot \mathrm{SmA} \cdot 117 \cdot \mathrm{I}\left({ }^{\circ} \mathrm{C}\right)$

41-SCN-12: $\mathrm{Cr} \cdot 53 \cdot \mathrm{SmA} \cdot 188 \cdot \mathrm{I}\left({ }^{\circ} \mathrm{C}\right)$

41-SCN-14: $\mathrm{Cr} \cdot 52 \cdot \mathrm{SmA} \cdot 250 \cdot$ dec. $\left({ }^{\circ} \mathrm{C}\right)$

41-SCN-16: $\mathrm{Cr} \cdot 62 \cdot \mathrm{SmA} \cdot 250 \cdot$ dec. $\left({ }^{\circ} \mathrm{C}\right)$

41-OTf-n $\left(\mathrm{X}^{-}=[\mathrm{OTf}]^{-}\right)$

41-OTf-8: $\mathrm{g} \cdot-44 \cdot \mathrm{I}\left({ }^{\circ} \mathrm{C}\right)$

41-OTf-10: $\mathrm{Cr}_{1} \cdot-13 \cdot \mathrm{Cr}_{2} \cdot 5 \cdot \mathrm{Cr}_{3} \cdot 14 \cdot \mathrm{Cr}_{4} \cdot 46 \cdot \mathrm{Cr}_{5} \cdot 56 \cdot \mathrm{I}\left({ }^{\circ} \mathrm{C}\right)$

41-OTf-12: $\mathrm{Cr} \cdot 67 \cdot \mathrm{I}\left({ }^{\circ} \mathrm{C}\right)$

41-OTf-14: $\mathrm{Cr}_{1} \cdot 24 \cdot \mathrm{Cr}_{2} \cdot 74 \cdot \mathrm{SmA} \cdot 111 \cdot \mathrm{I}\left({ }^{\circ} \mathrm{C}\right)$

41-OTf-16: $\mathrm{Cr}_{1} \cdot 41 \cdot \mathrm{Cr}_{2} \cdot 80 \cdot \mathrm{SmA} \cdot 148 \cdot \mathrm{I}\left({ }^{\circ} \mathrm{C}\right)$

41-NTf $\mathbf{H}_{2}-\boldsymbol{n}\left(\mathrm{X}^{-}=\left[\mathrm{NTf}_{2}\right]^{-}\right)$

41-NTf $\mathbf{N}_{2} \mathbf{- 8}: \mathrm{g} \cdot-59 \cdot \mathrm{Cr}_{1} \cdot-15 \cdot \mathrm{Cr}_{2} \cdot 27 \cdot \mathrm{I}\left({ }^{\circ} \mathrm{C}\right)$

41-NTf $\mathbf{N}_{2}-10: \mathrm{Cr}_{1} \cdot-24 \cdot \mathrm{Cr}_{2} \cdot 37 \cdot \mathrm{I}\left({ }^{\circ} \mathrm{C}\right)$

41-NTf $2-12: \mathrm{Cr} \cdot 50 \cdot \mathrm{I}\left({ }^{\circ} \mathrm{C}\right)$

41-NTf ${ }_{2}-14: \mathrm{Cr} \cdot 62 \cdot \mathrm{I}\left({ }^{\circ} \mathrm{C}\right)$

41-NTf $\mathbf{H}_{2}$-16: $\mathrm{Cr} \cdot 56 \cdot \mathrm{I}\left({ }^{\circ} \mathrm{C}\right)$

41-Ag(CN $)_{2}-12\left(\mathrm{X}^{-}=\left[\mathrm{Ag}(\mathrm{CN})_{2}\right]^{-} ; n=12\right): \mathrm{Cr} \cdot 61 \cdot \mathrm{SmA} \cdot 157 \cdot \mathrm{I}\left({ }^{\circ} \mathrm{C}\right)$

41-Au(CN) $)_{2}-12\left(\mathrm{X}^{-}=\left[\mathrm{Au}(\mathrm{CN})_{2}\right]^{-} ; n=12\right): \mathrm{Cr} \cdot 66 \cdot \mathrm{SmA} \cdot 112 \cdot \mathrm{I}\left({ }^{\circ} \mathrm{C}\right)$ 


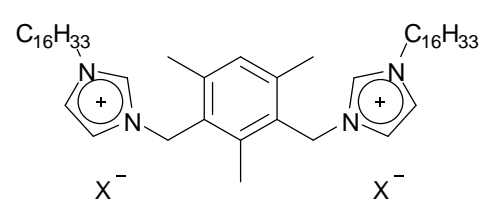

42-X $\left(\mathrm{X}^{-}=\mathrm{I}^{-},\left[\mathrm{BF}_{4}\right]^{-},\left[\mathrm{NTf}_{2}\right]^{-}\right)$

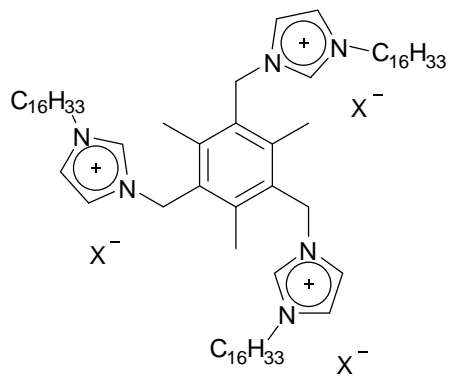

43-X $\left(\mathrm{X}^{-}=\mathrm{I}^{-},\left[\mathrm{BF}_{4}\right]^{-},\left[\mathrm{NTf}_{2}\right]^{-}\right)$

$$
\begin{gathered}
\text { 42-I }\left(\mathrm{X}^{-}=\mathrm{I}^{-}\right): \mathrm{Cr} \cdot 33 \cdot \mathrm{SmA} \cdot 157 \cdot \mathrm{I}\left({ }^{\circ} \mathrm{C}\right) \\
\text { 42-BF } \left.4 \text { ( } \mathrm{X}^{-}=\left[\mathrm{BF}_{4}\right]^{-}\right): \mathrm{Cr} \cdot 43 \cdot \mathrm{SmA} \cdot 150 \cdot \mathrm{I}\left({ }^{\circ} \mathrm{C}\right) \\
\text { 42-NTf } \\
2\left(\mathrm{X}^{-}=\left[\mathrm{NTf}_{2}\right]^{-}\right): \mathrm{Cr} \cdot 63 \cdot \mathrm{I}\left({ }^{\circ} \mathrm{C}\right)
\end{gathered}
$$

43-I $\left(\mathrm{X}^{-}=\mathrm{I}^{-}\right): \mathrm{Cr} \cdot 26 \cdot \mathrm{SmA} \cdot\left[\right.$ not reported; > 180] $\cdot$ dec. $\left({ }^{\circ} \mathrm{C}\right)$ 43-BF $4\left(\mathrm{X}^{-}=\left[\mathrm{BF}_{4}\right]^{-}\right): \mathrm{Cr} \cdot 34 \cdot \mathrm{SmA} \cdot\left[\right.$ not reported; > 180] $\cdot$ dec. $\left({ }^{\circ} \mathrm{C}\right)$ 43-NTf ${ }_{2}\left(\mathrm{X}^{-}=\left[\mathrm{NTf}_{2}\right]^{-}\right): \mathrm{Cr} \cdot(\mathrm{SmA} \cdot \mathrm{ca} .50 \cdot) \mathrm{ca} .68 \cdot \mathrm{I}\left({ }^{\circ} \mathrm{C}\right)$

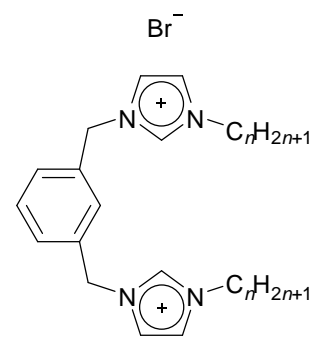

$\mathrm{Br}^{-}$

44-n

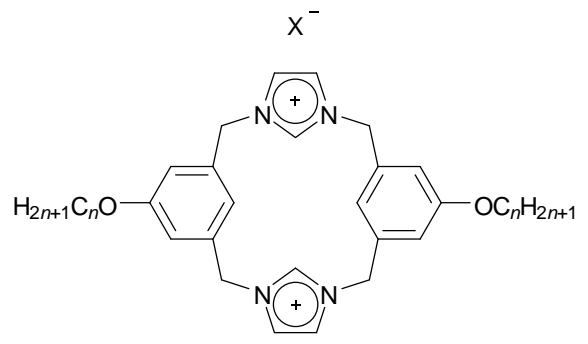

$x^{-}$

44-10: $\mathrm{SmX} \cdot 57 \cdot \mathrm{I}\left({ }^{\circ} \mathrm{C}\right)$

44-18: $\mathrm{Cr} \cdot 61 \cdot \mathrm{SmX} \cdot 250 \cdot \mathrm{I}\left({ }^{\circ} \mathrm{C}\right)$

$$
\text { 45-Br-n }\left(\mathrm{X}^{-}=\mathrm{Br}^{-}\right)
$$

45-Br-10: $\mathrm{Cr} \cdot 163 \cdot \mathrm{SmX} \cdot 255 \cdot \mathrm{I}\left({ }^{\circ} \mathrm{C}\right)$

45-Br-18: $\mathrm{Cr} \cdot 107 \cdot \mathrm{SmX} \cdot 311 \cdot$ dec. $\left({ }^{\circ} \mathrm{C}\right)$

45-BF ${ }_{4}-18\left(\mathrm{X}^{-}=\left[\mathrm{BF}_{4}\right]^{-} ; n=18\right): \mathrm{Cr} \cdot 113 \cdot \mathrm{SmX} \cdot 200 \cdot \mathrm{I}\left({ }^{\circ} \mathrm{C}\right)$

45-OTf-18 ( $\left.\mathrm{X}^{-}=[\mathrm{OTf}]^{-} ; n=18\right): \mathrm{Cr} \cdot 87 \cdot \mathrm{SmX}_{1} \cdot 99 \cdot \mathrm{SmX}_{2} \cdot 210 \cdot \mathrm{I}\left({ }^{\circ} \mathrm{C}\right)$

45-NTf $\mathbf{N}_{2}-\mathbf{1 8}\left(\mathrm{X}^{-}=\left[\mathrm{NTf}_{2}\right]^{-} ; n=18\right): \mathrm{Cr} \cdot 97 \cdot \mathrm{M} \cdot 106 \cdot \mathrm{Cub} \cdot\left[\right.$ not reported] $\cdot \mathrm{I}\left({ }^{\circ} \mathrm{C}\right)$ 


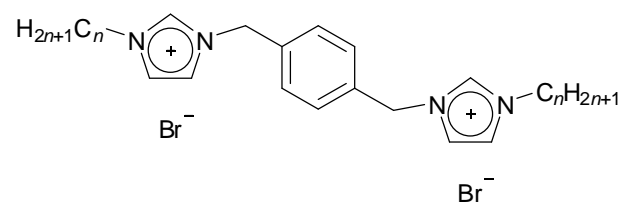

46-n

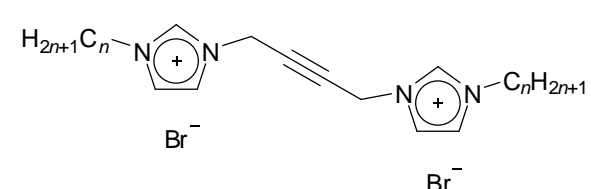

47-n

$$
\begin{gathered}
\text { 46-4: } \mathrm{Cr}_{1} \cdot 30 \cdot \mathrm{Cr}_{2} \cdot 72 \cdot \mathrm{I}\left({ }^{\circ} \mathrm{C}\right)\left(T_{\text {dec. }}=282{ }^{\circ} \mathrm{C}\right) \\
\text { 46-8: } \mathrm{Cr} \cdot 87 \cdot \mathrm{I}\left({ }^{\circ} \mathrm{C}\right)\left(T_{\text {dec. }}=286{ }^{\circ} \mathrm{C}\right) \\
\text { 46-10: } \mathrm{Cr}_{1} \cdot 35 \cdot \mathrm{Cr}_{2} \cdot 62 \cdot \mathrm{I}\left({ }^{\circ} \mathrm{C}\right)\left(T_{\text {dec. }}=277^{\circ} \mathrm{C}\right)
\end{gathered}
$$

46-12: $\mathrm{Cr}_{1} \cdot 33 \cdot \mathrm{Cr}_{2} \cdot 84 \cdot \mathrm{SmC}($ ? $) \cdot 162 \cdot \mathrm{SmA} \cdot 234 \cdot \mathrm{I} / \mathrm{dec} .\left({ }^{\circ} \mathrm{C}\right)\left(1^{\text {st }} \mathrm{DSC}\right.$ heating run; decomposition after melting; $T_{\text {dec. }}=290^{\circ} \mathrm{C}$ )

47-4: $\mathrm{Cr} \cdot 133 \cdot \mathrm{I}\left({ }^{\circ} \mathrm{C}\right)\left(1^{\text {st }} \mathrm{DSC}\right.$ heating run; decomposition after melting; $\left.T_{\text {dec. }}=165{ }^{\circ} \mathrm{C}\right)$

47-8: $\mathrm{Cr} \cdot 75 \cdot \mathrm{I}\left({ }^{\circ} \mathrm{C}\right)\left(1^{\mathrm{st}} \mathrm{DSC}\right.$ heating run; decomposition after melting; $\left.T_{\text {dec. }}=168{ }^{\circ} \mathrm{C}\right)$

47-10: $\mathrm{Cr} \cdot 80 \cdot \mathrm{SmC}\left(\right.$ ?) $\left({ }^{\circ} \mathrm{C}\right)\left(1^{\text {st }} \mathrm{DSC}\right.$ heating run $) / \mathrm{Cr} \cdot 75 \cdot \mathrm{SmC}($ ? $) \cdot$ ca. $178 \cdot \mathrm{dec} .\left({ }^{\circ} \mathrm{C}\right)\left(2^{\text {nd }} \mathrm{DSC}\right.$ heating run; decomposition after melting; $T_{\text {dec. }}=178{ }^{\circ} \mathrm{C}$ )

47-12: $\mathrm{Cr}_{1} \cdot 58 \cdot \mathrm{Cr}_{2} \cdot 81 \cdot \mathrm{SmC}(?)\left({ }^{\circ} \mathrm{C}\right)\left(1^{\text {st }} \mathrm{DSC}\right.$ heating run $) / \mathrm{Cr}_{1} \cdot 25 \cdot \mathrm{Cr}_{2} \cdot 69 \cdot \mathrm{SmC}\left(\right.$ ?) $\cdot$ ca. $185 \cdot$ dec. $\left({ }^{\circ} \mathrm{C}\right)$ $\left(2^{\text {nd }}\right.$ DSC heating run; decomposition after melting; $\left.T_{\text {dec. }}=185^{\circ} \mathrm{C}\right)$

$$
\begin{aligned}
& \mathrm{H}_{2 n+1} \mathrm{C}_{n}-{ }_{-}^{\mathrm{N}}-\left(\mathrm{CH}_{2}\right)_{m-n}-\mathrm{C}_{x} \mathrm{H}_{2 x+1} \\
& x^{-} \quad x^{-} \\
& \text {48-X-m/n/x }\left(\mathrm{X}^{-}=\mathrm{Br}^{-},\left[\mathrm{BF}_{4}\right]^{-},\left[\mathrm{NTf}_{2}\right]^{-}\right) \\
& \underbrace{+}_{\mathrm{X}^{-}} \\
& \text {49-X-m/n }\left(\mathrm{X}^{-}=\mathrm{Br}^{-},\left[\mathrm{BF}_{4}\right]^{-},\left[\mathrm{NTf}_{2}\right]^{-},\left[\mathrm{PF}_{6}\right]^{-},\left[\mathrm{ClO}_{4}\right]^{-}\right)
\end{aligned}
$$

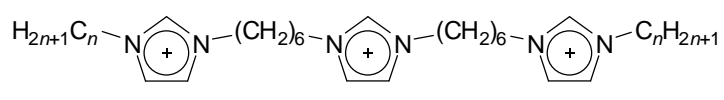

$$
\begin{aligned}
& \mathrm{x}^{-} \mathrm{x}^{-} \quad \mathrm{x}^{-} \\
& \text {50-X-n }\left(\mathrm{X}^{-}=\mathrm{Br}^{-},\left[\mathrm{BF}_{4}\right]^{-},\left[\mathrm{NTf}_{2}\right]^{-}\right) \\
& \text {48-Br-m/n/x }\left(\mathrm{X}^{-}=\mathrm{Br}^{-}\right)
\end{aligned}
$$




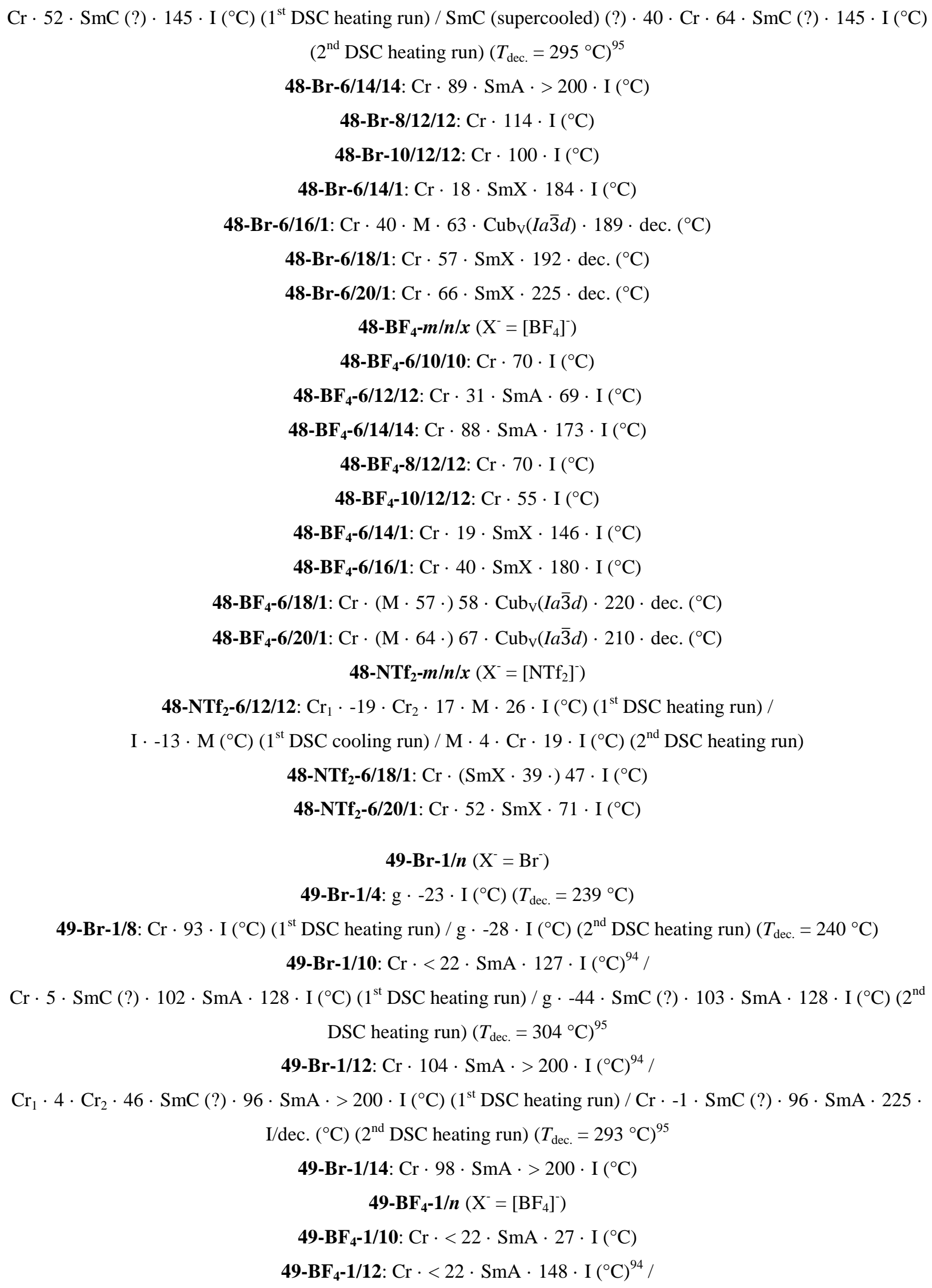




$$
\begin{gathered}
\mathrm{Cr} \cdot 6 \cdot \mathrm{T} \cdot 20 \cdot \mathrm{SmA} \cdot 140 \cdot \mathrm{I}\left({ }^{\circ} \mathrm{C}\right)\left(T_{\text {dec. }}=273{ }^{\circ} \mathrm{C}\right)^{95} \\
\text { 49- } \mathbf{B F}_{\mathbf{4}} \mathbf{- 1 / 1 4 :} \mathrm{Cr} \cdot 42 \cdot \mathrm{SmA} \cdot>200 \cdot \mathrm{I}\left({ }^{\circ} \mathrm{C}\right)
\end{gathered}
$$

49-PF $\mathbf{~}_{\mathbf{6}} \mathbf{- 1 / 1 2}\left(\mathrm{X}^{-}=\left[\mathrm{PF}_{6}\right]^{-}\right): \mathrm{Cr} \cdot 50 \cdot \mathrm{SmA} \cdot 147 \cdot \mathrm{I}\left({ }^{\circ} \mathrm{C}\right)\left(1^{\text {st }} \mathrm{DSC}\right.$ heating run $) / \mathrm{Cr} \cdot 2 \cdot \mathrm{SmA} \cdot 140 \cdot \mathrm{I}\left({ }^{\circ} \mathrm{C}\right)\left(2^{\mathrm{nd}} \mathrm{DSC}\right.$ heating run) $\left(T_{\text {dec. }}=246^{\circ} \mathrm{C}\right)$

49-NTf $\mathbf{2}_{2} \mathbf{- 1 / 1 2}\left(\mathrm{X}^{-}=\left[\mathrm{NTf}_{2}\right]^{-}\right): \mathrm{Cr}_{1} \cdot-27 \cdot \mathrm{Cr}_{2} \cdot 28 \cdot \mathrm{I}\left({ }^{\circ} \mathrm{C}\right)\left(1^{\text {st }} \mathrm{DSC}\right.$ heating run $) /$

$\mathrm{I} \cdot-27 \cdot \operatorname{Cr}\left({ }^{\circ} \mathrm{C}\right)\left(1^{\mathrm{st}} \mathrm{DSC}\right.$ cooling run $) /$

$\mathrm{Cr}_{1} \cdot-22 \cdot \mathrm{Cr}_{2} \cdot-17 \cdot \mathrm{M}_{1}(?) \cdot-13 \cdot \mathrm{M}_{2}(?) \cdot 2 \cdot \mathrm{Cr} \cdot 26 \cdot \mathrm{I}\left({ }^{\circ} \mathrm{C}\right)\left(2^{\text {nd }} \mathrm{DSC}\right.$ heating run $)\left(T_{\text {dec. }}=293{ }^{\circ} \mathrm{C}\right)$

49-ClO $\mathbf{4}_{4}-1 / 12\left(\mathrm{X}^{-}=\left[\mathrm{ClO}_{4}\right]^{-}\right): \mathrm{Cr} \cdot-1 \cdot \mathrm{T} \cdot 28 \cdot \mathrm{SmA} \cdot 126 \cdot \mathrm{I}\left({ }^{\circ} \mathrm{C}\right)\left(1^{\text {st }} \mathrm{DSC}\right.$ heating run $) / \mathrm{g} \cdot \mathrm{ca} \cdot-30 \cdot \mathrm{T} \cdot 4 \cdot \mathrm{SmA} \cdot$

$127 \cdot \mathrm{I}\left({ }^{\circ} \mathrm{C}\right)\left(2^{\text {nd }} \mathrm{DSC}\right.$ heating run $)\left(T_{\text {dec. }}=266^{\circ} \mathrm{C}\right)$

49-Br-2/12 $\left(\mathrm{X}^{-}=\mathrm{Br}^{-}\right): \mathrm{Cr} \cdot 73 \cdot \mathrm{SmA} \cdot 93 \cdot \mathrm{I}\left({ }^{\circ} \mathrm{C}\right)$

49-BF $\mathbf{H}_{\mathbf{4}}-\mathbf{2} / \mathbf{1 2}\left(\mathrm{X}^{-}=\left[\mathrm{BF}_{4}\right]^{-}\right): \mathrm{Cr} \cdot 29 \cdot \mathrm{SmA} \cdot 50 \cdot \mathrm{I}\left({ }^{\circ} \mathrm{C}\right)$

49-Br-3/12 $\left(\mathrm{X}^{-}=\mathrm{Br}^{-}\right): \mathrm{Cr} \cdot<22 \cdot \mathrm{SmA} \cdot 30 \cdot \mathrm{I}\left({ }^{\circ} \mathrm{C}\right)$

49-BF $\mathbf{4}^{-3 / 12}\left(\mathrm{X}^{-}=\left[\mathrm{BF}_{4}\right]^{-}\right): \mathrm{Cr} \cdot<22 \cdot \mathrm{SmA} \cdot 37 \cdot \mathrm{I}\left({ }^{\circ} \mathrm{C}\right)$

50-Br-n $\left(\mathrm{X}^{-}=\mathrm{Br}^{-}\right)$

50-Br-1: $\mathrm{Cr} \cdot 50 \cdot \mathrm{I}\left({ }^{\circ} \mathrm{C}\right)$

50-Br-4: $\mathrm{Cr} \cdot 114 \cdot \mathrm{I}\left({ }^{\circ} \mathrm{C}\right)$

50-Br-6: $\mathrm{Cr} \cdot 144 \cdot$ 'mixed LC' (?) $\cdot 150 \cdot \mathrm{I}\left({ }^{\circ} \mathrm{C}\right)$

50-Br-8: $\mathrm{Cr} \cdot 115 \cdot \mathrm{I}\left({ }^{\circ} \mathrm{C}\right)$

50-Br-10: $\mathrm{Cr} \cdot 120 \cdot$ 'mixed LC' $(?) \cdot 160 \cdot \mathrm{I}\left({ }^{\circ} \mathrm{C}\right)$

50-Br-12: $\mathrm{Cr} \cdot 110 \cdot \mathrm{I}\left({ }^{\circ} \mathrm{C}\right)$

50-Br-14: $\mathrm{Cr} \cdot 140 \cdot \mathrm{I}\left({ }^{\circ} \mathrm{C}\right)$

50-Br-16: [after initial annealing at $160{ }^{\circ} \mathrm{C}$ :] $\mathrm{Cr} \cdot 43 \cdot \mathrm{SmA} \cdot 142 \cdot \mathrm{I}\left({ }^{\circ} \mathrm{C}\right) /$ on cooling: $\mathrm{I} \cdot 40 \cdot \mathrm{SmA}\left({ }^{\circ} \mathrm{C}\right)$

50-Br-18: [after initial annealing at $160^{\circ} \mathrm{C}:$ ] $\mathrm{SmX} \cdot 58 \cdot \mathrm{SmA} \cdot 133 \cdot \mathrm{I}\left({ }^{\circ} \mathrm{C}\right) /$

$$
\text { on cooling: } \mathrm{I} \cdot 91 \cdot \mathrm{SmA} \cdot 53 \cdot \mathrm{SmX}\left({ }^{\circ} \mathrm{C}\right)
$$

50-Br-20: [after initial annealing at $160^{\circ} \mathrm{C}:$ ] $\mathrm{SmX} \cdot 70 \cdot \mathrm{SmA} \cdot 137 \cdot \mathrm{I}\left({ }^{\circ} \mathrm{C}\right) /$

$$
\begin{aligned}
& \text { on cooling: I } \cdot 97 \cdot \mathrm{SmA} \cdot 64 \cdot \mathrm{SmX}\left({ }^{\circ} \mathrm{C}\right) \\
& \text { 50-BF } \mathbf{4}_{\mathbf{4}} \boldsymbol{n}\left(\mathrm{X}^{-}=\left[\mathrm{BF}_{4}\right]^{-}\right) \\
& 50-\mathrm{BF}_{4}-\mathbf{4} \text { : liquid at r.t. } \\
& \text { 50-BF } 4 \text {-6: liquid at r.t. } \\
& \text { 50-BF } 4 \text {-8: } \mathrm{Cr} \cdot 56 \cdot \mathrm{I}\left({ }^{\circ} \mathrm{C}\right) \\
& \text { 50--BF } \mathbf{4}_{\mathbf{- 1 0}} \mathrm{Cr} \cdot 65 \cdot \mathrm{I}\left({ }^{\circ} \mathrm{C}\right) \\
& \text { 50-BF } \mathbf{4}_{\mathbf{4}} \text { 12: } \mathrm{SmA} \cdot 67 \cdot \mathrm{I}\left({ }^{\circ} \mathrm{C}\right) \\
& \text { 50-BF } \mathbf{- 1 4}_{\mathbf{4}} \mathrm{SmA} \cdot 118 \cdot \mathrm{I}\left({ }^{\circ} \mathrm{C}\right) \\
& \text { 50-BF } \text {-16: } \mathrm{Cr} \cdot 46 \cdot \mathrm{SmA} \cdot 184 \cdot \mathrm{I}\left({ }^{\circ} \mathrm{C}\right) \\
& \text { 50-BF }{ }_{4} \text {-18: } \mathrm{Cr} \cdot 48 \cdot \mathrm{SmX} \cdot 237 \cdot \mathrm{I}\left({ }^{\circ} \mathrm{C}\right) \\
& \text { 50-BF } \mathbf{4}_{4}-20: \mathrm{Cr} \cdot 70 \cdot \mathrm{SmX} \cdot>250 \cdot \mathrm{I}\left({ }^{\circ} \mathrm{C}\right)
\end{aligned}
$$


50-NTf $\mathbf{f}_{2}$-n $\left(\mathrm{X}^{-}=\left[\mathrm{NTf}_{2}\right]^{-}\right)$

50-NTf $2-4$ : liquid at r.t.

50-NTf $\mathbf{N}_{2} \mathbf{- 6}$ : liquid at r.t.

50-NTf $2-8$ : liquid at r.t.

50-NTf ${ }_{2}-\mathbf{1 0}: \mathrm{Cr} \cdot 55 \cdot \mathrm{I}\left({ }^{\circ} \mathrm{C}\right)$

50-NTf ${ }_{2}-12: \mathrm{Cr} \cdot 44 \cdot \mathrm{I}\left({ }^{\circ} \mathrm{C}\right)$

50-NTf ${ }_{2}-14: \mathrm{Cr} \cdot 49 \cdot \mathrm{I}\left({ }^{\circ} \mathrm{C}\right)$

50-NTf ${ }_{2}$-16: $\mathrm{Cr} \cdot 64 \cdot \mathrm{I}\left({ }^{\circ} \mathrm{C}\right)$

50-NTf ${ }_{2}$-18: $\mathrm{SmX} \cdot 68 \cdot \mathrm{I}\left({ }^{\circ} \mathrm{C}\right)$

50-NTf ${ }_{2}-20: \mathrm{SmA} \cdot 75 \cdot \mathrm{I}\left({ }^{\circ} \mathrm{C}\right)$

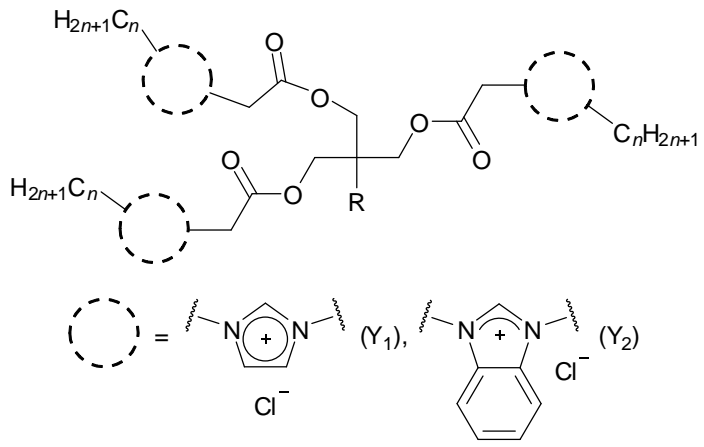

51- $\mathbf{C H}_{3}-\mathbf{Y}_{\boldsymbol{n}}-\boldsymbol{n}\left(\mathrm{R}=\mathrm{CH}_{3}\right)$

51- $\mathrm{CH}_{3}-\mathbf{Y}_{1}-10: \mathrm{SmA} \cdot 116 \cdot \mathrm{I}\left({ }^{\circ} \mathrm{C}\right)$

51- $\mathbf{C H}_{3}-\mathbf{Y}_{1}-12: \mathrm{SmA} \cdot 172 \cdot \mathrm{I}\left({ }^{\circ} \mathrm{C}\right)$

51- $\mathrm{CH}_{3}-\mathbf{Y}_{2}-10: \mathrm{Col} \cdot 37 \cdot \mathrm{I}\left({ }^{\circ} \mathrm{C}\right)$

51- $\mathrm{CH}_{3}-\mathbf{Y}_{2}-12: \mathrm{Col} \cdot 50 \cdot \mathrm{I}\left({ }^{\circ} \mathrm{C}\right)$

51-[4th cation]- $\mathbf{Y}_{\boldsymbol{n}}-\boldsymbol{n}$ (R = additional 'arm' with a cation)

51-[4th cation]-Y $\mathbf{1}^{-4}$ : LC? (unidentified mesophase; clearing point not detected)

51-[4th cation]-Y $\mathbf{Y}_{1}-6$ : $\mathrm{Col} \cdot\left[\right.$ not detected] $\cdot \mathrm{I}\left({ }^{\circ} \mathrm{C}\right)$

51-[4th cation]-Y $\mathbf{Y}_{\mathbf{1}} \mathbf{- 8}$ : $\mathrm{Col} \cdot\left[\right.$ not detected] $\cdot \mathrm{I}\left({ }^{\circ} \mathrm{C}\right)$

51-[4th cation]- $\mathbf{Y}_{1}-10$ : $\mathrm{Col}$ (or SmA?) $\cdot 176 \cdot \mathrm{I}\left({ }^{\circ} \mathrm{C}\right)$

51-[4th cation]- $\mathbf{Y}_{1}-12: \mathrm{Col}(?) \cdot 172 \cdot \mathrm{I}\left({ }^{\circ} \mathrm{C}\right)$

51-[4th cation]- $\mathbf{Y}_{2}-4: \mathrm{M} \cdot 107 \cdot \mathrm{I}\left({ }^{\circ} \mathrm{C}\right)$

51-[4th cation]- $\mathbf{Y}_{2}-\mathbf{6}$ : glassy state $(?) \cdot 159 \cdot \mathrm{I}\left({ }^{\circ} \mathrm{C}\right)$

51-[4th cation]- $\mathbf{Y}_{2}-\mathbf{8}$ : glassy state $(?) \cdot 120 \cdot \mathrm{I}\left({ }^{\circ} \mathrm{C}\right)$

51-[4th cation]- $\mathbf{Y}_{2}-10$ : glassy state $(?) \cdot 94 \cdot \mathrm{I}\left({ }^{\circ} \mathrm{C}\right)$

51-[4th cation]- $\mathbf{Y}_{2}-12$ : glassy state $(?) \cdot 117 \cdot \mathrm{I}\left({ }^{\circ} \mathrm{C}\right)$ 


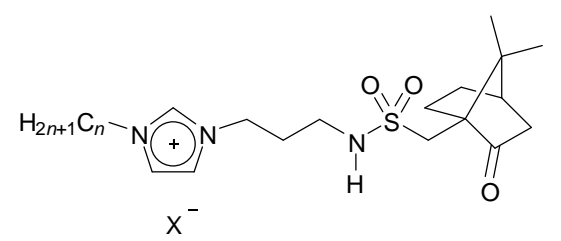

52-Br-12 ( $\left.\mathrm{X}^{-}=\mathrm{Br}^{-}, n=12\right)$ : not LC

52-I-n $\left(\mathrm{X}^{-}=\mathrm{I}^{-}\right)$

52-I-1: not LC

52-I-16: not LC

52- $\mathbf{C}_{12} \mathbf{H}_{25} \mathbf{O S O}_{3}-\boldsymbol{n}\left(\mathrm{X}^{-}=\left[\mathrm{C}_{12} \mathrm{H}_{25} \mathrm{OSO}_{3}\right]^{-}\right)$

52- $\mathrm{C}_{12} \mathrm{H}_{25} \mathrm{OSO}_{3}-1$ : not LC

52- $\mathrm{C}_{12} \mathbf{H}_{25} \mathbf{O S O}_{3}-12: \mathrm{Cr} \cdot 64 \cdot \mathrm{SmA} \cdot 80 \cdot \mathrm{I}\left({ }^{\circ} \mathrm{C}\right)$

52- $\mathrm{C}_{12} \mathbf{H}_{25} \mathrm{OSO}_{3}-16: \mathrm{Cr} \cdot 37 \cdot \mathrm{SmA} \cdot 108 \cdot \mathrm{I}\left({ }^{\circ} \mathrm{C}\right)$

52- $\mathbf{C}_{16} \mathbf{H}_{33} \mathbf{S O}_{3}-\boldsymbol{n}\left(\mathrm{X}^{-}=\left[\mathrm{C}_{16} \mathrm{H}_{33} \mathrm{SO}_{3}\right]^{-}\right)$

52- $\mathrm{C}_{16} \mathrm{H}_{33} \mathrm{SO}_{3}-1$ : not LC

52- $\mathbf{C}_{16} \mathbf{H}_{33} \mathrm{SO}_{3}-12: \mathrm{Cr} \cdot 31 \cdot \mathrm{SmA} \cdot 94 \cdot \mathrm{I}\left({ }^{\circ} \mathrm{C}\right)$

52- $\mathbf{C}_{16} \mathbf{H}_{33} \mathbf{S O}_{3}-16: \mathrm{Cr} \cdot 81 \cdot \mathrm{SmA} \cdot 123 \cdot \mathrm{I}\left({ }^{\circ} \mathrm{C}\right)$

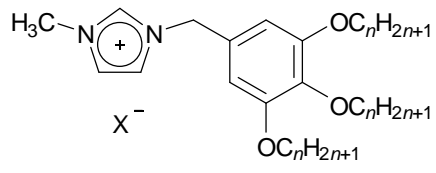

53-X-n $\left(\mathrm{X}^{-}=\mathrm{Cl}^{-},{ }^{96} \mathrm{Br}^{-}\right.$,

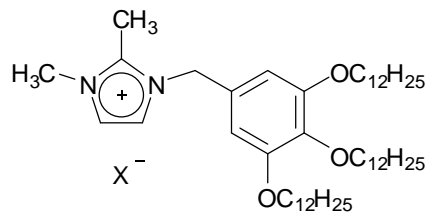

$\left.\left[\mathrm{BF}_{4}\right]^{-},\left[\mathrm{PF}_{6}\right]^{-},[\mathrm{OTf}]^{-},\left[\mathrm{NTf}_{2}\right]^{-}\right)$

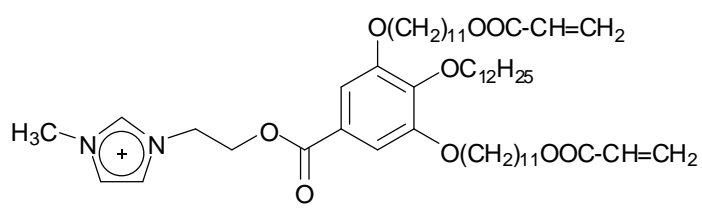

$\left[\mathrm{BF}_{4}\right]^{-}$

55

54-X $\left(\mathrm{X}^{-}=\left[\mathrm{BF}_{4}\right]^{-},\left[\mathrm{PF}_{6}\right]^{-},[\mathrm{OTf}]^{-},\left[\mathrm{NTf}_{2}\right]^{-}\right)$

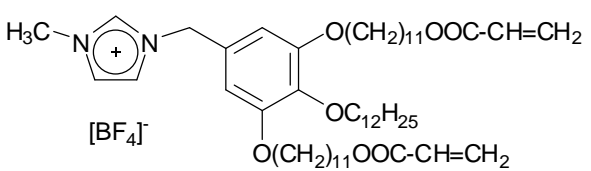

56

53-Cl-n $\left(\mathrm{X}^{-}=\mathrm{Cl}^{-}\right)$

53-Cl-8: $\mathrm{Cr} \cdot 52 \cdot \mathrm{Col}_{\mathrm{h}} \cdot 186 \cdot \mathrm{I}\left({ }^{\circ} \mathrm{C}\right)$

53-Cl-10: $\mathrm{Cr} \cdot 64 \cdot \mathrm{Col}_{\mathrm{h}} \cdot 199 \cdot \mathrm{I}\left({ }^{\circ} \mathrm{C}\right)$

53-Cl-12: $\mathrm{Cr} \cdot 70 \cdot \mathrm{Col}_{\mathrm{h}} \cdot 188 \cdot \mathrm{I}\left({ }^{\circ} \mathrm{C}\right)$

53-Cl-14: $\mathrm{Cr} \cdot 81 \cdot \mathrm{Col}_{\mathrm{h}} \cdot 163 \cdot \mathrm{I}\left({ }^{\circ} \mathrm{C}\right)$

53-Br-n $\left(\mathrm{X}^{-}=\mathrm{Br}^{-}\right)$

53-Br-8: $\mathrm{Cr} \cdot-26 \cdot \mathrm{Col}_{\mathrm{h}} \cdot 180 \cdot \mathrm{I}\left({ }^{\circ} \mathrm{C}\right)$ 
53-Br-12: $\mathrm{Cr} \cdot 73 \cdot \mathrm{Col}_{\mathrm{h}} \cdot 180 \cdot \mathrm{I}\left({ }^{\circ} \mathrm{C}\right)$

53-BF ${ }_{4}-\mathbf{n}\left(\mathrm{X}^{-}=\left[\mathrm{BF}_{4}\right]^{-}\right)$

53-BF ${ }_{4}-6: \mathrm{g} \cdot-35 \cdot \mathrm{Col}_{\mathrm{h}} \cdot 32 \cdot \mathrm{I}\left({ }^{\circ} \mathrm{C}\right)$

53-BF ${ }_{4}-8: \mathrm{g} \cdot-29 \cdot \mathrm{Col}_{\mathrm{h}} \cdot 133 \cdot \mathrm{I}\left({ }^{\circ} \mathrm{C}\right)$

53-BF $-10: \mathrm{Cr} \cdot 47 \cdot \mathrm{Col}_{\mathrm{h}} \cdot 173 \cdot \mathrm{I}\left({ }^{\circ} \mathrm{C}\right)$

53-BF 4 -12: $\mathrm{Cr} \cdot 17^{97} / 61^{96} \cdot \mathrm{Col}_{\mathrm{h}} \cdot 183^{97} / 185^{96} \cdot \mathrm{I}\left({ }^{\circ} \mathrm{C}\right)$

53-BF $-14: \mathrm{Cr}_{1} \cdot 53 \cdot \mathrm{Cr}_{2} \cdot 58 \cdot \mathrm{Cr}_{3} \cdot 72 \cdot \mathrm{Cr}_{4} \cdot\left[\right.$ not reported] $\cdot \mathrm{Col}_{\mathrm{h}} \cdot 178 \cdot \mathrm{I}\left({ }^{\circ} \mathrm{C}\right)$

53-BF $\mathbf{H}_{4}-16: \mathrm{Cr} \cdot 74 \cdot \mathrm{Col}_{\mathrm{h}} \cdot 177 \cdot \mathrm{I}\left({ }^{\circ} \mathrm{C}\right)$

53-BF 4 -18: $\mathrm{Cr} \cdot 88 \cdot \mathrm{Col}_{\mathrm{h}} \cdot 148 \cdot \mathrm{I}\left({ }^{\circ} \mathrm{C}\right)$

53-PF ${ }_{6}-\boldsymbol{n}\left(\mathrm{X}^{-}=\left[\mathrm{PF}_{6}\right]^{-}\right)$

53-PF 6 -8: g $\cdot-33 \cdot \mathrm{Col}_{\mathrm{h}} \cdot 78 \cdot \mathrm{I}\left({ }^{\circ} \mathrm{C}\right)$

53-PF $-12: \mathrm{Cr} \cdot 12 \cdot \mathrm{Col}_{\mathrm{h}} \cdot 151 \cdot \mathrm{I}\left({ }^{\circ} \mathrm{C}\right)$

53-PF $\mathbf{- 1 6 :} \mathrm{Cr} \cdot 55 \cdot \mathrm{Col}_{\mathrm{h}} \cdot 167 \cdot \mathrm{I}\left({ }^{\circ} \mathrm{C}\right)$

53-PF $-18: \mathrm{Cr} \cdot 68 \cdot \mathrm{Col}_{\mathrm{h}} \cdot 161 \cdot \mathrm{I}\left({ }^{\circ} \mathrm{C}\right)$

53-OTf-n $\left(\mathrm{X}^{-}=[\mathrm{OTf}]^{-}\right)$

53-OTf-8: $\mathrm{g} \cdot-56 \cdot \mathrm{I} \cdot-20$ (cold crystallization) $\cdot \mathrm{Cr} \cdot 1 \cdot \mathrm{I}\left({ }^{\circ} \mathrm{C}\right)$

53-OTf-12: $\mathrm{Cr} \cdot(\mathrm{SmA} \cdot 10 \cdot) 63 \cdot \mathrm{I}\left({ }^{\circ} \mathrm{C}\right)$

53-OTf-16: $\mathrm{Cr} \cdot 78 \cdot \mathrm{Col}_{\mathrm{h}} \cdot 117 \cdot \mathrm{I}\left({ }^{\circ} \mathrm{C}\right)$

53-OTf-18: $\mathrm{Cr} \cdot 86 \cdot \mathrm{Col}_{\mathrm{h}} \cdot 119 \cdot \mathrm{I}\left({ }^{\circ} \mathrm{C}\right)$

53-NTf ${ }_{2}-n\left(X^{-}=\left[\mathrm{NTf}_{2}\right]^{-}\right)$

53-NTf ${ }_{2}-8: \mathrm{g} \cdot-58 \cdot \mathrm{I} \cdot-19$ (cold crystallization) $\cdot \mathrm{Cr} \cdot 2 \cdot \mathrm{I}\left({ }^{\circ} \mathrm{C}\right)$

53-NTf $\mathbf{H}_{2}-\mathbf{1 0}: \mathrm{Cr}_{1} \cdot-13 \cdot \mathrm{Cr}_{2} \cdot 19 \cdot \mathrm{I}\left({ }^{\circ} \mathrm{C}\right)$

53-NTf $2-12: \mathrm{Cr} \cdot 37 \cdot \mathrm{I}\left({ }^{\circ} \mathrm{C}\right)$

53-NTf $2-14: \mathrm{Cr} \cdot 51 \cdot \mathrm{I}\left({ }^{\circ} \mathrm{C}\right)$

53-NTf 2 -16: $\mathrm{Cr} \cdot 55 \cdot \mathrm{Col}_{\mathrm{h}} \cdot 60 \cdot \mathrm{I}\left({ }^{\circ} \mathrm{C}\right)$

53-NTf $\mathbf{N A}_{2}$-18: $\mathrm{Cr} \cdot 66 \cdot \mathrm{Col}_{\mathrm{h}} \cdot 77 \cdot \mathrm{I}\left({ }^{\circ} \mathrm{C}\right)$

54-BF $4\left(\mathrm{X}^{-}=\left[\mathrm{BF}_{4}\right]^{-}\right): \mathrm{Cr} \cdot 19 \cdot \mathrm{Col}_{\mathrm{h}} \cdot 174 \cdot \mathrm{I}\left({ }^{\circ} \mathrm{C}\right)$

54-PF $\mathbf{F}_{6}\left(\mathrm{X}^{-}=\left[\mathrm{PF}_{6}\right]^{-}\right): \mathrm{Cr} \cdot 58 \cdot \mathrm{Col}_{\mathrm{h}} \cdot 155 \cdot \mathrm{I}\left({ }^{\circ} \mathrm{C}\right)$

54-OTf $\left(\mathrm{X}^{-}=[\mathrm{OTf}]\right): \mathrm{Cr} \cdot 66 \cdot \mathrm{Col}_{\mathrm{h}} \cdot 85 \cdot \mathrm{I}\left({ }^{\circ} \mathrm{C}\right)$

54-NTf ${ }_{2}\left(\mathrm{X}^{-}=\left[\mathrm{NTf}_{2}\right]^{-}\right): \mathrm{Cr} \cdot 51 \cdot \mathrm{I}\left({ }^{\circ} \mathrm{C}\right)$

55: $\mathrm{Cr} \cdot 20 \cdot \mathrm{Col}_{\mathrm{h}} \cdot 50 \cdot \mathrm{I}\left({ }^{\circ} \mathrm{C}\right)$

56: $\mathrm{Cr} \cdot 5 \cdot \mathrm{I}\left({ }^{\circ} \mathrm{C}\right)$ 


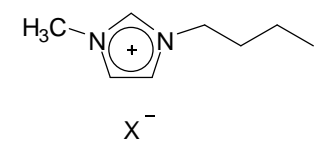

57-X $\left(\mathrm{X}^{-}=\mathrm{Br}^{-},\left[\mathrm{BF}_{4}\right]^{-}\right.$,

[Gly], [Ala]', [Leu]')

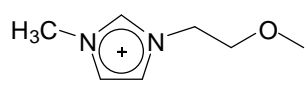

$x^{-}$

More information about mixtures of these ILs with neutral dihydroxy amphiphiles (NDA1-n, NDA2, NDA3, NDA4)

can be found in the main text and in Figure S8.

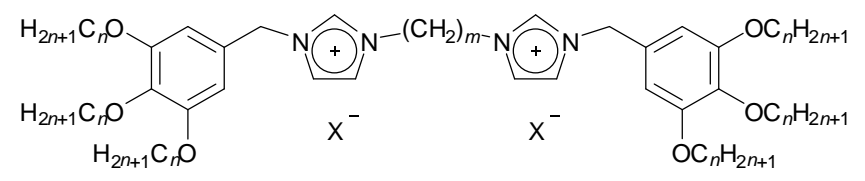

59-Cl-m/n $\left(\mathrm{X}^{-}=\mathrm{Cl}^{-}\right)$

59-Cl-4/8: $\mathrm{Cr} \cdot 46 \cdot \mathrm{Col}_{\mathrm{h}} \cdot 241 \cdot \mathrm{I} / \mathrm{dec} .\left({ }^{\circ} \mathrm{C}\right)$

59-Cl-4/12: $\mathrm{Cr}_{1} \cdot 49 \cdot \mathrm{Cr}_{2} \cdot 72 \cdot \mathrm{Col}_{\mathrm{h}} \cdot 213 \cdot \mathrm{I} / \mathrm{dec} .\left({ }^{\circ} \mathrm{C}\right)$

59-Cl-6/8: $\mathrm{Cr}_{1} \cdot 62 \cdot \mathrm{Cr}_{2} \cdot 81 \cdot \mathrm{Col}_{\mathrm{h}} \cdot 237 \cdot \mathrm{I} /$ dec. $\left({ }^{\circ} \mathrm{C}\right)$

59-Cl-6/12: $\mathrm{Cr}_{1} \cdot 36 \cdot \mathrm{Cr}_{2} \cdot 101 \cdot \mathrm{Col}_{\mathrm{h}} \cdot 221 \cdot \mathrm{I} /$ dec. $\left({ }^{\circ} \mathrm{C}\right)$

59-Cl-8/8: $\mathrm{Cr}_{1} \cdot-21 \cdot \mathrm{Cr}_{2} \cdot 34 \cdot \mathrm{Col}_{\mathrm{h}} \cdot 184 \cdot \mathrm{I}\left({ }^{\circ} \mathrm{C}\right)$

59-Cl-8/12: $\mathrm{Cr} \cdot 37 \cdot \mathrm{Col}_{\mathrm{h}} \cdot 216 \cdot \mathrm{I} /$ dec. $\left({ }^{\circ} \mathrm{C}\right)$

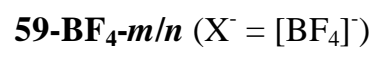

59-BF 4 -4/8: $\mathrm{Cr} \cdot 34 \cdot \mathrm{Col}_{\mathrm{h}} \cdot 238 \cdot \mathrm{I}\left({ }^{\circ} \mathrm{C}\right)$

59-BF 4 -4/12: $\mathrm{Cr} \cdot 4 \cdot \mathrm{Col}_{\mathrm{h}} \cdot 256 \cdot \mathrm{I} / \mathrm{dec} .\left({ }^{\circ} \mathrm{C}\right)$

59-BF 4 -6/8: $\mathrm{Cr} \cdot 41 \cdot \mathrm{Col}_{\mathrm{h}} \cdot 185 \cdot \mathrm{I}\left({ }^{\circ} \mathrm{C}\right)$

59-BF 4 -6/12: $\mathrm{Cr} \cdot 6 \cdot \mathrm{Col}_{\mathrm{h}} \cdot 246 \cdot \mathrm{I}\left({ }^{\circ} \mathrm{C}\right)$

59-BF $\mathbf{A}_{\mathbf{4}} \mathbf{- 8} / \mathbf{8}: \mathrm{Cr} \cdot 98 \cdot \mathrm{Col}_{\mathrm{h}} \cdot 135 \cdot \mathrm{I}\left({ }^{\circ} \mathrm{C}\right)$

59-BF $-\mathbf{- 8} / \mathbf{1 2}: \mathrm{Cr}_{1} \cdot 36 \cdot \mathrm{Cr}_{2} \cdot 51 \cdot \mathrm{Col}_{\mathrm{h}} \cdot 213 \cdot \mathrm{I}\left({ }^{\circ} \mathrm{C}\right)$

59-NTf $\mathbf{2}_{2}-\mathbf{m} / \mathbf{n}\left(\mathrm{X}^{-}=\left[\mathrm{NTf}_{2}\right]^{-}\right)$

59-NTf $\mathbf{2}_{2}-\mathbf{4} / \mathbf{8}: \mathrm{Cr} \cdot 43 \cdot \mathrm{I}\left({ }^{\circ} \mathrm{C}\right)$

59-NTf $\mathbf{2}_{2} \mathbf{- 4 / 1 2 :} \mathrm{Cr} \cdot 53 \cdot \mathrm{I}\left({ }^{\circ} \mathrm{C}\right)$

59-NTf ${ }_{2}-8 / 8: \mathrm{Cr} \cdot<-40 \cdot \mathrm{I}\left({ }^{\circ} \mathrm{C}\right)$

59-NTf $\mathbf{N}_{2} \mathbf{- 8} / \mathbf{1 2}: \mathrm{Cr}_{1} \cdot 27 \cdot \mathrm{Cr}_{2} \cdot 41 \cdot \mathrm{I}\left({ }^{\circ} \mathrm{C}\right)$ 


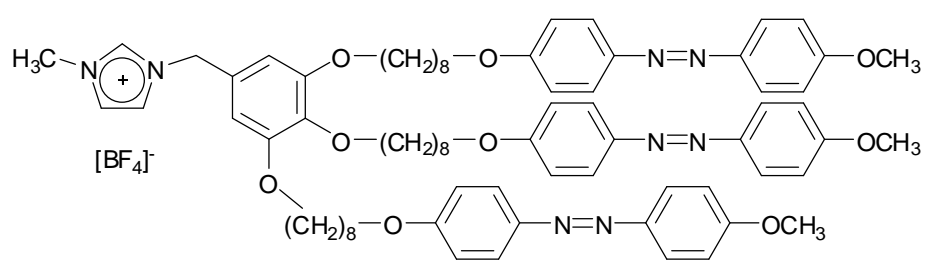

60: $\mathrm{Cr} \cdot 95 \cdot \mathrm{SmA} \cdot 140 \cdot \mathrm{N} \cdot 149 \cdot \mathrm{I}\left({ }^{\circ} \mathrm{C}\right)$

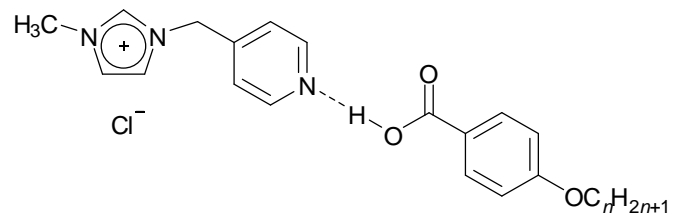

61-n

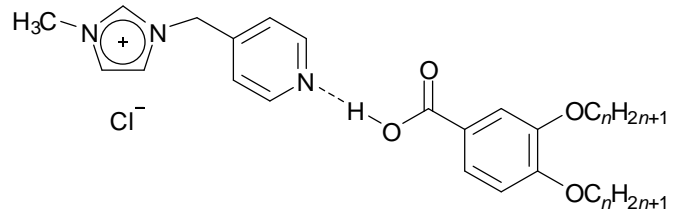

$62-n$

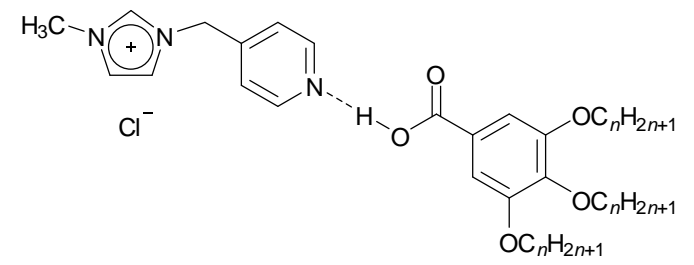

63-n

61-8: $\mathrm{Cr} \cdot 90 \cdot \mathrm{SmC} \cdot 117 \cdot \mathrm{I}\left({ }^{\circ} \mathrm{C}\right)$

61-12: $\mathrm{Cr} \cdot 91 \cdot \mathrm{SmC} \cdot 117 \cdot \mathrm{I}\left({ }^{\circ} \mathrm{C}\right)$

61-16: $\mathrm{Cr} \cdot 92 \cdot \mathrm{SmC} \cdot 118 \cdot \mathrm{I}\left({ }^{\circ} \mathrm{C}\right)$

62-8: $\mathrm{Cr} \cdot 104 \cdot \mathrm{Col}_{\mathrm{r}} \cdot 128 \cdot \mathrm{I}\left({ }^{\circ} \mathrm{C}\right)$

62-12: $\mathrm{Cr} \cdot 106 \cdot \mathrm{Col}_{\mathrm{r}} \cdot 135 \cdot \mathrm{I}\left({ }^{\circ} \mathrm{C}\right)$

62-16: $\mathrm{Cr} \cdot 123 \cdot \mathrm{I}\left({ }^{\circ} \mathrm{C}\right)$

63-8: $\mathrm{Cr} \cdot\left(\mathrm{Cub}_{\mathrm{I}} \cdot 87 \cdot\right) 98 \cdot \mathrm{I}\left({ }^{\circ} \mathrm{C}\right)$

63-12: $\mathrm{Cr} \cdot\left(\mathrm{Cub}_{\mathrm{I}} \cdot 72 \cdot\right) 90 \cdot \mathrm{I}\left({ }^{\circ} \mathrm{C}\right)$

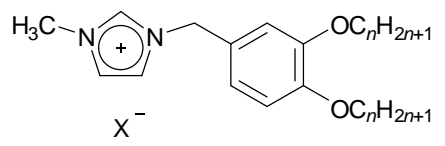

$$
\text { 64-Cl-n }\left(\mathrm{X}^{-}=\mathrm{Cl}^{-}\right)
$$

64-Cl-6: $\mathrm{Cr} \cdot 49 \cdot \mathrm{SmA} \cdot 180 \cdot \mathrm{I}\left({ }^{\circ} \mathrm{C}\right)$

64-Cl-12: $\mathrm{Cr} \cdot 90 \cdot \mathrm{Cub}_{\mathrm{V}}(\mathrm{Ia} \overline{3} d) \cdot 138 \cdot \mathrm{Col}_{\mathrm{h}} \cdot 200 \cdot \operatorname{dec} .\left({ }^{\circ} \mathrm{C}\right)$

64-Cl-16: $\mathrm{Cr} \cdot 95 \cdot \mathrm{Col}_{\mathrm{h}} \cdot 200 \cdot$ dec. $\left({ }^{\circ} \mathrm{C}\right)$

64-Cl-18: $\mathrm{Cr} \cdot 93 \cdot \mathrm{Col}_{\mathrm{h}} \cdot 200 \cdot$ dec. $\left({ }^{\circ} \mathrm{C}\right)$

64-Br-n $\left(\mathrm{X}^{-}=\mathrm{Br}^{-}\right)$ 
64-Br-6: $\mathrm{Cr} \cdot 9 \cdot \mathrm{SmA} \cdot 165 \cdot \mathrm{I}\left({ }^{\circ} \mathrm{C}\right)$

64-Br-12: $\mathrm{Cr} \cdot 81 \cdot \mathrm{Cub}_{\mathrm{V}}(\operatorname{Ia} \overline{3} d) \cdot 117 \cdot \mathrm{Col}_{\mathrm{h}} \cdot 200 \cdot$ dec. $\left({ }^{\circ} \mathrm{C}\right)$

64-Br-16: $\mathrm{Cr} \cdot 97 \cdot \mathrm{Col}_{\mathrm{h}} \cdot 200 \cdot$ dec. $\left({ }^{\circ} \mathrm{C}\right)$

64-Br-18: $\mathrm{Cr} \cdot 99 \cdot \mathrm{Col}_{\mathrm{h}} \cdot 200 \cdot \operatorname{dec}\left({ }^{\circ} \mathrm{C}\right)$ 
Scheme S1. Imidazolium-based ILCs that contain at least one rod-like mesogenic group that is connected to the cationic core via a flexible spacer.

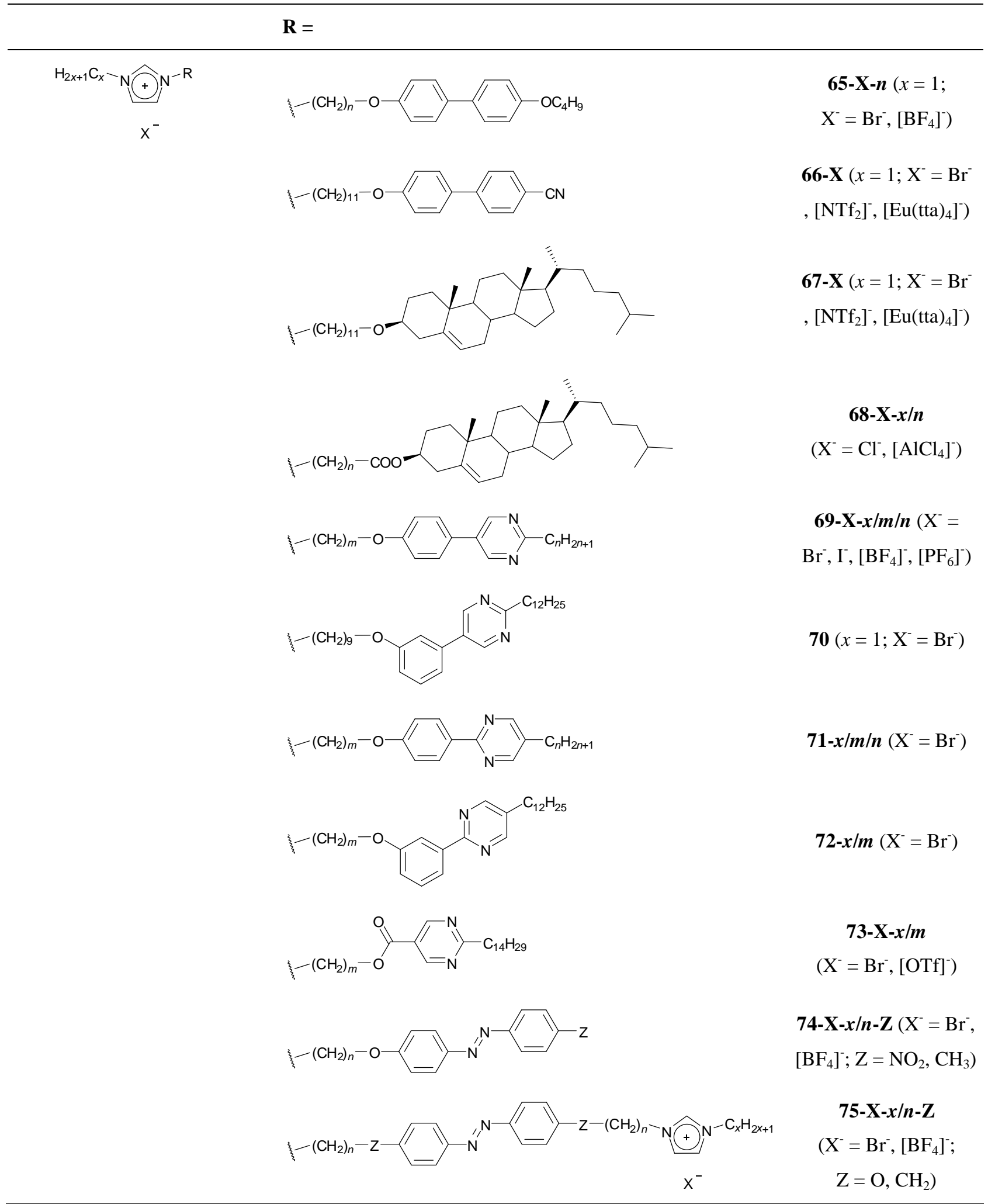




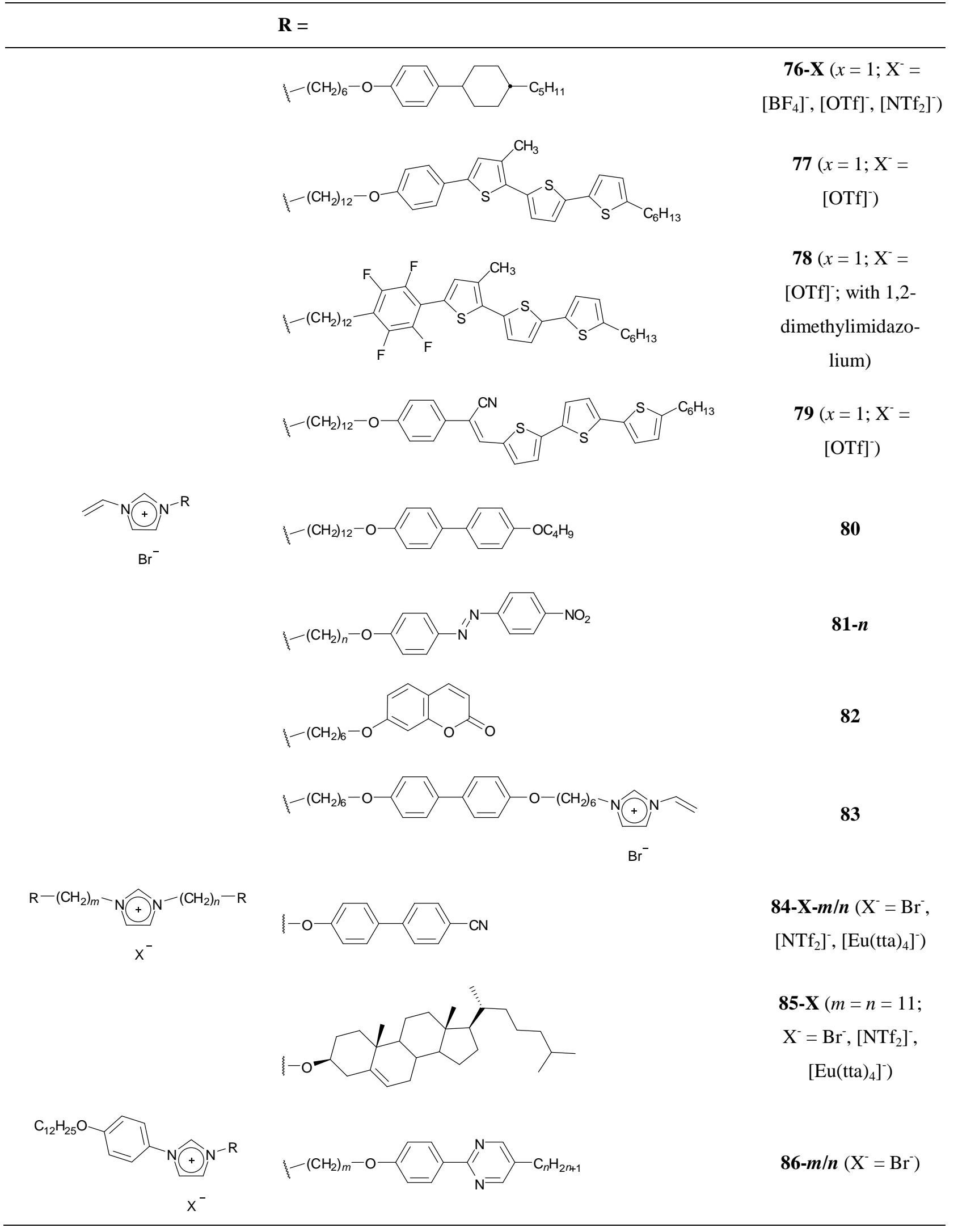




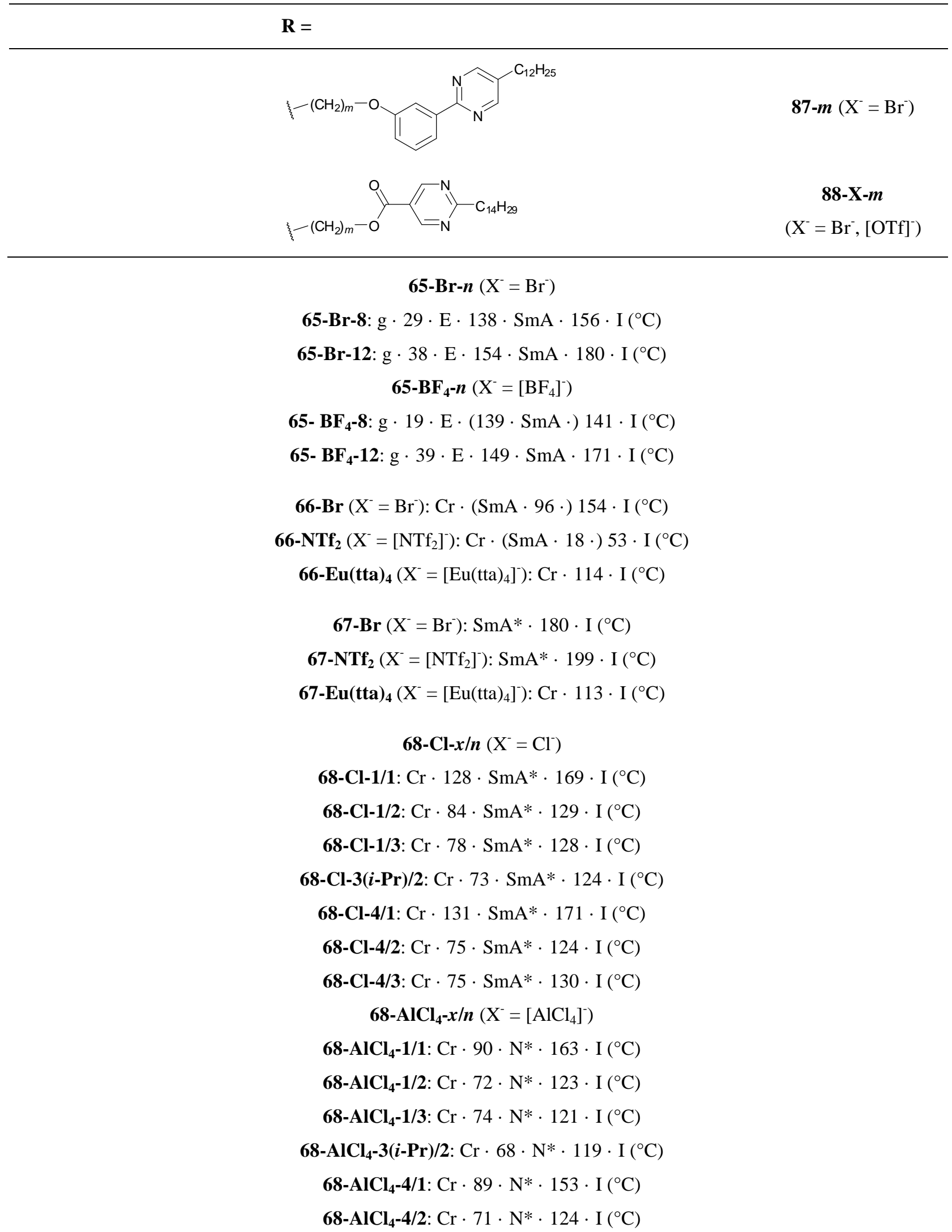


68- $\mathrm{AlCl}_{4}-\mathbf{4} / 3: \mathrm{Cr} \cdot 71 \cdot \mathrm{N}^{*} \cdot 113 \cdot \mathrm{I}\left({ }^{\circ} \mathrm{C}\right)$

69-Br-X/m/n $\left(\mathrm{X}^{-}=\mathrm{Br}^{-}\right)$

69-Br-1/4/12: $\mathrm{Cr} \cdot 88 \cdot \mathrm{SmA} \cdot 280 \cdot \mathrm{I}\left({ }^{\circ} \mathrm{C}\right)$

69-Br-1/5/12: $\mathrm{Cr} \cdot 88 \cdot \mathrm{SmA} \cdot 266 \cdot \mathrm{I}\left({ }^{\circ} \mathrm{C}\right)$

69-Br-1/6/12: $\mathrm{Cr} \cdot 65 \cdot \mathrm{SmA} \cdot 242 \cdot \mathrm{I}\left({ }^{\circ} \mathrm{C}\right)$

69-Br-1/7/12: $\mathrm{Cr} \cdot 66 \cdot \mathrm{SmA} \cdot 207 \cdot \mathrm{I}\left({ }^{\circ} \mathrm{C}\right)$

69-Br-1/8/12: $\mathrm{Cr} \cdot 74 \cdot \mathrm{SmA} \cdot 180 \cdot \mathrm{I}\left({ }^{\circ} \mathrm{C}\right)$

69-Br-1/9/12: $\mathrm{Cr} \cdot 72 \cdot \mathrm{SmA} \cdot 171^{98} / 169^{99} \cdot \mathrm{I}\left({ }^{\circ} \mathrm{C}\right)$

69-Br-1/4/6: $\mathrm{Cr} \cdot 143 \cdot \mathrm{I}\left({ }^{\circ} \mathrm{C}\right)$

69-Br-1/4/8: $\mathrm{Cr} \cdot 94 \cdot \mathrm{SmA} \cdot 220 \cdot \mathrm{I}\left({ }^{\circ} \mathrm{C}\right)$

69-Br-1/4/10: $\mathrm{Cr} \cdot 69 \cdot \mathrm{SmA} \cdot 270 \cdot \mathrm{I}\left({ }^{\circ} \mathrm{C}\right)$

69-Br-1/4/11: $\mathrm{Cr} \cdot 88 \cdot \mathrm{SmA} \cdot 278 \cdot \mathrm{I}\left({ }^{\circ} \mathrm{C}\right)$

69-Br-1/4/14: $\mathrm{Cr} \cdot 87 \cdot \mathrm{SmA} \cdot 286 \cdot \mathrm{I}\left({ }^{\circ} \mathrm{C}\right)$

69-Br-1/9/6: $\mathrm{Cr} \cdot 60 \cdot \mathrm{SmA} \cdot 90 \cdot \mathrm{I}\left({ }^{\circ} \mathrm{C}\right)$

69-Br-1/9/8: $\mathrm{Cr} \cdot 74 \cdot \mathrm{SmA} \cdot 117 \cdot \mathrm{I}\left({ }^{\circ} \mathrm{C}\right)$

69-Br-1/9/10: $\mathrm{Cr} \cdot 69 \cdot \mathrm{SmA} \cdot 139 \cdot \mathrm{I}\left({ }^{\circ} \mathrm{C}\right)$

69-Br-1/9/11: $\mathrm{Cr} \cdot 79 \cdot \mathrm{SmA} \cdot 152 \cdot \mathrm{I}\left({ }^{\circ} \mathrm{C}\right)$

69-Br-1/9/14: $\mathrm{Cr} \cdot 83 \cdot \mathrm{SmA} \cdot 181 \cdot \mathrm{I}\left({ }^{\circ} \mathrm{C}\right)$

69-Br-4/4/12: $\mathrm{Cr} \cdot 57 \cdot \mathrm{SmA} \cdot 131 \cdot \mathrm{I}\left({ }^{\circ} \mathrm{C}\right)$

69-Br-4/5/12: $\mathrm{Cr} \cdot 49 \cdot \mathrm{SmA} \cdot 126 \cdot \mathrm{I}\left({ }^{\circ} \mathrm{C}\right)$

69-Br-4/6/12: $\mathrm{Cr} \cdot 77 \cdot \mathrm{SmA} \cdot 106 \cdot \mathrm{I}\left({ }^{\circ} \mathrm{C}\right)$

69-Br-4/7/12: $\mathrm{Cr} \cdot 61 \cdot \mathrm{SmA} \cdot 93 \cdot \mathrm{I}\left({ }^{\circ} \mathrm{C}\right)$

69-Br-4/8/12: $\mathrm{Cr} \cdot 79 \cdot \mathrm{SmA} \cdot 90 \cdot \mathrm{I}\left({ }^{\circ} \mathrm{C}\right)$

69-Br-4/9/12: $\mathrm{Cr} \cdot 75 \cdot \mathrm{SmA} \cdot 90 \cdot \mathrm{I}\left({ }^{\circ} \mathrm{C}\right)$

69-Br-4/4/6: $\mathrm{Cr} \cdot 27 \cdot \mathrm{I}\left({ }^{\circ} \mathrm{C}\right)$

69-Br-4/4/8: $\mathrm{Cr} \cdot 45 \cdot \mathrm{I}\left({ }^{\circ} \mathrm{C}\right)$

69-Br-4/4/10: $\mathrm{Cr} \cdot 50 \cdot \mathrm{SmA} \cdot 80 \cdot \mathrm{I}\left({ }^{\circ} \mathrm{C}\right)$

69-Br-4/4/11: $\mathrm{Cr} \cdot 47 \cdot \mathrm{SmA} \cdot 105 \cdot \mathrm{I}\left({ }^{\circ} \mathrm{C}\right)$

69-Br-4/4/14: $\mathrm{Cr} \cdot 61 \cdot \mathrm{SmA} \cdot 162 \cdot \mathrm{I}\left({ }^{\circ} \mathrm{C}\right)$

69-Br-4/9/6: $\mathrm{Cr} \cdot 63 \cdot \mathrm{I}\left({ }^{\circ} \mathrm{C}\right)$

69-Br-4/9/8: $\mathrm{Cr} \cdot 75 \cdot \mathrm{I}\left({ }^{\circ} \mathrm{C}\right)$

69-Br-4/9/10: $\mathrm{Cr} \cdot 76 \cdot \mathrm{I}\left({ }^{\circ} \mathrm{C}\right)$

69-Br-4/9/11: $\mathrm{Cr} \cdot 73 \cdot \mathrm{SmA} \cdot 82 \cdot \mathrm{I}\left({ }^{\circ} \mathrm{C}\right)$

69-Br-4/9/14: $\mathrm{Cr} \cdot 75 \cdot \mathrm{SmA} \cdot 116 \cdot \mathrm{I}\left({ }^{\circ} \mathrm{C}\right)$

69-I-1/9/12 ( $\left.\mathrm{X}^{-}=\mathrm{I}^{-} ; x=1, m=9, n=12\right): \mathrm{Cr} \cdot 71 \cdot \mathrm{SmA} \cdot 150 \cdot \mathrm{I}\left({ }^{\circ} \mathrm{C}\right)$

69-BF $\mathbf{B}_{4} \mathbf{- 1 / 9} / 12\left(\mathrm{X}^{-}=\left[\mathrm{BF}_{4}\right]^{-} ; x=1, m=9, n=12\right): \mathrm{Cr} \cdot 68 \cdot \mathrm{SmA} \cdot 158 \cdot \mathrm{I}\left({ }^{\circ} \mathrm{C}\right)$

69-PF $\mathbf{F}_{\mathbf{6}} \mathbf{- 1 / 9 / 1 2}\left(\mathrm{X}^{-}=\left[\mathrm{PF}_{6}\right]^{-} ; x=1, m=9, n=12\right): \mathrm{Cr} \cdot 96 \cdot \mathrm{SmA} \cdot 147 \cdot \mathrm{I}\left({ }^{\circ} \mathrm{C}\right)$ 
69-PF $\mathbf{6}_{\mathbf{6}} \mathbf{- 1 / 6 / 1 2}\left(\mathrm{X}^{-}=\left[\mathrm{PF}_{6}\right]^{-} ; x=1, m=6, n=12\right): \mathrm{Cr} \cdot 100 \cdot \mathrm{SmA} \cdot 174 \cdot \mathrm{I}\left({ }^{\circ} \mathrm{C}\right)$

70: $\mathrm{Cr} \cdot 34 \cdot \mathrm{SmA} \cdot 114 \cdot \mathrm{I}\left({ }^{\circ} \mathrm{C}\right)$

71-1/4/8: $\mathrm{Cr} \cdot 99 \cdot \mathrm{SmA} \cdot 190 \cdot \mathrm{I}\left({ }^{\circ} \mathrm{C}\right)$

71-1/4/10: $\mathrm{Cr} \cdot 89 \cdot \mathrm{SmA} \cdot 220 \cdot \mathrm{I}\left({ }^{\circ} \mathrm{C}\right)$

71-1/4/12: $\mathrm{Cr} \cdot 112 \cdot \mathrm{SmA} \cdot 255 \cdot \mathrm{I}\left({ }^{\circ} \mathrm{C}\right)$

71-1/8/8: $\mathrm{Cr} \cdot 88 \cdot \mathrm{SmA} \cdot 148 \cdot \mathrm{I}\left({ }^{\circ} \mathrm{C}\right)$

71-1/8/10: $\mathrm{Cr} \cdot 71 \cdot \mathrm{SmC} \cdot 98 \cdot \mathrm{SmA} \cdot 177 \cdot \mathrm{I}\left({ }^{\circ} \mathrm{C}\right)$

71-1/8/12: $\mathrm{Cr} \cdot 104 \cdot \mathrm{SmC} \cdot 116 \cdot \mathrm{SmA} \cdot 197 \cdot \mathrm{I}\left({ }^{\circ} \mathrm{C}\right)$

71-4/4/8: $\mathrm{Cr} \cdot 56 \cdot \mathrm{I}\left({ }^{\circ} \mathrm{C}\right)$

71-4/4/10: $\mathrm{Cr} \cdot 97 \cdot \mathrm{I}\left({ }^{\circ} \mathrm{C}\right)$

71-4/4/12: $\mathrm{Cr} \cdot 46 \cdot \mathrm{SmA} \cdot 113 \cdot \mathrm{I}\left({ }^{\circ} \mathrm{C}\right)$

71-4/8/8: $\mathrm{Cr} \cdot 82 \cdot \mathrm{I}\left({ }^{\circ} \mathrm{C}\right)$

71-4/8/10: $\mathrm{Cr} \cdot 54 \cdot \mathrm{SmA} \cdot 87 \cdot \mathrm{I}\left({ }^{\circ} \mathrm{C}\right)$

71-4/8/12: $\mathrm{Cr} \cdot 69 \cdot \mathrm{SmA} \cdot 106 \cdot \mathrm{I}\left({ }^{\circ} \mathrm{C}\right)$

71-12/4/8: $\mathrm{Cr} \cdot(\mathrm{SmA} \cdot 100 \cdot) 110 \cdot \mathrm{I}\left({ }^{\circ} \mathrm{C}\right)$

71-12/4/10: $\mathrm{Cr} \cdot\left(\mathrm{SmA} \cdot 116(\right.$ ?) $\cdot) 112 \cdot \mathrm{I}\left({ }^{\circ} \mathrm{C}\right)$

71-12/4/12: $\mathrm{Cr} \cdot 111 \cdot \mathrm{SmA} \cdot 125 \cdot \mathrm{I}\left({ }^{\circ} \mathrm{C}\right)$

71-12/8/8: $\mathrm{Cr} \cdot 74 \cdot \mathrm{SmA} \cdot 79 \cdot \mathrm{I}\left({ }^{\circ} \mathrm{C}\right)$

71-12/8/10: $\mathrm{Cr} \cdot(\mathrm{SmA} \cdot 82(?) \cdot) 78 \cdot \mathrm{I}\left({ }^{\circ} \mathrm{C}\right)$

71-12/8/12: $\mathrm{Cr} \cdot 81 \cdot \mathrm{I}\left({ }^{\circ} \mathrm{C}\right)$

72-1/4: $\mathrm{Cr} \cdot 81 \cdot \mathrm{SmA} \cdot 185 \cdot \mathrm{I}\left({ }^{\circ} \mathrm{C}\right)$

72-1/8: $\mathrm{Cr} \cdot 52 \cdot \mathrm{SmA} \cdot 134 \cdot \mathrm{I}\left({ }^{\circ} \mathrm{C}\right)$

72-4/4: $\mathrm{Cr} \cdot 38 \cdot \mathrm{I}\left({ }^{\circ} \mathrm{C}\right)$

72-4/8: $\mathrm{Cr} \cdot 40 \cdot \mathrm{SmA} \cdot 89 \cdot \mathrm{I}\left({ }^{\circ} \mathrm{C}\right)$

72-12/4: $\mathrm{Cr} \cdot 55 \cdot \mathrm{SmA} \cdot 88 \cdot \mathrm{I}\left({ }^{\circ} \mathrm{C}\right)$

72-12/8: $\mathrm{Cr} \cdot 50 \cdot \mathrm{SmA} \cdot 54 \cdot \mathrm{I}\left({ }^{\circ} \mathrm{C}\right)$

73-Br- $x / m\left(\mathrm{X}^{-}=\mathrm{Br}^{-}\right)$

73-Br-1/4: $\mathrm{Cr} \cdot 44 \cdot \mathrm{SmA} \cdot 237 \cdot \mathrm{I}\left({ }^{\circ} \mathrm{C}\right)$

73-Br-1/8: $\mathrm{Cr} \cdot 42 \cdot \mathrm{SmA} \cdot 219 \cdot \mathrm{I}\left({ }^{\circ} \mathrm{C}\right)$

73-Br-12/4: $\mathrm{Cr} \cdot 41 \cdot \mathrm{SmA} \cdot 164 \cdot \mathrm{I}\left({ }^{\circ} \mathrm{C}\right)$

73-Br-12/8: $\mathrm{Cr} \cdot 55 \cdot \mathrm{SmA} \cdot 76 \cdot \mathrm{I}\left({ }^{\circ} \mathrm{C}\right)$

73-OTf- $x / m\left(\mathrm{X}^{-}=[\mathrm{OTf}]^{-}\right)$

73-OTf-1/4: $\mathrm{Cr} \cdot 35 \cdot \mathrm{SmA} \cdot 142 \cdot \mathrm{I}\left({ }^{\circ} \mathrm{C}\right)$

73-OTf-1/8: $\mathrm{Cr} \cdot 32 \cdot \mathrm{SmA} \cdot 79 \cdot \mathrm{I}\left({ }^{\circ} \mathrm{C}\right)$

73-OTf-12/4: $\mathrm{Cr} \cdot 25 \cdot \mathrm{SmA} \cdot 73 \cdot \mathrm{I}\left({ }^{\circ} \mathrm{C}\right)$

73-OTf-12/8: $\mathrm{Cr} \cdot 22 \cdot \mathrm{I}\left({ }^{\circ} \mathrm{C}\right)$ 


$$
\begin{aligned}
& \text { 74-Br-1/n- } \mathrm{NO}_{2}\left(\mathrm{X}^{-}=\mathrm{Br}^{-}, x=1, \mathrm{Z}=\mathrm{NO}_{2}\right) \\
& \text { 74-Br-1/3-NO }{ }_{2}: \mathrm{Cr} \cdot 177 \cdot \mathrm{I}\left({ }^{\circ} \mathrm{C}\right) \\
& \text { 74-Br-1/6-NO }{ }_{2}: \mathrm{Cr} \cdot 153 \cdot \mathrm{SmA} \cdot 165 \cdot \mathrm{I}\left({ }^{\circ} \mathrm{C}\right) \\
& \text { 74-Br-1/10-NO } \mathrm{N}_{2}: \mathrm{Cr} \cdot 110 \cdot \mathrm{SmA} \cdot 170 \cdot \mathrm{I}\left({ }^{\circ} \mathrm{C}\right) \\
& \text { 74-BF } \mathbf{H}_{4} \mathbf{- 1} / \mathbf{n}-\mathrm{NO}_{2}\left(\mathrm{X}^{-}=\left[\mathrm{BF}_{4}\right]^{-}, x=1, \mathrm{Z}=\mathrm{NO}_{2}\right) \\
& \text { 74-BF }{ }_{4}-\mathbf{1} / 3-\mathrm{NO}_{2}: \mathrm{Cr} \cdot 169 \cdot \mathrm{I}\left({ }^{\circ} \mathrm{C}\right) \\
& \text { 74-BF } \mathbf{H}_{4} \mathbf{- 1} / \mathbf{6}-\mathrm{NO}_{2}: \mathrm{Cr} \cdot 115 \cdot \mathrm{SmA} \cdot 149 \cdot \mathrm{I}\left({ }^{\circ} \mathrm{C}\right) \\
& \text { 74-BF }{ }_{4}-\mathbf{1} / \mathbf{1 0}-\mathrm{NO}_{2}: \mathrm{Cr} \cdot 104 \cdot \mathrm{SmA} \cdot 165 \cdot \mathrm{I}\left({ }^{\circ} \mathrm{C}\right) \\
& \text { 74-Br- } \mathbf{x} / \mathbf{n}-\mathrm{CH}_{3}\left(\mathrm{X}^{-}=\mathrm{Br}^{-}, \mathrm{Z}=\mathrm{CH}_{3}\right) \\
& \text { 74-Br-8/2-(- } \mathrm{CH}_{3}: \mathrm{Cr}_{1} \cdot 66 \cdot \mathrm{Cr}_{2} \cdot 138 \cdot \mathrm{I}\left({ }^{\circ} \mathrm{C}\right)
\end{aligned}
$$$$
\text { 74-Br-10/2- } \mathrm{CH}_{3}: \mathrm{Cr}_{1} \cdot 8 \cdot \mathrm{Cr}_{2} \cdot 92 \cdot \mathrm{Cr}_{3} \cdot 114 \cdot \mathrm{SmA} \cdot 147 \cdot \mathrm{I}\left({ }^{\circ} \mathrm{C}\right)
$$$$
\text { 74-Br-12/2- } \mathrm{CH}_{3}: \mathrm{Cr} \cdot 127 \cdot \mathrm{SmA} \cdot 167 \cdot \mathrm{I}\left({ }^{\circ} \mathrm{C}\right)
$$$$
\text { 74-Br-8/6- } \mathbf{C H}_{3}: \mathrm{Cr}_{1} \cdot 62 \cdot \mathrm{Cr}_{2} \cdot 75 \cdot \mathrm{I}\left({ }^{\circ} \mathrm{C}\right)
$$$$
\text { 74-Br-10/6- } \mathrm{CH}_{3}: \mathrm{Cr} \cdot 75 \cdot \mathrm{I}\left({ }^{\circ} \mathrm{C}\right)
$$$$
\text { 74-Br-12/6- } \mathrm{CH}_{3}: \mathrm{Cr}_{1} \cdot 93 \cdot \mathrm{Cr}_{2} \cdot 98 \cdot \mathrm{I}\left({ }^{\circ} \mathrm{C}\right)
$$$$
\text { 74-Br-14/6- } \mathrm{CH}_{3}: \mathrm{Cr}_{1} \cdot 25 \cdot \mathrm{Cr}_{2} \cdot 86 \cdot \mathrm{I}\left({ }^{\circ} \mathrm{C}\right)
$$$$
\text { 74-Br-16/6- } \mathrm{CH}_{3}: \mathrm{Cr} \cdot(\mathrm{SmA} \cdot 97 \cdot) 100 \cdot \mathrm{I}\left({ }^{\circ} \mathrm{C}\right)
$$$$
\text { 75-Br-1/n-O ( } \left.\mathrm{X}^{-}=\mathrm{Br}^{-}, x=1, \mathrm{Z}=\mathrm{O}\right)
$$

75-Br-1/6-O: $\mathrm{Cr}_{1} \cdot 154 \cdot \mathrm{Cr}_{2} \cdot\left[\right.$ not reported] $\cdot \mathrm{I}\left({ }^{\circ} \mathrm{C}\right)$

75-Br-1/10-O: $\mathrm{Cr}_{1} \cdot 77 \cdot \mathrm{Cr}_{2} \cdot 156 \cdot \mathrm{SmC} \cdot 169 \cdot \mathrm{I}\left({ }^{\circ} \mathrm{C}\right)$

75-BF $\mathbf{H}_{\mathbf{4}} \mathbf{- 1} \mathbf{n}-\mathbf{O}\left(\mathrm{X}^{-}=\left[\mathrm{BF}_{4}\right]^{-}, x=1, \mathrm{Z}=\mathrm{O}\right)$

$$
\text { 75- } \mathbf{B F}_{4}-\mathbf{- 1} / \mathbf{6}-\mathrm{O}: \mathrm{Cr} \cdot 143 \cdot \mathrm{I}\left({ }^{\circ} \mathrm{C}\right)
$$

75- $\mathrm{BF}_{4}-\mathbf{1} / \mathbf{1 0 - O}: \mathrm{Cr}_{1} \cdot 77 \cdot \mathrm{Cr}_{2} \cdot 98 \cdot \mathrm{Cr}_{3} \cdot 155 \cdot \mathrm{I}\left({ }^{\circ} \mathrm{C}\right)$

75-Br- $\mathbf{x} / \mathbf{0}-\mathbf{C H}_{2}\left(\mathrm{X}^{-}=\mathrm{Br}^{-}, n=0, \mathrm{Z}=\mathrm{CH}_{2}\right)$

75-Br-6/0- $\mathbf{C H}_{2}: \mathrm{Cr} \cdot 213 \cdot \mathrm{I} / \mathrm{dec} .\left({ }^{\circ} \mathrm{C}\right)$

75-Br-8/0-CH$: \mathbf{C r} \cdot 242 \cdot \mathrm{I} / \mathrm{dec} .\left({ }^{\circ} \mathrm{C}\right)$

75-Br-10/0-- $\mathrm{CH}_{2}: \mathrm{Cr}_{1} \cdot 144 \cdot \mathrm{Cr}_{2} \cdot 222 \cdot \mathrm{I} / \mathrm{dec} .\left({ }^{\circ} \mathrm{C}\right)$

75-Br-12/0- $\mathbf{C H}_{2}: \mathrm{Cr} \cdot 152 \cdot \mathrm{SmA} \cdot 181 \cdot \mathrm{I} / \mathrm{dec} .\left({ }^{\circ} \mathrm{C}\right)$

75-Br-14/0- $\mathbf{C H}_{2}: \mathrm{Cr}_{1} \cdot 76 \cdot \mathrm{Cr}_{2} \cdot 102 \cdot \mathrm{SmA} \cdot 185 \cdot \mathrm{I} /$ dec. $\left({ }^{\circ} \mathrm{C}\right)$

76- $\mathrm{BF}_{4}\left(\mathrm{X}^{-}=\left[\mathrm{BF}_{4}\right]^{-}\right): \mathrm{Cr} \cdot 35 \cdot \mathrm{SmA} \cdot 250 \cdot$ dec. $\left({ }^{\circ} \mathrm{C}\right)$ (data from $1^{\text {st }} \mathrm{DSC}$ cooling run, from $100{ }^{\circ} \mathrm{C}$, and from $2^{\text {nd }}$ DSC heating run)

$$
\text { 76-OTf }\left(\mathrm{X}^{-}=[\mathrm{OTf}]^{-}\right): \mathrm{Cr} \cdot 35 \cdot \mathrm{SmA} \cdot 148 \cdot \mathrm{I}\left({ }^{\circ} \mathrm{C}\right)\left(1^{\text {st }} \mathrm{DSC} \text { cooling run }\right)
$$

76-NTf ${ }_{2}\left(\mathrm{X}^{-}=\left[\mathrm{NTf}_{2}\right]^{-}\right): \mathrm{SmA} \cdot 64 \cdot \mathrm{I}\left({ }^{\circ} \mathrm{C}\right)\left(1^{\text {st }} \mathrm{DSC}\right.$ cooling run; melting point from $2^{\text {nd }}$ DSC heating run: $\left.\mathrm{ca} .42{ }^{\circ} \mathrm{C}\right)$

76-OTf/[C $\left.\mathrm{C}_{2} \mathrm{mim}\right][\mathrm{OTf}]$ : see ref. 100 for a full phase diagram

76-OTf/[C $\left.\mathrm{C}_{2} \mathrm{mim}\right][\mathrm{OTf}] / \mathrm{LiOTf}(70: 30: 0): \mathrm{Cr}+\mathrm{I} * \cdot 13 \cdot \mathrm{SmA} \cdot 107 \cdot \mathrm{I}\left({ }^{\circ} \mathrm{C}\right)\left(1^{\text {st }} \mathrm{DSC}\right.$ cooling run $)$

76-OTf $/\left[\mathrm{C}_{2} \mathrm{mim}\right][\mathrm{OTf}] / \mathrm{LiOTf}(70: 30: 3.75): \mathrm{Cr}+\mathrm{I}^{*} \cdot 10 \cdot \mathrm{SmA} \cdot 109 \cdot \mathrm{I}\left({ }^{\circ} \mathrm{C}\right)\left(1^{\text {st }} \mathrm{DSC}\right.$ cooling run $)$ 
76-OTf/[C $\left.\mathrm{C}_{2} \mathrm{mim}\right][\mathrm{OTf}] / \mathrm{LiOTf}(70: 30: 7.5): \mathrm{Cr}+\mathrm{I} * \cdot 6 \cdot \mathrm{SmA} \cdot 109 \cdot \mathrm{I}\left({ }^{\circ} \mathrm{C}\right)\left(1^{\text {st }} \mathrm{DSC}\right.$ cooling run $)$ 76-OTf/[C $\left.\mathrm{C}_{2} \mathrm{mim}\right][\mathrm{OTf}] / \mathrm{LiOTf}(70: 30: 15): \mathrm{SmA} \cdot 109 \cdot \mathrm{I}\left({ }^{\circ} \mathrm{C}\right)\left(1^{\text {st }} \mathrm{DSC}\right.$ cooling run $)$

76-OTf/[C $\left.\mathrm{C}_{2} \mathrm{mim}\right]\left[\mathrm{BF}_{4}\right]$ : see ref. 100 for a full phase diagram

76-OTf/[C $\left.\mathrm{C}_{2} \mathrm{mim}\right]\left[\mathrm{NTf}_{2}\right]$ : see ref. 100 for a full phase diagram * = biphasic mixture of crystalline and isotropic phases

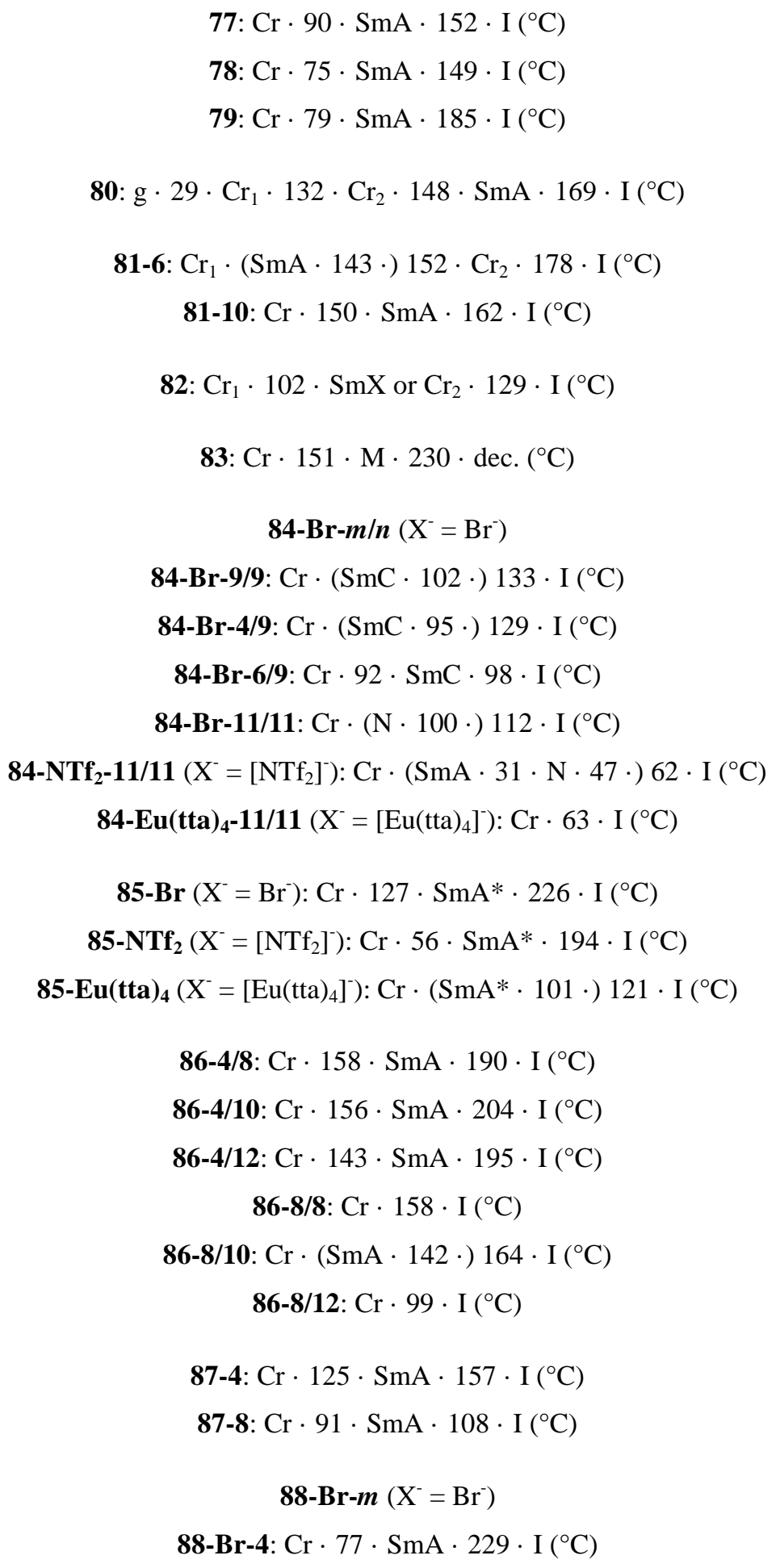


88-Br-8: $\mathrm{Cr} \cdot 101 \cdot \mathrm{SmA} \cdot 172 \cdot \mathrm{I}\left({ }^{\circ} \mathrm{C}\right)$

88-OTf-m $\left(\mathrm{X}^{-}=[\mathrm{OTf}]^{-}\right)$

88-OTf-4: $\mathrm{Cr} \cdot 90 \cdot \mathrm{SmA} \cdot 142 \cdot \mathrm{I}\left({ }^{\circ} \mathrm{C}\right)$

88-OTf-8: $\mathrm{Cr} \cdot 49 \cdot \mathrm{SmA} \cdot 72 \cdot \mathrm{I}\left({ }^{\circ} \mathrm{C}\right)$
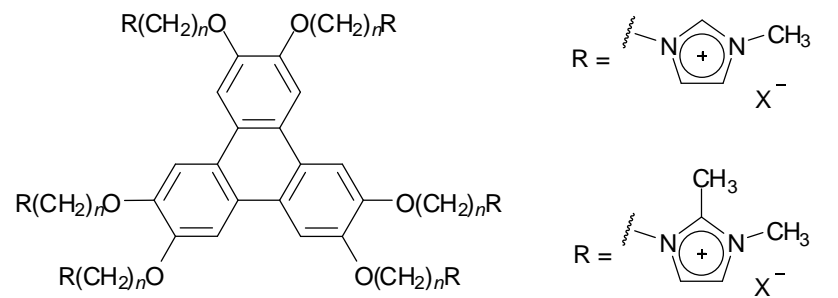

89-(1-Me-imid)-X-n

$\left(\mathrm{X}^{-}=\left[\mathrm{BF}_{4}\right]^{-},\left[\mathrm{PF}_{6}\right]^{-},\left[\mathrm{NTf}_{2}\right]^{-}\right)$

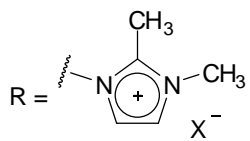

89-(1,2-Me-imid)-X-n

$\left(\mathrm{X}^{-}=\left[\mathrm{BF}_{4}\right]^{-},\left[\mathrm{PF}_{6}\right]^{-},\left[\mathrm{NTf}_{2}\right]^{-}\right)$

89-(1-Me-imid)-BF ${ }_{4}$-n $\left(\mathrm{X}^{-}=\left[\mathrm{BF}_{4}\right]^{-}\right)$

89-(1-Me-imid)-BF 4 -8: g $\cdot 2 \cdot \mathrm{I}\left({ }^{\circ} \mathrm{C}\right)$

89-(1-Me-imid)-BF $\mathbf{- 1 0 :} \mathrm{g} \cdot 10 \cdot \mathrm{Cub}_{\mathrm{V}}(\mathrm{Ia} \overline{3} d) \cdot 70 \cdot \mathrm{Col}_{\mathrm{h}} \cdot 114 \cdot \mathrm{I}\left({ }^{\circ} \mathrm{C}\right)$

89-(1-Me-imid)-BF $\mathbf{- 1 2 : ~ g ~} \cdot 16 \cdot \mathrm{Cub}_{\mathrm{V}}(\mathrm{Ia} \overline{3} d) \cdot 160 \cdot \mathrm{Col}_{\mathrm{h}} \cdot 180 \cdot \mathrm{I}\left({ }^{\circ} \mathrm{C}\right)$

89-(1-Me-imid)-BF $4 \mathbf{4}$ 14: $\mathrm{g} \cdot 24 \cdot \mathrm{Cub}_{\mathrm{V}}(\operatorname{Ia} \overline{3} d) \cdot 221 \cdot \mathrm{I}\left({ }^{\circ} \mathrm{C}\right)^{101} / \mathrm{g} \cdot 23 \cdot \mathrm{Cub}_{\mathrm{V}}(\operatorname{Ia} \overline{3} d) \cdot 218 \cdot \mathrm{I}\left({ }^{\circ} \mathrm{C}\right)^{102}$

89-(1-Me-imid)-PF $-\boldsymbol{n}\left(\mathrm{X}^{-}=\left[\mathrm{PF}_{6}\right]^{-}\right)$

89-(1-Me-imid)-PF $\mathbf{6}_{\mathbf{6}} \mathbf{- 1 0} \mathrm{g} \cdot 11 \cdot \mathrm{I}\left({ }^{\circ} \mathrm{C}\right)$

89-(1-Me-imid)-PF $\mathbf{6}-12: \mathrm{g} \cdot 5 \cdot \mathrm{Cub}_{\mathrm{V}}(\mathrm{Ia} \overline{3} d) \cdot 88 \cdot \mathrm{Col}_{\mathrm{h}} \cdot 129 \cdot \mathrm{I}\left({ }^{\circ} \mathrm{C}\right)$

89-(1-Me-imid)-PF $\mathbf{6}-14: \mathrm{g} \cdot 21 \cdot \mathrm{Cub}_{\mathrm{V}}(\mathrm{Ia} \overline{3} d) \cdot\left[\right.$ not reported] $\cdot \mathrm{Col}_{\mathrm{h}} \cdot 177 \cdot \mathrm{I}\left({ }^{\circ} \mathrm{C}\right)$

89-(1-Me-imid)-NTf $\mathbf{2}_{2} \boldsymbol{n}\left(\mathrm{X}^{-}=\left[\mathrm{NTf}_{2}\right]^{-}\right)$

89-(1-Me-imid)-NTf ${ }_{2}-10: \mathrm{g} \cdot-27 \cdot \mathrm{I}\left({ }^{\circ} \mathrm{C}\right)$

89-(1-Me-imid)-NTf ${ }_{2}-12: \mathrm{g} \cdot-14 \cdot \mathrm{I}\left({ }^{\circ} \mathrm{C}\right)$

89-(1-Me-imid)-NTf 2 -14: g $\cdot 1 \cdot \mathrm{Cub}_{\mathrm{V}}(\mathrm{Ia} \overline{3} d) \cdot 49 \cdot \mathrm{Col}_{\mathrm{h}} \cdot 94 \cdot \mathrm{I}\left({ }^{\circ} \mathrm{C}\right)$

89-(1,2-Me-imid)-BF ${ }_{4}-\boldsymbol{n}\left(\mathrm{X}^{-}=\left[\mathrm{BF}_{4}\right]^{-}\right)$

89-(1,2-Me-imid)-BF 4 -8: g $\cdot 8 \cdot \mathrm{I}\left({ }^{\circ} \mathrm{C}\right)$

89-(1,2-Me-imid)-BF 4 -10: g $\cdot 15 \cdot \mathrm{Col}_{\mathrm{h}} \cdot 126 \cdot \mathrm{I}\left({ }^{\circ} \mathrm{C}\right)^{101} / \mathrm{g} \cdot 19 \cdot \mathrm{Col}_{\mathrm{h}} \cdot 139 \cdot \mathrm{I}\left({ }^{\circ} \mathrm{C}\right)^{102}$

89-(1,2-Me-imid)-BF $\mathbf{4}_{\mathbf{4}} \mathbf{- 1 2} \mathrm{g} \cdot 7 \cdot \mathrm{Cub}_{\mathrm{V}}(\mathrm{Ia} \overline{3} d) \cdot 76 \cdot \mathrm{Col}_{\mathrm{h}} \cdot 188 \cdot \mathrm{I}\left({ }^{\circ} \mathrm{C}\right)$

89-(1,2-Me-imid)-BF $\mathbf{4}-14: \mathrm{g} \cdot 19 \cdot \mathrm{Cub}_{\mathrm{V}}(\operatorname{Pn} \overline{3} m) \cdot 129 \cdot \mathrm{Cub}_{\mathrm{V}}(\operatorname{Ia} \overline{3} d) \cdot 210 \cdot \mathrm{Col}_{\mathrm{h}} \cdot 226 \cdot \mathrm{I}\left({ }^{\circ} \mathrm{C}\right)$

89-(1,2-Me-imid)-PF $\mathbf{6}^{-n}\left(\mathrm{X}^{-}=\left[\mathrm{PF}_{6}\right]^{-}\right)$

89-(1,2-Me-imid)-PF $\mathbf{6}-10: \mathrm{g} \cdot-15 \cdot \mathrm{Col}_{\mathrm{h}} \cdot 57 \cdot \mathrm{I}\left({ }^{\circ} \mathrm{C}\right)$

89-(1,2-Me-imid)-PF $\mathbf{6}-12: \mathrm{g} \cdot 26 \cdot \mathrm{Col}_{\mathrm{h}} \cdot 151 \cdot \mathrm{I}\left({ }^{\circ} \mathrm{C}\right)$

89-(1,2-Me-imid)-PF $\mathbf{6}-14: \mathrm{g} \cdot 21 \cdot \mathrm{Cub}_{\mathrm{V}}(\mathrm{Ia} \overline{3} d) \cdot 143 \cdot \mathrm{Col}_{\mathrm{h}} \cdot 188 \cdot \mathrm{I}\left({ }^{\circ} \mathrm{C}\right)$

89-(1,2-Me-imid)-NTf ${ }_{2}-\boldsymbol{n}\left(\mathrm{X}^{-}=\left[\mathrm{NTf}_{2}\right]^{-}\right)$

89-(1,2-Me-imid)-NTf ${ }_{2}-10: \mathrm{g} \cdot-18 \cdot \mathrm{I}\left({ }^{\circ} \mathrm{C}\right)$

89-(1,2-Me-imid)-NTf 2 -12: g $\cdot-11 \cdot \mathrm{Col}_{\mathrm{h}} \cdot 74 \cdot \mathrm{I}\left({ }^{\circ} \mathrm{C}\right)$ 
89-(1,2-Me-imid)-NTf $\mathbf{2}_{\mathbf{2}} \mathbf{- 1 4}: \mathrm{g} \cdot 1 \cdot \mathrm{Cub}_{\mathrm{V}}(\mathrm{Ia} \overline{3} d) \cdot 39 \cdot \mathrm{Col}_{\mathrm{h}} \cdot 108 \cdot \mathrm{I}\left({ }^{\circ} \mathrm{C}\right)$

89-(1,2-Me-imid)-BF $\mathbf{4}-10+1$ wt.\% SWCNTs: g $\cdot 19 \cdot \mathrm{Col}_{\mathrm{h}} \cdot 134 \cdot \mathrm{I}\left({ }^{\circ} \mathrm{C}\right)$

89-(1,2-Me-imid)-BF $\mathbf{4}-10+3$ wt.\% SWCNTs: g $\cdot 20 \cdot \mathrm{Col}_{\mathrm{h}} \cdot 126 \cdot \mathrm{I}\left({ }^{\circ} \mathrm{C}\right)$

89-(1,2-Me-imid)-BF $\mathbf{4}-10+5$ wt.\% SWCNTs: g $\cdot 20 \cdot \mathrm{Col}_{\mathrm{h}} \cdot 126 \cdot \mathrm{I}\left({ }^{\circ} \mathrm{C}\right)$

89-(1-Me-imid)-BF $\mathbf{4}_{\mathbf{4}} \mathbf{- 1 4}+3$ wt.\% SWCNTs: g · $24 \cdot \mathrm{Cub}_{\mathrm{V}}(\mathrm{Ia} \overline{3} d) \cdot 222 \cdot \mathrm{I}\left({ }^{\circ} \mathrm{C}\right)$

89-(1-Me-imid)-BF $\mathbf{4}_{\mathbf{4}} \mathbf{1 4}+5$ wt.\% SWCNTs: g $\cdot 24 \cdot \mathrm{Cub}_{\mathrm{V}}(\mathrm{Ia} \overline{3} d) \cdot 208 \cdot \mathrm{I}\left({ }^{\circ} \mathrm{C}\right)$ 
Scheme S2. Imidazolium-based ILCs that contain at least one disk-like mesogenic group that is connected to the cationic core via a flexible spacer.

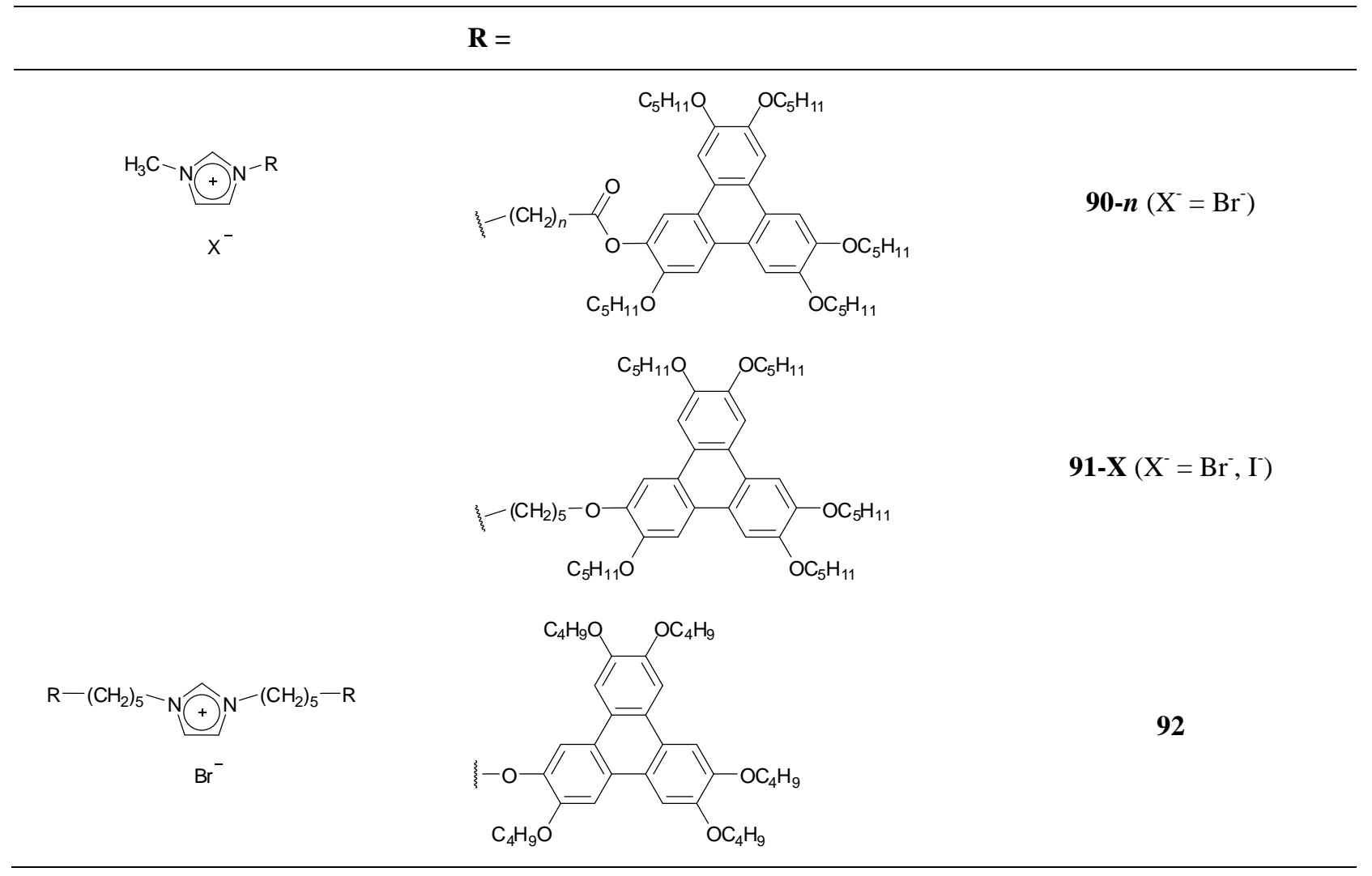

90-4: $\mathrm{Cr} \cdot 71 \cdot \mathrm{I}\left({ }^{\circ} \mathrm{C}\right)$

90-7: $\mathrm{g} \cdot-10 \cdot \mathrm{Col}_{\mathrm{ob}} \cdot 82 \cdot \mathrm{I}\left({ }^{\circ} \mathrm{C}\right)$

90-10: $\mathrm{Cr} \cdot-20 \cdot \mathrm{Col}_{\mathrm{r}} \cdot 110 \cdot \mathrm{I}\left({ }^{\circ} \mathrm{C}\right)$

91-Br $\left(\mathrm{X}^{-}=\mathrm{Br}\right): \mathrm{Cr} \cdot 67 \cdot \mathrm{Col} \cdot 101 \cdot \mathrm{I}\left({ }^{\circ} \mathrm{C}\right)$

91-I $\left(\mathrm{X}^{-}=\mathrm{I}^{-}\right): \mathrm{Cr} \cdot 64 \cdot \mathrm{Col} \cdot 83 \cdot \mathrm{I}\left({ }^{\circ} \mathrm{C}\right)$

92: $\mathrm{Cr} \cdot 84 \cdot \mathrm{Col}_{\mathrm{r}} \cdot 95 \cdot \mathrm{I}\left({ }^{\circ} \mathrm{C}\right)$

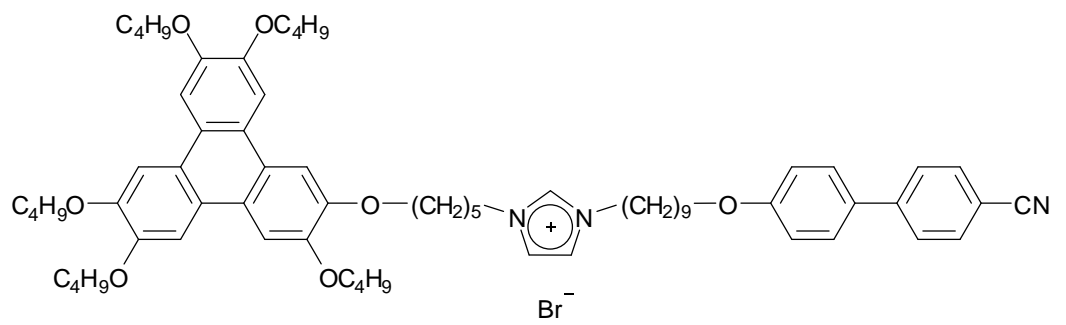

93: $\mathrm{Cr} \cdot 135 \cdot \mathrm{I}\left({ }^{\circ} \mathrm{C}\right)$ 
Scheme S3. LC symmetric gemini imidazolium salts tethered with mesogenic groups.

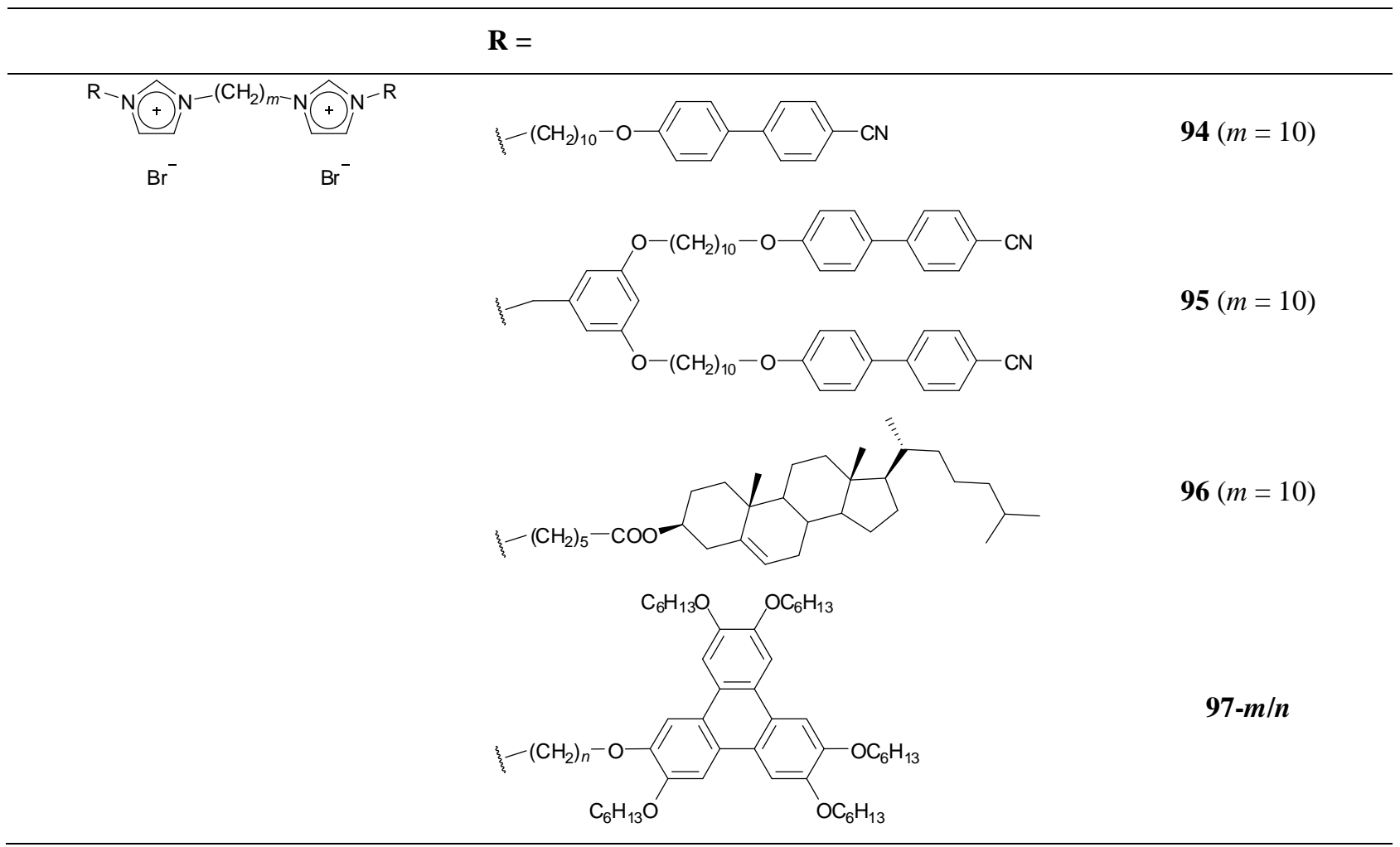

94: $\mathrm{Cr} \cdot(\mathrm{SmA} \cdot 55 \cdot) 55 \cdot \mathrm{I}\left({ }^{\circ} \mathrm{C}\right)$

95: $\mathrm{g} \cdot 51 \cdot \mathrm{SmA} \cdot 79 \cdot \mathrm{N} \cdot 81 \cdot \mathrm{I}\left({ }^{\circ} \mathrm{C}\right)$

96: $\mathrm{g} \cdot 39 \cdot \mathrm{SmA} \cdot 135 \cdot$ dec. $\left({ }^{\circ} \mathrm{C}\right)$

97-4/6: $\mathrm{Cr} \cdot 48 \cdot \mathrm{Col}_{\mathrm{h}} \cdot 180 \cdot \mathrm{I}\left({ }^{\circ} \mathrm{C}\right)$

97-5/12: $\mathrm{Cr} \cdot 60 \cdot \mathrm{Col}_{\mathrm{h}} \cdot 121 \cdot \mathrm{I}\left({ }^{\circ} \mathrm{C}\right)$

97-8/8: $\mathrm{Cr} \cdot 59 \cdot \mathrm{Col}_{\mathrm{h}} \cdot 87 \cdot \mathrm{I}\left({ }^{\circ} \mathrm{C}\right)$

97-8/9: $\mathrm{Cr} \cdot 59 \cdot \mathrm{Col}_{\mathrm{h}} \cdot 86 \cdot \mathrm{I}\left({ }^{\circ} \mathrm{C}\right)$

97-8/10: $\mathrm{Cr} \cdot 58 \cdot \mathrm{I}\left({ }^{\circ} \mathrm{C}\right)$

97-8/12: $\mathrm{Cr} \cdot 58 \cdot \mathrm{I}\left({ }^{\circ} \mathrm{C}\right)$ 


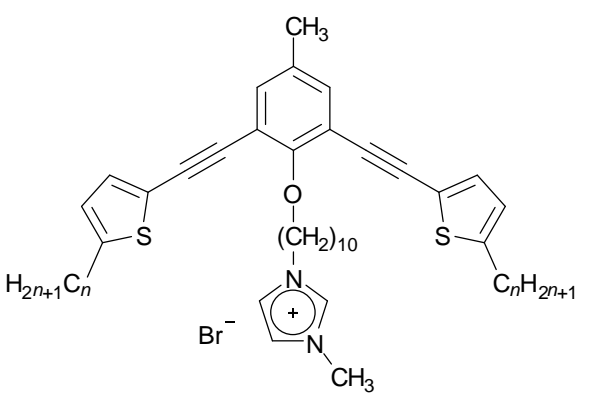

98-n

98-6: $\mathrm{Cr} \cdot 116 \cdot \mathrm{I}\left({ }^{\circ} \mathrm{C}\right)$

98-8: $\mathrm{Col}_{\mathrm{h}} \cdot 77 \cdot \mathrm{I}\left({ }^{\circ} \mathrm{C}\right)$

98-16: $\mathrm{Cr} \cdot 37 \cdot \mathrm{I}\left({ }^{\circ} \mathrm{C}\right)$ 
Scheme S4. LCs containing 1,3,4-oxadiazole moieties.

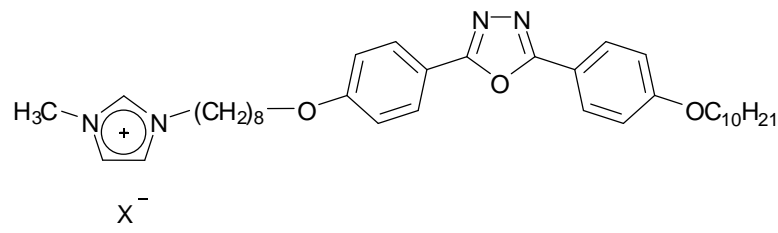

(-

$\mathrm{Br}^{-}$

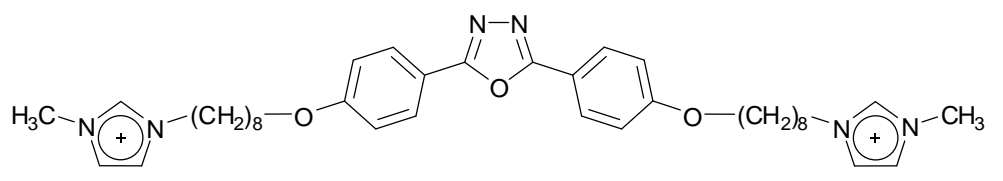

$\mathrm{Br}$
$\mathrm{Br}$

$$
\begin{gathered}
\text { 99-X }\left(\mathrm{X}^{-}=\mathrm{Br}^{-},\left[\mathrm{NO}_{3}\right]^{-},\left[\mathrm{BF}_{4}\right]^{-},\right. \\
\left.\left[\mathrm{ClO}_{4}\right]^{-},\left[\mathrm{C}_{12} \mathrm{H}_{25} \mathrm{OSO}_{3}\right]^{-}\right)
\end{gathered}
$$
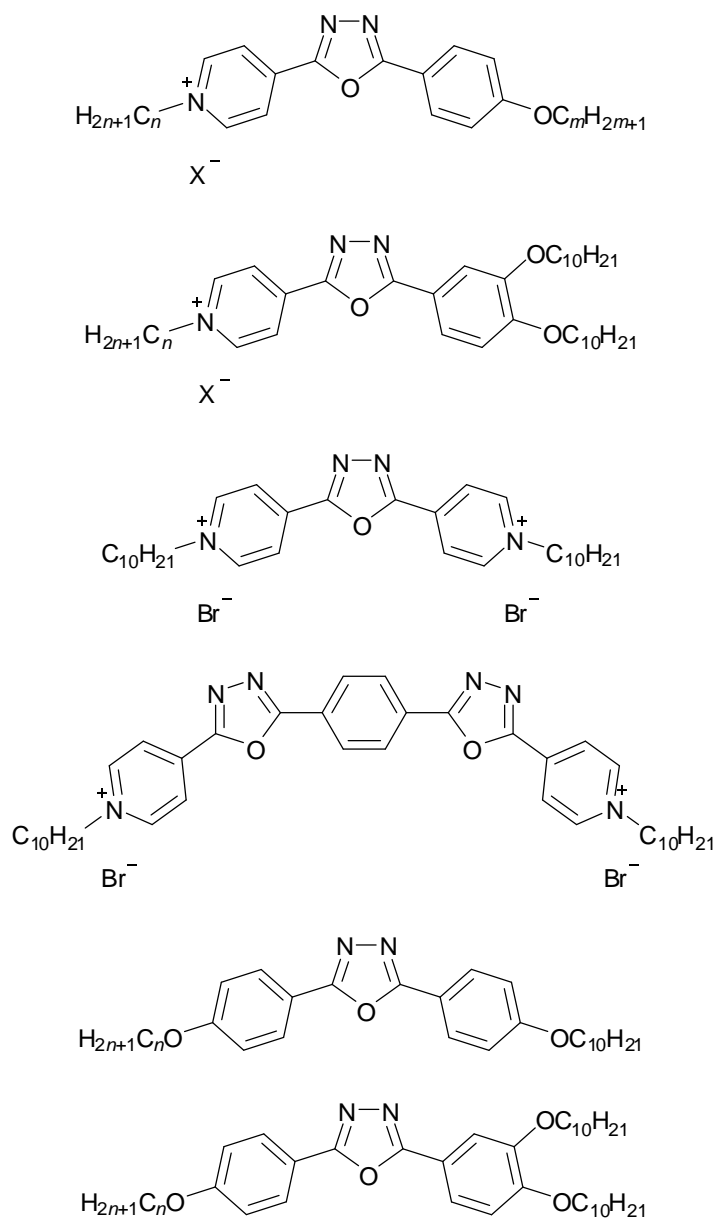

102-X-n/m $\left(\mathrm{X}^{-}=\mathrm{Br}^{-}, \mathrm{I}^{-},\left[\mathrm{NO}_{3}\right]^{-}\right.$, $\left.\left[\mathrm{BF}_{4}\right]^{-},\left[\mathrm{ClO}_{4}\right]^{-},\left[\mathrm{C}_{12} \mathrm{H}_{25} \mathrm{OSO}_{3}\right]^{-}\right)$

103-X-n $\left(\mathrm{X}^{-}=\mathrm{Br}^{-}, \mathrm{I}^{-}\right)$

107-n 


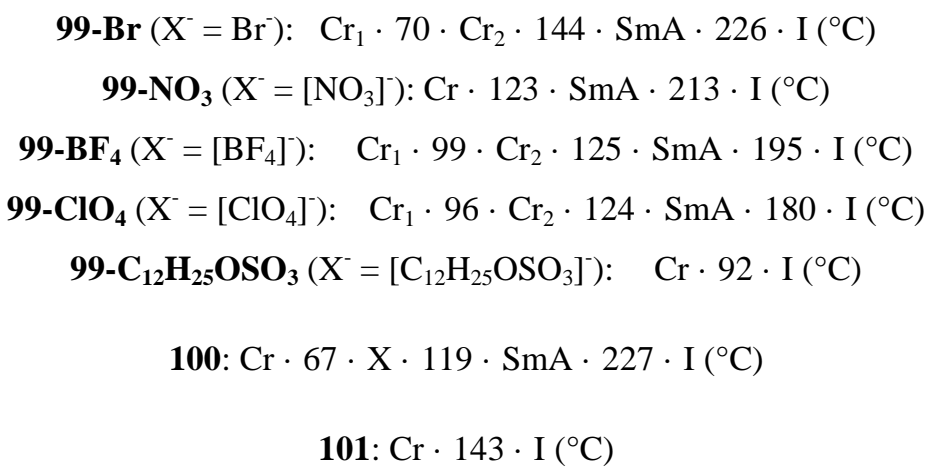




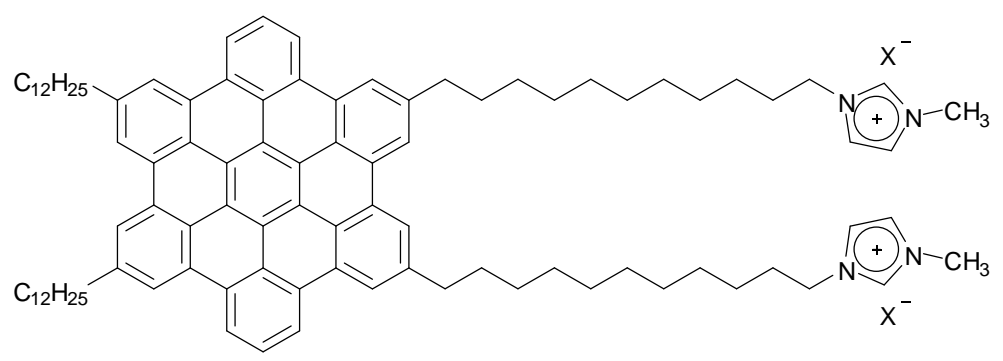

108-X $\left(\mathrm{X}^{-}=\mathrm{Cl}^{-},\left[\mathrm{BF}_{4}\right]^{-},\left[\mathrm{PF}_{6}\right]^{-}\right)$

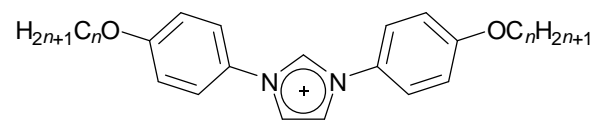

[OTf]

109-n

109-8: $\mathrm{Cr} \cdot 120 \cdot \mathrm{SmA} \cdot 126 \cdot \mathrm{I}\left({ }^{\circ} \mathrm{C}\right)$

109-10: $\mathrm{Cr} \cdot 116 \cdot \mathrm{SmA} \cdot 158 \cdot \mathrm{I}\left({ }^{\circ} \mathrm{C}\right)$

109-12: $\mathrm{Cr} \cdot 117 \cdot \mathrm{SmA} \cdot 176 \cdot \mathrm{I}\left({ }^{\circ} \mathrm{C}\right)$

109-14: $\mathrm{Cr} \cdot 118 \cdot \mathrm{SmA} \cdot 184 \cdot \mathrm{I}\left({ }^{\circ} \mathrm{C}\right)$

109-16: $\mathrm{Cr} \cdot 113 \cdot \mathrm{SmA} \cdot 191 \cdot \mathrm{I}\left({ }^{\circ} \mathrm{C}\right)$

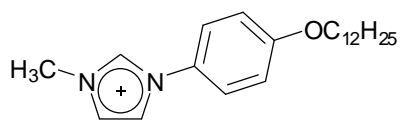

$x^{-}$

110-I $\left(\mathrm{X}^{-}=\mathrm{I}^{-}\right): \mathrm{Cr} \cdot 113 \cdot \mathrm{SmA} \cdot 250 \cdot \operatorname{dec} .\left({ }^{\circ} \mathrm{C}\right)$

110-BF $4\left(\mathrm{X}^{-}=\left[\mathrm{BF}_{4}\right]^{-}\right): \mathrm{Cr} \cdot 91 \cdot \mathrm{SmA} \cdot 230 \cdot \operatorname{dec} .\left({ }^{\circ} \mathrm{C}\right)$

110-PF $6\left(\mathrm{X}^{-}=\left[\mathrm{PF}_{6}\right]^{-}\right): \mathrm{Cr} \cdot 97 \cdot \mathrm{SmA} \cdot 163 \cdot \mathrm{I}\left({ }^{\circ} \mathrm{C}\right)$

110-OTf $\left(\mathrm{X}^{-}=[\mathrm{OTf}]^{-}\right): \mathrm{Cr} \cdot 77 \cdot \mathrm{SmA} \cdot 95 \cdot \mathrm{I}\left({ }^{\circ} \mathrm{C}\right)$

110-NTf $\mathbf{N}_{2}\left(\mathrm{X}^{-}=\left[\mathrm{NTf}_{2}\right]^{-}\right): \mathrm{Cr} \cdot 59 \cdot \mathrm{I}\left({ }^{\circ} \mathrm{C}\right)$ 


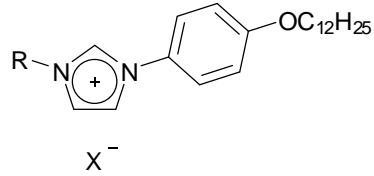

111-X $\left(\mathrm{X}^{-}=\mathrm{I}^{-},\left[\mathrm{BF}_{4}\right]^{-} ; \mathrm{R}=n-\mathrm{C}_{3} \mathrm{H}_{7}, n-\mathrm{C}_{12} \mathrm{H}_{25}\right.$,

3,7,11-trimethyldodecyl)

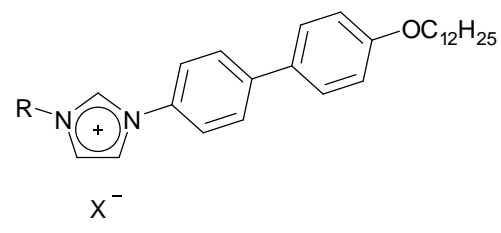

113-X $\left(\mathrm{X}^{-}=\mathrm{I}^{-},\left[\mathrm{BF}_{4}\right]^{-},\left[\mathrm{PF}_{6}\right]^{-},\left[\mathrm{ClO}_{4}\right]^{-},\left[\mathrm{C}_{12} \mathrm{H}_{25} \mathrm{SO}_{3}\right]^{-}\right.$;

$\mathrm{R}=n-\mathrm{C}_{3} \mathrm{H}_{7}, n-\mathrm{C}_{12} \mathrm{H}_{25}$, 3,7,11-trimethyldodecyl,

$\left(\mathrm{CH}_{2} \mathrm{CH}_{2} \mathrm{O}\right)_{3} \mathrm{CH}_{3}$, (S)-citronellyl)

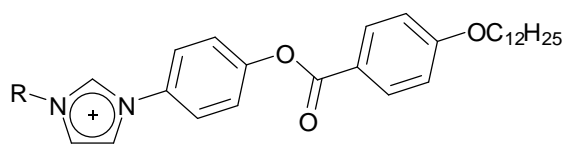

$x^{-}$

115-X $\left(\mathrm{X}^{-}=\mathrm{I}^{-},\left[\mathrm{BF}_{4}\right]^{-} ; \mathrm{R}=n-\mathrm{C}_{3} \mathrm{H}_{7}, n-\mathrm{C}_{12} \mathrm{H}_{25}\right.$,

3,7,11-trimethyldodecyl)

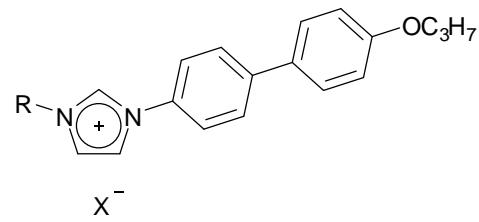

112-X $\left(\mathrm{X}^{-}=\mathrm{I}^{-},\left[\mathrm{BF}_{4}\right]^{-},\left[\mathrm{C}_{12} \mathrm{H}_{25} \mathrm{SO}_{3}\right]^{-} ; \mathrm{R}=n-\mathrm{C}_{3} \mathrm{H}_{7}\right.$, $n$ - $\mathrm{C}_{12} \mathrm{H}_{25}$, 3,7,11-trimethyldodecyl, $\left.\left(\mathrm{CH}_{2} \mathrm{CH}_{2} \mathrm{O}\right)_{3} \mathrm{CH}_{3}\right)$

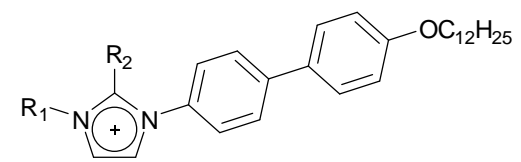

$x^{-}$

$$
\left.\mathrm{R}_{2}=\mathrm{CH}_{3}, \mathrm{C}_{2} \mathrm{H}_{5}, n-\mathrm{C}_{5} \mathrm{H}_{11}, \mathrm{Ph}\right)
$$

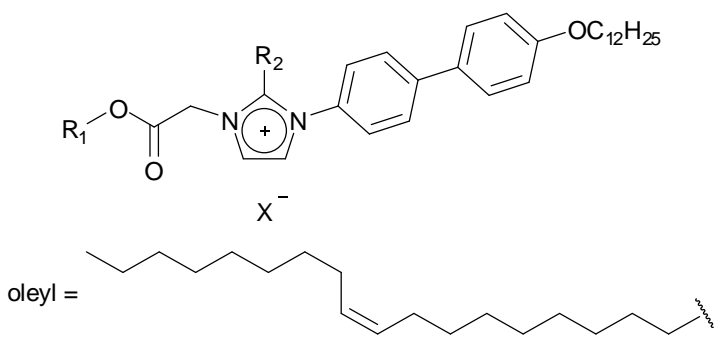

116-X $\left(\mathrm{X}^{-}=\mathrm{I}^{-},\left[\mathrm{BF}_{4}\right]^{-},\left[\mathrm{NTf}_{2}\right]^{-} ; \mathrm{R}_{1}=n-\mathrm{C}_{18} \mathrm{H}_{37}\right.$, oleyl;

$\mathrm{R}_{2}=\mathrm{H}, \mathrm{CH}_{3}, \mathrm{C}_{2} \mathrm{H}_{5}, n-\mathrm{C}_{3} \mathrm{H}_{7}, i-\mathrm{C}_{3} \mathrm{H}_{7}$ )

\section{1-I-(a-c) $\left(\mathrm{X}^{-}=\mathrm{I}^{-}\right)$}

111-I-a $\left(\mathrm{R}=n-\mathrm{C}_{3} \mathrm{H}_{7}\right): \mathrm{g}_{\mathrm{SmA}} \cdot 1 \cdot \mathrm{SmA} \cdot 57 \cdot \mathrm{I}\left({ }^{\circ} \mathrm{C}\right)$

111-I-b $\left(\mathrm{R}=n-\mathrm{C}_{12} \mathrm{H}_{25}\right): \mathrm{Cr} \cdot 85 \cdot \mathrm{SmA} \cdot 184 \cdot \mathrm{I}\left({ }^{\circ} \mathrm{C}\right)$

111-I-c (R = 3,7,11-trimethyldodecyl): $\mathrm{Cr} \cdot 83 \cdot \mathrm{SmA} \cdot 153 \cdot \mathrm{I}\left({ }^{\circ} \mathrm{C}\right)$

111-BF $4^{-}$(a-c) $\left(\mathrm{X}^{-}=\left[\mathrm{BF}_{4}\right]^{-}\right)$

111- $\mathrm{BF}_{4}-\mathbf{a}\left(\mathrm{R}=n-\mathrm{C}_{3} \mathrm{H}_{7}\right)$ : not LC

111- $-\mathrm{BF}_{4}-\mathbf{b}\left(\mathrm{R}=n-\mathrm{C}_{12} \mathrm{H}_{25}\right): \mathrm{Cr} \cdot 62 \cdot \mathrm{SmA} \cdot 141 \cdot \mathrm{I}\left({ }^{\circ} \mathrm{C}\right)$

111-BF $\mathbf{B F}_{4} \mathbf{- C}(\mathrm{R}=3,7,11$-trimethyldodecyl $): \mathrm{Cr} \cdot 69 \cdot \mathrm{SmA} \cdot 126 \cdot \mathrm{I}\left({ }^{\circ} \mathrm{C}\right)$

112-I-(a-d) $\left(\mathrm{X}^{-}=\mathrm{I}^{-}\right)$

112-I-a $\left(\mathrm{R}=n-\mathrm{C}_{3} \mathrm{H}_{7}\right): \mathrm{g}_{\text {Iso }} \cdot 36 \cdot \mathrm{I}\left({ }^{\circ} \mathrm{C}\right)$

112-I-b $\left(\mathrm{R}=n-\mathrm{C}_{12} \mathrm{H}_{25}\right): \mathrm{Cr} \cdot 120 \cdot \mathrm{SmA} \cdot 199 \cdot \mathrm{I}\left({ }^{\circ} \mathrm{C}\right)$

112-I-c (R = 3,7,11-trimethyldodecyl): $\mathrm{Cr} \cdot 143 \cdot \mathrm{SmA} \cdot 189 \cdot \mathrm{I}\left({ }^{\circ} \mathrm{C}\right)$ 


$$
\begin{aligned}
& \text { 112-I-d }\left(\mathrm{R}=\left(\mathrm{CH}_{2} \mathrm{CH}_{2} \mathrm{O}\right)_{3} \mathrm{CH}_{3}\right): \mathrm{g}_{\mathrm{Iso}} \cdot-24 \cdot \mathrm{I}\left({ }^{\circ} \mathrm{C}\right) \\
& \text { 112-BF } 4^{-}(\mathrm{a}-\mathrm{c})\left(\mathrm{X}^{-}=\left[\mathrm{BF}_{4}\right]^{-}\right) \\
& \text {112- }-\mathrm{BF}_{4}-\mathbf{a}\left(\mathrm{R}=n-\mathrm{C}_{12} \mathrm{H}_{25}\right): \mathrm{g}_{\mathrm{SmA}} \cdot-8 \cdot \mathrm{SmA} \cdot 121 \cdot \mathrm{I}\left({ }^{\circ} \mathrm{C}\right) \\
& \text { 112-BF }{ }_{4} \text {-b ( } \mathrm{R}=3,7,11 \text {-trimethyldodecyl): } \mathrm{Cr} \cdot 68 \cdot \mathrm{SmA} \cdot 132 \cdot \mathrm{I}\left({ }^{\circ} \mathrm{C}\right) \\
& \text { 112- }-\mathrm{BF}_{4}-\mathrm{C}\left(\mathrm{R}=\left(\mathrm{CH}_{2} \mathrm{CH}_{2} \mathrm{O}\right)_{3} \mathrm{CH}_{3}\right): \mathrm{g}_{\mathrm{Iso}} \cdot-30 \cdot \mathrm{I}\left({ }^{\circ} \mathrm{C}\right) \\
& \text { 112- } \mathrm{C}_{\mathbf{1 2}} \mathrm{H}_{25} \mathrm{SO}_{3} \text {-(a-b) }\left(\mathrm{X}^{-}=\left[\mathrm{C}_{12} \mathrm{H}_{25} \mathrm{SO}_{3}\right]^{-}\right) \\
& \text {112- } \mathrm{C}_{12} \mathrm{H}_{25} \mathrm{SO}_{3}-\mathbf{a}\left(\mathrm{R}=n-\mathrm{C}_{3} \mathrm{H}_{7}\right): \mathrm{Cr} \cdot 104 \cdot \mathrm{I}\left({ }^{\circ} \mathrm{C}\right) \\
& \text { 112- } \mathrm{C}_{12} \mathrm{H}_{25} \mathrm{SO}_{3}-\mathbf{b}\left(\mathrm{R}=n-\mathrm{C}_{12} \mathrm{H}_{25}\right): \mathrm{Cr} \cdot 135 \cdot \mathrm{I}\left({ }^{\circ} \mathrm{C}\right) \\
& \text { 113-I-(a-e) }\left(\mathrm{X}^{-}=\mathrm{I}^{-}\right) \\
& \text {113-I-a }\left(\mathrm{R}=n-\mathrm{C}_{3} \mathrm{H}_{7}\right): \mathrm{Cr} \cdot 93 \cdot \mathrm{SmA} \cdot 220 \cdot \mathrm{I} / \text { dec. }\left({ }^{\circ} \mathrm{C}\right) \\
& \text { 113-I-b }\left(\mathrm{R}=n-\mathrm{C}_{12} \mathrm{H}_{25}\right): \mathrm{Cr} \cdot 128 \cdot \mathrm{SmA} \cdot>250 \cdot \text { dec. }\left({ }^{\circ} \mathrm{C}\right) \\
& \text { 113-I-c ( } \mathrm{R}=3,7,11 \text {-trimethyldodecyl): } \mathrm{Cr} \cdot 151 \cdot \mathrm{SmA} \cdot 235 \cdot \mathrm{I}\left({ }^{\circ} \mathrm{C}\right) \\
& \text { 113-I-d }\left(\mathrm{R}=\left(\mathrm{CH}_{2} \mathrm{CH}_{2} \mathrm{O}\right)_{3} \mathrm{CH}_{3}\right): \mathrm{g}_{\mathrm{SmA}} \cdot-1 \cdot \mathrm{SmA} \cdot 70 \cdot \mathrm{I}\left({ }^{\circ} \mathrm{C}\right) \\
& \text { 113-I-e }\left(\mathrm{R}=(S) \text {-citronellyl): } \mathrm{Cr} \cdot 152 \cdot \mathrm{SmA} \cdot 222 \cdot \mathrm{I}\left({ }^{\circ} \mathrm{C}\right)\right. \\
& \text { 113-BF }- \text {-(a-e) }\left(\mathrm{X}^{-}=\left[\mathrm{BF}_{4}\right]^{-}\right) \\
& \text {113- } \mathbf{B F}_{4}-\mathbf{a}\left(\mathrm{R}=n-\mathrm{C}_{3} \mathrm{H}_{7}\right): \mathrm{g}_{\mathrm{SmA}} \cdot 8 \cdot \mathrm{SmA} \cdot 183 \cdot \mathrm{I}\left({ }^{\circ} \mathrm{C}\right) \\
& \text { 113- } \mathbf{B F}_{4}-\mathbf{b}\left(\mathrm{R}=n-\mathrm{C}_{12} \mathrm{H}_{25}\right): \mathrm{Cr} \cdot 94 \cdot \mathrm{SmA} \cdot 203 \cdot \mathrm{I}\left({ }^{\circ} \mathrm{C}\right) \\
& \text { 113-BF } \mathbf{B F}_{4} \mathbf{- C}\left(\mathrm{R}=3,7,11 \text {-trimethyldodecyl): } \mathrm{Cr} \cdot 94 \cdot \mathrm{SmA} \cdot 203 \cdot \mathrm{I}\left({ }^{\circ} \mathrm{C}\right)\right. \\
& \text { 113-BF } \mathbf{B F}_{4}-\mathbf{d}\left(\mathrm{R}=\left(\mathrm{CH}_{2} \mathrm{CH}_{2} \mathrm{O}\right)_{3} \mathrm{CH}_{3}\right): \mathrm{Cr} \cdot 8 \cdot \mathrm{SmA} \cdot 75 \cdot \mathrm{I}\left({ }^{\circ} \mathrm{C}\right) \\
& \text { 113-BF } \mathbf{B}_{4} \mathbf{- e}\left(\mathrm{R}=(\mathrm{S}) \text {-citronellyl): } \mathrm{Cr} \cdot 94 \cdot \mathrm{SmA} \cdot 168 \cdot \mathrm{I}\left({ }^{\circ} \mathrm{C}\right)\right. \\
& \text { 113-PF } \mathbf{P F}_{6}\left(\mathrm{X}^{-}=\left[\mathrm{PF}_{6}\right] ; \mathrm{R}=n-\mathrm{C}_{3} \mathrm{H}_{7}\right): \mathrm{Cr} \cdot 100 \cdot \mathrm{SmA} \cdot 140 \cdot \mathrm{I}\left({ }^{\circ} \mathrm{C}\right) \\
& \text { 113- } \mathrm{ClO}_{4}\left(\mathrm{X}^{-}=\left[\mathrm{ClO}_{4}\right]^{-} ; \mathrm{R}=n-\mathrm{C}_{3} \mathrm{H}_{7}\right): \mathrm{g}_{\mathrm{SmA}} \cdot 5 \cdot \mathrm{SmA} \cdot 171 \cdot \mathrm{I}\left({ }^{\circ} \mathrm{C}\right) \\
& \text { 113- } \mathrm{C}_{12} \mathbf{H}_{25} \mathrm{SO}_{3}\left(\mathrm{X}^{-}=\left[\mathrm{C}_{12} \mathrm{H}_{25} \mathrm{SO}_{3}\right]^{-} ; \mathrm{R}=n-\mathrm{C}_{3} \mathrm{H}_{7}\right): \mathrm{Cr} \cdot 105 \cdot \mathrm{SmA} \cdot 181 \cdot \mathrm{I}\left({ }^{\circ} \mathrm{C}\right) \\
& \text { 114-I-(a-g) }\left(\mathrm{X}^{-}=\mathrm{I}^{-}\right)
\end{aligned}
$$$$
\text { 114-I-a }\left(\mathrm{R}_{1}=n-\mathrm{C}_{3} \mathrm{H}_{7}, \mathrm{R}_{2}=\mathrm{CH}_{3}\right): \mathrm{SmX}(?) \cdot 46 \cdot \mathrm{SmA} \cdot 206 \cdot \mathrm{I}\left({ }^{\circ} \mathrm{C}\right)
$$$$
\text { 114-I-b }\left(\mathrm{R}_{1}=n-\mathrm{C}_{12} \mathrm{H}_{25}, \mathrm{R}_{2}=\mathrm{CH}_{3}\right): \mathrm{Cr} \cdot 107 \cdot \mathrm{SmA} \cdot 262 \cdot \mathrm{I} / \text { dec. }\left({ }^{\circ} \mathrm{C}\right)
$$$$
\text { 114-I-c }\left(\mathrm{R}_{1}=n-\mathrm{C}_{3} \mathrm{H}_{7}, \mathrm{R}_{2}=\mathrm{C}_{2} \mathrm{H}_{5}\right): \mathrm{g}_{\mathrm{SmA}} \cdot 18 \cdot \mathrm{SmA} \cdot 148 \cdot \mathrm{I}\left({ }^{\circ} \mathrm{C}\right)
$$$$
\text { 114-I-d }\left(\mathrm{R}_{1}=n-\mathrm{C}_{12} \mathrm{H}_{25}, \mathrm{R}_{2}=\mathrm{C}_{2} \mathrm{H}_{5}\right): \mathrm{Cr} \cdot 131 \cdot \mathrm{SmA} \cdot 197 \cdot \mathrm{I}\left({ }^{\circ} \mathrm{C}\right)
$$$$
\text { 114-I-e }\left(\mathrm{R}_{1}=n-\mathrm{C}_{3} \mathrm{H}_{7}, \mathrm{R}_{2}=n-\mathrm{C}_{5} \mathrm{H}_{11}\right): \mathrm{Cr} \cdot 90 \cdot \mathrm{I}\left({ }^{\circ} \mathrm{C}\right)
$$$$
\text { 114-I-f }\left(\mathrm{R}_{1}=n-\mathrm{C}_{12} \mathrm{H}_{25}, \mathrm{R}_{2}=n-\mathrm{C}_{5} \mathrm{H}_{11}\right): \mathrm{Cr} \cdot 123 \cdot \mathrm{I}\left({ }^{\circ} \mathrm{C}\right)
$$$$
\text { 114-I-g }\left(\mathrm{R}_{1}=n-\mathrm{C}_{12} \mathrm{H}_{25}, \mathrm{R}_{2}=\mathrm{Ph}\right): \mathrm{Cr} \cdot 87 \cdot \mathrm{I}\left({ }^{\circ} \mathrm{C}\right)
$$

$$
\text { 114-BF } \mathbf{H}^{-} \mathbf{-}(\mathbf{a}-\mathrm{g})\left(\mathrm{X}^{-}=\left[\mathrm{BF}_{4}\right]^{-}\right)
$$

114- $\mathrm{BF}_{4}-\mathbf{a}\left(\mathrm{R}_{1}=n-\mathrm{C}_{3} \mathrm{H}_{7}, \mathrm{R}_{2}=\mathrm{CH}_{3}\right): \mathrm{SmX}(?) \cdot 41 \cdot \mathrm{SmA} \cdot 145 \cdot \mathrm{I}\left({ }^{\circ} \mathrm{C}\right)$ 114-BF ${ }_{4}-\mathbf{b}\left(\mathrm{R}_{1}=n-\mathrm{C}_{12} \mathrm{H}_{25}, \mathrm{R}_{2}=\mathrm{CH}_{3}\right): \mathrm{Cr} \cdot 80 \cdot \mathrm{SmA} \cdot 219 \cdot \mathrm{I} /$ dec. $\left({ }^{\circ} \mathrm{C}\right)$

114- $\mathrm{BF}_{4}-\mathrm{C}\left(\mathrm{R}_{1}=n-\mathrm{C}_{3} \mathrm{H}_{7}, \mathrm{R}_{2}=\mathrm{C}_{2} \mathrm{H}_{5}\right): \mathrm{g}_{\mathrm{SmA}} \cdot-2 \cdot \mathrm{SmA} \cdot 123 \cdot \mathrm{I}\left({ }^{\circ} \mathrm{C}\right)$ 114- $\mathrm{BF}_{4}-\mathbf{d}\left(\mathrm{R}_{1}=n-\mathrm{C}_{12} \mathrm{H}_{25}, \mathrm{R}_{2}=\mathrm{C}_{2} \mathrm{H}_{5}\right): \mathrm{SmX}(?) \cdot 61 \cdot \mathrm{SmA} \cdot 149 \cdot \mathrm{I}\left({ }^{\circ} \mathrm{C}\right)$ 114- $\mathrm{BF}_{4}-\mathbf{e}\left(\mathrm{R}_{1}=n-\mathrm{C}_{3} \mathrm{H}_{7}, \mathrm{R}_{2}=n-\mathrm{C}_{5} \mathrm{H}_{11}\right): \mathrm{Cr} \cdot 76 \cdot \mathrm{I}\left({ }^{\circ} \mathrm{C}\right)$ 
114- $\mathrm{BF}_{4}-\mathbf{f}\left(\mathrm{R}_{1}=n-\mathrm{C}_{12} \mathrm{H}_{25}, \mathrm{R}_{2}=n-\mathrm{C}_{5} \mathrm{H}_{11}\right): \mathrm{Cr} \cdot 85 \cdot \mathrm{I}\left({ }^{\circ} \mathrm{C}\right)$

114- $\mathrm{BF}_{4}-\mathbf{g}\left(\mathrm{R}_{1}=n-\mathrm{C}_{12} \mathrm{H}_{25}, \mathrm{R}_{2}=\mathrm{Ph}\right): \mathrm{Cr} \cdot 82 \cdot \mathrm{I}\left({ }^{\circ} \mathrm{C}\right)$

115-I-(a-c) $\left(\mathrm{X}^{-}=\mathrm{I}^{-}\right)$

115-I-a $\left(\mathrm{R}=n-\mathrm{C}_{3} \mathrm{H}_{7}\right): \mathrm{Cr} \cdot 140 \cdot \mathrm{SmA} \cdot 214 \cdot \mathrm{I}\left({ }^{\circ} \mathrm{C}\right)$

115-I-b $\left(\mathrm{R}=n-\mathrm{C}_{12} \mathrm{H}_{25}\right): \mathrm{Cr} \cdot 160 \cdot \mathrm{SmA} \cdot 233 \cdot \mathrm{I}\left({ }^{\circ} \mathrm{C}\right)$

115-I-c (R = 3,7,11-trimethyldodecyl): $\mathrm{Cr} \cdot 170 \cdot \mathrm{SmA} \cdot 208 \cdot \mathrm{I}\left({ }^{\circ} \mathrm{C}\right)$

115-BF $\mathbf{B}^{-}(\mathrm{a}-\mathrm{c})\left(\mathrm{X}^{-}=\left[\mathrm{BF}_{4}\right]^{-}\right)$

115- $\mathbf{B F}_{4}-\mathbf{a}\left(\mathrm{R}=n-\mathrm{C}_{3} \mathrm{H}_{7}\right): \mathrm{g}_{\mathrm{SmA}} \cdot 8 \cdot \mathrm{SmA} \cdot 175 \cdot \mathrm{I}\left({ }^{\circ} \mathrm{C}\right)$

115- $\mathrm{BF}_{4}-\mathrm{b}\left(\mathrm{R}=n-\mathrm{C}_{12} \mathrm{H}_{25}\right): \mathrm{Cr} \cdot 130 \cdot \mathrm{SmA} \cdot 201 \cdot \mathrm{I}\left({ }^{\circ} \mathrm{C}\right)$

115-BF ${ }_{4}$-c $(\mathrm{R}=3,7,11$-trimethyldodecyl $): \mathrm{Cr} \cdot 123 \cdot \mathrm{SmA} \cdot 179 \cdot \mathrm{I}\left({ }^{\circ} \mathrm{C}\right)$

$$
\text { 116-BF }- \text {-(a-f) }\left(\mathrm{X}^{-}=\left[\mathrm{BF}_{4}\right]^{-}\right)
$$

116-BF ${ }_{4}-\mathbf{a}\left(\mathrm{R}_{1}=\right.$ oleyl (ca. 85\% cis), $\left.\mathrm{R}_{2}=\mathrm{H}\right): \mathrm{Cr} \cdot 76 \cdot \mathrm{SmA} \cdot 208 \cdot \mathrm{I}\left({ }^{\circ} \mathrm{C}\right)$

116- $\mathrm{BF}_{4}-\mathrm{b}\left(\mathrm{R}_{1}=\right.$ oleyl $($ ca. 85\% cis $\left.), \mathrm{R}_{2}=\mathrm{CH}_{3}\right):<-10 \cdot \mathrm{SmA} \cdot 160 \cdot \mathrm{I}\left({ }^{\circ} \mathrm{C}\right)$

116- $-\mathrm{BF}_{4}-\mathrm{cc}\left(\mathrm{R}_{1}=\right.$ oleyl (ca. 85\% cis), $\left.\mathrm{R}_{2}=\mathrm{C}_{2} \mathrm{H}_{5}\right):<-10 \cdot \mathrm{SmA} \cdot 140 \cdot \mathrm{I}\left({ }^{\circ} \mathrm{C}\right)$

116- $\mathrm{BF}_{4}-\mathrm{d}\left(\mathrm{R}_{1}=\right.$ oleyl (ca. 85\% cis), $\left.\mathrm{R}_{2}=n-\mathrm{C}_{3} \mathrm{H}_{7}\right):<-10 \cdot \mathrm{SmA} \cdot 79 \cdot \mathrm{I}\left({ }^{\circ} \mathrm{C}\right)$

116- $\mathrm{BF}_{4}-\mathbf{e}\left(\mathrm{R}_{1}=\right.$ oleyl (ca. 85\% cis), $\left.\mathrm{R}_{2}=i-\mathrm{C}_{3} \mathrm{H}_{7}\right): \mathrm{Cr} \cdot 63 \cdot \mathrm{SmA} \cdot 116 \cdot \mathrm{I}\left({ }^{\circ} \mathrm{C}\right)$

116- $\mathbf{B F}_{4}-\mathbf{f}\left(\mathrm{R}_{1}=n-\mathrm{C}_{18} \mathrm{H}_{37}, \mathrm{R}_{2}=n-\mathrm{C}_{3} \mathrm{H}_{7}\right): \mathrm{Cr} \cdot 59 \cdot \mathrm{SmA} \cdot 233 \cdot \mathrm{I}\left({ }^{\circ} \mathrm{C}\right)$

$$
\text { 116-NTf }{ }_{2}-(\mathbf{a}-\mathbf{f})\left(\mathrm{X}^{-}=\left[\mathrm{NTf}_{2}\right]^{-}\right)
$$

116-NTf ${ }_{2}$-a $\left(\mathrm{R}_{1}=\right.$ oleyl (ca. 85\% cis), $\left.\mathrm{R}_{2}=\mathrm{H}\right): \mathrm{Cr} \cdot 44 \cdot \mathrm{SmA} \cdot 99 \cdot \mathrm{I}\left({ }^{\circ} \mathrm{C}\right)$

116-NTf $\mathbf{N}_{2}$-b $\left(\mathrm{R}_{1}=\right.$ oleyl (ca. 85\% cis), $\left.\mathrm{R}_{2}=\mathrm{CH}_{3}\right): \mathrm{Cr} \cdot 23 \cdot \mathrm{SmA} \cdot 83 \cdot \mathrm{I}\left({ }^{\circ} \mathrm{C}\right)$

116-NTf $\mathbf{N}_{2}-\mathrm{c}\left(\mathrm{R}_{1}=\right.$ oleyl (ca. 85\% cis), $\left.\mathrm{R}_{2}=\mathrm{C}_{2} \mathrm{H}_{5}\right): \mathrm{Cr} \cdot 17$ (?) $\cdot \mathrm{SmA} \cdot 55 \cdot \mathrm{I}\left({ }^{\circ} \mathrm{C}\right)$

116-NTf $\mathbf{N}_{2}-\mathbf{d}\left(\mathrm{R}_{1}=\right.$ oleyl (ca. 85\% cis), $\left.\mathrm{R}_{2}=n-\mathrm{C}_{3} \mathrm{H}_{7}\right): \mathrm{Cr} \cdot(-2 \cdot \mathrm{SmA} \cdot 7 \cdot) 35 \cdot \mathrm{I}\left({ }^{\circ} \mathrm{C}\right)$

116-NTf - $_{2}-\mathbf{e}\left(\mathrm{R}_{1}=\right.$ oleyl (ca. 85\% cis), $\left.\mathrm{R}_{2}=i-\mathrm{C}_{3} \mathrm{H}_{7}\right): \mathrm{Cr} \cdot-2 \cdot \mathrm{SmA} \cdot 25 \cdot \mathrm{I}\left({ }^{\circ} \mathrm{C}\right)$

116- $\mathrm{NTf}_{2}-\mathbf{f}\left(\mathrm{R}_{1}=n-\mathrm{C}_{18} \mathrm{H}_{37}, \mathrm{R}_{2}=n-\mathrm{C}_{3} \mathrm{H}_{7}\right): \mathrm{Cr} \cdot 90 \cdot \mathrm{SmA} \cdot 110 \cdot \mathrm{I}\left({ }^{\circ} \mathrm{C}\right)$ 
Scheme S6. Imidazolium-based ILCs investigated by Cheng, Tschierske and co-workers.

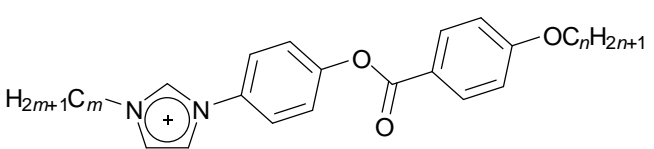

$\mathrm{Br}^{-}$

$117-m / n$

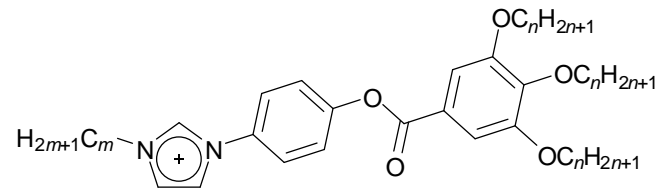

$\mathrm{Br}$

$119-m / n$

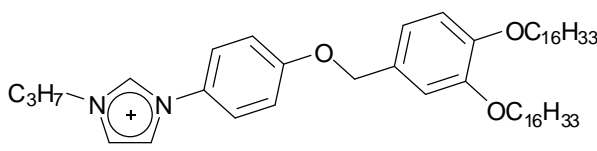

$\mathrm{Br}^{-}$

121

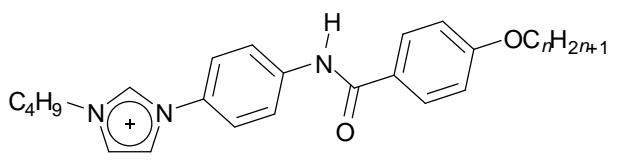

$\mathrm{Br}^{-}$

$123-m / n$

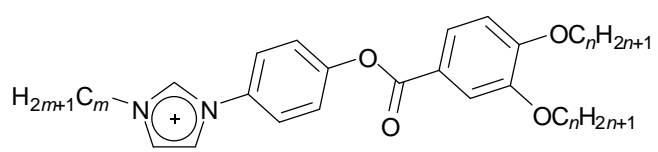

$\mathrm{Br}^{-}$

$118-m / n$

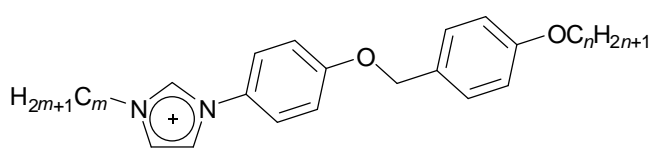

$\mathrm{Br}^{-}$

$120-m / n$

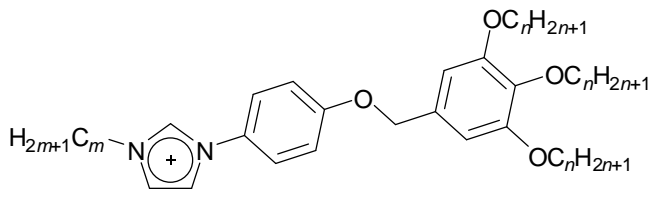

$\mathrm{Br}^{-}$

122-m/n

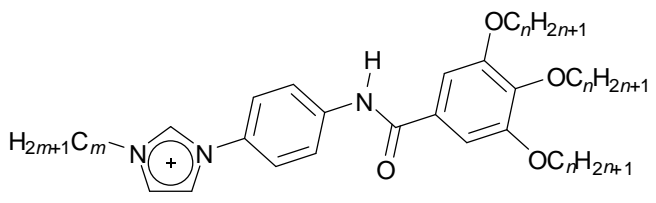

$\mathrm{Br}^{-}$

$124-m / n$

117-12/6: $\mathrm{Cr} \cdot 166 \cdot \mathrm{SmA} \cdot>200 \cdot$ dec. $\left({ }^{\circ} \mathrm{C}\right)$

117-18/6: $\mathrm{Cr} \cdot 172 \cdot \mathrm{SmA} \cdot>200 \cdot \operatorname{dec}\left({ }^{\circ} \mathrm{C}\right)$

117-4/8: $\mathrm{Cr} \cdot 199 \cdot \mathrm{I}\left({ }^{\circ} \mathrm{C}\right)$

117-12/8: $\mathrm{Cr} \cdot 190 \cdot \mathrm{SmA} \cdot>200 \cdot$ dec. $\left({ }^{\circ} \mathrm{C}\right)$

117-4/10: $\mathrm{Cr} \cdot\left(\mathrm{SmA}_{2} \cdot 154.5 \cdot \mathrm{N} \cdot 155 \cdot\right) 162 \cdot \mathrm{I}\left({ }^{\circ} \mathrm{C}\right)$

117-3/12: $\mathrm{Cr} \cdot 103 \cdot \mathrm{SmA}_{2} \cdot>200 \cdot \operatorname{dec} .\left({ }^{\circ} \mathrm{C}\right)$

117-4/12: $\mathrm{Cr}_{1} \cdot 89 \cdot \mathrm{Cr}_{2} \cdot 151 \cdot \mathrm{SmA}_{2} \cdot 196 \cdot \mathrm{I}\left({ }^{\circ} \mathrm{C}\right)$

117-12/12: $\mathrm{Cr} \cdot 169 \cdot \mathrm{SmA} \cdot>200 \cdot \operatorname{dec}\left({ }^{\circ} \mathrm{C}\right)$

117-18/12: $\mathrm{Cr}_{1} \cdot 112 \cdot \mathrm{Cr}_{2} \cdot 149 \cdot \mathrm{SmA} \cdot>200 \cdot$ dec. $\left({ }^{\circ} \mathrm{C}\right)$

117-6/18: $\mathrm{Cr} \cdot 165 \cdot \mathrm{SmA} \cdot>200 \cdot \operatorname{dec} .\left({ }^{\circ} \mathrm{C}\right)$

117-12/18: $\mathrm{Cr} \cdot 167 \cdot \mathrm{SmA} \cdot>200 \cdot$ dec. $\left({ }^{\circ} \mathrm{C}\right)$

118-4/12: $\mathrm{Cr} \cdot 51 \cdot \mathrm{SmA}_{2} \cdot>225 \cdot \operatorname{dec}\left({ }^{\circ} \mathrm{C}\right)$ 
118-8/12: $\mathrm{Cr} \cdot 62 \cdot \mathrm{SmA} \cdot 223 \cdot \mathrm{I}\left({ }^{\circ} \mathrm{C}\right)$

118-12/12: $\mathrm{Cr}_{1} \cdot 95 \cdot \mathrm{Cr}_{2} \cdot 106 \cdot \mathrm{SmA} \cdot 186 \cdot \mathrm{I}\left({ }^{\circ} \mathrm{C}\right)$

118-4/16: $\mathrm{Cr} \cdot 74 \cdot \mathrm{SmA}_{2} \cdot 170 \cdot \mathrm{I}\left({ }^{\circ} \mathrm{C}\right)$

119-3/12: $\mathrm{Cr} \cdot 92 \cdot \mathrm{Col}_{(\mathrm{h})} \cdot>200 \cdot$ dec. $\left({ }^{\circ} \mathrm{C}\right)$

119-4/12: $\mathrm{Cr} \cdot 111 \cdot \mathrm{Col}_{\mathrm{h}} \cdot 124 \cdot \mathrm{I}\left({ }^{\circ} \mathrm{C}\right)$

119-8/12: $\mathrm{Cr} \cdot 53 \cdot \mathrm{SmA} \cdot 76 \cdot \mathrm{I}\left({ }^{\circ} \mathrm{C}\right)$

119-14/12: $\mathrm{Cr}_{1} \cdot 47 \cdot \mathrm{Cr}_{2} \cdot 65 \cdot \mathrm{I}\left({ }^{\circ} \mathrm{C}\right)$

119-2/14: $\mathrm{Cr} \cdot 102 \cdot \mathrm{Cub}_{\mathrm{I}}(\operatorname{Pm} \overline{3} n) \cdot 225 \cdot \mathrm{I} /$ dec. $\left({ }^{\circ} \mathrm{C}\right)$

119-3/14: $\mathrm{Cr} \cdot 95 \cdot \mathrm{Col}_{(\mathrm{h})} \cdot 178 \cdot \mathrm{I}\left({ }^{\circ} \mathrm{C}\right) /$ on cooling: $\mathrm{I} \cdot 160 \cdot \mathrm{Cub}_{\mathrm{I}} \cdot<20 \cdot \mathrm{Cr}\left({ }^{\circ} \mathrm{C}\right)$

119-4/14: $\mathrm{Cr} \cdot 93 \cdot \mathrm{Col}_{\mathrm{h}} \cdot 120 \cdot \mathrm{I}\left({ }^{\circ} \mathrm{C}\right)$

119-14/14: $\mathrm{Cr} \cdot(\mathrm{SmA} \cdot 52 \cdot) 78 \cdot \mathrm{I}\left({ }^{\circ} \mathrm{C}\right)$

119-2/16: $\mathrm{Cr} \cdot 98 \cdot \mathrm{Cub}_{\mathrm{I}}(\operatorname{Pm} \overline{3} n) \cdot 225 \cdot \mathrm{I} /$ dec. $\left({ }^{\circ} \mathrm{C}\right)$

119-3/16: $\mathrm{Cr} \cdot 106 \cdot \mathrm{Cub}_{\mathrm{I}}(\operatorname{Pm} \overline{3} n) \cdot 202 \cdot \mathrm{I}\left({ }^{\circ} \mathrm{C}\right)$

119-4/16: $\mathrm{Cr} \cdot 109 \cdot \mathrm{Col}_{\mathrm{h}} \cdot 171 \cdot \mathrm{I}\left({ }^{\circ} \mathrm{C}\right)$

119-5/16: $\mathrm{Cr} \cdot 102 \cdot \mathrm{SmA} \cdot 118 \cdot \mathrm{I}\left({ }^{\circ} \mathrm{C}\right)$

119-6/16: $\mathrm{Cr} \cdot 82 \cdot \mathrm{SmA} \cdot 99 \cdot \mathrm{I}\left({ }^{\circ} \mathrm{C}\right)$

119-8/16: $\mathrm{Cr}_{1} \cdot 51 \cdot \mathrm{Cr}_{2} \cdot 57 \cdot \mathrm{SmA} \cdot 96 \cdot \mathrm{I}\left({ }^{\circ} \mathrm{C}\right)$

120-3/12: $\mathrm{Cr} \cdot 43 \cdot \mathrm{SmA}_{2} \cdot 139 \cdot \mathrm{I}\left({ }^{\circ} \mathrm{C}\right)$

120-4/12: $\mathrm{Cr} \cdot 81 \cdot \mathrm{SmA}_{2} \cdot 174 \cdot \mathrm{I}\left({ }^{\circ} \mathrm{C}\right)$

120-12/12: $\mathrm{Cr} \cdot 87 \cdot \mathrm{SmA} \cdot 212 \cdot \mathrm{I} /$ dec. $\left({ }^{\circ} \mathrm{C}\right)$

120-12/14: $\mathrm{Cr} \cdot 60 \cdot \mathrm{SmA} \cdot 224 \cdot \mathrm{I} /$ dec. $\left({ }^{\circ} \mathrm{C}\right)$

120-3/16: $\mathrm{Cr} \cdot 52 \cdot \mathrm{SmA}_{2} \cdot 213 \cdot \mathrm{I}\left({ }^{\circ} \mathrm{C}\right)$

120-6/18: $\mathrm{Cr} \cdot 68 \cdot \mathrm{SmA} \cdot 216 \cdot \mathrm{I} /$ dec. $\left({ }^{\circ} \mathrm{C}\right)$

121: $\mathrm{Cr} \cdot 72 \cdot \mathrm{Col}_{\mathrm{h}} \cdot 242 \cdot \mathrm{I}\left({ }^{\circ} \mathrm{C}\right)$

122-4/12: $\mathrm{Cr} \cdot 91 \cdot \mathrm{Col}_{\mathrm{h}} \cdot 109 \cdot \mathrm{I}\left({ }^{\circ} \mathrm{C}\right)$

122-5/12: $\mathrm{Cr} \cdot(\mathrm{SmA} \cdot 84 \cdot) 98 \cdot \mathrm{I}\left({ }^{\circ} \mathrm{C}\right)$

122-2/14: $\mathrm{Cr} \cdot 43 \cdot \mathrm{Cub}_{\mathrm{I}}(\operatorname{Pm} \overline{3} n) \cdot 177 \cdot \mathrm{I}\left({ }^{\circ} \mathrm{C}\right)$

122-3/14: $\mathrm{Cr} \cdot 68 \cdot \mathrm{Col}_{\mathrm{h}} \cdot 180 \cdot \mathrm{I}\left({ }^{\circ} \mathrm{C}\right)$

122-4/14: $\mathrm{Cr} \cdot 94 \cdot \mathrm{Col}_{\mathrm{h}} \cdot 133 \cdot \mathrm{I}\left({ }^{\circ} \mathrm{C}\right)$

122-5/14: $\mathrm{Cr} \cdot(\mathrm{SmA} \cdot 57 \cdot) 98 \cdot \mathrm{I}\left({ }^{\circ} \mathrm{C}\right)$

122-6/14: $\mathrm{Cr} \cdot(\mathrm{SmA} \cdot 68 \cdot) 96 \cdot \mathrm{I}\left({ }^{\circ} \mathrm{C}\right)$

122-2/16: $\mathrm{Cr} \cdot 96 \cdot \mathrm{Cub}_{\mathrm{I}}(\operatorname{Pm} \overline{3} n) \cdot 217 \cdot \mathrm{I}\left({ }^{\circ} \mathrm{C}\right)$

122-3/16: $\mathrm{Cr} \cdot 89 \cdot \mathrm{Cub}_{\mathrm{I}}(\operatorname{Pm} \overline{3} n) \cdot 184 \cdot \mathrm{I}\left({ }^{\circ} \mathrm{C}\right)$

122-4/16: $\mathrm{Cr} \cdot 100 \cdot \mathrm{Cub}_{\mathrm{I}}(\operatorname{Pm} \overline{3} n) \cdot 100 \cdot \mathrm{Col}_{\mathrm{h}} \cdot 148 \cdot \mathrm{I}\left({ }^{\circ} \mathrm{C}\right)$

122-5/16: $\mathrm{Cr} \cdot 97 \cdot \mathrm{I}\left({ }^{\circ} \mathrm{C}\right)$ 
122-3/18: $\mathrm{Cr} \cdot 94 \cdot \mathrm{Cub}_{\mathrm{I}}(\operatorname{Pm} \overline{3} n) \cdot 189 \cdot \mathrm{I}\left({ }^{\circ} \mathrm{C}\right)$

122-5/18: $\mathrm{Cr} \cdot 92 \cdot \mathrm{I}\left({ }^{\circ} \mathrm{C}\right)$

123-4/12: $\mathrm{Cr} \cdot 246 \cdot \mathrm{I} /$ dec. $\left({ }^{\circ} \mathrm{C}\right)$

123-4/16: $\mathrm{Cr} \cdot 242 \cdot \mathrm{I} /$ dec. $\left({ }^{\circ} \mathrm{C}\right)$

124-2/12: $\mathrm{Cr} \cdot 77 \cdot \mathrm{Col}_{\mathrm{h}} \cdot 183 \cdot \mathrm{I}\left({ }^{\circ} \mathrm{C}\right)$

124-3/12: $\mathrm{Cr} \cdot 93 \cdot \mathrm{Col}_{\mathrm{h}} \cdot 122 \cdot \mathrm{I}\left({ }^{\circ} \mathrm{C}\right)$

124-2/16: $\mathrm{Cr} \cdot 115 \cdot \mathrm{Col}_{\mathrm{h}} \cdot 189 \cdot \mathrm{I}\left({ }^{\circ} \mathrm{C}\right)$

124-4/16: $\mathrm{Cr} \cdot(\mathrm{SmA} \cdot 92 \cdot) 127 \cdot \mathrm{I}\left({ }^{\circ} \mathrm{C}\right)$

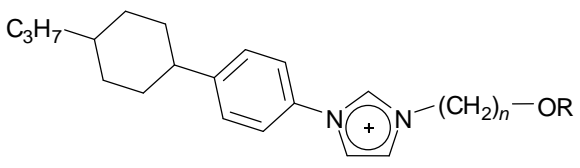

$\mathrm{Br}^{-}$
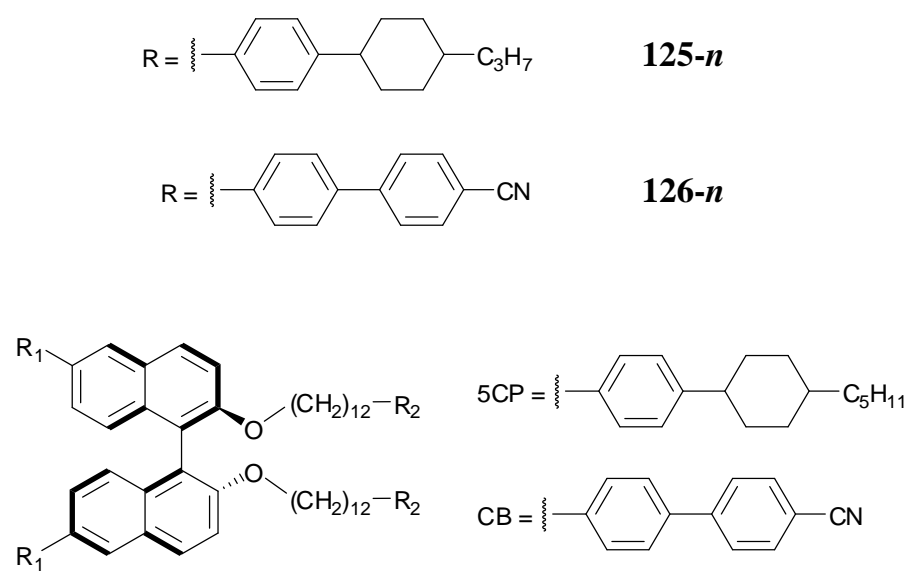

(R)-BND1: $\mathrm{R}_{1}=\mathrm{CB}, \mathrm{R}_{2}=\mathrm{CB}(\boldsymbol{( S ) - B N D 1}$ was also prepared)

(R)-BND2: $\mathrm{R}_{1}=5 \mathrm{CP}, \mathrm{R}_{2}=\mathrm{CB}(\boldsymbol{( S ) - B N D 2}$ was also prepared $)$

(R)-BND3: $\mathrm{R}_{1}=\mathrm{CB}, \mathrm{R}_{2}=5 \mathrm{CP}((\boldsymbol{S})$-BND3 was also prepared $)$

(R)-BND4: $\mathrm{R}_{1}=5 \mathrm{CP}, \mathrm{R}_{2}=5 \mathrm{CP}((\boldsymbol{S})$-BND4 was also prepared)

125-6: $\mathrm{Cr} \cdot 104 \cdot \mathrm{I}\left({ }^{\circ} \mathrm{C}\right)$

125-12: $\mathrm{Cr}_{1} \cdot 109 \cdot \mathrm{Cr}_{2} \cdot 128 \cdot \mathrm{SmX} \cdot 150 \cdot \mathrm{SmA} \cdot 176 \cdot \mathrm{I}\left({ }^{\circ} \mathrm{C}\right)$

126-6: $\mathrm{Cr} \cdot 53 \cdot \mathrm{N} \cdot 84 \cdot ? \cdot 91 \cdot \mathrm{I}\left({ }^{\circ} \mathrm{C}\right)$

126-12: $\mathrm{Cr} \cdot 43 \cdot \mathrm{N} \cdot 97 \cdot \mathrm{I}\left({ }^{\circ} \mathrm{C}\right)$

126-12/(R)-BND1 (100:5): Cr $\cdot 45 \cdot \mathrm{N}^{*} \cdot 94 \cdot \mathrm{I}\left({ }^{\circ} \mathrm{C}\right)$ 


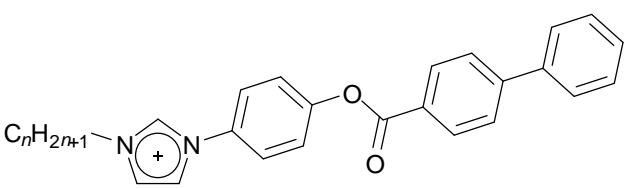

$\mathrm{Br}^{-}$

127-n

127-4: $\mathrm{Cr} \cdot 180 \cdot \mathrm{I}\left({ }^{\circ} \mathrm{C}\right)\left(2^{\text {nd }}\right.$ DSC heating run $)$

127-6: $\mathrm{Cr} \cdot 192 \cdot \mathrm{I} /$ on cooling: $\mathrm{I} \cdot 145 \cdot \mathrm{M} \cdot 141 \cdot \mathrm{Cr}\left({ }^{\circ} \mathrm{C}\right)\left(1^{\text {st }}\right.$ DSC heating/cooling cycle $)$

127-8: $\mathrm{Cr}_{1} \cdot 79 \cdot \mathrm{Cr}_{2} \cdot 186 \cdot \mathrm{I} /$ on cooling: $\mathrm{I} \cdot\left[\right.$ not reported] $\cdot \mathrm{M} \cdot 162 \cdot \mathrm{Cr}\left({ }^{\circ} \mathrm{C}\right)\left(1^{\text {st }} \mathrm{DSC}\right.$ heating/cooling cycle $)$

127-10: $\mathrm{Cr} \cdot 174 \cdot \mathrm{I} /$ on cooling: $\mathrm{I} \cdot 179 \cdot \mathrm{SmC}_{2}(?) \cdot 144 \cdot \mathrm{Cr}\left({ }^{\circ} \mathrm{C}\right)\left(2^{\text {nd }}\right.$ DSC heating/cooling cycle $)$

127-12: $\mathrm{Cr} \cdot 172 \cdot \mathrm{SmC}_{2} \cdot 203 \cdot \mathrm{I} /$ on cooling: $\mathrm{I} \cdot 206 \cdot \mathrm{SmC}_{2} \cdot\left[\right.$ no DSC signal] $\cdot \mathrm{Sm}\left(\mathrm{C}-\mathrm{T}^{\prime}\right)_{2} \cdot 127 \cdot$ tilted T’ $\left({ }^{\circ} \mathrm{C}\right)$

$\left(2^{\text {nd }}\right.$ DSC heating/cooling cycle)

127-14: $\mathrm{Cr} \cdot 153 \cdot \mathrm{SmC}_{2} \cdot 213 \cdot \mathrm{I} /$ on cooling: I $\cdot 213 \cdot \mathrm{SmC}_{2} \cdot$ [no DSC signal, only a baseline shift] $\cdot \mathrm{Sm}(\mathrm{C}-$

$\left.\mathrm{T}^{\prime}\right)_{2} /$ tilted T' $\left({ }^{\circ} \mathrm{C}\right)\left(2^{\text {nd }}\right.$ DSC heating/cooling cycle)

127-16: $\mathrm{Cr} \cdot 146 \cdot \mathrm{SmC}_{2} \cdot 226 \cdot \mathrm{I} /$ on cooling: $\mathrm{I} \cdot 224 \cdot \mathrm{SmC}_{2} \cdot$ [no DSC signal, only a baseline shift during the $1^{\text {st }}$ DSC cooling run $\cdot \operatorname{Sm}\left(\mathrm{C}-\mathrm{T}^{\prime}\right)_{2} /$ tilted T' $\left({ }^{\circ} \mathrm{C}\right)\left(2^{\text {nd }}\right.$ DSC heating/cooling cycle)

127-18: $\mathrm{Cr} \cdot 114 \cdot \mathrm{SmA}_{2} \cdot 229 \cdot \mathrm{I} /$ on cooling: $\mathrm{I} \cdot 227 \cdot \mathrm{SmA}_{2} \cdot$ [no DSC signal, only a baseline $\operatorname{shift}$ ] $\mathrm{Sm}(\mathrm{A}-$

$\left.\mathrm{T}^{\prime}\right)_{2} / \mathrm{T}^{\prime}\left({ }^{\circ} \mathrm{C}\right)\left(2^{\text {nd }} \mathrm{DSC}\right.$ heating/cooling cycle $)$

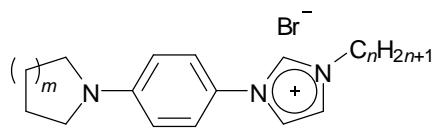

128-n/m

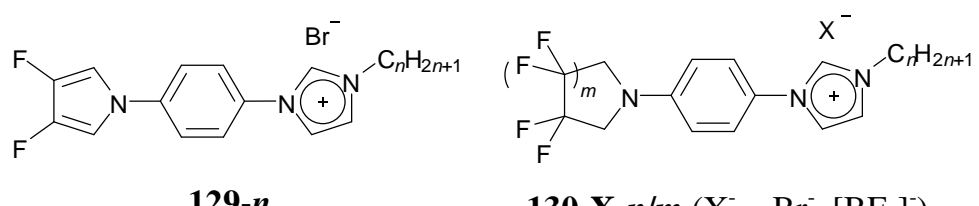

128-14/1: $\mathrm{Cr} \cdot 101 \cdot \mathrm{SmA} \cdot 124 \cdot \mathrm{I}\left({ }^{\circ} \mathrm{C}\right)$

128-18/1: $\mathrm{Cr}_{1} \cdot 106 \cdot \mathrm{Cr}_{2} \cdot 112 \cdot \mathrm{SmA} \cdot 122 \cdot \mathrm{I}\left({ }^{\circ} \mathrm{C}\right)$

128-14/2: $\mathrm{Cr} \cdot 64 \cdot \mathrm{SmA} \cdot 70 \cdot \mathrm{I}\left({ }^{\circ} \mathrm{C}\right)$

128-18/2: $\mathrm{Cr} \cdot 61 \cdot \mathrm{SmA} \cdot 72 \cdot \mathrm{I}\left({ }^{\circ} \mathrm{C}\right)$

128-14/3: $\mathrm{Cr} \cdot 37 \cdot \mathrm{I}\left({ }^{\circ} \mathrm{C}\right)$

129-14: $\mathrm{Cr} \cdot 136 \cdot \mathrm{SmA} \cdot 207 \cdot \mathrm{I}\left({ }^{\circ} \mathrm{C}\right)$

129-18: $\mathrm{Cr}_{1} \cdot 123 \cdot \mathrm{Cr}_{2} \cdot 135 \cdot \mathrm{SmA} \cdot 249 \cdot \mathrm{I} /$ dec. $\left({ }^{\circ} \mathrm{C}\right)$

130-Br-n/m $\left(\mathrm{X}^{-}=\mathrm{Br}^{-}\right)$ 
130-Br-14/1: $\mathrm{Cr} \cdot 89 \cdot \mathrm{SmA} \cdot 157 \cdot \mathrm{I}\left({ }^{\circ} \mathrm{C}\right)$

130-Br-18/1: $\mathrm{Cr} \cdot 108 \cdot \mathrm{SmA} \cdot 165 \cdot \mathrm{I}\left({ }^{\circ} \mathrm{C}\right)$

130-Br-14/2: $\mathrm{Cr} \cdot 106 \cdot \mathrm{SmA} \cdot 112 \cdot \mathrm{I}\left({ }^{\circ} \mathrm{C}\right)$

130-Br-18/2: $\mathrm{Cr} \cdot 108 \cdot \mathrm{SmA} \cdot 116 \cdot \mathrm{I}\left({ }^{\circ} \mathrm{C}\right)$

130-Br-14/3: $\mathrm{Cr}_{1} \cdot 146 \cdot \mathrm{Cr}_{2} \cdot 149 \cdot \mathrm{SmA} \cdot 180 \cdot \mathrm{I}\left({ }^{\circ} \mathrm{C}\right)$

130-Br-18/3: $\mathrm{Cr} \cdot 80 \cdot \mathrm{SmA} \cdot 182 \cdot \mathrm{I}\left({ }^{\circ} \mathrm{C}\right)$

130-BF $4-\mathbf{n} / \mathbf{1}\left(\mathrm{X}^{-}=\left[\mathrm{BF}_{4}\right]^{-}\right)$

130-BF 4 -14/1: $\mathrm{Cr} \cdot 84 \cdot \mathrm{SmA} \cdot 252 \cdot \mathrm{I}\left({ }^{\circ} \mathrm{C}\right)$

130-BF 4 -18/1: $\mathrm{Cr}_{1} \cdot 64 \cdot \mathrm{Cr}_{2} \cdot 84 \cdot \mathrm{SmA} \cdot 254 \cdot \mathrm{I}\left({ }^{\circ} \mathrm{C}\right)$

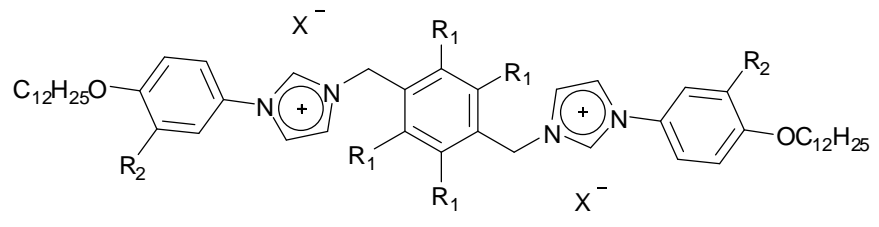

131-Br-R $\mathbf{R}_{\mathbf{1}} / \mathbf{R}_{\mathbf{2}}\left(\mathrm{X}^{-}=\mathrm{Br}^{-}\right)$

131-Br-H/H: $\mathrm{Cr} \cdot 106 \cdot \mathrm{SmA} \cdot>250 \cdot \operatorname{dec}\left({ }^{\circ} \mathrm{C}\right)$

131-Br-H/F: $\mathrm{Cr}_{1} \cdot 75 \cdot \mathrm{Cr}_{2} \cdot 133 \cdot \mathrm{M} \cdot 145 \cdot \mathrm{SmA} \cdot>250 \cdot$ dec. $\left({ }^{\circ} \mathrm{C}\right)$

131-NTf $\mathbf{N}_{\mathbf{2}}-\mathbf{R}_{\mathbf{1}} / \mathbf{R}_{\mathbf{2}}\left(\mathrm{X}^{-}=\left[\mathrm{NTf}_{2}\right]^{-}\right)$

131-NTf $\mathbf{N}_{2}$-H/F: $\mathrm{Cr} \cdot(\mathrm{SmÃ} \cdot 33 \cdot) 81 \cdot \mathrm{I}\left({ }^{\circ} \mathrm{C}\right)$

131- $\mathbf{N T f}_{2}$-F/H: $\mathrm{Cr}_{1} \cdot 63 \cdot \mathrm{Cr}_{2} \cdot(\mathrm{SmA} \cdot 79 \cdot) 82 \cdot \mathrm{I}\left({ }^{\circ} \mathrm{C}\right)$
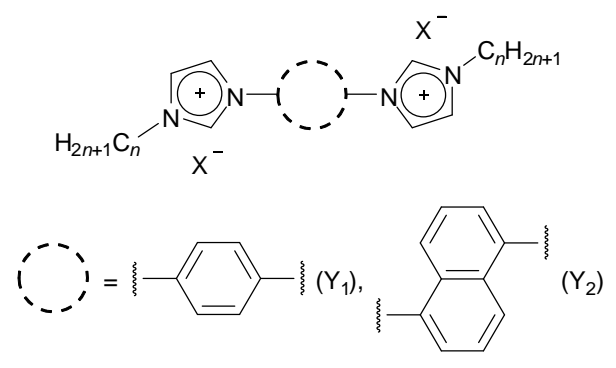

132-Br-Y $\mathbf{1}_{1}-\boldsymbol{n}\left(\mathrm{X}^{-}=\mathrm{Br}^{-}\right)$

132-Br-Y $\mathbf{1}_{1}-12: \mathrm{Cr} \cdot\left[\right.$ not reported] $\cdot$ dec. $\left({ }^{\circ} \mathrm{C}\right)$

132-Br-Y $\mathbf{Y}_{1}-16: \mathrm{Cr} \cdot\left[\right.$ not reported] $\cdot$ dec. $\left({ }^{\circ} \mathrm{C}\right)$

132-OTf-Y $\mathbf{1}_{1}-\boldsymbol{n}\left(\mathrm{X}^{-}=\left[\mathrm{OTf}^{-}\right)\right.$

132-OTf-Y $\mathbf{1}_{1}$-12: $\mathrm{Cr}_{1} \cdot 148 \cdot \mathrm{Cr}_{2} \cdot 169 \cdot \mathrm{SmC}(?) \cdot 184 \cdot \mathrm{I} \cdot \mathrm{ca} .250 \cdot$ dec. $\left({ }^{\circ} \mathrm{C}\right)$

132-OTf-Y $\mathbf{Y}_{1}-16: \mathrm{Cr}_{1} \cdot 141 \cdot \mathrm{Cr}_{2} \cdot 149 \cdot \mathrm{SmC}(?) \cdot \mathrm{ca} .250 \cdot$ dec. $\left({ }^{\circ} \mathrm{C}\right)$

$$
\text { 132-NTf } \mathbf{N}_{2}-\mathrm{Y}_{1}-\boldsymbol{n}\left(\mathrm{X}^{-}=\left[\mathrm{NTf}_{2}\right]^{-}\right)
$$

132-NTf $\mathbf{N}_{2}-\mathbf{Y}_{\mathbf{1}} \mathbf{- 1 2} \mathrm{Cr} \cdot 55 \cdot \mathrm{T}_{1}\left(\right.$ ?) $\cdot 105 \cdot \mathrm{T}_{2}\left(\right.$ ?) $\cdot 136 \cdot \mathrm{I} \cdot \mathrm{ca} .350 \cdot \operatorname{dec} .\left({ }^{\circ} \mathrm{C}\right)$ 132-NTf $\mathbf{N}_{2}-\mathbf{Y}_{\mathbf{1}} \mathbf{- 1 6}: \mathrm{Cr} \cdot 75 \cdot \mathrm{T}($ ?) $) 172 \cdot \mathrm{I} \cdot \mathrm{ca} .350 \cdot \operatorname{dec} .\left({ }^{\circ} \mathrm{C}\right)$ 
132-NTf $\mathbf{2}_{2}-\mathbf{Y}_{2}-\mathbf{1 2}: \mathrm{Cr}_{1} \cdot 87 \cdot \mathrm{Cr}_{2} \cdot 93 \cdot \mathrm{T} \cdot 123 \cdot \mathrm{I}\left({ }^{\circ} \mathrm{C}\right)$ (on cooling: $\mathrm{I} \cdot 117 \cdot \mathrm{T} \cdot 57 \cdot \mathrm{Cr}_{2} \cdot 55 \cdot \mathrm{Cr}_{1}\left({ }^{\circ} \mathrm{C}\right)$ )

132-NTf $\mathbf{2}_{2}-\mathbf{Y}_{2} \mathbf{- 1 6}: \mathrm{Cr} \cdot 94 \cdot \mathrm{T} \cdot 166 \cdot \mathrm{I}\left({ }^{\circ} \mathrm{C}\right)\left(\right.$ on cooling: $\mathrm{I} \cdot 162 \cdot \mathrm{T} \cdot 65 \cdot \mathrm{Cr}\left({ }^{\circ} \mathrm{C}\right)$ )
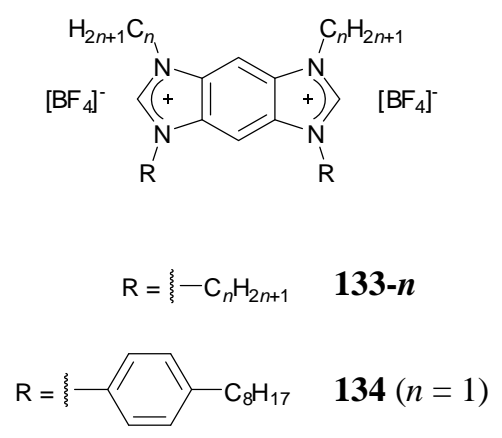

133-12: $\mathrm{Cr} \cdot 53 \cdot \mathrm{SmX} \cdot 194 \cdot \mathrm{I}\left({ }^{\circ} \mathrm{C}\right)$ (on cooling)

134: g $\cdot 188 \cdot \mathrm{Cub}_{\mathrm{V}} \cdot 238 \cdot \mathrm{I}\left({ }^{\circ} \mathrm{C}\right)$ (on cooling)

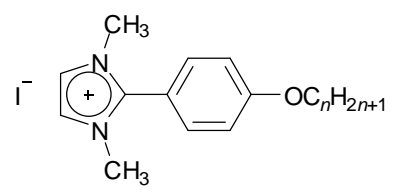

135-n

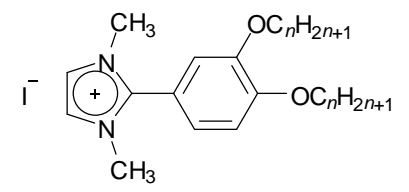

136-n

135-6: $\mathrm{Cr} \cdot 123 \cdot \mathrm{I}\left({ }^{\circ} \mathrm{C}\right)$

135-10: $\mathrm{Cr} \cdot 122 \cdot \mathrm{SmA} \cdot 152 \cdot \mathrm{I}\left({ }^{\circ} \mathrm{C}\right)$

135-14: $\mathrm{Cr} \cdot 120 \cdot \mathrm{SmA} \cdot 220 \cdot \mathrm{I}\left({ }^{\circ} \mathrm{C}\right)$

135-18: $\mathrm{Cr} \cdot 122 \cdot \mathrm{SmA} \cdot 243 \cdot \mathrm{I}\left({ }^{\circ} \mathrm{C}\right)$

136-6: $\mathrm{Cr}_{1} \cdot 33 \cdot \mathrm{Cr}_{2} \cdot 99 \cdot \mathrm{Cr}_{3} \cdot 106 \cdot \mathrm{SmX} \cdot 124 \cdot \mathrm{SmA} \cdot 147 \cdot \mathrm{I}\left({ }^{\circ} \mathrm{C}\right)$

136-10: $\mathrm{Cr}_{1} \cdot 66 \cdot \mathrm{Cr}_{2} \cdot 75 \cdot \mathrm{Cr}_{3} \cdot 113 \cdot \mathrm{SmA} \cdot 164 \cdot \mathrm{I}\left({ }^{\circ} \mathrm{C}\right)$

136-14: $\mathrm{Cr}_{1} \cdot 91 \cdot \mathrm{Cr}_{2} \cdot 115 \cdot \mathrm{SmA} \cdot 159 \cdot \mathrm{I}\left({ }^{\circ} \mathrm{C}\right)$

136-15: $\mathrm{Cr}_{1} \cdot 103 \cdot \mathrm{Cr}_{2} \cdot 118 \cdot \mathrm{Cub}_{\mathrm{V}}(\operatorname{Pm} \overline{3} m) \cdot 139 \cdot \mathrm{Col}_{\mathrm{h}} \cdot 172 \cdot \mathrm{I}\left({ }^{\circ} \mathrm{C}\right)$

136-16: $\mathrm{Cr}_{1} \cdot 94 \cdot \mathrm{Cr}_{2} \cdot\left(\mathrm{Cub}_{\mathrm{V}}(\operatorname{Pm} \overline{3} m) \cdot 96 \cdot\right) 118 \cdot \mathrm{Col}_{\mathrm{h}} \cdot 190 \cdot \mathrm{I}\left({ }^{\circ} \mathrm{C}\right)$

136-17: $\mathrm{Cr}_{1} \cdot 108 \cdot \mathrm{Cr}_{2} \cdot 120 \cdot \mathrm{Col}_{\mathrm{h}} \cdot 204 \cdot \mathrm{I}\left({ }^{\circ} \mathrm{C}\right)$

136-18: $\mathrm{Cr}_{1} \cdot 98 \cdot \mathrm{Cr}_{2} \cdot 103 \cdot \mathrm{Cr}_{3} \cdot 121 \cdot \mathrm{Col}_{\mathrm{h}} \cdot 208 \cdot \mathrm{I}\left({ }^{\circ} \mathrm{C}\right)$ 


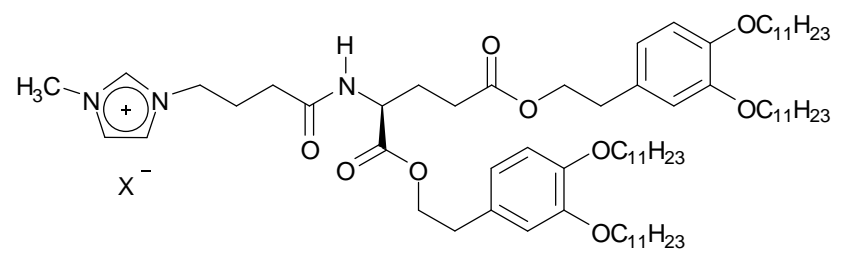

137-Br $\left(\mathrm{X}^{-}=\mathrm{Br}^{-}\right): \mathrm{Cr} \cdot 51 \cdot \mathrm{Col}_{\mathrm{h}} \cdot 136 \cdot \mathrm{Cub}_{\mathrm{I}}(\mathrm{Pm} \overline{3} n) \cdot>180 \cdot$ dec. $\left({ }^{\circ} \mathrm{C}\right)$ 137-NTf $2\left(\mathrm{X}^{-}=\left[\mathrm{NTf}_{2}\right]^{-}\right): \mathrm{Cr} \cdot 64 \cdot \mathrm{Col}_{\mathrm{h}} \cdot 99 \cdot \mathrm{I}\left({ }^{\circ} \mathrm{C}\right)$ 
Scheme S7. Dendrimeric ILCs investigated by Percec and co-workers.

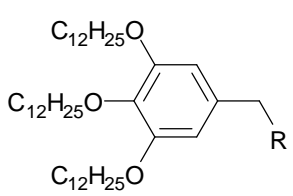

138-R $\left(\mathrm{AB}_{3}\right.$ first-generation dendron)

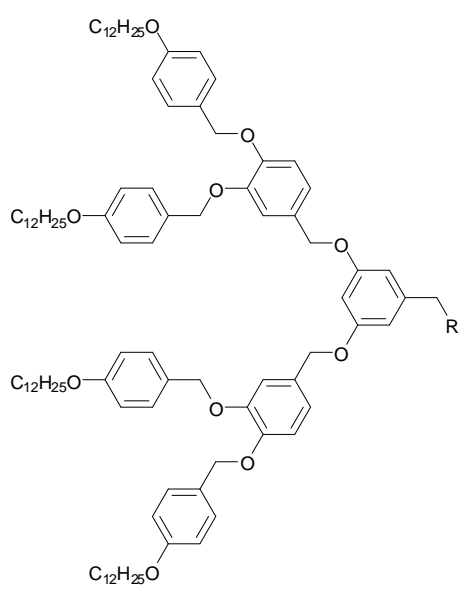

141-R $\left(\mathrm{AB}_{2}\right.$ second-generation dendron)

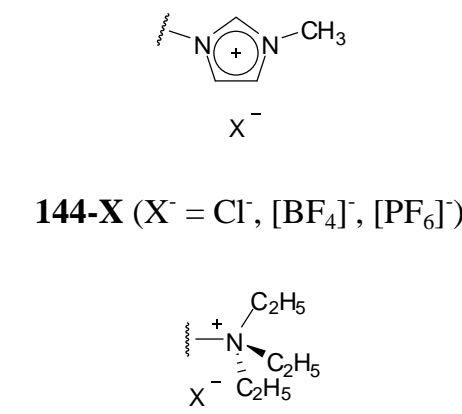

144-X $\left(\mathrm{X}^{-}=\mathrm{Cl}^{-},\left[\mathrm{BF}_{4}\right]^{-},\left[\mathrm{PF}_{6}\right]^{-}\right)$

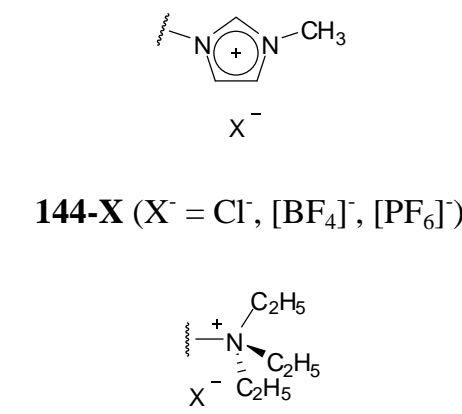

146-X $\left(\mathrm{X}^{-}=\mathrm{Cl}^{-},\left[\mathrm{BF}_{4}\right]^{-},\left[\mathrm{PF}_{6}\right]^{-}\right)$

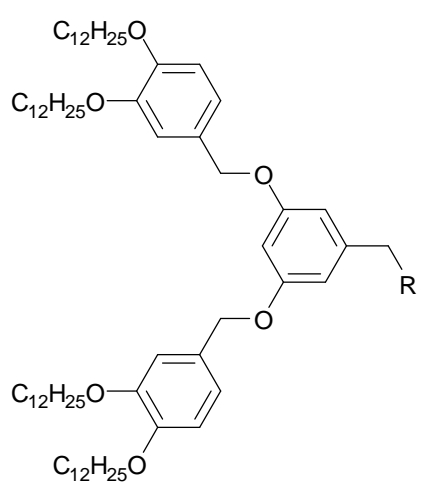

140-R $\left(\mathrm{AB}_{2}\right.$ second-generation dendron)

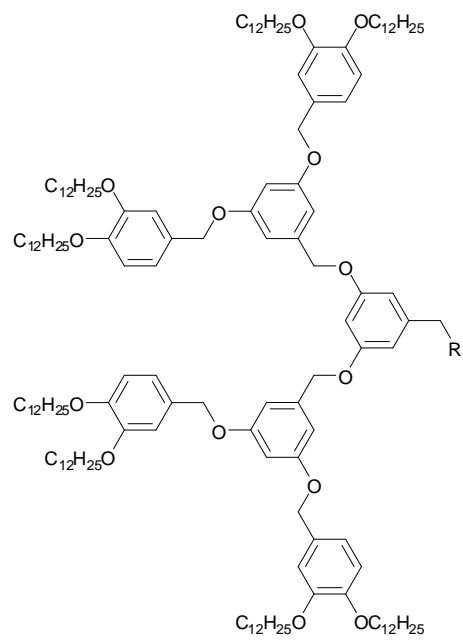

143-R $\left(\mathrm{AB}_{2}\right.$ third-generation dendron)

\section{Apex R groups:}

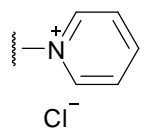

$\mathrm{Cl}^{-}$

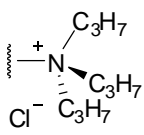

147 
Transition temperatures obtained from the first DSC heating run:

$$
\begin{gathered}
\text { 138-(144-Cl) }\left(\mathrm{X}^{-}=\mathrm{Cl}^{-}\right): \mathrm{Cr} \cdot 77 \cdot \mathrm{Col}_{\mathrm{h}} \cdot 180 \cdot \mathrm{I}\left({ }^{\circ} \mathrm{C}\right) \\
\begin{array}{r}
\text { 138-(144-BF } \\
\text { ) }
\end{array}\left(\mathrm{X}^{-}=\left[\mathrm{BF}_{4}\right]^{-}\right): \mathrm{Cr} \cdot 63 \cdot \mathrm{Col}_{\mathrm{h}} \cdot 181 \cdot \mathrm{I}\left({ }^{\circ} \mathrm{C}\right) \\
\text { 138-(144-PF })\left(\mathrm{X}^{-}=\left[\mathrm{PF}_{6}\right]\right): \mathrm{Cr} \cdot 53 \cdot \mathrm{Col}_{\mathrm{h}} \cdot 160 \cdot \mathrm{I}\left({ }^{\circ} \mathrm{C}\right) \\
\text { 138-(145): } \mathrm{Cr} \cdot 65 \cdot \mathrm{Col}_{\mathrm{h}} \cdot 166 \cdot \mathrm{I}\left({ }^{\circ} \mathrm{C}\right) \\
\text { 138-(146-Cl) }\left(\mathrm{X}^{-}=\mathrm{Cl}^{-}\right): \mathrm{Cr} \cdot 54 \cdot \mathrm{Col}_{\mathrm{h}} \cdot 162 \cdot \mathrm{I}\left({ }^{\circ} \mathrm{C}\right)
\end{gathered}
$$

138-(146-BF $\left.{ }_{4}\right)\left(\mathrm{X}^{-}=\left[\mathrm{BF}_{4}\right]^{-}\right): \mathrm{Cr}_{1} \cdot 48 \cdot \mathrm{Cr}_{2} \cdot 64 \cdot \mathrm{Col}_{\mathrm{h}} \cdot 132 \cdot \mathrm{I}\left({ }^{\circ} \mathrm{C}\right)$

138-(146-PF $\left.{ }_{6}\right)\left(\mathrm{X}^{-}=\left[\mathrm{PF}_{6}\right]^{-}\right): \mathrm{Cr}_{1} \cdot-21 \cdot \mathrm{Cr}_{2} \cdot 70 \cdot \mathrm{Col}_{\mathrm{r}}(\mathrm{p} 2 \mathrm{~mm}) \cdot 79 \cdot \mathrm{I}\left({ }^{\circ} \mathrm{C}\right)$

138-(147): $\mathrm{Cr}_{1} \cdot 50 \cdot \mathrm{Cr}_{2} \cdot 91 \cdot \mathrm{Col}_{\mathrm{h}} \cdot 107 \cdot \mathrm{I}\left({ }^{\circ} \mathrm{C}\right)$

139-(144-Cl) $\left(\mathrm{X}^{-}=\mathrm{Cl}^{-}\right): \mathrm{Cr}_{1} \cdot 10 \cdot \mathrm{Cr}_{2} \cdot 32 \cdot \mathrm{Cr}_{3} \cdot 70 \cdot \mathrm{Col}_{\mathrm{h}} \cdot 209 \cdot$ dec. $\left({ }^{\circ} \mathrm{C}\right)$

139-(144-BF 4$)\left(\mathrm{X}^{-}=\left[\mathrm{BF}_{4}\right]^{-}\right): \mathrm{Cr}_{1} \cdot-1.5 \cdot \mathrm{Cr}_{2} \cdot 7 \cdot \mathrm{Col}_{\mathrm{h}} \cdot 184 \cdot$ dec. $\left({ }^{\circ} \mathrm{C}\right)$

139-(144-PF 6$)\left(\mathrm{X}^{-}=\left[\mathrm{PF}_{6}\right]^{-}\right): \mathrm{Cr} \cdot 13 \cdot \mathrm{Col}_{\mathrm{h}} \cdot 184 \cdot \mathrm{I}\left({ }^{\circ} \mathrm{C}\right)$

$\left(2^{\text {nd }}\right.$ DSC heating run: $\left.\mathrm{g} \cdot 7 \cdot \mathrm{Col}_{\mathrm{r}}(\mathrm{p} 2 \mathrm{~mm}) \cdot 70 \cdot \mathrm{Col}_{\mathrm{h}} \cdot 183 \cdot \mathrm{I}\left({ }^{\circ} \mathrm{C}\right)\right)$

139-(145): $\mathrm{Cr}_{1} \cdot 15 \cdot \mathrm{Cr}_{2} \cdot 35 \cdot \mathrm{Cr}_{3} \cdot 57 \cdot \mathrm{Col}_{\mathrm{h}} \cdot 159 \cdot \mathrm{I}\left({ }^{\circ} \mathrm{C}\right)$

139-(146-Cl) $\left(\mathrm{X}^{-}=\mathrm{Cl}^{-}\right): \mathrm{Cr}_{1} \cdot-7 \cdot \mathrm{Cr}_{2} \cdot 74 \cdot \mathrm{Col}_{\mathrm{h}} \cdot 143 \cdot \mathrm{I}\left({ }^{\circ} \mathrm{C}\right)$

139-(146-BF 4$)\left(\mathrm{X}^{-}=\left[\mathrm{BF}_{4}\right]^{-}\right): \mathrm{Cr} \cdot 68 \cdot \mathrm{Col}_{\mathrm{h}} \cdot 150 \cdot \mathrm{I}\left({ }^{\circ} \mathrm{C}\right)$

139-(146-PF $6\left(\mathrm{X}^{-}=\left[\mathrm{PF}_{6}\right]\right): \mathrm{g} \cdot 92 \cdot \mathrm{Col}_{\mathrm{h}} \cdot 112 \cdot \mathrm{I}\left({ }^{\circ} \mathrm{C}\right)$

139-(147): $\mathrm{Cr}_{1} \cdot 19 \cdot \mathrm{Cr}_{2} \cdot 53 \cdot \mathrm{Cr}_{3} \cdot 78 \cdot \mathrm{Col}_{\mathrm{r}}(\mathrm{p} 2 \mathrm{~mm}) \cdot 115 \cdot \mathrm{I}\left({ }^{\circ} \mathrm{C}\right)$

$\left(2^{\text {nd }}\right.$ DSC heating run: $\left.\mathrm{Cr}_{1} \cdot 6 \cdot \mathrm{Cr}_{2} \cdot 72 \cdot \mathrm{Col}_{\mathrm{h}} \cdot 113 \cdot \mathrm{I}\left({ }^{\circ} \mathrm{C}\right)\right)$

140-(144-Cl) $\left(\mathrm{X}^{-}=\mathrm{Cl}^{-}\right): \mathrm{Cr} \cdot 47 \cdot \mathrm{Col}_{\mathrm{h}} \cdot 117 \cdot \mathrm{Cub}_{\mathrm{I}}(\operatorname{Pm} \overline{3} n) \cdot 215 \cdot$ dec. $\left({ }^{\circ} \mathrm{C}\right)$

140-(144-BF $\left.{ }_{4}\right)\left(\mathrm{X}^{-}=\left[\mathrm{BF}_{4}\right]^{-}\right): \mathrm{Cr}_{1} \cdot 40 \cdot \mathrm{Cr}_{2} \cdot 62 \cdot \mathrm{Cr}_{3} \cdot 90 \cdot \mathrm{Col}_{\mathrm{r}}(\mathrm{p} 2 \mathrm{~mm}) \cdot 108 \cdot \mathrm{Col}_{\mathrm{h}} \cdot 165 \cdot \mathrm{I}\left({ }^{\circ} \mathrm{C}\right)$

$\left(2^{\text {nd }}\right.$ DSC heating run: $\left.\mathrm{Cr} \cdot 50 \cdot \mathrm{Col}_{\mathrm{h}} \cdot 155 \cdot \mathrm{I}\left({ }^{\circ} \mathrm{C}\right)\right)$

140-(144-PF $)\left(\mathrm{X}^{-}=\left[\mathrm{PF}_{6}\right]^{-}\right): \mathrm{Cr}_{1} \cdot 41 \cdot \mathrm{Cr}_{2} \cdot 59 \cdot \mathrm{Cr}_{3} \cdot 99 \cdot \mathrm{Col}_{\mathrm{h}} \cdot 160 \cdot \mathrm{I}\left({ }^{\circ} \mathrm{C}\right)$

$\left(2^{\text {nd }}\right.$ DSC heating run: $\left.\mathrm{Cr} \cdot 43 \cdot \mathrm{Col}_{\mathrm{r}, 1}(p 2 m m) \cdot 84 \cdot \mathrm{Col}_{\mathrm{r}, 2}(p 2 m m) \cdot 100 \cdot \mathrm{Col}_{\mathrm{h}} \cdot 159 \cdot \mathrm{I}\left({ }^{\circ} \mathrm{C}\right)\right)$

140-(145): $\mathrm{Cr}_{1} \cdot 17 \cdot \mathrm{Cr}_{2} \cdot 92 \cdot \mathrm{Col}_{\mathrm{h}} \cdot 129 \cdot \mathrm{Cub}_{\mathrm{I}}(\operatorname{Pm} \overline{3} n) \cdot 164 \cdot \mathrm{I}\left({ }^{\circ} \mathrm{C}\right)$

$\left(2^{\text {nd }}\right.$ DSC heating run: $\mathrm{Cr}_{1} \cdot 31 \cdot \mathrm{Cr}_{2} \cdot 70 \cdot \mathrm{Col}_{\mathrm{h}} \cdot 160 \cdot \mathrm{I}\left({ }^{\circ} \mathrm{C}\right)$ )

140-(146-Cl) $\left(\mathrm{X}^{-}=\mathrm{Cl}^{-}\right): \mathrm{Cr} \cdot 109 \cdot \mathrm{Col}_{\mathrm{h}} \cdot 143 \cdot \mathrm{I}\left({ }^{\circ} \mathrm{C}\right)$

140-(146-BF 4$)\left(\mathrm{X}^{-}=\left[\mathrm{BF}_{4}\right]^{-}\right): \mathrm{Cr}_{1} \cdot 39 \cdot \mathrm{Cr}_{2} \cdot 52 \cdot \mathrm{Cr}_{3} \cdot 62 \cdot \mathrm{Cr}_{4} \cdot 125 \cdot \mathrm{Col}_{\mathrm{h}} \cdot 154 \cdot \mathrm{I}\left({ }^{\circ} \mathrm{C}\right)$

140-(146-PF $)\left(\mathrm{X}^{-}=\left[\mathrm{PF}_{6}\right]^{-}\right): \mathrm{Cr}_{1} \cdot 43 \cdot \mathrm{Cr}_{2} \cdot 66 \cdot \mathrm{Cr}_{3} \cdot 108 \cdot \mathrm{Col}_{\mathrm{h}} \cdot 130 \cdot \mathrm{I}\left({ }^{\circ} \mathrm{C}\right)$

140-(147): $\mathrm{Cr} \cdot 43 \cdot \mathrm{Col}_{\mathrm{h}} \cdot 108 \cdot \mathrm{I}\left({ }^{\circ} \mathrm{C}\right)$

141-(144-Cl) $\left(\mathrm{X}^{-}=\mathrm{Cl}^{-}\right): \mathrm{Cr}_{1} \cdot-17 \cdot \mathrm{Cr}_{2} \cdot 52 \cdot \mathrm{Col}_{\mathrm{h}} \cdot 203 \cdot \mathrm{I}\left({ }^{\circ} \mathrm{C}\right)$

141-(144-BF 4$)\left(\mathrm{X}^{-}=\left[\mathrm{BF}_{4}\right]^{-}\right): \mathrm{Cr} \cdot 40 \cdot \mathrm{Col}_{\mathrm{h}} \cdot 194 \cdot$ dec. $\left({ }^{\circ} \mathrm{C}\right)$

141-(144-PF $)\left(\mathrm{X}^{-}=\left[\mathrm{PF}_{6}\right]^{-}\right): \mathrm{Cr} \cdot 51 \cdot \mathrm{Col}_{\mathrm{h}} \cdot 198 \cdot \mathrm{I}\left({ }^{\circ} \mathrm{C}\right)$

142-(144-Cl) $\left(\mathrm{X}^{-}=\mathrm{Cl}^{-}\right): \mathrm{Cr} \cdot 46 \cdot \mathrm{Col}_{\mathrm{h}, 1} \cdot 74 \cdot \mathrm{Col}_{\mathrm{h}, 2} \cdot 218 \cdot \mathrm{I}\left({ }^{\circ} \mathrm{C}\right)$

142-(144-BF $\left.{ }_{4}\right)\left(\mathrm{X}^{-}=\left[\mathrm{BF}_{4}\right]^{-}\right): \mathrm{Cr} \cdot 46 \cdot \mathrm{Col}_{\mathrm{h}, 1} \cdot 91 \cdot \mathrm{Col}_{\mathrm{h}, 2} \cdot 182 \cdot$ dec. $\left({ }^{\circ} \mathrm{C}\right)$ 


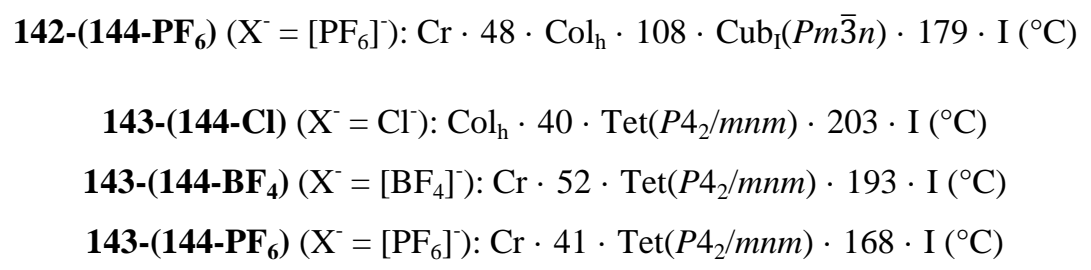

\section{Some additional structures}

The following structures and their transition temperatures were mentioned in a patent of Merck $\mathrm{GmbH}^{103}$

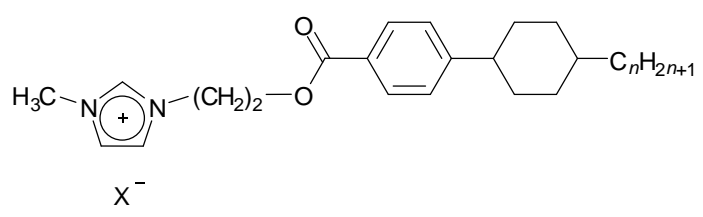

$$
\text { P1-Cl-n }\left(\mathrm{X}^{-}=\mathrm{Cl}^{-}\right)
$$

P1-Cl-3: $\mathrm{Cr} \cdot 158 \cdot \mathrm{SmA} \cdot 220 \cdot \mathrm{I}\left({ }^{\circ} \mathrm{C}\right)$

P1-Cl-7: $\mathrm{Cr} \cdot 131 \cdot \mathrm{SmA} \cdot 244 \cdot \mathrm{I}\left({ }^{\circ} \mathrm{C}\right)$

P1-Cl-11: $\mathrm{Cr} \cdot 50 \cdot \mathrm{SmA} \cdot 238 \cdot \mathrm{I}\left({ }^{\circ} \mathrm{C}\right)$

$\mathbf{P 1}_{-B_{4}-3}\left(\mathrm{X}^{-}=\left[\mathrm{BF}_{4}\right]^{-} ; n=3\right): \mathrm{Cr} \cdot 167 \cdot \mathrm{I}\left({ }^{\circ} \mathrm{C}\right)$

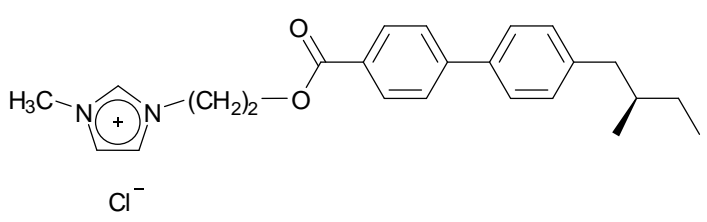

P2: waxy solid $\cdot 48 \cdot \mathrm{SmA}^{*} \cdot 199 \cdot \mathrm{I}\left({ }^{\circ} \mathrm{C}\right)$

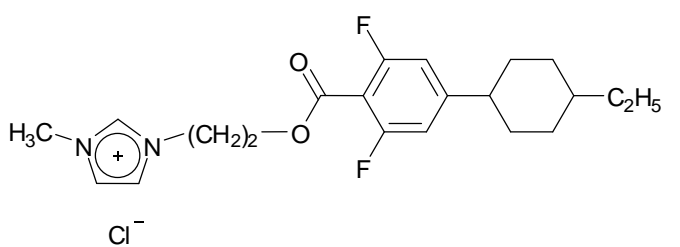

P3: $\mathrm{Cr} \cdot 229 \cdot \mathrm{I}\left({ }^{\circ} \mathrm{C}\right)$ 


\subsection{Ammonium-based ionic liquid crystals}

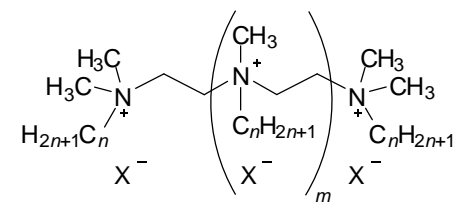

$$
\text { 148-Br-0/n }\left(\mathrm{X}^{-}=\mathrm{Br}^{-} ; m=0\right)
$$

148-Br-0/12: $\mathrm{Cr}_{1} \cdot 97 \cdot \mathrm{Cr}_{2} \cdot 105 \cdot \mathrm{Cr}_{3} \cdot 161 \cdot \mathrm{SmA}(?) \cdot\left[\right.$ not reported] $\cdot$ dec. $\left({ }^{\circ} \mathrm{C}\right)$

148-Br-0/14: $\mathrm{Cr}_{1} \cdot\left[\right.$ not reported] $\cdot \mathrm{Cr}_{2} \cdot\left[\right.$ not reported] $\cdot \mathrm{SmA}\left(\right.$ ?) $\cdot$ [not reported] $\cdot$ dec. $\left({ }^{\circ} \mathrm{C}\right)^{104} / \mathrm{Cr} \cdot 100 \cdot \mathrm{X}_{2} \cdot 160 \cdot$

$$
\mathrm{SmA} \cdot 180 \cdot \mathrm{I}\left({ }^{\circ} \mathrm{C}\right)^{105}
$$

148-Br-0/16: $\mathrm{Cr}_{1} \cdot$ [not reported] $\cdot \mathrm{Cr}_{2} \cdot$ [not reported] $\cdot \mathrm{SmA}\left(\right.$ ?) $\cdot$ [not reported] $\cdot$ dec. $\left({ }^{\circ} \mathrm{C}\right)$

148-Br-0/18: $\mathrm{Cr}_{1} \cdot 108 \cdot \mathrm{Cr}_{2} \cdot 170 \cdot \mathrm{SmA}\left(\right.$ ?) $\cdot$ [not reported] $\cdot$ dec. $\left({ }^{\circ} \mathrm{C}\right)$

148-I-0/14 $\left(\mathrm{X}^{-}=\mathrm{I}^{-} ; m=0\right): \mathrm{Cr} \cdot 105 \cdot \mathrm{X}_{1} \cdot 170 \cdot \mathrm{I}\left({ }^{\circ} \mathrm{C}\right)$

148-Cl-0/14 ( $\left.\mathrm{X}^{-}=\mathrm{Cl}^{-} ; \mathrm{m}=0\right): \mathrm{Cr} \cdot 55 \cdot \mathrm{X}_{2} \cdot 125 \cdot \mathrm{SmA} \cdot 185 \cdot \mathrm{I}\left({ }^{\circ} \mathrm{C}\right)$

148-F-0/14 ( $\left.\mathrm{X}^{-}=\mathrm{F}^{-} ; m=0\right): \mathrm{Cr} \cdot 37 \cdot \mathrm{SmA} \cdot 150 \cdot \operatorname{dec} .\left({ }^{\circ} \mathrm{C}\right)$

148- $\mathrm{NO}_{3}-\mathbf{- 0} / 14\left(\mathrm{X}^{-}=\left[\mathrm{NO}_{3}\right]^{-} ; m=0\right): \mathrm{Cr} \cdot 100 \cdot \mathrm{X}_{1} \cdot 180 \cdot$ dec. $\left({ }^{\circ} \mathrm{C}\right)$

148-CF $\mathbf{C F O}_{3} \mathbf{C O O} / \mathbf{1 4}\left(\mathrm{X}^{-}=\left[\mathrm{CF}_{3} \mathrm{COO}\right]^{-} ; m=0\right): \mathrm{Cr} \cdot 120 \cdot \mathrm{X}_{1} \cdot 175 \cdot \mathrm{I}\left({ }^{\circ} \mathrm{C}\right)$

148- $\mathrm{H}_{2} \mathrm{PO}_{4} \mathbf{- 0} / 14\left(\mathrm{X}^{-}=\left[\mathrm{H}_{2} \mathrm{PO}_{4}\right]^{-} ; m=0\right): \mathrm{Cr} \cdot 140 \cdot \mathrm{X}_{2} \cdot 200 \cdot \operatorname{dec} .\left({ }^{\circ} \mathrm{C}\right)$

148-HCOO-0/14 ( $\left.\mathrm{X}^{-}=[\mathrm{HCOO}]^{-} ; m=0\right): \mathrm{Cr} \cdot 80 \cdot \mathrm{X}_{2} \cdot 100 \cdot \mathrm{SmA} \cdot 160 \cdot \mathrm{I}\left({ }^{\circ} \mathrm{C}\right)$

148-CH $\mathbf{C H}_{3}$ COO-0/14 ( $\left.\mathrm{X}^{-}=\left[\mathrm{CH}_{3} \mathrm{COO}\right]^{-} ; m=0\right): \mathrm{Cr} \cdot 80 \cdot \mathrm{X}_{2} \cdot 105 \cdot \mathrm{SmA} \cdot 165 \cdot \mathrm{I}\left({ }^{\circ} \mathrm{C}\right)$

148- $\mathrm{C}_{2} \mathrm{H}_{5} \mathrm{COO-0/14}\left(\mathrm{X}^{-}=\left[\mathrm{C}_{2} \mathrm{H}_{5} \mathrm{COO}^{-} ; m=0\right): \mathrm{Cr} \cdot 80 \cdot \mathrm{X}_{2} \cdot 110 \cdot \mathrm{SmA} \cdot 180 \cdot \mathrm{I}\left({ }^{\circ} \mathrm{C}\right)\right.$

148- $\mathrm{C}_{5} \mathrm{H}_{11} \mathbf{C O O - 0 / 1 4}\left(\mathrm{X}^{-}=\left[\mathrm{C}_{5} \mathrm{H}_{11} \mathrm{COO}\right]^{-} ; m=0\right): \mathrm{Cr} \cdot 35 \cdot \mathrm{X}_{2} \cdot 90 \cdot \mathrm{SmA} \cdot 160 \cdot \mathrm{I}\left({ }^{\circ} \mathrm{C}\right)$

148- $\mathrm{C}_{7} \mathrm{H}_{15} \mathbf{C O O - 0 / 1 4}\left(\mathrm{X}^{-}=\left[\mathrm{C}_{7} \mathrm{H}_{15} \mathrm{COO}\right]^{-} ; m=0\right): \mathrm{Cr} \cdot 43 \cdot \mathrm{X}_{2} \cdot 93 \cdot \mathrm{SmA} \cdot 130 \cdot \mathrm{I}\left({ }^{\circ} \mathrm{C}\right)$

148- $\mathrm{C}_{9} \mathrm{H}_{19} \mathbf{C O O - 0 / 1 4}\left(\mathrm{X}^{-}=\left[\mathrm{C}_{9} \mathrm{H}_{19} \mathrm{COO}^{-} ; m=0\right): \mathrm{Cr} \cdot 60 \cdot \mathrm{X}_{2} \cdot 100 \cdot \mathrm{SmA} \cdot 135 \cdot \mathrm{I}\left({ }^{\circ} \mathrm{C}\right)\right.$

148- $\mathrm{C}_{11} \mathbf{H}_{23}$ COO-0/14 ( $\left.\mathrm{X}^{-}=\left[\mathrm{C}_{11} \mathrm{H}_{23} \mathrm{COO}\right]^{-} ; m=0\right)$ : $\mathrm{Cr} \cdot 80 \cdot \mathrm{X}_{2} \cdot 122 \cdot \mathrm{SmA} \cdot 142 \cdot \mathrm{I}\left({ }^{\circ} \mathrm{C}\right)$

148- $\mathbf{C}_{15} \mathbf{H}_{31} \mathbf{C O O - 0 / 1 4}\left(\mathrm{X}^{-}=\left[\mathrm{C}_{15} \mathrm{H}_{31} \mathrm{COO}\right]^{-} ; m=0\right): \mathrm{Cr} \cdot 95 \cdot \mathrm{X}_{2} \cdot 135 \cdot \mathrm{I}\left({ }^{\circ} \mathrm{C}\right)$

148- $\mathbf{C}_{12} \mathbf{H}_{25} \mathbf{O S O}_{3} \mathbf{- 0} / \mathbf{1 2}\left(\mathrm{X}^{-}=\left[\mathrm{C}_{12} \mathrm{H}_{25} \mathrm{OSO}_{3}\right]^{-} ; m=0\right)$ : see ref. 106

$\left[\mathrm{N}_{12,1,1,1}\right]\left[\mathrm{C}_{12} \mathrm{H}_{25} \mathrm{OSO}_{3}\right]$ : see ref. 106

$\left[\mathrm{N}_{12,12,1,1}\right]\left[\mathrm{C}_{12} \mathrm{H}_{25} \mathrm{OSO}_{3}\right]$ : see ref. 106

$\left[\mathrm{N}_{12,12,12,1}\right]\left[\mathrm{C}_{12} \mathrm{H}_{25} \mathrm{OSO}_{3}\right]$ : see ref. 106

148-(1-pentylheptyl)PhSO $\left.\mathbf{P}_{3} \mathbf{- 0 / 1 2}\left(\mathrm{X}^{-}=\text {[(1-pentylheptyl) } \mathrm{PhSO}_{3}\right]^{-} ; m=0\right)$ : see ref. 107

$\left[\mathrm{N}_{12,1,1,1}\right][(1-$ pentylheptyl)PhSO 3$]$ : see ref. 107

$\left[\mathrm{N}_{12,12,1,1}\right]\left[\left(1-\right.\right.$ pentylheptyl) $\left.\mathrm{PhSO}_{3}\right]$ : see ref. 107

$\left[\mathrm{N}_{12,12,12,1}\right]\left[(1-p e n t y l h e p t y l) \mathrm{PhSO}_{3}\right]$ : see ref. 107

148-cholate-0/12 ( $\mathrm{X}^{-}=$[cholate $\left.]^{-} ; m=0\right)$ : see ref. 108

$\left[\mathrm{N}_{12,1,1,1}\right][$ cholate]: see ref. 108

$\left[\mathrm{N}_{12,12,1,1}\right]$ [cholate]: see ref. 108 


\section{$\left[\mathrm{N}_{12,12,12,1}\right][$ cholate]: see ref. 108}

148-Br-1/12 $\left(\mathrm{X}^{-}=\mathrm{Br} ; m=1\right)$ : B (highly ordered) $\cdot 56 \cdot \mathrm{B}^{\prime}$ (highly ordered) $\cdot 126 \cdot \mathrm{B}$ '” (highly ordered) $\cdot$ ca. 221$229 \cdot \mathrm{SmA} / \mathrm{dec} .\left({ }^{\circ} \mathrm{C}\right)$

148- $\mathrm{C}_{\mathbf{1 2}} \mathbf{H}_{25} \mathbf{O S O}_{\mathbf{3}} \mathbf{- 1 / 1 2}\left(\mathrm{X}^{-}=\left[\mathrm{C}_{12} \mathrm{H}_{25} \mathrm{OSO}_{3}\right]^{-} ; m=1\right)$ : see ref. 106

148-(1-pentylheptyl)PhSO $\left.{ }_{3}-\mathbf{1 / 1 2}\left(\mathrm{X}^{-}=\text {[(1-pentylheptyl) } \mathrm{PhSO}_{3}\right]^{-} ; m=1\right)$ : see ref. 107

148-cholate-1/12 ( $\mathrm{X}^{-}=$[cholate $\left.]^{-} ; m=1\right)$ : see ref. 108

148-Br-2/12 ( $\left.\mathrm{X}^{-}=\mathrm{Br} ; m=2\right): \mathrm{T}$ (highly ordered) $\cdot$ ca. $274 \cdot$ dec. $\left({ }^{\circ} \mathrm{C}\right)$

148-(1-pentylheptyl) $\left.\mathbf{P h S O}_{3}-\mathbf{2} / \mathbf{1 2}\left(\mathrm{X}^{-}=\text {[(1-pentylheptyl) } \mathrm{PhSO}_{3}\right]^{-} ; m=2\right)$ : see ref. 107

148-cholate-2/12 ( $\mathrm{X}^{-}=$[cholate $\left.]^{-} ; m=2\right)$ : see ref. 108

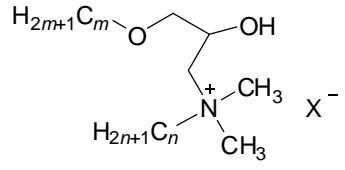

$$
\text { 149-Br-m/n }\left(\mathrm{X}^{-}=\mathrm{Br}^{-}\right)
$$

149-Br-10/8: $\mathrm{Cr} \cdot 81 \cdot \mathrm{SmA} \cdot 87 \cdot \mathrm{I}\left({ }^{\circ} \mathrm{C}\right)$

149-Br-12/8: $\mathrm{Cr} \cdot 77 \cdot \mathrm{SmA} \cdot 105 \cdot \mathrm{I}\left({ }^{\circ} \mathrm{C}\right)$

149-Br-14/8: $\mathrm{Cr} \cdot 75 \cdot \mathrm{SmA} \cdot 121 \cdot \mathrm{I}\left({ }^{\circ} \mathrm{C}\right)$

149-Br-16/8: $\mathrm{Cr} \cdot 81 \cdot \mathrm{SmA} \cdot 131 \cdot \mathrm{I}\left({ }^{\circ} \mathrm{C}\right)$

149-Br-14/10: $\mathrm{Cr} \cdot 82 \cdot \mathrm{SmA} \cdot 135 \cdot \mathrm{I}\left({ }^{\circ} \mathrm{C}\right)$

149-Br-12/12: $\mathrm{Cr} \cdot 90 \cdot \mathrm{SmA} \cdot 141 \cdot \mathrm{I}\left({ }^{\circ} \mathrm{C}\right)$

149-Br-10/14: $\mathrm{Cr} \cdot 88 \cdot \mathrm{SmA} \cdot 143 \cdot \mathrm{I}\left({ }^{\circ} \mathrm{C}\right)$

149-Br-m/1 $\left(\mathrm{X}^{-}=\mathrm{Br}^{-} ; n=1\right)$

149-Br-12/1: $\mathrm{Cr} \cdot 102 \cdot \mathrm{SmA} \cdot 238 \cdot \mathrm{I} / \mathrm{dec} .\left({ }^{\circ} \mathrm{C}\right)$

149-Br-14/1: $\mathrm{Cr} \cdot 106 \cdot \mathrm{SmA} \cdot 251 \cdot \mathrm{I} / \mathrm{dec} .\left({ }^{\circ} \mathrm{C}\right)$

149-Br-16/1: $\mathrm{Cr} \cdot 108 \cdot \mathrm{SmA} \cdot 251 \cdot \mathrm{I} / \mathrm{dec} .\left({ }^{\circ} \mathrm{C}\right)$

149-BF ${ }_{4}-\mathbf{m} / \mathbf{1}\left(\mathrm{X}^{-}=\left[\mathrm{BF}_{4}\right]^{-} ; n=1\right)$

149-BF ${ }_{4}-12 / 1: \mathrm{Cr} \cdot 68 \cdot \mathrm{SmA} \cdot 191 \cdot \mathrm{I}\left({ }^{\circ} \mathrm{C}\right)$

149-BF $\mathbf{4}^{-14} \mathbf{1 4}: \mathrm{Cr} \cdot 75 \cdot \mathrm{SmA} \cdot 215 \cdot \mathrm{I}\left({ }^{\circ} \mathrm{C}\right)$

149- $-\mathrm{BF}_{4}-16 / 1: \mathrm{Cr} \cdot 83 \cdot \mathrm{SmA} \cdot 224 \cdot \mathrm{I}\left({ }^{\circ} \mathrm{C}\right)$

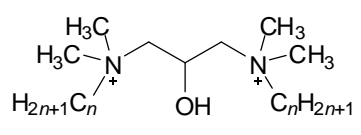

$\mathrm{Cl}^{-} \quad \mathrm{Cl}^{-}$

150-n

150-10: $\mathrm{Cr} \cdot 81 \cdot \mathrm{SmB} \cdot 120 \cdot \mathrm{SmA} \cdot \mathrm{ca} .160 \cdot$ dec. $\left({ }^{\circ} \mathrm{C}\right)$ 
150-12: $\mathrm{Cr} \cdot 83 \cdot \mathrm{SmB} \cdot 123 \cdot \mathrm{SmA} \cdot \mathrm{ca} .160 \cdot$ dec. $\left({ }^{\circ} \mathrm{C}\right)$

150-14: $\mathrm{Cr} \cdot 86 \cdot \mathrm{SmB} \cdot 122 \cdot \mathrm{SmA} \cdot \mathrm{ca} .160 \cdot$ dec. $\left({ }^{\circ} \mathrm{C}\right)$

150-16: $\mathrm{Cr} \cdot 89 \cdot \mathrm{SmB} \cdot 121 \cdot \mathrm{SmA} \cdot$ ca. $160 \cdot$ dec. $\left({ }^{\circ} \mathrm{C}\right)$

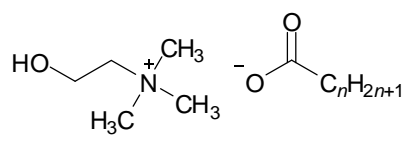

151-n

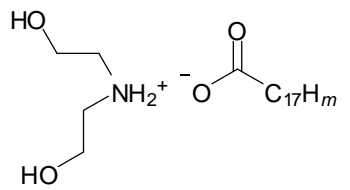

153-m $(m=33,35)$

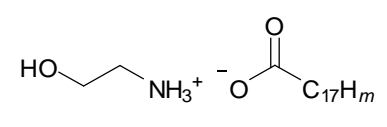

152-m $(m=33,35)$

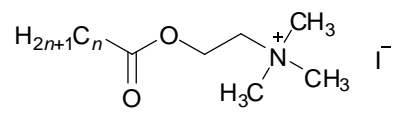

154-n

151-11: $\mathrm{Cr} \cdot 35 \cdot \mathrm{CrM}$ (semicrystalline) $\cdot 68 \cdot \mathrm{SmA} \cdot\left[\right.$ between $120^{\circ} \mathrm{C}$ and $150{ }^{\circ} \mathrm{C}$ ] $\mathrm{I} /$ dec. $\left(T_{\text {dec. }}=191{ }^{\circ} \mathrm{C}\right)\left({ }^{\circ} \mathrm{C}\right)$

151-13: $\mathrm{Cr} \cdot 36 \cdot \mathrm{CrM}$ (semicrystalline) $\cdot 78 \cdot \mathrm{SmA} \cdot$ [between $120^{\circ} \mathrm{C}$ and $\left.150^{\circ} \mathrm{C}\right] \cdot \mathrm{I} /$ dec. $\left(T_{\text {dec. }}=195{ }^{\circ} \mathrm{C}\right)\left({ }^{\circ} \mathrm{C}\right)$

151-15: $\mathrm{Cr} \cdot 44 \cdot \mathrm{CrM}$ (semicrystalline) $\cdot 86 \cdot \mathrm{SmA} \cdot$ [between $120^{\circ} \mathrm{C}$ and $150{ }^{\circ} \mathrm{C}$ ] $\mathrm{I} /$ dec. $\left(T_{\text {dec. }}=199{ }^{\circ} \mathrm{C}\right)\left({ }^{\circ} \mathrm{C}\right)$

151-17: $\mathrm{Cr} \cdot 48 \cdot \mathrm{CrM}$ (semicrystalline) $\cdot 93 \cdot \mathrm{SmA} \cdot\left[\right.$ between $120^{\circ} \mathrm{C}$ and $150{ }^{\circ} \mathrm{C}$ ] $\mathrm{I} /$ dec. $\left(T_{\text {dec. }}=202{ }^{\circ} \mathrm{C}\right)\left({ }^{\circ} \mathrm{C}\right)$

Phase diagrams for 152-33 (with an oleate anion), 152-35 (with a stearate anion), 153-33 (with an oleate anion) and

153-35 (with a stearate anion) (including non-equimolar mixtures of ethanolamine or diethanolamine with oleic acid, $\mathrm{C}_{17} \mathrm{H}_{33} \mathrm{COOH}$, or stearic acid, $\mathrm{C}_{17} \mathrm{H}_{35} \mathrm{COOH}$ ) can be found in ref. 109.

154-11: $\mathrm{Cr} \cdot 52 \cdot \mathrm{CrM}$ (semicrystalline) $/ \mathrm{X} \cdot 168 \cdot \mathrm{SmA} \cdot 197 \cdot$ dec. $\left({ }^{\circ} \mathrm{C}\right)\left(2^{\text {nd }}\right.$ DSC heating run $)$

154-13: $\mathrm{Cr} \cdot 59 \cdot \mathrm{CrM}$ (semicrystalline) $/ \mathrm{X} \cdot 162 \cdot \mathrm{SmA} \cdot 207 \cdot$ dec. $\left({ }^{\circ} \mathrm{C}\right)\left(2^{\text {nd }}\right.$ DSC heating run $)$

154-15: $\mathrm{Cr} \cdot 70 \cdot \mathrm{CrM}$ (semicrystalline) $/ \mathrm{X} \cdot 161 \cdot \mathrm{SmA} \cdot 209 \cdot$ dec. $\left({ }^{\circ} \mathrm{C}\right)\left(2^{\text {nd }}\right.$ DSC heating run $)$

154-17: $\mathrm{Cr} \cdot 80 \cdot \mathrm{CrM}$ (semicrystalline $) / \mathrm{X} \cdot 160 \cdot \mathrm{SmA} \cdot 209 \cdot$ dec. $\left({ }^{\circ} \mathrm{C}\right)\left(2^{\text {nd }} \mathrm{DSC}\right.$ heating run $)$

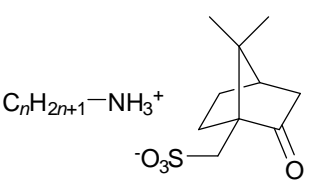

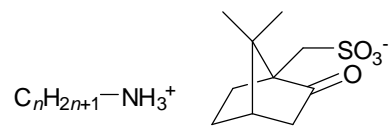

156-n

155-12: $\mathrm{Cr} \cdot 42 \cdot \mathrm{SmB}(?) \cdot 128 \cdot \mathrm{I}\left({ }^{\circ} \mathrm{C}\right)\left(1^{\text {st }} \mathrm{DSC}\right.$ heating run $)$

155-14: $\mathrm{Cr} \cdot 61 \cdot \mathrm{SmB}(?) \cdot 126 \cdot \mathrm{I}\left({ }^{\circ} \mathrm{C}\right)\left(1^{\text {st }} \mathrm{DSC}\right.$ heating run $)$

155-16: $\mathrm{Cr} \cdot 73 \cdot \mathrm{SmB}(?) \cdot 123 \cdot \mathrm{I}\left({ }^{\circ} \mathrm{C}\right)\left(1^{\text {st }} \mathrm{DSC}\right.$ heating run $)$

155-18: $\mathrm{Cr} \cdot 79 \cdot \mathrm{SmB}(?) \cdot 123 \cdot \mathrm{I}\left({ }^{\circ} \mathrm{C}\right)\left(1^{\text {st }} \mathrm{DSC}\right.$ heating run $)$

156-12: $\mathrm{Cr} \cdot 42 \cdot \mathrm{SmB}(?) \cdot 128 \cdot \mathrm{I}\left({ }^{\circ} \mathrm{C}\right)\left(1^{\mathrm{st}} \mathrm{DSC}\right.$ heating run $)$ 
156-14: $\mathrm{Cr} \cdot 63 \cdot \mathrm{SmB}(?) \cdot 120 \cdot \mathrm{I}\left({ }^{\circ} \mathrm{C}\right)\left(1^{\mathrm{st}} \mathrm{DSC}\right.$ heating run $)$

156-16: $\mathrm{Cr} \cdot 69 \cdot \mathrm{SmB}(?) \cdot 124 \cdot \mathrm{I}\left({ }^{\circ} \mathrm{C}\right)\left(1^{\text {st }} \mathrm{DSC}\right.$ heating run $)$

156-18: $\mathrm{Cr} \cdot 79 \cdot \mathrm{SmB}(?) \cdot 126 \cdot \mathrm{I}\left({ }^{\circ} \mathrm{C}\right)\left(1^{\mathrm{st}} \mathrm{DSC}\right.$ heating run $)$

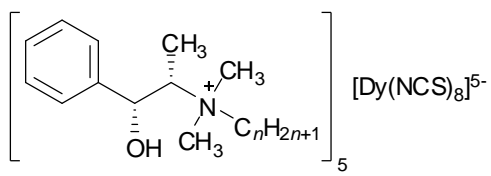

157-n

157-14: not LC

157-16: sample obtained by recrystallization from $\mathrm{CH}_{2} \mathrm{Cl}_{2}: \mathrm{Cr} \cdot 42 \cdot \mathrm{SmA} \cdot 67 \cdot \mathrm{I}\left({ }^{\circ} \mathrm{C}\right.$ ) (prior to melting desolvation of remaining $\mathrm{CH}_{2} \mathrm{Cl}_{2}$ occurs); sample obtained by recrystallization from EtOH: $\mathrm{Cr} \cdot 44 \cdot ? \cdot 75 \cdot \mathrm{I}\left({ }^{\circ} \mathrm{C}\right)$

157-18: sample obtained by recrystallization from $\mathrm{CH}_{2} \mathrm{Cl}_{2}: \mathrm{Cr} \cdot$ ? $\cdot \mathrm{SmA} \cdot 86 \cdot \mathrm{I}\left({ }^{\circ} \mathrm{C}\right)$ (prior to melting desolvation of remaining $\mathrm{CH}_{2} \mathrm{Cl}_{2}$ occurs); sample obtained by recrystallization from EtOH: $\mathrm{Cr} \cdot ? \cdot ? \cdot 98($ ? $) \cdot \mathrm{I}\left({ }^{\circ} \mathrm{C}\right)$

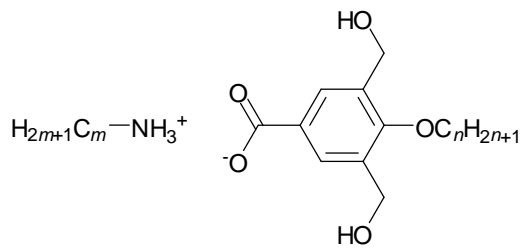

$158-m / n$

neutral benzoic acid precursor for $\mathbf{1 5 8 - m / 6 :} \mathrm{Cr} \cdot 155 \cdot \mathrm{I}\left({ }^{\circ} \mathrm{C}\right)$

neutral benzoic acid precursor for $\mathbf{1 5 8 - m / 1 2 :} \mathrm{Cr}_{1} \cdot 114 \cdot \mathrm{Cr}_{2} \cdot 131 \cdot \mathrm{I}\left({ }^{\circ} \mathrm{C}\right)$

neutral benzoic acid precursor for 158-m/18: $\mathrm{Cr}_{1} \cdot 122 \cdot \mathrm{Cr}_{2} \cdot 132 \cdot \mathrm{SmA} \cdot 135 \cdot \mathrm{I}\left({ }^{\circ} \mathrm{C}\right)$

158-12/6: $\mathrm{Cr}_{1} \cdot 44 \cdot \mathrm{Cr}_{2} \cdot 89 \cdot \mathrm{I}\left({ }^{\circ} \mathrm{C}\right)$

158-14/6: g $\cdot 41 \cdot \mathrm{Cr} \cdot 79 \cdot \mathrm{SmA} \cdot 103 \cdot \mathrm{I}\left({ }^{\circ} \mathrm{C}\right)$

158-16/6: $\mathrm{g} \cdot 64 \cdot \mathrm{Cr} \cdot 76 \cdot \mathrm{SmA} \cdot 110 \cdot \mathrm{I}\left({ }^{\circ} \mathrm{C}\right)$

158-18/6: $\mathrm{Cr}_{1} \cdot 22 \cdot \mathrm{Cr}_{2} \cdot 78 \cdot \mathrm{SmA} \cdot 122 \cdot \mathrm{I}\left({ }^{\circ} \mathrm{C}\right)$

158-12/12: $\mathrm{Cr}_{1} \cdot 10 \cdot \mathrm{Cr}_{2} \cdot 75 \cdot \mathrm{SmA} \cdot 123 \cdot \mathrm{I}\left({ }^{\circ} \mathrm{C}\right)$

158-14/12: $\mathrm{g} \cdot 14 \cdot \mathrm{Cr} \cdot 78 \cdot \mathrm{SmA} \cdot 127 \cdot \mathrm{I}\left({ }^{\circ} \mathrm{C}\right)$

158-16/12: $\mathrm{g} \cdot 12 \cdot \mathrm{Cr} \cdot 79 \cdot \mathrm{SmA} \cdot 126 \cdot \mathrm{I}\left({ }^{\circ} \mathrm{C}\right)$

158-18/12: $\mathrm{Cr}_{1} \cdot 22 \cdot \mathrm{Cr}_{2} \cdot 43 \cdot \mathrm{Cr}_{3} \cdot 57 \cdot \mathrm{Cr}_{4} \cdot 82 \cdot \mathrm{SmA} \cdot 127 \cdot \mathrm{I}\left({ }^{\circ} \mathrm{C}\right)$

158-18/12 (after mixing $\left(n-\mathrm{C}_{18} \mathrm{H}_{37}\right) \mathrm{NH}_{2}$ and the benzoic acid in a 1:1.9 molar ratio instead of a 1:1 ratio):

$\mathrm{Cr} \cdot$ ca. $15 \cdot \mathrm{SmA} \cdot \operatorname{ca} .130 \cdot \mathrm{I}\left({ }^{\circ} \mathrm{C}\right)$

158-12/18: $\mathrm{Cr} \cdot 86 \cdot \mathrm{SmA} \cdot 121 \cdot \mathrm{I}\left({ }^{\circ} \mathrm{C}\right)$

158-14/18: $\mathrm{Cr}_{1} \cdot 55 \cdot \mathrm{Cr}_{2} \cdot 83 \cdot \mathrm{SmA} \cdot 122 \cdot \mathrm{I}\left({ }^{\circ} \mathrm{C}\right)$

158-16/18: $\mathrm{Cr}_{1} \cdot 75 \cdot \mathrm{Cr}_{2} \cdot 89 \cdot \mathrm{SmA} \cdot 125 \cdot \mathrm{I}\left({ }^{\circ} \mathrm{C}\right)$

158-18/18: $\mathrm{Cr}_{1} \cdot 56 \cdot \mathrm{Cr}_{2} \cdot 87 \cdot \mathrm{SmA} \cdot 120 \cdot \mathrm{I}\left({ }^{\circ} \mathrm{C}\right)$ 


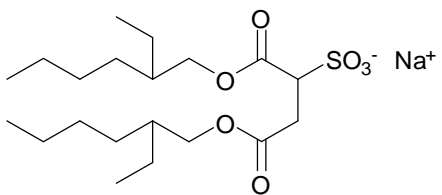

159

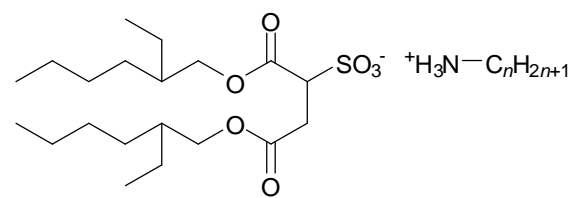

160-n

159: $<-48 \cdot \mathrm{Col}_{\mathrm{h}} \cdot 161 \cdot \mathrm{I}\left({ }^{\circ} \mathrm{C}\right)$

160-10: $<-63 \cdot \mathrm{Col}_{\mathrm{h}} \cdot 36 \cdot \mathrm{I}\left({ }^{\circ} \mathrm{C}\right)$

160-12: $<-48 \cdot \mathrm{Col}_{\mathrm{h}} \cdot 16 \cdot \mathrm{I}\left({ }^{\circ} \mathrm{C}\right)$

160-14: $\mathrm{Cr} \cdot \mathrm{ca} \cdot-33 \cdot \mathrm{Col}_{\mathrm{h}} \cdot-7 \cdot \mathrm{I}\left({ }^{\circ} \mathrm{C}\right)$

160-16: $\mathrm{Cr} \cdot\left[\right.$ not reported] $\cdot \mathrm{Col}_{\mathrm{h}} \cdot-16 \cdot \mathrm{I}\left({ }^{\circ} \mathrm{C}\right)$

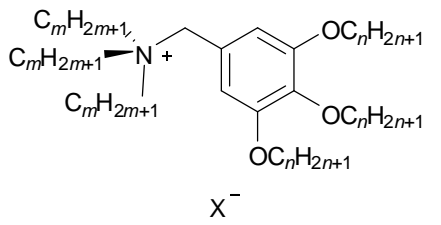

161-X-m/n $\left(\mathrm{X}^{-}=\left[\mathrm{BF}_{4}\right]^{-},\left[\mathrm{PF}_{6}\right]^{-},[\mathrm{OTf}]^{-}\right)$

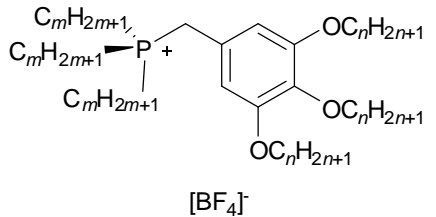

$162-m / n$

\footnotetext{
$161-\mathrm{BF}_{4}-\boldsymbol{m} / \boldsymbol{n}\left(\mathrm{X}^{-}=\left[\mathrm{BF}_{4}\right]^{-}\right)$

161-BF $\mathbf{- 1 / 1 0}: \mathrm{Cr} \cdot 60 \cdot \mathrm{Col}_{\mathrm{h}} \cdot 194 \cdot \mathrm{I}\left({ }^{\circ} \mathrm{C}\right)$

161-BF $\mathbf{B}_{4} \mathbf{1} / 12: \mathrm{Cr}_{1} \cdot 44 \cdot \mathrm{Cr}_{2} \cdot 60 \cdot \mathrm{Col}_{\mathrm{h}} \cdot 206 \cdot \mathrm{I}\left({ }^{\circ} \mathrm{C}\right)$

161-BF $\mathbf{4}-1 / 14: \mathrm{Cr} \cdot 57 \cdot \mathrm{Col}_{\mathrm{h}} \cdot 202 \cdot \mathrm{I}\left({ }^{\circ} \mathrm{C}\right)$

161-BF 4 -2/10: $\mathrm{Cr} \cdot 42 \cdot \mathrm{Cub}_{\mathrm{V}}(\operatorname{Ia} \overline{3} d) \cdot 82 \cdot \mathrm{I}\left({ }^{\circ} \mathrm{C}\right)$

161-BF 4 -2/12: $\mathrm{Cr} \cdot 32 \cdot \mathrm{Cub}_{\mathrm{V}}(\mathrm{Ia} \overline{3} d) \cdot 49 \cdot \mathrm{Col}_{\mathrm{h}} \cdot 126 \cdot \mathrm{I}\left({ }^{\circ} \mathrm{C}\right)$

161-BF $\mathbf{4}-\mathbf{2} / \mathbf{1 4}: \mathrm{Cr} \cdot 53 \cdot \mathrm{Col}_{\mathrm{h}} \cdot 142 \cdot \mathrm{I}\left({ }^{\circ} \mathrm{C}\right)$

161- $\mathrm{BF}_{4}-\mathbf{3} / \mathbf{1 0}: \mathrm{Cr} \cdot 82 \cdot \mathrm{I}\left({ }^{\circ} \mathrm{C}\right)$

161- $\mathrm{BF}_{4}-3 / 12: \mathrm{Cr} \cdot 80 \cdot \mathrm{I}\left({ }^{\circ} \mathrm{C}\right)$

161-BF $\mathbf{B}_{\mathbf{4}} \mathbf{3} / \mathbf{1 4}: \mathrm{Cr} \cdot 80 \cdot \mathrm{Col}_{\mathrm{h}} \cdot 91 \cdot \mathrm{I}\left({ }^{\circ} \mathrm{C}\right)$

161-PF $-\mathbf{m} / \boldsymbol{n}\left(\mathrm{X}^{-}=\left[\mathrm{PF}_{6}\right]^{-}\right)$

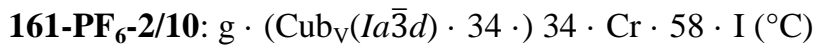

161-PF $-2 / 12: \mathrm{Cr} \cdot 64 \cdot \mathrm{Cub}_{\mathrm{V}}(\operatorname{Ia} \overline{3} d) \cdot 76 \cdot \mathrm{I}\left({ }^{\circ} \mathrm{C}\right)$

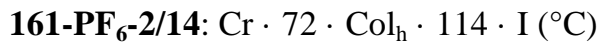

161-OTf-m/n ( $\mathrm{X}^{-}=\left[\mathrm{OTf}^{-}\right)$

161-OTf-2/10: $\mathrm{Cr} \cdot 45 \cdot \mathrm{I}\left({ }^{\circ} \mathrm{C}\right)$

161-OTf-2/12: $\mathrm{Cr}_{1} \cdot 53 \cdot \mathrm{Cr}_{2} \cdot 64 \cdot \mathrm{I}\left({ }^{\circ} \mathrm{C}\right)$

161-OTf-2/14: $\mathrm{Cr} \cdot 65 \cdot \mathrm{I}\left({ }^{\circ} \mathrm{C}\right)$
} 
$162-m / n$

162-1/10: $\mathrm{Cr} \cdot 55 \cdot \mathrm{Col}_{\mathrm{h}} \cdot 164 \cdot \mathrm{I}\left({ }^{\circ} \mathrm{C}\right)$

162-1/12: $\mathrm{Cr}_{1} \cdot 6 \cdot \mathrm{Cr}_{2} \cdot 34 \cdot \mathrm{Col}_{\mathrm{h}} \cdot 181 \cdot \mathrm{I}\left({ }^{\circ} \mathrm{C}\right)$

162-1/14: $\mathrm{Cr} \cdot 40 \cdot \mathrm{Col}_{\mathrm{h}} \cdot 164 \cdot \mathrm{I}\left({ }^{\circ} \mathrm{C}\right)$

162-2/10: $\mathrm{Cr}_{1} \cdot-17 \cdot \mathrm{Cr}_{2} \cdot 0 \cdot \mathrm{Cub}_{\mathrm{V}}(\mathrm{Ia} \overline{3} d) \cdot 48 \cdot \mathrm{I}\left({ }^{\circ} \mathrm{C}\right)$

162-2/12: $\mathrm{Cr} \cdot 31 \cdot \mathrm{Col}_{\mathrm{h}} \cdot 102 \cdot \mathrm{I}\left({ }^{\circ} \mathrm{C}\right)$

162-2/14: $\mathrm{Cr} \cdot 50 \cdot \mathrm{Col}_{\mathrm{h}} \cdot 127 \cdot \mathrm{I}\left({ }^{\circ} \mathrm{C}\right)$

162-3/10: $\mathrm{Cr} \cdot 31 \cdot \mathrm{Col}_{\mathrm{h}} \cdot 66 \cdot \mathrm{I}\left({ }^{\circ} \mathrm{C}\right)$

162-3/12: $\mathrm{Cr}_{1} \cdot 37 \cdot \mathrm{Cr}_{2} \cdot 62 \cdot \mathrm{Col}_{\mathrm{h}} \cdot 88 \cdot \mathrm{I}\left({ }^{\circ} \mathrm{C}\right)$

162-3/14: $\mathrm{Cr}_{1} \cdot 39 \cdot \mathrm{Cr}_{2} \cdot 64 \cdot \mathrm{Col}_{\mathrm{h}} \cdot 93 \cdot \mathrm{I}\left({ }^{\circ} \mathrm{C}\right)$

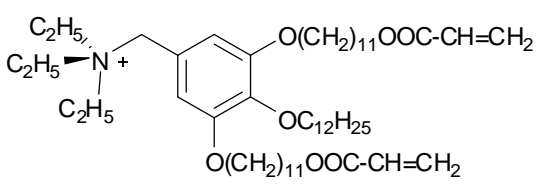

$\left[\mathrm{BF}_{4}\right]^{-}$

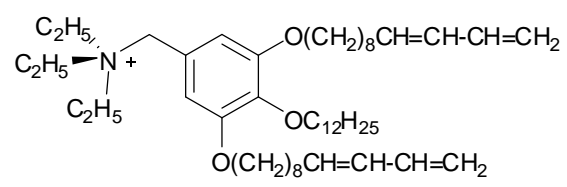

$\left[\mathrm{BF}_{4}\right]^{-}$

163

163: $\mathrm{Cr} \cdot[$ not reported $] \cdot \mathrm{I}\left({ }^{\circ} \mathrm{C}\right)$

164: $\mathrm{Cr} \cdot-5 \cdot \mathrm{Cub}_{\mathrm{V}}(\mathrm{Ia} \overline{3} d) \cdot 19 \cdot \mathrm{I}\left({ }^{\circ} \mathrm{C}\right)$

164/ $\mathrm{LiBF}_{4}(10: 1): \mathrm{Cr} \cdot-6 \cdot \mathrm{Cub}_{\mathrm{V}}(\mathrm{Ia} \overline{3} d) \cdot 22 \cdot \mathrm{I}\left({ }^{\circ} \mathrm{C}\right)$

164/ $\mathrm{LiBF}_{4}(4: 1): \mathrm{Cr}_{1} \cdot-38 \cdot \mathrm{Cr}_{2} \cdot-6 \cdot \mathrm{Cub}_{\mathrm{V}}(\mathrm{Ia} \overline{3} d) \cdot 19 \cdot \mathrm{Col}_{\mathrm{h}} \cdot 56 \cdot \mathrm{I}\left({ }^{\circ} \mathrm{C}\right)$

164/ $\mathrm{LiBF}_{4}(2: 1): \mathrm{Cr}_{1} \cdot-39 \cdot \mathrm{Cr}_{2} \cdot-8 \cdot \mathrm{Cub}_{\mathrm{V}}(\mathrm{Ia} \overline{3} d) \cdot 20 \cdot \mathrm{Col}_{\mathrm{h}} \cdot 76 \cdot \mathrm{I}\left({ }^{\circ} \mathrm{C}\right)$ 
Scheme S8. Ionic complexes of star-shaped tris(2-aminoethyl)amine (165) and benzoic acid derivatives, which were investigated by Kohmoto, Kishikawa and co-workers.

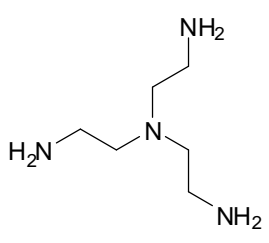

165

\section{Benzoic acid precursors:}

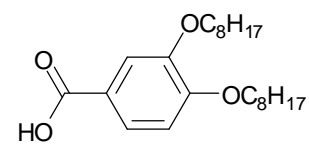

166

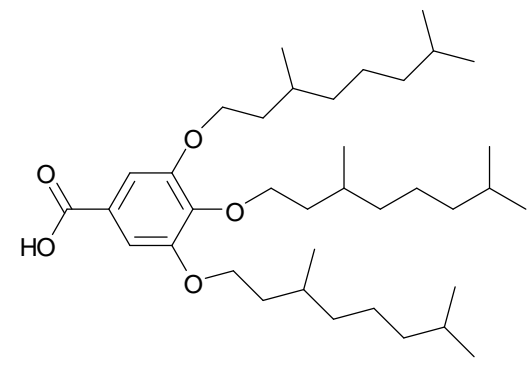

169

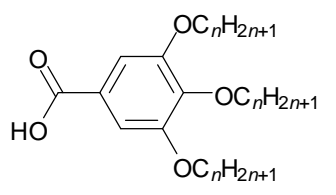

167-n

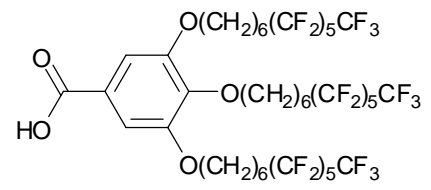

170

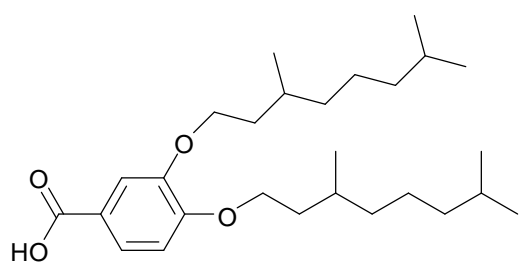

168

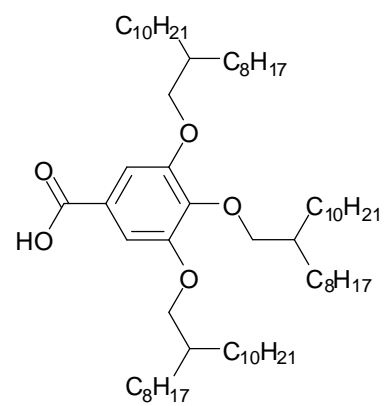

171

165/166 (1:0.5): $\mathrm{Cr} \cdot$ ca. $106 \cdot \mathrm{I}\left({ }^{\circ} \mathrm{C}\right)$

165/166 (1:1): $\mathrm{Cr} \cdot$ ca. $106 \cdot \mathrm{SmA} \cdot$ ca. $145 \cdot \mathrm{I}\left({ }^{\circ} \mathrm{C}\right)$

165/166 (1:1.5): $\mathrm{Cr} \cdot$ ca. $109 \cdot \mathrm{SmA} \cdot$ ca. $150 \cdot \mathrm{I}\left({ }^{\circ} \mathrm{C}\right)$

165/166 (1:2): $\mathrm{Cr} \cdot 111 \cdot \mathrm{SmA} \cdot 152 \cdot \mathrm{I}\left({ }^{\circ} \mathrm{C}\right)$

165/166 (1:2.5): $\mathrm{Cr} \cdot$ ca. $45 \cdot \mathrm{SmA} \cdot$ ca. $141 \cdot \mathrm{I}\left({ }^{\circ} \mathrm{C}\right)$

165/166 (1:3): $\mathrm{Cr} \cdot \mathrm{ca} .50 \cdot\left(\mathrm{SmA}+\mathrm{Col}_{\mathrm{h}}\right) \cdot \mathrm{ca} .129 \cdot \mathrm{I}\left({ }^{\circ} \mathrm{C}\right)$

165/166 (1:3.2): $\mathrm{Cr} \cdot\left[\right.$ not reported] $\cdot \mathrm{Col}_{\mathrm{h}} \cdot \mathrm{ca} .140 \cdot \mathrm{I}\left({ }^{\circ} \mathrm{C}\right)$

165/166 (1:3.5): $\mathrm{Cr} \cdot$ ca. $110 \cdot \mathrm{Col}_{\mathrm{h}} \cdot$ ca. $150 \cdot \mathrm{I}\left({ }^{\circ} \mathrm{C}\right)$

165/166 (1:3.8): $\mathrm{Cr} \cdot 94 \cdot \mathrm{Col}_{\mathrm{h}} \cdot 153 \cdot \mathrm{I}\left({ }^{\circ} \mathrm{C}\right)$

165/166 (1:4): $\mathrm{Cr} \cdot 94 \cdot \mathrm{Col}_{\mathrm{h}} \cdot 151 \cdot \mathrm{I}\left({ }^{\circ} \mathrm{C}\right)$

165/166 (1:4.5): $\mathrm{Cr} \cdot \mathrm{ca} .93 \cdot \mathrm{Col}_{\mathrm{h}} \cdot \mathrm{ca} .123 \cdot \mathrm{I}\left({ }^{\circ} \mathrm{C}\right)$

165/166 (1:5): $\mathrm{Cr} \cdot$ ca. $107 \cdot \mathrm{I}\left({ }^{\circ} \mathrm{C}\right)$ 
165/167-4 (1:3): $\mathrm{Cr}_{1} \cdot 64 \cdot \mathrm{Cr}_{2} \cdot 112 \cdot \mathrm{I}\left({ }^{\circ} \mathrm{C}\right)$

165/167-5 (1:2.3): $\mathrm{Cr} \cdot\left[\right.$ not reported] $\cdot \mathrm{Col}_{\mathrm{h}} \cdot 94 \cdot \mathrm{I}\left({ }^{\circ} \mathrm{C}\right)$

165/167-5 (1:2.6): $\mathrm{Cr} \cdot\left[\right.$ not reported] $\cdot \mathrm{Col}_{\mathrm{h}} \cdot 108 \cdot \mathrm{I}\left({ }^{\circ} \mathrm{C}\right)$

165/167-5 (1:3): $\mathrm{Cr} \cdot 93 \cdot \mathrm{Col}_{\mathrm{h}} \cdot 119 \cdot \mathrm{I}\left({ }^{\circ} \mathrm{C}\right)$

165/167-5 (1:3.4): $\mathrm{Cr} \cdot\left[\right.$ not reported] $\cdot \mathrm{Col}_{\mathrm{h}} \cdot 97 \cdot \mathrm{I}\left({ }^{\circ} \mathrm{C}\right)$

165/167-5 (1:4): $\mathrm{Cr} \cdot\left[\right.$ not reported] $\cdot \mathrm{Col}_{\mathrm{h}} \cdot 92 \cdot \mathrm{I}\left({ }^{\circ} \mathrm{C}\right)$

165/167-6 (1:2.6): $\mathrm{Cr} \cdot 90 \cdot \mathrm{Col}_{\mathrm{h}} \cdot 133 \cdot \mathrm{I}\left({ }^{\circ} \mathrm{C}\right)$

165/167-6 (1:3): $\mathrm{Cr} \cdot 93 \cdot \mathrm{Col}_{\mathrm{h}} \cdot 133 \cdot \mathrm{I}\left({ }^{\circ} \mathrm{C}\right)$

165/167-7 (1:2.3): g $84 \cdot \mathrm{Col}_{\mathrm{h}} \cdot 139 \cdot \mathrm{I}\left({ }^{\circ} \mathrm{C}\right)$

165/167-7 (1:3): $\mathrm{Cr} \cdot 86 \cdot \mathrm{Col}_{\mathrm{h}} \cdot 138 \cdot \mathrm{I}\left({ }^{\circ} \mathrm{C}\right)$

165/167-8 (1:1.9): g · [not reported] $\cdot \mathrm{Col}_{\mathrm{h}} \cdot \mathrm{ca} .128 \cdot \mathrm{I}\left({ }^{\circ} \mathrm{C}\right)$

165/167-8 (1:2.1): g · [not reported] $\cdot \mathrm{Col}_{\mathrm{h}} \cdot \mathrm{ca} .138 \cdot \mathrm{I}\left({ }^{\circ} \mathrm{C}\right)$

165/167-8 (1:2.2): g $85 \cdot \mathrm{Col}_{\mathrm{h}} \cdot 141 \cdot \mathrm{I}\left({ }^{\circ} \mathrm{C}\right)$

165/167-8 (1:2.3): g · [not reported] $\cdot \mathrm{Col}_{\mathrm{h}} \cdot \mathrm{ca} .139 \cdot \mathrm{I}\left({ }^{\circ} \mathrm{C}\right)$

165/167-8 (1:2.6): g · [not reported] $\cdot \mathrm{Col}_{\mathrm{h}} \cdot \mathrm{ca} .137 \cdot \mathrm{I}\left({ }^{\circ} \mathrm{C}\right)$

165/167-8 (1:3): $\mathrm{Cr} \cdot 74 \cdot \mathrm{Col}_{\mathrm{h}} \cdot 129 \cdot \mathrm{I}\left({ }^{\circ} \mathrm{C}\right)$

165/167-9 (1:2.1): g $82 \cdot \mathrm{Col}_{\mathrm{h}} \cdot 140 \cdot \mathrm{I}\left({ }^{\circ} \mathrm{C}\right)$

165/167-9 (1:3): $\mathrm{Cr} \cdot 100 \cdot \mathrm{Col}_{\mathrm{h}} \cdot 131 \cdot \mathrm{I}\left({ }^{\circ} \mathrm{C}\right)$

165/167-10 (1:2): g · [not reported] $\cdot \mathrm{Col}_{\mathrm{h}} \cdot 139 \cdot \mathrm{I}\left({ }^{\circ} \mathrm{C}\right)$

165/167-10 (1:3): $\mathrm{Cr} \cdot 37 \cdot \mathrm{Col}_{\mathrm{h}} \cdot 123 \cdot \mathrm{I}\left({ }^{\circ} \mathrm{C}\right)$

165/167-11 (1:3): $\mathrm{Cr} \cdot 31 \cdot \mathrm{Col}_{\mathrm{h}} \cdot 124 \cdot \mathrm{I}\left({ }^{\circ} \mathrm{C}\right)$

165/167-12 (1:2): $\mathrm{Cr} \cdot 21 \cdot \mathrm{Col}_{\mathrm{h}} \cdot 134 \cdot \mathrm{I}\left({ }^{\circ} \mathrm{C}\right)$

165/167-12 (1:3): $\mathrm{Cr} \cdot 8 \cdot \mathrm{Col}_{\mathrm{h}} \cdot 116 \cdot \mathrm{I}\left({ }^{\circ} \mathrm{C}\right)$

165/167-13 (1:3): $\mathrm{Cr} \cdot 25 \cdot \mathrm{Col}_{\mathrm{h}} \cdot 116 \cdot \mathrm{I}\left({ }^{\circ} \mathrm{C}\right)$

165/167-14 (1:2): $\mathrm{Cr} \cdot 42 \cdot \mathrm{Col}_{\mathrm{h}} \cdot 128 \cdot \mathrm{I}\left({ }^{\circ} \mathrm{C}\right)$

165/167-14 (1:3): $\mathrm{Cr} \cdot 37 \cdot \mathrm{Col}_{\mathrm{h}} \cdot 106 \cdot \mathrm{I}\left({ }^{\circ} \mathrm{C}\right)$

165/167-15 (1:3): $\mathrm{Cr} \cdot 47 \cdot \mathrm{Col}_{\mathrm{h}} \cdot 100 \cdot \mathrm{I}\left({ }^{\circ} \mathrm{C}\right)$

165/167-16 (1:2): $\mathrm{Cr} \cdot 58 \cdot \mathrm{Col}_{\mathrm{h}} \cdot 122 \cdot \mathrm{I}\left({ }^{\circ} \mathrm{C}\right)$

165/167-16 (1:3): $\mathrm{Cr} \cdot 54 \cdot \mathrm{Col}_{\mathrm{h}} \cdot 102 \cdot \mathrm{I}\left({ }^{\circ} \mathrm{C}\right)$

165/167-18 (1:2): $\mathrm{Cr} \cdot 67 \cdot \mathrm{Col}_{\mathrm{h}} \cdot 116 \cdot \mathrm{I}\left({ }^{\circ} \mathrm{C}\right)$

165/168 (1:3): g $\cdot$ [not reported] $\cdot \mathrm{Col}_{\mathrm{h}} \cdot 153 \cdot \mathrm{I}\left({ }^{\circ} \mathrm{C}\right)$

165/169 (1:2): g $\cdot$ [not reported] $\cdot \mathrm{Col}_{\mathrm{h}} \cdot 111 \cdot \mathrm{I}\left({ }^{\circ} \mathrm{C}\right)$

165/170 (1:0.5): $\mathrm{Cr} \cdot\left[\right.$ not reported] $\cdot \mathrm{Col}_{\mathrm{h}} \cdot \mathrm{ca} .145 \cdot \mathrm{I}\left({ }^{\circ} \mathrm{C}\right)$

165/170 (1:1): $\mathrm{Cr} \cdot\left[\right.$ not reported] $\cdot \mathrm{Col}_{\mathrm{h}} \cdot \mathrm{ca} .148 \cdot \mathrm{I}\left({ }^{\circ} \mathrm{C}\right)$

165/170 (1:1.3): $\mathrm{Cr} \cdot 53 \cdot \mathrm{Col}_{\mathrm{h}} \cdot 149 \cdot \mathrm{I}\left({ }^{\circ} \mathrm{C}\right)$

165/170 (1:1.5): $\mathrm{Cr} / \mathrm{g} \cdot \mathrm{ca} .49 \cdot \mathrm{Col}_{\mathrm{h}} \cdot \mathrm{ca} .146 \cdot \mathrm{I}\left({ }^{\circ} \mathrm{C}\right)$ 
165/170 (1:2): $\mathrm{Cr} / \mathrm{g} \cdot$ ca. $60 \cdot \mathrm{Col}_{\mathrm{h}} \cdot$ ca. $132 \cdot \mathrm{I}\left({ }^{\circ} \mathrm{C}\right)$

165/170 (1:2.5): $\mathrm{Cr} / \mathrm{g} \cdot \mathrm{ca} .74 \cdot \mathrm{Col}_{\mathrm{h}} \cdot \mathrm{ca} .110 \cdot \mathrm{I}\left({ }^{\circ} \mathrm{C}\right)$

165/170 (1:3): $\mathrm{Cr} / \mathrm{g} \cdot \mathrm{ca} .78 \cdot \mathrm{Col}_{\mathrm{h}} \cdot \mathrm{ca} .100 \cdot \mathrm{I}\left({ }^{\circ} \mathrm{C}\right)$

165/170 (1:3.3): $\mathrm{Cr} / \mathrm{g} \cdot \mathrm{ca} .72 \cdot\left(\mathrm{Col}_{\mathrm{h}}+\mathrm{Cub}_{\mathrm{I}}(\operatorname{Pm} \overline{3} n)\right) \cdot c a .108 \cdot\left(\mathrm{Cub}_{\mathrm{I}}(\operatorname{Pm} \overline{3} n)+\mathrm{I}\right) \cdot c a .138 \cdot \mathrm{I}\left({ }^{\circ} \mathrm{C}\right)$

165/170 (1:3.5): $\mathrm{Cr} / \mathrm{g} \cdot \mathrm{ca} .67 \cdot \mathrm{Cub}_{\mathrm{I}}(\mathrm{Pm} \overline{3} n) \cdot c a .145 \cdot \mathrm{I}\left({ }^{\circ} \mathrm{C}\right)$

165/170 (1:4): $\mathrm{Cr} / \mathrm{g} \cdot \mathrm{ca} .69 \cdot \mathrm{Cub}_{\mathrm{I}}(\mathrm{Pm} \overline{3} n) \cdot \mathrm{ca} .148 \cdot \mathrm{I}\left({ }^{\circ} \mathrm{C}\right)$

165/170 (1:4.5): $\mathrm{Cr} / \mathrm{g} \cdot \mathrm{ca} .63 \cdot \mathrm{Cub}_{\mathrm{I}}(\mathrm{Pm} \overline{3} n) \cdot \mathrm{ca} .151 \cdot \mathrm{I}\left({ }^{\circ} \mathrm{C}\right)$

165/170 (1:5): $\mathrm{Cr} / \mathrm{g} \cdot 67 \cdot \mathrm{Cub}_{\mathrm{I}}(\operatorname{Pm} \overline{3} n) \cdot 152 \cdot \mathrm{I}\left({ }^{\circ} \mathrm{C}\right)$

165/171 (1:2.5): g · [not reported] $\cdot \mathrm{Cub}_{\mathrm{I}}(\operatorname{Pm} \overline{3} n) \cdot$ ca $63 \cdot \mathrm{I}\left({ }^{\circ} \mathrm{C}\right)$

165/171 (1:3): g · [not reported] $\cdot \mathrm{Cub}_{\mathrm{I}}(P m \overline{3} n) \cdot c a .80 \cdot \mathrm{I}\left({ }^{\circ} \mathrm{C}\right)$

165/171 (1:3.5): g · [not reported] $\cdot \mathrm{Cub}_{\mathrm{I}}(\mathrm{Pm} \overline{3} n) \cdot \mathrm{ca} .86 \cdot \mathrm{I}\left({ }^{\circ} \mathrm{C}\right)$

165/171 (1:4): g · [not reported] $\cdot \mathrm{Cub}_{\mathrm{I}}(\mathrm{Pm} \overline{3} n) \cdot 89 \cdot \mathrm{I}\left({ }^{\circ} \mathrm{C}\right)$

165/171 (1:4.5): g · [not reported] $\cdot \mathrm{Cub}_{\mathrm{I}}(\operatorname{Pm} \overline{3} n) \cdot c a .89 \cdot \mathrm{I}\left({ }^{\circ} \mathrm{C}\right)$

165/171 (1:5): g · not reported] $\cdot \mathrm{Cub}_{\mathrm{I}}(\operatorname{Pm} \overline{3} n) \cdot c a .80 \cdot \mathrm{I}\left({ }^{\circ} \mathrm{C}\right)$

165/171 (1:5.5): g · [not reported] $\cdot \mathrm{Cub}_{\mathrm{I}}(\mathrm{Pm} \overline{3} n) \cdot \mathrm{ca} .66 \cdot \mathrm{I}\left({ }^{\circ} \mathrm{C}\right)$ 
Scheme S9. Ionic complexes of 2-aminoalcohols (and related compounds) and carboxylic acid derivatives, which were investigated by Saigo and co-workers.

2-Aminoalcohol precursors and related compounds:

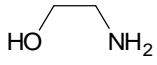

172a

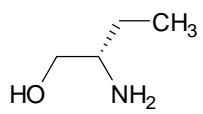

172d

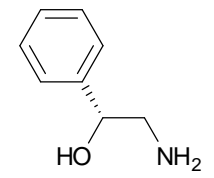

172g

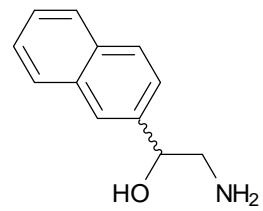

$172 \mathbf{j}$

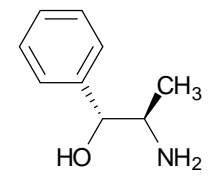

$172 \mathrm{~m}$

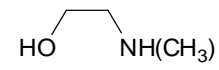

172p

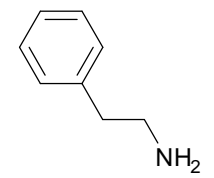

172s

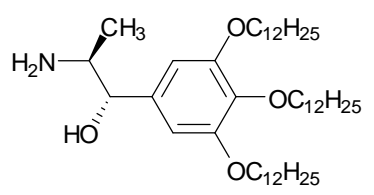

172v

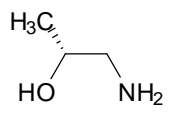

172b

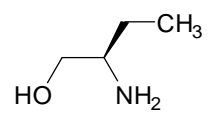

172e

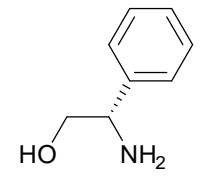

172h

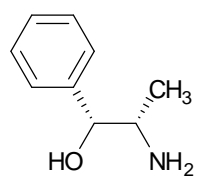

172k

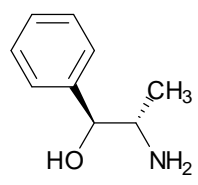

172n

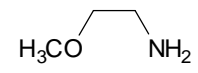

$172 q$

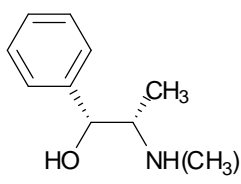

172t

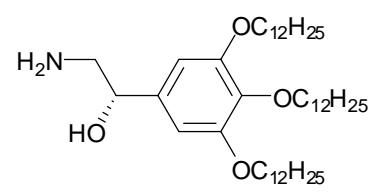

172w

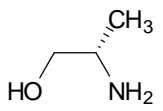

172c

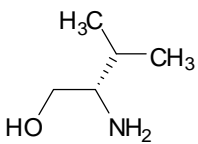

172f

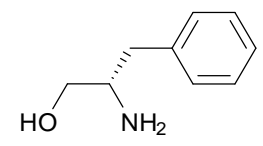

172i

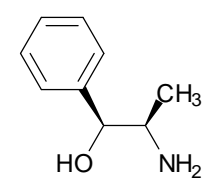

172I

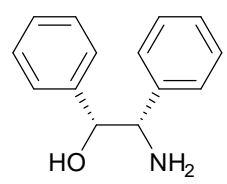

1720

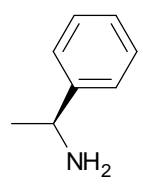

172r

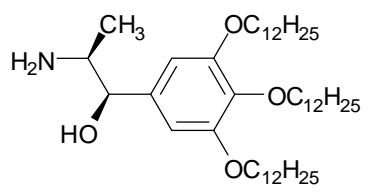

172u 


\section{Carboxylic acid precursors:}<smiles>CCOc1cc(C(=O)O)cc(OCC)c1OCC</smiles>

173<smiles>C=CC=CCCCOc1cc(C(=O)O)cc(OC)c1OCC=C</smiles>

174c<smiles>O=C(O)/C=C/c1ccccc1</smiles>

176<smiles>C=CC(=O)OCCOc1cc(C(=O)O)cc(OCc2ccccc2)c1OCC(=O)OCC</smiles>

174a<smiles>C=CC=CCCOc1cc(C(=O)O)cc(OCC)c1OCC=CC=C</smiles>

174d

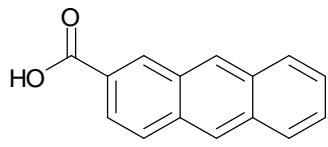

177<smiles>C=CCOC(=O)OCCOc1cc(C(=O)O)cc(OCC)c1OCC(=O)OCC</smiles>

174b

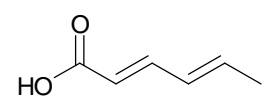

175

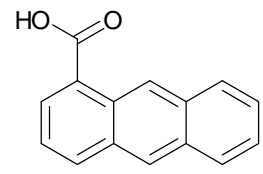

178

173/172a (1:1): $\mathrm{Cr}_{1} \cdot 16 \cdot \mathrm{Cr}_{2} \cdot 33 \cdot \mathrm{Cr}_{3} \cdot 41 \cdot \mathrm{Cr}_{4} \cdot 53 \cdot \mathrm{Col}_{\mathrm{h}} \cdot 105 \cdot \mathrm{I}\left({ }^{\circ} \mathrm{C}\right)$

173/172b (1:1): $\mathrm{Cr}_{1} \cdot 26 \cdot \mathrm{Cr}_{2} \cdot 31 \cdot \mathrm{Cr}_{3} \cdot 36 \cdot \mathrm{Cr}_{4} \cdot 40 \cdot \mathrm{Cr}_{5} \cdot 54 \cdot \mathrm{Col} \cdot 65 \cdot \mathrm{Col} \cdot 87 \cdot \mathrm{I}\left({ }^{\circ} \mathrm{C}\right)$

173/172c (1:1): $\mathrm{Cr} \cdot 42 \cdot \mathrm{Col}_{\mathrm{h}, 1} \cdot 50 \cdot \mathrm{Col}_{\mathrm{h}, 2} \cdot 83 \cdot \mathrm{I}\left({ }^{\circ} \mathrm{C}\right)$

173/172e (1:1): $\mathrm{Cr}_{1} \cdot 30 \cdot \mathrm{Cr}_{2} \cdot 35 \cdot \mathrm{Cr}_{3} \cdot 49 \cdot \mathrm{Cr}_{4} \cdot 58 \cdot \mathrm{I}\left({ }^{\circ} \mathrm{C}\right)$

173/172g (1:1): $\mathrm{Cr}_{1} \cdot 48 \cdot \mathrm{Cr}_{2} \cdot 80 \cdot \mathrm{Col} \cdot 91 \cdot \mathrm{I}\left({ }^{\circ} \mathrm{C}\right)$

173/172h (1:1): $\mathrm{Cr} \cdot 63 \cdot \mathrm{I}\left({ }^{\circ} \mathrm{C}\right)$

173/172l (1:1): $\mathrm{Cr}_{1} \cdot 26 \cdot \mathrm{Cr}_{2} \cdot 41 \cdot \mathrm{Cr}_{3} \cdot 57 \cdot \mathrm{Cr}_{4} \cdot 88 \cdot \mathrm{Col}_{\mathrm{r}} \cdot 104 \cdot \mathrm{I}\left({ }^{\circ} \mathrm{C}\right)$

173/172o (1:1): $\mathrm{Cr} \cdot 124 \cdot \mathrm{I}\left({ }^{\circ} \mathrm{C}\right)$

173/172p (1:1): $\mathrm{Cr}_{1} \cdot 34 \cdot \mathrm{Cr}_{2} \cdot 66 \cdot \mathrm{Col} \cdot 94 \cdot \mathrm{I}\left({ }^{\circ} \mathrm{C}\right)$

173/172q (1:1): $\mathrm{Cr}_{1} \cdot 41 \cdot \mathrm{Cr}_{2} \cdot 64 \cdot \mathrm{I}\left({ }^{\circ} \mathrm{C}\right)$

173/172r (1:1): $\mathrm{Cr}_{1} \cdot 33 \cdot \mathrm{Cr}_{2} \cdot 64 \cdot \mathrm{I}\left({ }^{\circ} \mathrm{C}\right)$

173/172s (1:1): $\mathrm{Cr}_{1} \cdot 21 \cdot \mathrm{Cr}_{2} \cdot 67 \cdot \mathrm{I}\left({ }^{\circ} \mathrm{C}\right)$

174a/172a (1:1): $\mathrm{Cr} \cdot 9 \cdot \mathrm{I}\left({ }^{\circ} \mathrm{C}\right)$

174a/172b (1:1): $\mathrm{Cr} \cdot 3 \cdot \mathrm{Col} \cdot 12 \cdot \mathrm{I}\left({ }^{\circ} \mathrm{C}\right)$

174a/172c (1:1): $\mathrm{Cr} \cdot 4 \cdot \mathrm{Col}_{\mathrm{h}} \cdot 38 \cdot \mathrm{I}\left({ }^{\circ} \mathrm{C}\right)$

174a/172g (1:1): $\mathrm{Cr} \cdot-14 \cdot \mathrm{M} \cdot 19 \cdot \mathrm{Col}$ (presumably monotropic) $\cdot 26 \cdot \mathrm{I}\left({ }^{\circ} \mathrm{C}\right){ }^{110} / \mathrm{Cr} \cdot 43 \cdot \mathrm{I}\left({ }^{\circ} \mathrm{C}\right){ }^{111}$

174a/172h (1:1): M $\cdot 43 \cdot \mathrm{I}\left({ }^{\circ} \mathrm{C}\right)$

174a/172i (1:1): $\mathrm{Cr} \cdot 45 \cdot \mathrm{I}\left({ }^{\circ} \mathrm{C}\right)$ 
174a/172k (1:1): $\mathrm{Col}_{\mathrm{r}} \cdot 73 \cdot \mathrm{I}\left({ }^{\circ} \mathrm{C}\right)$

174a/172l (1:1): $\mathrm{Cr} \cdot-15 \cdot \mathrm{Col}_{\mathrm{r}} \cdot 60 \cdot \mathrm{I}\left({ }^{\circ} \mathrm{C}\right)$

174a/172o (1:1): $\mathrm{Cr} \cdot 77 \cdot \mathrm{M} \cdot 91 \cdot \mathrm{I}\left({ }^{\circ} \mathrm{C}\right)$

174b/172k (1:1): $\mathrm{Col}_{\mathrm{r}} \cdot 77 \cdot \mathrm{I}\left({ }^{\circ} \mathrm{C}\right)$

174c/172k (1:1): $\mathrm{Col}_{\mathrm{r}} \cdot 99 \cdot \mathrm{I}\left({ }^{\circ} \mathrm{C}\right)$

174d/172k (1:1): $\mathrm{Col}_{\mathrm{r}} \cdot 103 \cdot \mathrm{I}\left({ }^{\circ} \mathrm{C}\right)$

175/172u (1:1): $\mathrm{Cr} \cdot$ ca. $62 \cdot \mathrm{I}\left({ }^{\circ} \mathrm{C}\right)$ (on cooling)

176/172u (1:1): $\mathrm{Cr} \cdot \mathrm{ca} .35 \cdot \mathrm{I}\left({ }^{\circ} \mathrm{C}\right)$ (on cooling)

177/172u (1:1): $\mathrm{Cr} \cdot 141 \cdot \mathrm{I}\left({ }^{\circ} \mathrm{C}\right)$ (on cooling)

178/172u (1:1): $\mathrm{Cr} \cdot \mathrm{ca} .81 \cdot \mathrm{I}\left({ }^{\circ} \mathrm{C}\right)$ (on cooling)

175/172v (1:1): SmA (monotropic) $\cdot 65 \cdot \mathrm{I}\left({ }^{\circ} \mathrm{C}\right)$ (on cooling)

176/172v (1:1): $\mathrm{SmX}$ (monotropic) $\cdot$ ca. $45 \cdot \mathrm{SmA} \cdot 82^{86}$ or ca. $87^{112} \cdot \mathrm{I}\left({ }^{\circ} \mathrm{C}\right)$ (on cooling)

177/172v (1:1): M (monotropic) $\cdot 59^{86}$ or $65^{112} \cdot \operatorname{SmA}$ (monotropic) $\cdot 100^{86}$ or $98^{112} \cdot \mathrm{I}\left({ }^{\circ} \mathrm{C}\right)$ (on cooling)

178/172v (1:1): $\mathrm{Col}_{\mathrm{r}}{ }^{86}$ or $\mathrm{Cr}^{112} \cdot 49 \cdot \mathrm{I}\left({ }^{\circ} \mathrm{C}\right)$ (on cooling)

175/172w (1:1): $\mathrm{SmX} \cdot$ ca. $53 \cdot \mathrm{SmA} \cdot$ ca. $108 \cdot \mathrm{I}\left({ }^{\circ} \mathrm{C}\right)$ (on cooling)

176/172w (1:1): $\mathrm{SmX} \cdot$ ca. $65 \cdot \mathrm{SmA} \cdot \mathrm{ca} .121 \cdot \mathrm{I}\left({ }^{\circ} \mathrm{C}\right)$ (on cooling)

177/172w (1:1): $\mathrm{SmX}$ (monotropic) $\cdot 60 \cdot \mathrm{SmA} \cdot 137 \cdot \mathrm{I}\left({ }^{\circ} \mathrm{C}\right)$ (on cooling)

178/172w (1:1): SmA (monotropic) $\cdot c a .86 \cdot \mathrm{I}\left({ }^{\circ} \mathrm{C}\right)$ (on cooling)

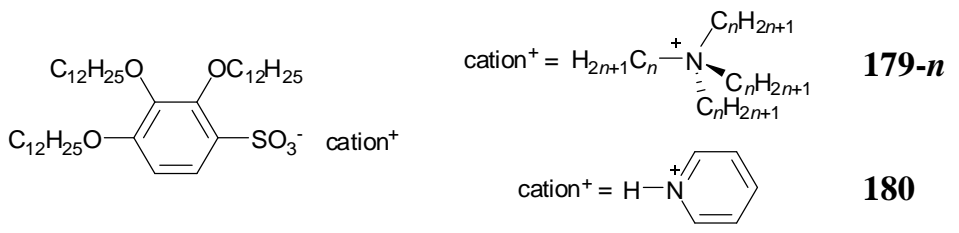

179-1: $\mathrm{Cr} \cdot 47 \cdot \mathrm{M}_{1}(\mathrm{Col} ?) \cdot 130 \cdot \mathrm{M}_{2}(\mathrm{Col} ?) \cdot 154 \cdot \mathrm{Col}_{\mathrm{h}} \cdot 231 \cdot \mathrm{I}\left({ }^{\circ} \mathrm{C}\right)$

179-2: $\mathrm{Cr} \cdot(\mathrm{M} \cdot 99 \cdot) 125 \cdot \mathrm{I}\left({ }^{\circ} \mathrm{C}\right)$

179-4: $\mathrm{Cr} \cdot 81 \cdot \mathrm{I}\left({ }^{\circ} \mathrm{C}\right)$

180: $\mathrm{Cr}_{1} \cdot 30 \cdot \mathrm{Cr}_{2} \cdot 53 \cdot \mathrm{Cr}_{3} \cdot 80 \cdot \mathrm{Col}_{\mathrm{h}} \cdot 122 \cdot \mathrm{I}\left({ }^{\circ} \mathrm{C}\right)\left(\right.$ dec. above $\left.150{ }^{\circ} \mathrm{C}\right)$

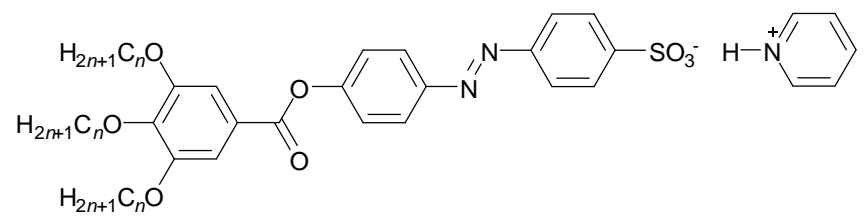

181-n

181-8: $\mathrm{SmX} \cdot 109 \cdot \mathrm{Col}_{\mathrm{h}(\mathrm{d})} \cdot 210 \cdot \mathrm{I}\left({ }^{\circ} \mathrm{C}\right)$

181-12: $\mathrm{SmX} \cdot 94 \cdot \mathrm{Col}_{\mathrm{h}(\mathrm{d})} \cdot 200 \cdot \mathrm{I}\left({ }^{\circ} \mathrm{C}\right)$ 


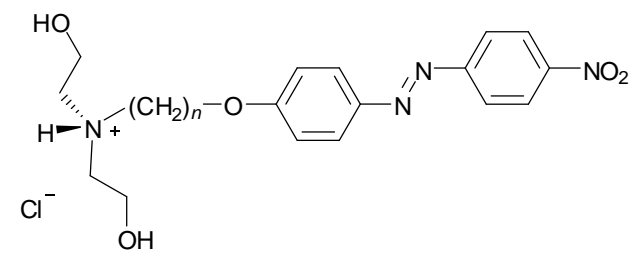

182-n

182-2: $\mathrm{Cr} \cdot 174 \cdot \mathrm{SmX} \cdot>210 \cdot \operatorname{dec}\left({ }^{\circ} \mathrm{C}\right)$

182-4: $\mathrm{Cr} \cdot 107 \cdot \mathrm{SmX} \cdot>210 \cdot \operatorname{dec}\left({ }^{\circ} \mathrm{C}\right)$

182-6: $\mathrm{Cr} \cdot 89 \cdot \mathrm{SmX} \cdot>210 \cdot$ dec. $\left({ }^{\circ} \mathrm{C}\right)$

182-8: $\mathrm{Cr} \cdot 98 \cdot \mathrm{SmX} \cdot>210 \cdot \operatorname{dec} .\left({ }^{\circ} \mathrm{C}\right)$

182-10: $\mathrm{Cr} \cdot 102 \cdot \mathrm{SmX} \cdot>210 \cdot$ dec. $\left({ }^{\circ} \mathrm{C}\right)$

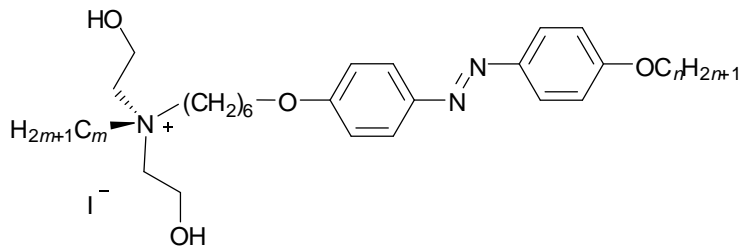

183-2/n $(m=2)$

183-2/4: $\mathrm{Cr} \cdot 118 \cdot \mathrm{SmA} \cdot 170 \cdot \mathrm{I}\left({ }^{\circ} \mathrm{C}\right)$

183-2/7: $\mathrm{Cr}_{1} \cdot 47 \cdot \mathrm{Cr}_{2} \cdot 107 \cdot \mathrm{SmC} \cdot 116 \cdot \mathrm{SmA} \cdot 128 \cdot \mathrm{I}\left({ }^{\circ} \mathrm{C}\right)$

183-m/2 $(m=1-4,7-10 ; n=2)$

183-4/2: $\mathrm{Cr} \cdot 32 \cdot \mathrm{SmC}\left(\right.$ ?) $\cdot 107 \cdot \mathrm{I}\left({ }^{\circ} \mathrm{C}\right)$

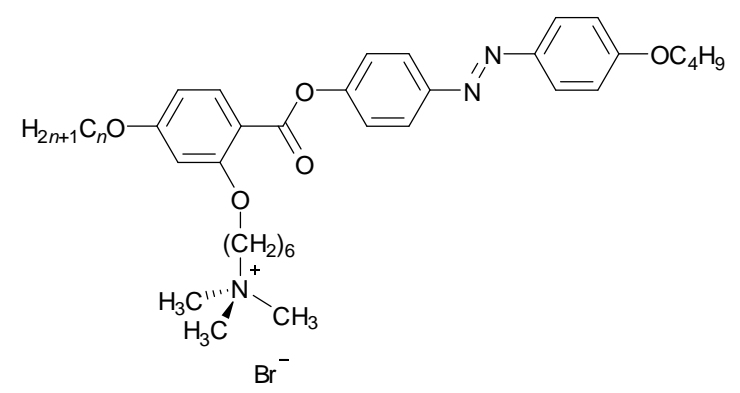

184-n

184-6: g $\cdot 23 \cdot \mathrm{N} \cdot 36 \cdot \mathrm{I}\left({ }^{\circ} \mathrm{C}\right)$

184-8: g $\cdot 25 \cdot \mathrm{N} \cdot 68 \cdot \mathrm{I}\left({ }^{\circ} \mathrm{C}\right)$

184-10: $\mathrm{g} \cdot 32 \cdot \mathrm{SmA} \cdot 87 \cdot \mathrm{I}\left({ }^{\circ} \mathrm{C}\right)$

184-12: g $31 \cdot \mathrm{SmA} \cdot 92 \cdot \mathrm{I}\left({ }^{\circ} \mathrm{C}\right)$ 


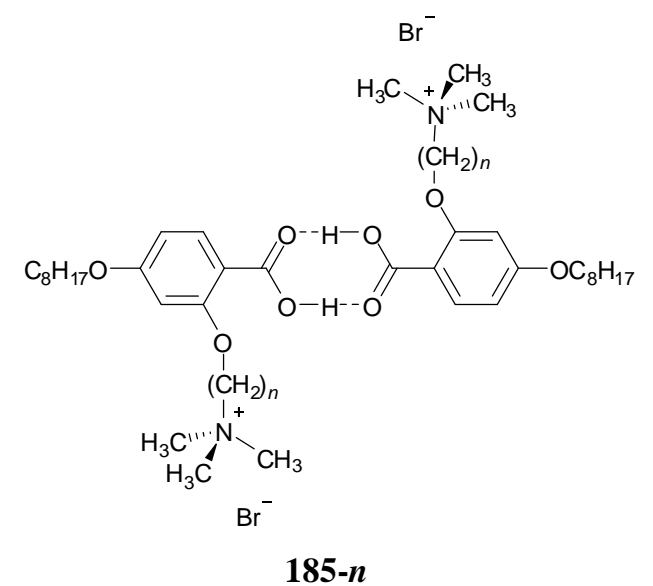

185-6: $\mathrm{g} \cdot 1 \cdot \mathrm{X} \cdot 153 \cdot \mathrm{SmA} \cdot 164 \cdot \mathrm{I}\left({ }^{\circ} \mathrm{C}\right)$

185-8: $\mathrm{g} \cdot 24 \cdot \mathrm{SmA} \cdot 86 \cdot \mathrm{I}\left({ }^{\circ} \mathrm{C}\right)$

185-10: $\mathrm{Cr}_{1} \cdot 25 \cdot \mathrm{Cr}_{2} \cdot 157 \cdot \mathrm{I}\left({ }^{\circ} \mathrm{C}\right)$

185-12: g $\cdot 12 \cdot \mathrm{Cr} \cdot 83 \cdot \mathrm{I}\left({ }^{\circ} \mathrm{C}\right)$

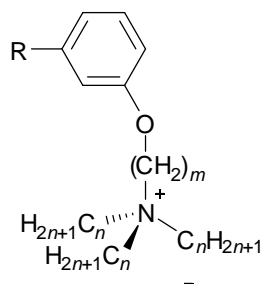

$\mathrm{Br}^{-}$

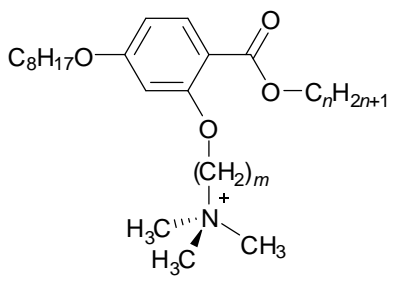

$\mathrm{Br}^{-}$

186-R-m/n

$187-m / n$

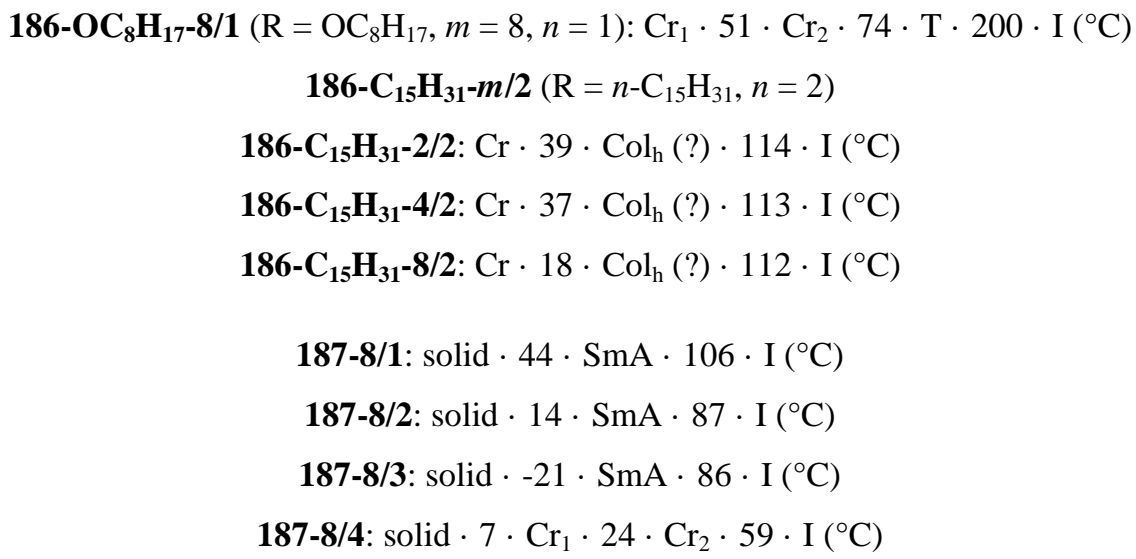

187-6/2: solid $\cdot$ [not reported] $\cdot \operatorname{SmA} \cdot \mathrm{ca} .75 \cdot \mathrm{I}\left({ }^{\circ} \mathrm{C}\right)$

187-10/2: solid $\cdot$ [not reported] $\cdot \mathrm{SmA} \cdot$ ca. $115 \cdot \mathrm{I}\left({ }^{\circ} \mathrm{C}\right)$

187-12/2: solid · [not reported] $\cdot \mathrm{SmA} \cdot$ ca. $135 \cdot \mathrm{I}\left({ }^{\circ} \mathrm{C}\right)$ 

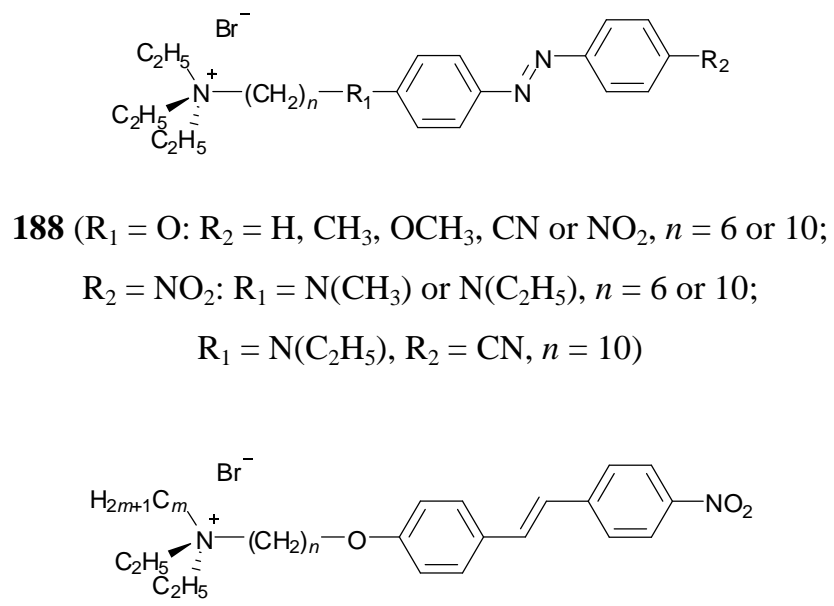

$189-m / n$

189-8/6: $\mathrm{Cr} \cdot(\mathrm{X} \cdot 46 \cdot \mathrm{SmX} \cdot 80 \cdot) 117 \cdot \mathrm{I}\left({ }^{\circ} \mathrm{C}\right)$

189-12/6: $\mathrm{Cr} \cdot 38 \cdot \mathrm{SmX} \cdot 120 \cdot \mathrm{I}\left({ }^{\circ} \mathrm{C}\right)$

189-12/10: $\mathrm{Cr} \cdot\left(\mathrm{X}_{1} \cdot 61 \cdot \mathrm{X}_{2} \cdot 82 \cdot\right) 88 \cdot \mathrm{SmX} \cdot 120\left({ }^{\circ} \mathrm{C}\right)$

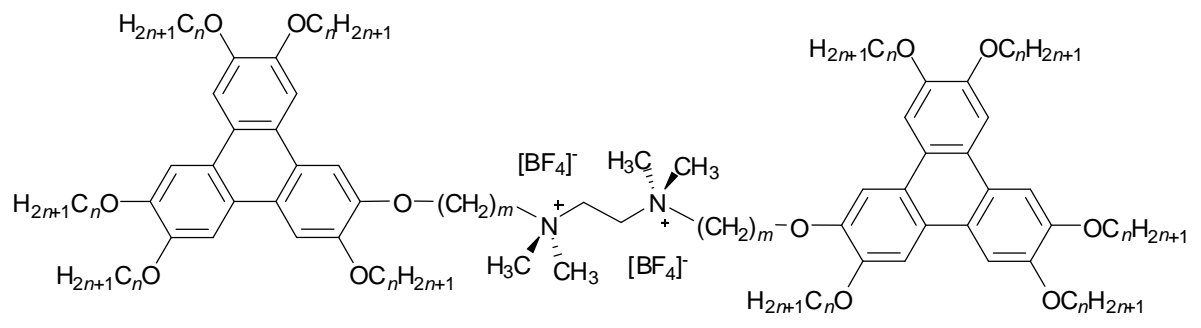

$190-m / n$

190-8/4: $\mathrm{Cr} \cdot 84 \cdot \mathrm{Col}_{\mathrm{h}}$ (monotropic?) $\cdot 90 \cdot \mathrm{I}\left({ }^{\circ} \mathrm{C}\right)$ (DSC peak temperatures)

190-12/6: $\mathrm{Cr} \cdot 52 \cdot \mathrm{Col}_{\mathrm{h}} \cdot 99 \cdot \mathrm{I}\left({ }^{\circ} \mathrm{C}\right)$ (DSC peak temperatures)
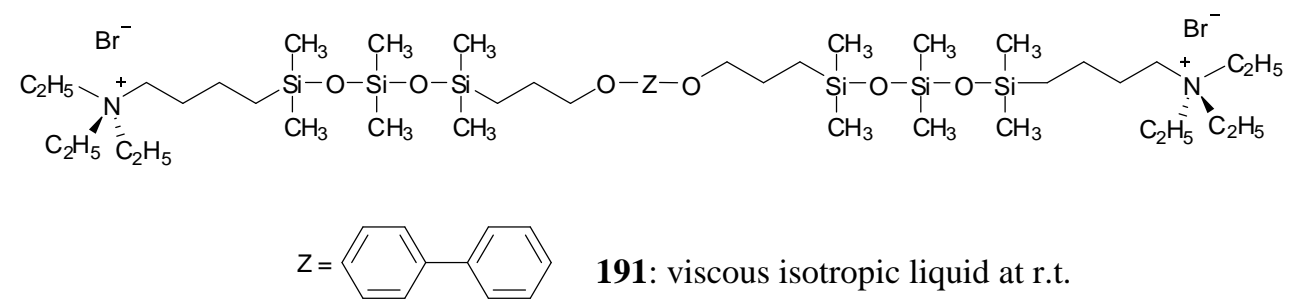

191: viscous isotropic liquid at r.t.

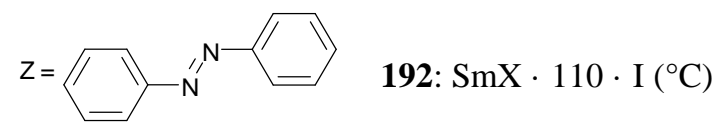



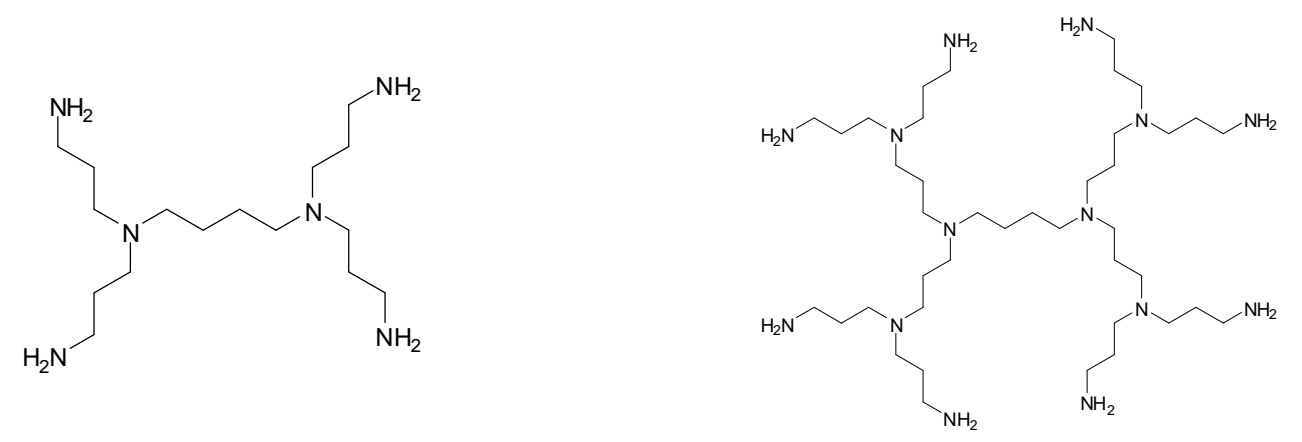

193 (PPI-G1)
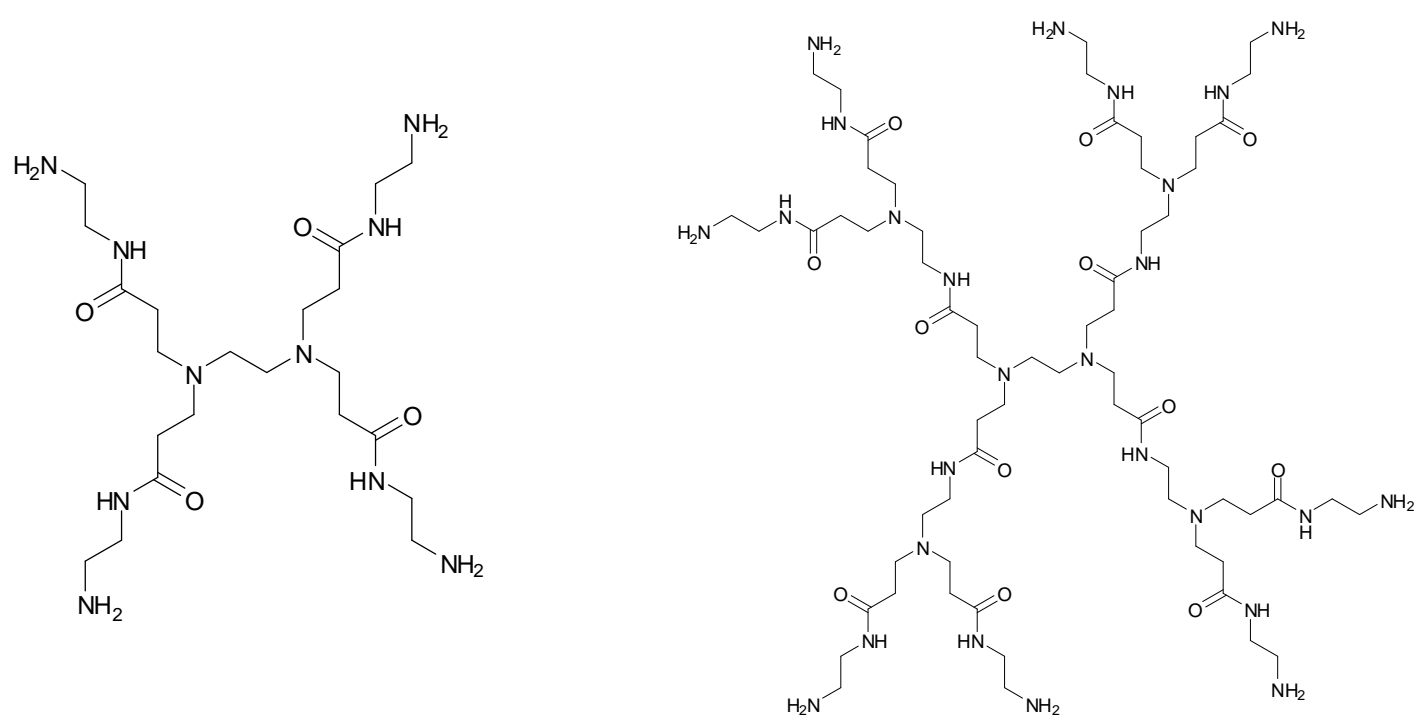

195 (PAMAM-G0)

196 (PAMAM-G1)

Remark: See Table S3 for the number of primary amino groups $\left(-\mathrm{NH}_{2}\right)$ and tertiary amino groups $\left(\mathrm{NR}_{3}\right)$ in the different generations of PPI and PAMAM dendrimers. 
Scheme S10. Carboxylic acid derivatives that were used by Serrano, Marcos, Barberá, Ros, Sánchez and co-workers, and by Sijbesma and co-workers, to make ionic complexes with PPI and PAMAM dendrimers of different generations (193, 194, 195, 196; see also Table S3).

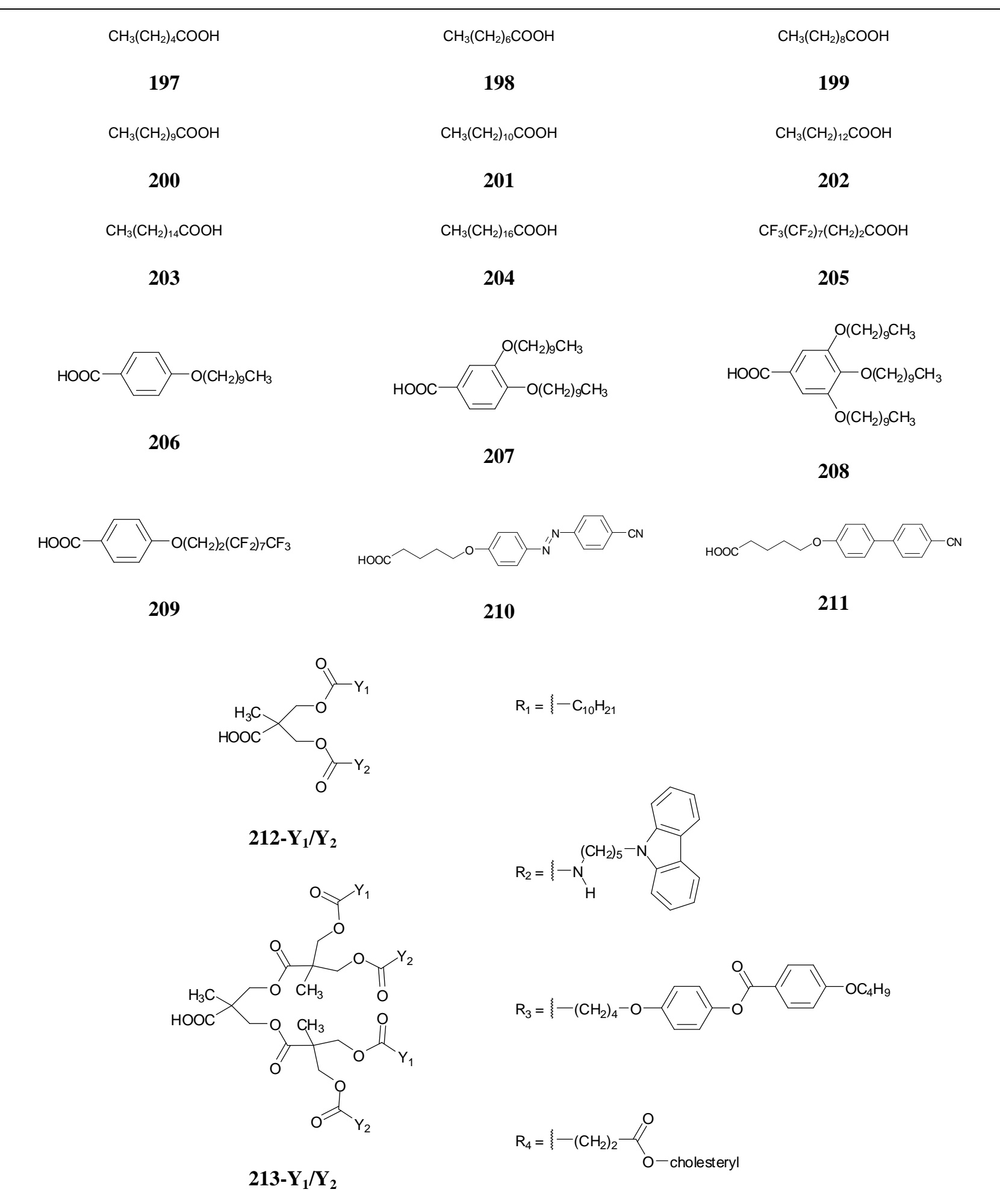




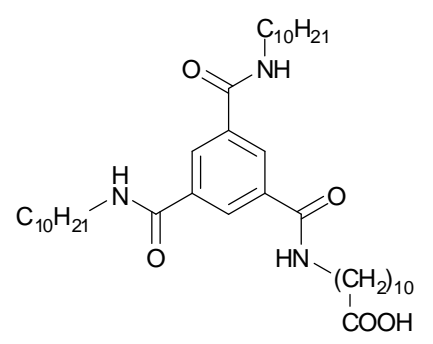

214

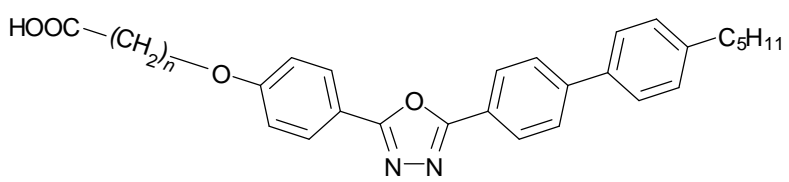

215-n

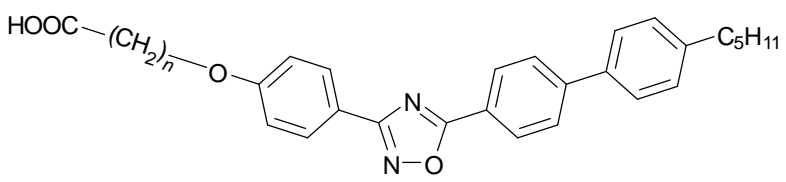

216-n

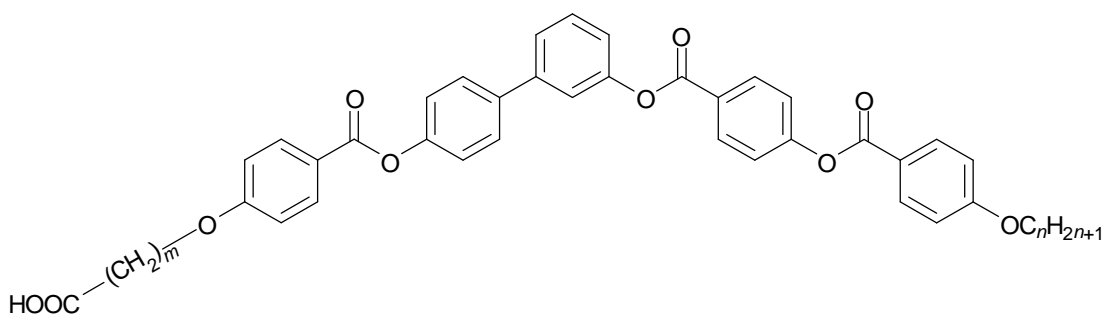

$217-m / n$

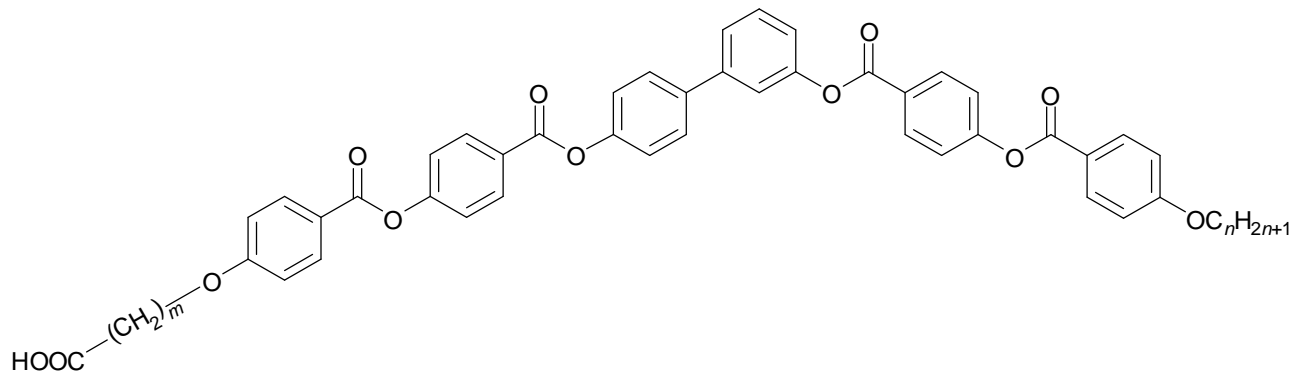

$218-m / n$

PPI-G1/(199) $)_{4}: \mathrm{SmA} \cdot 100 \cdot \mathrm{I}\left({ }^{\circ} \mathrm{C}\right)$

PPI-G1/(202) $)_{4}: \mathrm{Cr} \cdot 27 \cdot \mathrm{SmA} \cdot 116 \cdot \mathrm{I}\left({ }^{\circ} \mathrm{C}\right)$

PPI-G1/(204) $)_{4}: \mathrm{Cr}_{1} \cdot 60 \cdot \mathrm{Cr}_{2} \cdot 67 \cdot \mathrm{SmA} \cdot 116 \cdot \mathrm{I}\left({ }^{\circ} \mathrm{C}\right)$ 


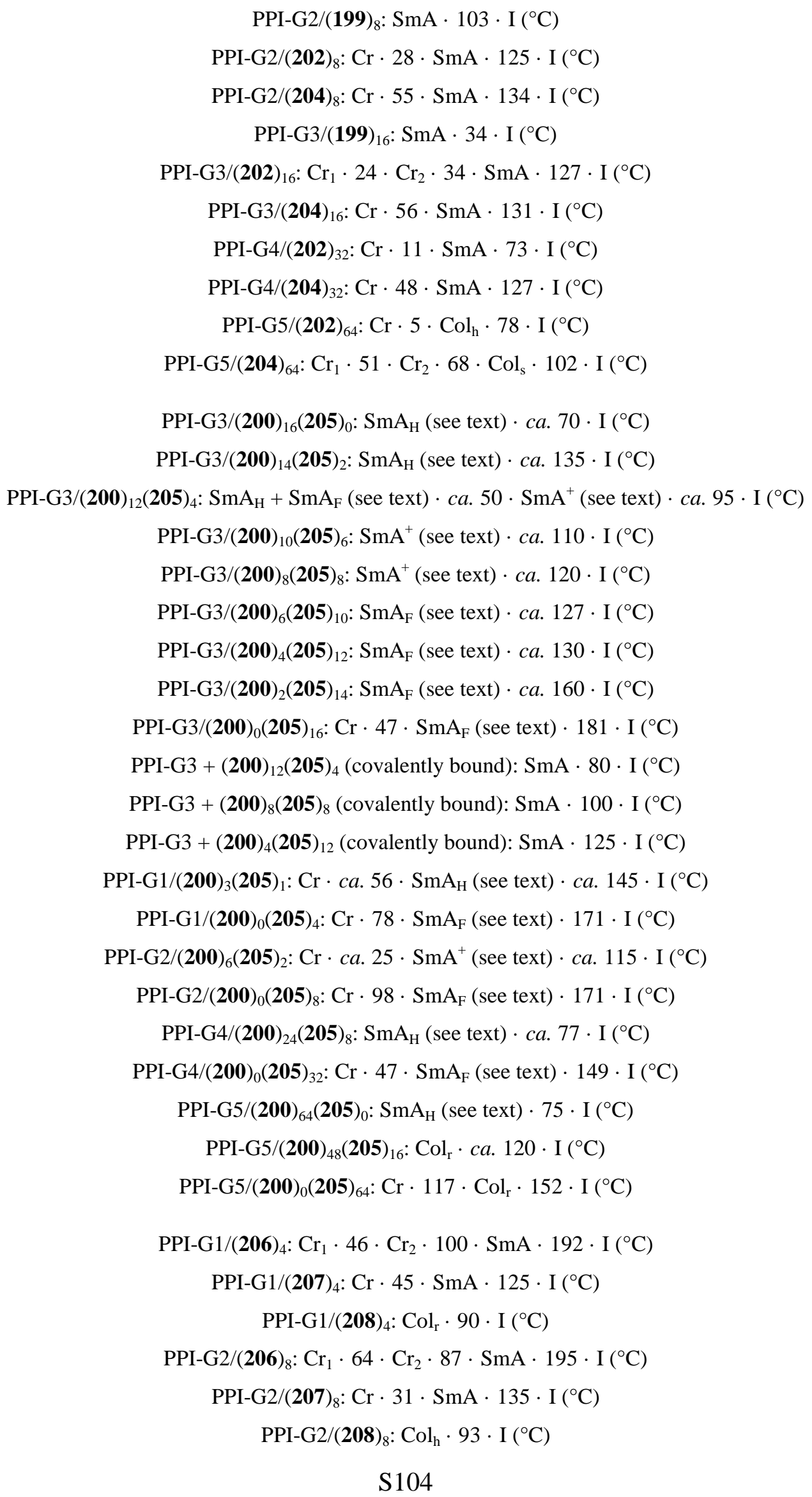




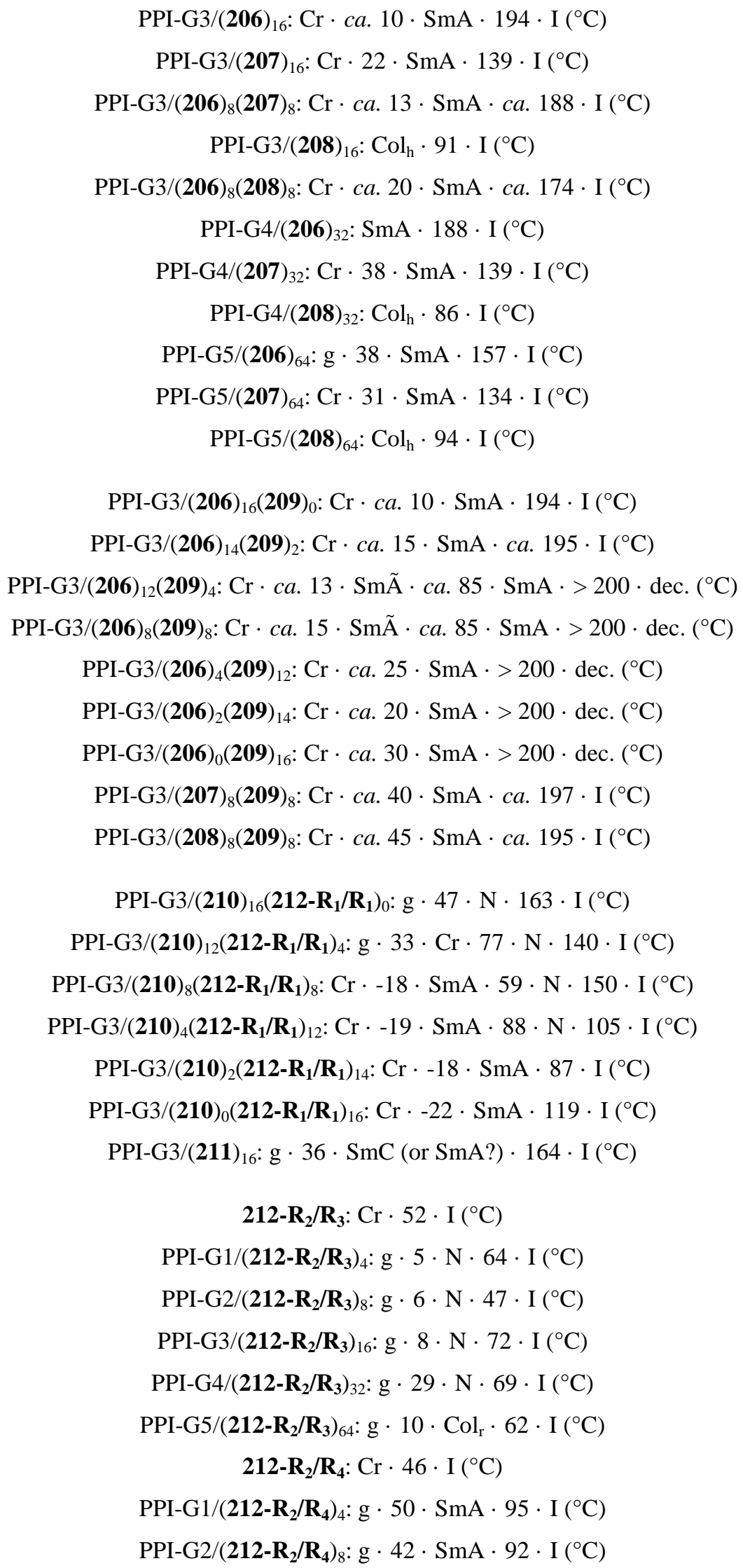




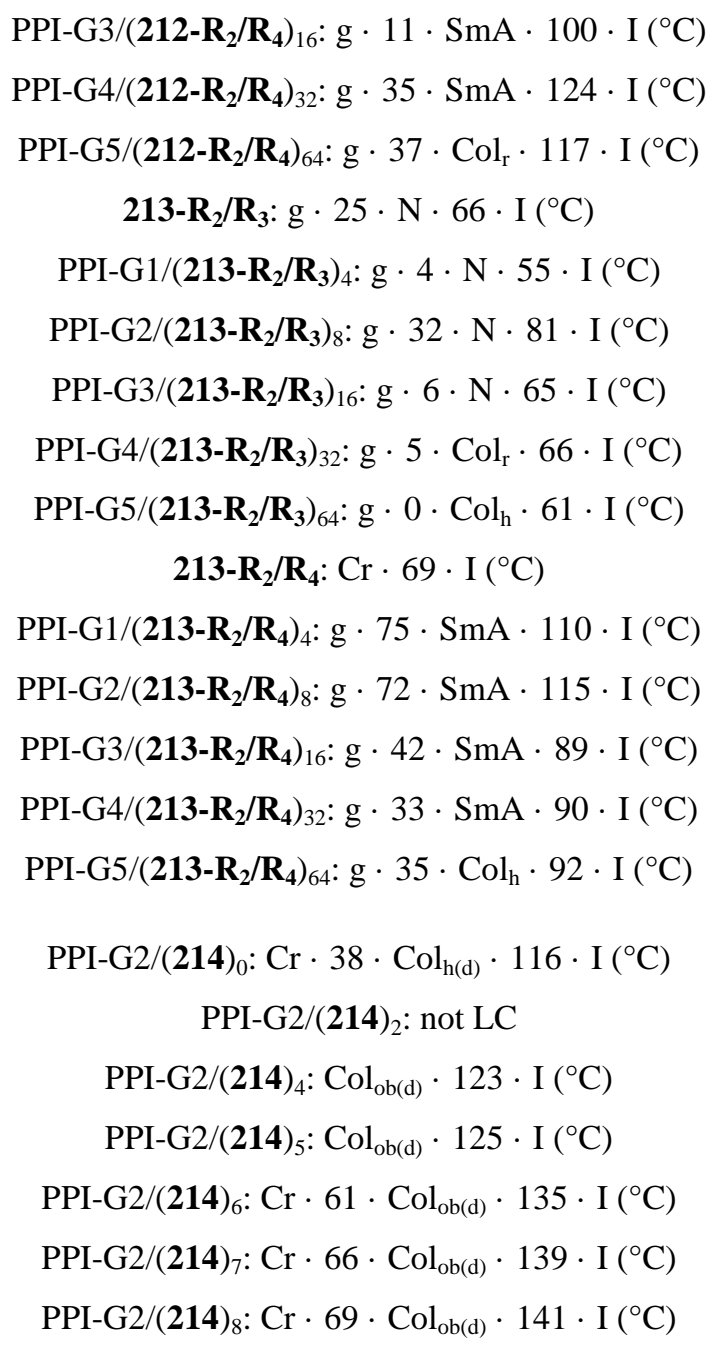

PPI-G2/(214) $)_{10}: \mathrm{Cr} \cdot 70 \cdot \mathrm{Col}\left(\mathrm{Col}_{\mathrm{ob}(\mathrm{d})}\right.$ with co-existing $\mathrm{Col}_{\mathrm{L}}$-type phase $) \cdot 114 \cdot \mathrm{Col}_{\mathrm{ob}(\mathrm{d})} \cdot 140 \cdot \mathrm{I}\left({ }^{\circ} \mathrm{C}\right)$ PPI-G2/(214) $)_{12}: \mathrm{Cr} \cdot 62 \cdot \mathrm{Col}\left(\mathrm{Col}_{\mathrm{ob}(\mathrm{d})}\right.$ with 2 co-existing $\mathrm{Col}_{\mathrm{L}}$-type phases) $\cdot 115 \cdot$

Col ('disordered' $\mathrm{Col}_{\mathrm{ob}(\mathrm{d})}$ ?) $\cdot 137 \cdot \mathrm{I}\left({ }^{\circ} \mathrm{C}\right)$

PPI-G2/(214) $)_{15}: \mathrm{Cr} \cdot 55 \cdot \mathrm{Col}\left(\mathrm{Col}_{\mathrm{ob}(\mathrm{d})}\right.$ with 2 co-existing $\mathrm{Col}_{\mathrm{L}}$-type phases $) \cdot 133 \cdot \mathrm{I}\left({ }^{\circ} \mathrm{C}\right)$

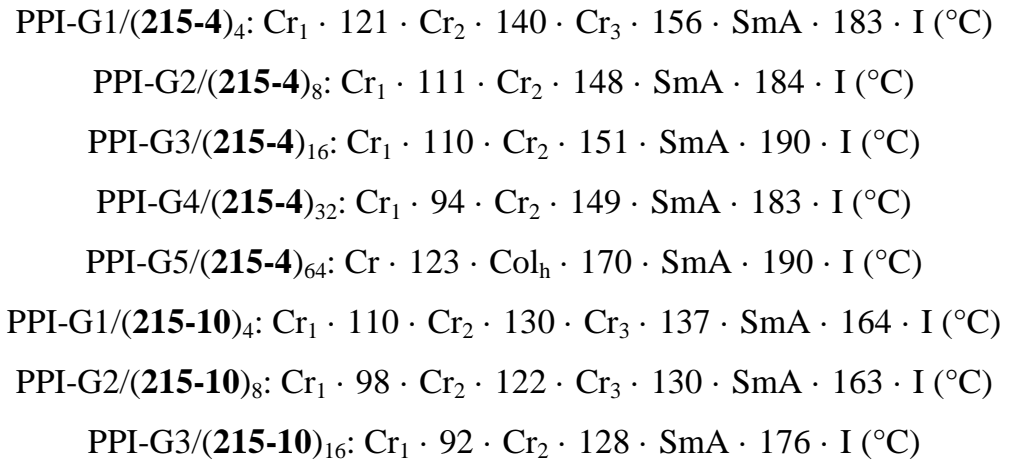




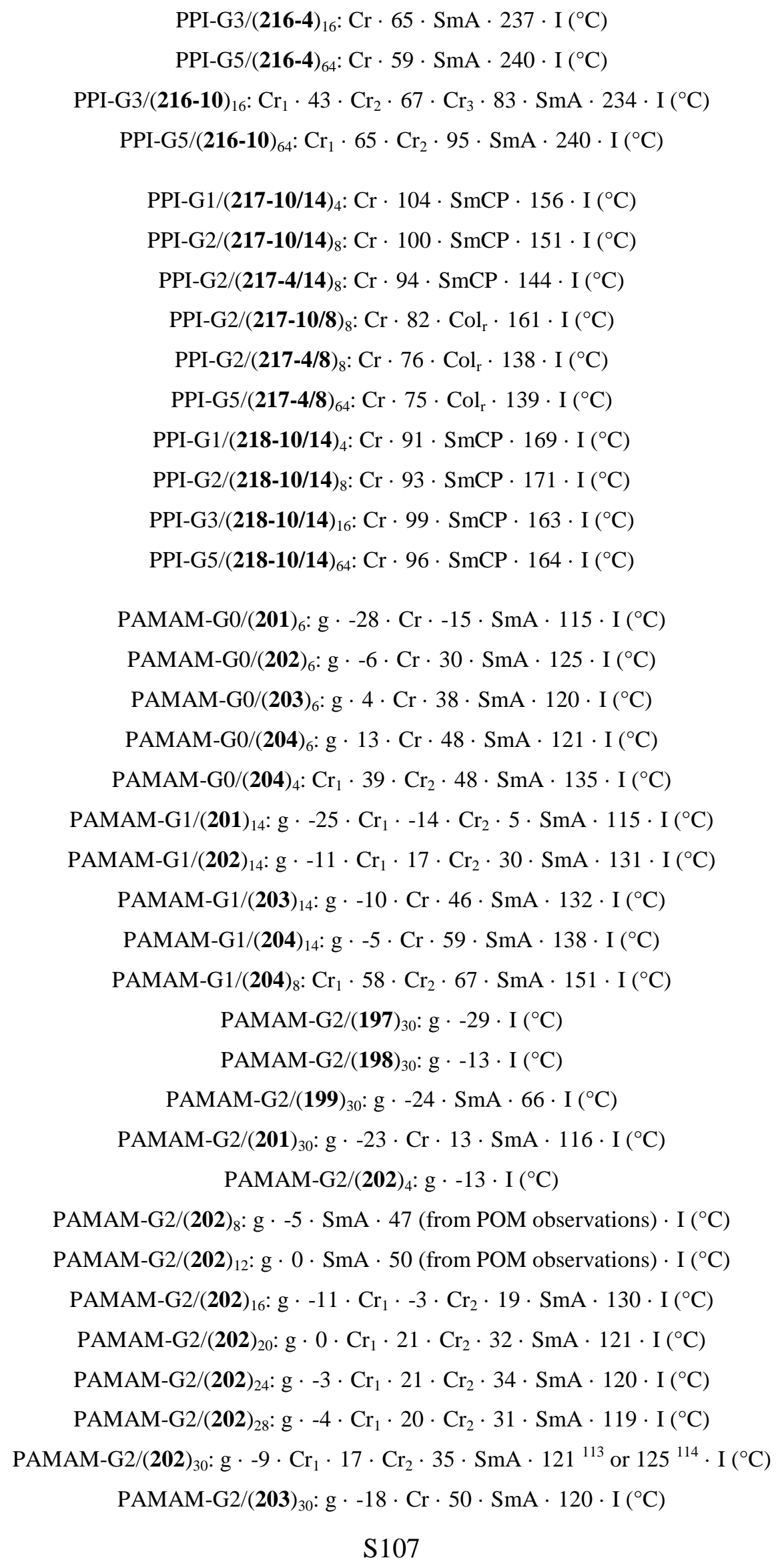




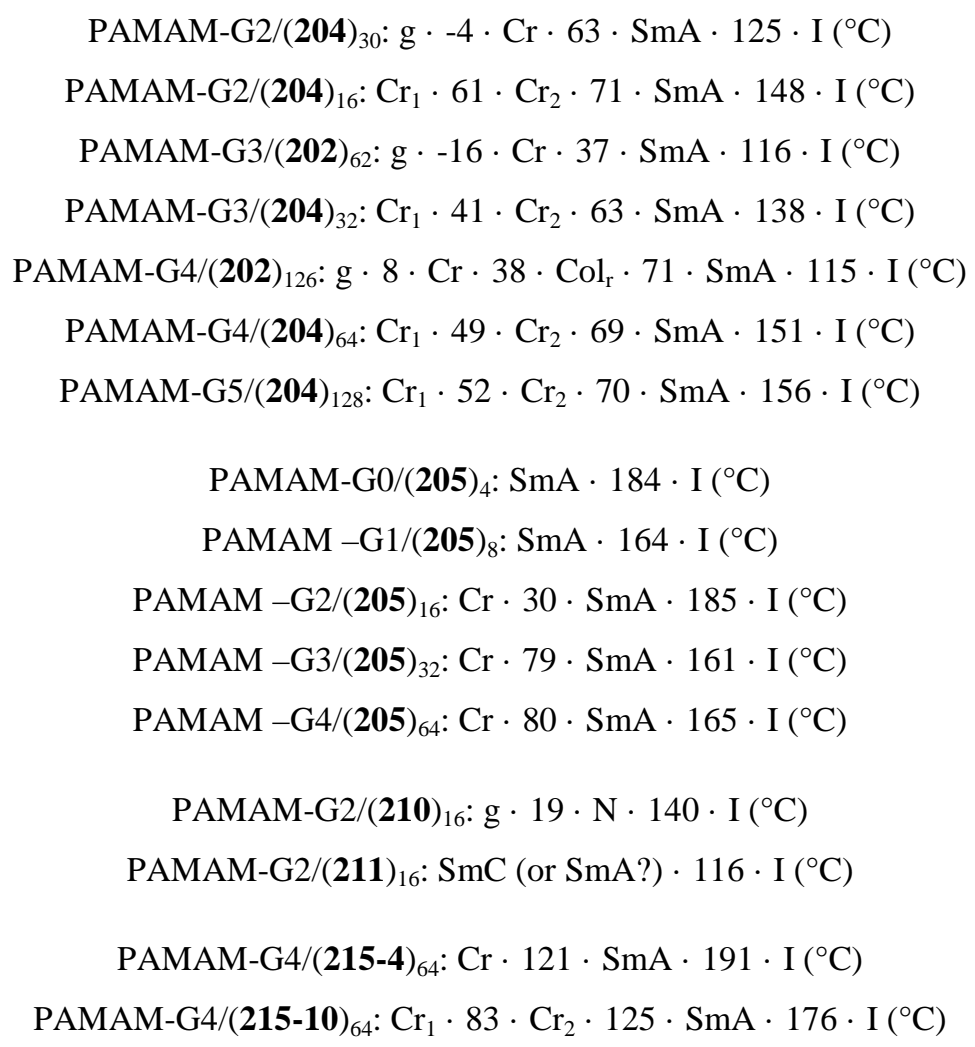


Scheme S11. Ammonium-based dendrons and dendrimers investigated by Mezzenga, Schlüter and co-workers.

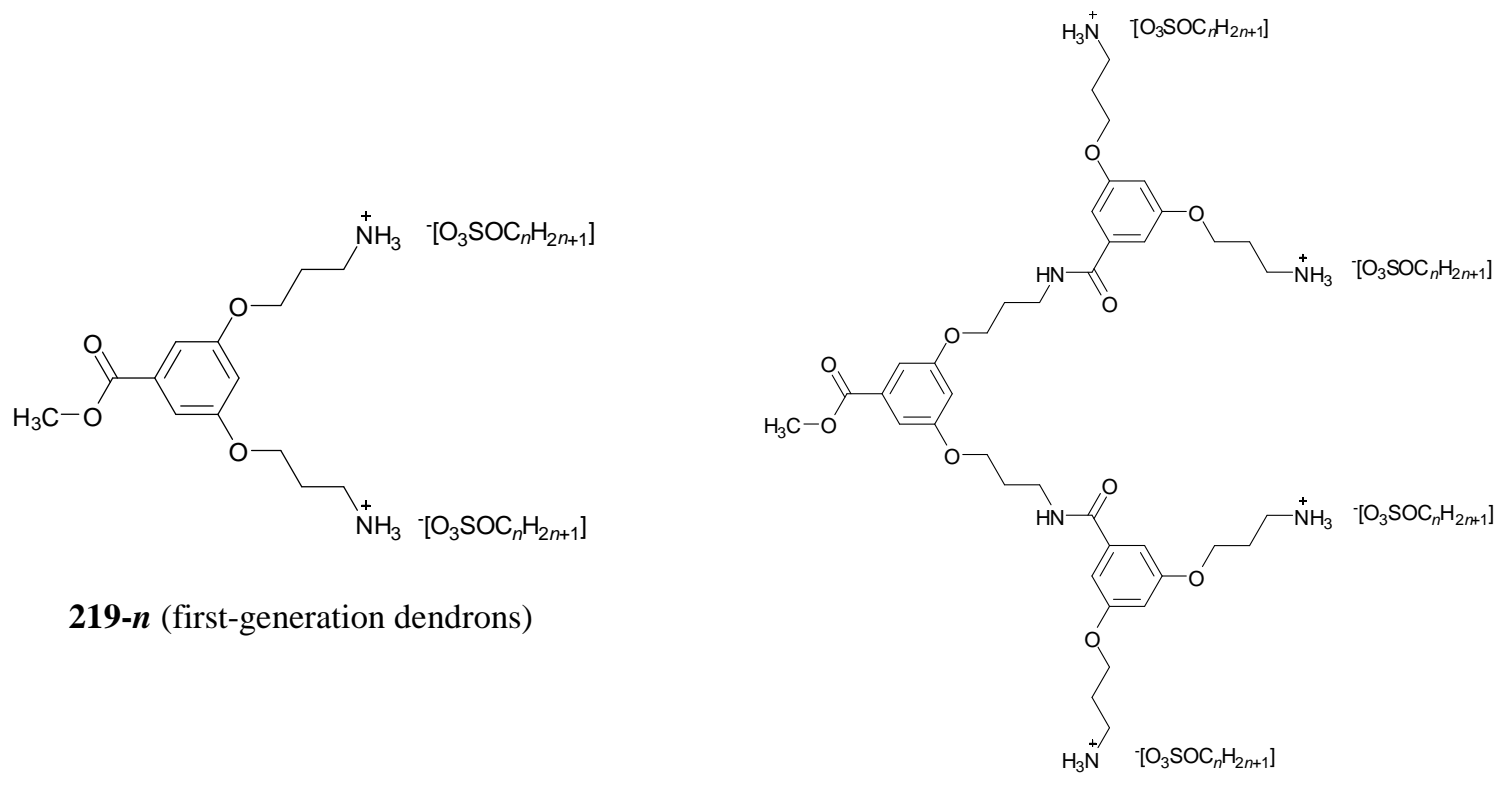

220-n (second-generation dendrons)

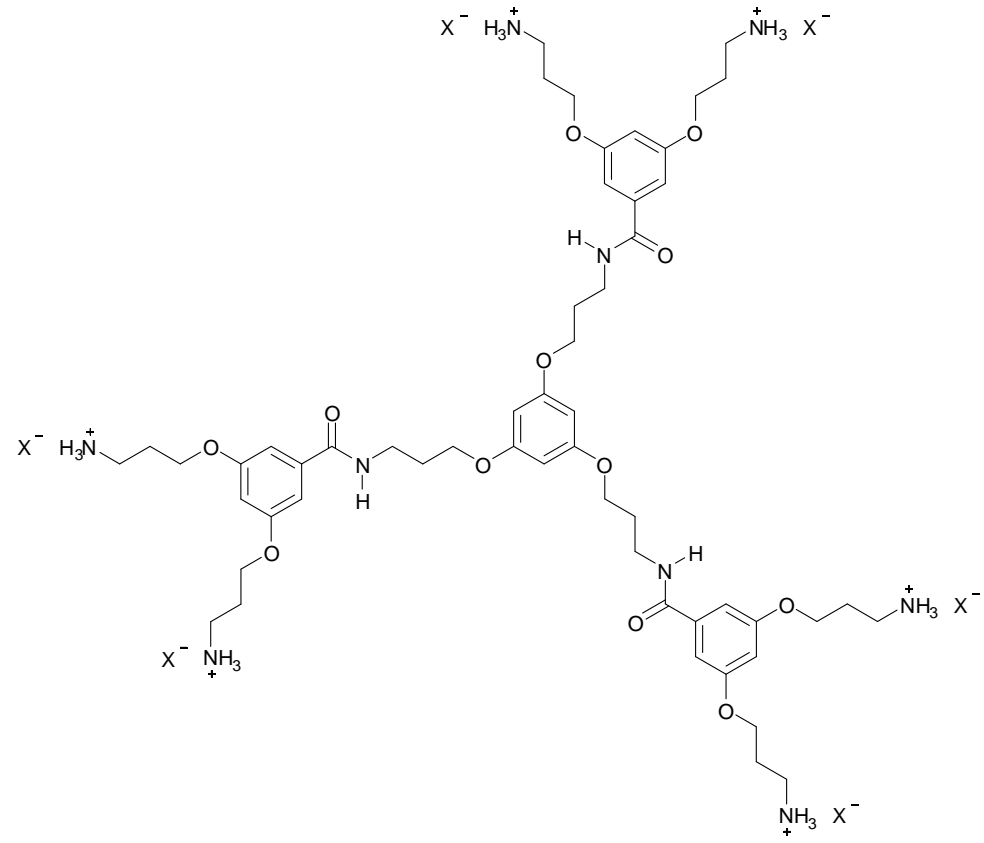

221-n ( $\left.\mathrm{X}^{-}=\left[\mathrm{C}_{n} \mathrm{H}_{2 n+1} \mathrm{OSO}_{3}\right]^{-}\right)$(first-generation dendrimers) 


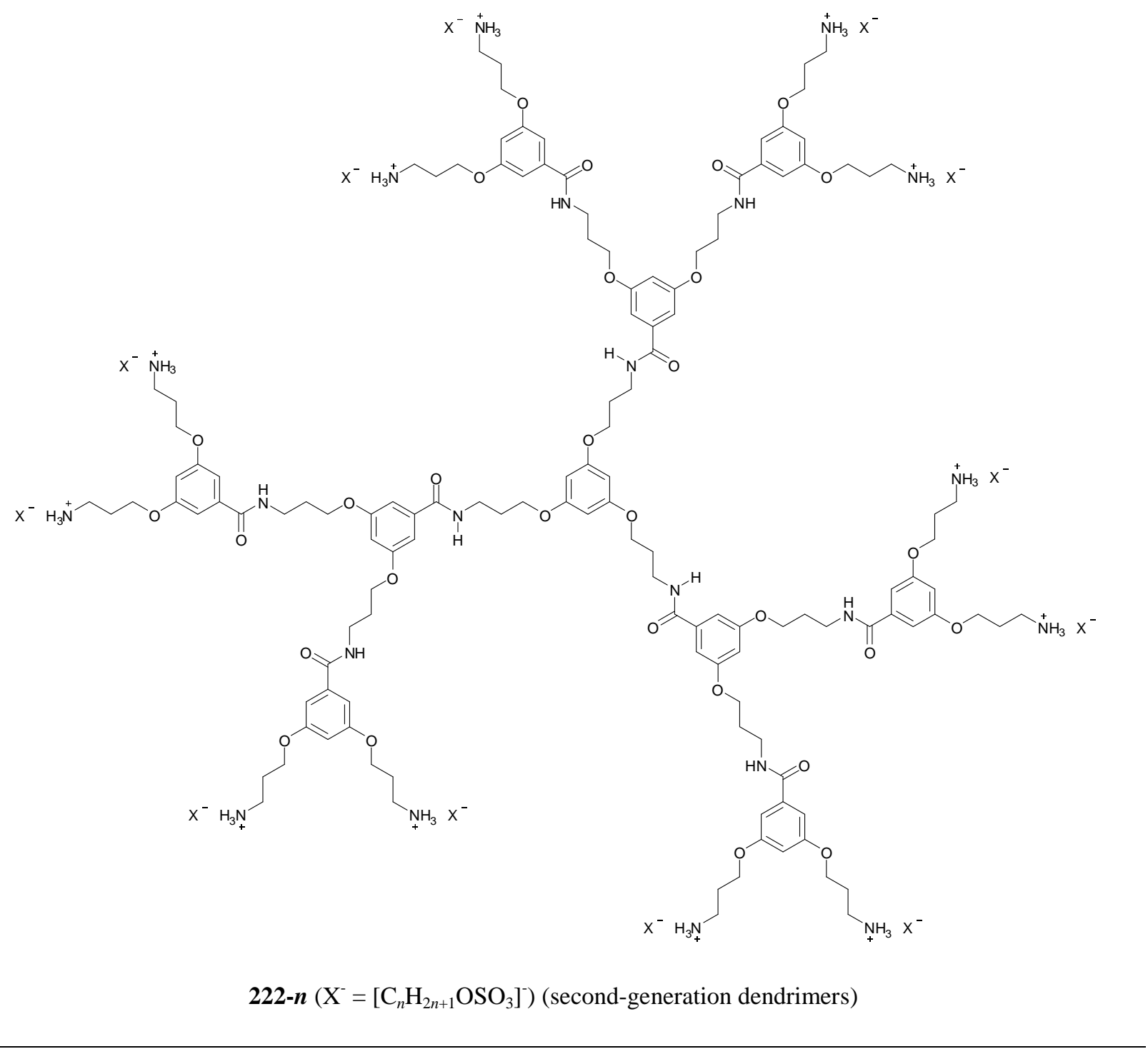

219-8: not LC

219-12: not LC

219-14: not LC

219-18: not LC

220-8: $\mathrm{Col}_{\mathrm{r}}$

220-12: $\mathrm{Col}_{h}$

220-14: $\mathrm{Col}_{\mathrm{h}}$

220-18: lamellar mesophase

221-8: not LC

221-12: $\mathrm{Col}_{h}$ 
221-14: $\mathrm{Col}_{\mathrm{h}}$

221-18: lamellar mesophase

222-8: not LC

222-12: $\mathrm{Col}_{\mathrm{h}}$

222-14: $\mathrm{Col}_{h}$

222-18: lamellar mesophase

Scheme S12. Ammonium-based structures studied by Klein Gebbink, van Koten and co-workers.

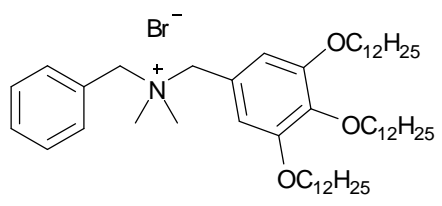

223

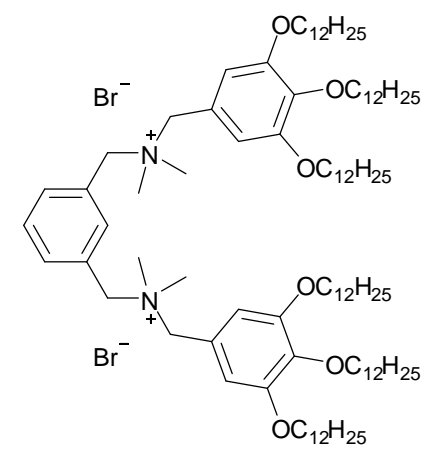

224

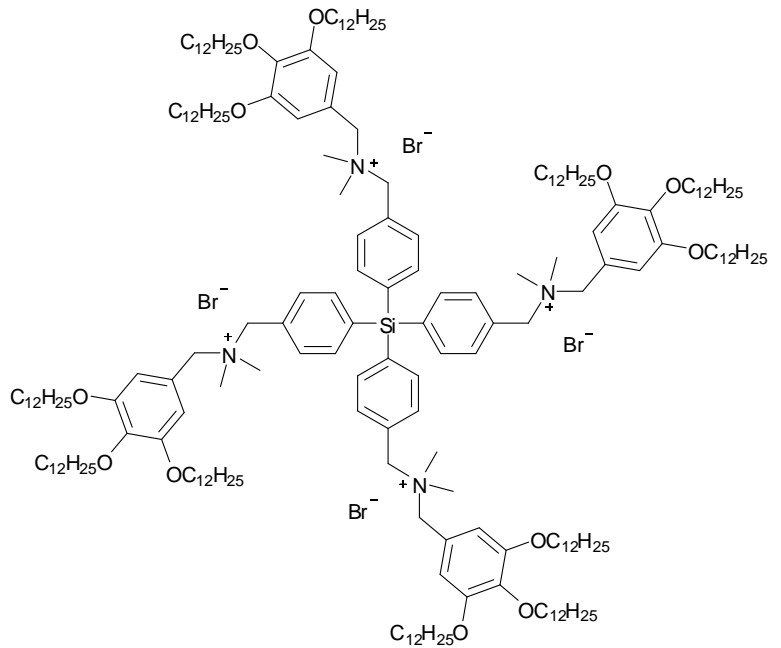

223: $\mathrm{M} \cdot 137 \cdot \mathrm{I}\left({ }^{\circ} \mathrm{C}\right)$

224: $\mathrm{M} \cdot 175 \cdot \operatorname{dec}\left({ }^{\circ} \mathrm{C}\right)$

225: M $\cdot 176 \cdot$ dec. $\left({ }^{\circ} \mathrm{C}\right)$

226: $\mathrm{M} \cdot 170 \cdot$ dec. $\left({ }^{\circ} \mathrm{C}\right)$ 


\subsection{Phosphonium-based ionic liquid crystals}

Scheme S13. Phosphonium-based ILCs investigated by Weiss and co-workers.

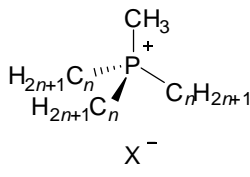

$$
\begin{gathered}
\text { 227-X-n }\left(\mathrm{X}^{-}=\mathrm{Cl}^{-}, \mathrm{Br}^{-}, \mathrm{I}^{-},\right. \\
{\left[\mathrm{NO}_{3}\right]^{-},\left[\mathrm{BF}_{4}\right]^{-},\left[\mathrm{PF}_{6}\right]^{-},} \\
\left.\left[\mathrm{NTf}_{2}\right]^{-},\left[\mathrm{ClO}_{4}\right]^{-}\right)
\end{gathered}
$$

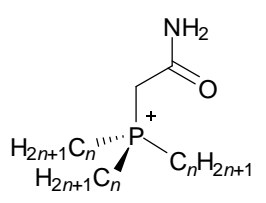

$\mathrm{Br}^{-}$

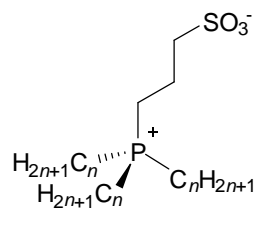

231-n

229-n
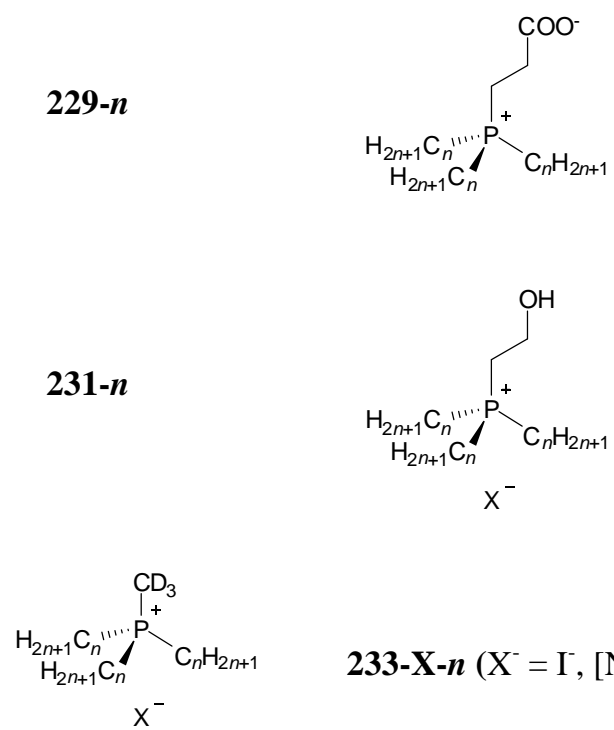

233-X-n $\left(\mathrm{X}^{-}=\mathrm{I}^{-},\left[\mathrm{NO}_{3}\right]^{-}\right)$
228-X-n $\left(\mathrm{X}^{-}=\mathrm{Cl}^{-}, \mathrm{Br}^{-}\right.$,

$\left.\left[\mathrm{PF}_{6}\right]^{-},\left[\mathrm{ClO}_{4}\right]^{-}\right)$

230-n

232-X-n $\left(\mathrm{X}^{-}=\mathrm{F}^{-}, \mathrm{Cl}^{-}, \mathrm{Br}^{-}\right.$,

$\left[\mathrm{NO}_{3}\right]^{-},\left[\mathrm{BF}_{4}\right]^{-},\left[\mathrm{NTf}_{2}\right]^{-}$,

$\left.\left[\mathrm{ClO}_{4}\right]^{-}\right)$

$$
\text { 227-Cl-n }\left(\mathrm{X}^{-}=\mathrm{Cl}^{-}\right)
$$

227-Cl-10: $\mathrm{Cr}$ (soft solid) $\cdot 103 \cdot \mathrm{I}\left({ }^{\circ} \mathrm{C}\right)\left(2^{\text {nd }} \mathrm{DSC}\right.$ heating run $)$

227-Cl-10 $\cdot \mathrm{H}_{2} \mathrm{O}: \mathrm{Cr}_{1} \cdot-34 \cdot \mathrm{Cr}_{2} \cdot(-2)-4$ (from POM observations) $\cdot \mathrm{SmA}_{2} \cdot 35 \cdot \mathrm{I}\left({ }^{\circ} \mathrm{C}\right)\left(1^{\text {st }} \mathrm{DSC} \text { cooling run }\right)^{72} / \mathrm{Cr}_{1} \cdot$ -

$$
17 \cdot \mathrm{Cr}_{2} \cdot 8 \cdot \mathrm{SmA}_{2} \cdot 103 \cdot \mathrm{I}\left({ }^{\circ} \mathrm{C}\right)\left(2^{\text {nd }} \text { DSC heating run }\right)^{115}
$$

227-Cl-10 $\mathrm{CH}_{3} \mathrm{OH}: \mathrm{Cr}_{1} \cdot-52 \cdot \mathrm{Cr}_{2} \cdot(-27)-(-17)$ (from $\mathrm{POM}$ observations) $\cdot \mathrm{SmA}_{2} \cdot 33 \cdot \mathrm{I}\left({ }^{\circ} \mathrm{C}\right)\left(1^{\text {st }} \mathrm{DSC}\right.$ cooling run)

227-Cl-10 $\mathrm{C}_{2} \mathrm{H}_{5} \mathrm{OH}: \mathrm{Cr}_{1} \cdot-63 \cdot \mathrm{Cr}_{2} \cdot(-25)-(-18)$ (from $\mathrm{POM}$ observations) $\cdot \mathrm{SmA}_{2} \cdot 14 \cdot \mathrm{I}\left({ }^{\circ} \mathrm{C}\right)\left(1^{\text {st }} \mathrm{DSC}\right.$ cooling run)

227-Cl-10 $\left(n-\mathrm{C}_{3} \mathrm{H}_{7} \mathrm{OH}\right): \mathrm{Cr}_{1} \cdot-61 \cdot \mathrm{Cr}_{2} \cdot(-28)-(-20)$ (from POM observations) $\cdot \mathrm{SmA}_{2}$.

$13 \cdot \mathrm{I}\left({ }^{\circ} \mathrm{C}\right)\left(1^{\text {st }} \mathrm{DSC}\right.$ cooling run $)$

227-Cl-10 $\cdot\left(n-\mathrm{C}_{4} \mathrm{H}_{9} \mathrm{OH}\right): \mathrm{Cr}_{1} \cdot-46 \cdot \mathrm{Cr}_{2} \cdot-20 \cdot \mathrm{SmA}_{2} \cdot 8 \cdot \mathrm{I}\left({ }^{\circ} \mathrm{C}\right)\left(1^{\text {st }} \mathrm{DSC}\right.$ cooling run $)$

[remark: See ref. 72 for more combinations of 227-Cl-10 with water and alcohols.]

227-Cl-14: $\mathrm{Cr}_{1}$ (soft solid) $\cdot 56 \cdot \mathrm{Cr}_{2}$ (soft solid) $\cdot 106 \cdot \mathrm{I}\left({ }^{\circ} \mathrm{C}\right)\left(2^{\text {nd }}\right.$ DSC heating run)

227-Cl-18: $\mathrm{Cr}_{1}$ (soft solid) $\cdot 86 \cdot \mathrm{Cr}_{2}$ (soft solid) $\cdot 101 \cdot \mathrm{I}\left({ }^{\circ} \mathrm{C}\right)\left(2^{\text {nd }}\right.$ DSC heating run)

[remark: See ref. 116 for equimolar mixtures of 227-Cl-14 and 227-Cl-18 with methanol.]

A phase diagram for mixtures of 227-Cl-10 with methanol is also displayed in Figure S14. 
227-Br-10: $\mathrm{Cr}$ (soft solid) $\cdot 98 \cdot \mathrm{I}\left({ }^{\circ} \mathrm{C}\right)\left(2^{\text {nd }}\right.$ DSC heating run)

227-Br-10 $\mathrm{H}_{2} \mathrm{O}: \mathrm{Cr}_{1} \cdot-36 \cdot \mathrm{Cr}_{2} \cdot(-13)-(-5)$ (from POM observations) $\cdot \mathrm{SmA}_{2} \cdot 57 \cdot \mathrm{I}\left({ }^{\circ} \mathrm{C}\right)\left(1^{\text {st }} \mathrm{DSC} \text { cooling run }\right)^{72} /$

$$
\mathrm{Cr}_{1} \cdot-9 \cdot \mathrm{Cr}_{2} \cdot-4 \cdot \mathrm{SmA}_{2} \cdot 95 \cdot \mathrm{I}\left({ }^{\circ} \mathrm{C}\right)\left(2^{\text {nd }} \mathrm{DSC} \text { heating run }\right)^{115}
$$

227-Br-10 $\mathrm{CH}_{3} \mathrm{OH}: \mathrm{Cr}_{1} \cdot-62 \cdot \mathrm{Cr}_{2} \cdot(-22)-(-15)$ (from $\mathrm{POM}$ observations) $\cdot \mathrm{SmA}_{2} \cdot 40 \cdot \mathrm{I}\left({ }^{\circ} \mathrm{C}\right)\left(1^{\text {st }} \mathrm{DSC}\right.$ cooling run)

227-Br-10 $\cdot\left(\mathrm{CH}_{3} \mathrm{OH}\right)_{1.5}: \mathrm{Cr}_{1} \cdot-62 \cdot \mathrm{Cr}_{2} \cdot(-23)-(-16)$ (from $\mathrm{POM}$ observations) $\cdot \mathrm{SmA}_{2} \cdot$

$$
35 \cdot \mathrm{I}\left({ }^{\circ} \mathrm{C}\right)\left(1^{\text {st }} \mathrm{DSC} \text { cooling run }\right)
$$

227-Br-10 $\mathrm{C}_{2} \mathrm{H}_{5} \mathrm{OH}: \mathrm{Cr}_{1} \cdot-53 \cdot \mathrm{Cr}_{2} \cdot(-29)-(-21)$ (from POM observations) $\cdot \mathrm{SmA}_{2} \cdot 31 \cdot \mathrm{I}\left({ }^{\circ} \mathrm{C}\right)\left(1^{\text {st }} \mathrm{DSC}\right.$ cooling run)

227-Br-10 $\cdot\left(n-\mathrm{C}_{3} \mathrm{H}_{7} \mathrm{OH}\right): \mathrm{Cr}_{1} \cdot-47 \cdot \mathrm{Cr}_{2} \cdot(-38)-(-29)$ (from POM observations) $\cdot \mathrm{SmA}_{2} \cdot$

$24 \cdot \mathrm{I}\left({ }^{\circ} \mathrm{C}\right)\left(1^{\text {st }} \mathrm{DSC}\right.$ cooling run $)$

227-Br-10 $\left(n-\mathrm{C}_{4} \mathrm{H}_{9} \mathrm{OH}\right): \mathrm{Cr}_{1} \cdot-50 \cdot \mathrm{Cr}_{2} \cdot-10 \cdot \mathrm{SmA}_{2} \cdot 22 \cdot \mathrm{I}\left({ }^{\circ} \mathrm{C}\right)\left(1^{\text {st }} \mathrm{DSC}\right.$ cooling run $)$

227-Br-10 $\cdot\left(n-\mathrm{C}_{5} \mathrm{H}_{11} \mathrm{OH}\right): \mathrm{Cr}_{1} \cdot-48 \cdot \mathrm{Cr}_{2} \cdot-8 \cdot \mathrm{SmA}_{2} \cdot 14 \cdot \mathrm{I}\left({ }^{\circ} \mathrm{C}\right)\left(1^{\text {st }}\right.$ DSC cooling run $)$

227-Br-10 $\cdot\left(n-\mathrm{C}_{6} \mathrm{H}_{13} \mathrm{OH}\right): \mathrm{Cr}_{1} \cdot-51 \cdot \mathrm{Cr}_{2} \cdot 6 \cdot \mathrm{SmA}_{2} \cdot 33 \cdot \mathrm{I}\left({ }^{\circ} \mathrm{C}\right)\left(1^{\text {st }} \mathrm{DSC}\right.$ cooling run $)$

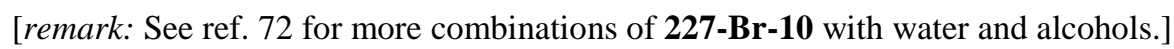

227-Br-14: $\mathrm{Cr}_{1} \cdot 50 \cdot \mathrm{Cr}_{2} \cdot 103 \cdot \mathrm{SmA}_{2} \cdot 119 \cdot \mathrm{I}\left({ }^{\circ} \mathrm{C}\right)\left(2^{\text {nd }}\right.$ DSC heating run $)$

227-Br-18: $\mathrm{Cr}_{1} \cdot 76 \cdot \mathrm{Cr}_{2} \cdot 99 \cdot \mathrm{SmA}_{2} \cdot 106 \cdot \mathrm{I}\left({ }^{\circ} \mathrm{C}\right)\left(2^{\text {nd }}\right.$ DSC heating run $)$

[remark: See ref. 116 for equimolar mixtures of 227-Br-14 and 227-Br-18 with methanol.]

Phase diagrams for mixtures of 227-Br-10 with methanol or ethylene glycol are also displayed in Figure S14.

$$
\text { 227-I-n }\left(\mathrm{X}^{-}=\mathrm{I}^{-}\right)
$$

227-I-10: $\mathrm{Cr}_{1} \cdot 40 \cdot \mathrm{Cr}_{2} \cdot 61 \cdot \mathrm{I}\left({ }^{\circ} \mathrm{C}\right)\left(1^{\text {st }} \mathrm{DSC}\right.$ heating run $)$

227-I-18: $\mathrm{Cr} \cdot 87 \cdot \mathrm{SmA}_{2} \cdot 113 \cdot \mathrm{I}\left({ }^{\circ} \mathrm{C}\right)\left(2^{\text {nd }}\right.$ DSC heating run $)$

$$
\text { 227- } \mathrm{NO}_{3}-\boldsymbol{n}\left(\mathrm{X}^{-}=\left[\mathrm{NO}_{3}\right]^{-}\right)
$$

227- $\mathrm{NO}_{3}-10: \mathrm{Cr}_{1} \cdot-10 \cdot \mathrm{Cr}_{2} \cdot 60 \cdot \mathrm{SmA}_{2} \cdot 83 \cdot \mathrm{I}\left({ }^{\circ} \mathrm{C}\right)\left(2^{\text {nd }}\right.$ DSC heating run $)$

227- $\mathrm{NO}_{3}-\mathbf{1 4}: \mathrm{Cr}_{1} \cdot 22 \cdot \mathrm{Cr}_{2} \cdot 49 \cdot \mathrm{SmA}_{2} \cdot 107 \cdot \mathrm{I}\left({ }^{\circ} \mathrm{C}\right)\left(2^{\text {nd }}\right.$ DSC heating run $)$

227- $\mathrm{NO}_{3}-\mathbf{- 1 8}: \mathrm{Cr}_{1} \cdot[$ not reported $] \cdot \mathrm{Cr}_{2} \cdot 66 \cdot \mathrm{SmA}_{2} \cdot 106 \cdot \mathrm{I}\left({ }^{\circ} \mathrm{C}\right)\left(2^{\text {nd }}\right.$ DSC heating run $)$

$$
\text { 227- } \mathbf{B F}_{4}-\boldsymbol{n}\left(\mathrm{X}^{-}=\left[\mathrm{BF}_{4}\right]^{-}\right)
$$

227-BF $4-10: \mathrm{Cr} \cdot 23 \cdot \mathrm{SmA}_{2} \cdot 45 \cdot \mathrm{I}\left({ }^{\circ} \mathrm{C}\right)\left(2^{\text {nd }}\right.$ DSC heating run $)$

227-BF $-14: \mathrm{Cr}_{1} \cdot 33 \cdot \mathrm{Cr}_{2} \cdot 56 \cdot \mathrm{SmA}_{2} \cdot 99 \cdot \mathrm{I}\left({ }^{\circ} \mathrm{C}\right)\left(2^{\text {nd }}\right.$ DSC heating run $)$

227-BF 4 -18: $\mathrm{Cr} \cdot 68 \cdot \mathrm{SmA}_{2} \cdot 105 \cdot \mathrm{I}\left({ }^{\circ} \mathrm{C}\right)\left(2^{\text {nd }}\right.$ DSC heating run $)$

$$
\text { 227-PF }-\mathbf{n}\left(\mathrm{X}^{-}=\left[\mathrm{PF}_{6}\right]^{-}\right)
$$

227-PF $\mathbf{F}_{6}-10: \mathrm{Cr} \cdot 48 \cdot \mathrm{I}\left({ }^{\circ} \mathrm{C}\right)\left(2^{\text {nd }}\right.$ DSC heating run $)$

227-PF $\mathbf{H}_{6}-\mathbf{1 4}: \mathrm{Cr} \cdot 47 \cdot \mathrm{SmA}_{2} \cdot 83 \cdot \mathrm{I}\left({ }^{\circ} \mathrm{C}\right)\left(2^{\text {nd }}\right.$ DSC heating run $)$

227-PF -1 -18: $\mathrm{Cr} \cdot 80 \cdot \mathrm{SmA}_{2} \cdot 96 \cdot \mathrm{I}\left({ }^{\circ} \mathrm{C}\right)\left(2^{\text {nd }}\right.$ DSC heating run $)$

$$
\text { 227-NTf } \mathbf{f}_{2}-\boldsymbol{n}\left(\mathrm{X}^{-}=\left[\mathrm{NTf}_{2}\right]^{-}\right)
$$

227-NTf $\mathbf{N}_{2}$-10: $\mathrm{Cr}_{1} \cdot-47 \cdot \mathrm{Cr}_{2} \cdot-29 \cdot \mathrm{Cr}_{3} \cdot-1 \cdot \mathrm{I}\left({ }^{\circ} \mathrm{C}\right)\left(1^{\text {st }}\right.$ DSC heating run $)$

227-NTf $\mathbf{2}_{2}$-14: $\mathrm{Cr} \cdot 46 \cdot \mathrm{I}\left({ }^{\circ} \mathrm{C}\right)\left(1^{\text {st }}\right.$ DSC heating run $)$

227-NTf ${ }_{2}$-18: $\mathrm{Cr} \cdot 67 \cdot \mathrm{I}\left({ }^{\circ} \mathrm{C}\right)\left(1^{\text {st }}\right.$ DSC heating run $)$ 


$$
\begin{gathered}
\text { 227-ClO }-\mathbf{n}\left(\mathrm{X}^{-}=\left[\mathrm{ClO}_{4}\right]^{-}\right) \\
\text {227-ClO }{ }_{4}-\mathbf{1 0}: \mathrm{Cr} \cdot 39 \cdot \mathrm{I}\left({ }^{\circ} \mathrm{C}\right)\left(2^{\text {nd }} \mathrm{DSC} \text { heating run }\right) \\
\text { 227- } \mathbf{C l O}_{4}-\mathbf{1 4}: \mathrm{Cr}_{1} \cdot 48 \cdot \mathrm{Cr}_{2} \cdot 58 \cdot \mathrm{SmA}_{2} \cdot 98 \cdot \mathrm{I}\left({ }^{\circ} \mathrm{C}\right)\left(2^{\text {nd }} \text { DSC heating run }\right) \\
\text { 227-ClO } \\
\text {-18: } \mathrm{Cr} \cdot 73 \cdot \mathrm{SmA}_{2} \cdot 105 \cdot \mathrm{I}\left({ }^{\circ} \mathrm{C}\right)\left(2^{\text {nd }} \text { DSC heating run }\right)
\end{gathered}
$$

[remark: See ref. 117 for the phase behavior of analogues of $P, P, P$-tris(n-alkyl)-P-methylphosphonium salts 227-X$\boldsymbol{n}$ with an ethyl, $n$-propyl, $n$-butyl, $n$-pentyl, $n$-dodecyl, $n$-octadecyl or benzyl substituent or a hydrogen atom instead of the methyl group. See ref. 118 for several mixtures of 227-X-n with (non-deuterated) alcohols, $\mathrm{CD}_{3} \mathrm{OD}$, (nondeuterated) acetonitrile, $\mathrm{CD}_{3} \mathrm{CN}$ and DMSO.]

$$
\begin{gathered}
\text { 228-Cl-n }\left(\mathrm{X}^{-}=\mathrm{Cl}^{-}\right) \\
\text {228-Cl-14: } \mathrm{Cr}_{1} \cdot 64 \cdot \mathrm{Cr}_{2} \cdot 99 \cdot \mathrm{I}\left({ }^{\circ} \mathrm{C}\right)\left(1^{\text {st }} \mathrm{DSC} \text { heating run }\right) \\
\text { 228-Cl-18: } \mathrm{Cr}_{1} \cdot 89 \cdot \mathrm{Cr}_{2} \cdot 98 \cdot \mathrm{I}\left({ }^{\circ} \mathrm{C}\right)\left(1^{\text {st }} \mathrm{DSC} \text { heating run }\right) \\
\text { 228-Br-n }\left(\mathrm{X}^{-}=\mathrm{Br}^{-}\right) \\
\text {228-Br-10: } \mathrm{Cr} \cdot 54 \cdot \mathrm{I}\left({ }^{\circ} \mathrm{C}\right)\left(1^{\text {st }} \mathrm{DSC} \text { heating run }\right) \\
\text { 228-Br-14: } \mathrm{Cr} \cdot 69 \cdot \mathrm{SmA}_{2} \cdot 88 \cdot \mathrm{I}\left({ }^{\circ} \mathrm{C}\right)\left(1^{\text {st }} \mathrm{DSC} \text { heating run }\right) \\
\text { 228-Br-18: } \mathrm{Cr}_{1} \cdot 68 \cdot \mathrm{Cr}_{2} \cdot 84 \cdot \mathrm{I}\left({ }^{\circ} \mathrm{C}\right)\left(1^{\text {st }} \mathrm{DSC} \text { heating run }\right)
\end{gathered}
$$

229-18: $\mathrm{Cr}_{1} \cdot\left[\right.$ not reported] $\cdot \mathrm{Cr}_{2} \cdot 61 \cdot \mathrm{I}\left({ }^{\circ} \mathrm{C}\right)\left(1^{\text {st }} \mathrm{DSC}\right.$ heating run $)$

\section{0-n}

230-6: $\mathrm{Cr} \cdot 161 \cdot \mathrm{I}\left({ }^{\circ} \mathrm{C}\right)\left(1^{\text {st }}\right.$ DSC heating run $)$

230-10: $\mathrm{Cr} \cdot 145 \cdot \mathrm{I}\left({ }^{\circ} \mathrm{C}\right)\left(1^{\text {st }} \mathrm{DSC}\right.$ heating run $)$

230-14: $\mathrm{Cr}_{1} \cdot 66 \cdot \mathrm{Cr}_{2} \cdot 131 \cdot \mathrm{I}\left({ }^{\circ} \mathrm{C}\right)\left(1^{\text {st }}\right.$ DSC heating run $)$

230-18: $\mathrm{Cr}_{1} \cdot[$ not reported $] \cdot \mathrm{Cr}_{2} \cdot 86 \cdot \mathrm{Cr}_{3} \cdot 120 \cdot \mathrm{I}\left({ }^{\circ} \mathrm{C}\right)\left(1^{\text {st }} \mathrm{DSC}\right.$ heating run $)$

231-n

231-10: $\mathrm{Cr}_{1} \cdot 45 \cdot \mathrm{Cr}_{2} \cdot 81 \cdot \mathrm{Cr}_{3} \cdot 148 \cdot \mathrm{I}\left({ }^{\circ} \mathrm{C}\right)\left(1^{\text {st }} \mathrm{DSC}\right.$ heating run $)$

231-14: $\mathrm{Cr}_{1} \cdot 47 \cdot \mathrm{Cr}_{2} \cdot 88 \cdot \mathrm{Cr}_{3} \cdot 133 \cdot \mathrm{I}\left({ }^{\circ} \mathrm{C}\right)\left(1^{\text {st }} \mathrm{DSC}\right.$ heating run $)$

231-18: $\mathrm{Cr}_{1} \cdot$ [not reported] $\cdot \mathrm{Cr}_{2} \cdot 100 \cdot \mathrm{Cr}_{3} \cdot 123 \cdot \mathrm{I}\left({ }^{\circ} \mathrm{C}\right)\left(1^{\text {st }}\right.$ DSC heating run $)$

$$
\text { 232-F-n }\left(\mathrm{X}^{-}=\mathrm{F}^{-}\right)
$$

232-F-14: $\mathrm{Cr} \cdot 30 \cdot \mathrm{I}\left({ }^{\circ} \mathrm{C}\right)\left(1^{\text {st }}\right.$ DSC heating run $)$

232-F-18: $\mathrm{Cr}_{1} \cdot 50 \cdot \mathrm{Cr}_{2} \cdot 58 \cdot \mathrm{Cr}_{3} \cdot 64 \cdot \mathrm{I}\left({ }^{\circ} \mathrm{C}\right)\left(1^{\text {st }} \mathrm{DSC}\right.$ heating run $)$

$$
\text { 232-Cl-n }\left(\mathrm{X}^{-}=\mathrm{Cl}^{-}\right)
$$

232-Cl-10: $\mathrm{Cr} \cdot 36 \cdot \mathrm{SmA}_{2} \cdot 75 \cdot \mathrm{I}\left({ }^{\circ} \mathrm{C}\right)\left(2^{\text {nd }}\right.$ DSC heating run $)$ 
232-Cl-14: $\mathrm{Cr}_{1} \cdot$ [not reported] $\cdot \mathrm{Cr}_{2} \cdot 59 \cdot \mathrm{SmA}_{2} \cdot 98 \cdot \mathrm{I}\left({ }^{\circ} \mathrm{C}\right)\left(2^{\text {nd }}\right.$ DSC heating run $)$

232-Cl-18: $\mathrm{Cr} \cdot 78 \cdot \mathrm{SmA}_{2} \cdot 94 \cdot \mathrm{I}\left({ }^{\circ} \mathrm{C}\right)\left(2^{\text {nd }} \mathrm{DSC}\right.$ heating run $)$

232-Br-n $\left(\mathrm{X}^{-}=\mathrm{Br}^{-}\right)$

232-Br-10: $\mathrm{Cr} \cdot 44 \cdot \mathrm{SmA}_{2} \cdot 66 \cdot \mathrm{I}\left({ }^{\circ} \mathrm{C}\right)\left(2^{\text {nd }} \mathrm{DSC}\right.$ heating run $)$

232-Br-14: $\mathrm{Cr}_{1} \cdot$ [not reported] $\cdot \mathrm{Cr}_{2} \cdot 49 \cdot \mathrm{SmA}_{2} \cdot 97 \cdot \mathrm{I}\left({ }^{\circ} \mathrm{C}\right)\left(2^{\text {nd }}\right.$ DSC heating run $)$

[remark: See ref. 118 for mixtures of 232-Br-14 with $\mathrm{CD}_{3} \mathrm{OD}$ and $\mathrm{CD}_{3} \mathrm{CN}$.]

232-Br-18: $\mathrm{Cr}_{1} \cdot 64 \cdot \mathrm{Cr}_{2} \cdot 67 \cdot \mathrm{Cr}_{3} \cdot 69 \cdot \mathrm{I}\left({ }^{\circ} \mathrm{C}\right)\left(2^{\text {nd }} \mathrm{DSC}\right.$ heating run $)$

$$
\text { 232- } \mathrm{NO}_{3}-\boldsymbol{n}\left(\mathrm{X}^{-}=\left[\mathrm{NO}_{3}\right]^{-}\right)
$$

232- $\mathrm{NO}_{3}-10: \mathrm{Cr} \cdot 33 \cdot \mathrm{I}\left({ }^{\circ} \mathrm{C}\right)\left(1^{\text {st }}\right.$ DSC heating run $)$

232- $\mathrm{NO}_{3}-14: \mathrm{Cr}_{1} \cdot 34 \cdot \mathrm{Cr}_{2} \cdot 38 \cdot \mathrm{I}\left({ }^{\circ} \mathrm{C}\right)\left(1^{\text {st }} \mathrm{DSC}\right.$ heating run $)$

$$
\text { 232-BF }- \text {-n }\left(\mathrm{X}^{-}=\left[\mathrm{BF}_{4}\right]^{-}\right)
$$

232- $\mathrm{BF}_{4}-10: \mathrm{Cr}_{1} \cdot-28 \cdot \mathrm{Cr}_{2} \cdot 1 \cdot \mathrm{I}\left({ }^{\circ} \mathrm{C}\right)\left(1^{\text {st }} \mathrm{DSC}\right.$ heating run $)$

232-BF ${ }_{4}-14: \mathrm{Cr} \cdot 39 \cdot \mathrm{SmA}_{2} \cdot 60 \cdot \mathrm{I}\left({ }^{\circ} \mathrm{C}\right)\left(1^{\text {st }} \mathrm{DSC}\right.$ heating run $)$

232-BF $-\mathbf{B F}_{4}$-18: $\mathrm{Cr}_{1} \cdot$ [not reported] $\cdot \mathrm{Cr}_{2} \cdot 61 \cdot \mathrm{I}\left({ }^{\circ} \mathrm{C}\right)\left(1^{\text {st }} \mathrm{DSC}\right.$ heating run $)$

$$
\text { 232-NTf } \mathbf{f}_{2}-\boldsymbol{n}\left(\mathrm{X}^{-}=\left[\mathrm{NTf}_{2}\right]^{-}\right)
$$

232-NTf 2 -14: $\mathrm{Cr} \cdot 30 \cdot \mathrm{I}\left({ }^{\circ} \mathrm{C}\right)\left(1^{\text {st }}\right.$ DSC heating run $)$

232-NTf $\mathbf{N}_{2}$-18: $\mathrm{Cr} \cdot 55 \cdot \mathrm{I}\left({ }^{\circ} \mathrm{C}\right)\left(1^{\text {st }}\right.$ DSC heating run $)$

$$
\text { 232- } \mathrm{ClO}_{4}-\boldsymbol{n}\left(\mathrm{X}^{-}=\left[\mathrm{ClO}_{4}\right]^{-}\right)
$$

232-ClO 4 -14: $\mathrm{Cr}_{1} \cdot 38 \cdot \mathrm{Cr}_{2} \cdot 45 \cdot \mathrm{I}\left({ }^{\circ} \mathrm{C}\right)\left(1^{\text {st }} \mathrm{DSC}\right.$ heating run $)$

232- $\mathrm{ClO}_{4}-1$ 18: $\mathrm{Cr}_{1} \cdot$ [not reported] $\cdot \mathrm{Cr}_{2} \cdot 61 \cdot \mathrm{I}\left({ }^{\circ} \mathrm{C}\right)\left(1^{\text {st }} \mathrm{DSC}\right.$ heating run $)$

233-I-14 ( $\left.\mathrm{X}^{-}=\mathrm{I}^{-} ; n=14\right): \mathrm{Cr}_{1} \cdot 38 \cdot \mathrm{Cr}_{2} \cdot 63 \cdot \mathrm{SmA}_{2} \cdot 112 \cdot \mathrm{I}\left({ }^{\circ} \mathrm{C}\right)\left(1^{\text {st }} \mathrm{DSC}\right.$ heating run $)$

$$
\text { 233- } \mathrm{NO}_{3}-\boldsymbol{n}\left(\mathrm{X}^{-}=\left[\mathrm{NO}_{3}\right]^{-}\right)
$$

233- $\mathrm{NO}_{3}-10: \mathrm{Cr} \cdot 51 \cdot \mathrm{SmA}_{2} \cdot 82 \cdot \mathrm{I}\left({ }^{\circ} \mathrm{C}\right)$ (from POM observations)

233- $\mathrm{NO}_{3}-14: \mathrm{Cr} \cdot 40 \cdot \mathrm{SmA}_{2} \cdot 98 \cdot \mathrm{I}\left({ }^{\circ} \mathrm{C}\right)$ (from POM observations)

233- $\mathrm{NO}_{3}-18: \mathrm{Cr} \cdot 64 \cdot \mathrm{SmA}_{2} \cdot 104 \cdot \mathrm{I}\left({ }^{\circ} \mathrm{C}\right)($ from POM observations) 


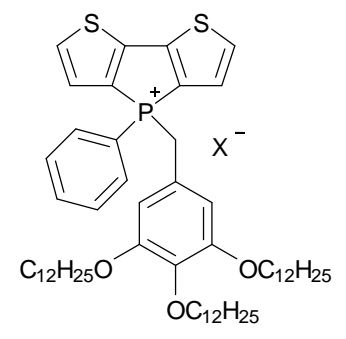

234-X $\left(\mathrm{X}^{-}=\mathrm{Br}^{-},\left[\mathrm{BF}_{4}\right]^{-}\right.$,

$\left.\left[\mathrm{B}\left(\mathrm{C}_{6} \mathrm{H}_{5}\right)_{4}\right]^{-},[\mathrm{OTf}]^{-}\right)$

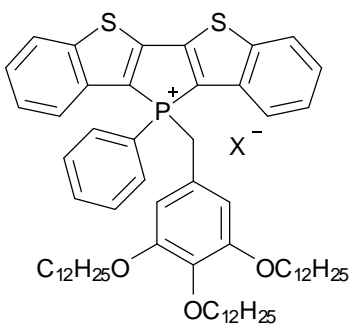

236-X $\left(\mathrm{X}^{-}=\mathrm{Br}^{-},\left[\mathrm{BF}_{4}\right]^{-}\right)$

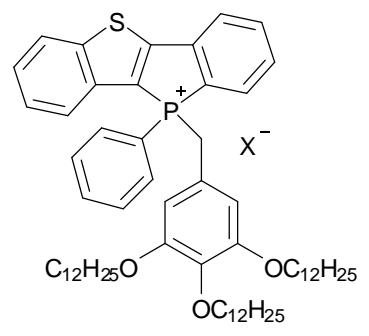

235-X $\left(\mathrm{X}^{-}=\mathrm{Br}^{-},\left[\mathrm{BF}_{4}\right]^{-}\right)$

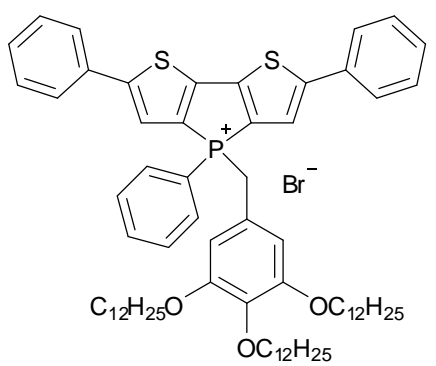

237

234-Br $\left(\mathrm{X}^{-}=\mathrm{Br}^{-}\right): \mathrm{Cr}_{1} \cdot 13 \cdot \mathrm{Cr}_{2} \cdot 37 \cdot \mathrm{X} \cdot 76 \cdot \mathrm{SmX} \cdot 205 \cdot \mathrm{I}\left({ }^{\circ} \mathrm{C}\right)$

234-BF $4\left(\mathrm{X}^{-}=\left[\mathrm{BF}_{4}\right]^{-}\right): \mathrm{Cr} \cdot-1 \cdot \mathrm{X} \cdot 41 \cdot \mathrm{SmX} \cdot 168 \cdot \mathrm{I}\left({ }^{\circ} \mathrm{C}\right)$

234-B $\left(\mathrm{C}_{6} \mathbf{H}_{5}\right)_{4}\left(\mathrm{X}^{-}=\left[\mathrm{B}\left(\mathrm{C}_{6} \mathrm{H}_{5}\right)_{4}\right]^{-}\right): \mathrm{Cr} \cdot 58 \cdot \mathrm{X} \cdot 108 \cdot \mathrm{I}\left({ }^{\circ} \mathrm{C}\right)$

234-OTf $\left(\mathrm{X}^{-}=[\mathrm{OTf}]^{-}\right): \mathrm{Cr}_{1} \cdot 16 \cdot \mathrm{Cr}_{2} \cdot 43 \cdot \mathrm{X} \cdot 98 \cdot \mathrm{I}\left({ }^{\circ} \mathrm{C}\right)$

235-Br $\left(\mathrm{X}^{-}=\mathrm{Br}^{-}\right): \mathrm{Cr} \cdot 18 \cdot \mathrm{X} \cdot 53 \cdot \mathrm{SmX} \cdot 173 \cdot \mathrm{I}\left({ }^{\circ} \mathrm{C}\right)$

235- $\mathrm{BF}_{4}\left(\mathrm{X}^{-}=\left[\mathrm{BF}_{4}\right]^{-}\right): \mathrm{Cr} \cdot 5 \cdot \mathrm{M} \cdot 123 \cdot \mathrm{I}\left({ }^{\circ} \mathrm{C}\right)$

236-Br $\left(\mathrm{X}^{-}=\mathrm{Br}^{-}\right): \mathrm{g} \cdot 31 \cdot \mathrm{M} \cdot 88 \cdot \mathrm{X} \cdot 140 \cdot \mathrm{I}\left({ }^{\circ} \mathrm{C}\right)$

236- $\mathrm{BF}_{4}\left(\mathrm{X}^{-}=\left[\mathrm{BF}_{4}\right]^{-}\right): \mathrm{g} \cdot 26 \cdot \mathrm{M} \cdot 95 \cdot \mathrm{X} \cdot 129 \cdot \mathrm{I}\left({ }^{\circ} \mathrm{C}\right)$

237: $\mathrm{g} \cdot 40 \cdot \mathrm{M}_{1} \cdot 90 \cdot \mathrm{Cr} \cdot 138 \cdot \mathrm{M}_{2} \cdot 150 \cdot \mathrm{I}\left({ }^{\circ} \mathrm{C}\right)$
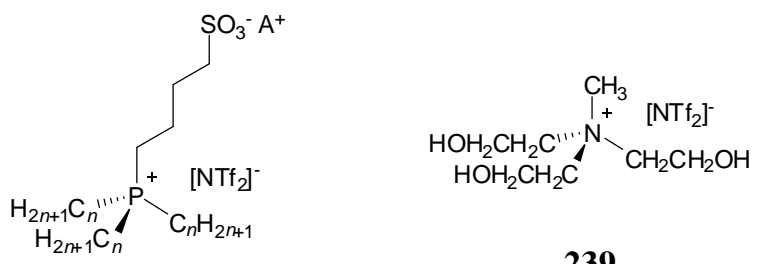

239

238-A-n $\left(\mathrm{A}^{+}=\mathrm{Li}^{+}, \mathrm{H}^{+}\right)$

Phase diagrams for mixtures of 238-Li-6 $\left(\mathrm{A}^{+}=\mathrm{Li}^{+}\right)$and $\mathbf{2 3 9}$, and 238- $\mathbf{- i - 8}\left(\mathrm{A}^{+}=\mathrm{Li}^{+}\right)$and $\mathbf{2 3 9}$ are displayed in Figure S15. 


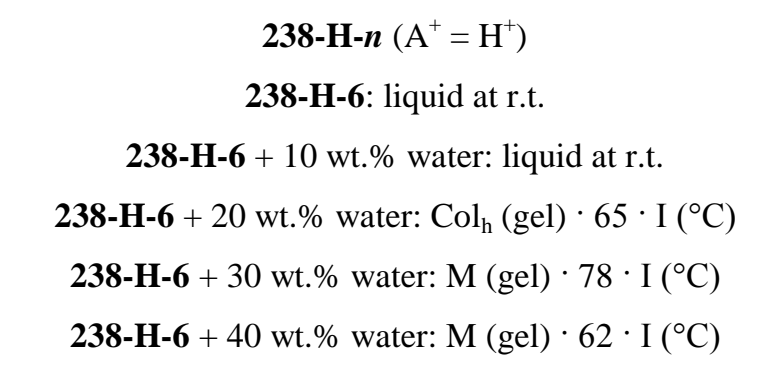

238-H-6 + 50 wt.\% water: non-mesomorphic gel at r.t.

238-H-8: liquid at r.t.

238-H-8 + 10 wt.\% water: $\mathrm{M}($ gel $) \cdot 70 \cdot \mathrm{I}\left({ }^{\circ} \mathrm{C}\right)$

238-H-8 + 20 wt. \% water: $\mathrm{Col}_{\mathrm{h}}(\mathrm{gel}) \cdot 78 \cdot \mathrm{I}\left({ }^{\circ} \mathrm{C}\right)$

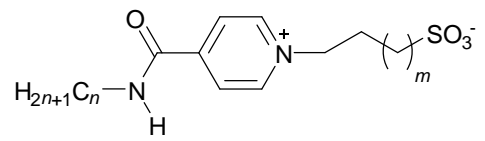

\section{$240-n / m$}

240-12/1: $\mathrm{Cr} \cdot\left[\right.$ not reported] $\cdot \mathrm{SmA} \cdot>200 \cdot \mathrm{I}\left({ }^{\circ} \mathrm{C}\right)$

240-14/1: $\mathrm{Cr} \cdot\left[\right.$ not reported] $\cdot \mathrm{SmA} \cdot>200 \cdot \mathrm{I}\left({ }^{\circ} \mathrm{C}\right)$

240-16/1: $\mathrm{Cr} \cdot\left[\right.$ not reported] $\cdot \mathrm{SmA} \cdot>200 \cdot \mathrm{I}\left({ }^{\circ} \mathrm{C}\right)$

240-18/1: $\mathrm{Cr} \cdot\left[\right.$ not reported] $\cdot \mathrm{SmA} \cdot>200 \cdot \mathrm{I}\left({ }^{\circ} \mathrm{C}\right)$

(240-12/1)/HNTf $2(1: 1): \quad \mathrm{Cr} \cdot c a .20 \cdot \mathrm{Cub}_{\mathrm{V}}(\mathrm{Ia} \overline{3} d) \cdot c a .85 \cdot \mathrm{I}\left({ }^{\circ} \mathrm{C}\right) /$ on cooling: $\mathrm{I} \cdot \mathrm{ca} .80 \cdot \mathrm{Col} \cdot \mathrm{ca} .45 \cdot \mathrm{Cub}(\mathrm{Ia} \overline{3} d)$ ca. $-5 \cdot \operatorname{Cr}\left({ }^{\circ} \mathrm{C}\right)$ (transition temperatures can vary up to $5 \%$ depending on the absorption of moisture (2-3 wt.\% water))

(240-14/1)/ $\operatorname{HNTf}_{2}(1: 1): \quad \mathrm{Cr} \cdot c a .10 \cdot \mathrm{Cub}_{\mathrm{V}}(\mathrm{Ia} \overline{3} d) \cdot c a .90 \cdot \mathrm{I}\left({ }^{\circ} \mathrm{C}\right)$ (on cooling) (transition temperatures can vary up to $5 \%$ depending on the absorption of moisture (2-3 wt.\% water))

(240-16/1)/HNTf 2 (1:1): $\quad \mathrm{Cr} \cdot c a .35 \cdot \mathrm{Cub}_{\mathrm{V}}(\mathrm{Ia} \overline{3} d) \cdot c a .60 \cdot \mathrm{SmA} \cdot$ [not reported] $\cdot \mathrm{I}\left({ }^{\circ} \mathrm{C}\right)$ (on cooling) (transition temperatures can vary up to 5\% depending on the absorption of moisture (2-3 wt.\% water))

(240-18/1)/ $\mathrm{HNTf}_{2}(1: 1): \quad \mathrm{Cr} \cdot \mathrm{ca} .45 \cdot \mathrm{SmA} \cdot$ [not reported] $\cdot \mathrm{I}\left({ }^{\circ} \mathrm{C}\right)$ (on cooling) (transition temperatures can vary up to 5\% depending on the absorption of moisture (2-3 wt.\% water))

For 240-n/2 and for mixtures of 240-n/m $(m=1,2)$ with LiCl, LiBF$_{4}, \mathrm{LiOTf}_{\mathrm{LiNTf}}$ and $\mathrm{HNTf}_{2}$ : see ref. 119

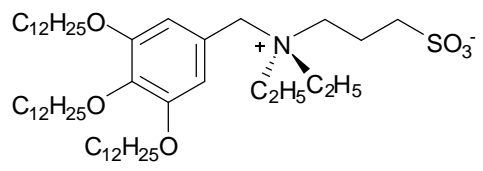

241: $\mathrm{Cr} \cdot 25 \cdot \mathrm{Col}_{\mathrm{h}} \cdot 200 \cdot \operatorname{dec}\left({ }^{\circ} \mathrm{C}\right)$

241/ $\mathrm{PhSO}_{3} \mathrm{H}(90: 10): \mathrm{Cub}_{\mathrm{V}}(\mathrm{Ia} \overline{3} d)+\mathrm{Col}_{\mathrm{h}} \cdot 78 \cdot \mathrm{Col}_{\mathrm{h}} \cdot 200 \cdot$ dec. $\left({ }^{\circ} \mathrm{C}\right)$ (from POM observations)

241/ $\mathrm{PhSO}_{3} \mathrm{H}$ (80:20): $\mathrm{Cr} \cdot 46$ [broad transition] $\cdot \mathrm{Cub}_{\mathrm{V}}(\mathrm{Ia} \overline{3} d) \cdot 97 \cdot \mathrm{Col}_{\mathrm{h}} \cdot 200 \cdot \mathrm{dec}$ ( $\left.{ }^{\circ} \mathrm{C}\right)($ from POM observations) 241/ $\mathrm{PhSO}_{3} \mathrm{H}$ (70:30): $\mathrm{Cr} \cdot 53$ [broad transition] $\cdot \mathrm{Cub}_{\mathrm{V}}(\mathrm{Ia} \overline{3} d) \cdot 115 \cdot \mathrm{Col}_{\mathrm{h}} \cdot 200 \cdot$ dec. $\left({ }^{\circ} \mathrm{C}\right)($ from POM observations) 
241/ $\mathrm{PhSO}_{3} \mathrm{H}(60: 40): \mathrm{Cr} \cdot 67$ [broad transition] $\cdot \mathrm{Cub}_{\mathrm{v}}(\mathrm{Ia} \overline{3} \mathrm{~d}) \cdot 130 \cdot \mathrm{Col}_{\mathrm{h}} \cdot 200 \cdot \mathrm{dec} .\left({ }^{\circ} \mathrm{C}\right)$ (from POM observations) 241/ $\mathrm{PhSO}_{3} \mathrm{H}$ (50:50): $\mathrm{Cr} \cdot 80$ [broad transition] $\cdot \mathrm{Cub}_{\mathrm{V}}(\mathrm{Ia} \overline{3} d) \cdot 119 \cdot \mathrm{I}\left({ }^{\circ} \mathrm{C}\right)$ (from POM observations) 241/ $\mathrm{PhSO}_{3} \mathrm{H}$ (40:60): $\mathrm{Cr} \cdot 45 \cdot$ [phase separation] $\left({ }^{\circ} \mathrm{C}\right)$ (from POM observations) 


\subsection{Pyridinium-based ionic liquid crystals}

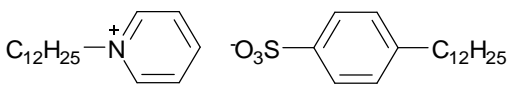

242: $(\mathrm{Sm}) \mathrm{X}(?) \cdot 107 \cdot \mathrm{SmA} \cdot 152 \cdot \mathrm{I}\left({ }^{\circ} \mathrm{C}\right)$
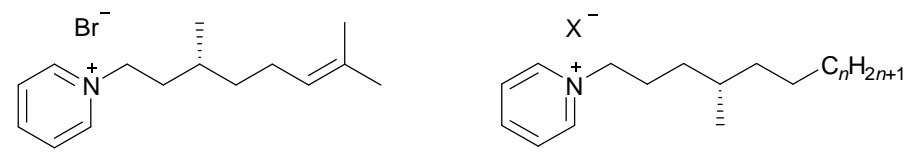

243

$$
\begin{aligned}
& \text { 244-X-n }\left(\mathrm{X}^{-}=\mathrm{Br}^{-}, \mathrm{I}^{-},\left[\mathrm{BF}_{4}\right]^{-},\right. \\
& \left.\left[\mathrm{PF}_{6}\right]^{-},[\mathrm{SCN}]^{-},\left[\mathrm{CH}_{3} \mathrm{COO}\right]^{-}\right)
\end{aligned}
$$

243: $\mathrm{g} \cdot-56 \cdot \mathrm{I}\left({ }^{\circ} \mathrm{C}\right)$

244-Br-n $\left(\mathrm{X}^{-}=\mathrm{Br}^{-}\right)$

244-Br-6: $\mathrm{Cr} \cdot-27 \cdot \mathrm{SmA} \cdot 127 \cdot \mathrm{I}\left({ }^{\circ} \mathrm{C}\right)$

244-Br-7: $\mathrm{Cr} \cdot-8 \cdot \mathrm{SmA} \cdot 184 \cdot \mathrm{I}\left({ }^{\circ} \mathrm{C}\right)$

244-Br-8: $\mathrm{Cr} \cdot-1 \cdot \mathrm{SmA} \cdot 184 \cdot \mathrm{I}\left({ }^{\circ} \mathrm{C}\right)$

244-Br-9: $\mathrm{Cr} \cdot-1 \cdot \mathrm{SmA} \cdot 173 \cdot \mathrm{I}\left({ }^{\circ} \mathrm{C}\right)$

244-I-9 ( $\left.\mathrm{X}^{-}=\mathrm{I}^{-}, n=9\right): \mathrm{Cr}_{1} \cdot-6 \cdot \mathrm{Cr}_{2} \cdot 41 \cdot \mathrm{SmA} \cdot 171 \cdot \mathrm{I}\left({ }^{\circ} \mathrm{C}\right)$

244-BF $\mathbf{B F}_{4} \mathbf{- 9}\left(\mathrm{X}^{-}=\left[\mathrm{BF}_{4}\right]^{-}, n=9\right): \mathrm{Cr}_{1} \cdot-19 \cdot \mathrm{Cr}_{2} \cdot 1 \cdot \mathrm{SmA} \cdot 140 \cdot \mathrm{I}\left({ }^{\circ} \mathrm{C}\right)$

244-PF $\mathbf{P F}_{6} \mathbf{- 9}\left(\mathrm{X}^{-}=\left[\mathrm{PF}_{6}\right]^{-}, n=9\right): \mathrm{Cr}_{1} \cdot 63 \cdot \mathrm{Cr}_{2} \cdot 80 \cdot \mathrm{Cr}_{3} \cdot 126 \cdot \mathrm{I}\left({ }^{\circ} \mathrm{C}\right)$

244-SCN-9 (X $\left.=[\mathrm{SCN}]^{-}, n=9\right): \mathrm{Cr}_{1} \cdot 2 \cdot \mathrm{Cr}_{2} \cdot 21 \cdot \mathrm{SmA} \cdot 135 \cdot \mathrm{I}\left({ }^{\circ} \mathrm{C}\right)$

244-CH COO-9 $_{3}$ ( $\left.{ }^{-}=\left[\mathrm{CH}_{3} \mathrm{COO}\right]^{-}, n=9\right): \mathrm{Cr} \cdot 0 \cdot \mathrm{SmA} \cdot 162 \cdot \mathrm{I}\left({ }^{\circ} \mathrm{C}\right)$

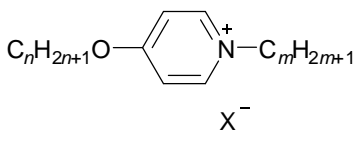

245-Br-m/n $\left(\mathrm{X}^{-}=\mathrm{Br}^{-}\right)$

245-Br-10/10: $\mathrm{Cr} \cdot 63 \cdot \mathrm{SmA} \cdot 69 \cdot \mathrm{I}\left({ }^{\circ} \mathrm{C}\right)$

245-Br-12/12: $\mathrm{Cr} \cdot 70 \cdot \mathrm{SmA} \cdot 120 \cdot \mathrm{I}\left({ }^{\circ} \mathrm{C}\right)$

245-Br-14/14: $\mathrm{Cr} \cdot 77 \cdot \mathrm{SmA} \cdot 136 \cdot \mathrm{I}\left({ }^{\circ} \mathrm{C}\right)$

245-Br-16/16: $\mathrm{Cr} \cdot 79 \cdot \mathrm{SmA} \cdot 133 \cdot \mathrm{I}\left({ }^{\circ} \mathrm{C}\right)$

245-Br-18/18: $\mathrm{Cr} \cdot 87 \cdot \mathrm{SmA} \cdot 133 \cdot \mathrm{I}\left({ }^{\circ} \mathrm{C}\right)$

245- $\mathbf{B F}_{4}-\boldsymbol{m} / \boldsymbol{n}\left(\mathrm{X}^{-}=\left[\mathrm{BF}_{4}\right]^{-}\right)$

245-BF $\mathbf{H}_{\mathbf{4}}$ 10/10: $\mathrm{Cr} \cdot 47 \cdot \mathrm{I}\left({ }^{\circ} \mathrm{C}\right)$

245-BF $-12 / 12: \mathrm{Cr} \cdot 39 \cdot \mathrm{SmA} \cdot 77 \cdot \mathrm{I}\left({ }^{\circ} \mathrm{C}\right)$ 
245-BF ${ }_{4} \mathbf{- 1 4} / \mathbf{1 4}: \mathrm{Cr} \cdot 51 \cdot \mathrm{SmA} \cdot 98 \cdot \mathrm{I}\left({ }^{\circ} \mathrm{C}\right)$

245-BF $\mathbf{H}^{-16 / 16:} \mathrm{Cr} \cdot 63 \cdot \mathrm{SmA} \cdot 114 \cdot \mathrm{I}\left({ }^{\circ} \mathrm{C}\right)$

245-BF ${ }_{4}-18 / 18: \mathrm{Cr} \cdot 70 \cdot \mathrm{SmA} \cdot 128 \cdot \mathrm{I}\left({ }^{\circ} \mathrm{C}\right)$

245-PF ${ }_{6}-\mathbf{m} / \mathbf{n}\left(\mathrm{X}^{-}=\left[\mathrm{PF}_{6}\right]^{-}\right)$

245- $\mathrm{PF}_{\mathbf{6}}-\mathbf{- 1 0} / \mathbf{1 0}: \mathrm{Cr} \cdot 38 \cdot \mathrm{I}\left({ }^{\circ} \mathrm{C}\right)$

245- $\mathrm{PF}_{\mathbf{6}}-\mathbf{1 2} / \mathbf{1 2} \mathrm{Cr} \cdot(\mathrm{SmA} \cdot 53 \cdot) 57 \cdot \mathrm{I}\left({ }^{\circ} \mathrm{C}\right)$

245- $\mathrm{PF}_{\mathbf{6}} \mathbf{- 1 4} / \mathbf{1 4}: \mathrm{Cr} \cdot 67 \cdot \mathrm{SmA} \cdot 94 \cdot \mathrm{I}\left({ }^{\circ} \mathrm{C}\right)$

245- $\mathbf{P F}_{\mathbf{6}} \mathbf{- 1 6 / 1 6 :} \mathrm{Cr} \cdot 77 \cdot \mathrm{SmA} \cdot 115 \cdot \mathrm{I}\left({ }^{\circ} \mathrm{C}\right)$

245-PF $\mathbf{6}-18 / 18: \mathrm{Cr} \cdot 84 \cdot \mathrm{SmA} \cdot 129 \cdot \mathrm{I}\left({ }^{\circ} \mathrm{C}\right)$

245-Cl-m/0 ( $\left.\mathrm{X}^{-}=\mathrm{Cl}^{-}, n=0\right)$

245-Cl-12/0: $\mathrm{Cr} \cdot 74 \cdot \mathrm{I}\left({ }^{\circ} \mathrm{C}\right)$

245-Cl-14/0: $\mathrm{Cr} \cdot 79 \cdot \mathrm{I}\left({ }^{\circ} \mathrm{C}\right)$

245-Cl-16/0: $\mathrm{Cr} \cdot 85 \cdot \mathrm{SmA} \cdot 115 \cdot \mathrm{I}\left({ }^{\circ} \mathrm{C}\right)$

245-Cl-18/0: $\mathrm{Cr} \cdot 87 \cdot \mathrm{SmA} \cdot 157 \cdot \mathrm{I}\left({ }^{\circ} \mathrm{C}\right)$

$$
\text { 245-Cl-0/n }\left(\mathrm{X}^{-}=\mathrm{Cl}^{-}, m=0\right)
$$

245-Cl-0/12: $\mathrm{Cr}_{1} \cdot 92 \cdot \mathrm{Cr}_{2} \cdot 122 \cdot \mathrm{SmA} \cdot \mathrm{I} / \mathrm{dec}\left({ }^{\circ} \mathrm{C}\right)$

245-Cl-0/14: $\mathrm{Cr}_{1} \cdot 95 \cdot \mathrm{Cr}_{2} \cdot 122 \cdot \mathrm{SmA} \cdot 139 \cdot \mathrm{I} / \mathrm{dec}\left({ }^{\circ} \mathrm{C}\right)$

245-Cl-0/16: $\mathrm{Cr}_{1} \cdot 95 \cdot \mathrm{Cr}_{2} \cdot 124 \cdot \mathrm{SmA} \cdot 142 \cdot \mathrm{I} / \mathrm{dec}\left({ }^{\circ} \mathrm{C}\right)$

245-Cl-0/18: $\mathrm{Cr}_{1} \cdot 93 \cdot \mathrm{Cr}_{2} \cdot 124 \cdot \mathrm{SmA} \cdot 143 \cdot \mathrm{I} / \mathrm{dec}\left({ }^{\circ} \mathrm{C}\right)$

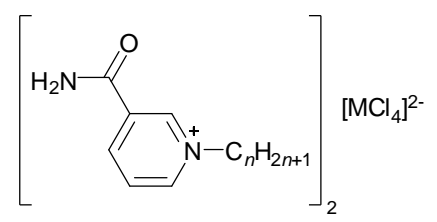

$$
\text { 246-Cu-n }\left(\mathrm{M}=\mathrm{Cu}^{2+}\right)
$$

246-Cu-12: $\mathrm{Cr} \cdot 100 \cdot \mathrm{SmA} \cdot 135 \cdot \mathrm{I}\left({ }^{\circ} \mathrm{C}\right)$

246-Cu-14: $\mathrm{Cr} \cdot 98 \cdot \mathrm{SmA} \cdot 163 \cdot \mathrm{I}\left({ }^{\circ} \mathrm{C}\right)$

246-Cu-16: $\mathrm{Cr} \cdot 112 \cdot \mathrm{SmA} \cdot 200 \cdot \mathrm{I} / \mathrm{dec} .\left({ }^{\circ} \mathrm{C}\right)$

$$
\text { 246-Zn-n }\left(\mathrm{M}=\mathrm{Zn}^{2+}\right)
$$

246-Zn-12: $\mathrm{Cr} \cdot 112 \cdot \mathrm{SmA} \cdot 142 \cdot \mathrm{I}\left({ }^{\circ} \mathrm{C}\right)$

246-Zn-14: $\mathrm{Cr} \cdot 111 \cdot \mathrm{SmA} \cdot 169 \cdot \mathrm{I}\left({ }^{\circ} \mathrm{C}\right)$

246-Zn-16: $\mathrm{Cr} \cdot 114 \cdot \mathrm{SmA} \cdot 220 \cdot \mathrm{I} / \mathrm{dec} .\left({ }^{\circ} \mathrm{C}\right)$ 


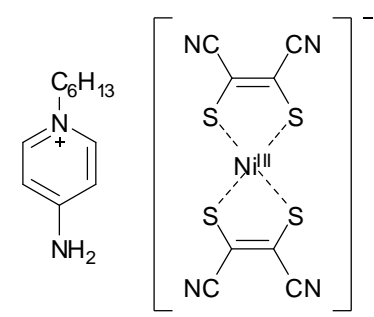

247: not entirely clear whether it is a thermotropic LC

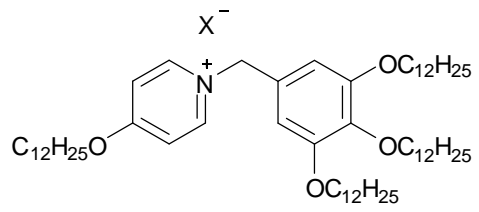

248-X

248-Br $\left(\mathrm{X}^{-}=\mathrm{Br}^{-}\right): \mathrm{Cr} \cdot 63 \cdot\left(\mathrm{Col}_{2} \cdot 74 \cdot\right) \mathrm{Col}_{1} \cdot 108 \cdot \mathrm{I}\left({ }^{\circ} \mathrm{C}\right)$

248- $\mathrm{NO}_{3}\left(\mathrm{X}^{-}=\left[\mathrm{NO}_{3}\right]^{-}\right): \mathrm{Cr} \cdot 87 \cdot \mathrm{I}\left({ }^{\circ} \mathrm{C}\right)$

248- $-\mathrm{BF}_{4}\left(\mathrm{X}^{-}=\left[\mathrm{BF}_{4}\right]^{-}\right): \mathrm{Cr} \cdot 59 \cdot \mathrm{I}\left({ }^{\circ} \mathrm{C}\right)$

248-PF $\mathbf{P F}_{6}\left(\mathrm{X}^{-}=\left[\mathrm{PF}_{6}\right]^{-}\right): \mathrm{Cr}_{1} \cdot 72 \cdot \mathrm{Cr}_{2} \cdot 80 \cdot \mathrm{I}\left({ }^{\circ} \mathrm{C}\right)$

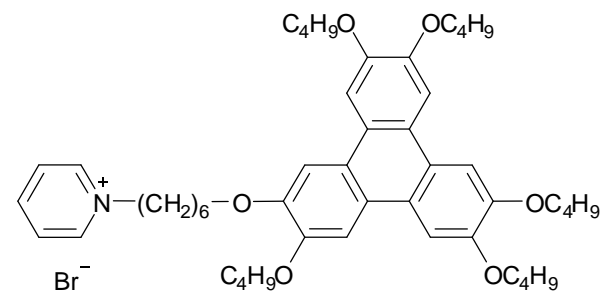

249: $\mathrm{Cr} \cdot 84 \cdot \mathrm{Col} \cdot 95 \cdot \mathrm{I}\left({ }^{\circ} \mathrm{C}\right)$

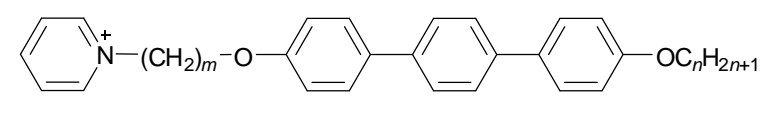

$\mathrm{Br}^{-}$

250-m/n

250-8/14: $\mathrm{Cr} \cdot 116 \cdot \mathrm{SmX}_{1} \cdot 172 \cdot \mathrm{SmX}_{2} \cdot 188 \cdot \mathrm{SmC} \cdot 220 \cdot \mathrm{I} /$ dec. $\left({ }^{\circ} \mathrm{C}\right)$

250-12/14: $\mathrm{Cr} \cdot 116 \cdot \mathrm{SmX}_{1} \cdot 187 \cdot \mathrm{SmX}_{2} \cdot 207 \cdot \mathrm{SmC} \cdot 220 \cdot \mathrm{I} /$ dec. $\left({ }^{\circ} \mathrm{C}\right)$

250-8/16: $\mathrm{Cr} \cdot 120 \cdot \mathrm{SmX}_{1} \cdot 178 \cdot \mathrm{SmX}_{2} \cdot 189 \cdot \mathrm{SmC} \cdot 220 \cdot \mathrm{I} /$ dec. $\left({ }^{\circ} \mathrm{C}\right)$

250-12/16: $\mathrm{Cr} \cdot 120 \cdot \mathrm{SmX}_{1} \cdot 186 \cdot \mathrm{SmX}_{2} \cdot 206 \cdot \mathrm{SmC} \cdot 220 \cdot \mathrm{I} /$ dec. $\left({ }^{\circ} \mathrm{C}\right)$ 


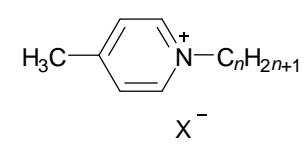

251-X-n $\left(\mathrm{X}^{-}=\mathrm{Br}^{-}, \mathrm{I}^{-}\right)$

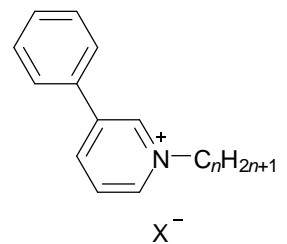

253-X-n $\left(\mathrm{X}^{-}=\mathrm{Br}^{-}, \mathrm{I}^{-}\right)$

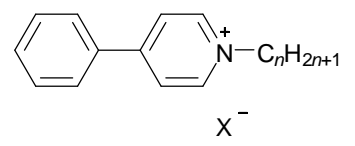

252-X-n $\left(\mathrm{X}^{-}=\mathrm{Cl}^{-}, \mathrm{Br}^{-}, \mathrm{I}^{-}\right.$,

$\left[\mathrm{B}\left(\mathrm{C}_{6} \mathrm{H}_{5}\right)_{4}\right]^{-}$, [OTs] $\left.]^{-}\right)$

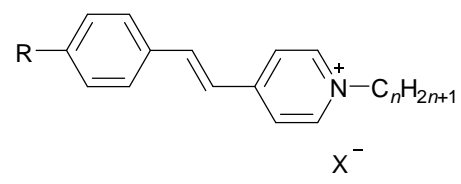

254-X-n-R $\left(\mathrm{X}^{-}=\mathrm{Br}^{-}, \mathrm{I}^{-}\right.$;

$\left.\mathrm{R}=\mathrm{OH}, \mathrm{OCH}_{3}, \mathrm{H}\right)$

251-Br-n $\left(\mathrm{X}^{-}=\mathrm{Br}^{-}\right)$

251-Br-14: $\mathrm{Cr} \cdot 77 \cdot \mathrm{I}\left({ }^{\circ} \mathrm{C}\right)$

251-Br-16: $\mathrm{Cr} \cdot 85 \cdot \mathrm{SmA} \cdot 119 \cdot \mathrm{I}\left({ }^{\circ} \mathrm{C}\right)$

251-Br-18: $\mathrm{Cr} \cdot 82 \cdot \mathrm{SmA} \cdot 168 \cdot \mathrm{I}\left({ }^{\circ} \mathrm{C}\right)$

251-Br-20: $\mathrm{Cr} \cdot 84 \cdot \mathrm{SmA} \cdot 197 \cdot \mathrm{I}\left({ }^{\circ} \mathrm{C}\right)$

251-Br-22: $\mathrm{Cr} \cdot 91 \cdot \mathrm{SmA} \cdot 207 \cdot \mathrm{I}\left({ }^{\circ} \mathrm{C}\right)$

$$
\text { 251-I-n }\left(\mathrm{X}^{-}=\mathrm{I}^{-}\right)
$$

251-I-16: $\mathrm{Cr} \cdot 67 \cdot \mathrm{SmA} \cdot 104 \cdot \mathrm{I}\left({ }^{\circ} \mathrm{C}\right)$

251-I-18: $\mathrm{Cr} \cdot 84 \cdot \mathrm{SmA} \cdot 142 \cdot \mathrm{I}\left({ }^{\circ} \mathrm{C}\right)$

252-Cl-22 $\left(\mathrm{X}^{-}=\mathrm{Cl}^{-} ; n=22\right): \mathrm{Cr} \cdot 72 \cdot \mathrm{SmA} \cdot 213 \cdot \mathrm{I}\left({ }^{\circ} \mathrm{C}\right)$

252-Br-n $\left(\mathrm{X}^{-}=\mathrm{Br}^{-}\right)$

252-Br-16: $\mathrm{Cr} \cdot 86 \cdot \mathrm{I}\left({ }^{\circ} \mathrm{C}\right)$

252-Br-18: $\mathrm{Cr} \cdot 89 \cdot \mathrm{M} \cdot 114 \cdot \mathrm{I}\left({ }^{\circ} \mathrm{C}\right)$

252-Br-20: $\mathrm{Cr} \cdot 95 \cdot \mathrm{M} \cdot 134 \cdot \mathrm{SmA} \cdot 156 \cdot \mathrm{I}\left({ }^{\circ} \mathrm{C}\right)$

252-Br-22: $\mathrm{Cr} \cdot 98 \cdot \mathrm{M} \cdot 109 \cdot \mathrm{SmA} \cdot 179 \cdot \mathrm{I}\left({ }^{\circ} \mathrm{C}\right)$

252-I-n $\left(\mathrm{X}^{-}=\mathrm{I}^{-}\right)$

252-I-16: $\mathrm{Cr} \cdot 83 \cdot \mathrm{I}\left({ }^{\circ} \mathrm{C}\right)$

252-I-18: $\mathrm{Cr} \cdot 90 \cdot \mathrm{SmA} \cdot 98 \cdot \mathrm{I}\left({ }^{\circ} \mathrm{C}\right)$

252-I-22: $\mathrm{Cr} \cdot 95 \cdot \mathrm{SmA} \cdot 161 \cdot \mathrm{I}\left({ }^{\circ} \mathrm{C}\right)$

252-B $\left(\mathrm{C}_{6} \mathbf{H}_{5}\right)_{4}-22\left(\mathrm{X}^{-}=\left[\mathrm{B}\left(\mathrm{C}_{6} \mathrm{H}_{5}\right)_{4}\right]^{-} ; n=22\right): \mathrm{Cr} \cdot 78 \cdot \mathrm{SmA} \cdot 151 \cdot \mathrm{I}\left({ }^{\circ} \mathrm{C}\right)$

252-OTs-22 ( $\left.\mathrm{X}^{-}=[\mathrm{OTs}]^{-} ; n=22\right): \mathrm{Cr} \cdot 94 \cdot \mathrm{I}\left({ }^{\circ} \mathrm{C}\right)$

253-Br-n $\left(\mathrm{X}^{-}=\mathrm{Br}^{-}\right)$

253-Br-16: $\mathrm{Cr} \cdot 99 \cdot \mathrm{I}\left({ }^{\circ} \mathrm{C}\right)$

253-Br-18: $\mathrm{Cr} \cdot 45 \cdot \mathrm{SmA} \cdot 82 \cdot \mathrm{I}\left({ }^{\circ} \mathrm{C}\right)$

253-Br-20: $\mathrm{Cr} \cdot 54 \cdot \mathrm{SmA} \cdot 131 \cdot \mathrm{I}\left({ }^{\circ} \mathrm{C}\right)$ 
253-Br-22: $\mathrm{Cr}_{1} \cdot 67 \cdot \mathrm{Cr}_{2} \cdot 106 \cdot \mathrm{SmA} \cdot 149 \cdot \mathrm{I}\left({ }^{\circ} \mathrm{C}\right)$

253-I-n $\left(\mathrm{X}^{-}=\mathrm{I}^{-}\right)$

253-I-16: $\mathrm{Cr} \cdot 77 \cdot \mathrm{I}\left({ }^{\circ} \mathrm{C}\right)$

253-I-18: $\mathrm{Cr} \cdot 44 \cdot \mathrm{SmA} \cdot 65 \cdot \mathrm{I}\left({ }^{\circ} \mathrm{C}\right)$

254-Br-n-R $\left(\mathrm{X}^{-}=\mathrm{Br}^{-}\right)$

254-Br-14-OH: $\mathrm{Cr} \cdot 173 \cdot \mathrm{SmX} \cdot 186 \cdot \mathrm{I}\left({ }^{\circ} \mathrm{C}\right)$

254-Br-16-OH: $\mathrm{Cr} \cdot 177 \cdot \mathrm{SmA} \cdot 221 \cdot \mathrm{I} / \mathrm{dec}\left({ }^{\circ} \mathrm{C}\right)$

254-Br-18-OH: $\mathrm{Cr} \cdot 172 \cdot \mathrm{SmA} \cdot 230 \cdot \mathrm{I} / \mathrm{dec}\left({ }^{\circ} \mathrm{C}\right)$

254-Br-20-OH: Cr $\cdot 172 \cdot \mathrm{SmA} \cdot 260 \cdot \mathrm{I} / \operatorname{dec}\left({ }^{\circ} \mathrm{C}\right)$

254-Br-22-OH: $\mathrm{Cr} \cdot 176 \cdot \mathrm{SmA} \cdot 245 \cdot \mathrm{I} / \mathrm{dec}\left({ }^{\circ} \mathrm{C}\right)$

254-Br-14-OCH $: \mathrm{Cr} \cdot 231 \cdot \mathrm{SmA} \cdot 247 \cdot \mathrm{I} / \mathrm{dec}\left({ }^{\circ} \mathrm{C}\right)$

254-Br-16-OCH $: \mathrm{Cr} \cdot 54 \cdot \mathrm{SmA} \cdot 227 \cdot \mathrm{I} / \mathrm{dec}\left({ }^{\circ} \mathrm{C}\right)$

254-Br-18-OCH $3: \mathrm{Cr} \cdot 63 \cdot \mathrm{SmA} \cdot 255 \cdot \mathrm{I} / \mathrm{dec}\left({ }^{\circ} \mathrm{C}\right)$

254-Br-20-OCH $3: \mathrm{Cr} \cdot 68 \cdot \mathrm{SmA} \cdot 227 \cdot \mathrm{I} / \mathrm{dec}\left({ }^{\circ} \mathrm{C}\right)$

254-Br-22-OCH $3: \mathrm{Cr} \cdot 75 \cdot \mathrm{SmA} \cdot 214 \cdot \mathrm{I} / \mathrm{dec}\left({ }^{\circ} \mathrm{C}\right)$

254-Br-14-H: $\mathrm{Cr} \cdot 209 \cdot \mathrm{I} / \mathrm{dec}\left({ }^{\circ} \mathrm{C}\right)$

254-Br-16-H: $\mathrm{Cr} \cdot 200 \cdot \mathrm{I}\left({ }^{\circ} \mathrm{C}\right)$

254-Br-18-H: $\mathrm{Cr} \cdot 153 \cdot \mathrm{SmA} \cdot 217 \cdot \mathrm{I} / \mathrm{dec}\left({ }^{\circ} \mathrm{C}\right)$

254-Br-20-H: $\mathrm{Cr} \cdot 141 \cdot \mathrm{SmA} \cdot 216 \cdot \mathrm{I} / \mathrm{dec}\left({ }^{\circ} \mathrm{C}\right)$

254-Br-22-H: $\mathrm{Cr} \cdot 149 \cdot \mathrm{SmA} \cdot 247 \cdot \mathrm{I} / \mathrm{dec}\left({ }^{\circ} \mathrm{C}\right)$

254-I-n-R $\left(\mathrm{X}^{-}=\mathrm{I}^{-}\right)$

254-I-16-OH: $\mathrm{Cr} \cdot 143 \cdot \mathrm{SmA} \cdot 181 \cdot \mathrm{I}\left({ }^{\circ} \mathrm{C}\right)$

254-I-18-OH: $\mathrm{Cr} \cdot 130 \cdot \mathrm{SmA} \cdot 184 \cdot \mathrm{I}\left({ }^{\circ} \mathrm{C}\right)$

254-I-18-OCH ${ }_{3}: \mathrm{Cr}_{1} \cdot 67 \cdot \mathrm{Cr}_{2} \cdot 245 \cdot \mathrm{I} / \mathrm{dec}\left({ }^{\circ} \mathrm{C}\right)$

254-I-18-H: $\mathrm{Cr} \cdot 189 \cdot \mathrm{I}\left({ }^{\circ} \mathrm{C}\right)$

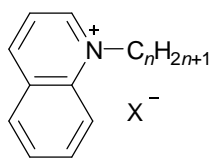

255-X-n $\left(\mathrm{X}^{-}=\mathrm{Br}^{-},\left[\mathrm{BF}_{4}\right]^{-},\left[\mathrm{PF}_{6}\right]^{-}\right.$,

$\left[\mathrm{C}_{12} \mathrm{H}_{25} \mathrm{OSO}_{3}\right]^{-}$, [DOSS] (for $n=1,4$ ),

[DcHSS] $]^{-}($for $n=1)$, [DHSS] $]^{-}($for $n=1)$ )

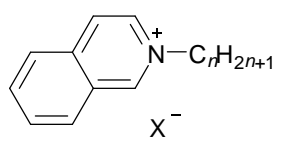

256-X-n $\left(\mathrm{X}^{-}=\mathrm{Br}^{-},\left[\mathrm{BF}_{4}\right]^{-},\left[\mathrm{PF}_{6}\right]^{-}\right.$,

$\left[\mathrm{C}_{12} \mathrm{H}_{25} \mathrm{OSO}_{3}\right]^{-}$, [DOSS]' (for $n=1,4$ ),

[DcHSS] ${ }^{-}$(for $\left.n=1\right)$, [DHSS] $($ for $n=1)$ )

255-Br-n $\left(\mathrm{X}^{-}=\mathrm{Br}^{-}\right)$(see also Figure S16 for a phase diagram)

255-Br-4: $\mathrm{Cr} \cdot 157 \cdot \mathrm{I}\left({ }^{\circ} \mathrm{C}\right)$

255-Br-12: $\mathrm{Cr} \cdot 102 \cdot \mathrm{I}\left({ }^{\circ} \mathrm{C}\right)$

255-Br-14: $\mathrm{Cr} \cdot 108 \cdot \mathrm{I}\left({ }^{\circ} \mathrm{C}\right)$ 
255-Br-16: $\mathrm{Cr} \cdot(\mathrm{SmA} \cdot 73 \cdot) 112 \cdot \mathrm{I}\left({ }^{\circ} \mathrm{C}\right)$

255-Br-17: $\mathrm{Cr}_{1} \cdot(\mathrm{SmA} \cdot 74 \cdot) 98 \cdot \mathrm{Cr}_{2} \cdot 117 \cdot \mathrm{I}\left({ }^{\circ} \mathrm{C}\right)$

255-Br-18: $\mathrm{Cr} \cdot 111 \cdot \mathrm{SmA} \cdot 169 \cdot \mathrm{I}\left({ }^{\circ} \mathrm{C}\right)$

255-Br-19: $\mathrm{Cr}_{1} \cdot 100 \cdot \mathrm{Cr}_{2} \cdot 105 \cdot \mathrm{Cr}_{3} \cdot 119 \cdot \mathrm{SmA} \cdot 197 \cdot \mathrm{I}\left({ }^{\circ} \mathrm{C}\right)$

255-Br-20: $\mathrm{Cr}_{1} \cdot 88 \cdot \mathrm{Cr}_{2} \cdot 111 \cdot \mathrm{SmA} \cdot 209 \cdot \mathrm{I}\left({ }^{\circ} \mathrm{C}\right)$

255-Br-22: $\mathrm{Cr}_{1} \cdot 71 \cdot \mathrm{Cr}_{2} \cdot 104 \cdot \mathrm{Cr}_{3} \cdot 116 \cdot \mathrm{SmA} \cdot 210 \cdot \mathrm{I}\left({ }^{\circ} \mathrm{C}\right)$

255-BF $\mathbf{H}_{\mathbf{-}} \boldsymbol{n}\left(\mathrm{X}^{-}=\left[\mathrm{BF}_{4}\right]^{-}\right)$

255- $-\mathrm{BF}_{4}-\mathbf{1 2}: \mathrm{Cr}_{1} \cdot 25 \cdot \mathrm{Cr}_{2} \cdot 47 \cdot \mathrm{I}\left({ }^{\circ} \mathrm{C}\right)$

255- $\mathrm{BF}_{\mathbf{4}}-14: \mathrm{Cr} \cdot 68 \cdot \mathrm{I}\left({ }^{\circ} \mathrm{C}\right)$

255-BF $-\mathbf{B}_{\mathbf{4}}$ 16: $\mathrm{Cr}_{1} \cdot 39 \cdot \mathrm{Cr}_{2} \cdot(\mathrm{SmA} \cdot 47 \cdot) 77 \cdot \mathrm{I}\left({ }^{\circ} \mathrm{C}\right)$

255-BF $-17: \mathrm{Cr}_{1} \cdot 33 \cdot \mathrm{Cr}_{2} \cdot(\mathrm{SmA} \cdot 60 \cdot) 85 \cdot \mathrm{I}\left({ }^{\circ} \mathrm{C}\right)$

255-BF $-18: \mathrm{Cr}_{1} \cdot 44 \cdot \mathrm{Cr}_{2} \cdot(\mathrm{SmA} \cdot 56 \cdot) 76 \cdot \mathrm{I}\left({ }^{\circ} \mathrm{C}\right)$

255-BF $-\mathbf{B F}_{\mathbf{4}}$ 19: $\mathrm{Cr}_{1} \cdot 42 \cdot \mathrm{Cr}_{2} \cdot 91 \cdot \mathrm{SmA} \cdot 121 \cdot \mathrm{I}\left({ }^{\circ} \mathrm{C}\right)$

255-BF $\mathbf{B}_{\mathbf{4}} \mathbf{2 0}: \mathrm{Cr}_{1} \cdot 50 \cdot \mathrm{Cr}_{2} \cdot 86 \cdot \mathrm{SmA} \cdot 153 \cdot \mathrm{I}\left({ }^{\circ} \mathrm{C}\right)$

255-BF $\mathbf{B}_{\mathbf{4}}$-22: $\mathrm{Cr}_{1} \cdot 58 \cdot \mathrm{Cr}_{2} \cdot 92 \cdot \mathrm{SmA} \cdot 190 \cdot \mathrm{I}\left({ }^{\circ} \mathrm{C}\right)$

255- $\mathbf{P F}_{\mathbf{6}}-\boldsymbol{n}\left(\mathrm{X}^{-}=\left[\mathrm{PF}_{6}\right]^{-}\right)$(see also Figure S16 for a phase diagram)

255- $\mathbf{P F}_{\mathbf{6}}-\mathbf{1 2}: \mathrm{Cr}_{1} \cdot 37 \cdot \mathrm{Cr}_{2} \cdot 69 \cdot \mathrm{I}\left({ }^{\circ} \mathrm{C}\right)$

255-PF $-\mathbf{F}_{\mathbf{6}}$ 14: $\mathrm{Cr}_{1} \cdot 22 \cdot \mathrm{Cr}_{2} \cdot 43 \cdot \mathrm{Cr}_{3} \cdot 68 \cdot \mathrm{Cr}_{4} \cdot 81 \cdot \mathrm{I}\left({ }^{\circ} \mathrm{C}\right)$

255-PF $-\mathbf{P F}_{\mathbf{6}} \mathbf{- 1 6} \mathrm{Cr}_{1} \cdot 55 \cdot \mathrm{Cr}_{2} \cdot 81 \cdot \mathrm{I}\left({ }^{\circ} \mathrm{C}\right)$

255- $\mathbf{P F}_{\mathbf{6}} \mathbf{- 1 7}: \mathrm{Cr}_{1} \cdot 54 \cdot \mathrm{Cr}_{2} \cdot 80 \cdot \mathrm{Cr}_{3} \cdot 86 \cdot \mathrm{I}\left({ }^{\circ} \mathrm{C}\right)$

255-PF $-\mathbf{P F}_{6} \mathbf{- 1 8} \mathrm{Cr}_{1} \cdot 74 \cdot \mathrm{Cr}_{2} \cdot 86 \cdot \mathrm{I}\left({ }^{\circ} \mathrm{C}\right)$

255-PF $-\mathbf{P F}_{6}$ 19: $\mathrm{Cr}_{1} \cdot 64 \cdot \mathrm{Cr}_{2} \cdot 87 \cdot \mathrm{I}\left({ }^{\circ} \mathrm{C}\right)$

255-PF $\mathbf{P F}_{\mathbf{6}}-\mathbf{2 0} \mathrm{Cr}_{1} \cdot 74 \cdot \mathrm{Cr}_{2} \cdot 90 \cdot \mathrm{SmA} \cdot 102 \cdot \mathrm{I}\left({ }^{\circ} \mathrm{C}\right)$

255-PF $\mathbf{~}_{\mathbf{6}}-22: \mathrm{Cr}_{1} \cdot 74 \cdot \mathrm{Cr}_{2} \cdot 90 \cdot \mathrm{SmA} \cdot 139 \cdot \mathrm{I}\left({ }^{\circ} \mathrm{C}\right)$

255- $\mathbf{C}_{12} \mathbf{H}_{25} \mathbf{O S O}_{3}-\boldsymbol{n}\left(\mathrm{X}^{-}=\left[\mathrm{C}_{12} \mathrm{H}_{25} \mathrm{OSO}_{3}\right]^{-}\right)$(see also Figure $\mathrm{S} 16$ for a phase diagram)

255- $\mathrm{C}_{12} \mathbf{H}_{25} \mathbf{O S O}_{3} \mathbf{- 1 2}: \mathrm{Cr} \cdot 87 \cdot \mathrm{SmA} \cdot 110 \cdot \mathrm{I}\left({ }^{\circ} \mathrm{C}\right)$

255- $\mathbf{C}_{12} \mathbf{H}_{25} \mathbf{O S O}_{3}-\mathbf{1 4}: \mathrm{Cr} \cdot 103 \cdot \mathrm{SmA} \cdot 127 \cdot \mathrm{I}\left({ }^{\circ} \mathrm{C}\right)$

255- $\mathrm{C}_{12} \mathbf{H}_{25} \mathbf{O S O}_{3}-16: \mathrm{Cr}_{1} \cdot 88 \cdot \mathrm{Cr}_{2} \cdot 97 \cdot \mathrm{SmA} \cdot 136 \cdot \mathrm{I}\left({ }^{\circ} \mathrm{C}\right)$

255- $\mathrm{C}_{12} \mathrm{H}_{25} \mathbf{O S O}_{3}-17: \mathrm{Cr}_{1} \cdot 80 \cdot \mathrm{Cr}_{2} \cdot 94 \cdot \mathrm{SmA} \cdot 139 \cdot \mathrm{I}\left({ }^{\circ} \mathrm{C}\right)$

255- $\mathrm{C}_{12} \mathbf{H}_{25} \mathbf{O S O}_{3}-18: \mathrm{Cr} \cdot 85 \cdot \mathrm{SmA} \cdot 142 \cdot \mathrm{I}\left({ }^{\circ} \mathrm{C}\right)$

255- $\mathrm{C}_{12} \mathbf{H}_{25} \mathrm{OSO}_{3}-\mathbf{1 9}: \mathrm{Cr}_{1} \cdot 75 \cdot \mathrm{Cr}_{2} \cdot 90 \cdot \mathrm{SmA} \cdot 143 \cdot \mathrm{I}\left({ }^{\circ} \mathrm{C}\right)$

255- $\mathrm{C}_{12} \mathrm{H}_{25} \mathrm{OSO}_{3}-\mathbf{2 0}: \mathrm{Cr} \cdot 84 \cdot \mathrm{SmA} \cdot 140 \cdot \mathrm{I}\left({ }^{\circ} \mathrm{C}\right)$

255- $\mathbf{C}_{12} \mathbf{H}_{25}$ OSO $_{3}-22: \mathrm{Cr} \cdot 87 \cdot \mathrm{SmA} \cdot 146 \cdot \mathrm{I}\left({ }^{\circ} \mathrm{C}\right)$

255-I-1 $\left(\mathrm{X}^{-}=\mathrm{I}^{-}, n=1\right): \mathrm{Cr} \cdot 144 \cdot \mathrm{I}\left({ }^{\circ} \mathrm{C}\right)$

255-DOSS-1 $\left(\mathrm{X}^{-}=[\mathrm{DOSS}]^{-}, n=1\right): \mathrm{SmA} \cdot 57 \cdot \mathrm{I}\left({ }^{\circ} \mathrm{C}\right)$

255-DOSS-4 ( $\left.\mathrm{X}^{-}=[\mathrm{DOSS}]^{-}, n=4\right): \mathrm{Cr} \cdot 59 \cdot \mathrm{I}\left({ }^{\circ} \mathrm{C}\right)$

255-DcHSS-1 ( $\mathrm{X}^{-}=\left[\mathrm{DcHSS}^{-}, n=1\right): \mathrm{Cr} \cdot 131 \cdot \mathrm{I}\left({ }^{\circ} \mathrm{C}\right)$

255-DHSS-1 ( $\left.\mathrm{X}^{-}=[\mathrm{DHSS}]^{-}, n=1\right): \mathrm{Cr} \cdot(\mathrm{SmA} \cdot 23 \cdot) 63 \cdot \mathrm{I}\left({ }^{\circ} \mathrm{C}\right)$ 


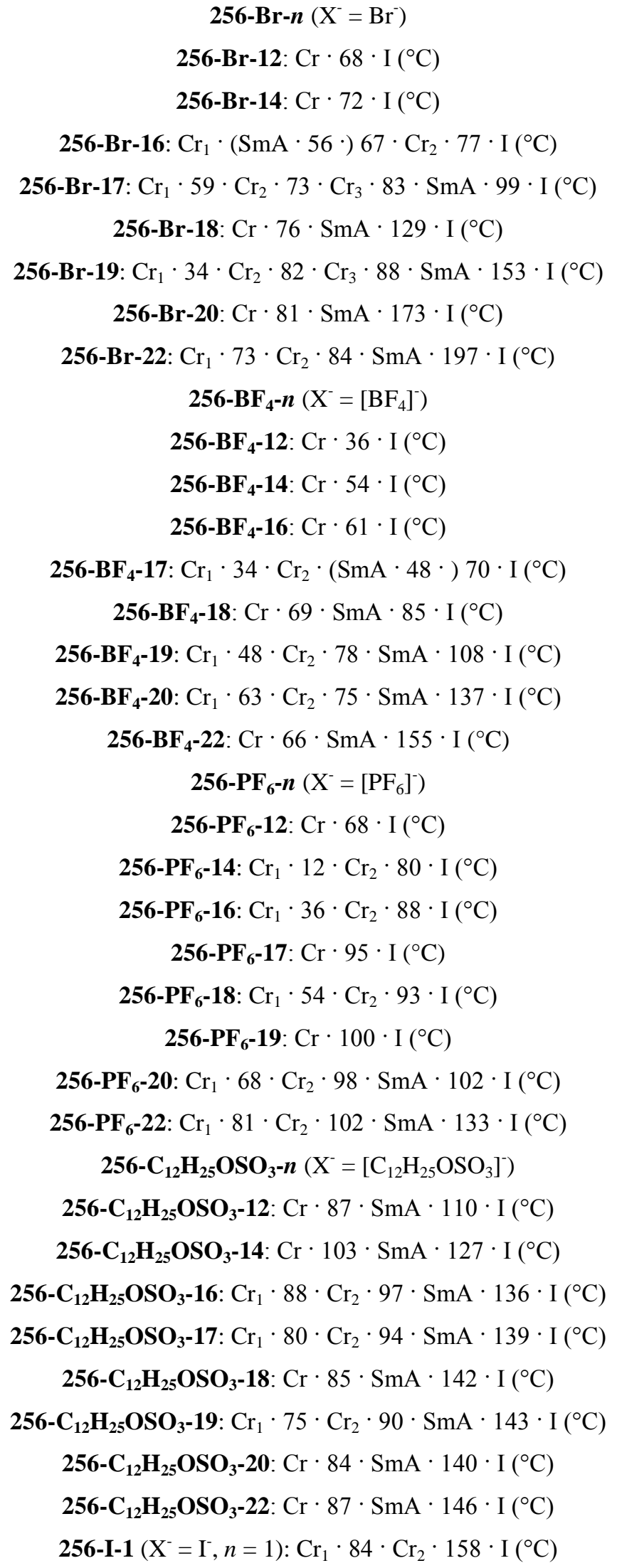


256-DOSS-1 ( $\left.\mathrm{X}^{-}=[\mathrm{DOSS}]^{-}, n=1\right): \mathrm{SmA} \cdot 35 \cdot \mathrm{I}\left({ }^{\circ} \mathrm{C}\right)$

256-DcHSS-1 $\left(\mathrm{X}^{-}=\right.$[DcHSS $\left.^{-}, n=1\right)$ : liquid at r.t.

256-DHSS-1 ( $\left.\mathrm{X}^{-}=[\mathrm{DHSS}]^{-}, n=1\right): \mathrm{Cr} \cdot 90 \cdot \mathrm{I}\left({ }^{\circ} \mathrm{C}\right)$
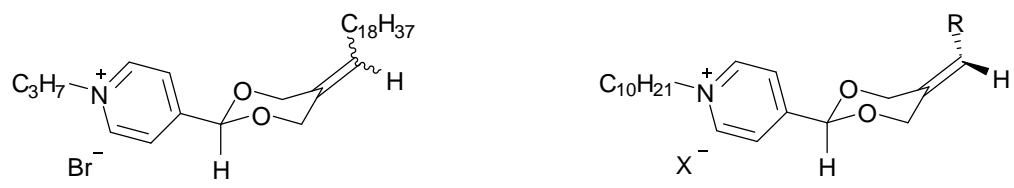

257 (racemic mixture)

258-X-R $\left(\mathrm{X}^{-}=\mathrm{Br}^{-}, \mathrm{I}^{-},\left[\mathrm{BF}_{4}\right]^{-},\left[\mathrm{PF}_{6}\right]^{-},\left[\mathrm{NTf}_{2}\right]^{-}\right.$;

$\mathrm{R}=\mathrm{Br}, \mathrm{C}_{6} \mathrm{H}_{5}, n-\mathrm{C}_{8} \mathrm{H}_{17}, n-\mathrm{C}_{18} \mathrm{H}_{37}$; both racemic mixtures and enantiopure products)

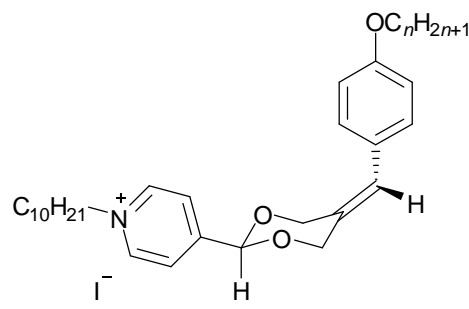

259-n (both racemic mixtures and an enantiopure product)

257 (racemic mixture): $\mathrm{Cr} \cdot 52 \cdot \mathrm{SmX} \cdot 149 \cdot \mathrm{I}\left({ }^{\circ} \mathrm{C}\right)$

258-Br-R $\left(\mathrm{X}^{-}=\mathrm{Br}^{-}\right)$

258-Br-Br (RS (racemic mixture)): $\mathrm{g} \cdot-23 \cdot \mathrm{I}\left({ }^{\circ} \mathrm{C}\right)$

258-Br-Br $(R): \mathrm{g} \cdot-23 \cdot \mathrm{I}\left({ }^{\circ} \mathrm{C}\right)$

258-Br- $\mathrm{C}_{8} \mathrm{H}_{17}$ (RS (racemic mixture)): $\mathrm{SmC} \cdot 55 \cdot \mathrm{I}\left({ }^{\circ} \mathrm{C}\right)$

258-I-R $\left(\mathrm{X}^{-}=\mathrm{I}^{-}\right)$

258-I-Br (RS (racemic mixture)): g $\cdot-20 \cdot \mathrm{I}\left({ }^{\circ} \mathrm{C}\right)$

258-I-Ph (RS (racemic mixture)): $\mathrm{g} \cdot-1 \cdot \mathrm{I}\left({ }^{\circ} \mathrm{C}\right)$

258-I-Ph $(R): \mathrm{g} \cdot-6 \cdot \mathrm{I}\left({ }^{\circ} \mathrm{C}\right)$

258-I- $\mathbf{C}_{8} \mathbf{H}_{17}\left(R S\right.$ (racemic mixture)): $\mathrm{SmX} \cdot 44 / 55 \cdot \mathrm{I}\left({ }^{\circ} \mathrm{C}\right)$

258-I- $\mathrm{C}_{8} \mathrm{H}_{17}(R): \mathrm{N}^{*} \cdot 35 / 40 \cdot \mathrm{I}\left({ }^{\circ} \mathrm{C}\right)$

258-I- $\mathbf{C}_{18} \mathbf{H}_{37}\left(R S\right.$ (racemic mixture)): $\mathrm{SmX} \cdot 41 \cdot \mathrm{N} \cdot 90 \cdot \mathrm{I} / \mathrm{dec} .\left({ }^{\circ} \mathrm{C}\right)$

258-I- $\mathbf{C}_{18} \mathbf{H}_{37}(R): \mathrm{SmX}_{1} \cdot 50 \cdot \mathrm{SmX}_{2} \cdot 90 \cdot \mathrm{I}\left({ }^{\circ} \mathrm{C}\right)$

258- $\mathbf{B F}_{4}-\mathbf{R}\left(\mathrm{X}^{-}=\left[\mathrm{BF}_{4}\right]^{-}\right)$

258- $-\mathrm{BF}_{4}-\mathrm{Br}\left(R S\right.$ (racemic mixture)): $\mathrm{g} \cdot-30 \cdot \mathrm{I}\left({ }^{\circ} \mathrm{C}\right)$

258- $\mathbf{B F}_{4}-\mathbf{C}_{8} \mathbf{H}_{17}\left(R S\right.$ (racemic mixture)): $\mathrm{g} \cdot-25 \cdot \mathrm{SmX} \cdot 92 \cdot \mathrm{I}\left({ }^{\circ} \mathrm{C}\right)$

258-PF $\mathbf{F}_{6}-\mathbf{R}\left(\mathrm{X}^{-}=\left[\mathrm{PF}_{6}\right]^{-}\right)$ 
258- $\mathbf{P F}_{6}-\mathbf{B r}\left(R S\right.$ (racemic mixture)): $\mathrm{g} \cdot-17 \cdot \mathrm{I}\left({ }^{\circ} \mathrm{C}\right)$

258- $\mathrm{PF}_{6}-\mathrm{C}_{8} \mathrm{H}_{17}\left(R S\right.$ (racemic mixture)): $\mathrm{g} \cdot-35 \cdot \mathrm{I}\left({ }^{\circ} \mathrm{C}\right)$

258-NTf ${ }_{2}-\mathbf{R}\left(\mathrm{X}^{-}=\left[\mathrm{NTf}_{2}\right]^{-}\right)$

258-NTf $2-B r\left(R S\right.$ (racemic mixture)): $\mathrm{g} \cdot-40 \cdot \mathrm{I}\left({ }^{\circ} \mathrm{C}\right)$

258-NTf $\mathbf{N}_{2}$-Br $(R): \mathrm{g} \cdot-39 \cdot \mathrm{I}\left({ }^{\circ} \mathrm{C}\right)$

258- $\mathrm{NTf}_{2}-\mathrm{C}_{8} \mathrm{H}_{17}$ (RS (racemic mixture)): $\mathrm{g} \cdot-43 \cdot \mathrm{I}\left({ }^{\circ} \mathrm{C}\right)$

259-1 (RS (racemic mixture)): g $\cdot 17 \cdot \mathrm{I}\left({ }^{\circ} \mathrm{C}\right)$

259-4 (RS (racemic mixture)): $\mathrm{N} \cdot 47 \cdot \mathrm{I}\left({ }^{\circ} \mathrm{C}\right)$

259-4 (R): $\mathrm{N}^{*} \cdot 39 / 45 \cdot \mathrm{I}\left({ }^{\circ} \mathrm{C}\right)$

259-8 (RS (racemic mixture)): $\mathrm{g} \cdot 22 \cdot \mathrm{SmX} \cdot 92 \cdot \mathrm{I}\left({ }^{\circ} \mathrm{C}\right)$

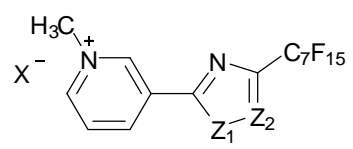

260-I-Z $\mathbf{Z}_{1} / \mathbf{Z}_{2}\left(\mathrm{X}^{-}=\mathrm{I}^{-}\right)$

260-I-O/N: $\mathrm{Cr} \cdot 97 \cdot \mathrm{SmA} \cdot 150 \cdot \mathrm{I}\left({ }^{\circ} \mathrm{C}\right)$

260-I-N/O: $\mathrm{Cr} \cdot 113 \cdot \mathrm{SmA} \cdot 135 \cdot \mathrm{I}\left({ }^{\circ} \mathrm{C}\right)$

260-OTf- $\mathbf{Z}_{1} / \mathbf{Z}_{2}\left(\mathrm{X}^{-}=[\mathrm{OTf}]^{-}\right)$

260-OTf-O/N: $\mathrm{Cr} \cdot 111 \cdot \mathrm{I}\left({ }^{\circ} \mathrm{C}\right)$

260-OTf-N/O: $\mathrm{Cr} \cdot 122 \cdot \mathrm{I}\left({ }^{\circ} \mathrm{C}\right)$

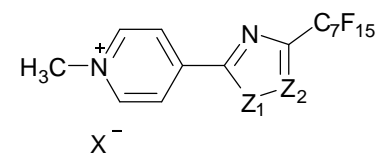

261-I-Z $/ Z_{1}\left(\mathrm{Z}^{-}=\mathrm{I}^{-}\right)$

261-I-O/N: $\mathrm{Cr}_{1} \cdot 74 \cdot \mathrm{Cr}_{2} \cdot 96 \cdot \mathrm{I}\left({ }^{\circ} \mathrm{C}\right)$

261-I-N/O: $\mathrm{Cr} \cdot 99 \cdot \mathrm{I}\left({ }^{\circ} \mathrm{C}\right)$

261-OTf- $Z_{1} / Z_{2}\left(X^{-}=[O T f]^{-}\right)$

261-OTf-O/N: $\mathrm{Cr}_{1} \cdot 84 \cdot \mathrm{Cr}_{2} \cdot 99 \cdot \mathrm{I}\left({ }^{\circ} \mathrm{C}\right)$

261-OTf-N/O: $\mathrm{Cr} \cdot 134 \cdot \mathrm{I}\left({ }^{\circ} \mathrm{C}\right)$

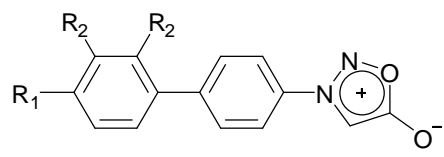

262-(a-m)

262-a $\left(\mathrm{R}_{1}=\mathrm{C}_{2} \mathrm{H}_{5}, \mathrm{R}_{2}=\mathrm{H}\right): \mathrm{Cr} \cdot 146 \cdot \mathrm{I}\left({ }^{\circ} \mathrm{C}\right)$ 
262-b $\left(\mathrm{R}_{1}=n-\mathrm{C}_{3} \mathrm{H}_{7}, \mathrm{R}_{2}=\mathrm{H}\right): \mathrm{Cr} \cdot(\mathrm{N} \cdot 92 \cdot) 125 \cdot \mathrm{I}\left({ }^{\circ} \mathrm{C}\right)$

262-c $\left(\mathrm{R}_{1}=n-\mathrm{C}_{4} \mathrm{H}_{9}, \mathrm{R}_{2}=\mathrm{H}\right): \mathrm{Cr} \cdot 120 \cdot \mathrm{I}\left({ }^{\circ} \mathrm{C}\right)$

262-d $\left(\mathrm{R}_{1}=n-\mathrm{C}_{5} \mathrm{H}_{11}, \mathrm{R}_{2}=\mathrm{H}\right): \mathrm{Cr} \cdot(\mathrm{SmA} \cdot 104 \cdot \mathrm{N} \cdot 110 \cdot) 114 \cdot \mathrm{I}\left({ }^{\circ} \mathrm{C}\right)$

262-e $\left(\mathrm{R}_{1}=n-\mathrm{C}_{6} \mathrm{H}_{13}, \mathrm{R}_{2}=\mathrm{H}\right): \mathrm{Cr} \cdot 107 \cdot \mathrm{SmA} \cdot 118 \cdot \mathrm{I}\left({ }^{\circ} \mathrm{C}\right)$

262-f $\left(\mathrm{R}_{1}=n-\mathrm{C}_{7} \mathrm{H}_{15}, \mathrm{R}_{2}=\mathrm{H}\right): \mathrm{Cr} \cdot 101 \cdot \mathrm{SmA} \cdot 135 \cdot \mathrm{I}\left({ }^{\circ} \mathrm{C}\right)$

262-g $\left(\mathrm{R}_{1}=n-\mathrm{C}_{8} \mathrm{H}_{17}, \mathrm{R}_{2}=\mathrm{H}\right): \mathrm{Cr} \cdot 99 \cdot \mathrm{SmA} \cdot 145 \cdot \mathrm{I}\left({ }^{\circ} \mathrm{C}\right)$

262-h $\left(\mathrm{R}_{1}=n-\mathrm{C}_{9} \mathrm{H}_{19}, \mathrm{R}_{2}=\mathrm{H}\right): \mathrm{Cr} \cdot 96 \cdot \mathrm{SmA} \cdot 156 \cdot \mathrm{I}\left({ }^{\circ} \mathrm{C}\right)$

262-i $\left(\mathrm{R}_{1}=n-\mathrm{C}_{10} \mathrm{H}_{21}, \mathrm{R}_{2}=\mathrm{H}\right): \mathrm{Cr} \cdot 98 \cdot \mathrm{SmA} \cdot 159 \cdot \mathrm{I}\left({ }^{\circ} \mathrm{C}\right)$

262-j $\left(\mathrm{R}_{1}=\mathrm{OC}_{8} \mathrm{H}_{17}, \mathrm{R}_{2}=\mathrm{F}\right): \mathrm{Cr} \cdot 90 \cdot \mathrm{SmA} \cdot 136 \cdot \mathrm{I}\left({ }^{\circ} \mathrm{C}\right)$

262-k $\left(\mathrm{R}_{1}=\mathrm{OC}_{10} \mathrm{H}_{21}, \mathrm{R}_{2}=\mathrm{F}\right): \mathrm{Cr} \cdot 92 \cdot \mathrm{SmA} \cdot 145 \cdot \mathrm{I}\left({ }^{\circ} \mathrm{C}\right)$

262-I $\left(\mathrm{R}_{1}=(1 S)-1\right.$-methylheptyloxy, $\left.\mathrm{R}_{2}=\mathrm{F}\right): \mathrm{Cr} \cdot\left(\mathrm{SmX}^{*} \cdot 49 \cdot\right) 51 \cdot \mathrm{I}\left({ }^{\circ} \mathrm{C}\right)$

262-m $\left(\mathrm{R}_{1}=(1 R)-1\right.$-methylheptyloxy, $\left.\mathrm{R}_{2}=\mathrm{F}\right): \mathrm{Cr} \cdot 48 \cdot \mathrm{I}\left({ }^{\circ} \mathrm{C}\right)$

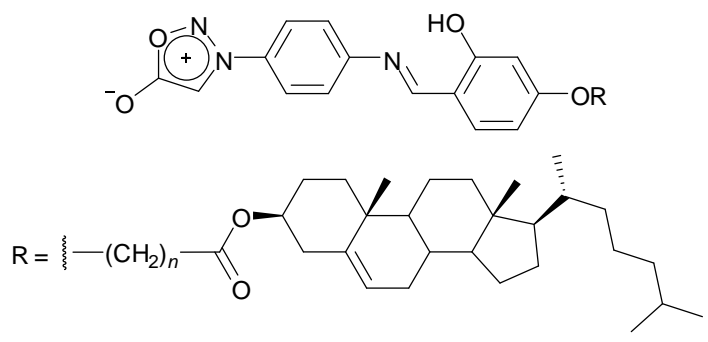

263-n

263-3: $\mathrm{Cr} \cdot 230 \cdot \mathrm{SmA} * 265 \cdot \mathrm{I} /$ dec. $\left({ }^{\circ} \mathrm{C}\right)$

263-4: $\mathrm{Cr} \cdot 162 \cdot \mathrm{SmA}^{*} \cdot 210 \cdot \mathrm{I} /$ dec. $\left({ }^{\circ} \mathrm{C}\right)$

263-5: $\mathrm{Cr} \cdot 170 \cdot \mathrm{SmA}^{*} \cdot 215 \cdot \mathrm{I} /$ dec. $\left({ }^{\circ} \mathrm{C}\right)$

263-7: $\mathrm{Cr} \cdot 242 \cdot \mathrm{SmA}^{*} \cdot 270 \cdot \mathrm{I} /$ dec $\left({ }^{\circ} \mathrm{C}\right)$

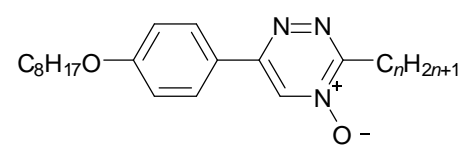

264-n

264-3: $\mathrm{Cr}_{1} \cdot 85 \cdot \mathrm{Cr}_{2} \cdot 95 \cdot \mathrm{SmA} \cdot 120 \cdot \mathrm{I}\left({ }^{\circ} \mathrm{C}\right)$

264-5: $\mathrm{Cr}_{1} \cdot 89 \cdot \mathrm{Cr}_{2} \cdot 104 \cdot \mathrm{SmA} \cdot 121 \cdot \mathrm{I}\left({ }^{\circ} \mathrm{C}\right)$

264-6: $\mathrm{Cr}_{1} \cdot 77 \cdot \mathrm{Cr}_{2} \cdot 100 \cdot \mathrm{SmC} \cdot 110 \cdot \mathrm{SmA} \cdot 121 \cdot \mathrm{I}\left({ }^{\circ} \mathrm{C}\right)$

264-7: $\mathrm{Cr}_{1} \cdot 72 \cdot \mathrm{Cr}_{2} \cdot 95 \cdot \mathrm{SmC} \cdot 116 \cdot \mathrm{SmA} \cdot 124 \cdot \mathrm{I}\left({ }^{\circ} \mathrm{C}\right)$

264-8: $\mathrm{Cr} \cdot 97 \cdot \mathrm{SmC} \cdot 117 \cdot \mathrm{SmA} \cdot 123 \cdot \mathrm{I}\left({ }^{\circ} \mathrm{C}\right)$ 


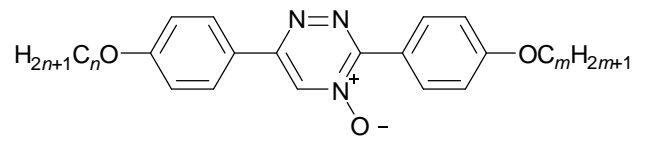

\section{5-n/m}

265-4/4: $\mathrm{Cr} \cdot 131 \cdot \mathrm{SmC} \cdot 186 \cdot \mathrm{N} \cdot 233 \cdot \mathrm{I}\left({ }^{\circ} \mathrm{C}\right)$

265-4/8: $\mathrm{Cr} \cdot 100 \cdot \mathrm{SmC} \cdot 206 \cdot \mathrm{N} \cdot 225 \cdot \mathrm{I}\left({ }^{\circ} \mathrm{C}\right)$

265-4/12: $\mathrm{Cr} \cdot 86 \cdot \mathrm{SmC} \cdot 215 \cdot \mathrm{I}\left({ }^{\circ} \mathrm{C}\right)$

265-8/2: $\mathrm{Cr} \cdot 129 \cdot \mathrm{SmC} \cdot 204 \cdot \mathrm{N} \cdot 230 \cdot \mathrm{I}\left({ }^{\circ} \mathrm{C}\right)$

265- 8/4: $\mathrm{Cr} \cdot 101 \cdot \mathrm{SmC} \cdot 215 \cdot \mathrm{N} \cdot 222 \cdot \mathrm{I}\left({ }^{\circ} \mathrm{C}\right)$

265-8/6: $\mathrm{Cr} \cdot 94 \cdot \mathrm{SmC} \cdot 219 \cdot \mathrm{I}\left({ }^{\circ} \mathrm{C}\right)$

265-8/8: $\mathrm{Cr} \cdot 89 \cdot \mathrm{SmC} \cdot 213 \cdot \mathrm{I}\left({ }^{\circ} \mathrm{C}\right)$

265-8/10: $\mathrm{Cr} \cdot 93 \cdot \mathrm{SmC} \cdot 214 \cdot \mathrm{I}\left({ }^{\circ} \mathrm{C}\right)$

265-8/12: $\mathrm{Cr} \cdot 93 \cdot \mathrm{SmC} \cdot 208 \cdot \mathrm{I}\left({ }^{\circ} \mathrm{C}\right)$

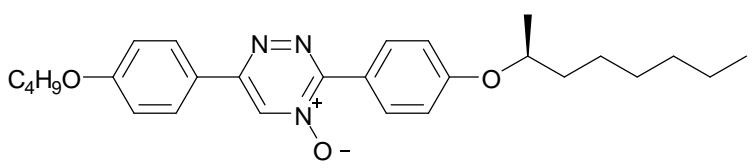

266: $\mathrm{Cr} \cdot 98 \cdot \mathrm{SmC}^{*} \cdot 140 \cdot \mathrm{SmA} \cdot 156 \cdot \mathrm{I}\left({ }^{\circ} \mathrm{C}\right)$

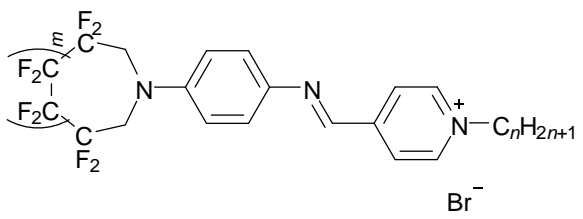

\section{$267-m / n$}

267-0/14: $\mathrm{Cr} \cdot 87 \cdot \mathrm{M} \cdot 156 \cdot \mathrm{I} \cdot 232 \cdot \operatorname{dec}\left({ }^{\circ} \mathrm{C}\right)$

267-0/16: $\mathrm{Cr} \cdot 90 \cdot \mathrm{M} \cdot 159 \cdot \mathrm{SmA} \cdot 175 \cdot \mathrm{I} \cdot 233 \cdot \mathrm{dec} .\left({ }^{\circ} \mathrm{C}\right)$

267-0/18: $\mathrm{Cr} \cdot 95 \cdot \mathrm{M} \cdot 154 \cdot \mathrm{SmA} \cdot 193 \cdot \mathrm{I} \cdot 205 \cdot \operatorname{dec}\left({ }^{\circ} \mathrm{C}\right)$

267-1/14: $\mathrm{Cr} \cdot 80 \cdot \mathrm{I}\left({ }^{\circ} \mathrm{C}\right)$

267-1/16: $\mathrm{Cr} \cdot 80 \cdot \mathrm{I}\left({ }^{\circ} \mathrm{C}\right)$

267-1/18: $\mathrm{Cr} \cdot 84 \cdot \mathrm{I}\left({ }^{\circ} \mathrm{C}\right)$ 

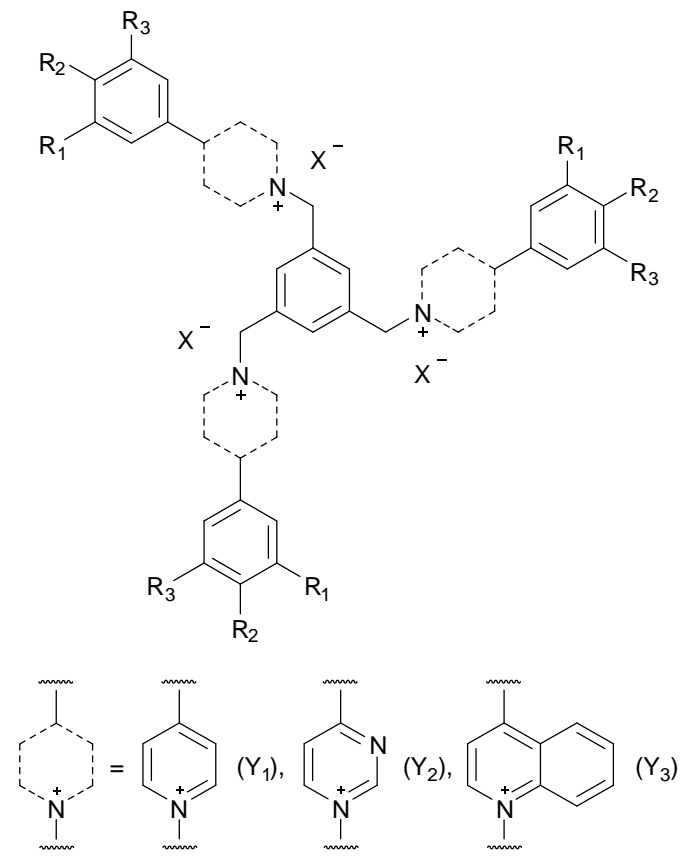

268-Br-Y-Y $-\mathbf{a}\left(\mathrm{X}^{-}=\mathrm{Br}^{-}, \mathrm{R}_{1}=\mathrm{R}_{2}=\mathrm{R}_{3}=\mathrm{OC}_{12} \mathrm{H}_{25}\right): \mathrm{Cr} \cdot 77 \cdot \mathrm{Col}_{\mathrm{h}} \cdot 179 \cdot \mathrm{Cub}_{\mathrm{I}}(\mathrm{Pm} \overline{3} n) \cdot 245 \cdot \mathrm{I}\left({ }^{\circ} \mathrm{C}\right)$

268-BF ${ }_{4}-\mathrm{Y}_{1}-\mathbf{a}\left(\mathrm{X}^{-}=\left[\mathrm{BF}_{4}\right]^{-}, \mathrm{R}_{1}=\mathrm{R}_{2}=\mathrm{R}_{3}=\mathrm{OC}_{12} \mathrm{H}_{25}\right): \mathrm{Cr} \cdot-3 \cdot \mathrm{Col}_{\mathrm{h}} \cdot 160 \cdot \mathrm{I}\left({ }^{\circ} \mathrm{C}\right)$

$$
\text { 268-PF } \mathbf{F}_{\mathbf{6}}-\mathrm{Y}_{\boldsymbol{n}}-\mathbf{( a - c )}\left(\mathrm{X}^{-}=\left[\mathrm{PF}_{6}\right]^{-}\right)
$$

268- $\mathrm{PF}_{6}-\mathrm{Y}_{\mathbf{1}}-\mathbf{a}\left(\mathrm{R}_{1}=\mathrm{R}_{2}=\mathrm{R}_{3}=\mathrm{OC}_{12} \mathrm{H}_{25}\right): \mathrm{Cr}_{1} \cdot 13 \cdot \mathrm{Cr}_{2} \cdot 24 \cdot \mathrm{Col}_{\mathrm{r}} \cdot 245 \cdot \mathrm{I}\left({ }^{\circ} \mathrm{C}\right)$

268- $-\mathrm{PF}_{6}-\mathrm{Y}_{1}-\mathbf{b}\left(\mathrm{R}_{1}=\mathrm{R}_{2}=\mathrm{OC}_{12} \mathrm{H}_{25}, \mathrm{R}_{3}=\mathrm{H}\right): \mathrm{Cr} \cdot 48 \cdot \mathrm{Col}_{\mathrm{r}} \cdot 120 \cdot \mathrm{Col} \cdot 240 \cdot \mathrm{I}\left({ }^{\circ} \mathrm{C}\right)$

268- $\mathbf{P F}_{6}-\mathrm{Y}_{1}-\mathbf{C}\left(\mathrm{R}_{1}=\mathrm{R}_{3}=\mathrm{H}, \mathrm{R}_{3}=\mathrm{N}\left(\mathrm{C}_{12} \mathrm{H}_{25}\right)_{2}\right): \mathrm{Cr} \cdot-30 \cdot \mathrm{Col}_{\mathrm{r}} \cdot 105 \cdot \mathrm{Cub} \cdot 180 \cdot \mathrm{I}\left({ }^{\circ} \mathrm{C}\right)$

268- $\mathrm{PF}_{6}-\mathrm{Y}_{2}-\mathbf{a}\left(\mathrm{R}_{1}=\mathrm{R}_{2}=\mathrm{R}_{3}=\mathrm{OC}_{12} \mathrm{H}_{25}\right): \mathrm{Cr} \cdot-32 \cdot \mathrm{Col}_{\mathrm{r}} \cdot 240 \cdot \mathrm{I}\left({ }^{\circ} \mathrm{C}\right)$

268- $\mathrm{PF}_{6}-\mathrm{Y}_{2}-\mathbf{b}\left(\mathrm{R}_{1}=\mathrm{R}_{2}=\mathrm{OC}_{12} \mathrm{H}_{25}, \mathrm{R}_{3}=\mathrm{H}\right): \mathrm{Cr} \cdot 86 \cdot \mathrm{Col}_{\mathrm{r}} \cdot 210 \cdot \mathrm{I}\left({ }^{\circ} \mathrm{C}\right)$

268- $\mathbf{P F}_{6}-\mathrm{Y}_{2}-\mathbf{c}\left(\mathrm{R}_{1}=\mathrm{R}_{3}=\mathrm{H}, \mathrm{R}_{3}=\mathrm{N}\left(\mathrm{C}_{12} \mathrm{H}_{25}\right)_{2}\right): \mathrm{g} \cdot-10 \cdot \mathrm{Col}_{\mathrm{h}} \cdot 176 \cdot \mathrm{I}\left({ }^{\circ} \mathrm{C}\right)$

268- $\mathrm{PF}_{6}-\mathrm{Y}_{3}-\mathbf{a}\left(\mathrm{R}_{1}=\mathrm{R}_{2}=\mathrm{R}_{3}=\mathrm{OC}_{12} \mathrm{H}_{25}\right): \mathrm{Cr} \cdot-37 \cdot \mathrm{Col}_{\mathrm{h}} \cdot 223 \cdot \mathrm{I}\left({ }^{\circ} \mathrm{C}\right)$

268- $\mathbf{P F}_{6}-\mathrm{Y}_{3}-\mathbf{b}\left(\mathrm{R}_{1}=\mathrm{R}_{2}=\mathrm{OC}_{12} \mathrm{H}_{25}, \mathrm{R}_{3}=\mathrm{H}\right): \mathrm{Cr} \cdot 56 \cdot \mathrm{Col}_{\mathrm{r}} \cdot 191 \cdot \mathrm{I}\left({ }^{\circ} \mathrm{C}\right)$

268- $\mathbf{P F}_{6}-\mathrm{Y}_{3}-\mathbf{c}\left(\mathrm{R}_{1}=\mathrm{R}_{3}=\mathrm{H}, \mathrm{R}_{3}=\mathrm{N}\left(\mathrm{C}_{12} \mathrm{H}_{25}\right)_{2}\right): \mathrm{g} \cdot 79 \cdot \mathrm{Col}_{\mathrm{h}} \cdot 210 \cdot \mathrm{I}\left({ }^{\circ} \mathrm{C}\right)$ 


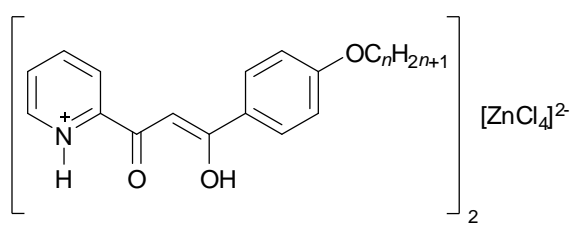

269-n

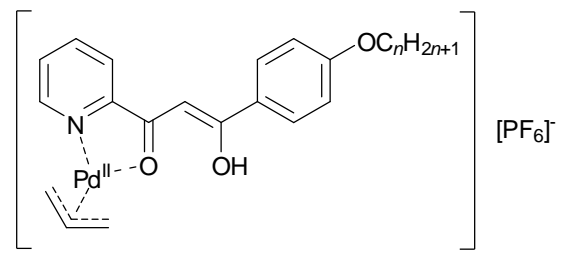

271-n

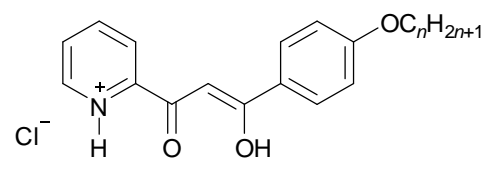

270-n

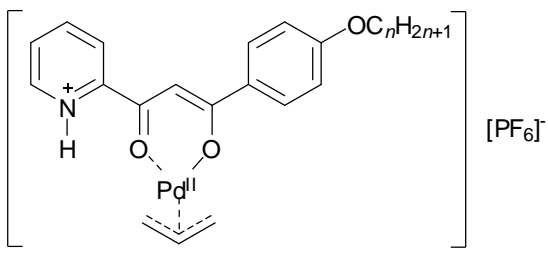

272-n

269-10: $\mathrm{Cr}_{1} \cdot 77 \cdot \mathrm{Cr}_{2} \cdot 100 \cdot \mathrm{X} \cdot 127 \cdot \mathrm{SmA} \cdot 224 \cdot \mathrm{I} / \mathrm{dec}\left({ }^{\circ} \mathrm{C}\right)$

269-12: $\mathrm{Cr} \cdot 95 \cdot \mathrm{X} \cdot 130 \cdot \mathrm{SmA} 230 \cdot \mathrm{I} / \mathrm{dec}\left({ }^{\circ} \mathrm{C}\right)$

269-14: $\mathrm{Cr}_{1} \cdot 62 \cdot \mathrm{Cr}_{2} \cdot 94 \cdot \mathrm{X} \cdot 111 \cdot \mathrm{SmA} \cdot 255 \cdot \mathrm{I} / \mathrm{dec}\left({ }^{\circ} \mathrm{C}\right)$

269-16: $\mathrm{Cr}_{1} \cdot 77 \cdot \mathrm{Cr}_{2} \cdot 97 \cdot \mathrm{X} \cdot 127 \cdot \mathrm{SmA} \cdot 233 \cdot \mathrm{I} / \mathrm{dec}\left({ }^{\circ} \mathrm{C}\right)$

269-18: $\mathrm{Cr}_{1} \cdot 88 \cdot \mathrm{Cr}_{2} \cdot 109 \cdot \mathrm{X} \cdot 118 \cdot \mathrm{SmA} \cdot 226 \cdot \mathrm{I} / \mathrm{dec}\left({ }^{\circ} \mathrm{C}\right)$

270-10: $\mathrm{Cr}_{1} \cdot 65 \cdot \mathrm{Cr}_{2} \cdot 149 \cdot \mathrm{I}\left({ }^{\circ} \mathrm{C}\right)$

270-12: $\mathrm{Cr}_{1} \cdot 56 \cdot \mathrm{Cr}_{2} \cdot 79 \cdot \mathrm{Cr}_{3} \cdot 149 \cdot \mathrm{I}\left({ }^{\circ} \mathrm{C}\right)$

270-14: $\mathrm{Cr}_{1} \cdot 64 \cdot \mathrm{Cr}_{2} \cdot 75 \cdot \mathrm{Cr}_{3} \cdot 148 \cdot \mathrm{I}\left({ }^{\circ} \mathrm{C}\right)$

270-16: $\mathrm{Cr}_{1} \cdot 70 \cdot \mathrm{Cr}_{2} \cdot 79 \cdot \mathrm{Cr}_{3} \cdot 144 \cdot \mathrm{I}\left({ }^{\circ} \mathrm{C}\right)$

270-18: $\mathrm{Cr}_{1} \cdot 56 \cdot \mathrm{Cr}_{2} \cdot 75 \cdot \mathrm{Cr}_{3} \cdot 132 \cdot \mathrm{I}\left({ }^{\circ} \mathrm{C}\right)$

271-12: $\mathrm{Cr} \cdot 180 \cdot \mathrm{I}\left({ }^{\circ} \mathrm{C}\right)$

271-14: $\mathrm{Cr}_{1} \cdot 76 \cdot \mathrm{Cr}_{2} \cdot 173 \cdot \mathrm{I}\left({ }^{\circ} \mathrm{C}\right)$

271-16: $\mathrm{Cr}_{1} \cdot 90 \cdot \mathrm{Cr}_{2} \cdot 155 \cdot \mathrm{SmC} \cdot 172 \cdot \mathrm{I}\left({ }^{\circ} \mathrm{C}\right)$

271-18: $\mathrm{Cr}_{1} \cdot 87 \cdot \mathrm{Cr}_{2} \cdot 144 \cdot \mathrm{SmC} \cdot 153 \cdot \mathrm{I}\left({ }^{\circ} \mathrm{C}\right)$

272-12: $\mathrm{Cr} \cdot 174 \cdot \mathrm{I}\left({ }^{\circ} \mathrm{C}\right)$

272-14: $\mathrm{Cr}_{1} \cdot 84 \cdot \mathrm{Cr}_{2} \cdot 158 \cdot \mathrm{SmC} \cdot 170 \cdot \mathrm{I}\left({ }^{\circ} \mathrm{C}\right)$

272-16: $\mathrm{Cr}_{1} \cdot 78 \cdot \mathrm{Cr}_{2} \cdot 107 \cdot \mathrm{SmC} \cdot 160 \cdot \mathrm{I}\left({ }^{\circ} \mathrm{C}\right)$

272-18: $\mathrm{Cr} \cdot 94 \cdot \mathrm{SmC} \cdot 144 \cdot \mathrm{I}\left({ }^{\circ} \mathrm{C}\right)$ 
Scheme S14. ILCs based on 1,10-disubstituted [closo-1- $\left.\mathrm{CB}_{9} \mathrm{H}_{8}\right]^{-}$and 1,12-disubstituted [closo-1- $\left.\mathrm{CB}_{11} \mathrm{H}_{10}\right]^{-}$clusters, investigated by Kaszynski and co-workers.

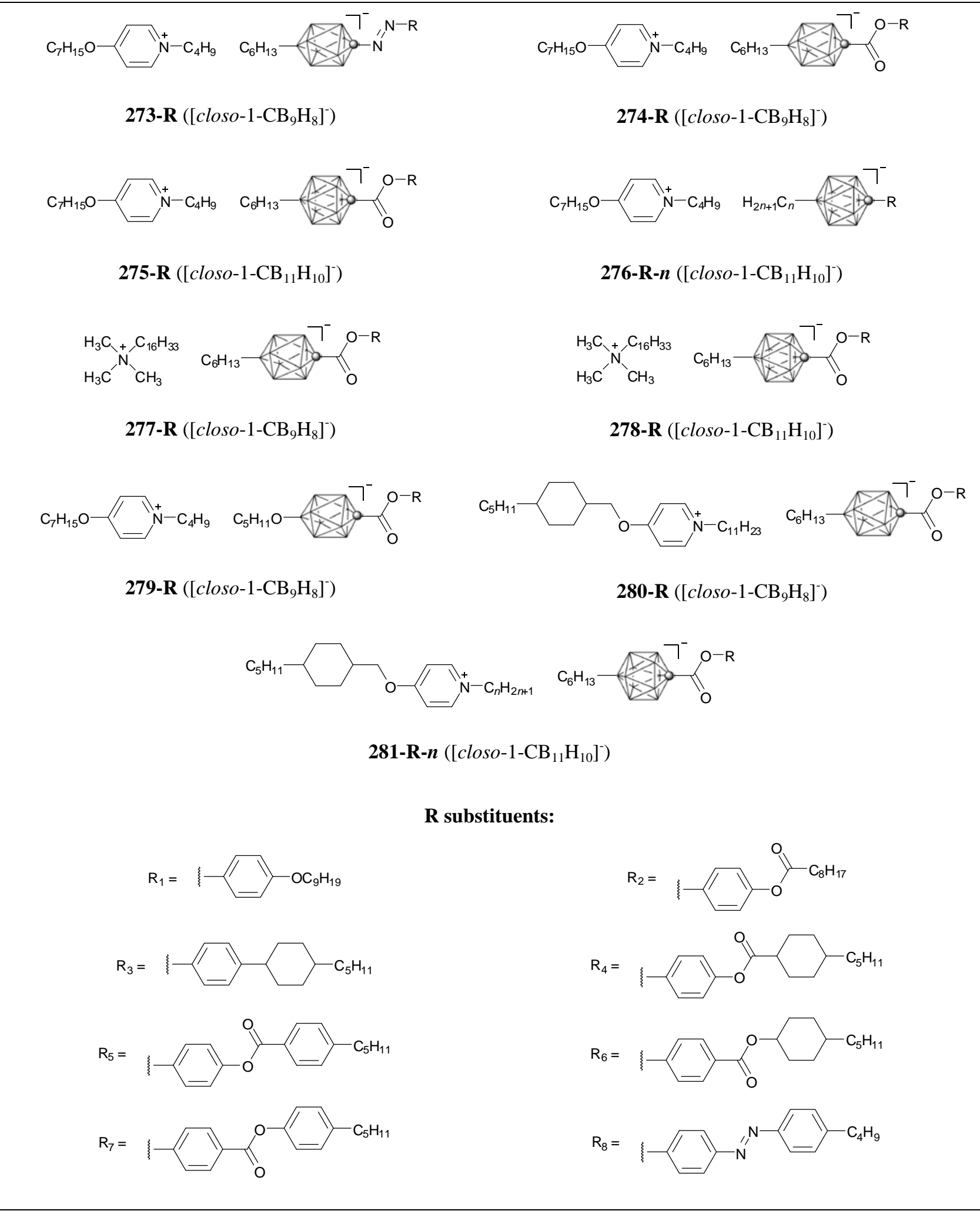



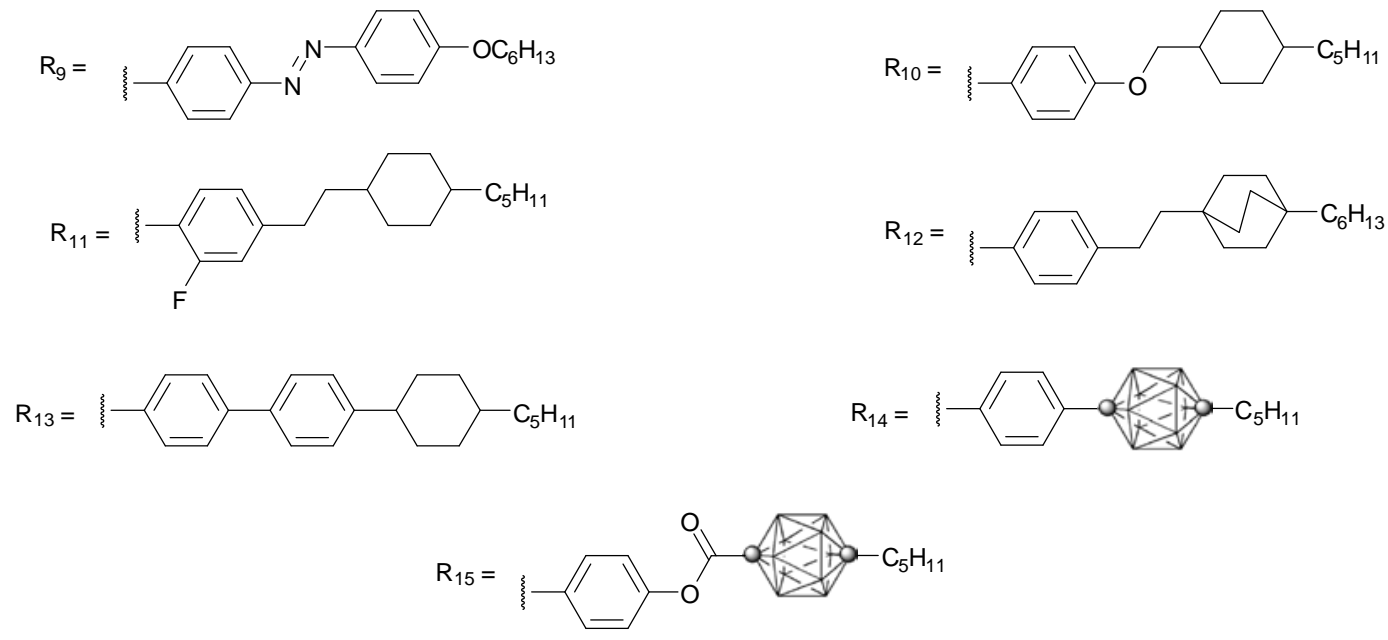

273-R $\mathbf{1}: \mathrm{Cr} \cdot 93 \cdot \mathrm{SmA} \cdot 115 \cdot \mathrm{I}\left({ }^{\circ} \mathrm{C}\right)$

273-R $: \mathbf{C r} \cdot 96 \cdot \mathrm{I}\left({ }^{\circ} \mathrm{C}\right)$

273- $\mathbf{R}_{4}: \mathrm{Cr}_{1} \cdot 70 \cdot \mathrm{Cr}_{2} \cdot 106 \cdot \mathrm{SmA} \cdot 139 \cdot \mathrm{I}\left({ }^{\circ} \mathrm{C}\right)$

273-R $\mathbf{R}_{15}: \mathrm{Cr} \cdot 136 \cdot \mathrm{SmA} \cdot 151 \cdot \mathrm{I}\left({ }^{\circ} \mathrm{C}\right)$

274-R $\mathrm{R}_{1}: \mathrm{Cr}_{1} \cdot 53 \cdot \mathrm{Cr}_{2} \cdot 127 \cdot \mathrm{Cr}_{3} \cdot 151 \cdot \mathrm{I}\left({ }^{\circ} \mathrm{C}\right)$

274-R $\mathrm{R}_{2}: \mathrm{Cr}_{1} \cdot 64 \cdot \mathrm{Cr}_{2} \cdot 101 \cdot \mathrm{M} \cdot 107 \cdot \mathrm{I}\left({ }^{\circ} \mathrm{C}\right)$

274- $\mathbf{R}_{3}: \mathrm{Cr} \cdot 128 \cdot\left(\mathrm{M}_{1} \cdot 129 \cdot\right) \mathrm{M}_{2} \cdot 153 \cdot \mathrm{SmA} \cdot 163 \cdot \mathrm{I}\left({ }^{\circ} \mathrm{C}\right)$

274-R $\mathbf{R}_{4}: \mathrm{Cr} \cdot 102 \cdot \mathrm{SmA} \cdot 164 \cdot \mathrm{I}\left({ }^{\circ} \mathrm{C}\right)$

274- $\mathbf{R}_{\mathbf{8}}: \mathbf{C r} \cdot 131 \cdot \mathrm{I}$

274-R $\mathbf{R}_{\mathbf{9}} \mathrm{Cr}_{1} \cdot 32 \cdot \mathrm{Cr}_{2} \cdot 123 \cdot \mathrm{SmA} \cdot 132 \cdot \mathrm{N} \cdot 137 \cdot \mathrm{I}\left({ }^{\circ} \mathrm{C}\right)$

274-R $\mathbf{R}_{11}: \mathrm{Cr} \cdot 87 \cdot \mathrm{SmA} \cdot 148 \cdot \mathrm{I}\left({ }^{\circ} \mathrm{C}\right)$

274-R $\mathbf{R}_{12}: \mathrm{Cr} \cdot 123 \cdot \mathrm{M}_{1} \cdot 129 \cdot \mathrm{M}_{2} \cdot 132 \cdot \mathrm{SmA} \cdot 189 \cdot \mathrm{I}\left({ }^{\circ} \mathrm{C}\right)$

274-R $\mathrm{R}_{14}: \mathrm{Cr} \cdot 120 \cdot \mathrm{M}_{1} \cdot 145 \cdot \mathrm{M}_{2} \cdot 166 \cdot \mathrm{SmA} \cdot 187 \cdot \mathrm{I}\left({ }^{\circ} \mathrm{C}\right)$

274-R $\mathrm{R}_{15}: \mathrm{Cr}_{1} \cdot 94 \cdot \mathrm{M} \cdot 162 \cdot \mathrm{SmA} \cdot 180 \cdot \mathrm{I}\left({ }^{\circ} \mathrm{C}\right)$

275- $\mathbf{R}_{2}: \mathrm{Cr} \cdot 117 \cdot \mathrm{I}\left({ }^{\circ} \mathrm{C}\right)$

275- $\mathbf{R}_{3}: \mathrm{Cr}_{1} \cdot 162 \cdot \mathrm{Cr}_{2} \cdot 178 \cdot \mathrm{I}\left({ }^{\circ} \mathrm{C}\right)$

275-R $\mathbf{R}_{4}: \mathrm{Cr}_{1} \cdot 109 \cdot \mathrm{Cr}_{2} \cdot 119 \cdot \mathrm{SmA} \cdot 161 \cdot \mathrm{I}\left({ }^{\circ} \mathrm{C}\right)$

275-R $\mathbf{R}: \mathbf{C r} \cdot(\mathrm{SmA} \cdot 115 \cdot) 125 \cdot \mathrm{I}\left({ }^{\circ} \mathrm{C}\right)$

275-R $: \mathrm{Cr} \cdot(\mathrm{SmX} \cdot 87 \cdot \mathrm{N} \cdot 93 \cdot) 104 \cdot \mathrm{I}\left({ }^{\circ} \mathrm{C}\right)$

275-R $\mathbf{8}: \mathbf{C r} \cdot 155 \cdot \mathrm{I}$

275-R $\mathbf{g}: \mathrm{Cr} \cdot(\mathrm{SmA} \cdot 133 \cdot) 134 \cdot \mathrm{N} \cdot 144 \cdot \mathrm{I}\left({ }^{\circ} \mathrm{C}\right)$

275-R $\mathbf{R}_{11}: \mathrm{Cr} \cdot 99 \cdot \mathrm{SmA} \cdot 146 \cdot \mathrm{I}\left({ }^{\circ} \mathrm{C}\right)$

275-R $\mathbf{R}_{\mathbf{1 2}}: \mathrm{Cr} \cdot 150 \cdot \mathrm{SmA} \cdot 182 \cdot \mathrm{I}\left({ }^{\circ} \mathrm{C}\right)$

276-R $\mathbf{- 6}$ : $\mathrm{Cr} \cdot 136 \cdot \mathrm{SmA} \cdot 139 \cdot \mathrm{I}\left({ }^{\circ} \mathrm{C}\right)$ 


$$
\begin{aligned}
& \text { 276-R } \mathbf{R}_{5}-\mathbf{6}: \mathrm{Cr} \cdot(\mathrm{SmA} \cdot 83 \cdot) 109 \cdot \mathrm{I}\left({ }^{\circ} \mathrm{C}\right) \\
& \text { 276-R } \mathbf{R}_{\mathbf{6}} \mathbf{- 6}: \mathrm{Cr} \cdot(\mathrm{SmA} \cdot 92 \cdot) 108 \cdot \mathrm{I}\left({ }^{\circ} \mathrm{C}\right) \\
& \text { 276-R }-6: \mathbf{C r} \cdot(\mathrm{SmA} \cdot 75 \cdot) 92 \cdot \mathrm{I}\left({ }^{\circ} \mathrm{C}\right) \\
& \text { 276-R } \mathbf{1 0}^{-6} \mathbf{6}: \mathrm{Cr} \cdot 105 \cdot \mathrm{SmA} \cdot 141 \cdot \mathrm{I}\left({ }^{\circ} \mathrm{C}\right) \\
& \text { 276-R } \mathbf{6}-12: \mathrm{Cr} \cdot 71 \cdot \mathrm{SmA} \cdot 137 \cdot \mathrm{I}\left({ }^{\circ} \mathrm{C}\right) \\
& \text { 277-R } \mathrm{R}_{11}: \mathrm{Cr}_{1} \cdot 64 \cdot \mathrm{Cr}_{2} \cdot 169 \cdot \mathrm{I}\left({ }^{\circ} \mathrm{C}\right) \\
& \text { 277- } \mathbf{R}_{13}: \mathrm{Cr} \cdot<20 \cdot \mathrm{M} \cdot 184 \cdot \mathrm{SmA} \cdot 229 \cdot \mathrm{I}\left({ }^{\circ} \mathrm{C}\right) \\
& \text { 278-R } \mathrm{R}_{13}: \mathrm{Cr} \cdot 36 \cdot \mathrm{M}_{1} \cdot 211 \cdot \mathrm{M}_{2} \cdot 216 \cdot \mathrm{SmA} \cdot 226 \cdot \operatorname{dec} .\left({ }^{\circ} \mathrm{C}\right) \\
& \text { 279-R } \mathbf{g}: \mathrm{Cr} \cdot 107 \cdot \mathrm{I}\left({ }^{\circ} \mathrm{C}\right)
\end{aligned}
$$

280- $\mathbf{R}_{2}: \mathrm{Cr} \cdot 126 \cdot \mathrm{E} \cdot 163 \cdot \mathrm{SmA} \cdot 207 \cdot \mathrm{I}\left({ }^{\circ} \mathrm{C}\right)$ (the cationic part with a $\mathrm{Br}^{-}$counterion shows a SmA phase and another, more ordered mesophase)

281-R $-\mathbf{2 1 1}: \mathrm{Cr} \cdot 85 \cdot \mathrm{X} \cdot 148 \cdot \mathrm{E} \cdot 200 \cdot \mathrm{SmA} \cdot 210 \cdot \mathrm{I}\left({ }^{\circ} \mathrm{C}\right)$

281- $\mathbf{R}_{2}-\mathbf{4}: \mathrm{Cr}_{1} \cdot 77 \cdot \mathrm{Cr}_{2} \cdot 132 \cdot \mathrm{E} \cdot 172 \cdot \mathrm{I}\left({ }^{\circ} \mathrm{C}\right)$

Scheme S15. Zwitterions based on boron clusters, investigated by Kaszynski and co-workers. More examples can be found in other references (see main text).

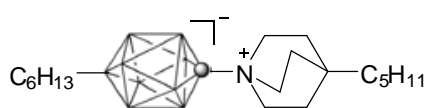

282

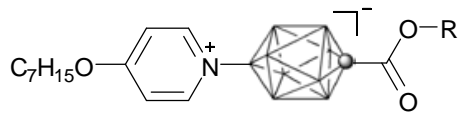

284-R

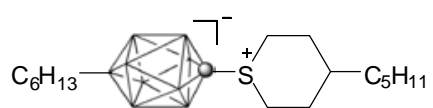

283

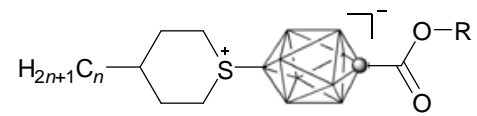

285-n-R

\section{R substituents:}
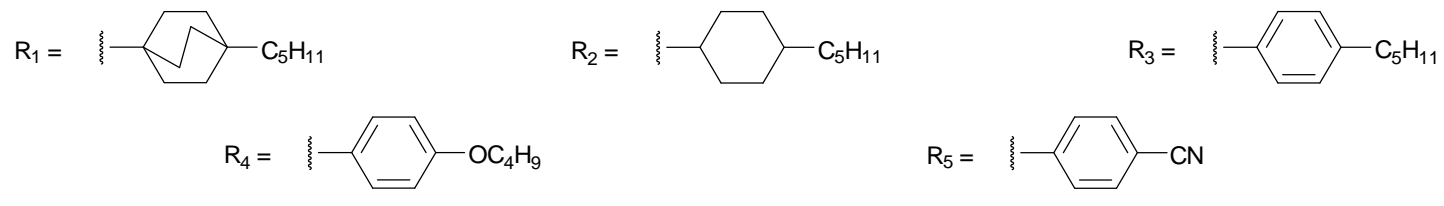

$$
\begin{aligned}
& \text { 282: } \mathrm{Cr}_{1} \cdot 109 \cdot \mathrm{Cr}_{2} \cdot 363 \cdot \mathrm{I} / \mathrm{dec} .\left({ }^{\circ} \mathrm{C}\right) \\
& \text { 283: } \mathrm{Cr}_{1} \cdot 66 \cdot \mathrm{Cr}_{2} \cdot 209 \cdot \mathrm{I}\left({ }^{\circ} \mathrm{C}\right) \\
& \text { 284-R } \mathbf{R}_{\mathbf{1}}: \mathrm{Cr} \cdot 161 \cdot \mathrm{N} \cdot 231 \cdot \mathrm{I}\left({ }^{\circ} \mathrm{C}\right) \\
& \text { 284-R } \mathbf{R}_{2} \mathrm{Cr} \cdot 148 \cdot \mathrm{N} \cdot 161 \cdot \mathrm{I}\left({ }^{\circ} \mathrm{C}\right) \\
& \text { 284-R }: \mathrm{Cr} \cdot(\mathrm{N} \cdot 114 \cdot) 120 \cdot \mathrm{I}
\end{aligned}
$$


284-R $\mathbf{R}_{\mathbf{4}}: \mathrm{Cr} \cdot 122 \cdot \mathrm{N} \cdot 156 \cdot \mathrm{I}\left({ }^{\circ} \mathrm{C}\right)$

284-R $\mathbf{5}: \mathrm{Cr} \cdot(\mathrm{N} \cdot 128 \cdot) 129 \cdot \mathrm{I}$

285-5-R: $: \mathrm{Cr} \cdot 97 \cdot \mathrm{I}\left({ }^{\circ} \mathrm{C}\right)$

285-5-R $\mathbf{4}: \mathrm{Cr} \cdot(\mathrm{N} \cdot 97 \cdot)$ 101 $\cdot \mathrm{I}$

285-3-R $\mathbf{4}: \mathrm{Cr} \cdot(\mathrm{N} \cdot 96 \cdot) 111 \cdot \mathrm{I}$ 


\subsection{4,4'-Bipyridinium-based ionic liquid crystals}

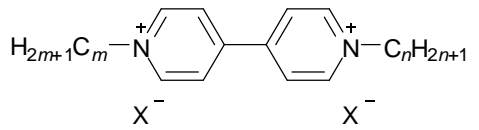

286-Br-m/n $\left(\mathrm{X}^{-}=\mathrm{Br}^{-}\right)$

286-Br-7/7: $\mathrm{Cr}_{1} \cdot 107 \cdot \mathrm{Cr}_{2} \cdot 277 \cdot \mathrm{dec} .\left({ }^{\circ} \mathrm{C}\right)$

286-Br-8/8: $\mathrm{Cr}_{1} \cdot 129 \cdot \mathrm{Cr}_{2} \cdot 280 \cdot \operatorname{dec} .\left({ }^{\circ} \mathrm{C}\right)$

286-NTf $2-\boldsymbol{m} / \boldsymbol{n}\left(\mathrm{X}^{-}=\left[\mathrm{NTf}_{2}\right]^{-}\right)$

286-NTf $\mathbf{2}_{\mathbf{2}}$ 1/1: $\mathrm{Cr} \cdot 132 \cdot \mathrm{I}\left({ }^{\circ} \mathrm{C}\right)$

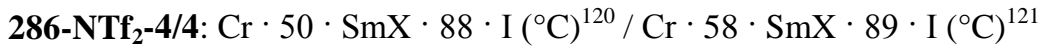

286-NTf $\mathbf{2}_{2}-5 / 5: \mathrm{Cr} \cdot-4 \cdot \mathrm{SmX} \cdot 42 \cdot \mathrm{I}\left({ }^{\circ} \mathrm{C}\right)$

286-NTf $\mathbf{2}-\mathbf{6} / \mathbf{6}: \mathrm{Cr} \cdot 58 \cdot \mathrm{SmX} \cdot 78 \cdot \mathrm{I}\left({ }^{\circ} \mathrm{C}\right)$

286-NTf $\mathbf{N}_{2}-7 / 7: \mathrm{Cr} \cdot 41 \cdot \mathrm{SmX} \cdot 112 \cdot \mathrm{I}\left({ }^{\circ} \mathrm{C}\right)$

286-NTf $\mathbf{2}-\mathbf{8} / \mathbf{8}: \mathrm{Cr} \cdot 37 \cdot \mathrm{SmX} \cdot 136 \cdot \mathrm{I}\left({ }^{\circ} \mathrm{C}\right)$

286-NTf $\mathbf{N}_{2}$ 12/12: $\mathrm{Cr} \cdot 18 \cdot \mathrm{SmX}_{1} \cdot 119 \cdot \mathrm{SmX}_{2} \cdot 202 \cdot \mathrm{SmX}_{3} \cdot 356 \cdot \operatorname{dec} .\left({ }^{\circ} \mathrm{C}\right)^{120} / \mathrm{Cr} \cdot 33 \cdot \mathrm{SmX}_{1} \cdot 130 \cdot \mathrm{SmX}_{2} \cdot 200 \cdot$

$\mathrm{SmX}_{3} \cdot[$ not reported $] \cdot \operatorname{dec} .\left({ }^{\circ} \mathrm{C}\right)^{121}$

286-NTf $\mathbf{2}_{\mathbf{2}} \mathbf{1 4 / 1 4}: \mathrm{Cr} \cdot 42 \cdot \mathrm{SmX} \cdot 218 \cdot \mathrm{I}\left({ }^{\circ} \mathrm{C}\right)\left(1^{\text {st }} \mathrm{DSC} \text { heating run }\right)^{122}$

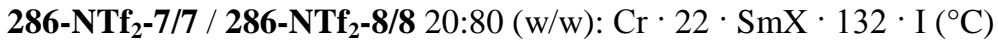

286-NTf ${ }_{2}$-3/11: $\mathrm{Cr} \cdot 44 \cdot \mathrm{I}\left({ }^{\circ} \mathrm{C}\right)$

286-NTf ${ }_{2}-4 / 8: \mathrm{Cr} \cdot 57 \cdot \mathrm{I}\left({ }^{\circ} \mathrm{C}\right)$

286-NTf ${ }_{2}-\mathbf{4} / 11: \mathrm{Cr} \cdot 45 \cdot \mathrm{I}\left({ }^{\circ} \mathrm{C}\right)$

286-NTf 2 -5/8: $\mathrm{Cr} \cdot(\mathrm{SmX} \cdot \mathrm{ca} .52 \cdot) 63 \cdot \mathrm{I}\left({ }^{\circ} \mathrm{C}\right)$

286-NTf $\mathbf{2}_{2}$-6/8: $\mathrm{Cr} \cdot 24 \cdot \mathrm{SmX} \cdot 104 \cdot \mathrm{I}\left({ }^{\circ} \mathrm{C}\right)$

286-NTf ${ }_{2}-6 / 9: \mathrm{Cr} \cdot 8 \cdot \mathrm{SmX} \cdot 106 \cdot \mathrm{I}\left({ }^{\circ} \mathrm{C}\right)$

286-NTf $\mathbf{2}_{2} \mathbf{7 / 8}: \mathrm{Cr} \cdot 25 \cdot \mathrm{SmX} \cdot 121 \cdot \mathrm{I}\left({ }^{\circ} \mathrm{C}\right)$

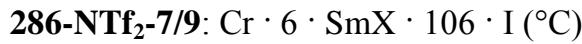

286-NTf $\mathbf{2}_{2}-\mathbf{7 / 1 0}: \mathrm{Cr} \cdot 1 \cdot \mathrm{SmX} \cdot 130 \cdot \mathrm{I}\left({ }^{\circ} \mathrm{C}\right)$

286-NTf ${ }_{2}-7 / 11: \mathrm{Cr} \cdot-0.4 \cdot \mathrm{SmX} \cdot 136 \cdot \mathrm{I}\left({ }^{\circ} \mathrm{C}\right)$

286-NTf ${ }_{2}-\mathbf{8} / \mathbf{9}: \mathrm{Cr} \cdot 25 \cdot \mathrm{SmX} \cdot 140 \cdot \mathrm{I}\left({ }^{\circ} \mathrm{C}\right)$

286-NTf ${ }_{2}-\mathbf{8} / \mathbf{1 0}: \mathrm{Cr} \cdot 4 \cdot \mathrm{SmX} \cdot 147 \cdot \mathrm{I}\left({ }^{\circ} \mathrm{C}\right)$

286-NTf $\mathbf{2}_{\mathbf{2}} \mathbf{- 8 / 1 1 :} \mathrm{Cr} \cdot 4 \cdot \mathrm{SmX} \cdot 130 \cdot \mathrm{I}\left({ }^{\circ} \mathrm{C}\right)$

286-NTf $\mathbf{2}_{\mathbf{2}}$ 14/2: $\mathrm{Cr} \cdot 71 \cdot \mathrm{I}\left({ }^{\circ} \mathrm{C}\right)\left(1^{\text {st }} \text { DSC heating run }\right)^{122}$ 


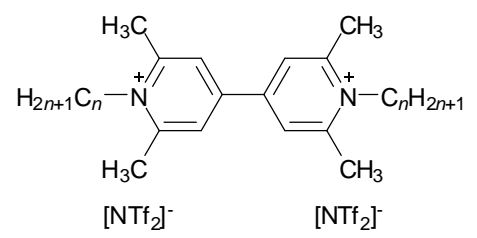

287-n

287-8: $\mathrm{Cr} \cdot 98 \cdot \mathrm{I}\left({ }^{\circ} \mathrm{C}\right)$

287-10: $\mathrm{Cr}_{1} \cdot 84 \cdot \mathrm{Cr}_{2} \cdot 95 \cdot \mathrm{I}\left({ }^{\circ} \mathrm{C}\right)$

287-12: $\mathrm{Cr}_{1} \cdot 40 \cdot \mathrm{Cr}_{2} \cdot 51 \cdot \mathrm{Cr}_{3} \cdot 75 \cdot \mathrm{Cr}_{4} \cdot 90 \cdot \mathrm{Cr}_{5} \cdot 95 \cdot \mathrm{I}\left({ }^{\circ} \mathrm{C}\right)$

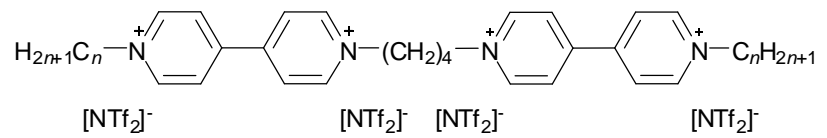

\section{8-n}

288-12: $\mathrm{Cr} \cdot 88 \cdot \mathrm{SmX} \cdot 154 \cdot \mathrm{SmA} \cdot 183 \cdot \mathrm{I}\left({ }^{\circ} \mathrm{C}\right)\left(1^{\text {st }} \mathrm{DSC}\right.$ heating run $)$

288-14: $\mathrm{Cr} \cdot 84 \cdot \mathrm{SmX} \cdot 153 \cdot \mathrm{SmA} \cdot 229 \cdot \mathrm{I}\left({ }^{\circ} \mathrm{C}\right)\left(1^{\text {st }}\right.$ DSC heating run $)$

288-16: $\mathrm{Cr} \cdot 79 \cdot \mathrm{SmX} \cdot 152 \cdot \mathrm{SmA} \cdot 264 \cdot \mathrm{I}\left({ }^{\circ} \mathrm{C}\right)\left(1^{\text {st }} \mathrm{DSC}\right.$ heating run $)$
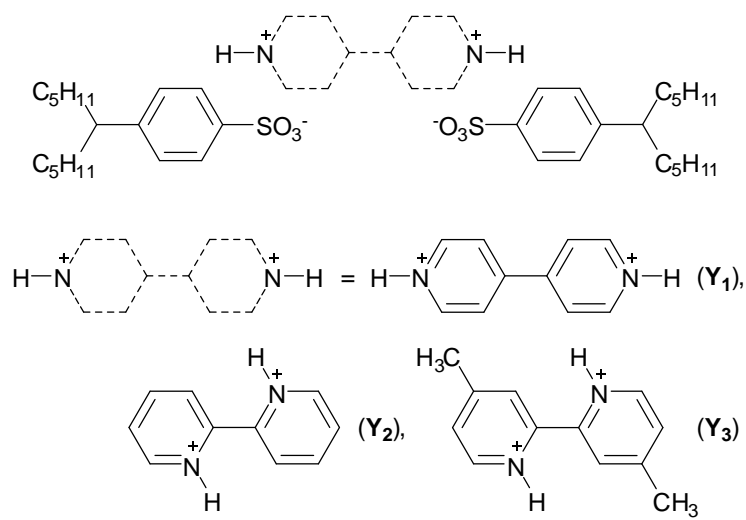

289-Y ${ }_{1}: \mathrm{Cr} \cdot(\mathrm{M} \cdot[$ not reported $] \cdot) 248 \cdot \mathrm{I}\left({ }^{\circ} \mathrm{C}\right)$

289-Y $\mathbf{Y}_{2}: \mathrm{Cr} \cdot 120 \cdot \mathrm{I}\left({ }^{\circ} \mathrm{C}\right)$

289-Y $\mathbf{Y}_{3}: \mathrm{Cr} \cdot 114 \cdot \mathrm{I}\left({ }^{\circ} \mathrm{C}\right)$

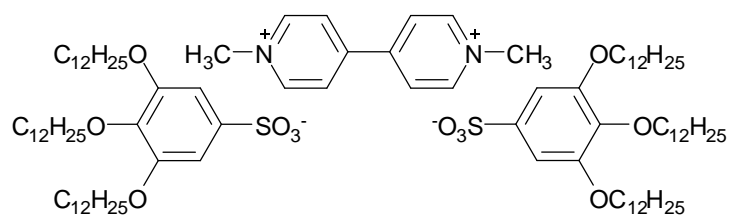

290: $\mathrm{Cr} \cdot 65 \cdot \mathrm{M}_{1}\left(\mathrm{Col}_{\mathrm{h}}\right.$ ? $) \cdot 270 \cdot \mathrm{I} /$ dec. $\left({ }^{\circ} \mathrm{C}\right)\left(1^{\text {st }} \mathrm{DSC}\right.$ heating run $)$ 


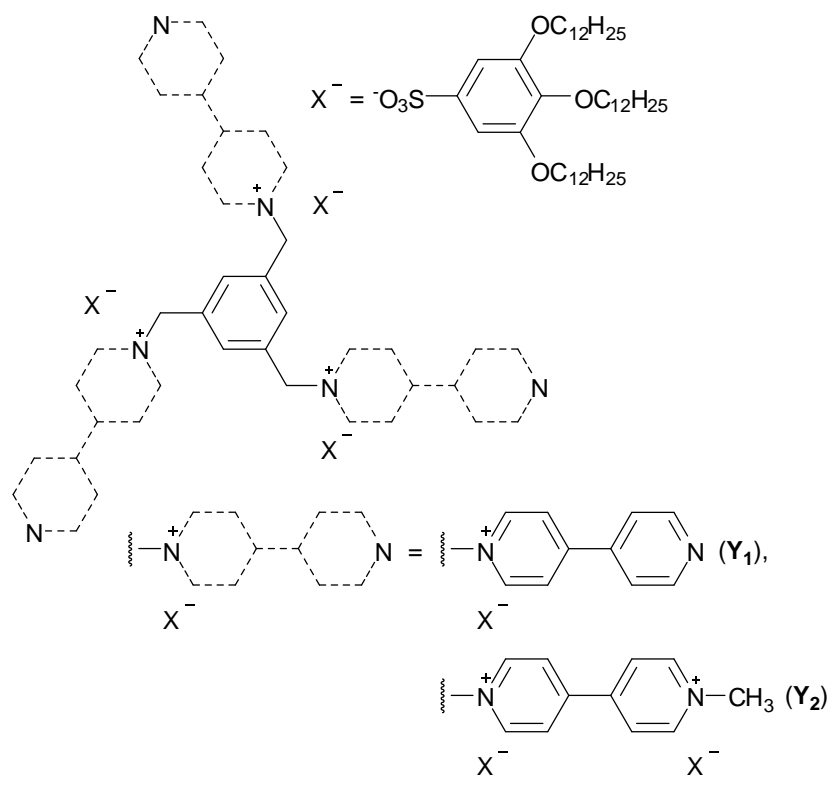

291- $\mathrm{Y}_{1}: \mathrm{Cr} \cdot 37 \cdot \mathrm{M}_{2} \cdot 230 \cdot \mathrm{I} / \mathrm{dec} .\left({ }^{\circ} \mathrm{C}\right)\left(1^{\text {st }}\right.$ DSC heating run $)$ 291- $\mathbf{Y}_{2}: \mathrm{Cr} \cdot 39 \cdot \mathrm{M}_{2} \cdot 230 \cdot \mathrm{I} / \mathrm{dec} .\left({ }^{\circ} \mathrm{C}\right)\left(1^{\text {st }} \mathrm{DSC}\right.$ heating run $)$

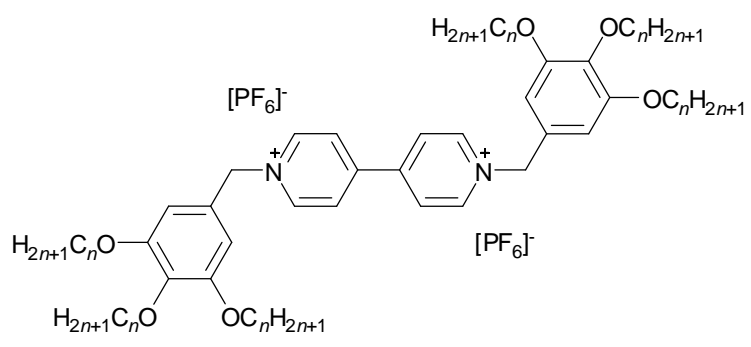

292-n

292-8: $\mathrm{Cr} \cdot-15 \cdot \mathrm{Col}_{\mathrm{h}} \cdot 160 \cdot \operatorname{dec} .\left({ }^{\circ} \mathrm{C}\right)$

292-12: $\mathrm{Cr} \cdot 47 \cdot \mathrm{Col}_{\mathrm{r}} \cdot 160 \cdot \operatorname{dec} .\left({ }^{\circ} \mathrm{C}\right)$

292-16: $\mathrm{Cr} \cdot 64 \cdot \mathrm{Col}_{\mathrm{r}} \cdot 160 \cdot \operatorname{dec} .\left({ }^{\circ} \mathrm{C}\right)$

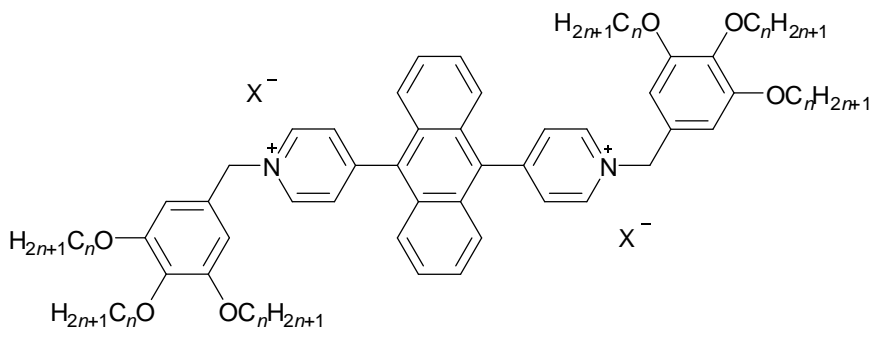

293-Br-n $\left(\mathrm{X}^{-}=\mathrm{Br}^{-}\right)$

293-Br-8: $\mathrm{Cr} \cdot 197 \cdot \mathrm{Col}_{\mathrm{r}} \cdot 228 \cdot$ dec. $\left({ }^{\circ} \mathrm{C}\right)$

293-Br-12: $\mathrm{Cr} \cdot 203 \cdot \mathrm{Col}_{\mathrm{r}} \cdot 235 \cdot$ dec. $\left({ }^{\circ} \mathrm{C}\right)$ 
293-BF ${ }_{4}-\boldsymbol{n}\left(\mathrm{X}^{-}=\left[\mathrm{BF}_{4}\right]^{-}\right)$

293-BF 4 -8: $\mathrm{Cr} \cdot 181 \cdot \mathrm{Col}_{\mathrm{r}} \cdot 218 \cdot \operatorname{dec} .\left({ }^{\circ} \mathrm{C}\right)$

293-BF 4 -12: $\mathrm{Cr} \cdot 185 \cdot \mathrm{Col}_{\mathrm{r}} \cdot 205 \cdot \operatorname{dec} .\left({ }^{\circ} \mathrm{C}\right)$

293-PF $\mathbf{6}_{6}-\boldsymbol{n}\left(\mathrm{X}^{-}=\left[\mathrm{PF}_{6}\right]^{-}\right)$

293-PF $\mathbf{F}_{6} \mathbf{- 8}: \mathrm{Cr} \cdot(\mathrm{SmX}(?) \cdot 151 \cdot) 216 \cdot \mathrm{I}\left({ }^{\circ} \mathrm{C}\right)$

293-PF $-12: \mathrm{Cr} \cdot\left(\mathrm{Col}_{\mathrm{r}} \cdot 148 \cdot\right) 220 \cdot \mathrm{I}\left({ }^{\circ} \mathrm{C}\right)$

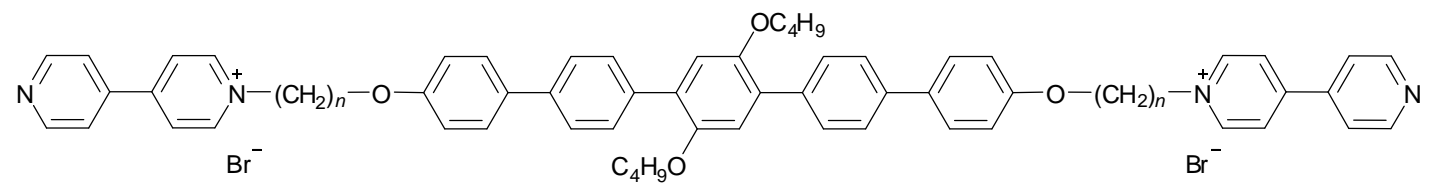

294-n

294-10: $\mathrm{Cr} \cdot\left[\right.$ not reported] $\cdot \mathrm{SmF} / \mathrm{SmI}($ ?; only observed on heating $) \cdot 100 \cdot \mathrm{SmA} \cdot 185(?) \cdot \mathrm{I} / \mathrm{dec} .\left({ }^{\circ} \mathrm{C}\right)$ 294-12: $\mathrm{Cr} \cdot\left[\right.$ not reported] $\cdot \mathrm{SmF} / \mathrm{SmI}(?) \cdot 100 \cdot \mathrm{SmA} \cdot 160-175 \cdot \mathrm{I} / \mathrm{dec} .\left({ }^{\circ} \mathrm{C}\right)$

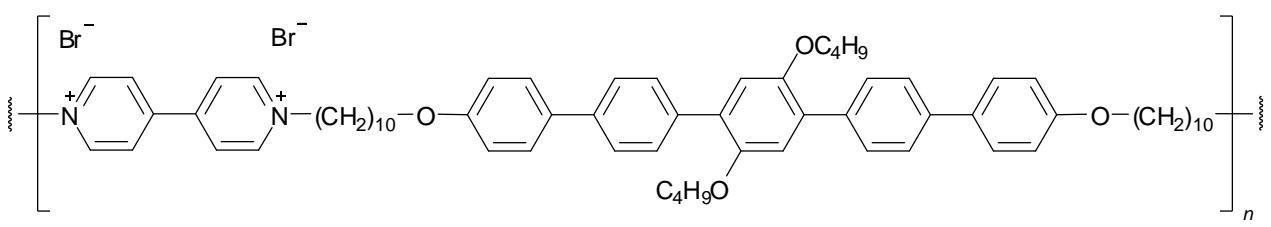

295: $\mathrm{SmC} \cdot 80-120 \cdot \mathrm{SmA} \cdot 190 \cdot \mathrm{I}\left({ }^{\circ} \mathrm{C}\right)$

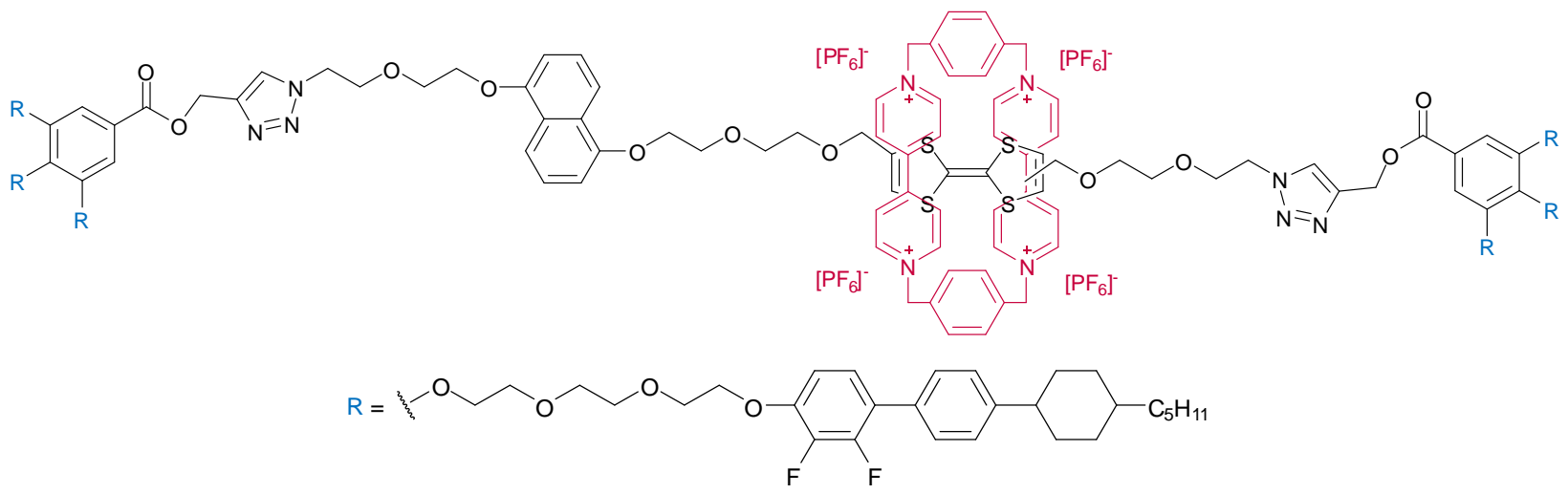

296: $\mathrm{g} \cdot 10 \cdot \mathrm{SmA} \cdot 150 \cdot \operatorname{dec}\left({ }^{\circ} \mathrm{C}\right)$ 

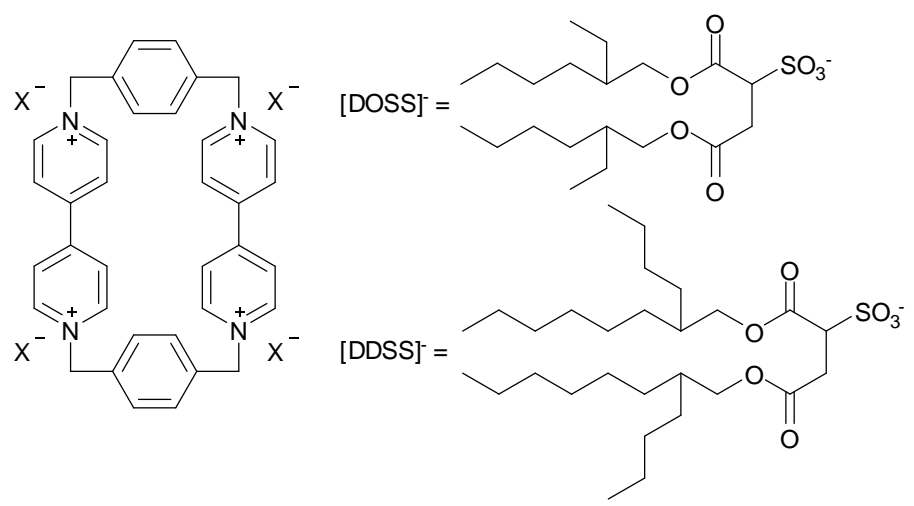

297-DOSS $\left(\mathrm{X}^{-}=[\text {DOSS }]^{-}\right): \mathrm{Cr} \cdot 265 \cdot$ dec. $\left({ }^{\circ} \mathrm{C}\right)$

297-DOSS/[Na][DOSS] (1:0.5): $\mathrm{L}_{\mathrm{Col}} \cdot 230 \cdot \mathrm{I} \cdot 265$ dec. $\left({ }^{\circ} \mathrm{C}\right)$

297-DOSS/[Na][DOSS] (1:1): $\mathrm{L}_{\mathrm{Col}} \cdot 220 \cdot \mathrm{I} \cdot 265$ dec. $\left({ }^{\circ} \mathrm{C}\right)$

297-DOSS/[Na][DOSS] (1:4): $\mathrm{Col}_{\mathrm{h}} \cdot 210 \cdot \mathrm{I} \cdot 265 \mathrm{dec} .\left({ }^{\circ} \mathrm{C}\right)$

297-DOSS/TTF (1:1): $\mathrm{Cr} \cdot 265 \cdot$ dec. $\left({ }^{\circ} \mathrm{C}\right)$

297-DDSS $\left(\mathrm{X}^{-}=[\mathrm{DDSS}]^{-}\right): \mathrm{Col}_{\mathrm{r}} \cdot 230 \cdot \mathrm{I} \cdot 245 \cdot$ dec. $\left({ }^{\circ} \mathrm{C}\right)$

297-DDSS/TTF (1:1): $\mathrm{L}_{\mathrm{Col}} \cdot 210 \cdot \mathrm{I} \cdot 245 \cdot$ dec. $\left({ }^{\circ} \mathrm{C}\right)$

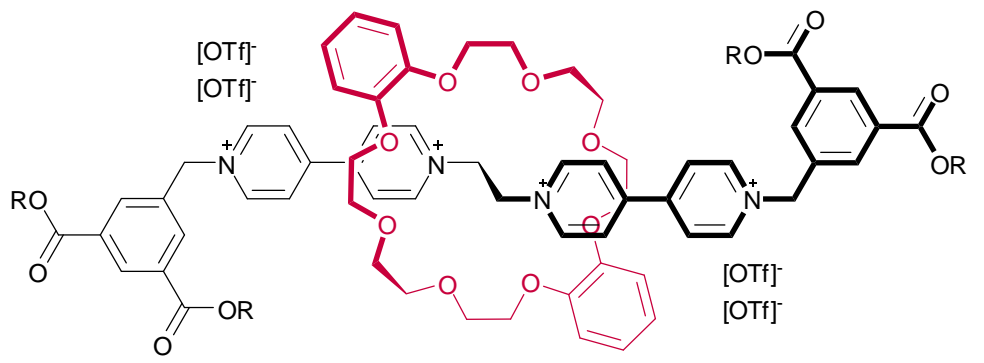

298-(a-e) (without macrocycle)

298-a $\left(\mathrm{R}=n-\mathrm{C}_{6} \mathrm{H}_{13}\right): \mathrm{X} \cdot>170 \cdot \operatorname{dec} .\left({ }^{\circ} \mathrm{C}\right)$

298-b $\left(\mathrm{R}=n-\mathrm{C}_{9} \mathrm{H}_{19}\right): \mathrm{X}_{1} \cdot 38 \cdot \mathrm{X}_{2} \cdot>170 \cdot \operatorname{dec} .\left({ }^{\circ} \mathrm{C}\right)$

298-c $\left(\mathrm{R}=n-\mathrm{C}_{12} \mathrm{H}_{25}\right): \mathrm{X}_{1} \cdot 49 \cdot \mathrm{X}_{2} \cdot>170 \cdot$ dec. $\left({ }^{\circ} \mathrm{C}\right)$

298-d $\left(\mathrm{R}=n-\mathrm{C}_{16} \mathrm{H}_{33}\right): \mathrm{X}_{1} \cdot 44-70 \cdot \mathrm{X}_{2} \cdot 113 \cdot \mathrm{X}_{3, \mathrm{~L}} \cdot>170 \cdot \operatorname{dec} .\left({ }^{\circ} \mathrm{C}\right)\left(1^{\text {st }} \mathrm{DSC}\right.$ heating run $) /$

$\mathrm{X}_{4, \mathrm{~L}} \cdot 28 \cdot \mathrm{X}_{5, \mathrm{~L}} \cdot 45 \cdot \mathrm{X}_{3, \mathrm{~L}} \cdot>170 \cdot \operatorname{dec} .\left({ }^{\circ} \mathrm{C}\right)$ (2nd DSC heating run of non-decomposed sample)

298-e (R = 2-hexyldecyl): $\mathrm{X} \cdot>170 \cdot$ dec. $\left({ }^{\circ} \mathrm{C}\right)$

298-crown-(a-e) (with macrocycle)

298-crown-a $\left(\mathrm{R}=n-\mathrm{C}_{6} \mathrm{H}_{13}\right): \mathrm{X} \cdot 81 \cdot \mathrm{I}\left({ }^{\circ} \mathrm{C}\right)$

298-crown-b $\left(\mathrm{R}=n-\mathrm{C}_{9} \mathrm{H}_{19}\right): \mathrm{X} \cdot 118 \cdot \mathrm{I}\left({ }^{\circ} \mathrm{C}\right)$

298-crown-c $\left(\mathrm{R}=n-\mathrm{C}_{12} \mathrm{H}_{25}\right): \mathrm{X}_{\mathrm{L}} \cdot 53 \cdot \mathrm{SmA} \cdot 137 \cdot \mathrm{I}\left({ }^{\circ} \mathrm{C}\right)\left(1^{\text {st }} \mathrm{DSC}\right.$ heating run $) /$

$\mathrm{SmA} \cdot 137 \cdot \mathrm{I}\left({ }^{\circ} \mathrm{C}\right)\left(2^{\text {nd }}\right.$ DSC heating run $)$

298-crown-d $\left(\mathrm{R}=n-\mathrm{C}_{16} \mathrm{H}_{33}\right): \mathrm{SmX}_{1} \cdot 47 \cdot \mathrm{SmA} \cdot>190 \cdot$ dec. $\left({ }^{\circ} \mathrm{C}\right)\left(1^{\text {st }} \mathrm{DSC}\right.$ heating run $) /$ 
$\mathrm{SmX}_{2} \cdot 12 \cdot \mathrm{SmA} \cdot>190 \cdot$ dec. $\left({ }^{\circ} \mathrm{C}\right)\left(2^{\text {nd }}\right.$ DSC heating run of non-decomposed sample $)$

298-crown-e ( $\mathrm{R}=2$-hexyldecyl): $\mathrm{X}_{1} \cdot 72 \cdot \mathrm{X}_{2} \cdot 176 \cdot \mathrm{I}\left({ }^{\circ} \mathrm{C}\right)\left(1^{\text {st }} \mathrm{DSC}\right.$ heating run $) /$

$\mathrm{X}_{2} \cdot 176 \cdot \mathrm{I}\left({ }^{\circ} \mathrm{C}\right)\left(2^{\text {nd }} \mathrm{DSC}\right.$ heating run (after the $1^{\text {st }} \mathrm{DSC}$ heating run the $\mathrm{X}_{2}$ phase only forms again upon heating at $124^{\circ} \mathrm{C}$ ))

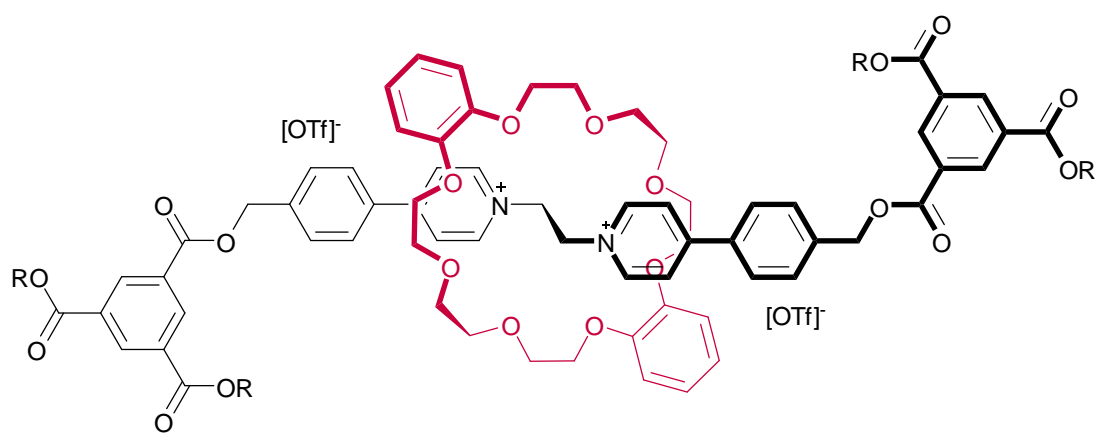

299-a (without macrocycle) $\left(\mathrm{R}=n-\mathrm{C}_{12} \mathrm{H}_{25}\right): \mathrm{X}_{1} \cdot 74 \cdot \mathrm{X}_{2} \cdot 166 \cdot \mathrm{I} / \mathrm{dec} .\left({ }^{\circ} \mathrm{C}\right)\left(1^{\text {st }}\right.$ DSC heating run $) /$ $\mathrm{X}_{3} \cdot 72 \cdot \mathrm{X}_{2} \cdot 166 \cdot \mathrm{I} /$ dec. $\left({ }^{\circ} \mathrm{C}\right)\left(2^{\text {nd }} \mathrm{DSC}\right.$ heating run $)$

299-crown-(a-b) (with macrocycle) 299-crown-a $\left(\mathrm{R}=n-\mathrm{C}_{12} \mathrm{H}_{25}\right): \mathrm{SmA} \cdot 111 \cdot \mathrm{I}\left({ }^{\circ} \mathrm{C}\right)$

299-crown-b $\left(\mathrm{R}=\left(\mathrm{CH}_{2}\right)_{4}\left(\mathrm{Si}\left(\mathrm{CH}_{3}\right)_{2} \mathrm{O}\right)_{2}\left(\mathrm{Si}\left(\mathrm{CH}_{3}\right)_{3}\right)\right): \mathrm{SmA} \cdot 151 \cdot \mathrm{I}\left({ }^{\circ} \mathrm{C}\right)$

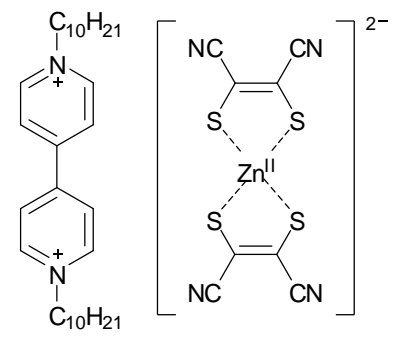

300: $\mathrm{Cr} \cdot 142 \cdot \mathrm{N} \cdot 155 \cdot \mathrm{I}\left({ }^{\circ} \mathrm{C}\right)$

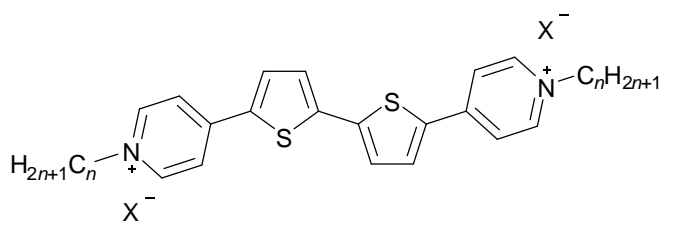

301-I-n $\left(\mathrm{X}^{-}=\mathrm{I}^{-}\right)$

301-I-8: $\mathrm{Cr} \cdot \mathrm{ca} .183 \cdot \mathrm{I}\left({ }^{\circ} \mathrm{C}\right)$

301-I-9: $\mathrm{Cr} \cdot \mathrm{ca} .185 \cdot \mathrm{I}\left({ }^{\circ} \mathrm{C}\right)$

301-I-10: $\mathrm{Cr} \cdot \mathrm{ca} .195 \cdot \mathrm{I}\left({ }^{\circ} \mathrm{C}\right)$

301-I-11: $\mathrm{Cr} \cdot \mathrm{ca} .188 \cdot \mathrm{I}\left({ }^{\circ} \mathrm{C}\right)$ 
301-I-12: $\mathrm{Cr}_{1} \cdot 84$ (broad transition) $\cdot \mathrm{Cr}_{2} \cdot 150 \cdot \mathrm{SmA} \cdot$ ca. $255 \cdot \mathrm{I} /$ dec. $\left({ }^{\circ} \mathrm{C}\right)$

$$
\text { 301-NTf } \mathbf{N}_{2}-\boldsymbol{n}\left(\mathrm{X}^{-}=\left[\mathrm{NTf}_{2}\right]^{-}\right)
$$

301-NTf $\mathbf{N}_{\mathbf{2}} \mathbf{- 8}: \mathrm{Cr} \cdot 125 \cdot \mathrm{I}\left({ }^{\circ} \mathrm{C}\right)$

301-NTf $\mathbf{2}_{2} \mathbf{- 9}: 1^{\text {st }}$ DSC heating/cooling cycle: $\mathrm{Cr}_{1} \cdot 68 \cdot \mathrm{Cr}_{2} \cdot 114 \cdot \mathrm{Col}_{\mathrm{ro}, 1}(p 2 g g) \cdot 132 \cdot \mathrm{I}\left({ }^{\circ} \mathrm{C}\right) /$ on cooling: $\cdot 129 \cdot$

$$
\mathrm{Col}_{\mathrm{ro}, 1}(p 2 g g) \cdot 109 \cdot \mathrm{Col}_{\mathrm{ro}, 2}(p 2 g g)\left({ }^{\circ} \mathrm{C}\right) ;
$$

$2^{\text {nd }}$ DSC heating/cooling cycle: $\mathrm{Col}_{\mathrm{ro}, 2}(p 2 g g) \cdot c a .122 \cdot \mathrm{Col}_{\mathrm{ro}, 1}(p 2 g g) \cdot c a .132 \cdot \mathrm{I}\left({ }^{\circ} \mathrm{C}\right) /$ on cooling: $\mathrm{I} \cdot c a .128 \cdot$

$$
\mathrm{Col}_{\mathrm{ro}, 1}(p 2 g g) \cdot \mathrm{ca} .110 \cdot \mathrm{Col}_{\mathrm{ro}, 2}(p 2 g g)\left({ }^{\circ} \mathrm{C}\right)
$$

301-NTf $\mathbf{2}_{2} \mathbf{- 1 0}$ : $1^{\text {st }}$ DSC heating/cooling cycle: $\mathrm{Cr}_{1} \cdot 63 \cdot \mathrm{Cr}_{2} \cdot 115 \cdot \mathrm{L}_{\mathrm{Col}} \cdot 137 \cdot \mathrm{I}\left({ }^{\circ} \mathrm{C}\right) /$ on cooling: $\cdot 134 \cdot \mathrm{SmA} \cdot$

$$
115 \cdot \mathrm{L}_{\mathrm{Col}}\left({ }^{\circ} \mathrm{C}\right) \text {; }
$$

$2^{\text {nd }}$ DSC heating/cooling cycle: vitrified $\mathrm{L}_{\mathrm{Col}} \cdot c a .110 \cdot \mathrm{L}_{\mathrm{Col}} \cdot c a .136 \cdot \mathrm{I}\left({ }^{\circ} \mathrm{C}\right) /$ on cooling: $\mathrm{I} \cdot c a .135 \cdot \mathrm{SmA} \cdot c a$.

$$
120 \cdot \mathrm{L}_{\mathrm{Col}}\left({ }^{\circ} \mathrm{C}\right)
$$

301-NTf -11: $^{\text {st }}$ DSC heating/cooling cycle: $\mathrm{Cr}_{1} \cdot 63 \cdot \mathrm{Cr}_{2} \cdot 117 \cdot \mathrm{Col}_{\mathrm{rd}} \cdot 152 \cdot \mathrm{I}\left({ }^{\circ} \mathrm{C}\right) /$ on cooling: $\mathrm{I} \cdot 146 \cdot \mathrm{SmA} \cdot$

$114 \cdot$ solid with $\mathrm{Col}_{\mathrm{rd}}$ structure or vitrified SmA phase $\left({ }^{\circ} \mathrm{C}\right)$ (depending on the alignment conditions);

$2^{\text {nd }}$ DSC heating/cooling cycle: solid with $\mathrm{Col}_{\mathrm{rd}}$ structure or vitrified SmA phase $\cdot c a .115 \cdot \mathrm{SmA} \cdot \mathrm{ca} \cdot 150 \cdot \mathrm{I}\left({ }^{\circ} \mathrm{C}\right) /$ on cooling: I $\cdot$ ca. $148 \cdot \mathrm{SmA} \cdot$ ca. $110 \cdot$ vitrified $\mathrm{SmA}$ phase $\left({ }^{\circ} \mathrm{C}\right)$

301-NTf -12: $^{\text {st }}$ DSC heating/cooling cycle: $\mathrm{Cr}_{1} \cdot 43 \cdot \mathrm{Cr}_{2} \cdot 121 \cdot \mathrm{Col}_{\mathrm{r}} \cdot 167 \cdot \mathrm{SmA} \cdot 180 \cdot \mathrm{I}\left({ }^{\circ} \mathrm{C}\right) /$ on cooling: $\cdot$ ? $\cdot$ $\mathrm{SmA} \cdot 132 \cdot$ glassy phase $\cdot 108 \cdot$ crystalline solid $\left({ }^{\circ} \mathrm{C}\right)$; $2^{\text {nd }}$ DSC heating run: $\mathrm{Cr} \cdot \mathrm{ca} .120 \cdot \mathrm{SmA} \cdot \mathrm{ca} .185 \cdot \mathrm{I}\left({ }^{\circ} \mathrm{C}\right)$ 


\subsection{New cationic cores for ionic liquid crystals}

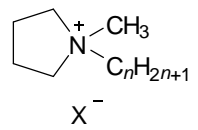

$x^{-}$

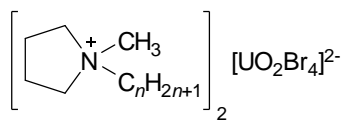

303-n

302-X-n $\left(\mathrm{X}^{-}=\mathrm{Br}^{-},\left[\mathrm{BF}_{4}\right]^{-},\left[\mathrm{PF}_{6}\right]^{-}\right.$,

$\left.\left[\mathrm{NTf}_{2}\right]^{-},[\mathrm{SCN}]^{-},\left[\mathrm{Eu}(\mathrm{tta})_{4}\right]^{-}\right)$

302-Br-n $\left(\mathrm{X}^{-}=\mathrm{Br}^{-}\right)$

302-Br-8: $\mathrm{Cr} \cdot 159 \cdot \mathrm{I}\left({ }^{\circ} \mathrm{C}\right)$

302-Br-10: $\mathrm{Cr}_{1} \cdot 41 \cdot \mathrm{Cr}_{2} \cdot 162 \cdot \mathrm{I}\left({ }^{\circ} \mathrm{C}\right)$

302-Br-11: $\mathrm{Cr} \cdot 27 \cdot \mathrm{T}_{1} \cdot 70 \cdot \mathrm{T}_{2} \cdot 171 \cdot \mathrm{I}\left({ }^{\circ} \mathrm{C}\right)$

302-Br-12: $\mathrm{Cr} \cdot 53 \cdot \mathrm{T}_{1} \cdot 78 \cdot \mathrm{T}_{2} \cdot 191 \cdot \mathrm{I}\left({ }^{\circ} \mathrm{C}\right)$

302-Br-13: $\mathrm{Cr}_{1} \cdot 53 \cdot \mathrm{Cr}_{2} \cdot 59 \cdot \mathrm{T}_{1} \cdot 79 \cdot \mathrm{T}_{2} \cdot 201 \cdot \mathrm{I}\left({ }^{\circ} \mathrm{C}\right)$

302-Br-14: $\mathrm{Cr} \cdot 62 \cdot \mathrm{T}_{1} \cdot 80 \cdot \mathrm{T}_{2} \cdot 208 \cdot \mathrm{I}\left({ }^{\circ} \mathrm{C}\right)$

302-Br-15: $\mathrm{Cr} \cdot 74 \cdot\left(\mathrm{T}_{1} \cdot 83 \cdot\right) \mathrm{T}_{2} \cdot 214 \cdot \mathrm{I}\left({ }^{\circ} \mathrm{C}\right)$

302-Br-16: $\mathrm{Cr}_{1} \cdot 66 \cdot \mathrm{Cr}_{2} \cdot 75 \cdot\left(\mathrm{T}_{1} \cdot 77 \cdot\right) \mathrm{T}_{2} \cdot 217 \cdot \mathrm{I}\left({ }^{\circ} \mathrm{C}\right)$

302-Br-17: $\mathrm{Cr}_{1} \cdot 38 \cdot \mathrm{Cr}_{2} \cdot 82 \cdot \mathrm{T} \cdot 218 \cdot \mathrm{I}\left({ }^{\circ} \mathrm{C}\right)$

302-Br-18: $\mathrm{Cr}_{1} \cdot 42 \cdot \mathrm{Cr}_{2} \cdot 82 \cdot \mathrm{T} \cdot 217 \cdot \mathrm{SmA} \cdot 229 \cdot \mathrm{I}\left({ }^{\circ} \mathrm{C}\right)$

302-Br-19: $\mathrm{Cr}_{1} \cdot 35 \cdot \mathrm{Cr}_{2} \cdot 89 \cdot \mathrm{T} \cdot 216 \cdot \mathrm{SmA} \cdot 233 \cdot \mathrm{I}\left({ }^{\circ} \mathrm{C}\right)$

302-Br-20: $\mathrm{Cr} \cdot 89 \cdot \mathrm{T} \cdot 214 \cdot \mathrm{SmA} \cdot 233 \cdot \mathrm{I}\left({ }^{\circ} \mathrm{C}\right)$

302-BF ${ }_{4}^{-18}\left(\mathrm{X}^{-}=\left[\mathrm{BF}_{4}\right]^{-}, n=18\right): \mathrm{Cr}_{1} \cdot 47 \cdot \mathrm{Cr}_{2} \cdot 92 \cdot \mathrm{T} \cdot 166 \cdot \mathrm{I}\left({ }^{\circ} \mathrm{C}\right)$

302-PF $\mathbf{P}_{6}-18\left(\mathrm{X}^{-}=\left[\mathrm{PF}_{6}\right]^{-}, n=18\right): \mathrm{Cr} \cdot 95 \cdot \mathrm{T} \cdot 145 \cdot \mathrm{I}\left({ }^{\circ} \mathrm{C}\right)$

302-NTf ${ }_{2}-18\left(\mathrm{X}^{-}=\left[\mathrm{NTf}_{2}\right]^{-}, n=18\right): \mathrm{Cr}_{1} \cdot 34 \cdot \mathrm{Cr}_{2} \cdot 72 \cdot \mathrm{I}\left({ }^{\circ} \mathrm{C}\right)$

302-SCN-18 $\left(\mathrm{X}^{-}=[\mathrm{SCN}]^{-}, n=18\right): \mathrm{Cr}_{1} \cdot 37 \cdot \mathrm{Cr}_{2} \cdot 86 \cdot \mathrm{SmA} \cdot 164 \cdot \mathrm{I}\left({ }^{\circ} \mathrm{C}\right)$

302-Eu(tta) $)_{4}-18\left(\mathrm{X}^{-}=\left[\mathrm{Eu}(\mathrm{tta})_{4}\right]^{-}, n=18\right): \mathrm{Cr} \cdot 121 \cdot \mathrm{I}\left({ }^{\circ} \mathrm{C}\right)$

303-8: $\mathrm{Cr} \cdot 67 \cdot \mathrm{I}\left({ }^{\circ} \mathrm{C}\right)$

303-10: $\mathrm{Cr}_{1} \cdot 57 \cdot \mathrm{Cr}_{2} \cdot 54 \cdot \mathrm{I}\left({ }^{\circ} \mathrm{C}\right)$

303-12: $\mathrm{Cr}_{1} \cdot 61 \cdot \mathrm{Cr}_{2} \cdot 108 \cdot \mathrm{I}\left({ }^{\circ} \mathrm{C}\right)$

303-13: $\mathrm{Cr}_{1} \cdot 45 \cdot \mathrm{Cr}_{2} \cdot 109 \cdot \mathrm{I}\left({ }^{\circ} \mathrm{C}\right)$

303-14: $\mathrm{Cr}_{1} \cdot 49 \cdot \mathrm{Cr}_{2} \cdot 55 \cdot \mathrm{E} \cdot\left(\mathrm{M}\left(\mathrm{L}_{\text {col }}\right.\right.$ ? $) \cdot[$ too difficult to determine] $\cdot) 133 \cdot \mathrm{I}\left({ }^{\circ} \mathrm{C}\right)$

303-15: $\mathrm{Cr} \cdot 60 \cdot \mathrm{E} \cdot\left(\mathrm{M}\left(\mathrm{L}_{\mathrm{col}}\right.\right.$ ? $) \cdot[$ too difficult to determine] $\cdot) 147 \cdot \mathrm{I}\left({ }^{\circ} \mathrm{C}\right)$

303-16: $\mathrm{Cr} \cdot 64 \cdot \mathrm{E} \cdot\left(\mathrm{M}\left(\mathrm{L}_{\mathrm{col}}\right.\right.$ ? $\left.) \cdot 151 \cdot\right) 160 \cdot \mathrm{I}\left({ }^{\circ} \mathrm{C}\right)$

303-17: $\mathrm{Cr}_{1} \cdot 69 \cdot \mathrm{Cr}_{2} \cdot 72 \cdot \mathrm{E} \cdot\left(\mathrm{M}\left(\mathrm{L}_{\mathrm{col}}\right.\right.$ ? $) \cdot[$ too difficult to determine] $\cdot) 169 \cdot \mathrm{I}\left({ }^{\circ} \mathrm{C}\right)$

303-18: $\mathrm{Cr}_{1} \cdot 62 \cdot \mathrm{Cr}_{2} \cdot 72 \cdot \mathrm{E} \cdot 172 \cdot \mathrm{I}\left({ }^{\circ} \mathrm{C}\right)$

303-19: $\mathrm{Cr}_{1} \cdot 75 \cdot \mathrm{Cr}_{2} \cdot 80 \cdot \mathrm{E} \cdot 185 \cdot \mathrm{I}\left({ }^{\circ} \mathrm{C}\right)$

303-20: $\mathrm{Cr}_{1} \cdot 41 \cdot \mathrm{Cr}_{2} \cdot 69 \cdot \mathrm{Cr}_{3} \cdot 83 \cdot \mathrm{E} \cdot 186 \cdot \mathrm{SmA} \cdot 267 \cdot \mathrm{I}\left({ }^{\circ} \mathrm{C}\right)$ 


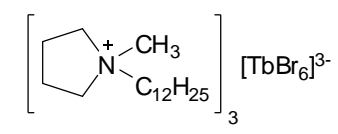

304: $\mathrm{g} \cdot-5 \cdot \operatorname{SmA}(?) \cdot \mathrm{ca} .160 \cdot \mathrm{I}\left({ }^{\circ} \mathrm{C}\right)$

$$
\left.\left[\mathrm{F}^{-\mathrm{H}^{-}}{ }^{-\mathrm{F}} \mathrm{H}_{-\mathrm{F}}\right]^{+}-\mathrm{CH}_{n}\right]^{-}
$$

\section{5-n}

305-10: $\mathrm{Cr} \cdot-12 \cdot \mathrm{I}\left({ }^{\circ} \mathrm{C}\right)$

305-12: $\mathrm{Cr} \cdot 32 \cdot \mathrm{I}\left({ }^{\circ} \mathrm{C}\right)$

305-14: $\mathrm{Cr} \cdot 54 \cdot \mathrm{SmA} \cdot 80 \cdot \mathrm{I}\left({ }^{\circ} \mathrm{C}\right)$

305-16: $\mathrm{Cr} \cdot 64 \cdot \mathrm{SmA} \cdot 126 \cdot \mathrm{I}\left({ }^{\circ} \mathrm{C}\right)$

305-18: $\mathrm{Cr} \cdot 72 \cdot \mathrm{SmA} \cdot 163 \cdot \mathrm{I}\left({ }^{\circ} \mathrm{C}\right)$

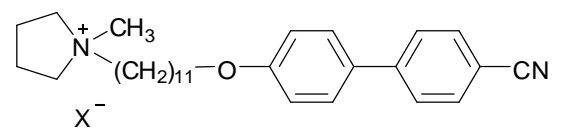

306- $\mathrm{Br}\left(\mathrm{X}^{-}=\mathrm{Br}\right): \mathrm{Cr} \cdot(\mathrm{SmA} \cdot 86 \cdot) 148 \cdot \mathrm{I}\left({ }^{\circ} \mathrm{C}\right)$

306-NTf $\mathrm{N}_{2}\left(\mathrm{X}^{-}=\left[\mathrm{NTf}_{2}\right]^{-}\right): \mathrm{Cr} \cdot(\mathrm{SmA} \cdot 15 \cdot) 56 \cdot \mathrm{I}\left({ }^{\circ} \mathrm{C}\right)$

306-Eu(tta $)_{4}\left(\mathrm{X}^{-}=\left[\mathrm{Eu}(\mathrm{tta})_{4}\right]^{-}\right): \mathrm{g} \cdot 47 \cdot \mathrm{I}\left({ }^{\circ} \mathrm{C}\right)$

306-UO ${ }_{2} \mathrm{Br}_{4}\left(\mathrm{X}^{-}=\left[\mathrm{UO}_{2} \mathrm{Br}_{4}\right]^{2-}\right.$ (with 2 cations)): $\mathrm{Cr} \cdot 172 \cdot \mathrm{I}\left({ }^{\circ} \mathrm{C}\right)$

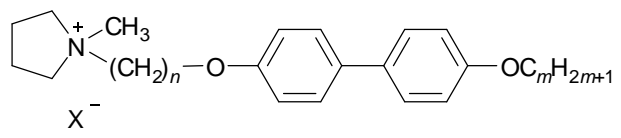

$$
\text { 307-Br-n/m }\left(\mathrm{X}^{-}=\mathrm{Br}^{-}\right)
$$

307-Br-4/4: $\mathrm{Cr} \cdot\left(\mathrm{M}_{1} \cdot 142 \cdot \mathrm{M}_{2} \cdot 143 \cdot\right) 166 \cdot \mathrm{I}\left({ }^{\circ} \mathrm{C}\right)$

307-Br-4/10: $\mathrm{Cr}_{1} \cdot 98 \cdot \mathrm{Cr}_{2} \cdot 143 \cdot \mathrm{E} \cdot 159 \cdot \mathrm{SmA} \cdot 225-235 \cdot \operatorname{dec}\left({ }^{\circ} \mathrm{C}\right)$

307-Br-10/4: $\mathrm{Cr} \cdot 179 \cdot \mathrm{I}\left({ }^{\circ} \mathrm{C}\right)$

307-Br-10/10: $\mathrm{Cr}_{1} \cdot 72 \cdot \mathrm{Cr}_{2} \cdot 125 \cdot \mathrm{E} \cdot 162 \cdot \mathrm{SmA} \cdot 197 \cdot \mathrm{I}\left({ }^{\circ} \mathrm{C}\right)$

307-NTf $\mathbf{2}_{2}-\mathbf{n} / \mathbf{m}\left(\mathrm{X}^{-}=\left[\mathrm{NTf}_{2}\right]^{-}\right)$

307-NTf ${ }_{2}-4 / 4: \mathrm{Cr} \cdot 76 \cdot \mathrm{I}\left({ }^{\circ} \mathrm{C}\right)$

307-NTf $\mathbf{N}_{2} \mathbf{4} / \mathbf{1 0}: \mathrm{Cr} \cdot(\mathrm{SmA} \cdot 60 \cdot) 80 \cdot \mathrm{I}\left({ }^{\circ} \mathrm{C}\right)$

307-NTf $\mathrm{N}_{2}-10 / 4: \mathrm{Cr}_{1} \cdot 13 \cdot \mathrm{Cr}_{2} \cdot 129 \cdot \mathrm{I}\left({ }^{\circ} \mathrm{C}\right)$

307-NTf ${ }_{2}-10 / 10: \mathrm{Cr} \cdot 110 \cdot \mathrm{I}\left({ }^{\circ} \mathrm{C}\right)$ 


$$
\begin{gathered}
\text { 307-Eu(tta) })_{4}-\mathbf{n} / \mathbf{m}\left(\mathrm{X}^{-}=\left[\mathrm{Eu}(\mathrm{tta})_{4}\right]^{-}\right) \\
\text {307-Eu(tta) })_{4}-\mathbf{4} / \mathbf{1 0}: \mathrm{g} \cdot 34 \cdot \mathrm{I}\left({ }^{\circ} \mathrm{C}\right)
\end{gathered}
$$

307-Eu(tta) $)_{4}-10 / 10: \mathrm{g} \cdot 29 \cdot \mathrm{I}\left({ }^{\circ} \mathrm{C}\right)$

307- $\mathrm{UO}_{2} \mathrm{Br}_{4}-\mathbf{n} / \mathbf{m}\left(\mathrm{X}^{-}=\left[\mathrm{UO}_{2} \mathrm{Br}_{4}\right]^{2-}\right.$ (with 2 cations))

307-UO $\mathbf{O}_{2} \mathrm{Br}_{4}-\mathbf{4} / \mathbf{4}: \mathrm{Cr} \cdot 103 \cdot \mathrm{I}\left({ }^{\circ} \mathrm{C}\right)$

307-UO $\mathbf{O}_{2} \mathbf{B r}_{\mathbf{4}} \mathbf{- 4 / 1 0 :} \mathrm{Cr} \cdot 112 \cdot \mathrm{SmA} \cdot 225-235 \cdot \operatorname{dec}\left({ }^{\circ} \mathrm{C}\right)$

307-UO $\mathbf{O}_{2} \mathbf{B r}_{\mathbf{4}}-\mathbf{1 0} / \mathbf{1 0}: \mathrm{Cr}_{1} \cdot 75 \cdot \mathrm{Cr}_{2} \cdot 120 \cdot \mathrm{SmC} \cdot 126 \cdot \mathrm{SmA} \cdot 225-235 \cdot \operatorname{dec}\left({ }^{\circ} \mathrm{C}\right)$

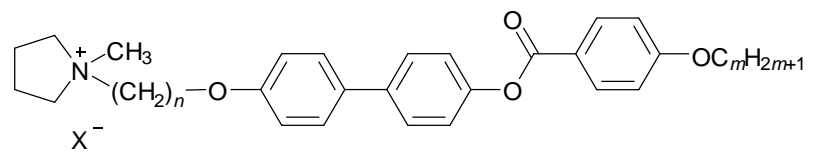 \\ 308-Br-n/m $\left(\mathrm{X}^{-}=\mathrm{Br}^{-}\right)$ \\ 308-Br-4/4: $\mathrm{Cr}_{1} \cdot 59 \cdot \mathrm{Cr}_{2} \cdot 103 \cdot \mathrm{Cr}_{3} \cdot 208 \cdot \mathrm{SmA} \cdot 225-235 \cdot \operatorname{dec}\left({ }^{\circ} \mathrm{C}\right)$ \\ 308-Br-4/10: $\mathrm{Cr}_{1} \cdot 41 \cdot \mathrm{Cr}_{2} \cdot 132 \cdot \mathrm{Cr}_{3} \cdot 143 \cdot \mathrm{X} \cdot 197 \cdot \mathrm{E} \cdot 203 \cdot \mathrm{SmC} \cdot 214 \cdot \mathrm{SmA} \cdot 225-235 \cdot \operatorname{dec}\left({ }^{\circ} \mathrm{C}\right)$ \\ 308-Br-10/4: $\mathrm{Cr}_{1} \cdot 108 \cdot \mathrm{Cr}_{2} \cdot 122 \cdot \mathrm{Cr}_{3} \cdot 196 \cdot \mathrm{SmA} \cdot 215 \cdot \mathrm{I}\left({ }^{\circ} \mathrm{C}\right)$ \\ 308-Br-10/10: $\mathrm{Cr} \cdot 109 \cdot \mathrm{X} \cdot 183 \cdot \mathrm{E} \cdot 190 \cdot \mathrm{SmC} \cdot 225 \cdot \mathrm{SmA} \cdot 225-235 \cdot \operatorname{dec}\left({ }^{\circ} \mathrm{C}\right)$ \\ 308-NTf $\mathbf{2}_{2}-\mathbf{n} / \mathbf{m}\left(\mathrm{X}^{-}=\left[\mathrm{NTf}_{2}\right]^{-}\right)$ \\ 308-NTf $2-4 / 4: \mathrm{Cr}_{1} \cdot 33 \cdot \mathrm{Cr}_{2} \cdot 101 \cdot \mathrm{SmA} \cdot 114 \cdot \mathrm{I}\left({ }^{\circ} \mathrm{C}\right)$ \\ 308-NTf $\mathbf{N}_{2}-\mathbf{4} / \mathbf{1 0}: \mathrm{Cr}_{1} \cdot 100 \cdot \mathrm{Cr}_{2} \cdot 106 \cdot \mathrm{SmA} \cdot 143 \cdot \mathrm{I}\left({ }^{\circ} \mathrm{C}\right)$ \\ 308-NTf $\mathbf{N}_{2} \mathbf{- 1 0} / 4: \mathrm{Cr} \cdot(\mathrm{SmA} \cdot 148 \cdot) 154 \cdot \mathrm{I}\left({ }^{\circ} \mathrm{C}\right)$ \\ 308-NTf $\mathbf{N}_{2} \mathbf{- 1 0} / \mathbf{1 0}: \mathrm{Cr} \cdot(\mathrm{M} \cdot 109 \cdot) 116 \cdot \mathrm{SmC} \cdot 125 \cdot \mathrm{SmA} \cdot 154 \cdot \mathrm{I}\left({ }^{\circ} \mathrm{C}\right)$ \\ 308-Eu(tta) $)_{4}-\mathbf{n} / \mathbf{m}\left(\mathrm{X}^{-}=\left[\mathrm{Eu}(\mathrm{tta})_{4}\right]^{-}\right)$ \\ 308-Eu(tta) $)_{4}-4 / 4: \mathrm{g} \cdot 55 \cdot \mathrm{I}\left({ }^{\circ} \mathrm{C}\right)$ \\ 308-Eu(tta) $)_{4}-\mathbf{4} / \mathbf{1 0}: \mathrm{g} \cdot 43 \cdot \mathrm{I}\left({ }^{\circ} \mathrm{C}\right)$ \\ 308-UO $\mathbf{O}_{2} \mathrm{Br}_{4}-\mathbf{n} / \mathbf{m}\left(\mathrm{X}^{-}=\left[\mathrm{UO}_{2} \mathrm{Br}_{4}\right]^{2-}\right.$ (with 2 cations)) \\ 308-UO $\mathbf{O}_{2} \mathbf{B r}_{4}-\mathbf{4} / \mathbf{4}: \mathrm{Cr} \cdot 219 \cdot \mathrm{SmA} \cdot 225 \cdot \operatorname{dec}\left({ }^{\circ} \mathrm{C}\right)$ \\ 308-UO ${ }_{2} \mathbf{B r}_{4}-\mathbf{4} / \mathbf{1 0}: \mathrm{Cr} \cdot(\mathrm{SmC} \cdot 183 \cdot) \cdot 185 \cdot \mathrm{SmA} \cdot 225-235 \cdot \operatorname{dec}\left({ }^{\circ} \mathrm{C}\right)$ \\ 308- $\mathrm{UO}_{2} \mathrm{Br}_{4}-\mathbf{1 0} / 4: \mathrm{Cr}_{1} \cdot 86 \cdot \mathrm{Cr}_{2} \cdot 205 \cdot \mathrm{SmA} \cdot 237 \cdot \mathrm{I}\left({ }^{\circ} \mathrm{C}\right)$ \\ 308-UO $\mathbf{O}_{2} \mathbf{B r}_{4} \mathbf{- 1 0} / \mathbf{1 0}: \mathrm{Cr}_{1} \cdot 118 \cdot \mathrm{Cr}_{2} \cdot 158 \cdot \mathrm{SmF} / \mathrm{SmI} \cdot 201 \cdot \mathrm{SmC} \cdot 223 \cdot \mathrm{SmA} \cdot 225-235 \cdot \operatorname{dec}\left({ }^{\circ} \mathrm{C}\right)$
}




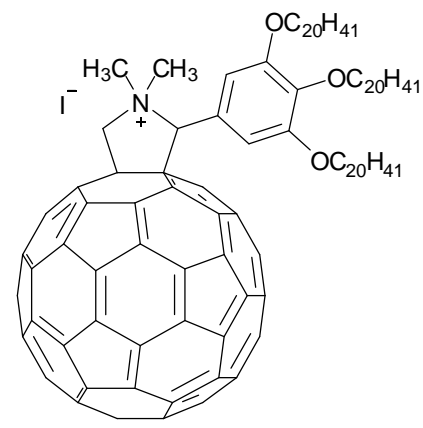

309: $\mathrm{Cr} \cdot 60 \cdot \mathrm{SmX} \cdot 188 \cdot \mathrm{I}\left({ }^{\circ} \mathrm{C}\right)$

neutral precursor of $\mathbf{3 0 9}$ (only one methyl group on the pyrrolidine nitrogen atom):

$\mathrm{Cr} \cdot 62 \cdot \mathrm{SmX} \cdot 193 \cdot \mathrm{I}\left({ }^{\circ} \mathrm{C}\right)$

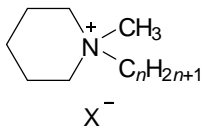

310-X-n

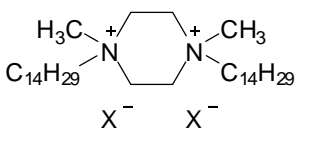

312-X

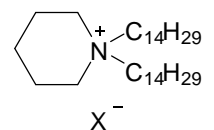

311-X

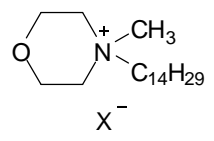

313-X

$$
\text { 310-Br-n }\left(\mathrm{X}^{-}=\mathrm{Br}^{-}\right)
$$

310-Br-8: $\mathrm{Cr} \cdot 104 \cdot \mathrm{X} \cdot 198 \cdot \mathrm{I}\left({ }^{\circ} \mathrm{C}\right)$

310-Br-10: $\mathrm{Cr} \cdot 98 \cdot \mathrm{X} \cdot 203 \cdot \mathrm{I}\left({ }^{\circ} \mathrm{C}\right)$

310-Br-12: $\mathrm{Cr} \cdot 82 \cdot \mathrm{X} \cdot 206 \cdot \mathrm{I}\left({ }^{\circ} \mathrm{C}\right)$

310-Br-14: $\mathrm{Cr} \cdot 88 \cdot \mathrm{T} \cdot 213 \cdot \mathrm{I}\left({ }^{\circ} \mathrm{C}\right)$

310-Br-16: $\mathrm{Cr}_{1} \cdot 76 \cdot \mathrm{Cr}_{2} \cdot 94 \cdot \mathrm{T} \cdot 220 \cdot \mathrm{I}\left({ }^{\circ} \mathrm{C}\right)$

310-Br-18: $\mathrm{Cr}_{1} \cdot 51 \cdot \mathrm{Cr}_{2} \cdot 100 \cdot \mathrm{T} \cdot 225 \cdot \mathrm{I}\left({ }^{\circ} \mathrm{C}\right)$

310-BF ${ }_{4}^{-14}\left(\mathrm{X}^{-}=\left[\mathrm{BF}_{4}\right]^{-}, n=14\right): \mathrm{Cr}_{1} \cdot 41 \cdot \mathrm{Cr}_{2} \cdot 62 \cdot \mathrm{Cr}_{3} \cdot 84 \cdot \mathrm{T} \cdot 109 \cdot \mathrm{I}\left({ }^{\circ} \mathrm{C}\right)$

310-PF $\mathbf{P}_{6}-14\left(\mathrm{X}^{-}=\left[\mathrm{PF}_{6}\right]^{-}, n=14\right): \mathrm{Cr}_{1} \cdot 82 \cdot \mathrm{Cr}_{2} \cdot 86 \cdot \mathrm{I}\left({ }^{\circ} \mathrm{C}\right)$

310-NTf $2-14\left(\mathrm{X}^{-}=\left[\mathrm{NTf}_{2}\right]^{-}, n=14\right): \mathrm{Cr} \cdot 41 \cdot \mathrm{I}\left({ }^{\circ} \mathrm{C}\right)$

310- $\mathrm{C}_{12} \mathrm{H}_{25} \mathbf{O S O}_{3}-\mathbf{1 4}\left(\mathrm{X}^{-}=\left[\mathrm{C}_{12} \mathrm{H}_{25} \mathrm{OSO}_{3}\right]^{-}, n=14\right): \mathrm{Cr}_{1} \cdot 51 \cdot \mathrm{Cr}_{2} \cdot 63 \cdot \mathrm{Cr}_{3} \cdot 85 \cdot \mathrm{X} \cdot 170 \cdot \mathrm{I}\left({ }^{\circ} \mathrm{C}\right)$

310-DOSS-14 ( $\left.\mathrm{X}^{-}=[\mathrm{DOSS}]^{-}, n=14\right): \mathrm{Cr} \cdot 51 \cdot \mathrm{I}\left({ }^{\circ} \mathrm{C}\right)$

310-DcHSS-14 ( $\left.\mathrm{X}^{-}=[\mathrm{DcHSS}], n=14\right): \mathrm{Cr} \cdot 56 \cdot \mathrm{I}\left({ }^{\circ} \mathrm{C}\right)$

310-DHSS-14 ( $\left.\mathrm{X}^{-}=[\mathrm{DHSS}]^{-}, n=14\right): \mathrm{Cr}_{1} \cdot 41 \cdot \mathrm{Cr}_{2} \cdot 65 \cdot \mathrm{I}\left({ }^{\circ} \mathrm{C}\right)$ 
311-Br $\left(\mathrm{X}^{-}=\mathrm{Br}^{-}\right): \mathrm{Cr}_{1} \cdot 70 \cdot \mathrm{Cr}_{2} \cdot 89 \cdot \mathrm{SmX} \cdot 181 \cdot \mathrm{I}\left({ }^{\circ} \mathrm{C}\right)$

$311-\mathrm{BF}_{4}\left(\mathrm{X}^{-}=\left[\mathrm{BF}_{4}\right]^{-}\right): \mathrm{Cr} \cdot 77 \cdot \mathrm{SmX} \cdot 127 \cdot \mathrm{I}\left({ }^{\circ} \mathrm{C}\right)$

311-PF $6\left(\mathrm{X}^{-}=\left[\mathrm{PF}_{6}\right]^{-}\right): \mathrm{Cr} \cdot 80 \cdot \mathrm{SmX} \cdot 119 \cdot \mathrm{I}\left({ }^{\circ} \mathrm{C}\right)$

311-NTf $\mathbf{N}_{2}\left(\mathrm{X}^{-}=\left[\mathrm{NTf}_{2}\right]^{-}\right)$: IL at r.t.

311- $\mathbf{C}_{12} \mathbf{H}_{25} \mathbf{O S O}_{3}\left(\mathrm{X}^{-}=\left[\mathrm{C}_{12} \mathrm{H}_{25} \mathrm{OSO}_{3}\right]^{-}\right): \mathrm{Cr} \cdot 114 \cdot \mathrm{Col}_{\mathrm{h}} \cdot 163 \cdot \mathrm{I}\left({ }^{\circ} \mathrm{C}\right)$

311-DOSS $\left(\mathrm{X}^{-}=[\mathrm{DOSS}]^{-}\right): \mathrm{Cr}_{1} \cdot 23 \cdot \mathrm{I}$ and $\mathrm{Cr}_{2} \cdot 53 \cdot \mathrm{I}\left({ }^{\circ} \mathrm{C}\right)$

311-DcHSS $\left(\mathrm{X}^{-}=[\mathrm{DcHSS}]\right): \mathrm{Cr} \cdot 45 \cdot \mathrm{I}\left({ }^{\circ} \mathrm{C}\right)$

311-DHSS $\left(\mathrm{X}^{-}=[\mathrm{DHSS}]^{-}\right): \mathrm{Cr} \cdot 106 \cdot \mathrm{I}\left({ }^{\circ} \mathrm{C}\right)$

312- $\mathrm{Br}\left(\mathrm{X}^{-}=\mathrm{Br}^{-}\right): \mathrm{Cr}_{1} \cdot 72 \cdot \mathrm{Cr}_{2} \cdot 104 \cdot \mathrm{Cr}_{3} \cdot 250 \cdot \operatorname{dec}\left({ }^{\circ} \mathrm{C}\right)$

312- $\mathrm{BF}_{4}\left(\mathrm{X}^{-}=\left[\mathrm{BF}_{4}\right]^{-}\right): \mathrm{Cr}_{1} \cdot 53 \cdot \mathrm{Cr}_{2} \cdot 92 \cdot \mathrm{Cr}_{3} \cdot 250 \cdot \operatorname{dec}\left({ }^{\circ} \mathrm{C}\right)$

312-PF $\mathbf{P}_{6}\left(\mathrm{X}^{-}=\left[\mathrm{PF}_{6}\right]\right): \mathrm{Cr}_{1} \cdot 82 \cdot \mathrm{Cr}_{2} \cdot 105 \cdot \mathrm{Cr}_{3} \cdot 250 \cdot \operatorname{dec}\left({ }^{\circ} \mathrm{C}\right)$

312-NTf ${ }_{2}\left(\mathrm{X}^{-}=\left[\mathrm{NTf}_{2}\right]^{-}\right): \mathrm{Cr}_{1} \cdot 60 \cdot \mathrm{Cr}_{2} \cdot 197 \cdot \mathrm{I}\left({ }^{\circ} \mathrm{C}\right)$

312- $\mathbf{C}_{12} \mathbf{H}_{25} \mathbf{O S O}_{3}\left(\mathrm{X}^{-}=\left[\mathrm{C}_{12} \mathrm{H}_{25} \mathrm{OSO}_{3}\right]^{-}\right): \mathrm{Cr}_{1} \cdot 33 \cdot \mathrm{Cr}_{2} \cdot 44 \cdot \mathrm{Cr}_{3} \cdot 66 \cdot \mathrm{SmA} \cdot 250 \cdot \operatorname{dec}\left({ }^{\circ} \mathrm{C}\right)$

312-DOSS $\left(\mathrm{X}^{-}=[\mathrm{DOSS}]^{-}\right): \mathrm{Cr} \cdot 32 \cdot \mathrm{SmA} \cdot 102 \cdot \mathrm{I}\left({ }^{\circ} \mathrm{C}\right)$

312-DcHSS $\left(\mathrm{X}^{-}=[\mathrm{DcHSS}]^{-}\right): \mathrm{Cr} \cdot 95 \cdot \mathrm{SmX} \cdot 126 \cdot \mathrm{SmA} \cdot 158 \cdot \mathrm{I}\left({ }^{\circ} \mathrm{C}\right)$

312-DHSS $\left(\mathrm{X}^{-}=[\mathrm{DHSS}]\right): \mathrm{Cr} \cdot 74 \cdot \mathrm{SmA} \cdot 169 \cdot \mathrm{I}\left({ }^{\circ} \mathrm{C}\right)$

313-Br $\left(\mathrm{X}^{-}=\mathrm{Br}^{-}\right): \mathrm{Cr} \cdot 97 \cdot \mathrm{X} \cdot 138 \cdot \mathrm{SmX} \cdot 221 \cdot \mathrm{SmA} \cdot 230 \cdot \mathrm{I}\left({ }^{\circ} \mathrm{C}\right)$

$313-\mathrm{BF}_{4}\left(\mathrm{X}^{-}=\left[\mathrm{BF}_{4}\right]^{-}\right): \mathrm{Cr} \cdot 82 \cdot \mathrm{SmX} \cdot 120 \cdot \mathrm{SmA} \cdot 137 \cdot \mathrm{I}\left({ }^{\circ} \mathrm{C}\right)$

313-PF ${ }_{6}\left(\mathrm{X}^{-}=\left[\mathrm{PF}_{6}\right]^{-}\right): \mathrm{Cr}_{1} \cdot 100 \cdot \mathrm{Cr}_{2} \cdot 119 \cdot \mathrm{SmX} \cdot 162 \cdot \mathrm{I}\left({ }^{\circ} \mathrm{C}\right)$

313-NTf $2\left(\mathrm{X}^{-}=\left[\mathrm{NTf}_{2}\right]^{-}\right): \mathrm{Cr} \cdot 36 \cdot \mathrm{I}\left({ }^{\circ} \mathrm{C}\right)$

313- $\mathrm{C}_{12} \mathbf{H}_{25} \mathbf{O S O}_{3}\left(\mathrm{X}^{-}=\left[\mathrm{C}_{12} \mathrm{H}_{25} \mathrm{OSO}_{3}\right]^{-}\right): \mathrm{Cr}_{1} \cdot 53 \cdot \mathrm{Cr}_{2} \cdot 80 \cdot \mathrm{Cr}_{3} \cdot 108 \cdot \mathrm{SmX} \cdot 181 \cdot \mathrm{SmA} \cdot 202 \cdot \mathrm{I}\left({ }^{\circ} \mathrm{C}\right)$

313-DOSS $\left(\mathrm{X}^{-}=[\mathrm{DOSS}]^{-}\right): \mathrm{Col}_{\mathrm{h}} \cdot 137 \cdot \mathrm{I}\left({ }^{\circ} \mathrm{C}\right)$

313-DcHSS $\left(\mathrm{X}^{-}=[\mathrm{DcHSS}]^{-}\right): \mathrm{Col}_{\mathrm{h}} \cdot 120 \cdot \mathrm{I}\left({ }^{\circ} \mathrm{C}\right)$

313-DHSS $\left(\mathrm{X}^{-}=[\mathrm{DHSS}]^{-}\right): \mathrm{Col}_{\mathrm{h}} \cdot 147 \cdot \mathrm{I}\left({ }^{\circ} \mathrm{C}\right)$

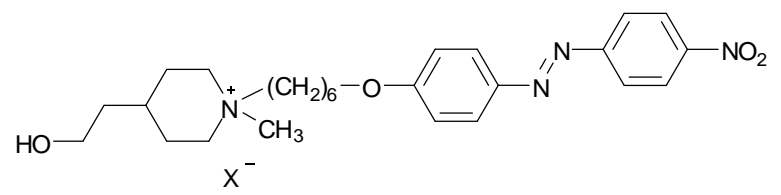

314-Br $\left(\mathrm{X}^{-}=\mathrm{Br}\right): \mathrm{Cr} \cdot 78 \cdot \mathrm{SmA} \cdot 172 \cdot \mathrm{I}\left({ }^{\circ} \mathrm{C}\right)$

314-I $\left(\mathrm{X}^{-}=\mathrm{I}^{\mathrm{I}}\right): \mathrm{g} \cdot 33 \cdot \mathrm{SmA} \cdot 149 \cdot \mathrm{I}\left({ }^{\circ} \mathrm{C}\right)$

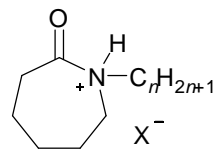

315-OTs-n $\cdot \mathrm{H}_{2} \mathrm{O}\left(\mathrm{X}^{-}=[\mathrm{OTs}]^{-}\right)$ 
315-OTs-6 $\cdot \mathrm{H}_{2} \mathrm{O}: \mathrm{Cr}_{1} \cdot 51 \cdot \mathrm{Cr}_{2} \cdot 85 \cdot \mathrm{Cr}_{3} \cdot 95 \cdot \mathrm{I}\left({ }^{\circ} \mathrm{C}\right)$

315-OTs-8 $\mathrm{H}_{2} \mathrm{O}: \mathrm{Cr}_{1} \cdot 84 \cdot \mathrm{Cr}_{2} \cdot(\mathrm{SmA} \cdot 96 \cdot) 97($ ?) $) \cdot \mathrm{I}\left({ }^{\circ} \mathrm{C}\right)$

315-OTs-10 $\mathrm{H}_{2} \mathrm{O}: \mathrm{Cr}_{1} \cdot 92 \cdot \mathrm{Cr}_{2} \cdot 98 \cdot \mathrm{SmA} \cdot 109 \cdot \mathrm{I}\left({ }^{\circ} \mathrm{C}\right)$

315-OTs-12 $\mathrm{H}_{2} \mathrm{O}: \mathrm{Cr} \cdot 98 \cdot \mathrm{SmA} \cdot 118 \cdot \mathrm{I}\left({ }^{\circ} \mathrm{C}\right)$

315-OTs-16 $\mathrm{H}_{2} \mathrm{O}: \mathrm{Cr} \cdot 104 \cdot \mathrm{SmA} \cdot 129 \cdot \mathrm{I}\left({ }^{\circ} \mathrm{C}\right)$

315-OTs-18 $\cdot \mathrm{H}_{2} \mathrm{O}: \mathrm{Cr} \cdot 106 \cdot \mathrm{SmA} \cdot 132 \cdot \mathrm{I}\left({ }^{\circ} \mathrm{C}\right)$

315- $\mathbf{C H}_{3} \mathrm{SO}_{3}-\boldsymbol{n} \cdot \mathrm{H}_{2} \mathrm{O}\left(\mathrm{X}^{-}=\left[\mathrm{CH}_{3} \mathrm{SO}_{3}\right]^{-}\right)$

315- $\mathrm{CH}_{3} \mathrm{SO}_{3}-6 \cdot \mathrm{H}_{2} \mathrm{O}: \mathrm{Cr} \cdot 127 \cdot \mathrm{I}\left({ }^{\circ} \mathrm{C}\right)$

315- $\mathrm{CH}_{3} \mathrm{SO}_{3}-\mathbf{1 6} \cdot \mathrm{H}_{2} \mathrm{O}: \mathrm{Cr} \cdot 113 \cdot \mathrm{SmA} \cdot 135 \cdot \mathrm{I}\left({ }^{\circ} \mathrm{C}\right)$

315- $\mathrm{CH}_{3} \mathrm{SO}_{3}-\mathbf{1 8} \cdot \mathrm{H}_{2} \mathrm{O}: \mathrm{Cr}_{1} \cdot 62 \cdot \mathrm{Cr}_{2} \cdot(\mathrm{SmA} \cdot 112 \cdot) 118($ ? $) \cdot \mathrm{I}\left({ }^{\circ} \mathrm{C}\right)$

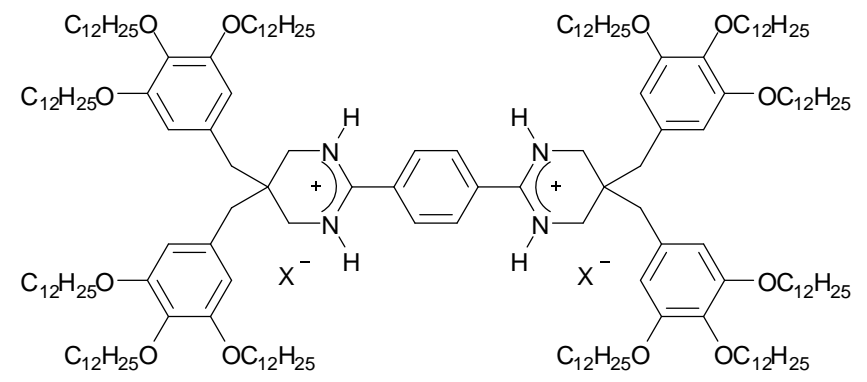

316-Cl $\left(\mathrm{X}^{-}=\mathrm{Cl}^{-}\right): \mathrm{Cr} \cdot 12 \cdot \mathrm{Col}_{\mathrm{r}} \cdot 80 \cdot \mathrm{I}\left({ }^{\circ} \mathrm{C}\right)$

316-Ag(CN $)_{2}\left(\mathrm{X}^{-}=\left[\mathrm{Ag}(\mathrm{CN})_{2}\right]^{-}\right): \mathrm{g} \cdot 64 \cdot \mathrm{Col}_{\mathrm{h}} \cdot 110 \cdot \mathrm{I}\left({ }^{\circ} \mathrm{C}\right)$

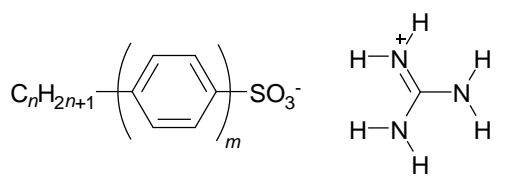

$317-m / n$

317-0/n $(n=6,8,10,12,14,16,18)$ : almost no exact transition temperatures are given 317-0/18: $\mathrm{Cr} \cdot 97 \cdot \mathrm{SmX}_{1} \cdot(155) \cdot \mathrm{SmX}_{2} \cdot 158 \cdot \mathrm{SmA} \cdot\left[\right.$ not reported] $\cdot \mathrm{I} /$ dec. $\left({ }^{\circ} \mathrm{C}\right)$

317-1/n $(n=0-12,14,16)$ : no exact transition temperatures are given 317-2/n $(n=6-10,12,14,16)$ : no exact transition temperatures are given

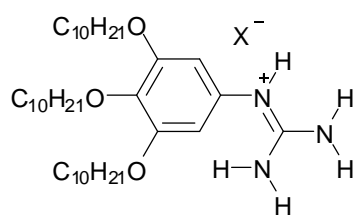

318-Cl $\left(\mathrm{X}^{-}=\mathrm{Cl}^{-}\right): \mathrm{Cr} \cdot 78 \cdot \mathrm{Col}_{\mathrm{h}} \cdot 114 \cdot \mathrm{Cub}_{\mathrm{I}}(\mathrm{Pm} \overline{3} n) \cdot 165 \cdot \mathrm{I}\left({ }^{\circ} \mathrm{C}\right)$

318-BF $4\left(\mathrm{X}^{-}=\left[\mathrm{BF}_{4}\right]^{-}\right): \mathrm{Cr} \cdot 37 \cdot \mathrm{Col}_{\mathrm{h}} \cdot 173 \cdot \mathrm{I}\left({ }^{\circ} \mathrm{C}\right)$ 
318- $\mathrm{NO}_{3}\left(\mathrm{X}^{-}=\left[\mathrm{NO}_{3}\right]^{-}\right): \mathrm{Cr} \cdot 97 \cdot \mathrm{Col}_{\mathrm{h}} \cdot 140 \cdot \mathrm{I}\left({ }^{\circ} \mathrm{C}\right)$

318-HC $\equiv \mathbf{C}-\mathbf{C O O}\left(\mathrm{X}^{-}=[\mathrm{HC} \equiv \mathrm{C}-\mathrm{COO}]^{-}\right): \mathrm{Col}_{\mathrm{r}} \cdot 93 \cdot \mathrm{I}\left({ }^{\circ} \mathrm{C}\right)$ 
Scheme S16. Guanidinium-based ILCs investigated by Laschat and co-workers.

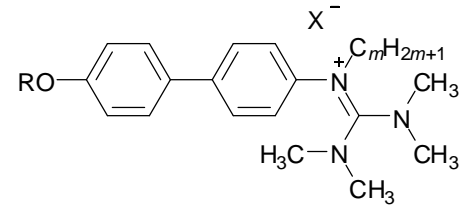

319-X-(a-k)-m ( $\mathrm{X}^{-}=\mathrm{Cl}^{-}, \mathrm{Br}^{-}, \mathrm{I}^{-},\left[\mathrm{BF}_{4}\right]^{-},\left[\mathrm{PF}_{6}\right]^{-}$,

$[\mathrm{SCN}]^{-},\left[\mathrm{B}\left(\mathrm{C}_{6} \mathrm{H}_{5}\right)_{4}\right]^{-} ; \mathrm{R}=n-\mathrm{C}_{\mathrm{n}} \mathrm{H}_{2 \mathrm{n}+1}(n=7-14,16)$,

(S)-citronellyl, 3,7,11,15-tetramethylhexadecyl; $m=0$-3)

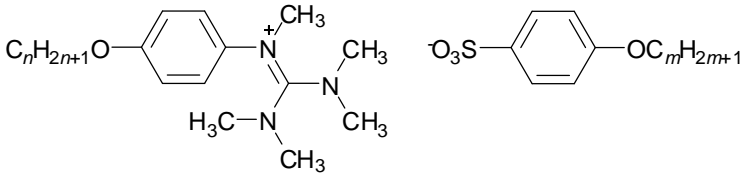

$321-n / m$

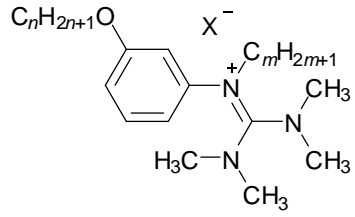

323-X-n/m ( $\left.\mathrm{X}^{-}=\mathrm{Cl}^{-}, \mathrm{Br}^{-}, \mathrm{I}^{-},\left[\mathrm{BF}_{4}\right]^{-},\left[\mathrm{PF}_{6}\right]^{-},[\mathrm{SCN}]^{-}\right)$

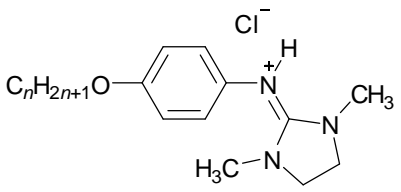

$325-n$

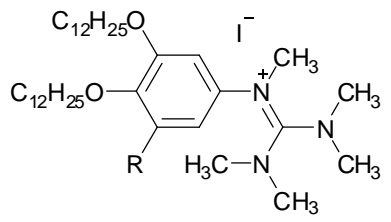

327-R $\left(\mathrm{R}=\mathrm{H}\right.$ or $\left.\mathrm{OC}_{12} \mathrm{H}_{25}\right)$

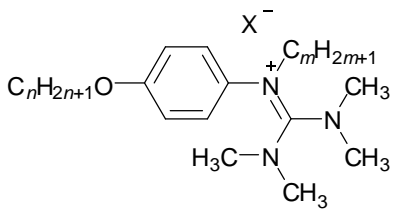

320-X-n/m (X- = $\mathrm{Cl}^{-}, \mathrm{I}^{-} ; n=8$,

$10-12,14,16,18,20 ; m=0,1)$

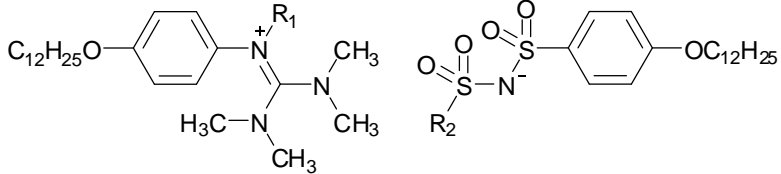

322- $\mathbf{R}_{\mathbf{1}} / \mathbf{R}_{\mathbf{2}}\left(\mathrm{R}_{1}=\mathrm{H}, \mathrm{CH}_{3} ; \mathrm{R}_{2}=\mathrm{CH}_{3}, \mathrm{CF}_{3}\right)$

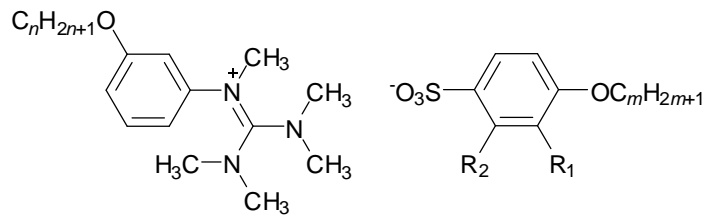

324- $\mathbf{R}_{\mathbf{1}} / \mathbf{R}_{\mathbf{2}}-\mathbf{n} / \boldsymbol{m}\left(\mathrm{R}_{1}, \mathrm{R}_{2}=\mathrm{H}\right.$ or $\left.\mathrm{OC}_{12} \mathrm{H}_{25}\right)$

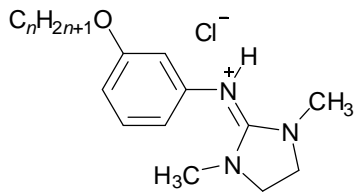

326-n<smiles></smiles>

328-(a-c)/(d-g) $\left(\mathrm{R}_{1}, \mathrm{R}_{2}, \mathrm{R}_{3}, \mathrm{R}_{4}, \mathrm{R}_{5}=\mathrm{H}\right.$ or $\left.\mathrm{OC}_{12} \mathrm{H}_{25}\right)$ 


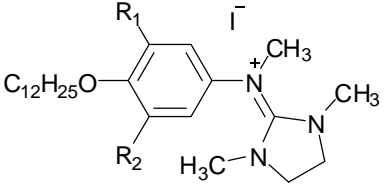

329- $\mathbf{R}_{1} / \mathbf{R}_{2}\left(\mathrm{R}_{1}, \mathrm{R}_{2}=\mathrm{H}\right.$ or $\left.\mathrm{OC}_{12} \mathrm{H}_{25}\right)$

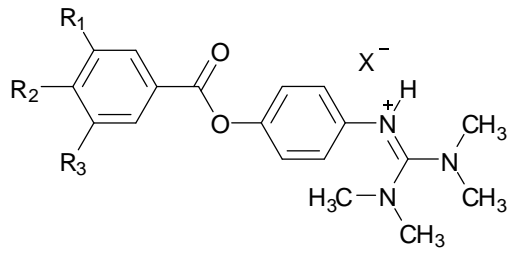

331-X-(a-f) $\left(\mathrm{X}^{-}=\mathrm{Cl}^{-},[\mathrm{OTf}]^{-},\left[\mathrm{BF}_{4}\right]^{-},\left[\mathrm{PF}_{6}\right]^{-}\right.$;

$\mathrm{R}_{1}, \mathrm{R}_{2}, \mathrm{R}_{3}=\mathrm{OC}_{n} \mathrm{H}_{2 n+1}$ or $\mathrm{H}$ )

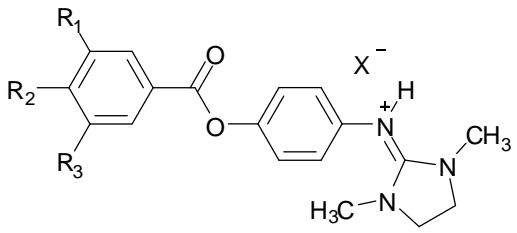

333-X-(a-h) $\left(\mathrm{X}^{-}=\mathrm{Cl}^{-}\right.$, [OTf];

$\mathrm{R}_{1}, \mathrm{R}_{2}, \mathrm{R}_{3}=\mathrm{OC}_{n} \mathrm{H}_{2 n+1}$ or $\left.\mathrm{H}\right)$

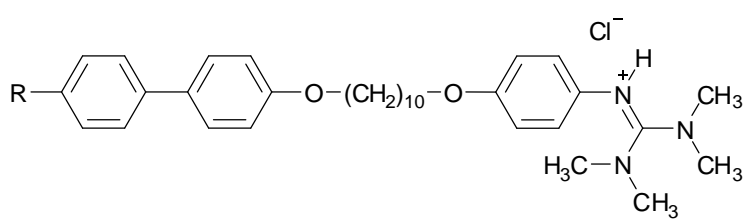

335-R $\left(\mathrm{R}=\mathrm{CN}\right.$ or $\left.\mathrm{OC}_{10} \mathrm{H}_{21}\right)$

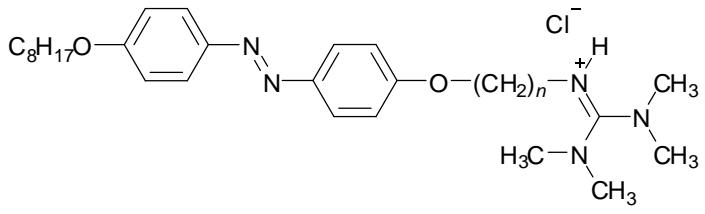

337-n<smiles>[R]c1cc([N+](C)=C2N(C)CCN2C)cc([Se]=O)c1OCCC</smiles>

330-(a-c)/(d-g) $\left(\mathrm{R}_{1}, \mathrm{R}_{2}, \mathrm{R}_{3}, \mathrm{R}_{4}, \mathrm{R}_{5}=\mathrm{H}\right.$ or $\left.\mathrm{OC}_{12} \mathrm{H}_{25}\right)$<smiles>[Y]N=C(N(C)C)N(C)C</smiles>

332-X-n $\left(\mathrm{X}^{-}=\mathrm{Cl}^{-}\right.$, [OTf] $\left.{ }^{-}\right)$

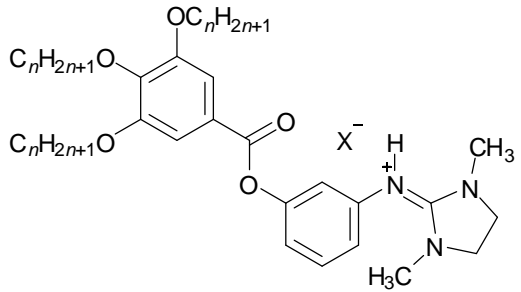

334-X-n $\left(\mathrm{X}^{-}=\mathrm{Cl}^{-}, \mathrm{OOTf}^{-}\right)$

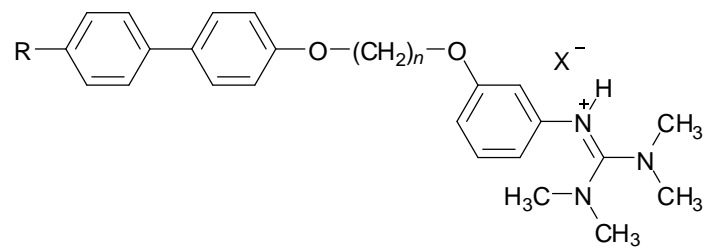

336-X-R-n ( $\mathrm{X}^{-}=\mathrm{Cl}^{-}, \mathrm{Br}^{-}, \mathrm{I}^{-},\left[\mathrm{BF}_{4}\right]^{-},\left[\mathrm{PF}_{6}\right]^{-}$, [OTf]',

[CH $\left.\mathrm{COO}_{3}\right]^{-},[\mathrm{SCN}]^{-} ; \mathrm{R}=\mathrm{CN}$ or $\left.\mathrm{OC}_{10} \mathrm{H}_{21}\right)$

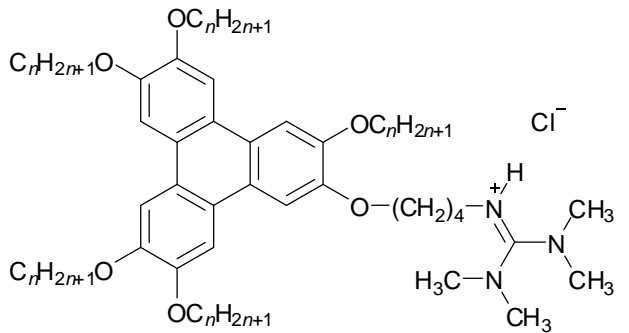

338-n 


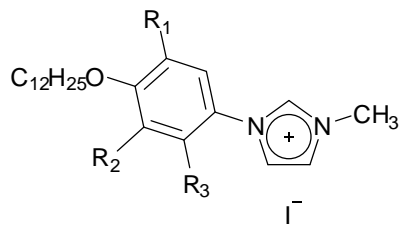

339-(a-d) $\left(\mathrm{R}_{1}, \mathrm{R}_{2}, \mathrm{R}_{3}=\mathrm{H}\right.$ or $\left.\mathrm{OC}_{12} \mathrm{H}_{25}\right)$
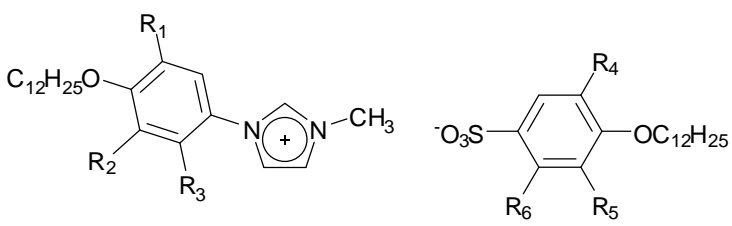

340-(a-e) $\left(\mathrm{R}_{1}, \mathrm{R}_{2}, \mathrm{R}_{3}, \mathrm{R}_{4}, \mathrm{R}_{5}, \mathrm{R}_{6}=\mathrm{H}\right.$ or $\left.\mathrm{OC}_{12} \mathrm{H}_{25}\right)$

$$
\text { 319-Cl-(a-k)-0 ( } \left.\mathrm{X}^{-}=\mathrm{Cl}^{-} ; m=0\right)
$$

319-Cl-a-0 $\left(\mathrm{R}=n-\mathrm{C}_{7} \mathrm{H}_{15}\right): \mathrm{Cr} \cdot 145 \cdot \mathrm{I}\left({ }^{\circ} \mathrm{C}\right)$

319-Cl-b-0 $\left(\mathrm{R}=n-\mathrm{C}_{8} \mathrm{H}_{17}\right): \mathrm{g} \cdot 39 \cdot \mathrm{SmA} \cdot 119 \cdot \mathrm{I}\left({ }^{\circ} \mathrm{C}\right)$

319-Cl-c-0 $\left(\mathrm{R}=n-\mathrm{C}_{9} \mathrm{H}_{19}\right): \mathrm{g} \cdot 26 \cdot \mathrm{SmA} \cdot 180 \cdot \mathrm{I}\left({ }^{\circ} \mathrm{C}\right)$

319-Cl-d-0 $\left(\mathrm{R}=n-\mathrm{C}_{10} \mathrm{H}_{21}\right): \mathrm{g} \cdot 46 \cdot \mathrm{Cr}_{1} \cdot 111 \cdot \mathrm{Cr}_{2} \cdot 133 \cdot \mathrm{SmA} \cdot 181 \cdot \mathrm{I}\left({ }^{\circ} \mathrm{C}\right)^{123} / \mathrm{g} \cdot 33 \cdot \mathrm{SmA} \cdot 193 \cdot \mathrm{I}\left({ }^{\circ} \mathrm{C}\right)^{124}$

319-Cl-e-0 $\left(\mathrm{R}=n-\mathrm{C}_{11} \mathrm{H}_{23}\right): \mathrm{g} \cdot 40 \cdot \mathrm{SmA} \cdot 193 \cdot \mathrm{I}\left({ }^{\circ} \mathrm{C}\right)$

319-Cl-f-0 $\left(\mathrm{R}=n-\mathrm{C}_{12} \mathrm{H}_{25}\right): \mathrm{g} \cdot 42 \cdot \mathrm{SmA} \cdot 208 \cdot \mathrm{I}\left({ }^{\circ} \mathrm{C}\right)$

319-Cl-g-0 $\left(\mathrm{R}=n-\mathrm{C}_{13} \mathrm{H}_{27}\right): \mathrm{g} \cdot 40 \cdot \mathrm{SmA} \cdot 220 \cdot \mathrm{I}\left({ }^{\circ} \mathrm{C}\right)$

319-Cl-h-0 $\left(\mathrm{R}=n-\mathrm{C}_{14} \mathrm{H}_{19}\right): \mathrm{g} \cdot 40 \cdot \mathrm{SmA} \cdot 234 \cdot \mathrm{I}\left({ }^{\circ} \mathrm{C}\right)$

319-Cl-i-0 $\left(\mathrm{R}=n-\mathrm{C}_{16} \mathrm{H}_{33}\right): \mathrm{Cr} \cdot 43^{123} / 47^{124} \cdot \mathrm{SmA} \cdot 223^{123} / 231^{124} \cdot \mathrm{I}\left({ }^{\circ} \mathrm{C}\right)$

319-Cl-j-0 (R = (S)-citronellyl): SmA $\cdot 169 \cdot \mathrm{I}\left({ }^{\circ} \mathrm{C}\right)$

319-Cl-k-0 (R = 3,7,11,15-tetramethylhexadecyl): $\mathrm{g} \cdot 28 \cdot \mathrm{SmA} \cdot 221 \cdot \mathrm{I}\left({ }^{\circ} \mathrm{C}\right)$

319-Br-(d,f,h,i)-0 ( $\left.\mathrm{X}^{-}=\mathrm{Br}^{-} ; m=0\right)$

319-Br-d-0 $\left(\mathrm{R}=n-\mathrm{C}_{10} \mathrm{H}_{21}\right): \mathrm{g} \cdot 44 \cdot \mathrm{SmA} \cdot 162 \cdot \mathrm{I}\left({ }^{\circ} \mathrm{C}\right)$

319-Br-f-0 $\left(\mathrm{R}=n-\mathrm{C}_{12} \mathrm{H}_{25}\right): \mathrm{g} \cdot 19 \cdot \mathrm{SmA} \cdot 204 \cdot \mathrm{I}\left({ }^{\circ} \mathrm{C}\right)$

319-Br-h-0 $\left(\mathrm{R}=n-\mathrm{C}_{14} \mathrm{H}_{29}\right): \mathrm{g} \cdot 54 \cdot \mathrm{SmA} \cdot 244 \cdot \mathrm{I}\left({ }^{\circ} \mathrm{C}\right)$

319-Br-i-0 $\left(\mathrm{R}=n-\mathrm{C}_{16} \mathrm{H}_{33}\right): \mathrm{g} \cdot 47 \cdot \mathrm{SmA} \cdot 232 \cdot \mathrm{I}\left({ }^{\circ} \mathrm{C}\right)$

319-I-(d,f,g,h,i)-m ( $\left.\mathrm{X}^{-}=\mathrm{I}^{-}\right)$

319-I-d-0 $\left(\mathrm{R}=n-\mathrm{C}_{10} \mathrm{H}_{21}\right): \mathrm{g} \cdot 9 \cdot \mathrm{SmA} \cdot 141 \cdot \mathrm{I}\left({ }^{\circ} \mathrm{C}\right)$

319-I-f-0 $\left(\mathrm{R}=n-\mathrm{C}_{12} \mathrm{H}_{25}\right): \mathrm{g} \cdot 16 \cdot \mathrm{SmA} \cdot 187 \cdot \mathrm{I}\left({ }^{\circ} \mathrm{C}\right)$

319-I-h-0 $\left(\mathrm{R}=n-\mathrm{C}_{14} \mathrm{H}_{29}\right): \mathrm{g} \cdot 38 \cdot \mathrm{SmA} \cdot 210 \cdot \mathrm{I}\left({ }^{\circ} \mathrm{C}\right)$

319-I-i-0 $\left(\mathrm{R}=n-\mathrm{C}_{16} \mathrm{H}_{33}\right): \mathrm{g} \cdot 41 \cdot \mathrm{SmA} \cdot 237 \cdot \mathrm{I}\left({ }^{\circ} \mathrm{C}\right)$

319-I-d-1 $\left(\mathrm{R}=n-\mathrm{C}_{10} \mathrm{H}_{21}\right): \mathrm{Cr} \cdot 171 \cdot \mathrm{I}\left({ }^{\circ} \mathrm{C}\right)$

319-I-f-1 $\left(\mathrm{R}=n-\mathrm{C}_{12} \mathrm{H}_{25}\right): \mathrm{Cr} \cdot 173 \cdot \mathrm{I}\left({ }^{\circ} \mathrm{C}\right)$

319-I-g-1 ( $\left.\mathrm{R}=n-\mathrm{C}_{13} \mathrm{H}_{27}\right): \mathrm{Cr}_{1} \cdot 153 \cdot \mathrm{Cr}_{2} \cdot 166 \cdot \mathrm{SmA} \cdot 213 \cdot \mathrm{I}\left({ }^{\circ} \mathrm{C}\right)$

319-I-h-1 $\left(\mathrm{R}=n-\mathrm{C}_{14} \mathrm{H}_{29}\right): \mathrm{Cr}_{1} \cdot 150 \cdot \mathrm{Cr}_{2} \cdot 164 \cdot \mathrm{SmA} \cdot 232 \cdot \mathrm{I}\left({ }^{\circ} \mathrm{C}\right)$

319-I-i-1 $\left(\mathrm{R}=n-\mathrm{C}_{16} \mathrm{H}_{33}\right): \mathrm{Cr} \cdot 153 \cdot \mathrm{SmA} \cdot 255 \cdot \mathrm{I}\left({ }^{\circ} \mathrm{C}\right)$

319-I-d-2 $\left(\mathrm{R}=n-\mathrm{C}_{10} \mathrm{H}_{21}\right): \mathrm{Cr} \cdot \mathrm{ca} .147 \cdot \mathrm{I}\left({ }^{\circ} \mathrm{C}\right)$

319-I-f-2 $\left(\mathrm{R}=n-\mathrm{C}_{12} \mathrm{H}_{25}\right): \mathrm{Cr}_{1} \cdot 144 \cdot \mathrm{Cr}_{2} \cdot 151 \cdot \mathrm{I}\left({ }^{\circ} \mathrm{C}\right)$

319-I-g-2 $\left(\mathrm{R}=n-\mathrm{C}_{13} \mathrm{H}_{27}\right): \mathrm{Cr}_{1} \cdot 103 \cdot \mathrm{Cr}_{2} \cdot 112 \cdot \mathrm{Cr}_{3} \cdot 145 \cdot \mathrm{SmA} \cdot 165 \cdot \mathrm{I}\left({ }^{\circ} \mathrm{C}\right)$

319-I-h-2 $\left(\mathrm{R}=n-\mathrm{C}_{14} \mathrm{H}_{29}\right): \mathrm{Cr}_{1} \cdot 109 \cdot \mathrm{Cr}_{2} \cdot 137 \cdot \mathrm{Cr}_{3} \cdot 150 \cdot \mathrm{SmA} \cdot 187 \cdot \mathrm{I}\left({ }^{\circ} \mathrm{C}\right)$ 


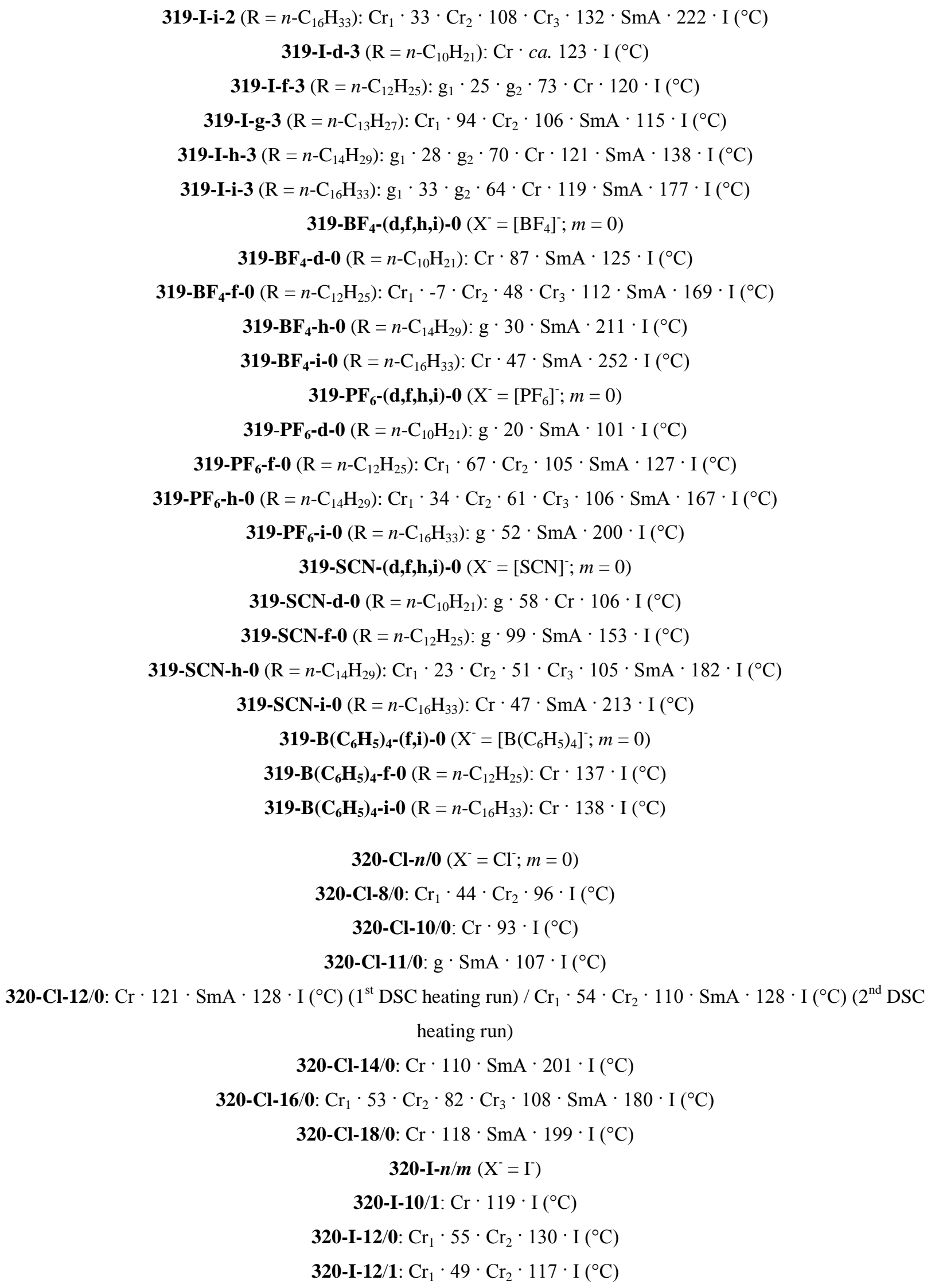




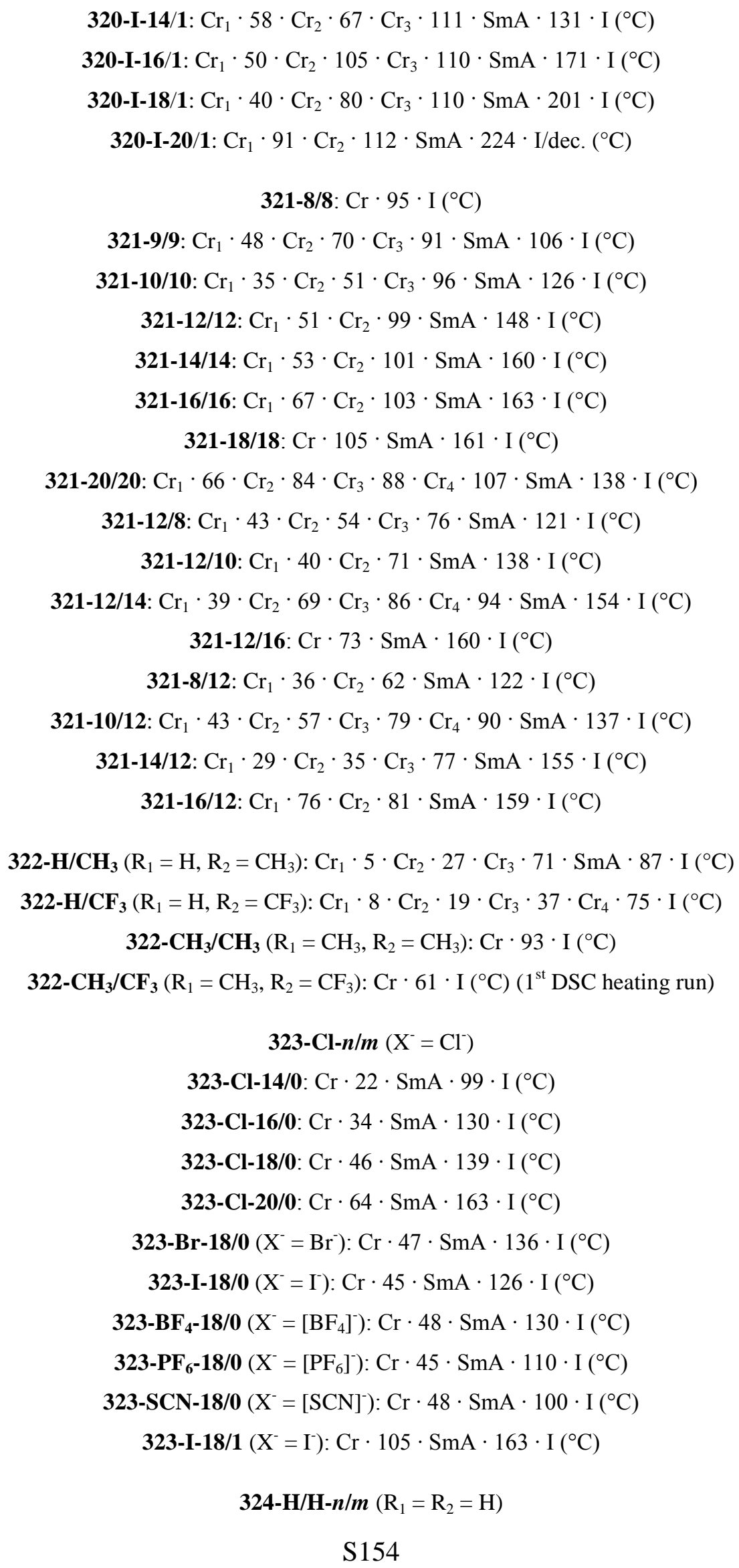


324-H/H-12/18: $\mathrm{Cr} \cdot 22 \cdot \mathrm{SmA} \cdot 113 \cdot \mathrm{I}\left({ }^{\circ} \mathrm{C}\right)$

324-H/H-14/14: $\mathrm{Cr} \cdot 22 \cdot \mathrm{Col}_{\mathrm{r}} \cdot 87 \cdot \mathrm{I}\left({ }^{\circ} \mathrm{C}\right)$

324-H/H-14/18: $\mathrm{Cr} \cdot 38 \cdot \mathrm{SmA} \cdot 116 \cdot \mathrm{I}\left({ }^{\circ} \mathrm{C}\right)$

324-H/H-16/18: $\mathrm{Cr} \cdot 50 \cdot \mathrm{Col}_{\mathrm{r}} \cdot 119 \cdot \mathrm{I}\left({ }^{\circ} \mathrm{C}\right)$

324-H/H-18/14: $\mathrm{Cr} \cdot 42 \cdot \mathrm{Col}_{\mathrm{r}} \cdot 101 \cdot \mathrm{I}\left({ }^{\circ} \mathrm{C}\right)$

324-H/H-18/16: $\mathrm{Cr} \cdot 53 \cdot \mathrm{Col}_{\mathrm{r}} \cdot 119 \cdot \mathrm{I}\left({ }^{\circ} \mathrm{C}\right)$

324-H/H-18/18: $\mathrm{Cr} \cdot 58 \cdot \mathrm{Col}_{\mathrm{r}} \cdot 122 \cdot \mathrm{I}\left({ }^{\circ} \mathrm{C}\right)$

324-H/H-20/18: $\mathrm{Cr} \cdot 65 \cdot \mathrm{SmA} \cdot 129 \cdot \mathrm{I}\left({ }^{\circ} \mathrm{C}\right)$

324-OC ${ }_{12} \mathrm{H}_{25} / \mathbf{H}-\mathbf{1 8} / \mathbf{1 2}\left(\mathrm{R}_{1}=\mathrm{OC}_{12} \mathrm{H}_{25}, \mathrm{R}_{2}=\mathrm{H}\right): \mathrm{Cr} \cdot 42 \cdot \mathrm{SmA} \cdot 80 \cdot \mathrm{I}\left({ }^{\circ} \mathrm{C}\right)$

324-OC ${ }_{12} \mathrm{H}_{25} / \mathrm{OC}_{12} \mathrm{H}_{25}-\mathbf{1 8} / \mathbf{1 2}\left(\mathrm{R}_{1}=\mathrm{R}_{2}=\mathrm{OC}_{12} \mathrm{H}_{25}\right): \mathrm{Cr} \cdot 17 \cdot \mathrm{Col}_{\mathrm{h}} \cdot 72 \cdot \mathrm{I}\left({ }^{\circ} \mathrm{C}\right)$

325-14: $\mathrm{Cr} \cdot 121 \cdot \mathrm{SmA} \cdot 205 \cdot \mathrm{I}\left({ }^{\circ} \mathrm{C}\right)$

325-18: $\mathrm{Cr} \cdot 111 \cdot \mathrm{SmA} \cdot 222 \cdot \mathrm{I}\left({ }^{\circ} \mathrm{C}\right)$

326-14: $\mathrm{Cr} \cdot 61 \cdot \mathrm{SmA} \cdot 145 \cdot \mathrm{I}\left({ }^{\circ} \mathrm{C}\right)$

326-18: $\mathrm{Cr} \cdot 76 \cdot \mathrm{SmA} \cdot 157 \cdot \mathrm{I}\left({ }^{\circ} \mathrm{C}\right)$

327-H $(\mathrm{R}=\mathrm{H}): \mathrm{Cr} \cdot 60 \cdot \mathrm{SmA} \cdot 150 \cdot \mathrm{I}\left({ }^{\circ} \mathrm{C}\right)$

$327-\mathrm{OC}_{12} \mathbf{H}_{25}\left(\mathrm{R}=\mathrm{OC}_{12} \mathrm{H}_{25}\right): \mathrm{Cr} \cdot 55 \cdot \mathrm{Col}_{\mathrm{h}} \cdot 129 \cdot \mathrm{I}\left({ }^{\circ} \mathrm{C}\right)$

328-a/(d-g) $\left(\mathrm{R}_{1}=\mathrm{R}_{2}=\mathrm{H}\right)$

328-a/d $\left(\mathrm{R}_{3}=\mathrm{R}_{4}=\mathrm{R}_{5}=\mathrm{H}\right): \mathrm{Cr} \cdot 99 \cdot \mathrm{SmA} \cdot 148 \cdot \mathrm{I}\left({ }^{\circ} \mathrm{C}\right)$

328-a/e $\left(\mathrm{R}_{3}=\mathrm{OC}_{12} \mathrm{H}_{25}, \mathrm{R}_{4}=\mathrm{R}_{5}=\mathrm{H}\right): \mathrm{Cr} \cdot 87 \cdot \mathrm{SmA} \cdot 119 \cdot \mathrm{I}\left({ }^{\circ} \mathrm{C}\right)$

328-a/f $\left(\mathrm{R}_{3}=\mathrm{R}_{4}=\mathrm{OC}_{12} \mathrm{H}_{25}, \mathrm{R}_{5}=\mathrm{H}\right): \mathrm{Cr} \cdot 85 \cdot \mathrm{Col}_{\mathrm{h}} \cdot 116 \cdot \mathrm{I}\left({ }^{\circ} \mathrm{C}\right)$

328-a/g $\left(\mathrm{R}_{3}=\mathrm{H}, \mathrm{R}_{4}=\mathrm{R}_{5}=\mathrm{OC}_{12} \mathrm{H}_{25}\right): \mathrm{Cr} \cdot 76 \cdot \mathrm{Col}_{\mathrm{h}} \cdot 113 \cdot \mathrm{I}\left({ }^{\circ} \mathrm{C}\right)$

328-b/(d-g) $\left(\mathrm{R}_{1}=\mathrm{OC}_{12} \mathrm{H}_{25}, \mathrm{R}_{2}=\mathrm{H}\right)$

328-b/d $\left(\mathrm{R}_{3}=\mathrm{R}_{4}=\mathrm{R}_{5}=\mathrm{H}\right): \mathrm{Cr} \cdot 74 \cdot \mathrm{SmA} \cdot 93 \cdot \mathrm{I}\left({ }^{\circ} \mathrm{C}\right)$

328-b/e $\left(\mathrm{R}_{3}=\mathrm{OC}_{12} \mathrm{H}_{25}, \mathrm{R}_{4}=\mathrm{R}_{5}=\mathrm{H}\right)$ : $\mathrm{Cr} \cdot 54 \cdot \mathrm{Col}_{\mathrm{h}} \cdot 144 \cdot \mathrm{I}\left({ }^{\circ} \mathrm{C}\right)$

328-b/f $\left(\mathrm{R}_{3}=\mathrm{R}_{4}=\mathrm{OC}_{12} \mathrm{H}_{25}, \mathrm{R}_{5}=\mathrm{H}\right): \mathrm{Cr} \cdot 29 \cdot \mathrm{Col}_{\mathrm{h}} \cdot 128 \cdot \mathrm{I}\left({ }^{\circ} \mathrm{C}\right)$

328-b/g $\left(\mathrm{R}_{3}=\mathrm{H}, \mathrm{R}_{4}=\mathrm{R}_{5}=\mathrm{OC}_{12} \mathrm{H}_{25}\right): \mathrm{Cr} \cdot 27 \cdot \mathrm{Col}_{\mathrm{h}} \cdot 115 \cdot \mathrm{I}\left({ }^{\circ} \mathrm{C}\right)$

$$
\text { 328-c/(d-e) }\left(\mathrm{R}_{1}=\mathrm{R}_{2}=\mathrm{OC}_{12} \mathrm{H}_{25}\right)
$$

328-c/d $\left(\mathrm{R}_{3}=\mathrm{R}_{4}=\mathrm{R}_{5}=\mathrm{H}\right): \mathrm{Cr} \cdot 51 \cdot \mathrm{Col}_{\mathrm{h}} \cdot 134 \cdot \mathrm{I}\left({ }^{\circ} \mathrm{C}\right)$

328-c/e $\left(\mathrm{R}_{3}=\mathrm{OC}_{12} \mathrm{H}_{25}, \mathrm{R}_{4}=\mathrm{R}_{5}=\mathrm{H}\right): \mathrm{Cr} \cdot 19 \cdot \mathrm{Col}_{\mathrm{h}} \cdot 114 \cdot \mathrm{I}\left({ }^{\circ} \mathrm{C}\right)$

329-H/H $\left(\mathrm{R}_{1}=\mathrm{R}_{2}=\mathrm{H}\right): \mathrm{Cr} \cdot 90 \cdot \mathrm{SmA} \cdot 115 \cdot \mathrm{I}\left({ }^{\circ} \mathrm{C}\right)$

329-OC ${ }_{12} \mathrm{H}_{25} / \mathrm{H}\left(\mathrm{R}_{1}=\mathrm{OC}_{12} \mathrm{H}_{25}, \mathrm{R}_{2}=\mathrm{H}\right): \mathrm{Cr} \cdot 107 \cdot \mathrm{SmA} \cdot 136 \cdot \mathrm{I}\left({ }^{\circ} \mathrm{C}\right)$

329-OC ${ }_{12} \mathbf{H}_{25} / \mathbf{O C}_{12} \mathbf{H}_{25}\left(\mathrm{R}_{1}=\mathrm{R}_{2}=\mathrm{OC}_{12} \mathrm{H}_{25}\right): \mathrm{Cr} \cdot 52 \cdot \mathrm{Col}_{\mathrm{h}} \cdot 129 \cdot \mathrm{I}\left({ }^{\circ} \mathrm{C}\right)$

330-a/(d-g) $\left(\mathrm{R}_{1}=\mathrm{R}_{2}=\mathrm{H}\right)$

330-a/d $\left(\mathrm{R}_{3}=\mathrm{R}_{4}=\mathrm{R}_{5}=\mathrm{H}\right): \mathrm{Cr} \cdot 100 \cdot \mathrm{SmA} \cdot 154 \cdot \mathrm{I}\left({ }^{\circ} \mathrm{C}\right)$

330-a/e $\left(\mathrm{R}_{3}=\mathrm{OC}_{12} \mathrm{H}_{25}, \mathrm{R}_{4}=\mathrm{R}_{5}=\mathrm{H}\right): \mathrm{Cr} \cdot 80 \cdot \mathrm{SmA} \cdot 113 \cdot \mathrm{I}\left({ }^{\circ} \mathrm{C}\right)$ 


$$
\begin{aligned}
& \text { 330-a/f }\left(\mathrm{R}_{3}=\mathrm{R}_{4}=\mathrm{OC}_{12} \mathrm{H}_{25}, \mathrm{R}_{5}=\mathrm{H}\right): \mathrm{Cr} \cdot 68 \cdot \mathrm{Col}_{\mathrm{h}} \cdot 140 \cdot \mathrm{I}\left({ }^{\circ} \mathrm{C}\right) \\
& \text { 330-a/g }\left(\mathrm{R}_{3}=\mathrm{H}, \mathrm{R}_{4}=\mathrm{R}_{5}=\mathrm{OC}_{12} \mathrm{H}_{25}\right): \mathrm{Cr} \cdot 38 \cdot \mathrm{Col}_{\mathrm{h}} \cdot 124 \cdot \mathrm{I}\left({ }^{\circ} \mathrm{C}\right) \\
& \text { 330-b/(e-g) }\left(\mathrm{R}_{1}=\mathrm{OC}_{12} \mathrm{H}_{25}, \mathrm{R}_{2}=\mathrm{H}\right) \\
& \text { 330-b/e }\left(\mathrm{R}_{3}=\mathrm{OC}_{12} \mathrm{H}_{25}, \mathrm{R}_{4}=\mathrm{R}_{5}=\mathrm{H}\right): \mathrm{Cr} \cdot 49 \cdot \mathrm{Col}_{\mathrm{h}} \cdot 153 \cdot \mathrm{I}\left({ }^{\circ} \mathrm{C}\right) \\
& \text { 330-b/f }\left(\mathrm{R}_{3}=\mathrm{R}_{4}=\mathrm{OC}_{12} \mathrm{H}_{25}, \mathrm{R}_{5}=\mathrm{H}\right): \mathrm{Cr} \cdot 55 \cdot \mathrm{Col}_{\mathrm{h}} \cdot 119 \cdot \mathrm{I}\left({ }^{\circ} \mathrm{C}\right) \\
& \text { 330-b/g }\left(\mathrm{R}_{3}=\mathrm{H}, \mathrm{R}_{4}=\mathrm{R}_{5}=\mathrm{OC}_{12} \mathrm{H}_{25}\right): \mathrm{Cr} \cdot 24 \cdot \mathrm{Col}_{\mathrm{h}} \cdot 108 \cdot \mathrm{I}\left({ }^{\circ} \mathrm{C}\right) \\
& \text { 330-c/(d-f) }\left(\mathrm{R}_{1}=\mathrm{R}_{2}=\mathrm{OC}_{12} \mathrm{H}_{25}\right) \\
& \text { 330-c/d }\left(\mathrm{R}_{3}=\mathrm{R}_{4}=\mathrm{R}_{5}=\mathrm{H}\right): \mathrm{Cr} \cdot 51 \cdot \mathrm{Col}_{\mathrm{h}} \cdot 132 \cdot \mathrm{I}\left({ }^{\circ} \mathrm{C}\right) \\
& \text { 330-c/e }\left(\mathrm{R}_{3}=\mathrm{OC}_{12} \mathrm{H}_{25}, \mathrm{R}_{4}=\mathrm{R}_{5}=\mathrm{H}\right): \mathrm{Cr}_{1} \cdot 78 \cdot \mathrm{Cr}_{2} \cdot 90 \cdot \mathrm{Col}_{\mathrm{h}} \cdot 106 \cdot \mathrm{I}\left({ }^{\circ} \mathrm{C}\right) \\
& \text { 330-c/f }\left(\mathrm{R}_{3}=\mathrm{R}_{4}=\mathrm{OC}_{12} \mathrm{H}_{25}, \mathrm{R}_{5}=\mathrm{H}\right): \mathrm{Cr} \cdot 66 \cdot \text { [plastic phase] } \cdot 81 \cdot \mathrm{I}\left({ }^{\circ} \mathrm{C}\right) \\
& \text { 331-Cl-(a-f) }\left(\mathrm{X}^{-}=\mathrm{Cl}^{-}\right)
\end{aligned}
$$

331-Cl-a $\left(\mathrm{R}_{1}=\mathrm{H}, \mathrm{R}_{2}=\mathrm{OC}_{12} \mathrm{H}_{25}, \mathrm{R}_{3}=\mathrm{H}\right): \mathrm{Cr} \cdot 90 \cdot \mathrm{SmA} \cdot 198 \cdot \mathrm{I} /$ dec. $\left({ }^{\circ} \mathrm{C}\right)$

331-Cl-b $\left(\mathrm{R}_{1}=\mathrm{OC}_{12} \mathrm{H}_{25}, \mathrm{R}_{2}=\mathrm{OC}_{12} \mathrm{H}_{25}, \mathrm{R}_{3}=\mathrm{H}\right): \mathrm{Cr} \cdot 50 \cdot \mathrm{Col}_{\mathrm{h}} \cdot 185 \cdot \mathrm{I} /$ dec. $\left({ }^{\circ} \mathrm{C}\right)$

331-Cl-c $\left(\mathrm{R}_{1}=\mathrm{OC}_{10} \mathrm{H}_{21}, \mathrm{R}_{2}=\mathrm{OC}_{10} \mathrm{H}_{21}, \mathrm{R}_{3}=\mathrm{OC}_{10} \mathrm{H}_{21}\right): \mathrm{g} \cdot 13 \cdot \mathrm{Col}_{\mathrm{h}} \cdot 205 \cdot \mathrm{I} /$ dec. $\left({ }^{\circ} \mathrm{C}\right)$

331-Cl-d $\left(\mathrm{R}_{1}=\mathrm{OC}_{12} \mathrm{H}_{25}, \mathrm{R}_{2}=\mathrm{OC}_{12} \mathrm{H}_{25}, \mathrm{R}_{3}=\mathrm{OC}_{12} \mathrm{H}_{25}\right): \mathrm{g} \cdot 33 \cdot \mathrm{Col}_{\mathrm{h}} \cdot 218 \cdot \mathrm{I} /$ dec. $\left({ }^{\circ} \mathrm{C}\right)$

331-Cl-e $\left(\mathrm{R}_{1}=\mathrm{OC}_{14} \mathrm{H}_{29}, \mathrm{R}_{2}=\mathrm{OC}_{14} \mathrm{H}_{29}, \mathrm{R}_{3}=\mathrm{OC}_{14} \mathrm{H}_{29}\right): \mathrm{g} \cdot 33 \cdot \mathrm{Col}_{\mathrm{h}} \cdot 218 \cdot \mathrm{I} / \mathrm{dec} .\left({ }^{\circ} \mathrm{C}\right)$

331-Cl-f $\left(\mathrm{R}_{1}=\mathrm{OC}_{16} \mathrm{H}_{33}, \mathrm{R}_{2}=\mathrm{OC}_{16} \mathrm{H}_{33}, \mathrm{R}_{3}=\mathrm{OC}_{16} \mathrm{H}_{33}\right): \mathrm{g} \cdot 58 \cdot \mathrm{Col}_{\mathrm{h}} \cdot 190 \cdot \mathrm{I} /$ dec. $\left({ }^{\circ} \mathrm{C}\right)$

331- $\mathrm{BF}_{4}-\mathbf{d}\left(\mathrm{X}^{-}=\left[\mathrm{BF}_{4}\right]^{-} ; \mathrm{R}_{1}=\mathrm{OC}_{12} \mathrm{H}_{25}, \mathrm{R}_{2}=\mathrm{OC}_{12} \mathrm{H}_{25}, \mathrm{R}_{3}=\mathrm{OC}_{12} \mathrm{H}_{25}\right): \mathrm{Cr} \cdot 33 \cdot \mathrm{Col} \cdot 228 \cdot \operatorname{dec} .\left({ }^{\circ} \mathrm{C}\right)$

331-OTf-(a-f) $\left(\mathrm{X}^{-}=[\mathrm{OTf}]^{-}\right)$

331-OTf-a $\left(\mathrm{R}_{1}=\mathrm{H}, \mathrm{R}_{2}=\mathrm{OC}_{12} \mathrm{H}_{25}, \mathrm{R}_{3}=\mathrm{H}\right)$ : $\mathrm{Cr} \cdot 110 \cdot \mathrm{I}\left({ }^{\circ} \mathrm{C}\right)$

331-OTf-b $\left(\mathrm{R}_{1}=\mathrm{OC}_{12} \mathrm{H}_{25}, \mathrm{R}_{2}=\mathrm{OC}_{12} \mathrm{H}_{25}, \mathrm{R}_{3}=\mathrm{H}\right): \mathrm{Cr} \cdot 89 \cdot \mathrm{SmA} \cdot 124 \cdot \mathrm{I}\left({ }^{\circ} \mathrm{C}\right)$

331-OTf-c $\left(\mathrm{R}_{1}=\mathrm{OC}_{10} \mathrm{H}_{21}, \mathrm{R}_{2}=\mathrm{OC}_{10} \mathrm{H}_{21}, \mathrm{R}_{3}=\mathrm{OC}_{10} \mathrm{H}_{21}\right): \mathrm{g} \cdot 80 \cdot \mathrm{I}\left({ }^{\circ} \mathrm{C}\right)$

331-OTf-d $\left(\mathrm{R}_{1}=\mathrm{OC}_{12} \mathrm{H}_{25}, \mathrm{R}_{2}=\mathrm{OC}_{12} \mathrm{H}_{25}, \mathrm{R}_{3}=\mathrm{OC}_{12} \mathrm{H}_{25}\right): \mathrm{Cr} \cdot 43 \cdot \mathrm{Col}_{\mathrm{h}} \cdot 123 \cdot \mathrm{I}\left({ }^{\circ} \mathrm{C}\right)$

331-OTf-e $\left(\mathrm{R}_{1}=\mathrm{OC}_{14} \mathrm{H}_{29}, \mathrm{R}_{2}=\mathrm{OC}_{14} \mathrm{H}_{29}, \mathrm{R}_{3}=\mathrm{OC}_{14} \mathrm{H}_{29}\right)$ : g $\cdot 41 \cdot \mathrm{Col}_{\mathrm{h}} \cdot 146 \cdot \mathrm{I}\left({ }^{\circ} \mathrm{C}\right)$

331-OTf-f $\left(\mathrm{R}_{1}=\mathrm{OC}_{16} \mathrm{H}_{33}, \mathrm{R}_{2}=\mathrm{OC}_{16} \mathrm{H}_{33}, \mathrm{R}_{3}=\mathrm{OC}_{16} \mathrm{H}_{33}\right): \mathrm{Cr} \cdot 65 \cdot \mathrm{Col}_{\mathrm{h}} \cdot 156 \cdot \mathrm{I}\left({ }^{\circ} \mathrm{C}\right)$

331- $\mathrm{PF}_{6}-\mathbf{d}\left(\mathrm{X}^{-}=\left[\mathrm{PF}_{6}\right] ; \mathrm{R}_{1}=\mathrm{OC}_{12} \mathrm{H}_{25}, \mathrm{R}_{2}=\mathrm{OC}_{12} \mathrm{H}_{25}, \mathrm{R}_{3}=\mathrm{OC}_{12} \mathrm{H}_{25}\right): \mathrm{Cr} \cdot 47 \cdot \mathrm{Col} \cdot 182 \cdot \mathrm{I} / \mathrm{dec} .\left({ }^{\circ} \mathrm{C}\right)$

$$
\text { 332-Cl-n }\left(\mathrm{X}^{-}=\mathrm{Cl}^{-}\right)
$$

332-Cl-10: g $\cdot 4 \cdot \mathrm{Col}_{\mathrm{h}} \cdot 168 \cdot \mathrm{I} / \mathrm{dec} .\left({ }^{\circ} \mathrm{C}\right)$

332-Cl-12: $\mathrm{Cr} \cdot 36 \cdot \mathrm{Col}_{\mathrm{h}} \cdot 170 \cdot \mathrm{I} / \mathrm{dec} .\left({ }^{\circ} \mathrm{C}\right)$

332-Cl-14: $\mathrm{Cr} \cdot 39 \cdot \mathrm{Col}_{\mathrm{h}} \cdot 168 \cdot \mathrm{I} /$ dec. $\left({ }^{\circ} \mathrm{C}\right)$

332-Cl-16: $\mathrm{Cr} \cdot 55 \cdot \mathrm{Col}_{\mathrm{h}} \cdot 162 \cdot \mathrm{I} / \mathrm{dec} .\left({ }^{\circ} \mathrm{C}\right)$

332-OTf-n $\left(\mathrm{X}^{-}=[\mathrm{OTf}]^{-}\right)$

332-OTf-10: $\mathrm{Cr} \cdot 55 \cdot \mathrm{I}\left({ }^{\circ} \mathrm{C}\right)$

332-OTf-12: $\mathrm{Cr} \cdot 24 \cdot \mathrm{Col}_{\mathrm{h}} \cdot 94 \cdot \mathrm{I}\left({ }^{\circ} \mathrm{C}\right)$

332-OTf-14: $\mathrm{Cr} \cdot 43 \cdot \mathrm{Col}_{\mathrm{h}} \cdot 108 \cdot \mathrm{I}\left({ }^{\circ} \mathrm{C}\right)$

332-OTf-16: $\mathrm{Cr} \cdot 55 \cdot \mathrm{Col}_{\mathrm{h}} \cdot 117 \cdot \mathrm{I}\left({ }^{\circ} \mathrm{C}\right)$ 


$$
\text { 333-Cl-(a-h) }\left(\mathrm{X}^{-}=\mathrm{Cl}^{-}\right)
$$

333-Cl-a $\left(\mathrm{R}_{1}=\mathrm{H}, \mathrm{R}_{2}=\mathrm{OC}_{12} \mathrm{H}_{25}, \mathrm{R}_{3}=\mathrm{H}\right): \mathrm{Cr} \cdot 92 \cdot \mathrm{SmA} \cdot 223 \cdot \mathrm{I} /$ dec. $\left({ }^{\circ} \mathrm{C}\right)$

333-Cl-b $\left(\mathrm{R}_{1}=\mathrm{OC}_{12} \mathrm{H}_{25}, \mathrm{R}_{2}=\mathrm{OC}_{12} \mathrm{H}_{25}, \mathrm{R}_{3}=\mathrm{H}\right): \mathrm{Cr} \cdot 90 \cdot \mathrm{Col}_{\mathrm{h}} \cdot 218 \cdot \mathrm{I} / \mathrm{dec} .\left({ }^{\circ} \mathrm{C}\right)$

333-Cl-c $\left(\mathrm{R}_{1}=\mathrm{OC}_{6} \mathrm{H}_{13}, \mathrm{R}_{2}=\mathrm{OC}_{6} \mathrm{H}_{13}, \mathrm{R}_{3}=\mathrm{OC}_{6} \mathrm{H}_{13}\right): \mathrm{Cr} \cdot 193 \cdot \mathrm{I} /$ dec. $\left({ }^{\circ} \mathrm{C}\right)$

333-Cl-d $\left(\mathrm{R}_{1}=\mathrm{OC}_{8} \mathrm{H}_{17}, \mathrm{R}_{2}=\mathrm{OC}_{8} \mathrm{H}_{17}, \mathrm{R}_{3}=\mathrm{OC}_{8} \mathrm{H}_{17}\right): \mathrm{Cr} \cdot 141 \cdot \mathrm{Col}_{\mathrm{h}} \cdot 205 \cdot \mathrm{I} / \mathrm{dec} .\left({ }^{\circ} \mathrm{C}\right)$

333-Cl-e $\left(\mathrm{R}_{1}=\mathrm{OC}_{10} \mathrm{H}_{21}, \mathrm{R}_{2}=\mathrm{OC}_{10} \mathrm{H}_{21}, \mathrm{R}_{3}=\mathrm{OC}_{10} \mathrm{H}_{21}\right): \mathrm{Cr} \cdot 93 \cdot \mathrm{Col}_{\mathrm{h}} \cdot 218 \cdot \mathrm{I} / \mathrm{dec} .\left({ }^{\circ} \mathrm{C}\right)$

333-Cl-f $\left(\mathrm{R}_{1}=\mathrm{OC}_{12} \mathrm{H}_{25}, \mathrm{R}_{2}=\mathrm{OC}_{12} \mathrm{H}_{25}, \mathrm{R}_{3}=\mathrm{OC}_{12} \mathrm{H}_{25}\right): \mathrm{Cr} \cdot 61 \cdot \mathrm{Col}_{\mathrm{h}} \cdot 212 \cdot \mathrm{I} / \mathrm{dec} .\left({ }^{\circ} \mathrm{C}\right)$

333-Cl-g $\left(\mathrm{R}_{1}=\mathrm{OC}_{14} \mathrm{H}_{29}, \mathrm{R}_{2}=\mathrm{OC}_{14} \mathrm{H}_{29}, \mathrm{R}_{3}=\mathrm{OC}_{14} \mathrm{H}_{29}\right): \mathrm{Cr} \cdot 42 \cdot \mathrm{Col}_{\mathrm{h}} \cdot 219 \cdot \mathrm{I} / \mathrm{dec} .\left({ }^{\circ} \mathrm{C}\right)$

333-Cl-h $\left(\mathrm{R}_{1}=\mathrm{OC}_{16} \mathrm{H}_{29}, \mathrm{R}_{2}=\mathrm{OC}_{16} \mathrm{H}_{29}, \mathrm{R}_{3}=\mathrm{OC}_{16} \mathrm{H}_{29}\right): \mathrm{Cr} \cdot 78 \cdot \mathrm{Col}_{\mathrm{h}} \cdot 206 \cdot \mathrm{I} / \mathrm{dec} .\left({ }^{\circ} \mathrm{C}\right)$

333-OTf-(a-h) $\left(\mathrm{X}^{-}=[\mathrm{OTf}]^{-}\right)$

333-OTf-a $\left(\mathrm{R}_{1}=\mathrm{H}, \mathrm{R}_{2}=\mathrm{OC}_{12} \mathrm{H}_{25}, \mathrm{R}_{3}=\mathrm{H}\right)$ : $\mathrm{Cr} \cdot 113 \cdot \mathrm{I}\left({ }^{\circ} \mathrm{C}\right)$

333-OTf-b $\left(\mathrm{R}_{1}=\mathrm{OC}_{12} \mathrm{H}_{25}, \mathrm{R}_{2}=\mathrm{OC}_{12} \mathrm{H}_{25}, \mathrm{R}_{3}=\mathrm{H}\right): \mathrm{Cr} \cdot 99 \cdot \mathrm{SmA} \cdot 134 \cdot \mathrm{I}\left({ }^{\circ} \mathrm{C}\right)$

333-OTf-c $\left(\mathrm{R}_{1}=\mathrm{OC}_{6} \mathrm{H}_{13}, \mathrm{R}_{2}=\mathrm{OC}_{6} \mathrm{H}_{13}, \mathrm{R}_{3}=\mathrm{OC}_{6} \mathrm{H}_{13}\right)$ : $\mathrm{Cr} \cdot 67 \cdot \mathrm{I}\left({ }^{\circ} \mathrm{C}\right)$

333-OTf-d $\left(\mathrm{R}_{1}=\mathrm{OC}_{8} \mathrm{H}_{17}, \mathrm{R}_{2}=\mathrm{OC}_{8} \mathrm{H}_{17}, \mathrm{R}_{3}=\mathrm{OC}_{8} \mathrm{H}_{17}\right): \mathrm{Cr} \cdot 7 \cdot \mathrm{Col}_{\mathrm{h}} \cdot 79 \cdot \mathrm{I}\left({ }^{\circ} \mathrm{C}\right)$

333-OTf-e $\left(\mathrm{R}_{1}=\mathrm{OC}_{10} \mathrm{H}_{21}, \mathrm{R}_{2}=\mathrm{OC}_{10} \mathrm{H}_{21}, \mathrm{R}_{3}=\mathrm{OC}_{10} \mathrm{H}_{21}\right): \mathrm{Cr} \cdot 77 \cdot \mathrm{Col}_{\mathrm{h}} \cdot 103 \cdot \mathrm{I}\left({ }^{\circ} \mathrm{C}\right)$

333-OTf-f $\left(\mathrm{R}_{1}=\mathrm{OC}_{12} \mathrm{H}_{25}, \mathrm{R}_{2}=\mathrm{OC}_{12} \mathrm{H}_{25}, \mathrm{R}_{3}=\mathrm{OC}_{12} \mathrm{H}_{25}\right): \mathrm{Cr} \cdot 31 \cdot \mathrm{Col}_{\mathrm{h}} \cdot 144 \cdot \mathrm{I}\left({ }^{\circ} \mathrm{C}\right)$

333-OTf-g $\left(\mathrm{R}_{1}=\mathrm{OC}_{14} \mathrm{H}_{29}, \mathrm{R}_{2}=\mathrm{OC}_{14} \mathrm{H}_{29}, \mathrm{R}_{3}=\mathrm{OC}_{14} \mathrm{H}_{29}\right): \mathrm{Cr} \cdot 47 \cdot \mathrm{Col}_{\mathrm{h}} \cdot 163 \cdot \mathrm{I}\left({ }^{\circ} \mathrm{C}\right)$

333-OTf-h $\left(\mathrm{R}_{1}=\mathrm{OC}_{16} \mathrm{H}_{29}, \mathrm{R}_{2}=\mathrm{OC}_{16} \mathrm{H}_{29}, \mathrm{R}_{3}=\mathrm{OC}_{16} \mathrm{H}_{29}\right): \mathrm{Cr} \cdot 50 \cdot \mathrm{Col}_{\mathrm{h}} \cdot 167 \cdot \mathrm{I}\left({ }^{\circ} \mathrm{C}\right)$

334-Cl-n $\left(\mathrm{X}^{-}=\mathrm{Cl}^{-}\right)$

334-Cl-8: $\mathrm{g} \cdot 8 \cdot \mathrm{Col}_{\mathrm{h}} \cdot 162 \cdot \mathrm{I} / \mathrm{dec} .\left({ }^{\circ} \mathrm{C}\right)$

334-Cl-10: $\mathrm{Cr} \cdot 50 \cdot \mathrm{Col}_{\mathrm{h}} \cdot 179 \cdot \mathrm{I} / \mathrm{dec} .\left({ }^{\circ} \mathrm{C}\right)$

334-Cl-12: $\mathrm{Cr} \cdot 49 \cdot \mathrm{Col}_{\mathrm{h}} \cdot 171 \cdot \mathrm{I} / \mathrm{dec} .\left({ }^{\circ} \mathrm{C}\right)$

334-Cl-14: $\mathrm{Cr} \cdot 54 \cdot \mathrm{Col}_{\mathrm{h}} \cdot 173 \cdot \mathrm{I} / \mathrm{dec} .\left({ }^{\circ} \mathrm{C}\right)$

334-Cl-16: $\mathrm{Cr} \cdot 64 \cdot \mathrm{Col}_{\mathrm{h}} \cdot 183 \cdot \mathrm{I} / \mathrm{dec} .\left({ }^{\circ} \mathrm{C}\right)$

334-OTf-n $\left(\mathrm{X}^{-}=[\mathrm{OTf}]^{-}\right)$

334-OTf-8: $\mathrm{g} \cdot 17 \cdot \mathrm{I}\left({ }^{\circ} \mathrm{C}\right)$

334-OTf-10: $\mathrm{Cr} \cdot 41 \cdot \mathrm{Col}_{\mathrm{h}} \cdot 64 \cdot \mathrm{I}\left({ }^{\circ} \mathrm{C}\right)$

334-OTf-12: $\mathrm{Cr} \cdot 19 \cdot \mathrm{Col}_{\mathrm{h}} \cdot 104 \cdot \mathrm{I}\left({ }^{\circ} \mathrm{C}\right)$

334-OTf-14: $\mathrm{Cr} \cdot 42 \cdot \mathrm{Col}_{\mathrm{h}} \cdot 121 \cdot \mathrm{I}\left({ }^{\circ} \mathrm{C}\right)$

334-OTf-16: $\mathrm{Cr} \cdot 56 \cdot \mathrm{Col}_{\mathrm{h}} \cdot 131 \cdot \mathrm{I}\left({ }^{\circ} \mathrm{C}\right)$

335-CN $(\mathrm{R}=\mathrm{CN}): \mathrm{Cr} \cdot 135 \cdot \mathrm{I}\left({ }^{\circ} \mathrm{C}\right)\left(1^{\text {st }}\right.$ DSC heating run $)$

335- $\mathbf{O C}_{10} \mathbf{H}_{21}\left(\mathrm{R}=\mathrm{OC}_{10} \mathrm{H}_{21}\right): \mathrm{Cr} \cdot 144 \cdot \mathrm{SmA} \cdot 167 \cdot \mathrm{I}\left({ }^{\circ} \mathrm{C}\right)\left(1^{\text {st }} \mathrm{DSC}\right.$ heating run $)$

336-Cl-R-n $\left(\mathrm{X}^{-}=\mathrm{Cl}^{-}\right)$

336-Cl-CN-10 $(\mathrm{R}=\mathrm{CN})$ : $\mathrm{Cr} \cdot 149 \cdot \mathrm{I}\left({ }^{\circ} \mathrm{C}\right)\left(1^{\text {st }} \mathrm{DSC}\right.$ heating run $)$

336-Cl-OC ${ }_{10} \mathbf{H}_{21}-\mathbf{4}\left(\mathrm{R}=\mathrm{OC}_{10} \mathrm{H}_{21}\right): \mathrm{Cr} \cdot 184 \cdot \mathrm{I}\left({ }^{\circ} \mathrm{C}\right)\left(1^{\text {st }}\right.$ DSC heating run $)$

336-Cl-OC ${ }_{10} \mathbf{H}_{21}-6\left(\mathrm{R}=\mathrm{OC}_{10} \mathrm{H}_{21}\right): \mathrm{Cr} \cdot(\mathrm{SmA} \cdot 141 \cdot) 150 \cdot \mathrm{I}\left({ }^{\circ} \mathrm{C}\right)\left(1^{\mathrm{st}} \mathrm{DSC}\right.$ heating/cooling cycle $)$ 
336-Cl-OC ${ }_{10} \mathrm{H}_{21}-\mathbf{8}\left(\mathrm{R}=\mathrm{OC}_{10} \mathrm{H}_{21}\right): \mathrm{Cr} \cdot(\mathrm{SmA} \cdot 136 \cdot) 154 \cdot \mathrm{I}\left({ }^{\circ} \mathrm{C}\right)\left(1^{\mathrm{st}} \mathrm{DSC}\right.$ heating/cooling cycle $)$ 336-Cl-OC $\mathbf{1 0}_{\mathbf{1 0}} \mathbf{H}_{\mathbf{2 1}}-\mathbf{1 0}\left(\mathrm{R}=\mathrm{OC}_{10} \mathrm{H}_{21}\right): \mathrm{Cr} \cdot 133 \cdot \mathrm{SmA} \cdot 149 \cdot \mathrm{I}\left({ }^{\circ} \mathrm{C}\right)\left(1^{\text {st }} \mathrm{DSC}\right.$ heating run $)$

336-Br-OC $\mathbf{O C}_{10} \mathrm{H}_{21}-\mathbf{1 0}\left(\mathrm{X}^{-}=\mathrm{Br}^{-}, \mathrm{R}=\mathrm{OC}_{10} \mathrm{H}_{21}\right): \mathrm{Cr} \cdot 123 \cdot \mathrm{SmA} \cdot 128 \cdot \mathrm{I}\left({ }^{\circ} \mathrm{C}\right)\left(1^{\text {st }} \mathrm{DSC}\right.$ heating run $)$ 336-I-OC ${ }_{10} \mathbf{H}_{21}-10\left(\mathrm{X}^{-}=\mathrm{I}^{-}, \mathrm{R}=\mathrm{OC}_{10} \mathrm{H}_{21}\right): \mathrm{Cr} \cdot(\mathrm{SmA} \cdot 127 \cdot) 124 \cdot \mathrm{I}\left({ }^{\circ} \mathrm{C}\right)\left(1^{\text {st }} \mathrm{DSC}\right.$ heating/cooling cycle $)$

336- $-\mathbf{B F}_{4}-\mathbf{O C}_{10} \mathbf{H}_{21}-10\left(\mathrm{X}^{-}=\left[\mathrm{BF}_{4}\right]^{-}, \mathrm{R}=\mathrm{OC}_{10} \mathrm{H}_{21}\right): \mathrm{Cr} \cdot 129 \cdot \mathrm{SmA} \cdot 135 \cdot \mathrm{I}\left({ }^{\circ} \mathrm{C}\right)\left(1^{\text {st }} \mathrm{DSC}\right.$ heating run $)$ 336- $\mathbf{P F}_{6}-\mathrm{OC}_{10} \mathbf{H}_{21}-\mathbf{1 0}\left(\mathrm{X}^{-}=\left[\mathrm{PF}_{6}\right]^{-}, \mathrm{R}=\mathrm{OC}_{10} \mathrm{H}_{21}\right): \mathrm{Cr} \cdot 127 \cdot \mathrm{I}\left({ }^{\circ} \mathrm{C}\right)\left(1^{\text {st }}\right.$ DSC heating run $)$ 336-OTf-OC $\mathbf{1 0}_{\mathbf{1 0}} \mathbf{H}_{21}-\mathbf{1 0}\left(\mathrm{X}^{-}=[\mathrm{OTf}]^{-}, \mathrm{R}=\mathrm{OC}_{10} \mathrm{H}_{21}\right): \mathrm{Cr} \cdot 117 \cdot \mathrm{I}\left({ }^{\circ} \mathrm{C}\right)\left(1^{\text {st }} \mathrm{DSC}\right.$ heating run $)$ 336- $\mathbf{C H}_{3} \mathbf{C O O}-\mathbf{O C}_{10} \mathbf{H}_{21} \mathbf{- 1 0}\left(\mathrm{X}^{-}=\left[\mathrm{CH}_{3} \mathrm{COO}\right]^{-}, \mathrm{R}=\mathrm{OC}_{10} \mathrm{H}_{21}\right): \mathrm{Cr} \cdot 87 \cdot \mathrm{I}\left({ }^{\circ} \mathrm{C}\right)\left(1^{\text {st }}\right.$ DSC heating run $)$ 336-SCN-OC ${ }_{10} \mathbf{H}_{21}-10\left(\mathrm{X}^{-}=[\mathrm{SCN}]^{-}, \mathrm{R}=\mathrm{OC}_{10} \mathrm{H}_{21}\right): \mathrm{Cr} \cdot(\mathrm{SmA} \cdot 127 \cdot) 125 \cdot \mathrm{I}\left({ }^{\circ} \mathrm{C}\right)\left(1^{\text {st }}\right.$ DSC heating $/$ cooling cycle $)$

$$
\begin{gathered}
\text { 337-3: } \mathrm{Cr} \cdot 118 \cdot \mathrm{SmA} \cdot 139 \cdot \mathrm{I}\left({ }^{\circ} \mathrm{C}\right) \\
\text { 337-4: } \mathrm{Cr} \cdot 125 \cdot \mathrm{SmA} \cdot 151 \cdot \mathrm{I}\left({ }^{\circ} \mathrm{C}\right) \\
\text { 337-5: } \mathrm{Cr} \cdot 125 \cdot \mathrm{SmA} \cdot 150 \cdot \mathrm{I}\left({ }^{\circ} \mathrm{C}\right) \\
\text { 337-6: } \mathrm{Cr} \cdot 117 \cdot \mathrm{SmA} \cdot 137 \cdot \mathrm{I}\left({ }^{\circ} \mathrm{C}\right) \\
\text { 337-7: } \mathrm{Cr} \cdot 111 \cdot \mathrm{SmA} \cdot 129 \cdot \mathrm{I}\left({ }^{\circ} \mathrm{C}\right) \\
\text { 337-8: } \mathrm{Cr} \cdot 100 \cdot \mathrm{SmA} \cdot 125 \cdot \mathrm{I}\left({ }^{\circ} \mathrm{C}\right) \\
\text { 338-5: } \mathrm{Cr} \cdot 53 \cdot \mathrm{M} \cdot 58 \cdot \mathrm{I}\left({ }^{\circ} \mathrm{C}\right) \\
\text { 338-6: } \mathrm{Cr} \cdot 46 \cdot \mathrm{Col}_{\mathrm{r}, 1} \cdot 81 \cdot \mathrm{Col}_{\mathrm{r}, 2} \cdot 106 \cdot \mathrm{I}\left({ }^{\circ} \mathrm{C}\right) \\
\text { 338-7: } \mathrm{Cr} \cdot 47 \cdot \mathrm{Cr}_{2} \cdot 55 \cdot \mathrm{Col}_{\mathrm{r}, 2} \cdot 95 \cdot \mathrm{I}\left({ }^{\circ} \mathrm{C}\right) \\
\text { 338-8: } \mathrm{Cr} \cdot 44 \cdot \mathrm{Col}_{\mathrm{r}, 2 \rightarrow 3} \cdot 103 \cdot \mathrm{I}\left({ }^{\circ} \mathrm{C}\right) \\
\text { 338-9: } \mathrm{Cr}_{1} \cdot 41 \cdot \mathrm{Cr}_{2} \cdot 49 \cdot \mathrm{Col}_{\mathrm{r}, 3} \cdot 115 \cdot \mathrm{I}\left({ }^{\circ} \mathrm{C}\right)
\end{gathered}
$$$$
\text { 338-10: } \mathrm{Cr} \cdot 45 \cdot \mathrm{Col}_{\mathrm{r}, 3} \cdot 91 \cdot \mathrm{I}\left({ }^{\circ} \mathrm{C}\right)
$$

339-a $\left(\mathrm{R}_{1}=\mathrm{R}_{2}=\mathrm{R}_{3}=\mathrm{H}\right): \mathrm{Cr} \cdot 113 \cdot \mathrm{SmA} \cdot 250 \cdot \operatorname{dec} \cdot\left({ }^{\circ} \mathrm{C}\right)$ 339-b $\left(\mathrm{R}_{1}=\mathrm{OC}_{12} \mathrm{H}_{25}, \mathrm{R}_{2}=\mathrm{R}_{3}=\mathrm{H}\right): \mathrm{Cr} \cdot 98 \cdot \mathrm{Col}_{\mathrm{h}} \cdot 222 \cdot \operatorname{dec} .\left({ }^{\circ} \mathrm{C}\right)$ 339-c $\left(\mathrm{R}_{1}=\mathrm{R}_{2}=\mathrm{OC}_{12} \mathrm{H}_{25}, \mathrm{R}_{3}=\mathrm{H}\right): \mathrm{Cr} \cdot 101 \cdot \mathrm{Col}_{\mathrm{h}, 1} \cdot 149 \cdot \mathrm{Col}_{\mathrm{h}, 2} \cdot 155 \cdot \mathrm{I}\left({ }^{\circ} \mathrm{C}\right)$ 339-d $\left(\mathrm{R}_{1}=\mathrm{H}, \mathrm{R}_{2}=\mathrm{R}_{3}=\mathrm{OC}_{12} \mathrm{H}_{25}\right): \mathrm{Cr} \cdot 77 \cdot \mathrm{Col}_{\mathrm{h}} \cdot 153 \cdot \mathrm{I}\left({ }^{\circ} \mathrm{C}\right)$

340-a $\left(\mathrm{R}_{1}=\mathrm{R}_{2}=\mathrm{R}_{3}=\mathrm{R}_{4}=\mathrm{R}_{5}=\mathrm{R}_{6}=\mathrm{H}\right): \mathrm{Cr} \cdot 121 \cdot \mathrm{SmA} \cdot 216 \cdot \mathrm{I}\left({ }^{\circ} \mathrm{C}\right)$ 340-b $\left(\mathrm{R}_{1}=\mathrm{R}_{4}=\mathrm{OC}_{12} \mathrm{H}_{25}, \mathrm{R}_{2}=\mathrm{R}_{3}=\mathrm{R}_{5}=\mathrm{R}_{6}=\mathrm{H}\right): \mathrm{Cr} \cdot 35 \cdot \mathrm{Col}_{\mathrm{h}} \cdot 176 \cdot \mathrm{I}\left({ }^{\circ} \mathrm{C}\right)$ 340-c $\left(\mathrm{R}_{1}=\mathrm{R}_{2}=\mathrm{R}_{4}=\mathrm{R}_{5}=\mathrm{OC}_{12} \mathrm{H}_{25}, \mathrm{R}_{3}=\mathrm{R}_{6}=\mathrm{H}\right): \mathrm{Cr} \cdot 100 \cdot \mathrm{Cub} \cdot 146 \cdot \mathrm{I}\left({ }^{\circ} \mathrm{C}\right)$ 340-d $\left(\mathrm{R}_{1}=\mathrm{R}_{4}=\mathrm{H}, \mathrm{R}_{2}=\mathrm{R}_{3}=\mathrm{R}_{5}=\mathrm{R}_{6}=\mathrm{OC}_{12} \mathrm{H}_{25}\right): \mathrm{Cr} \cdot 17 \cdot \mathrm{Col}_{\mathrm{h}} \cdot 63 \cdot \mathrm{I}\left({ }^{\circ} \mathrm{C}\right)$ 340-e $\left(\mathrm{R}_{1}=\mathrm{R}_{6}=\mathrm{H}, \mathrm{R}_{2}=\mathrm{R}_{3}=\mathrm{R}_{4}=\mathrm{R}_{5}=\mathrm{OC}_{12} \mathrm{H}_{25}\right): \mathrm{Cr} \cdot 7 \cdot \mathrm{Cub} \cdot 146 \cdot \mathrm{I}\left({ }^{\circ} \mathrm{C}\right)$ 
Scheme S17. Isomerization process of compound $\mathbf{3 4 1}$ upon addition of $p$-toluenesulfonic acid. ${ }^{125}$

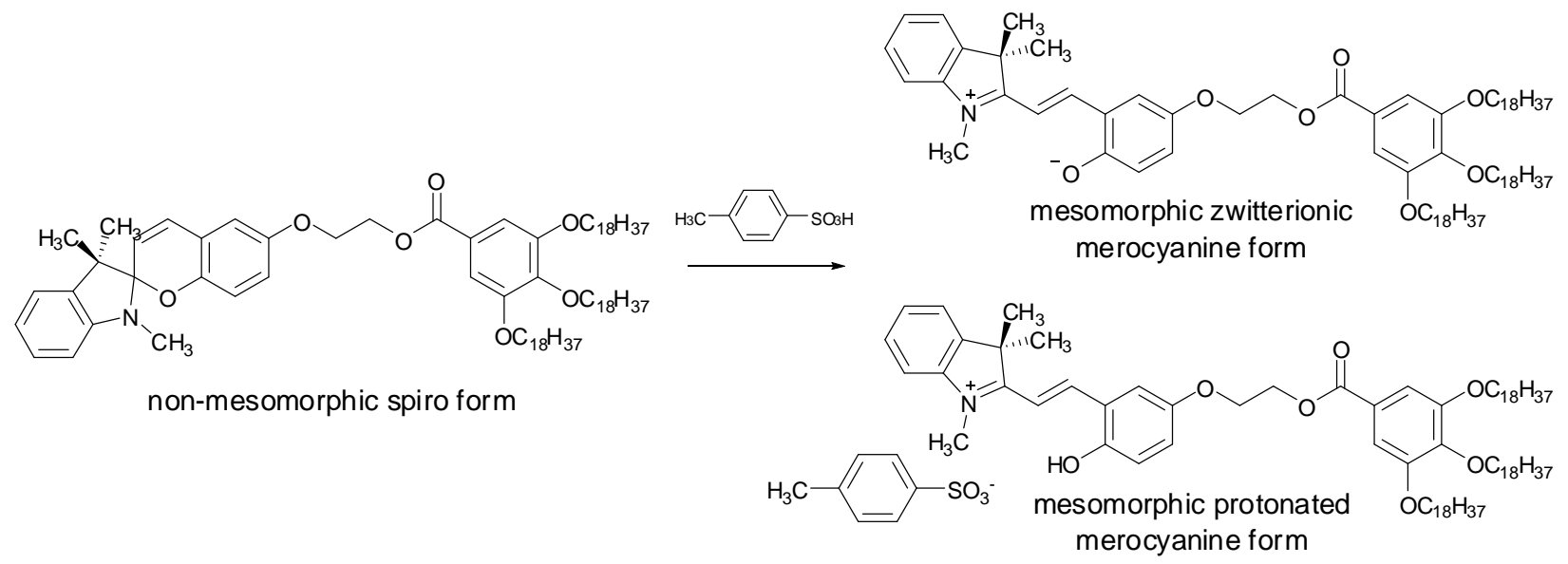

341: $\mathrm{Cr} \cdot 40 \cdot \mathrm{I}\left({ }^{\circ} \mathrm{C}\right)$

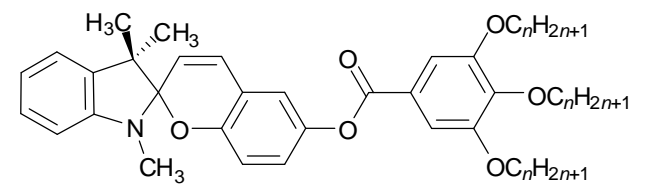

342-n

342-8: $\mathrm{Cr} \cdot-4 \cdot \mathrm{I}\left({ }^{\circ} \mathrm{C}\right)$

342-10: $\mathrm{Cr} \cdot 39 \cdot \mathrm{I}\left({ }^{\circ} \mathrm{C}\right)$

342-12: $\mathrm{Cr}_{1} \cdot 24 \cdot \mathrm{Cr}_{2} \cdot 49 \cdot \mathrm{Cr}_{3} \cdot 59 \cdot \mathrm{I}\left({ }^{\circ} \mathrm{C}\right)$

342-14: $\mathrm{Cr}_{1} \cdot 41 \cdot \mathrm{Cr}_{2} \cdot 49 \cdot \mathrm{I}\left({ }^{\circ} \mathrm{C}\right)$

342-16: $\mathrm{Cr} \cdot 60 \cdot \mathrm{I}\left({ }^{\circ} \mathrm{C}\right)$

342-18: $\mathrm{Cr} \cdot 64 \cdot \mathrm{I}\left({ }^{\circ} \mathrm{C}\right)$ 
Scheme S18. Acidic compounds that were tested by Kato and co-workers for the induction of a LC phase in mixtures with 341 or 342-n.

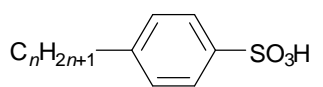

343-n

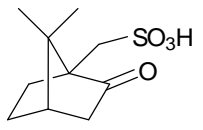

346
$\mathrm{CF}_{3} \mathrm{SO}_{3} \mathrm{H}$

344

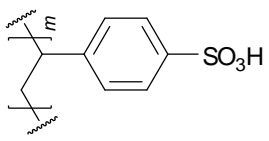

347
$\mathrm{C}_{10} \mathrm{H}_{21} \mathrm{SO}_{3} \mathrm{H}$

345

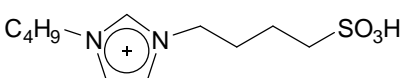

$\mathrm{x}^{-}$

$348-\mathrm{X}\left(\mathrm{X}^{-}=\left[\mathrm{BF}_{4}\right]^{-},[\mathrm{OTf}]^{-}\right)$

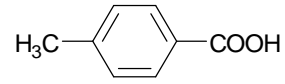

349

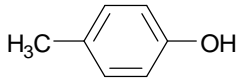

350

341/(343-1) (1:1): $\mathrm{Cr} \cdot 55 \cdot \mathrm{Cr}+\mathrm{Col}_{\mathrm{h}} \cdot 60 \cdot \mathrm{Col}_{\mathrm{h}} \cdot 119 \cdot \mathrm{I}\left({ }^{\circ} \mathrm{C}\right)$

341/(343-1) (3:2): $\mathrm{Cr} \cdot 56 \cdot \mathrm{Cr}+\mathrm{Col}_{\mathrm{h}} \cdot 62 \cdot \mathrm{Col}_{\mathrm{h}} \cdot 133 \cdot \mathrm{I}\left({ }^{\circ} \mathrm{C}\right)$

341/(343-1) (2:1): $\mathrm{Cr} \cdot 56 \cdot \mathrm{Cr}+\mathrm{Col}_{\mathrm{h}} \cdot 63 \cdot \mathrm{Col}_{\mathrm{h}} \cdot 96 \cdot \mathrm{I}\left({ }^{\circ} \mathrm{C}\right)$

341/(343-12) (1:1): $\mathrm{Cr}_{1} \cdot 23 \cdot \mathrm{Cr}_{2} \cdot 29 \cdot \mathrm{Cr}_{3} \cdot 46 \cdot \mathrm{I}\left({ }^{\circ} \mathrm{C}\right)$

341/349 (1:1): phase separation

341/350 (1:1): $\mathrm{Cr} \cdot 48 \cdot \mathrm{I}\left({ }^{\circ} \mathrm{C}\right)$

342-8/(343-1) (1:1): Cr $\cdot-1 \cdot \mathrm{Col}_{\mathrm{h}} \cdot 102 \cdot \mathrm{I}\left({ }^{\circ} \mathrm{C}\right)$

342-10/(343-1) (1:1): $\mathrm{Cr}_{1} \cdot 4 \cdot \mathrm{Cr}_{2} \cdot 19 \cdot \mathrm{Col}_{\mathrm{h}} \cdot 100 \cdot \mathrm{I}\left({ }^{\circ} \mathrm{C}\right)$

342-12/(343-1) (1:1): $\mathrm{Cr} \cdot 34 \cdot \mathrm{Col}_{\mathrm{h}} \cdot 105 \cdot \mathrm{I}\left({ }^{\circ} \mathrm{C}\right)$

342-14/(343-1) (1:1): $\mathrm{Cr}_{1} \cdot 34 \cdot \mathrm{Cr}_{2} \cdot 53 \cdot \mathrm{Col}_{\mathrm{h}} \cdot 112 \cdot \mathrm{I}\left({ }^{\circ} \mathrm{C}\right)$

342-16/(343-1) (1:1): $\mathrm{Cr}_{1} \cdot 50 \cdot \mathrm{Cr}_{2} \cdot 58 \cdot \mathrm{Cr}_{3} \cdot 63 \cdot \mathrm{Col}_{\mathrm{h}} \cdot 134 \cdot \mathrm{I}\left({ }^{\circ} \mathrm{C}\right)$

342-18/(343-1) (1:1): $\mathrm{Cr} \cdot 54 \cdot \mathrm{Col}_{\mathrm{h}} \cdot 148 \cdot \mathrm{I}\left({ }^{\circ} \mathrm{C}\right)$

342-18/344 (1:1): $\mathrm{Cr} \cdot 74 \cdot \mathrm{Col}_{\mathrm{h}} \cdot 102 \cdot \mathrm{I}\left({ }^{\circ} \mathrm{C}\right)$

342-18/345 (1:1): $\mathrm{Cr}_{1} \cdot 34 \cdot \mathrm{Cr}_{2} \cdot 45 \cdot \mathrm{Cr}_{3} \cdot 66 \cdot \mathrm{I}\left({ }^{\circ} \mathrm{C}\right)$

342-18/346 (1:1): $\mathrm{Cr} \cdot 58 \cdot \mathrm{Col}_{\mathrm{h}} \cdot 75 \cdot \mathrm{I}\left({ }^{\circ} \mathrm{C}\right)$

342-18/347 (1:1): $\mathrm{Cr}_{1} \cdot 48 \cdot \mathrm{Cr}_{2} \cdot 54 \cdot \mathrm{I}\left({ }^{\circ} \mathrm{C}\right)$

342-18/348--BF 4 (1:1): $\mathrm{Cr}_{1} \cdot 48 \cdot \mathrm{Cr}_{2} \cdot 68 \cdot \mathrm{Col}_{\mathrm{h}} \cdot 186 \cdot \mathrm{I}\left({ }^{\circ} \mathrm{C}\right)$

342-18/348-OTf (1:1): $\mathrm{Cr} \cdot 47 \cdot \mathrm{Col}_{\mathrm{h}} \cdot 174 \cdot \mathrm{I}\left({ }^{\circ} \mathrm{C}\right)$ 


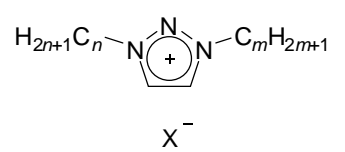

351-Cl-1/12 $\left(\mathrm{X}^{-}=\mathrm{Cl}^{-}\right): \mathrm{Cr} \cdot 89 \cdot \mathrm{SmX} \cdot 108 \cdot \mathrm{SmA} \cdot 159 \cdot \mathrm{I}\left({ }^{\circ} \mathrm{C}\right)$

$$
\text { 351-Br-n/m }\left(\mathrm{X}^{-}=\mathrm{Br}^{-}\right)
$$

351-Br-1/12: $\mathrm{Cr} \cdot 93 \cdot \mathrm{SmX} \cdot 102 \cdot \mathrm{SmA} \cdot 155 \cdot \mathrm{I}\left({ }^{\circ} \mathrm{C}\right)$

351-Br-2/12: $\mathrm{Cr} \cdot-8 \cdot \mathrm{SmX} \cdot 57 \cdot \mathrm{SmA} \cdot 85 \cdot \mathrm{I}\left({ }^{\circ} \mathrm{C}\right)$

351-Br-4/12: $\mathrm{Cr} \cdot\left(\mathrm{SmX}_{1} \cdot 41 \cdot \mathrm{SmX}_{2} \cdot 48 \cdot\right) 52 \cdot \mathrm{I}\left({ }^{\circ} \mathrm{C}\right)$

351-Br-5/12: $\mathrm{Cr} \cdot 43 \cdot \mathrm{SmX}_{1} \cdot 63 \cdot \mathrm{SmX}_{2} \cdot 75 \cdot \mathrm{I}\left({ }^{\circ} \mathrm{C}\right)$

351-Br-6/12: $\mathrm{Cr}_{1} \cdot-1 \cdot \mathrm{Cr}_{2} \cdot 26 \cdot \mathrm{SmX}_{1} \cdot 68 \cdot \mathrm{SmX}_{2} \cdot 87 \cdot \mathrm{I}\left({ }^{\circ} \mathrm{C}\right)$

351-Br-7/12: $\mathrm{Cr}_{1} \cdot 27 \cdot \mathrm{Cr}_{2} \cdot 35 \cdot \mathrm{SmX}_{1} \cdot\left(\mathrm{SmX}_{2} \cdot 89 \cdot\right) 91 \cdot \mathrm{SmA}(?) \cdot 109 \cdot \mathrm{I}\left({ }^{\circ} \mathrm{C}\right)$

351-Br-8/12: $\mathrm{Cr} \cdot 42 \cdot \mathrm{SmC}\left(\right.$ ?) $\cdot 102 \cdot \mathrm{I}\left({ }^{\circ} \mathrm{C}\right)$

351-Br-10/12: $\mathrm{Cr} \cdot 66 \cdot \mathrm{SmC}(?) \cdot 112 \cdot \mathrm{I}\left({ }^{\circ} \mathrm{C}\right)$

351-Br-12/12: $\mathrm{Cr}_{1} \cdot 31 \cdot \mathrm{Cr}_{2} \cdot 82 \cdot \mathrm{SmC}(?) \cdot 114 \cdot \mathrm{I}\left({ }^{\circ} \mathrm{C}\right)$

351-Br-14/12: $\mathrm{Cr} \cdot 81 \cdot \mathrm{SmC}(?) \cdot 104 \cdot \mathrm{I}\left({ }^{\circ} \mathrm{C}\right)$

351-Br-10/10: $\mathrm{Cr} \cdot 69 \cdot \mathrm{SmC}(?) \cdot 113 \cdot \mathrm{I}\left({ }^{\circ} \mathrm{C}\right)$

351-Br-12/12: $\mathrm{Cr}_{1} \cdot 31 \cdot \mathrm{Cr}_{2} \cdot 82 \cdot \mathrm{SmC}($ ? $) \cdot 114 \cdot \mathrm{I}\left({ }^{\circ} \mathrm{C}\right)$

351-Br-14/14: $\mathrm{Cr}_{1} \cdot 29 \cdot \mathrm{Cr}_{2} \cdot 92 \cdot \mathrm{SmC}(?) \cdot 104 \cdot \mathrm{I}\left({ }^{\circ} \mathrm{C}\right)$

$$
\text { 351-I-n/m }\left(\mathrm{X}^{-}=\mathrm{I}^{-}\right)
$$

351-I-1/12: $\mathrm{Cr} \cdot 83 \cdot \mathrm{SmA} \cdot 135 \cdot \mathrm{I}\left({ }^{\circ} \mathrm{C}\right)$

351-I-12/12: $\mathrm{Cr}_{1} \cdot 60 \cdot \mathrm{Cr}_{2} \cdot 76 \cdot \mathrm{SmX} \cdot 88 \cdot \mathrm{SmA} \cdot 95$ (from $1^{\text {st }} \mathrm{DSC}$ heating run) $\cdot \mathrm{I}\left({ }^{\circ} \mathrm{C}\right.$ )

351- $\mathrm{I}_{3}-\boldsymbol{n} / \mathbf{m}\left(\mathrm{X}^{-}=\mathrm{I}_{3}^{-}\right)$

351-I $\mathbf{I}_{3}-1 / 12: \mathrm{Cr} \cdot 49 \cdot \mathrm{I}\left({ }^{\circ} \mathrm{C}\right)$

351-I $-12 / 12: \mathrm{Cr}_{1} \cdot 44 \cdot \mathrm{Cr}_{2} \cdot 52 \cdot \mathrm{I}\left({ }^{\circ} \mathrm{C}\right)$

351-PF $\mathbf{- 1 / 1 2}\left(\mathrm{X}^{-}=\left[\mathrm{PF}_{6}\right]^{-}\right): \mathrm{Cr}_{1} \cdot 61 \cdot \mathrm{Cr}_{2} \cdot 78 \cdot \mathrm{SmA} \cdot 113 \cdot \mathrm{I}\left({ }^{\circ} \mathrm{C}\right)$

351-NTf2-n/m ( $\left.\mathrm{X}^{-}=\left[\mathrm{NTf}_{2}\right]^{-}\right)$

351-NTf $\mathbf{2}_{2}$ 1/12: $\mathrm{Cr} \cdot 13 \cdot \mathrm{I}\left({ }^{\circ} \mathrm{C}\right)$

351-NTf $\mathbf{2}_{2}$-12/12: $\mathrm{Cr} \cdot 26 \cdot \mathrm{SmC}(?) \cdot 43 \cdot \mathrm{I}\left({ }^{\circ} \mathrm{C}\right)$

351-BF $\mathbf{H}_{\mathbf{4}} \mathbf{- 1 2 / 1 2}\left(\mathrm{X}^{-}=\left[\mathrm{BF}_{4}\right]^{-}\right): \mathrm{Cr}_{1} \cdot 60 \cdot \mathrm{Cr}_{2} \cdot 71 \cdot \mathrm{SmX} \cdot 80 \cdot \mathrm{SmA} \cdot 92 \cdot \mathrm{I}\left({ }^{\circ} \mathrm{C}\right)$

351-SbF $\mathbf{6}_{\mathbf{6}} \mathbf{- 1 2 / 1 2}\left(\mathrm{X}^{-}=\left[\mathrm{SbF}_{6}\right]^{-}\right): \mathrm{Cr} \cdot 84 \cdot \mathrm{SmC}(?) \cdot 100 \cdot \mathrm{I}\left({ }^{\circ} \mathrm{C}\right)$

351-N(CN $)_{2} \mathbf{- 1 2} / 12\left(\mathrm{X}^{-}=\left[\mathrm{N}(\mathrm{CN})_{2}\right]^{-}\right): \mathrm{Cr} \cdot 82 \cdot \mathrm{SmC}(?) \cdot 102 \cdot \mathrm{I}\left({ }^{\circ} \mathrm{C}\right)$ 


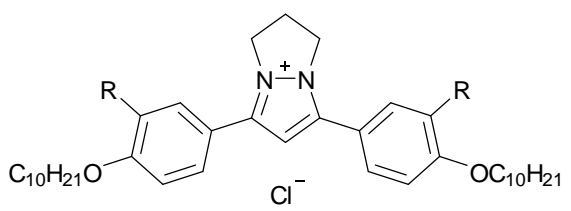

352-R

352-H $(\mathrm{R}=\mathrm{H}): \mathrm{Cr} \cdot 35 \cdot \mathrm{SmB} \cdot[$ not reported $] \cdot \mathrm{SmA} \cdot 208 \cdot \mathrm{I}\left({ }^{\circ} \mathrm{C}\right)$ (on cooling)

352-OC ${ }_{10} \mathrm{H}_{21}\left(\mathrm{R}=\mathrm{OC}_{10} \mathrm{H}_{21}\right): 1^{\text {st }}$ DSC heating run: $\mathrm{Cr}_{1} \cdot 119 \cdot \mathrm{Col}_{\mathrm{h}} \cdot 158-165$ (melting of the second crystal polymorph, $\mathrm{Cr}_{2}$, also occurs in this temperature range $) \cdot \mathrm{I}\left({ }^{\circ} \mathrm{C}\right) / 1^{\text {st }} \mathrm{DSC}$ cooling run: $\mathrm{I} \cdot 158 \cdot \mathrm{Col}_{\mathrm{h}} \cdot 105 \cdot \mathrm{Cr}$

(crystallization of the second crystal polymorph might take place before reaching $105^{\circ} \mathrm{C}$ on cooling)

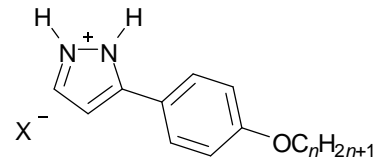

$$
\text { 353-Cl-n }\left(\mathrm{X}^{-}=\mathrm{Cl}^{-}\right)
$$

353-Cl-8: $\mathrm{Cr} \cdot(\mathrm{SmA} \cdot 150 \cdot) 150 \cdot \mathrm{I}\left({ }^{\circ} \mathrm{C}\right)$

353-Cl-10: $\mathrm{Cr}_{1} \cdot\left(\mathrm{Cr}_{2} \cdot 135 \cdot\right) 150 \cdot \mathrm{SmA} \cdot 166 \cdot \mathrm{I}\left({ }^{\circ} \mathrm{C}\right)$

353-Cl-12: $\mathrm{Cr} \cdot 144 \cdot \mathrm{SmA} \cdot 170 \cdot \mathrm{I}\left({ }^{\circ} \mathrm{C}\right)$

353-Cl-14: $\mathrm{Cr}_{1} \cdot\left(\mathrm{Cr}_{2} \cdot 128 \cdot\right) 140 \cdot \mathrm{SmA} \cdot 170 \cdot \mathrm{I}\left({ }^{\circ} \mathrm{C}\right)$

353-Cl-16: $\mathrm{Cr}_{1} \cdot\left(\mathrm{Cr}_{2} \cdot 123 \cdot\right) 140 \cdot \mathrm{SmA} \cdot 167 \cdot \mathrm{I}\left({ }^{\circ} \mathrm{C}\right)$

353-Cl-18: $\mathrm{Cr}_{1} \cdot\left(\mathrm{Cr}_{2} \cdot 129 \cdot\right) 138 \cdot \mathrm{SmA} \cdot 166 \cdot \mathrm{I}\left({ }^{\circ} \mathrm{C}\right)$

353-BF $\mathbf{H}_{4} \boldsymbol{n}\left(\mathrm{X}^{-}=\left[\mathrm{BF}_{4}\right]^{-}\right)$

353-BF 4 -8: $\mathrm{Cr} \cdot 86 \cdot \mathrm{SmA} \cdot 110 \cdot \mathrm{I}\left({ }^{\circ} \mathrm{C}\right)$

353-BF $\mathbf{H}_{\mathbf{4}} \mathbf{- 1 0}: \mathrm{Cr}_{1} \cdot 80 \cdot \mathrm{Cr}_{2} \cdot 123 \cdot \mathrm{SmA} \cdot 165 \cdot \mathrm{I}\left({ }^{\circ} \mathrm{C}\right)$

353-BF $-12: \mathrm{Cr}_{1} \cdot 91 \cdot \mathrm{Cr}_{2} \cdot 122 \cdot \mathrm{SmA} \cdot 182 \cdot \mathrm{I}\left({ }^{\circ} \mathrm{C}\right)$

353-BF $\mathbf{B}_{\mathbf{4}} \mathbf{1 4}: \mathrm{Cr}_{1} \cdot 98 \cdot \mathrm{Cr}_{2} \cdot 122 \cdot \mathrm{SmA} \cdot 187 \cdot \mathrm{I}\left({ }^{\circ} \mathrm{C}\right)$

353-BF $\mathbf{- 1 6}: \mathrm{Cr}_{1} \cdot\left(\mathrm{Cr}_{2} \cdot 90 \cdot\right) 104 \cdot \mathrm{SmA} \cdot 179 \cdot \mathrm{I}\left({ }^{\circ} \mathrm{C}\right)$

353-BF 4 -18: $\mathrm{Cr} \cdot 102 \cdot \mathrm{SmA} \cdot 162 \cdot \mathrm{I}\left({ }^{\circ} \mathrm{C}\right)$

353-SbF $\mathbf{6}^{-} \boldsymbol{n}\left(\mathrm{X}^{-}=\left[\mathrm{SbF}_{6}\right]^{-}\right)$

353-SbF 6 -8: $\mathrm{Cr}_{1} \cdot 100 \cdot \mathrm{Cr}_{2} \cdot 112 \cdot \mathrm{Cr}_{3} \cdot 147 \cdot \mathrm{SmA} \cdot\left[\right.$ not determined] $\cdot \mathrm{I}\left({ }^{\circ} \mathrm{C}\right)$

353-SbF $\mathbf{6}-10: \mathrm{Cr}_{1} \cdot 91 \cdot \mathrm{Cr}_{2} \cdot 104 \cdot \mathrm{Cr}_{3} \cdot 151 \cdot \mathrm{SmA} \cdot\left[\right.$ not determined] $\cdot \mathrm{I}\left({ }^{\circ} \mathrm{C}\right)$

353-SbF $\mathbf{6}_{\mathbf{6}}$ 12: $\mathrm{Cr}_{1} \cdot 114 \cdot \mathrm{Cr}_{2} \cdot 153 \cdot \mathrm{SmA} \cdot$ n.d. $\cdot \mathrm{I}\left({ }^{\circ} \mathrm{C}\right)$

353-SbF $\mathbf{6}-14: \mathrm{Cr}_{1} \cdot 113 \cdot \mathrm{Cr}_{2} \cdot 144 \cdot \mathrm{SmA} \cdot 166 \cdot \mathrm{I}\left({ }^{\circ} \mathrm{C}\right)$

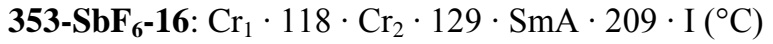

353-SbF $\mathbf{6}_{\mathbf{6}} \mathbf{- 1 8}: \mathrm{Cr}_{1} \cdot 121 \cdot \mathrm{Cr}_{2} \cdot 132 \cdot \mathrm{SmA} \cdot 199 \cdot \mathrm{I}\left({ }^{\circ} \mathrm{C}\right)$

353-ReO ${ }_{4}^{-n}\left(\mathrm{X}^{-}=\left[\mathrm{ReO}_{4}\right]^{-}\right)$

353-ReO 4 -8: $\mathrm{Cr}_{1} \cdot 124 \cdot \mathrm{Cr}_{2} \cdot 155 \cdot \mathrm{SmA} \cdot 164 \cdot \mathrm{I}\left({ }^{\circ} \mathrm{C}\right)$

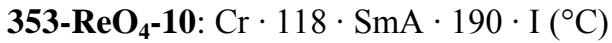


353- $\mathbf{R e O}_{4}-12: \mathrm{Cr} \cdot 118 \cdot \mathrm{SmA} \cdot 208 \cdot \mathrm{I}\left({ }^{\circ} \mathrm{C}\right)$

353-ReO ${ }_{4}-14: \mathrm{Cr} \cdot 114 \cdot \mathrm{SmA} \cdot 195 \cdot \mathrm{I}\left({ }^{\circ} \mathrm{C}\right)$

353-ReO ${ }_{4}-16: \mathrm{Cr}_{1} \cdot 87 \cdot \mathrm{Cr}_{2} \cdot 112 \cdot \mathrm{SmA} \cdot 162 \cdot \mathrm{I}\left({ }^{\circ} \mathrm{C}\right)$

353-ReO ${ }_{4}-18: \mathrm{Cr}_{1} \cdot\left(\mathrm{Cr}_{2} \cdot 104 \cdot\right) 148 \cdot \mathrm{SmA} \cdot 148 \cdot \mathrm{I}\left({ }^{\circ} \mathrm{C}\right)$

353-OTf-n $\left(\mathrm{X}^{-}=[\mathrm{OTf}]^{-}\right)$

353-OTf-8: $\mathrm{Cr}_{1} \cdot 72 \cdot \mathrm{Cr}_{2} \cdot 155 \cdot \mathrm{I}\left({ }^{\circ} \mathrm{C}\right)$

353-OTf-10: $\mathrm{Cr}_{1} \cdot 83 \cdot \mathrm{Cr}_{2} \cdot 86 \cdot \mathrm{Cr}_{3} \cdot 110 \cdot \mathrm{Cr}_{4} \cdot 146 \cdot \mathrm{I}\left({ }^{\circ} \mathrm{C}\right)$

353-OTf-12: $\mathrm{Cr}_{1} \cdot 100 \cdot \mathrm{Cr}_{2} \cdot 106 \cdot \mathrm{Cr}_{3} \cdot 142 \cdot \mathrm{I}\left({ }^{\circ} \mathrm{C}\right)$

353-OTf-14: $\mathrm{Cr}_{1} \cdot 81 \cdot \mathrm{Cr}_{2} \cdot 107 \cdot \mathrm{Cr}_{3} \cdot 132 \cdot \mathrm{I}\left({ }^{\circ} \mathrm{C}\right)$

353-OTf-16: $\mathrm{Cr}_{1} \cdot 115 \cdot \mathrm{Cr}_{2} \cdot 138 \cdot \mathrm{I}\left({ }^{\circ} \mathrm{C}\right)$

353-OTf-18: $\mathrm{Cr}_{1} \cdot 89 \cdot \mathrm{Cr}_{2} \cdot 116 \cdot \mathrm{Cr}_{3} \cdot 127 \cdot \mathrm{I}\left({ }^{\circ} \mathrm{C}\right)$

353-OTs-n $\left(\mathrm{X}^{-}=[\mathrm{OTs}]^{-}\right)$

353-OTs-8: $\mathrm{Cr}_{1} \cdot 60 \cdot \mathrm{Cr}_{2} \cdot 110 \cdot \mathrm{SmA} \cdot 161 \cdot \mathrm{I}\left({ }^{\circ} \mathrm{C}\right)$

353-OTs-10: $\mathrm{Cr}_{1} \cdot 64 \cdot \mathrm{Cr}_{2} \cdot 113 \cdot \mathrm{Cr}_{3} \cdot 125 \cdot \mathrm{Cr}_{4} \cdot 151 \cdot \mathrm{I}\left({ }^{\circ} \mathrm{C}\right)$

353-OTs-12: $\mathrm{Cr}_{1} \cdot 70 \cdot \mathrm{Cr}_{2} \cdot 111 \cdot \mathrm{Cr}_{3} \cdot 117 \cdot \mathrm{Cr}_{4} \cdot 148 \cdot \mathrm{I}\left({ }^{\circ} \mathrm{C}\right)$

353-OTs-14: $\mathrm{Cr}_{1} \cdot 77 \cdot \mathrm{Cr}_{2} \cdot 113 \cdot \mathrm{Cr}_{3} \cdot 123 \cdot \mathrm{Cr}_{4} \cdot 147 \cdot \mathrm{I}\left({ }^{\circ} \mathrm{C}\right)$

353-OTs-16: $\mathrm{Cr}_{1} \cdot 87 \cdot \mathrm{Cr}_{2} \cdot 124 \cdot \mathrm{Cr}_{3} \cdot 149 \cdot \mathrm{I}\left({ }^{\circ} \mathrm{C}\right)$

353-OTs-18: $\mathrm{Cr}_{1} \cdot 90 \cdot \mathrm{Cr}_{2} \cdot 94 \cdot \mathrm{Cr}_{3} \cdot 124 \cdot \mathrm{Cr}_{4} \cdot 144 \cdot \mathrm{I}\left({ }^{\circ} \mathrm{C}\right)$
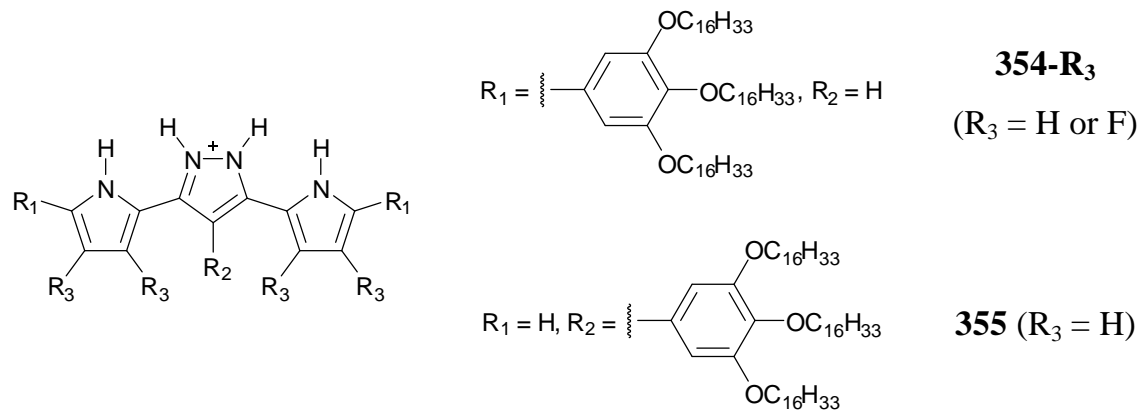

[354-H] $\left[\mathrm{CF}_{3} \mathrm{COO}\right]\left(\mathrm{R}_{3}=\mathrm{H}\right): \mathrm{Cr} \cdot 66 \cdot \mathrm{Col}_{\mathrm{r}} / \mathrm{Cub}\left(\right.$ not clear) $\cdot 85 \cdot \mathrm{Cub}(\mathrm{Pn} \overline{3} m) \cdot 96 \cdot \mathrm{I}\left({ }^{\circ} \mathrm{C}\right)(\mathrm{DSC}$ cooling run)

[354-F] $\left[\mathrm{CF}_{3} \mathrm{COO}\right]\left(\mathrm{R}_{3}=\mathrm{F}\right): \mathrm{Cr} \cdot 68 \cdot \mathrm{Col}_{\mathrm{h}} \cdot 131 \cdot \mathrm{I}\left({ }^{\circ} \mathrm{C}\right)(\mathrm{DSC}$ cooling run)

[355][CF $\left.\mathrm{CF}_{3} \mathrm{COO}\right]$ : see ref. 126

$[355]_{2}\left[\mathrm{OOC}\left(\mathrm{CF}_{2}\right)_{4} \mathrm{COO}\right]$ : see ref. 126

$[355]_{2}\left[\mathrm{OOC}\left(\mathrm{CF}_{2}\right)_{8} \mathrm{COO}\right]$ : see ref. 126 


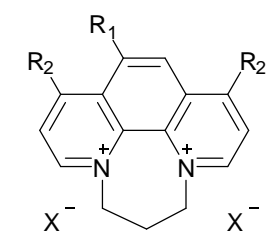

356-X-R $/ \mathbf{R}_{2}$

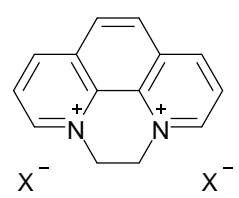

357-X

356- $\mathbf{C}_{12} \mathbf{H}_{25} \mathbf{O S O}_{3}-\mathbf{R}_{\mathbf{1}} / \mathbf{H}\left(\mathrm{X}^{-}=\left[\mathrm{C}_{12} \mathrm{H}_{25} \mathrm{OSO}_{3}\right]^{-}, \mathrm{R}_{2}=\mathrm{H}\right)$

356- $\mathbf{C}_{12} \mathbf{H}_{25} \mathbf{O S O}_{3}-\mathbf{H} / \mathbf{H}: \mathrm{Cr}_{1} \cdot 88 \cdot \mathrm{Cr}_{2} \cdot 133 \cdot \mathrm{Cr}_{3} \cdot 143 \cdot \mathrm{SmE} \cdot 200 \cdot \operatorname{dec}\left({ }^{\circ} \mathrm{C}\right)$

356- $\mathbf{C}_{12} \mathbf{H}_{25} \mathbf{O S O}_{3}-\mathbf{C H}_{3} / \mathbf{H}: \mathrm{Cr}_{1} \cdot 87 \cdot \mathrm{Cr}_{2} \cdot 149 \cdot \mathrm{SmE} \cdot 200 \cdot \operatorname{dec}\left({ }^{\circ} \mathrm{C}\right)$

356- $\mathbf{C}_{12} \mathbf{H}_{25} \mathbf{O S O}_{3}-\mathbf{C l} / \mathbf{H}: \mathrm{Cr}_{1} \cdot 40 \cdot \mathrm{Cr}_{2} \cdot 62 \cdot \mathrm{Cr}_{3} \cdot 100 \cdot \mathrm{Cr}_{4} \cdot 185 \cdot \mathrm{SmE} \cdot 200 \cdot \operatorname{dec}\left({ }^{\circ} \mathrm{C}\right)$

356-DOSS-R $\mathbf{R}_{\mathbf{1}} / \mathbf{H}\left(\mathrm{X}^{-}=[\mathrm{DOSS}]^{-}, \mathrm{R}_{2}=\mathrm{H}\right)$

356-DOSS-H/H: $\mathrm{Cr} \cdot 81 \cdot \mathrm{SmA} \cdot 171 \cdot \mathrm{I}\left({ }^{\circ} \mathrm{C}\right)$

356-DOSS- $\mathrm{CH}_{3} / \mathrm{H}: \mathbf{C r} \cdot 63 \cdot \mathrm{SmA} \cdot 157 \cdot \mathrm{I}\left({ }^{\circ} \mathrm{C}\right)$

356-DOSS-Cl/H: $\mathrm{Cr} \cdot 65 \cdot \mathrm{SmA} \cdot 146 \cdot \mathrm{I}\left({ }^{\circ} \mathrm{C}\right)$

356- $\mathbf{C}_{12} \mathbf{H}_{25} \mathbf{O S O}_{3}-\mathbf{H} / \mathbf{P h}\left(\mathrm{X}^{-}=\left[\mathrm{C}_{12} \mathrm{H}_{25} \mathrm{OSO}_{3}\right]^{-}, \mathrm{R}_{1}=\mathrm{H}, \mathrm{R}_{2}=\mathrm{Ph}\right): \mathrm{Cr}_{1} \cdot 34 \cdot \mathrm{Cr}_{2} \cdot 190 \cdot \mathrm{I}\left({ }^{\circ} \mathrm{C}\right)$

356-DOSS-H/Ph $\left(\mathrm{X}^{-}=[\mathrm{DOSS}]^{-}, \mathrm{R}_{1}=\mathrm{H}, \mathrm{R}_{2}=\mathrm{Ph}\right): \mathrm{g} \cdot 66 \cdot \mathrm{I}\left({ }^{\circ} \mathrm{C}\right)$

357- $\mathbf{C}_{12} \mathbf{H}_{25} \mathbf{O S O}_{3}\left(\mathrm{X}^{-}=\left[\mathrm{C}_{12} \mathrm{H}_{25} \mathrm{OSO}_{3}\right]^{-}\right): \mathrm{Cr}_{1} \cdot 41 \cdot \mathrm{Cr}_{2} \cdot 93 \cdot \mathrm{Cr}_{3} \cdot 200 \cdot \operatorname{dec}\left({ }^{\circ} \mathrm{C}\right)$

357-DOSS $\left(\mathrm{X}^{-}=[\mathrm{DOSS}]^{-}\right): \mathrm{Cr} \cdot 116 \cdot \mathrm{SmA} \cdot 182 \cdot \mathrm{I}\left({ }^{\circ} \mathrm{C}\right)$
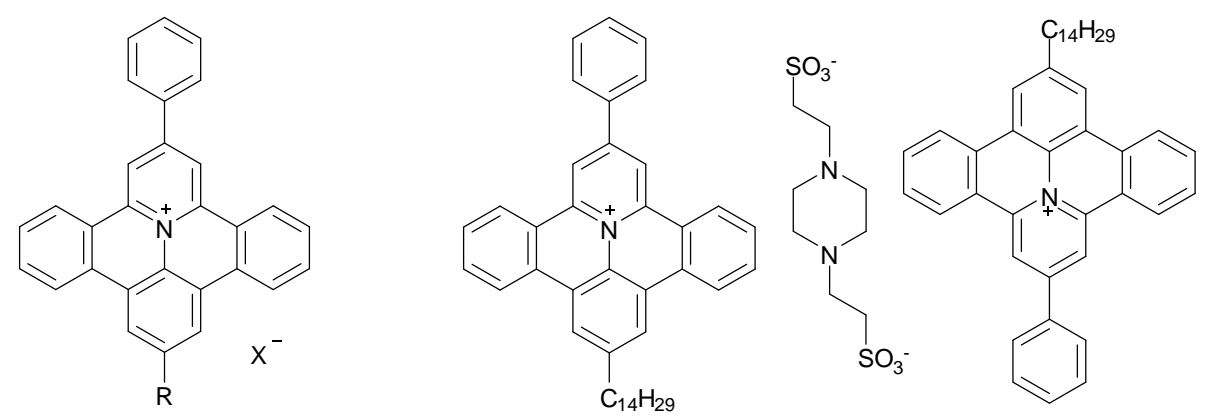

358-X(n)-R $\left(\mathrm{X}^{-}=\left[\mathrm{C}_{n} \mathrm{H}_{2 n+1} \mathrm{SO}_{3}\right]^{-}\right.$,

359

$\left[\mathrm{C}_{12} \mathrm{H}_{25} \mathrm{PhSO}_{3}\right]^{-} ; \mathrm{R}=\mathrm{H}$ or $\left.n-\mathrm{C}_{14} \mathrm{H}_{29}\right)$

358- $\mathrm{C}_{12} \mathrm{H}_{25} \mathrm{SO}_{3}-\mathrm{H}\left(\mathrm{X}^{-}=\left[\mathrm{C}_{12} \mathrm{H}_{25} \mathrm{SO}_{3}\right]^{-}, \mathrm{R}=\mathrm{H}\right): \mathrm{Col} \cdot 180 \cdot \mathrm{I}\left({ }^{\circ} \mathrm{C}\right)$

358- $\mathbf{C}_{12} \mathbf{H}_{25} \mathbf{P h S O}_{3}-\mathbf{H}\left(\mathrm{X}^{-}=\left[\mathrm{C}_{12} \mathrm{H}_{25} \mathrm{PhSO}_{3}\right]^{-}, \mathrm{R}=\mathrm{H}\right): \mathrm{Col} \cdot 210 \cdot \mathrm{I}\left({ }^{\circ} \mathrm{C}\right)$ 


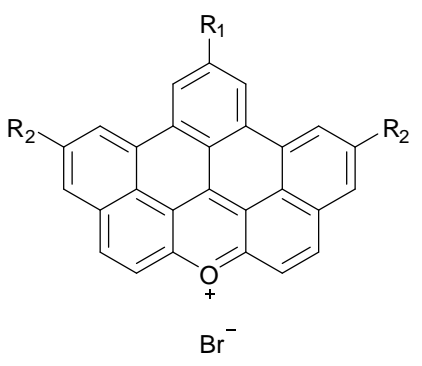

360-(a-b)

360-a $\left(\mathrm{R}_{1}=\mathrm{H}, \mathrm{R}_{2}=n-\mathrm{C}_{12} \mathrm{H}_{25}\right): \mathrm{Col}_{\mathrm{h}}$, no phase transitions detected by DSC between $-100{ }^{\circ} \mathrm{C}$ and $300{ }^{\circ} \mathrm{C}$ 360-b $\left(\mathrm{R}_{1}=\mathrm{R}_{2}=n-\mathrm{C}_{12} \mathrm{H}_{25}\right): \mathrm{Col}_{h}$, no phase transitions detected by DSC between $-100{ }^{\circ} \mathrm{C}$ and $300{ }^{\circ} \mathrm{C}$ 
Scheme S19. ILCs based on receptor-anion complexes, investigated by Maeda and co-workers.

Anionic receptor-anion complexes that were combined with planar TATA cations:

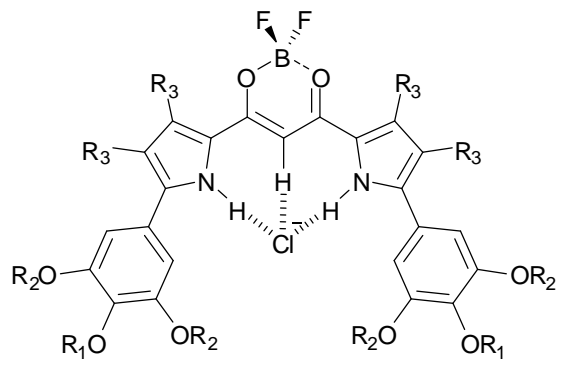

361-(a-f)- $\mathbf{R}_{3} \cdot \mathrm{Cl}^{-}\left(\mathrm{R}_{1}, \mathrm{R}_{2}=n-\mathrm{C}_{n} \mathrm{H}_{2 n+1}(n=12,16)\right.$,

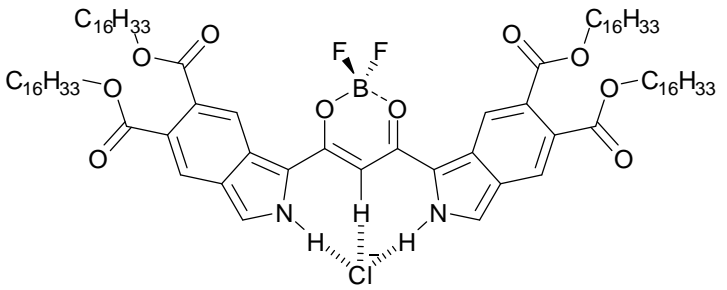

$362 \cdot \mathrm{Cl}^{-}$

$\left(\mathrm{CH}_{2}\right)_{6}\left(\mathrm{CF}_{2}\right)_{5} \mathrm{CF}_{3},(\mathrm{~S})$-3,7-dimethyloctyl; $\left.\mathrm{R}_{3}=\mathrm{H}, \mathrm{CH}_{3}, \mathrm{~F}\right)$
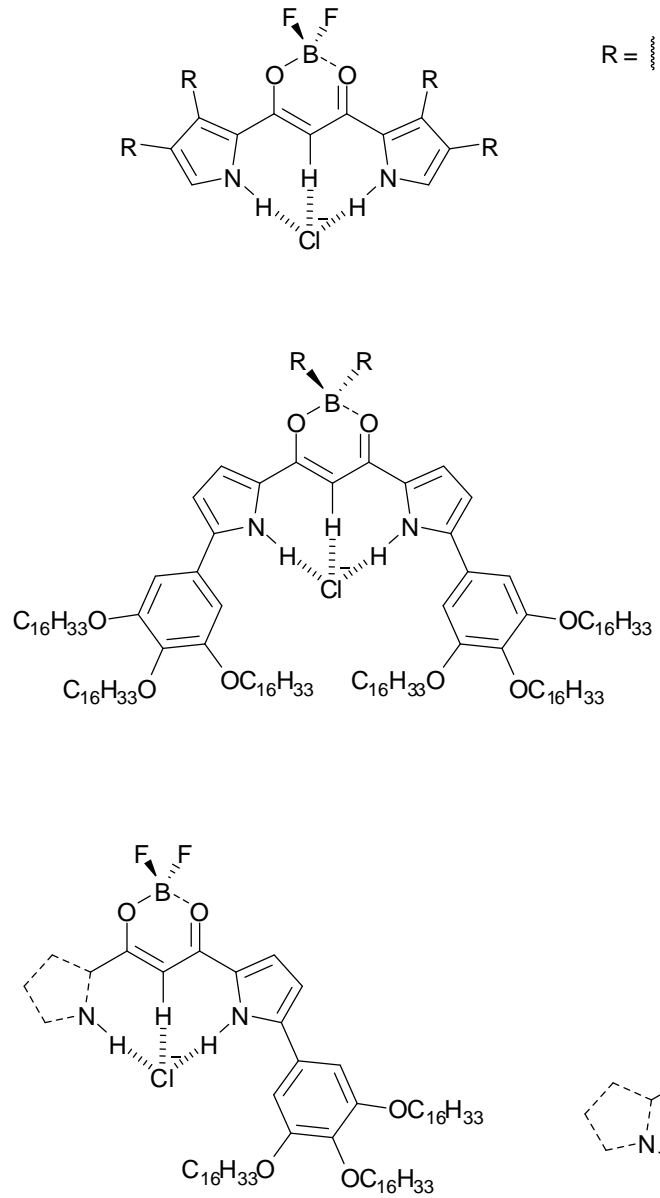

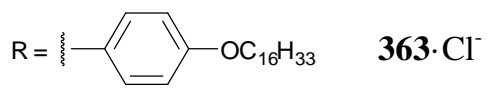

$\mathrm{R}=\mathrm{O}-\mathrm{OC}_{16} \mathrm{H}_{33}$

$364 \cdot \mathrm{Cl}^{-}$

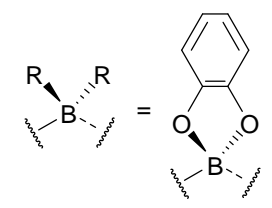

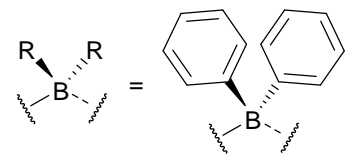

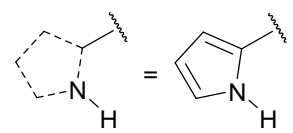

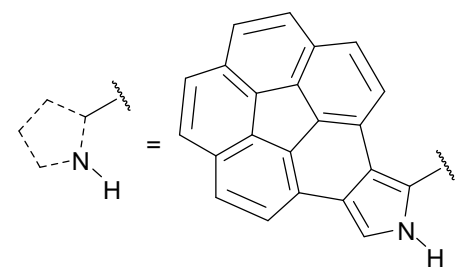

$366 \cdot \mathrm{Cl}^{-}$

$367 \cdot \mathrm{Cl}^{-}$

$365 \cdot \mathrm{Cl}^{-}$

$368 \cdot \mathrm{Cl}^{-}$ 
Planar 4,8,12-trialkyl-4,8,12-triazatriangulenium (TATA) cations:

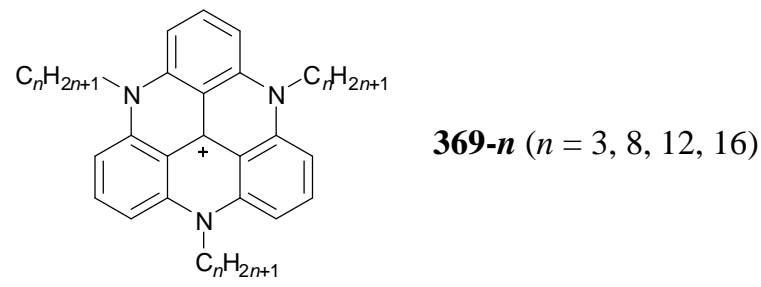

Other ILCs based on anionic receptor-anion complexes:

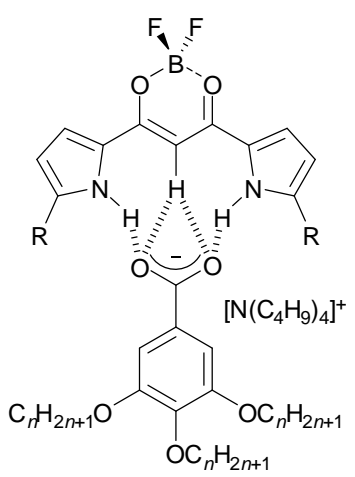

370-n-R $(\mathrm{R}=\mathrm{H}, \mathrm{Ph})$

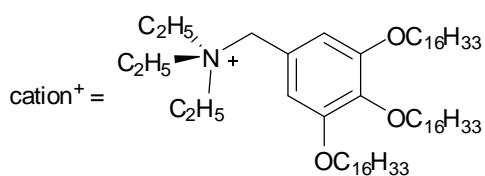

$371-\mathbf{R}_{\mathbf{1}} / \mathbf{R}_{2}$

$\left(\mathrm{R}_{1}=\mathrm{H}, \mathrm{F}\right.$;

$\left.\mathrm{R}_{2}=\mathrm{H}, \mathrm{Ph}\right)$
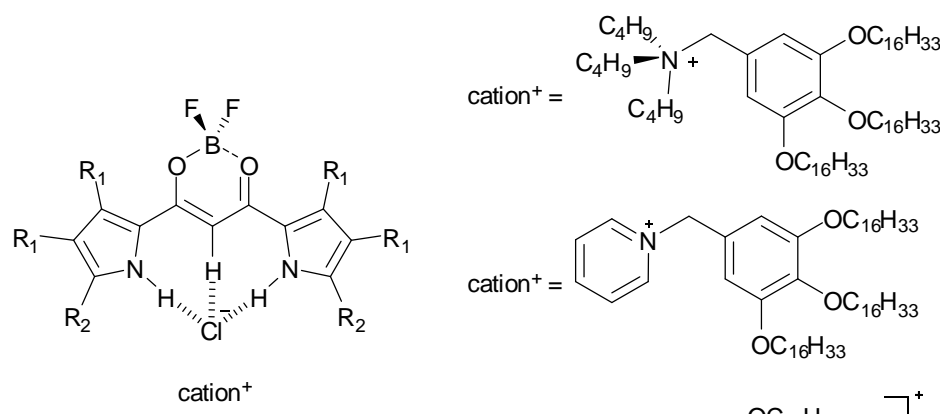

$372-\mathbf{R}_{\mathbf{1}} / \mathbf{R}_{\mathbf{2}}$

$\left(\mathrm{R}_{1}=\mathrm{H}, \mathrm{F}\right.$;

$\left.\mathrm{R}_{2}=\mathrm{H}, \mathrm{Ph}\right)$

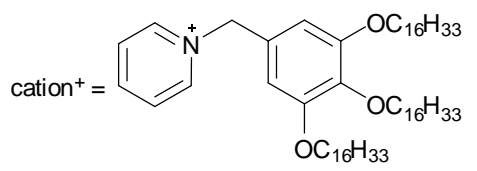

$373-\mathbf{R}_{\mathbf{1}} / \mathbf{R}_{\mathbf{2}}$

$\left(\mathrm{R}_{1}=\mathrm{H}, \mathrm{F}\right.$;

$\left.\mathrm{R}_{2}=\mathrm{H}, \mathrm{Ph}\right)$

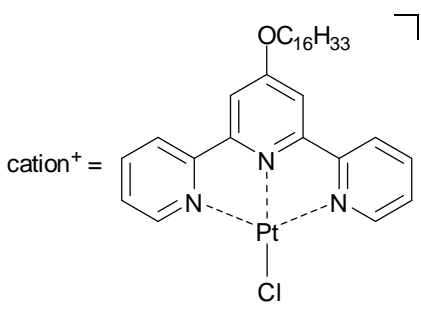

$374\left(\mathrm{R}_{1}=\mathrm{H}\right.$,

$\mathrm{R}_{2}=3,4,5-$

$\left.\left(\mathrm{C}_{16} \mathrm{H}_{33} \mathrm{O}\right)_{3} \mathrm{Ph}\right)$

Cationic receptor-anion complexes that were used to prepare ILCs:
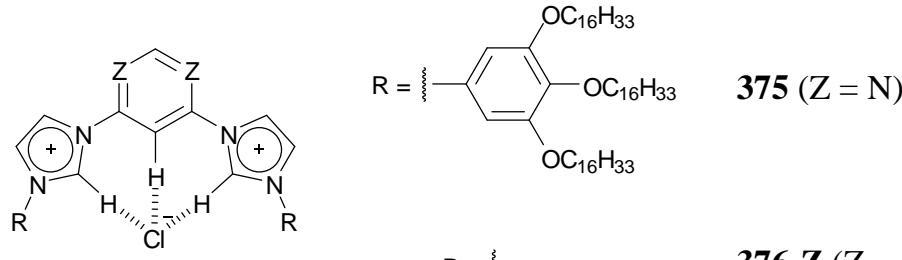

$$
\mathrm{R}=-\mathrm{C}_{16} \mathrm{H}_{33} \quad \text { 376-Z }(\mathrm{Z}=\mathrm{CH}, \mathrm{N})
$$




$$
\begin{aligned}
& \text { 361-a- } \mathbf{R}_{3}\left(\mathrm{R}_{1}=\mathrm{R}_{2}=n-\mathrm{C}_{16} \mathrm{H}_{33}\right) \\
& \text { 361-a-H: } \mathrm{Cr}_{1} \cdot 37 \cdot \mathrm{Cr}_{2} \cdot 51 \cdot \mathrm{Col}_{\mathrm{h}} \cdot 171 \cdot \mathrm{I}\left({ }^{\circ} \mathrm{C}\right) \\
& \text { 361-a- } \mathrm{CH}_{3}: \mathrm{Cr} \cdot 32 \cdot \mathrm{Col}_{\mathrm{h}} \cdot 101 \cdot \mathrm{I}\left({ }^{\circ} \mathrm{C}\right) \\
& \text { 361-a-F: } \mathrm{Cr}_{1} \cdot 33 \cdot \mathrm{Cr}_{2} \cdot 45 \cdot \mathrm{Col}_{\mathrm{h}} \cdot 204 \cdot \mathrm{I}\left({ }^{\circ} \mathrm{C}\right) \\
& \text { 361-a-H· } \mathrm{Cl}^{-} / 369-3 \text { (n-octane xerogel): } \mathrm{Cr} \cdot 44 \cdot \mathrm{Col}_{\mathrm{h}} \cdot 96 \cdot \mathrm{I}\left({ }^{\circ} \mathrm{C}\right)
\end{aligned}
$$

361-a- $\mathrm{CH}_{3} \cdot \mathrm{Cl}^{-} / \mathbf{3 6 9}-3$ (precipitate obtained from $n$-octane): $\mathrm{Cr}_{1} \cdot 40 \cdot \mathrm{Cr}_{2} \cdot 43 \cdot \mathrm{Col}_{\mathrm{h}} \cdot 153 \cdot \mathrm{I}\left({ }^{\circ} \mathrm{C}\right)$ 361-a-F $\cdot \mathrm{Cl}^{-} / \mathbf{3 6 9 - 3}$ (precipitate obtained from $n$-octane): $\mathrm{Cr} \cdot 45 \cdot \mathrm{Col}_{\mathrm{h}} \cdot 146 \cdot \mathrm{I}\left({ }^{\circ} \mathrm{C}\right)$

361-a-H $\cdot \mathrm{Cl}^{-} / \mathbf{3 6 9 - 1 6}$ (precipitate obtained from $n$-octane): $\mathrm{L}_{\mathrm{Col}}$ (no exact transition temperatures are given)

$$
\begin{aligned}
& \text { 361-a-H· } \mathrm{Cl}^{-} /\left[\mathrm{N}_{8,1,1,1}\right]^{+}: \mathrm{Cr} \cdot 43 \cdot \mathrm{Col}_{\mathrm{h}} \cdot 75 \cdot \mathrm{I}\left({ }^{\circ} \mathrm{C}\right) \\
& \text { 361-a-H· } \mathrm{Cl}^{-} /\left[\mathrm{N}_{12,1,1,1}\right]^{+}: \mathrm{Cr} \cdot 43 \cdot \mathrm{Col}_{\mathrm{h}} \cdot 74 \cdot \mathrm{I}\left({ }^{\circ} \mathrm{C}\right) \\
& \text { 361-a-He } \mathrm{Cl}^{-} /\left[\mathrm{N}_{16,1,1,1}\right]^{+}: \mathrm{Cr} \cdot 45 \cdot \mathrm{Col}_{\mathrm{h}} \cdot 73 \cdot \mathrm{I}\left({ }^{\circ} \mathrm{C}\right) \\
& \text { 361-a-He } \mathrm{Cl}^{-} /\left[\mathrm{N}_{8,8,1,1}\right]^{+}: \mathrm{Cr} \cdot 39 \cdot \mathrm{Col}_{\mathrm{h}} \cdot 63 \cdot \mathrm{I}\left({ }^{\circ} \mathrm{C}\right) \\
& \text { 361-a-H· } \mathrm{Cl}^{-} /\left[\mathrm{N}_{12,12,1,1}\right]^{+}: \mathrm{Cr} \cdot 36 \cdot \mathrm{Col}_{\mathrm{h}} \cdot 70 \cdot \mathrm{I}\left({ }^{\circ} \mathrm{C}\right) \\
& \text { 361-a-H· } \mathrm{Cl}^{-} /\left[\mathrm{N}_{18,18,1,1}\right]^{+}: \mathrm{Cr} \cdot 45 \cdot \mathrm{Col}_{\mathrm{h}} \cdot 81 \cdot \mathrm{I}\left({ }^{\circ} \mathrm{C}\right) \\
& \text { 361-a-H· } \mathrm{Cl}^{-} /\left[\mathrm{N}_{4,4,4,1}\right]^{+}: \mathrm{Cr} \cdot 46 \cdot \mathrm{Col}_{\mathrm{h}} \cdot 62 \cdot \mathrm{I}\left({ }^{\circ} \mathrm{C}\right) \\
& \text { 361-a-He } \mathrm{Cl}^{-} /\left[\mathrm{N}_{8,8,8,1,1}\right]^{+}: \mathrm{Cr} \cdot 35 \cdot \mathrm{I}\left({ }^{\circ} \mathrm{C}\right) \\
& \text { 361-a-He } \mathrm{Cl}^{-} /\left[\mathrm{N}_{12,12,12,1}\right]^{+}: \mathrm{Cr} \cdot 31 \cdot \mathrm{I}\left({ }^{\circ} \mathrm{C}\right) \\
& \text { 361-a-He } \mathrm{Cl}^{-} /\left[\mathrm{N}_{4,4,4,4}\right]^{+}: \mathrm{Cr} \cdot 47 \cdot \mathrm{Col}_{\mathrm{h}} \cdot 84 \cdot \mathrm{I}\left({ }^{\circ} \mathrm{C}\right)
\end{aligned}
$$

361-a-He $\mathrm{Cl}^{-} /\left[\mathrm{N}_{8,8,8,8}\right]^{+}: \mathrm{Cr}_{1} \cdot 33 \cdot \mathrm{Cr}_{2}$ (solid phase with a $\mathrm{Col}_{\mathrm{r}}$ structure) $\cdot 46 \cdot \mathrm{I}\left({ }^{\circ} \mathrm{C}\right)$

361-a-H· $\mathrm{Cl}^{-} /\left[\mathrm{N}_{12,12,12,12}\right]^{+}: \mathrm{Cr}_{1} \cdot 28 \cdot \mathrm{Cr}_{2}$ (solid phase with a $\mathrm{Col}_{\mathrm{r}}$ structure) $\cdot 42 \cdot \mathrm{I}\left({ }^{\circ} \mathrm{C}\right)$

361-b-H $\left(\mathrm{R}_{1}=\mathrm{R}_{2}=n-\mathrm{C}_{12} \mathrm{H}_{25}\right): \mathrm{I} \cdot 176 \cdot \mathrm{Col}_{\mathrm{h}} \cdot ? \cdot \mathrm{Cr}\left({ }^{\circ} \mathrm{C}\right)$ (DSC cooling run)

361-b-H· $\mathrm{Cl}^{-} / 369-3\left(\mathrm{R}_{1}=\mathrm{R}_{2}=n-\mathrm{C}_{12} \mathrm{H}_{25}\right): \mathrm{I} \cdot 79 \cdot \mathrm{Col}_{\mathrm{h}} \cdot 17 \cdot$ [unidentified phase] $\left({ }^{\circ} \mathrm{C}\right)$ (DSC cooling run)

361-c-H $\left(\mathrm{R}_{1}=\mathrm{R}_{2}=\left(\mathrm{CH}_{2}\right)_{6}\left(\mathrm{CF}_{2}\right)_{5} \mathrm{CF}_{3}\right): \mathrm{I} \cdot 167 \cdot \mathrm{Col}_{\mathrm{h}(\mathrm{d})} \cdot 119 \cdot \mathrm{Col}_{\mathrm{h}(\mathrm{o})} \cdot 75 \cdot \mathrm{Cr}\left({ }^{\circ} \mathrm{C}\right)(\mathrm{DSC}$ cooling run)

361-c-He $\mathrm{Cl}^{-} / 369-3\left(\mathrm{R}_{1}=\mathrm{R}_{2}=\left(\mathrm{CH}_{2}\right)_{6}\left(\mathrm{CF}_{2}\right)_{5} \mathrm{CF}_{3}\right): \mathrm{I} \cdot 153 \cdot \mathrm{Col}_{\mathrm{h}} \cdot$ ? $\cdot \mathrm{Cr}\left({ }^{\circ} \mathrm{C}\right)(\mathrm{DSC}$ cooling run)

361-d-He $\mathrm{Cl}^{-} / \mathbf{3 6 9}-\mathbf{3}\left(\mathrm{R}_{1}=\mathrm{R}_{2}=(S)-3,7-\right.$ dimethyloctyl): not $\mathrm{LC}$

361-e-H· $\mathrm{Cl}^{-} / 369-3\left(\mathrm{R}_{1}=n-\mathrm{C}_{16} \mathrm{H}_{33}, \mathrm{R}_{2}=(S)\right.$-3,7-dimethyloctyl): not $\mathrm{LC}$

361-f-H· $\mathrm{Cl}^{-} / 369-3\left(\mathrm{R}_{1}=(S)\right.$-3,7-dimethyloctyl, $\left.\mathrm{R}_{2}=n-\mathrm{C}_{16} \mathrm{H}_{33}\right)$ : not $\mathrm{LC}$

361-a-H· $\mathrm{Cl}^{-} / 375: \mathrm{I} \cdot 119 \cdot \mathrm{M} \cdot 29 \cdot \mathrm{Cr}\left({ }^{\circ} \mathrm{C}\right)(\mathrm{DSC}$ cooling run)

361-a-H· $\mathrm{Cl}^{-} / 376-(\mathrm{CH}): \mathrm{I} \cdot 104 \cdot \mathrm{Col}_{\mathrm{h}} \cdot 47 \cdot \mathrm{Cr}\left({ }^{\circ} \mathrm{C}\right)$ (DSC cooling run)

361-a-H· $\mathrm{Cl}^{-} / 376-\mathrm{N}: \mathrm{I} \cdot 148 \cdot \mathrm{Col}_{\mathrm{h}} \cdot 34 \cdot \mathrm{Cr}\left({ }^{\circ} \mathrm{C}\right)(\mathrm{DSC}$ cooling run)

[375][Cl]: I $\cdot 158 \cdot \mathrm{Col}_{\mathrm{h}} \cdot 45 \cdot \mathrm{Cr}\left({ }^{\circ} \mathrm{C}\right)$ (DSC cooling run)

[376-(CH)][Cl]: I $\cdot 214$ (from POM) $\cdot$ lamellar phase $\cdot 84 \cdot \mathrm{Cr}\left({ }^{\circ} \mathrm{C}\right)$ (DSC cooling run)

[376-N][Cl]: I $\cdot 156 \cdot$ lamellar phase $\cdot 24 \cdot \mathrm{Cr}\left({ }^{\circ} \mathrm{C}\right)($ DSC cooling run)

362 $\cdot \mathrm{Cl}^{-} / 369-3: \mathrm{Cr}_{1} \cdot 24 \cdot \mathrm{Cr}_{2} / \mathrm{Col}_{\mathrm{s}}(?) \cdot 59 \cdot \mathrm{Col}_{\mathrm{s}} \cdot 102 \cdot \mathrm{I}\left({ }^{\circ} \mathrm{C}\right)$

363. $\mathrm{Cl}^{-} / 369-3: \mathrm{Cr} \cdot 15 \cdot \mathrm{I}\left({ }^{\circ} \mathrm{C}\right)$

364 $\mathrm{Cl}^{-} / 369-3: \mathrm{Cr} \cdot 57 \cdot \mathrm{M} \cdot 70 \cdot \mathrm{I}\left({ }^{\circ} \mathrm{C}\right)$ 
365 $\mathrm{Cl}^{-} / \mathbf{3 6 9}$-3 (precipitate obtained from 1,4-dioxane): $\mathrm{Cr} \cdot 44 \cdot \mathrm{Col}_{\mathrm{r}, 1} \cdot 58 \cdot \mathrm{Col}_{\mathrm{r}, 2} \cdot 86 \cdot$ [phase type not reported] $\cdot 91$ - I $\left({ }^{\circ} \mathrm{C}\right)$

366 $\mathrm{Cl}^{-} / 369-3$ (precipitate obtained from 1,4-dioxane): $\mathrm{Cr} \cdot 74 \cdot \mathrm{I}\left({ }^{\circ} \mathrm{C}\right)$ (with complex phase behavior prior to the melting point)

367: I $\cdot 136 \cdot$ LC phase with lamellar structure $\cdot 90 \cdot \operatorname{Cr}\left({ }^{\circ} \mathrm{C}\right)$ (DSC cooling run)

367 $\mathrm{Cl}^{-} / 369-3$ : lamellar phase at $100{ }^{\circ} \mathrm{C}, \mathrm{Col}_{\mathrm{r}}$ phase at $25^{\circ} \mathrm{C}$

368: $\mathrm{I} \cdot 152 \cdot \mathrm{Col}_{\mathrm{r}} \cdot 127 \cdot \mathrm{LC}$ phase with lamellar structure $\cdot 19 \cdot \mathrm{Col}_{\mathrm{h}}\left({ }^{\circ} \mathrm{C}\right)$ (DSC cooling run)

368. $\mathrm{Cl}^{-} /\left[\mathrm{N}_{4,4,4,4}\right]^{+}$(precipitate obtained from acetone): lamellar phase

368. $\mathrm{Cl}^{-} / 369-3$ : not LC

370-16-H: $\mathrm{Cr} \cdot 42 \cdot \mathrm{M}_{1} \cdot 56 \cdot \mathrm{M}_{2} \cdot 103 \cdot \mathrm{I}\left({ }^{\circ} \mathrm{C}\right)$

370-18-H: $\mathrm{Cr} \cdot 67 \cdot \mathrm{M}_{1} \cdot 73 \cdot \mathrm{M}_{2} \cdot 101 \cdot \mathrm{I}\left({ }^{\circ} \mathrm{C}\right)$

370-20-H: $\mathrm{Cr} \cdot 67 \cdot \mathrm{M}_{1} \cdot 81 \cdot \mathrm{M}_{2} \cdot 96 \cdot \mathrm{I}\left({ }^{\circ} \mathrm{C}\right)$

370-16-Ph: $\mathrm{Cr} \cdot 40 \cdot \mathrm{M}_{1} \cdot 61 \cdot \mathrm{M}_{2} \cdot 81 \cdot \mathrm{I}\left({ }^{\circ} \mathrm{C}\right)$

370-18-Ph: $\mathrm{Cr} \cdot 53 \cdot \mathrm{M}_{1} \cdot 64 \cdot \mathrm{M}_{2} \cdot 86 \cdot \mathrm{I}\left({ }^{\circ} \mathrm{C}\right)$

370-20-Ph: $\mathrm{Cr} \cdot 61 \cdot \mathrm{M}_{1} \cdot 82 \cdot \mathrm{M}_{2} \cdot 89 \cdot \mathrm{I}\left({ }^{\circ} \mathrm{C}\right)$

161-Cl-2/16: $\mathrm{Cr}_{1}$ (with $\mathrm{Col}_{\mathrm{h}}$ structure) $\cdot 39 \cdot \mathrm{Cr}_{2}$ (with $\mathrm{Col}_{\mathrm{h}}$ structure) $\cdot 64 \cdot \mathrm{Col}_{\mathrm{h}} \cdot 129 \cdot \mathrm{I}\left({ }^{\circ} \mathrm{C}\right)$

371-H/H: $\mathrm{Cr}$ (with $\mathrm{Col}_{\mathrm{h}}$ structure) $\cdot 50 \cdot \mathrm{Col}_{\mathrm{h}} \cdot 133 \cdot \mathrm{I}\left({ }^{\circ} \mathrm{C}\right)$

371-H/Ph: $\mathrm{Cr}_{1} \cdot 37 \cdot \mathrm{Cr}_{2} \cdot 46 \cdot \mathrm{I}\left({ }^{\circ} \mathrm{C}\right)$

371-F/H: $\mathrm{Cr}_{1} \cdot 7 \cdot \mathrm{Cr}_{2}$ (with $\mathrm{Col}_{\mathrm{h}}$ structure) $\cdot 36 \cdot \mathrm{Col}_{\mathrm{h}} \cdot 102 \cdot \mathrm{I}\left({ }^{\circ} \mathrm{C}\right)$

371-F/Ph: $\mathrm{Cr}_{1}$ (with $\mathrm{Col}_{\mathrm{r}}$ structure) $\cdot 29 \cdot \mathrm{Cr}_{2}$ (with $\mathrm{Col}_{\mathrm{r}}$ structure) $\cdot 51 \cdot \mathrm{I}\left({ }^{\circ} \mathrm{C}\right)$

161-Cl-4/16: $\mathrm{Cr}_{1}$ (with $\mathrm{Col}_{\mathrm{h}}$ structure) $\cdot 42 \cdot \mathrm{Cr}_{2} \cdot 54 \cdot \mathrm{Cr}_{3} \cdot 76 \cdot \mathrm{I}\left({ }^{\circ} \mathrm{C}\right)\left(1^{\text {st }}\right.$ DSC cooling run: $\mathrm{I} \cdot 81 \cdot \mathrm{Col} / \mathrm{h} \cdot 39 \cdot \mathrm{Cr}$ (with $\mathrm{Col}_{\mathrm{h}}$ structure) $\left({ }^{\circ} \mathrm{C}\right)$ )

372-H/H: Cr (with lamellar structure) $\cdot 44 \cdot \mathrm{I}\left({ }^{\circ} \mathrm{C}\right)$

372-H/Ph: $\mathrm{Cr}$ (with $\mathrm{Col}_{\mathrm{h}}$ structure) $\cdot 39 \cdot \mathrm{Col}_{\mathrm{h}} \cdot 143 \cdot \mathrm{I}\left({ }^{\circ} \mathrm{C}\right)$

372-F/H: $\mathrm{Cr}_{1}$ (with $\mathrm{Col}_{\mathrm{h}}$ structure) $\cdot 39 \cdot \mathrm{Cr}_{2}$ (with $\mathrm{Col}_{\mathrm{h}}$ structure) $\cdot 60 \cdot \mathrm{Cr}_{3}$ (with $\mathrm{Col}_{\mathrm{h}}$ structure) $\cdot 92 \cdot \mathrm{I}\left({ }^{\circ} \mathrm{C}\right)$

372-F/Ph: Cr (with lamellar structure) $\cdot 31 \cdot \mathrm{M}_{1}$ (with $\mathrm{Col}_{\mathrm{r}}$ structure) $\cdot 70 \cdot \mathrm{I}\left({ }^{\circ} \mathrm{C}\right)\left(1^{\text {st }} \mathrm{DSC}\right.$ cooling run: $\mathrm{I} \cdot 74 \cdot \mathrm{M}_{2} \cdot$ $37 \cdot \mathrm{Cr}$ (with lamellar structure) $\left({ }^{\circ} \mathrm{C}\right)$ )

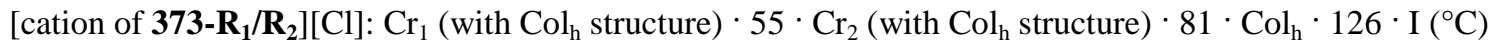

373-H/H: Cr (with $\mathrm{Col}_{\mathrm{h}}$ structure) $\cdot 54 \cdot \mathrm{Col}_{\mathrm{h}} \cdot 134 \cdot \mathrm{I}\left({ }^{\circ} \mathrm{C}\right)$

373-H/Ph: $\mathrm{Cr}_{1} \cdot 48 \cdot \mathrm{Cr}_{2} \cdot 127 \cdot \mathrm{I}\left({ }^{\circ} \mathrm{C}\right)$

373-F/H: $\mathrm{Cr}$ (with $\mathrm{Col}_{\mathrm{h}}$ structure) $\cdot 39 \cdot \mathrm{Col}_{\mathrm{h}} \cdot 53 \cdot \mathrm{I}\left({ }^{\circ} \mathrm{C}\right)$

373-F/Ph: $\mathrm{Cr}_{1}$ (with $\mathrm{Col}_{\mathrm{h}}$ structure) $\cdot 30 \cdot \mathrm{Cr}_{2}$ (with $\mathrm{Col}_{\mathrm{h}}$ structure) $\cdot 46 \cdot \mathrm{I}\left({ }^{\circ} \mathrm{C}\right)$

374: $\mathrm{Cr}$ (with $\mathrm{Col}_{\mathrm{h}}$ structure) $\cdot 28 \cdot \mathrm{Col}_{\mathrm{h}} \cdot 195 \cdot \mathrm{I}\left({ }^{\circ} \mathrm{C}\right)$ 


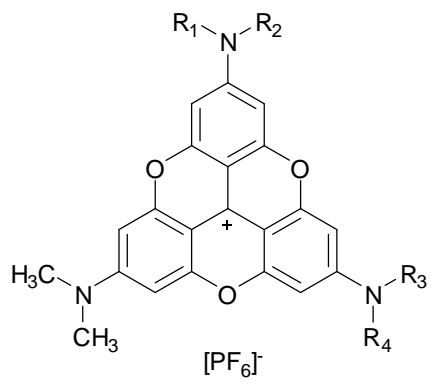

377-(a-c)

$$
\begin{gathered}
\text { 377-a }\left(\mathrm{R}_{1}=\mathrm{R}_{2}=n-\mathrm{C}_{10} \mathrm{H}_{21}, \mathrm{R}_{3}=\mathrm{R}_{4}=\mathrm{CH}_{3}\right) \\
\text { 377-b }\left(\mathrm{R}_{1}=\mathrm{R}_{3}=n-\mathrm{C}_{10} \mathrm{H}_{21}, \mathrm{R}_{2}=\mathrm{R}_{4}=\mathrm{CH}_{3}\right) \\
\text { 377-c }\left(\mathrm{R}_{1}=\mathrm{R}_{2}=\mathrm{R}_{3}=\mathrm{R}_{4}=n-\mathrm{C}_{10} \mathrm{H}_{21}\right)
\end{gathered}
$$
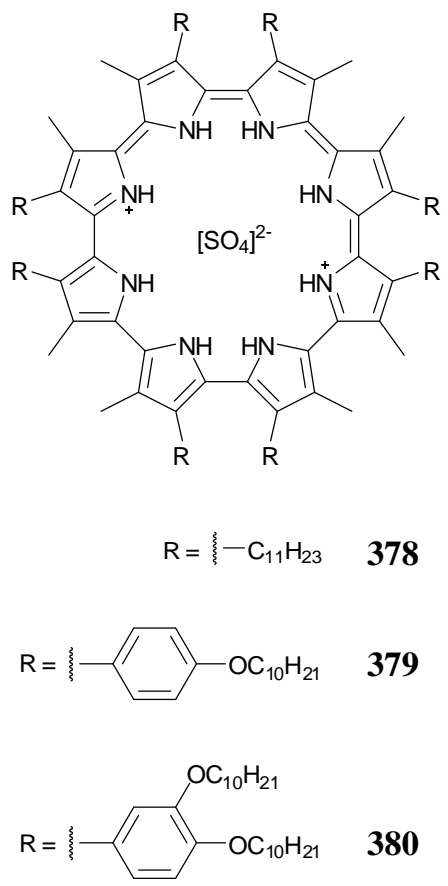

378: $\mathrm{Cr} \cdot 73 \cdot \mathrm{I}\left({ }^{\circ} \mathrm{C}\right)$

379: g $\cdot$ ca. $150 \cdot \mathrm{I}\left({ }^{\circ} \mathrm{C}\right)$

380: $\mathrm{g} \cdot \mathrm{ca} .50 \cdot \mathrm{I}\left({ }^{\circ} \mathrm{C}\right)$

378/TNF (1:1) (TNF = 2,4,7-trinitrofluorenone): $\mathrm{Col}_{\mathrm{h}} \cdot 74 \cdot \mathrm{I}\left({ }^{\circ} \mathrm{C}\right)$

378/TNB (1:2) $(\mathrm{TNB}=1,3,5$-trinitrobenzene $): \mathrm{Cr} \cdot 86 \cdot \mathrm{I}\left({ }^{\circ} \mathrm{C}\right)$

379/TNB (1:1): [phase separation: TNB microcrystals dispersed in the cyclo[8]pyrrole glass] $\cdot c a .200 \cdot \mathrm{Col}_{\mathrm{h}} \cdot \mathrm{ca}$. 250-270 $\cdot$ dec. $\left({ }^{\circ} \mathrm{C}\right)$ (once formed, the $\mathrm{Col}_{\mathrm{h}}$ phase remains unchanged on cooling to $25^{\circ} \mathrm{C}$ )

379/TNP (1:1) (TNP = 2,4,6-trinitrophenol): $\mathrm{Col}_{\mathrm{h}} \cdot$ ca. $250-270 \cdot \operatorname{dec} .\left({ }^{\circ} \mathrm{C}\right)$

380/TNB (1:1): $\mathrm{Col}_{\mathrm{h}} \cdot 171 \cdot \mathrm{I}\left({ }^{\circ} \mathrm{C}\right)$ 


\subsection{Ionic liquid crystals formed by 'ionic self-assembly’ (ISA)}

Scheme S20. Components of LC ISA compounds with functional anions, that have been reported since 2005 (see Scheme S21 for LC ISA compounds with polyoxometalate anions).

Functional anions:
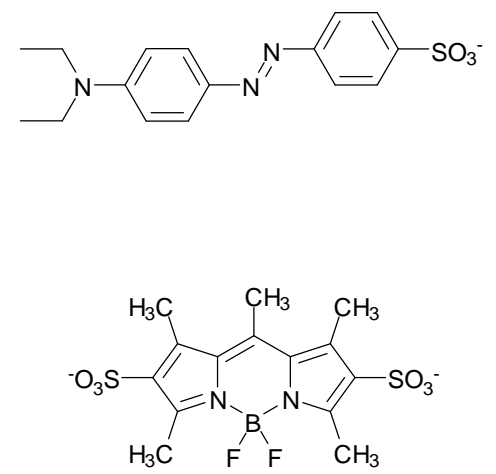

(c)
381<smiles>O=S(=O)(O)c1ccc2cc3cc(S(=O)(=O)O)ccc3cc2c1</smiles>

383<smiles>O=S(=O)([O-])c1ccc(C2=C3C=CC(=N3)C=CC(c3ccc(S(=O)(=O)O)cc3)=C3C=CC(=N3)C(c3ccc(S(=O)(=O)O)cc3)=c3ccc([nH]3)=C(c3ccc(S(=O)(=O)[O-])cc3)c3ccc2[nH]3)cc1</smiles>

384
385

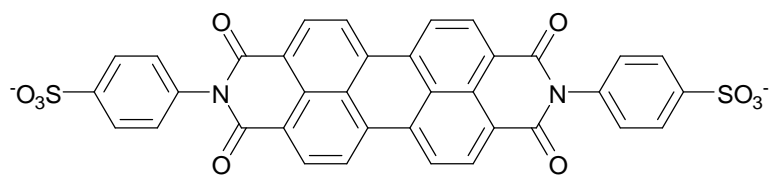




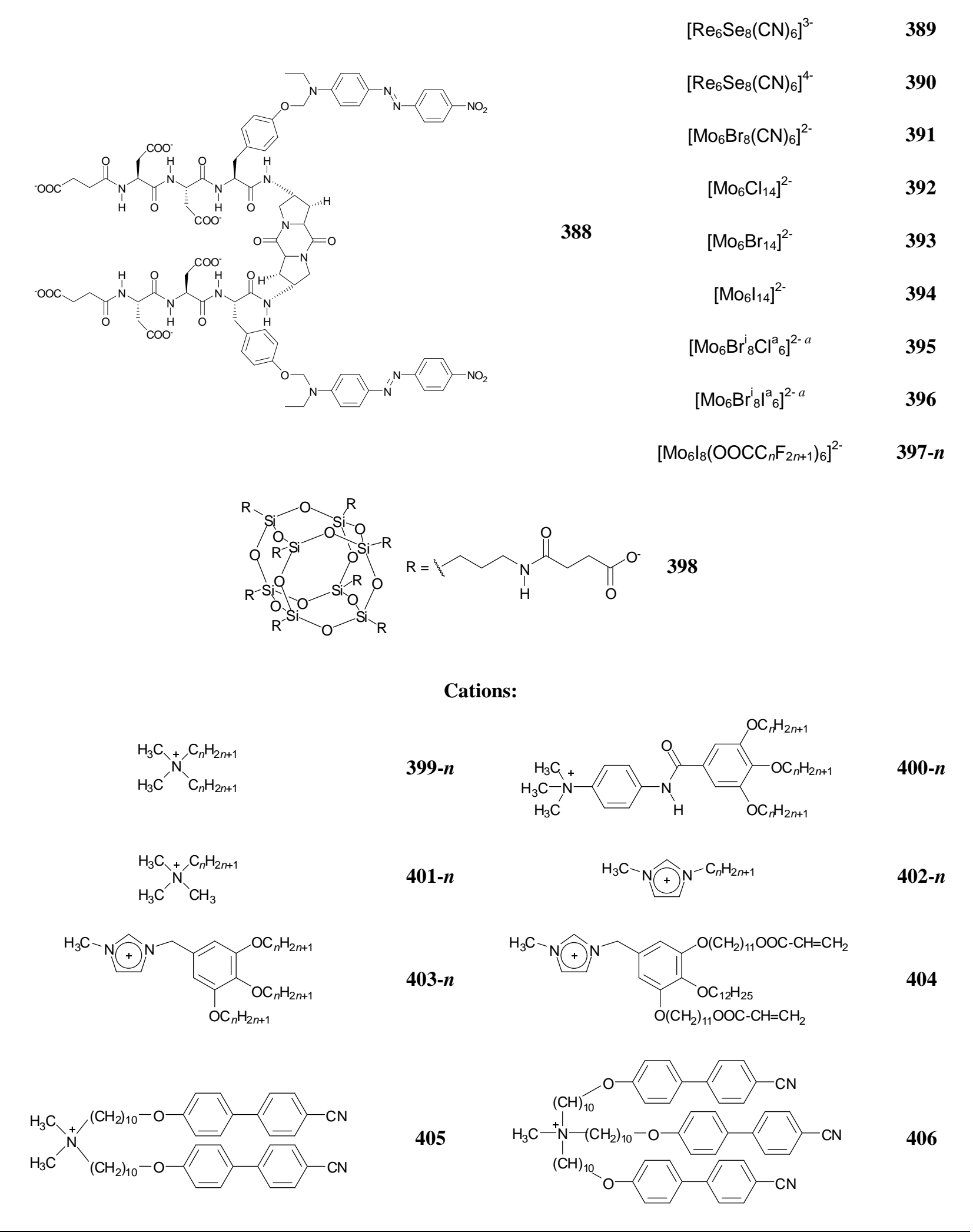



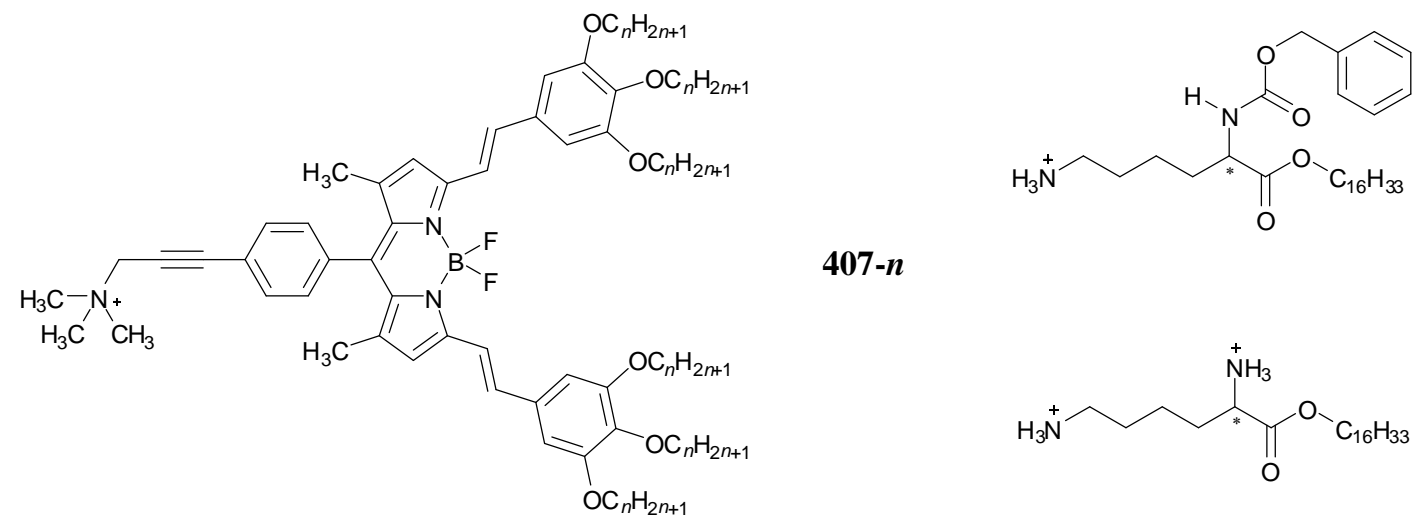

408

407-n

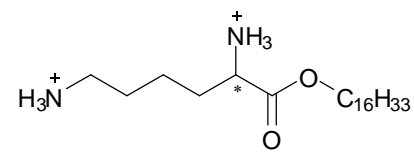

409

\footnotetext{
${ }^{a}$ Superscript 'i’ denotes 'inner’ ligands; superscript ‘a' denotes ‘apical’ ligands.
}

381-(399-12): $\mathrm{g} \cdot-5 \cdot \mathrm{Col}_{\mathrm{r}} \cdot 65 \cdot \mathrm{SmA}_{2} \cdot 160 \cdot \mathrm{SmA} \cdot>180 \cdot \operatorname{dec}\left({ }^{\circ} \mathrm{C}\right)$

381-(399-16): g $\cdot c a .30 \cdot \mathrm{Col}_{\mathrm{s}} \cdot \mathrm{ca} .150 \cdot \mathrm{N}_{\mathrm{C}} \cdot>180 \cdot$ dec. $\left({ }^{\circ} \mathrm{C}\right)$

382-(403-8) $2: \mathrm{Cr} \cdot\left(\mathrm{Col}_{\mathrm{h}} \cdot 73 \cdot\right) 129 \cdot \mathrm{I}$

382-(403-12) $)_{2}: \mathrm{Col}_{\mathrm{h}} \cdot 193 \cdot \mathrm{I}\left({ }^{\circ} \mathrm{C}\right)$

382-(403-16) $)_{2}: \mathrm{Cr} \cdot 47 \cdot \mathrm{Col}_{\mathrm{h}} \cdot 240 \cdot \operatorname{dec}\left({ }^{\circ} \mathrm{C}\right)$

382-(400-8) $)_{2}: \mathrm{Cr}_{1} \cdot 20 \cdot \mathrm{Cr}_{2} \cdot 90 \cdot \mathrm{Cr}_{3} \cdot 180 \cdot \operatorname{dec}\left({ }^{\circ} \mathrm{C}\right)$

382-(400-12) $)_{2}: \mathrm{Cr}_{1} \cdot 66 \cdot \mathrm{Cr}_{2} \cdot 85 \cdot \mathrm{Cr}_{3} \cdot 180 \cdot \operatorname{dec}\left({ }^{\circ} \mathrm{C}\right)$

382-(400-14) $)_{2}: \mathrm{Cr}_{1} \cdot 77 \cdot \mathrm{Cr}_{2} \cdot 99 \cdot \mathrm{Cr}_{3} \cdot 170 \cdot \operatorname{dec}\left({ }^{\circ} \mathrm{C}\right)$

382-(400-16) $)_{2}: \mathrm{Cr}_{1} \cdot 85 \cdot \mathrm{Cr}_{2} \cdot 106 \cdot \mathrm{Cr}_{3} \cdot 170 \cdot \operatorname{dec}\left({ }^{\circ} \mathrm{C}\right)$

382-(399-16) $)_{2}: \mathrm{Cr}_{1} \cdot 49 \cdot \mathrm{Cr}_{2} \cdot 170 \cdot \operatorname{dec}\left({ }^{\circ} \mathrm{C}\right)$

382-(399-18) $)_{2}: \mathrm{Cr}_{1} \cdot 45 \cdot \mathrm{Cr}_{2} \cdot 180 \cdot \operatorname{dec}\left({ }^{\circ} \mathrm{C}\right)$

383-(400-12) $)_{2}: \mathrm{Col}_{\mathrm{h}} \cdot 180 \cdot \operatorname{dec}\left({ }^{\circ} \mathrm{C}\right)$

383-(400-14) $)_{2}: \mathrm{Cr} \cdot-2 \cdot \mathrm{Col}_{\mathrm{h}} \cdot 180 \cdot \operatorname{dec}\left({ }^{\circ} \mathrm{C}\right)$

383-(400-16) $)_{2}: \mathrm{Cr} \cdot 11 \cdot \mathrm{Col}_{\mathrm{h}} \cdot 180 \cdot \operatorname{dec}\left({ }^{\circ} \mathrm{C}\right)$

383-(403-8) $)_{2}: \mathrm{Cr} \cdot 25 \cdot \mathrm{Col}_{\mathrm{h}} \cdot 128 \cdot \mathrm{I}\left({ }^{\circ} \mathrm{C}\right)$

383-(403-12) $)_{2}: \mathrm{Col}_{\mathrm{h}} \cdot 130 \cdot \mathrm{I}\left({ }^{\circ} \mathrm{C}\right)$

383-(403-16) $)_{2}: \mathrm{Cr} \cdot 48 \cdot \mathrm{Col}_{\mathrm{h}} \cdot\left[\right.$ not reported] $\cdot \mathrm{I}\left({ }^{\circ} \mathrm{C}\right)$

383-(404) $)_{2}$ : not LC

383-(407-8) $)_{2}: \mathrm{Cr} \cdot 155 \cdot \mathrm{Col}_{\mathrm{h}} \cdot \mathrm{ca} .215 \cdot \mathrm{dec} .\left({ }^{\circ} \mathrm{C}\right)\left(1^{\text {st }} \mathrm{DSC}\right.$ heating run $)$

383-(407-12) $)_{2}: \mathrm{Cr} \cdot 77 \cdot \mathrm{Col}_{\mathrm{h}} \cdot \mathrm{ca} .210 \cdot \mathrm{dec} .\left({ }^{\circ} \mathrm{C}\right)\left(1^{\text {st }} \mathrm{DSC}\right.$ heating run $)$

383-(407-16) $)_{2}: \mathrm{Cr} \cdot 19 \cdot \mathrm{Col}_{\mathrm{h}} \cdot \mathrm{ca} .190 \cdot \operatorname{dec} .\left({ }^{\circ} \mathrm{C}\right)\left(2^{\text {nd }} \mathrm{DSC}\right.$ heating run $)$

384-(400-8) $)_{4}: \mathrm{Col}_{\mathrm{h}} \cdot 170 \cdot \operatorname{dec}\left({ }^{\circ} \mathrm{C}\right)$

384-(400-12) $)_{4}: \mathrm{Col}_{\mathrm{h}} \cdot 170 \cdot \operatorname{dec}\left({ }^{\circ} \mathrm{C}\right)$ 
385-(399-12) $)_{3}: \mathrm{SmA} \cdot 180 \cdot \operatorname{dec}\left({ }^{\circ} \mathrm{C}\right)$
385-(399-16) $)_{3}: \mathrm{SmA} \cdot 180 \cdot \operatorname{dec}\left({ }^{\circ} \mathrm{C}\right)$
385-(400-12) $)_{3}: \mathrm{Col}_{\mathrm{h}} \cdot 180 \cdot \operatorname{dec}\left({ }^{\circ} \mathrm{C}\right)$
385-(400-14) $)_{3}: \mathrm{Col}_{\mathrm{h}} \cdot 180 \cdot \operatorname{dec}\left({ }^{\circ} \mathrm{C}\right)$
385-(400-16) $)_{3}: \mathrm{Col}_{\mathrm{h}} \cdot 180 \cdot \operatorname{dec}\left({ }^{\circ} \mathrm{C}\right)$
$(386-\mathrm{Ph})-(399-12)_{4}(\mathrm{R}=\mathrm{Ph}): \operatorname{not} \mathrm{LC}$

(386-Ph)-(399-16) $)_{4}(\mathrm{R}=\mathrm{Ph}): \mathrm{Cr} \cdot 38 \cdot \mathrm{SmX}($ ? $) \cdot\left[\right.$ not reported] $\cdot \mathrm{I}\left({ }^{\circ} \mathrm{C}\right)$

(386- $\left.\mathrm{C}_{11} \mathrm{H}_{23}\right)-(399-12)_{4}\left(\mathrm{R}=n-\mathrm{C}_{11} \mathrm{H}_{23}\right): \mathrm{Cr} \cdot\left[\right.$ not reported] $\cdot \mathrm{SmX}\left(\right.$ ?) $\cdot$ [not reported] $\cdot \mathrm{I}\left({ }^{\circ} \mathrm{C}\right)$

$\left(\mathbf{3 8 6}-\mathrm{C}_{11} \mathrm{H}_{23}\right)-(399-16)_{4}\left(\mathrm{R}=n-\mathrm{C}_{11} \mathrm{H}_{23}\right): \mathrm{Cr} \cdot 0 \cdot \mathrm{SmX}\left(\right.$ ?) $\cdot\left[\right.$ not reported] $\cdot \mathrm{I}\left({ }^{\circ} \mathrm{C}\right)$

387-(408) $)_{2}: \mathrm{Cr} \cdot 28 \cdot \mathrm{L}_{\mathrm{Col}} \cdot 125 \cdot \operatorname{dec} .\left({ }^{\circ} \mathrm{C}\right)$

387-409: $\mathrm{L}_{\mathrm{Col}} \cdot 125 \cdot \operatorname{dec} .\left({ }^{\circ} \mathrm{C}\right)$

388-(399-16) 6 : $\mathrm{X} \cdot 40 \cdot \mathrm{SmX} \cdot\left[\right.$ not reported] $\cdot \mathrm{I}\left({ }^{\circ} \mathrm{C}\right)$

388-(401-16) $:$ : ca. $20 \cdot \mathrm{SmX} \cdot\left[\right.$ not reported] $\cdot \mathrm{I}\left({ }^{\circ} \mathrm{C}\right)$

[405][Br]: $\mathrm{Cr} \cdot(\mathrm{N} \cdot 111 \cdot) 117 \cdot \mathrm{I}\left({ }^{\circ} \mathrm{C}\right)$

389-(405) $)_{3}: 1^{\text {st }}$ DSC heating run: $\mathrm{Cr}_{1} \cdot 41 \cdot \mathrm{Cr}_{2} \cdot 121 \cdot \mathrm{Cr}_{3} \cdot 165 \cdot \mathrm{I}\left({ }^{\circ} \mathrm{C}\right) ; 1^{\text {st }}$ DSC cooling run and subsequent

heating/cooling cycles: $\mathrm{g} \cdot(\mathrm{SmA} \cdot 133 \cdot) 139 \cdot \mathrm{I}\left({ }^{\circ} \mathrm{C}\right)$

390-(405) $)_{4}: 1^{\text {st }}$ DSC heating run: $\mathrm{Cr}_{1} \cdot 156 \cdot \mathrm{Cr}_{2} \cdot 173 \cdot \mathrm{I}\left({ }^{\circ} \mathrm{C}\right) ; 1^{\text {st }} \mathrm{DSC}$ cooling run and subsequent heating/cooling cycles: $\mathrm{Cr} \cdot(\mathrm{M} \cdot 143 \cdot) 153 \cdot \mathrm{I}\left({ }^{\circ} \mathrm{C}\right)$

391-(405) $)_{2}$ : not LC

392-(405) $)_{2}$ not LC

[406][Br]: $2^{\text {nd }}$ cooling run: $\mathrm{I} \cdot 113 \cdot \mathrm{N} \cdot 103 \cdot \mathrm{SmA} \cdot 35 \cdot \mathrm{g}\left({ }^{\circ} \mathrm{C}\right)$

389-(406) $)_{3}: 2^{\text {nd }}$ cooling run: $\mathrm{I} \cdot 112 \cdot \mathrm{SmA} \cdot 44 \cdot \mathrm{g}\left({ }^{\circ} \mathrm{C}\right)$

390-(406) $)_{4}: 2^{\text {nd }}$ cooling run: I $\cdot 124 \cdot \operatorname{SmA} \cdot 26 \cdot \mathrm{g}\left({ }^{\circ} \mathrm{C}\right)$

391-(406) $)_{2}: 2^{\text {nd }}$ cooling run: $\mathrm{I} \cdot 97 \cdot \mathrm{N} \cdot 90 \cdot \mathrm{SmA} \cdot 47 \cdot \mathrm{g}\left({ }^{\circ} \mathrm{C}\right)$

392-(406) $)_{2}: 2^{\text {nd }}$ heating run: $\mathrm{g} \cdot 43 \cdot \mathrm{N} \cdot 97 \cdot \mathrm{I}\left({ }^{\circ} \mathrm{C}\right) ; 2^{\text {nd }}$ cooling run: $\mathrm{I} \cdot 71 \cdot \mathrm{N} \cdot 44 \cdot \mathrm{g}\left({ }^{\circ} \mathrm{C}\right)$

393-(406) $)_{2}: 2^{\text {nd }}$ heating run: $\mathrm{g} \cdot 52 \cdot \mathrm{N} \cdot 97 \cdot \mathrm{I}\left({ }^{\circ} \mathrm{C}\right) ; 2^{\text {nd }}$ cooling run: $\mathrm{I} \cdot 87 \cdot \mathrm{N} \cdot 48 \cdot \mathrm{g}\left({ }^{\circ} \mathrm{C}\right)$

394-(406) $)_{2}: 2^{\text {nd }}$ heating run: $\mathrm{g} \cdot 31 \cdot \mathrm{N} \cdot 95 \cdot \mathrm{I}\left({ }^{\circ} \mathrm{C}\right) ; 2^{\text {nd }}$ cooling run: $\mathrm{I} \cdot 78 \cdot \mathrm{N} \cdot 27 \cdot \mathrm{g}\left({ }^{\circ} \mathrm{C}\right)$

395-(406) $)_{2}: 2^{\text {nd }}$ heating run: $\mathrm{g} \cdot 47 \cdot \mathrm{N} \cdot 99 \cdot \mathrm{I}\left({ }^{\circ} \mathrm{C}\right) ; 2^{\text {nd }}$ cooling run: $\mathrm{I} \cdot 71 \cdot \mathrm{N} \cdot 46 \cdot \mathrm{g}\left({ }^{\circ} \mathrm{C}\right)$

396-(406) $)_{2}: 2^{\text {nd }}$ heating run: $\mathrm{g} \cdot 44 \cdot \mathrm{N} \cdot 97 \cdot \mathrm{I}\left({ }^{\circ} \mathrm{C}\right) ; 2^{\text {nd }}$ cooling run: $\mathrm{I} \cdot 90 \cdot \mathrm{N} \cdot 40 \cdot \mathrm{g}\left({ }^{\circ} \mathrm{C}\right)$

(397-1)-(406) $)_{2}: 1^{\text {st }}$ cooling run: $\mathrm{I} \cdot 64 \cdot \mathrm{N} \cdot 22 \cdot \mathrm{g}\left({ }^{\circ} \mathrm{C}\right)$

(397-2)-(406) $)_{2}: 1^{\text {st }}$ cooling run: $\mathrm{I} \cdot 64 \cdot \mathrm{N} \cdot 20 \cdot \mathrm{g}\left({ }^{\circ} \mathrm{C}\right)$

(397-3)-(406) $)_{2}: 1^{\text {st }}$ cooling run: $\mathrm{I} \cdot 61 \cdot \mathrm{N} \cdot 19 \cdot \mathrm{g}\left({ }^{\circ} \mathrm{C}\right)$

398-(402-4) $)_{8} \mathrm{Cr} \cdot 23 \cdot \mathrm{I}\left({ }^{\circ} \mathrm{C}\right)$

398-(402-6) $)_{8} \mathrm{Cr} \cdot 18 \cdot \mathrm{I}\left({ }^{\circ} \mathrm{C}\right)$

398-(402-8) $: \mathrm{Cr} \cdot ? \cdot \mathrm{I}\left({ }^{\circ} \mathrm{C}\right)$ 


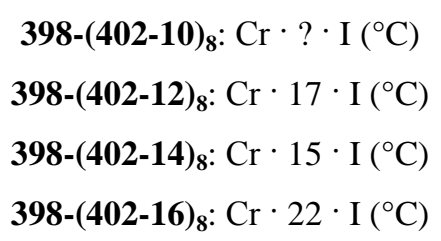

398-(402-18) $)_{8}: \mathrm{Cr} \cdot 45 \cdot \mathrm{SmX}$ [continues to exist until at least $\left.100{ }^{\circ} \mathrm{C}\right] \cdot 208 \cdot \mathrm{dec} .\left({ }^{\circ} \mathrm{C}\right)$

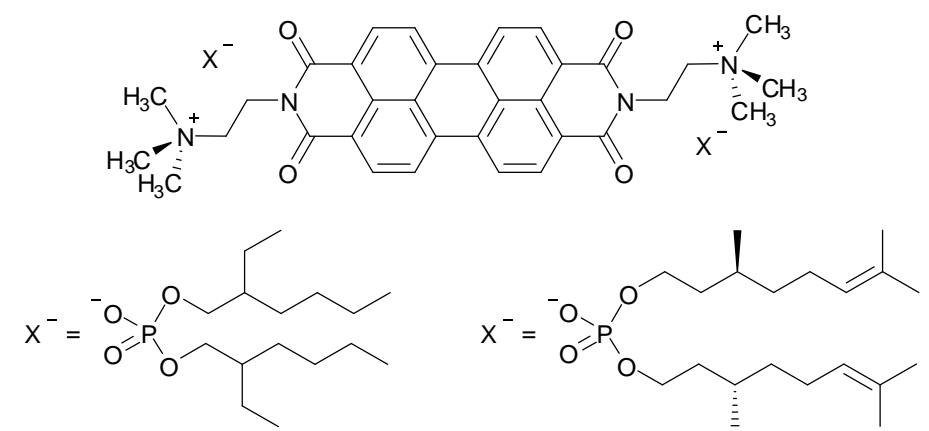

410

411

410: $\mathrm{Col}_{\mathrm{ob}}$ (no exact transition temperatures are given)

411: $\mathrm{Cr} \cdot 150 \cdot \mathrm{Col}_{\mathrm{ob}} \cdot 220 \cdot \operatorname{dec} .\left({ }^{\circ} \mathrm{C}\right)$
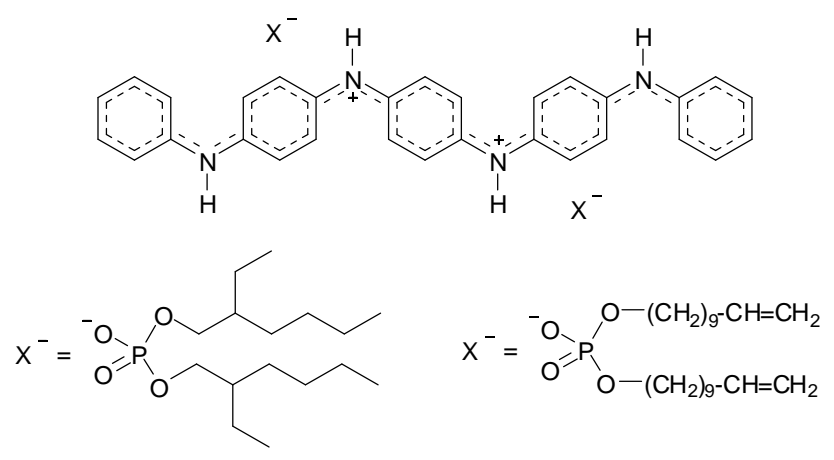

413

412

412: $\mathrm{Col}_{\mathrm{r}} \cdot \mathrm{ca} .80 \cdot \mathrm{I}\left({ }^{\circ} \mathrm{C}\right)$

413: $\mathrm{Col}_{\mathrm{r}} \cdot \mathrm{ca} .70 \cdot \mathrm{I}\left({ }^{\circ} \mathrm{C}\right)$

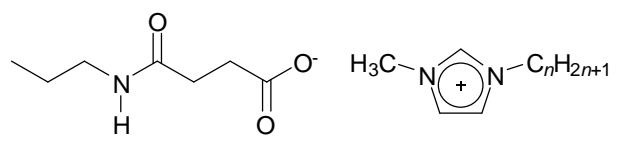

414-n

414-4: $\mathrm{Cr} \cdot 51 \cdot \mathrm{I}(?)\left({ }^{\circ} \mathrm{C}\right)$

414-6: $\mathrm{Cr} \cdot$ ? $\cdot \mathrm{I}($ ? $)\left({ }^{\circ} \mathrm{C}\right)$ 
414-8: $\mathrm{Cr} \cdot 9 \cdot \mathrm{I}(?)\left({ }^{\circ} \mathrm{C}\right)$

414-10: $\mathrm{Cr} \cdot 31 / 47 \cdot \mathrm{I}\left(\right.$ ?) $\left({ }^{\circ} \mathrm{C}\right)$

414-12: $\mathrm{Cr} \cdot 36 / 42 \cdot \mathrm{I}($ ? $)\left({ }^{\circ} \mathrm{C}\right)$

414-14: $\mathrm{Cr} \cdot 43 \cdot \mathrm{I}\left(\right.$ ?) $\left({ }^{\circ} \mathrm{C}\right)$

414-16: $\mathrm{Cr} \cdot 52 \cdot \mathrm{I}\left(\right.$ ?) $\left({ }^{\circ} \mathrm{C}\right)$

414-18: $\mathrm{Cr} \cdot(<) 59 \cdot \mathrm{SmX} \cdot 83 \cdot \mathrm{I}\left({ }^{\circ} \mathrm{C}\right)$ 
Scheme S21. Components of LC ISA compounds with polyoxometalate anions, that have been reported since 2005.

\section{Functional polyoxometalate anions:}

\begin{tabular}{|c|c|c|c|c|c|}
\hline$\left[\mathrm{ZnH}_{6} \mathrm{Mo}_{6} \mathrm{O}_{24}\right]^{3-}$ & 415 & {$\left[\mathrm{PMO}_{12} \mathrm{O}_{40}\right]^{3-}$} & 416 & {$\left[\mathrm{PW}_{12} \mathrm{O}_{40}\right]^{3-}$} & 417 \\
\hline$\left[\mathrm{SiW}_{12} \mathrm{O}_{40}\right]^{4-}$ & 418 & {$\left[\mathrm{GeW}_{12} \mathrm{O}_{40}\right]^{4-}$} & 419 & {$\left[\mathrm{BW}_{12} \mathrm{O}_{40}\right]^{5-}$} & 420 \\
\hline $\begin{array}{c}{\left[\mathrm{PW}_{11} \mathrm{O}_{39} \mathrm{Rh}\right.} \\
\left.\mathrm{CH}_{2} \mathrm{C}(\mathrm{O}) \mathrm{OH}\right]^{5-}\end{array}$ & 421 & {$\left[\mathrm{SiW}_{11} \mathrm{CoO}_{39}\right]^{6-}$} & 422 & {$\left[\mathrm{MnMo}_{9} \mathrm{O}_{32}\right]^{6-}$} & 423 \\
\hline $\begin{array}{l}{\left[\mathrm{Mo}_{2} \mathrm{O}_{4}\left(\left(\mathrm{Mo}_{2} \mathrm{O}_{6}\right) \mathrm{NH}_{3}\right.\right.} \\
\left.\left.\left(\mathrm{CH}_{2}\right)_{3} \mathrm{C}(\mathrm{O})\left(\mathrm{PO}_{3}\right)_{2}\right)_{2}\right]^{6-}\end{array}$ & 424 & $\begin{array}{l}{\left[\mathrm{M}\left[\mathrm{SiW}_{11} \mathrm{O}_{39}\right]\right]^{7^{-}}} \\
\left(\mathrm{M}=\mathrm{Na}^{+} \text {or } \mathrm{K}^{+}\right)\end{array}$ & 425 & {$\left[\mathrm{CeMo}_{12} \mathrm{O}_{42}\right]^{8-}$} & 426 \\
\hline $\begin{array}{c}{\left[\mathrm{Mo}_{2} \mathrm{O}_{4}\left(\left(\mathrm{Mo}_{2} \mathrm{O}_{6}\right) \mathrm{CH}_{3}\right.\right.} \\
\left.\left.\mathrm{C}(\mathrm{O})\left(\mathrm{PO}_{3}\right)_{2}\right)_{2}\right]^{8-}\end{array}$ & 427 & {$\left[\mathrm{EuW}_{10} \mathrm{O}_{36}\right]^{9-}$} & 428 & {$\left[\mathrm{Na}_{2}\left[\mathrm{P}_{2} \mathrm{~W}_{15} \mathrm{O}_{56}\right]\right]^{10-}$} & 429 \\
\hline$\left[\mathrm{Mn}_{4}\left(\mathrm{VW}_{9} \mathrm{O}_{34}\right)_{2}\left(\mathrm{H}_{2} \mathrm{O}\right)_{2}\right]^{10-}$ & 430 & {$\left[\mathrm{Eu}\left(\mathrm{PW}_{11} \mathrm{O}_{39}\right)_{2}\right]^{11-}$} & 431 & {$\left[\mathrm{Ge}_{2} \mathrm{Mo}_{16} \mathrm{O}_{58}\right]^{12-}$} & 432 \\
\hline$\left[\mathrm{EuP}_{5} \mathrm{~W}_{30} \mathrm{O}_{110}\right]^{12-}$ & 433 & {$\left[\mathrm{Eu}\left(\mathrm{SiW}_{9} \mathrm{Mo}_{2} \mathrm{O}_{39}\right)_{2}\right]^{13-}$} & 434 & {$\left[\mathrm{Eu}\left(\mathrm{SiW}_{11} \mathrm{O}_{39}\right)_{2}\right]^{13-}$} & 435 \\
\hline$\left[\mathrm{Tb}\left(\mathrm{SiW}_{11} \mathrm{O}_{39}\right)_{2}\right]^{13-}$ & 436 & {$\left[\mathrm{Eu}\left(\mathrm{BW}_{11} \mathrm{O}_{39}\right)_{2}\right]^{15-}$} & 437 & $\begin{array}{c}{\left[\mathrm{Ni}_{9}(\mathrm{OH})_{3}\left(\mathrm{H}_{2} \mathrm{O}\right)_{6}\left(\mathrm{HPO}_{4}\right)_{2}\right.} \\
\left.\left(\mathrm{PW}_{9} \mathrm{O}_{34}\right)_{3}\right]^{16-}\end{array}$ & 438 \\
\hline $\begin{array}{c}{\left[\left(\mathrm{Mo}_{4} \mathrm{O}_{4} \mathrm{~S}_{4}(\mathrm{OH})_{2}\left(\mathrm{H}_{2} \mathrm{O}\right)_{3}\right)_{2}\right.} \\
\left.\left(\mathrm{P}_{8} \mathrm{~W}_{48} \mathrm{O}_{184}\right)\right]^{36-}\end{array}$ & 439 & $\begin{array}{c}{\left[\mathrm{Mo}_{132} \mathrm{O}_{372}\left(\mathrm{CH}_{3} \mathrm{COO}\right)_{30}\right.} \\
\left.\left(\mathrm{H}_{2} \mathrm{O}\right)_{72}\right]^{42-}\end{array}$ & 440 & $\begin{array}{c}{\left[\mathrm{Mo}_{132} \mathrm{O}_{312} \mathrm{~S}_{60}\left(\mathrm{SO}_{4}\right)_{x}\right.} \\
\left.\left(\mathrm{H}_{2} \mathrm{O}\right)_{132-2 x}\right]^{(12+2 x)-}\end{array}$ & $\begin{array}{l}441-x(x \\
=23,30)\end{array}$ \\
\hline
\end{tabular}

Cations (see also Scheme S20):
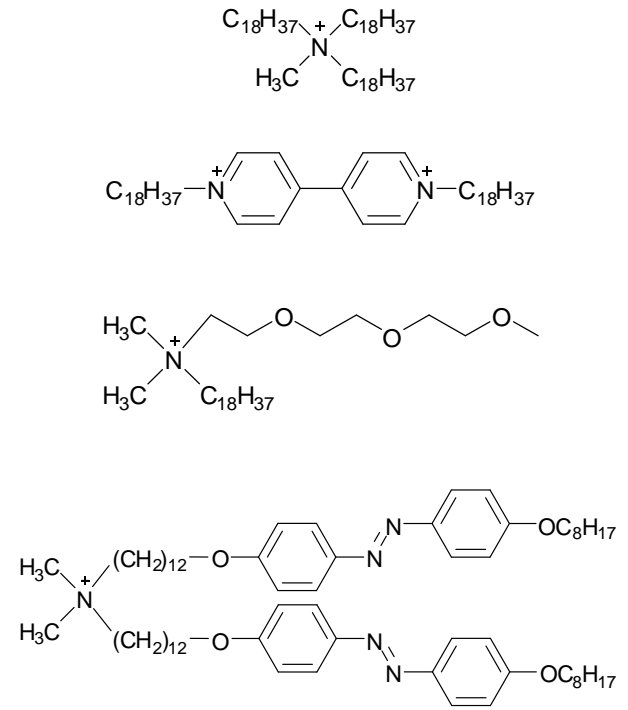

442

444

446

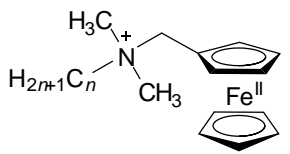

448

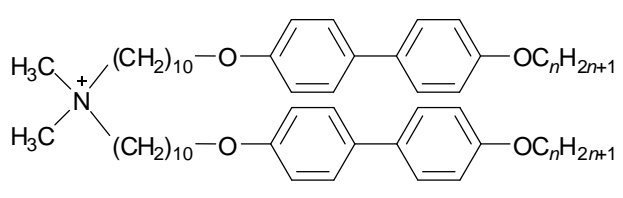

443-n

445-n

447-n

449-n 


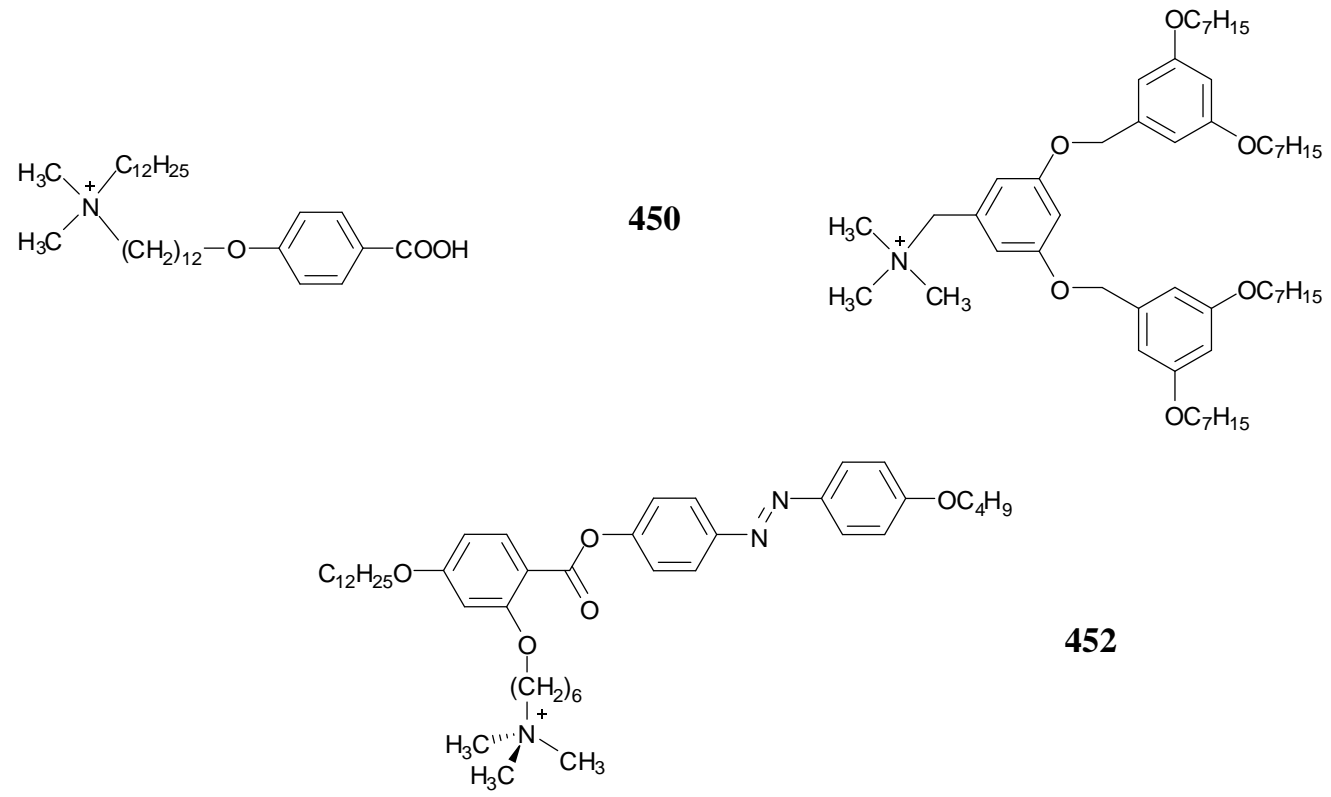

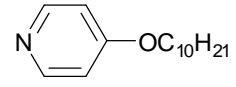

453

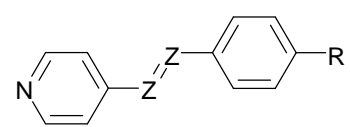

454-(a-d) ( $\mathrm{Z}=\mathrm{CH}, \mathrm{N} ; \mathrm{R}=$ $\mathrm{NO}_{2}, \mathrm{OC}_{10} \mathrm{H}_{21}, \mathrm{OC}_{16} \mathrm{H}_{33}$, 3,4,5- $\left(\mathrm{C}_{16} \mathrm{H}_{33} \mathrm{O}\right)_{3}$-benzyloxy)

415-(449-8) $)_{3} \mathrm{Cr} \cdot 131 \cdot \mathrm{SmC} \cdot 159 \cdot \mathrm{SmA} \cdot 190 \cdot \mathrm{I}\left({ }^{\circ} \mathrm{C}\right)$

416-(446) $)_{3}: \mathrm{Cr}_{1} \cdot-22 \cdot \mathrm{Cr}_{2}\left({ }^{\circ} \mathrm{C}\right)$

417-(443-8) $:$ not LC

417-(448) $)_{3}$ (protonated): $\mathrm{Cr} \cdot 110 \cdot \mathrm{SmB} \cdot 198 \cdot \mathrm{I}\left({ }^{\circ} \mathrm{C}\right)\left(\mathrm{H}_{3} \mathrm{PW}_{12} \mathrm{O}_{40}\right.$ precursor)

417-(448) $)_{3}$ (protonated): $\mathrm{Cr} \cdot 110 \cdot \mathrm{SmB} \cdot 196 \cdot \mathrm{I}\left({ }^{\circ} \mathrm{C}\right)\left(\mathrm{Na}_{3} \mathrm{PW}_{12} \mathrm{O}_{40}\right.$ precursor)

417-(448) $)_{3}$ (deprotonated): $\mathrm{Cr} \cdot 109 \cdot \mathrm{SmB} \cdot 188 \cdot \mathrm{I}\left({ }^{\circ} \mathrm{C}\right)\left(\mathrm{H}_{3} \mathrm{PW}_{12} \mathrm{O}_{40}\right.$ precursor)

417-(449-8) $: \mathrm{Cr}_{1} \cdot 128 \cdot \mathrm{Cr}_{2} \cdot 191 \cdot \mathrm{SmX} \cdot 208 \cdot \mathrm{SmB} \cdot 247 \cdot \mathrm{I}\left({ }^{\circ} \mathrm{C}\right)$

417-(450) $)_{3}: \mathrm{Cr} \cdot 87 \cdot \mathrm{SmB} \cdot 160 \cdot \mathrm{I}\left({ }^{\circ} \mathrm{C}\right)$

(phase behavior of 450: $\mathrm{Cr} \cdot 113 \cdot \mathrm{SmC} \cdot 143 \cdot \mathrm{SmA} \cdot 174 \cdot \mathrm{I}\left({ }^{\circ} \mathrm{C}\right)$ )

417-(450/454-b (1:1) $)_{3}\left(\mathrm{Z}=\mathrm{CH}, \mathrm{R}=\mathrm{OC}_{10} \mathrm{H}_{21}\right): \mathrm{Cr} \cdot 55 \cdot \mathrm{SmB} \cdot 127 \cdot \mathrm{I}\left({ }^{\circ} \mathrm{C}\right)$

418-(443-4) $)_{4}: \mathrm{Cr}_{1}$ (with micellar cubic structure) $\cdot 291 \cdot \mathrm{Cr}_{2} \cdot[$ not reported $] \cdot \mathrm{I}\left({ }^{\circ} \mathrm{C}\right)\left(T_{\text {dec. }}=340^{\circ} \mathrm{C}\right)$

418-(443-6) $)_{4}: \mathrm{Cr}_{1} \cdot 165 \cdot \mathrm{Cr}_{2}$ (with micellar cubic structure) $\cdot\left[\right.$ not reported] $\cdot \mathrm{I}\left({ }^{\circ} \mathrm{C}\right)\left(T_{\text {dec. }}=260{ }^{\circ} \mathrm{C}\right)$ 418-(443-8) $)_{4}: \mathrm{Cr}_{1} \cdot 62 \cdot \mathrm{Cr}_{2} \cdot 90 \cdot \mathrm{M}$ (with pseudo-lamellar structure) $\cdot 135 \cdot \mathrm{I}\left({ }^{\circ} \mathrm{C}\right)\left(T_{\text {dec. }}=220^{\circ} \mathrm{C}\right)$ 
418-(443-10) $)_{4}: \mathrm{Cr} \cdot(\mathrm{M}$ (with pseudo-lamellar structure) $\cdot 170 \cdot) 178 \cdot \mathrm{I}\left({ }^{\circ} \mathrm{C}\right)\left(T_{\text {dec. }}=220^{\circ} \mathrm{C}\right)$

$$
\text { 418-(446) })_{4}: \mathrm{Cr}_{1} \cdot-34 \cdot \mathrm{Cr}_{2} \cdot 55 \cdot \mathrm{I}\left({ }^{\circ} \mathrm{C}\right)
$$

418-(448) $)_{4}$ (protonated): $\mathrm{Cr}_{1} \cdot 109 \cdot \mathrm{Cr}_{2} \cdot 164 \cdot \mathrm{SmC} \cdot 197 \cdot \mathrm{SmA} \cdot 205 \cdot \mathrm{I}\left({ }^{\circ} \mathrm{C}\right)\left(\mathrm{H}_{4} \mathrm{SiW}_{12} \mathrm{O}_{40}\right.$ precursor $)$

418-(448) $)_{4}$ (deprotonated): $\mathrm{Cr}_{1} \cdot 109 \cdot \mathrm{Cr}_{2} \cdot 128 \cdot \mathrm{Cr}_{3} \cdot 169 \cdot \mathrm{SmB} \cdot 210 \cdot \mathrm{I}\left({ }^{\circ} \mathrm{C}\right)\left(\mathrm{K}_{4} \mathrm{SiW}_{12} \mathrm{O}_{40}\right.$ precursor $)$ 418-(450) $)_{4}: \mathrm{Cr}_{1} \cdot 75 \cdot \mathrm{Cr}_{2} \cdot 93 \cdot \mathrm{SmX} \cdot 142 \cdot \mathrm{I}\left({ }^{\circ} \mathrm{C}\right)$

418-(450/454-a (1:1) $)_{4}\left(\mathrm{Z}=\mathrm{CH}, \mathrm{R}=\mathrm{NO}_{2}\right): \mathrm{Cr}_{1} \cdot 54 \cdot \mathrm{Cr}_{2} \cdot 80 \cdot \mathrm{SmA} \cdot 104 \cdot \mathrm{I}\left({ }^{\circ} \mathrm{C}\right)$ 418-(450/454-b (1:1)) $)_{4}\left(\mathrm{Z}=\mathrm{CH}, \mathrm{R}=\mathrm{OC}_{10} \mathrm{H}_{21}\right): \mathrm{Cr} \cdot 120 \cdot \mathrm{SmB} \cdot 150 \cdot \mathrm{I}\left({ }^{\circ} \mathrm{C}\right)$

418-(450/454-c (1:1)) $)_{4}\left(\mathrm{Z}=\mathrm{N}, \mathrm{R}=\mathrm{OC}_{16} \mathrm{H}_{33}\right): \mathrm{Cr} \cdot 72 \cdot \mathrm{SmA} \cdot 123 \cdot \mathrm{I}\left({ }^{\circ} \mathrm{C}\right)$

418-(450/454-d (1:1) $)_{4}\left(\mathrm{Z}=\mathrm{N}, \mathrm{R}=3,4,5-\left(\mathrm{C}_{16} \mathrm{H}_{33} \mathrm{O}\right)_{3}\right.$-benzyloxy): $\mathrm{Cr}_{1} \cdot 47 \cdot \mathrm{Cr}_{2} \cdot 67 \cdot \mathrm{SmC} \cdot 152 \cdot \mathrm{I}\left({ }^{\circ} \mathrm{C}\right)$ 418-(451) 4 : $\mathrm{Cr} \cdot\left[\right.$ not reported] $\cdot \mathrm{SmX}$ or not LC (?) $\cdot\left[\right.$ not reported] $\cdot \mathrm{I}\left({ }^{\circ} \mathrm{C}\right)$

419-(443-8) $)_{4} \mathrm{Cr} \cdot 80 \cdot \mathrm{SmX} \cdot 115 \cdot \mathrm{I}\left({ }^{\circ} \mathrm{C}\right)$

420-(443-8) $)_{5}: \mathrm{g} \cdot 0 \cdot \mathrm{SmX} \cdot>150 \cdot \mathrm{I}\left({ }^{\circ} \mathrm{C}\right)\left(2^{\text {nd }}\right.$ DSC heating run $)$

420-(446) $)_{5}: \mathrm{Cr} \cdot-13 \cdot \mathrm{X} \cdot 164 \cdot \mathrm{SmB} \cdot 211 \cdot \mathrm{I}\left({ }^{\circ} \mathrm{C}\right)$

420-(449-8) $)_{5} \mathrm{Cr}_{1} \cdot 110 \cdot \mathrm{Cr}_{2} \cdot 130 \cdot \mathrm{SmC} \cdot 182 \cdot \mathrm{X} \cdot 196 \cdot \mathrm{SmA} \cdot 223 \cdot \mathrm{I}\left({ }^{\circ} \mathrm{C}\right)$

420-(451) $)_{5}$ : $\mathrm{Cr} \cdot\left[\right.$ not reported] $\cdot \mathrm{SmX}$ or not LC (?) · [not reported] $\cdot \mathrm{I}\left({ }^{\circ} \mathrm{C}\right)$

420-(452) $)_{5}: \mathrm{I} \cdot 129 \cdot \mathrm{N} \cdot 30 \cdot \operatorname{solid}\left({ }^{\circ} \mathrm{C}\right)\left(1^{\text {st }} \mathrm{DSC}\right.$ cooling run)

421-(399-18) $)_{5} \cdot 28 \mathrm{H}_{2} \mathrm{O}: \mathrm{Cr}_{1} \cdot 22 \cdot \mathrm{Cr}_{2} \cdot 29 \cdot \mathrm{Cr}_{3} \cdot 36 \cdot \mathrm{SmA} \cdot>143 \cdot \mathrm{I}\left({ }^{\circ} \mathrm{C}\right)$

422-(452) $)_{6}: \mathrm{I} \cdot 153 \cdot \mathrm{N} \cdot 28 \cdot \operatorname{solid}\left({ }^{\circ} \mathrm{C}\right)\left(1^{\text {st }} \mathrm{DSC}\right.$ cooling run)

423-(399-18) ${ }_{6} \cdot 16 \mathrm{H}_{2} \mathrm{O}: \mathrm{Cr}_{1} \cdot 31 \cdot \mathrm{Cr}_{2} \cdot 49 \cdot \mathrm{SmA} \cdot 148 \cdot \mathrm{I}\left({ }^{\circ} \mathrm{C}\right)$

424-(399-18) $\cdot 16 \mathrm{H}_{2} \mathrm{O}: \mathrm{Cr}_{1} \cdot 32 \cdot \mathrm{Cr}_{2} \cdot 49 \cdot \mathrm{SmA} \cdot 102 \cdot \mathrm{I}\left({ }^{\circ} \mathrm{C}\right)$

425-(451) $)_{7}: \mathrm{Cr} \cdot\left[\right.$ not reported] $\cdot \mathrm{Col}_{\mathrm{h}}$ or not LC (?) $\cdot\left[\right.$ not reported] $\cdot \mathrm{I}\left({ }^{\circ} \mathrm{C}\right)$

426-(399-18) $\cdot 9 \mathrm{H}_{2} \mathrm{O}: \mathrm{Cr} \cdot 50 \cdot \mathrm{SmX} \cdot 137 \cdot \mathrm{I}\left({ }^{\circ} \mathrm{C}\right)$

427-(399-18) $)_{8} \cdot 8 \mathrm{H}_{2} \mathrm{O}: \mathrm{Cr} \cdot 26 \cdot \mathrm{SmA} \cdot 142 \cdot \mathrm{I}\left({ }^{\circ} \mathrm{C}\right)$

428-(448) $)_{9}: \mathrm{Cr} \cdot 88 / 91 \cdot \mathrm{SmX}_{1} \cdot 114 \cdot \mathrm{SmX}_{2} \cdot 164 \cdot \mathrm{I}\left({ }^{\circ} \mathrm{C}\right)$

428-(450) $)_{9}: \mathrm{Cr} \cdot 137 \cdot \mathrm{SmA} \cdot 160 \cdot \mathrm{I}\left({ }^{\circ} \mathrm{C}\right)$

429-(451) $)_{10}: \mathrm{Cr} \cdot$ [not reported] $\cdot \mathrm{Col}_{\mathrm{h}}$ or not LC (?) $\cdot\left[\right.$ not reported] $\cdot \mathrm{I}\left({ }^{\circ} \mathrm{C}\right)$

430-(452) $)_{10}: \mathrm{I} \cdot 168 \cdot \mathrm{N} \cdot 29 \cdot \operatorname{solid}\left({ }^{\circ} \mathrm{C}\right)\left(1^{\text {st }}\right.$ DSC cooling run $)$

431-(448) $)_{11}: \mathrm{Cr} \cdot 87 / 91 \cdot \mathrm{SmX}_{1} \cdot 115 \cdot \mathrm{SmX}_{2} \cdot 177 \cdot \mathrm{I}\left({ }^{\circ} \mathrm{C}\right)$

431-(450) $)_{11}: \mathrm{Cr} \cdot 117 \cdot \mathrm{SmA} \cdot 155 \cdot \mathrm{I}\left({ }^{\circ} \mathrm{C}\right)$

432-(399-18) $)_{12} \cdot 16 \mathrm{H}_{2} \mathrm{O}: \mathrm{Cr}_{1} \cdot 49 \cdot \mathrm{Cr}_{2} \cdot 61 \cdot \mathrm{SmX}_{1} \cdot 75 \cdot \mathrm{SmX}_{2} \cdot 82 \cdot \mathrm{SmX}_{3}(=\mathrm{SmA}) \cdot 116 \cdot \mathrm{I}\left({ }^{\circ} \mathrm{C}\right)$

433-(399-12) $)_{12}: \mathrm{Cr} \cdot-61 \cdot \mathrm{SmX} \cdot 220 \cdot \operatorname{dec}\left({ }^{\circ} \mathrm{C}\right)$

433-(399-14) $)_{12}: \mathrm{Cr} \cdot-35 \cdot \mathrm{SmX} \cdot 220 \cdot \operatorname{dec}\left({ }^{\circ} \mathrm{C}\right)$

433-(399-16) $)_{12}: \mathrm{Cr} \cdot-8 \cdot \mathrm{SmX} \cdot 220 \cdot \operatorname{dec}\left({ }^{\circ} \mathrm{C}\right)$

433-(399-18) $)_{12}: \mathrm{Cr} \cdot 22 \cdot \mathrm{SmX} \cdot 220 \cdot \operatorname{dec}\left({ }^{\circ} \mathrm{C}\right)$

433-(401-16) $)_{12}: \mathrm{Cr} \cdot-25 \cdot \mathrm{SmX} \cdot 220 \cdot \operatorname{dec}\left({ }^{\circ} \mathrm{C}\right)$

433-(401-18) $)_{12}: \mathrm{Cr} \cdot 22 \cdot \mathrm{SmX} \cdot 220 \cdot \operatorname{dec}\left({ }^{\circ} \mathrm{C}\right)$

433-(442) $)_{12}: \mathrm{Cr} \cdot 28 \cdot \mathrm{X} \cdot 77 \cdot \mathrm{SmX} \cdot 220 \cdot \operatorname{dec}\left({ }^{\circ} \mathrm{C}\right)$

433-(443-4) $12: \mathrm{X} \cdot 200 \cdot \operatorname{dec}\left({ }^{\circ} \mathrm{C}\right)$ 
433-(444) $)_{12}: \mathrm{Cr} \cdot-27 \cdot \mathrm{X} \cdot 200 \cdot \operatorname{dec}\left({ }^{\circ} \mathrm{C}\right)$

433-(447-11) $)_{12}$ : hexagonally ordered mesophase $\cdot 130 \cdot \operatorname{dec}\left({ }^{\circ} \mathrm{C}\right)$

433-(447-15) $)_{12}$ : hexagonally ordered mesophase $\cdot 130 \cdot \operatorname{dec}\left({ }^{\circ} \mathrm{C}\right)$

434-(399-12) $)_{13}: \operatorname{SmX}\left(\right.$ probably glassy state) $\cdot 220 \cdot \operatorname{dec}\left({ }^{\circ} \mathrm{C}\right)$

434-(399-16) $)_{13}: \mathrm{Cr} \cdot 15 \cdot \mathrm{SmX}$ (probably glassy state) $\cdot 220 \cdot \operatorname{dec}\left({ }^{\circ} \mathrm{C}\right)$

434-(401-12) $)_{13}$ : hexagonally ordered phase (probably glassy state) $\cdot 180 \cdot \operatorname{dec}\left({ }^{\circ} \mathrm{C}\right)$

434-(401-16) $)_{13}$ : phase of cubic symmetry (probably glassy state) $\cdot 180 \cdot \operatorname{dec}\left({ }^{\circ} \mathrm{C}\right)$

434-(444) $)_{13}: \operatorname{SmX}$ (probably glassy state) $\cdot 180 \cdot \operatorname{dec}\left({ }^{\circ} \mathrm{C}\right)$

434-(447-11) $)_{13}$ : X (probably glassy state) $\cdot 130 \cdot \operatorname{dec}\left({ }^{\circ} \mathrm{C}\right)$

434-(447-15) $)_{13}$ : X (probably glassy state) $\cdot 130 \cdot \operatorname{dec}\left({ }^{\circ} \mathrm{C}\right)$

435-(450) $)_{13}: \mathrm{Cr} \cdot 105 \cdot \mathrm{SmC} \cdot 126 \cdot \mathrm{SmA} \cdot 153 \cdot \mathrm{I}\left({ }^{\circ} \mathrm{C}\right)$

436-(448) $)_{13}: \mathrm{Cr} \cdot 88 / 91 \cdot \mathrm{SmX}_{1} \cdot 114 \cdot \mathrm{SmX}_{2} \cdot 185 \cdot \mathrm{I}\left({ }^{\circ} \mathrm{C}\right)$

437-(449-6) $)_{15}: \mathrm{Cr}_{1} \cdot 101 \cdot \mathrm{Cr}_{2} \cdot 131 \cdot \mathrm{SmC} \cdot 182 \cdot \mathrm{SmA} \cdot 215 \cdot \mathrm{I}\left({ }^{\circ} \mathrm{C}\right)$

437-(449-8) $)_{15}: \mathrm{Cr}_{1} \cdot 66 \cdot \mathrm{Cr}_{2} \cdot 119 \cdot \mathrm{SmC} \cdot 184 \cdot \mathrm{SmA} \cdot 216 \cdot \mathrm{I}\left({ }^{\circ} \mathrm{C}\right)$

437-(449-10) $)_{15}: \mathrm{Cr}_{1} \cdot 78 \cdot \mathrm{Cr}_{2} \cdot 112 \cdot \mathrm{SmC} \cdot 182 \cdot \mathrm{SmA} \cdot 217 \cdot \mathrm{I}\left({ }^{\circ} \mathrm{C}\right)$

437-(449-12) $)_{15}: \mathrm{Cr}_{1} \cdot 74 \cdot \mathrm{Cr}_{2} \cdot 106 \cdot \mathrm{SmC} \cdot 182 \cdot \mathrm{SmA} \cdot 217 \cdot \mathrm{I}\left({ }^{\circ} \mathrm{C}\right)$

438-(399-18) $)_{16} \cdot 36 \mathrm{H}_{2} \mathrm{O}: \mathrm{Cr}_{1} \cdot 30 \cdot \mathrm{Cr}_{2} \cdot 55 \cdot \mathrm{SmX} \cdot 75 \cdot \mathrm{SmA} \cdot 125 \cdot \mathrm{I}\left({ }^{\circ} \mathrm{C}\right)$

439-(399-18) ${ }_{19} \mathbf{K}_{2} \mathbf{N a}_{2} \mathrm{Li}_{5} \mathbf{H}_{8} \cdot 10 \mathrm{H}_{2} \mathrm{O}: \mathrm{g} \cdot 20 \cdot \mathrm{SmA} \cdot>200 \cdot$ dec. $\left({ }^{\circ} \mathrm{C}\right)$

439-(445-12) ${ }_{25} \mathrm{~K}_{2} \mathrm{NaLi}_{4} \mathbf{H}_{4} \cdot 20 \mathrm{H}_{2} \mathrm{O}: \mathrm{g} \cdot\left[\right.$ [not reported] $\cdot \mathrm{SmA}$ (highly ordered) $\cdot>200 \cdot$ dec. $\left({ }^{\circ} \mathrm{C}\right.$ )

439-(445-14) ${ }_{26} \mathbf{K}_{2} \mathbf{N a}_{2} \mathbf{L i}_{3} \mathrm{H}_{3} \cdot 35 \mathrm{H}_{2} \mathrm{O}: \mathrm{g} \cdot-3 \cdot \mathrm{SmA}$ (highly ordered) $\cdot>200 \cdot \operatorname{dec}\left({ }^{\circ} \mathrm{C}\right)$

439-(445-16) ${ }_{23} \mathrm{~K}_{2} \mathrm{Na}_{3} \mathrm{Li}_{3} \mathrm{H}_{5} \cdot 40 \mathrm{H}_{2} \mathrm{O}: \mathrm{g} \cdot-4 \cdot \mathrm{SmA}$ (highly ordered) $\cdot>200 \cdot \operatorname{dec}\left({ }^{\circ} \mathrm{C}\right)$

439-(445-18) ${ }_{24} \mathbf{K}_{2} \mathbf{N a L i}_{3} \mathbf{H}_{6} \cdot 25 \mathrm{H}_{2} \mathrm{O}: \mathrm{g} \cdot 38 \cdot \mathrm{SmA}$ (highly ordered) $\cdot>200 \cdot$ dec. $\left({ }^{\circ} \mathrm{C}\right.$ )

439-(445-20) ${ }_{24} \mathbf{K}_{2} \mathbf{N a L i}_{3} \mathbf{H}_{6} \cdot 15 \mathrm{H}_{2} \mathrm{O}: \mathrm{g} \cdot 46 \cdot \mathrm{SmA}$ (highly ordered) $\cdot>200 \cdot$ dec. $\left({ }^{\circ} \mathrm{C}\right)$

440-(399-18) ${ }_{36}\left(\mathbf{N H}_{4}\right)_{6} \cdot 75 \mathrm{H}_{2} \mathrm{O}: \mathrm{g} \cdot 9 \cdot \mathrm{SmX} \cdot>220 \cdot$ dec. $\left({ }^{\circ} \mathrm{C}\right)$

(441-23)-(399-18) ${ }_{44}\left(\mathbf{N H}_{4}\right)_{14}: \mathrm{g} \cdot 9 \cdot \mathrm{SmX}($ SmA-like $) \cdot>220 \cdot$ dec. $\left({ }^{\circ} \mathrm{C}\right)$

(441-30)-(399-18) $)_{\mathbf{5 6}}\left(\mathbf{N H}_{4}\right)_{\mathbf{1 6}} \cdot 15 \mathrm{H}_{2} \mathrm{O}: \mathrm{g} \cdot \mathbf{9} \cdot \mathrm{SmX}(\mathrm{SmA}-\mathrm{like}) \cdot>220 \cdot$ dec. $\left({ }^{\circ} \mathrm{C}\right)$ 

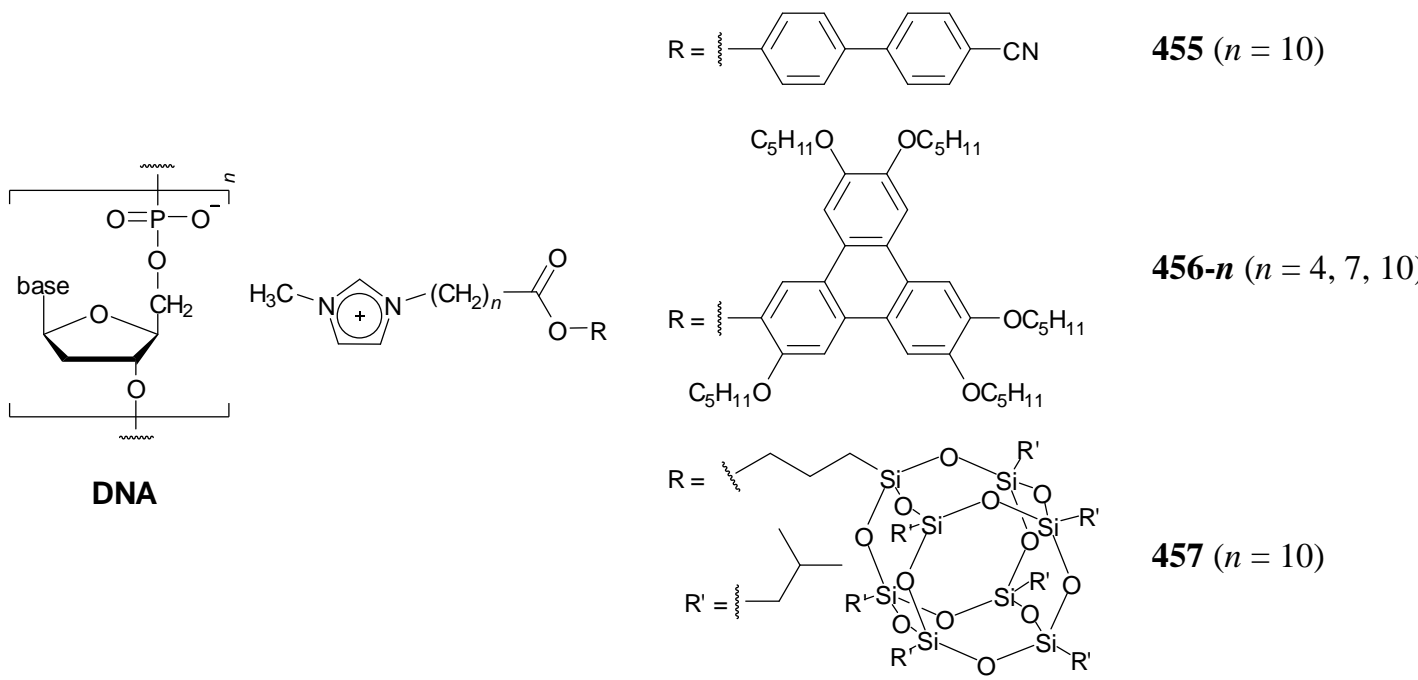

455: $\mathrm{g} \cdot 53 \cdot \mathrm{SmA}$ lamello-columnar phase $\left(\mathrm{L}_{\alpha}{ }^{\mathrm{C}}\right)($ see text $) \cdot$ ca. $200 \cdot$ dec. $\left({ }^{\circ} \mathrm{C}\right)$

456-4: $\mathrm{g} \cdot 8 \cdot \mathrm{Col}_{\mathrm{ob}}($ see text $) \cdot \mathrm{ca} .200 \cdot$ dec. $\left({ }^{\circ} \mathrm{C}\right)$

456-7: $\mathrm{g} \cdot 37 \cdot \mathrm{Col}_{\mathrm{L}}\left(\mathrm{L}_{\alpha}{ }^{\mathrm{C}}\right)$ (see text) $\cdot \mathrm{ca} .200 \cdot$ dec. $\left({ }^{\circ} \mathrm{C}\right)$

456-10: $\mathrm{g} \cdot 36 \cdot \mathrm{Col}_{\mathrm{L}}\left(\mathrm{L}_{\alpha}{ }^{\mathrm{C}}\right)$ (see text) $\cdot \mathrm{ca} .200 \cdot$ dec. $\left({ }^{\circ} \mathrm{C}\right)$

457: $\mathrm{Cr} \cdot 162$ (melting of POSS crystals) $\cdot \mathrm{Col}_{\mathrm{h}}\left(\mathrm{H}_{\mathrm{II}}{ }^{\mathrm{C}}\right)$ (see text) $\cdot$ ca. $200 \cdot \operatorname{dec} .\left({ }^{\circ} \mathrm{C}\right.$ )

See ref. 127 for the phase behavior of thermotropic ionic LC complexes of genetically engineered, supercharged elastin-like polypeptides (ELPs, with 9, 18, 36, 72 or 144 negative charges) with $N, N$-bis(n-alkyl)- $N, N$ dimethylammonium cations. 


\subsection{Structures mentioned in section about applications of ionic liquid crystals}

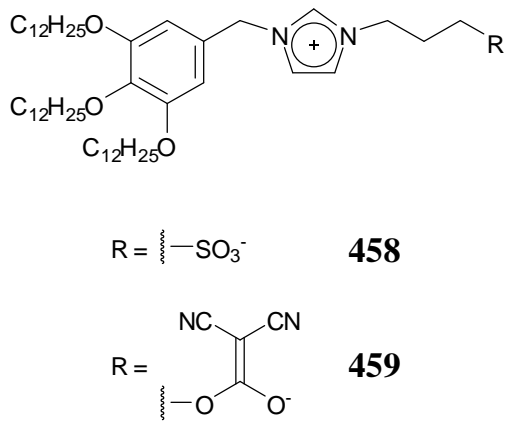

458: $\mathrm{Col}_{\mathrm{h}} \cdot 207 \cdot$ dec. $\left({ }^{\circ} \mathrm{C}\right)$

458/LiNTf 2 (90:10): $\mathrm{Col}_{\mathrm{h}} \cdot 200 \cdot$ dec. $\left({ }^{\circ} \mathrm{C}\right)$

458/LiNTf 2 (80:20): $\mathrm{Col}_{\mathrm{h}} \cdot 200 \cdot$ dec. $\left({ }^{\circ} \mathrm{C}\right)$

458/LiNTf 2 (70:30): $\mathrm{Col}_{\mathrm{h}} \cdot 200 \cdot$ dec. $\left({ }^{\circ} \mathrm{C}\right)$

458/LiNTf 2 (60:40): $\mathrm{Col}_{\mathrm{h}} \cdot 200 \cdot$ dec. $\left({ }^{\circ} \mathrm{C}\right)$

458/LiNTf 2 (50:50): $\mathrm{Col}_{\mathrm{h}} \cdot 200 \cdot$ dec. $\left({ }^{\circ} \mathrm{C}\right)$

458/LiNTf 2 (80:20) +5 wt.\% * propylene carbonate: $\operatorname{Col}_{\mathrm{h}}$ (microscopic observation until $120{ }^{\circ} \mathrm{C}$; dec. at ca. $200{ }^{\circ} \mathrm{C}$ )

458/LiNTf 2 (80:20) + 10 wt.\% * propylene carbonate: $\mathrm{Col}_{\mathrm{h}}$ (microscopic observation until $12{ }^{\circ} \mathrm{C}$; dec. at ca.

$\left.200{ }^{\circ} \mathrm{C}\right)$

458/LiNTf 2 (50:50) +5 wt.\% * propylene carbonate: $\operatorname{Col}_{h}$ (microscopic observation until $120{ }^{\circ} \mathrm{C}$; dec. at ca. $200{ }^{\circ} \mathrm{C}$ )

458/LiNTf $(50: 50)+10$ wt.\% * propylene carbonate: Col $_{\mathrm{h}}$ (microscopic observation until $120{ }^{\circ} \mathrm{C}$; dec. at ca.

$\left.200{ }^{\circ} \mathrm{C}\right)$

458/LiNTf $2+\geq 15$ wt. $\% *$ propylene carbonate: biphasic

* with respect to the mass of the zwitterion

459: $\mathrm{Col}_{\mathrm{h}} \cdot 217 \cdot$ dec. $\left({ }^{\circ} \mathrm{C}\right)$

459/LiNTf $(90: 10): \mathrm{Col}_{\mathrm{h}} \cdot 190 \cdot$ dec. $\left({ }^{\circ} \mathrm{C}\right)$

459/LiNTf 2 (80:20): $\mathrm{Col}_{\mathrm{h}} \cdot 190 \cdot$ dec. $\left({ }^{\circ} \mathrm{C}\right)$

459/LiNTf 2 (70:30): $\mathrm{Col}_{\mathrm{h}} \cdot 190 \cdot \operatorname{dec} .\left({ }^{\circ} \mathrm{C}\right)$

459/LiNTf 2 (60:40): $\mathrm{Col}_{\mathrm{h}} \cdot 190 \cdot \operatorname{dec} .\left({ }^{\circ} \mathrm{C}\right)$

459/LiNTf 2 (50:50): $\operatorname{Cub}_{\mathrm{V}}(\mathrm{Ia} \overline{3} d) \cdot 65-88 \cdot \mathrm{Col}_{\mathrm{h}} \cdot 190 \cdot$ dec. $\left({ }^{\circ} \mathrm{C}\right)$

459/LiNTf $2(80: 20)+5$ wt.\% * propylene carbonate: $\mathrm{Cub}_{\mathrm{V}}(\mathrm{Ia} \overline{3} d) \cdot 50-68 \cdot \mathrm{Col}_{\mathrm{h}} \cdot 120\left({ }^{\circ} \mathrm{C}\right)$ (microscopic observation until $120^{\circ} \mathrm{C}$; dec. at ca. $200^{\circ} \mathrm{C}$ )

459/ $\operatorname{LiNTf}_{2}(80: 20)+10$ wt.\% * propylene carbonate: $\mathrm{Cub}_{\mathrm{V}}(\mathrm{Ia} \overline{3} d) \cdot 50-75 \cdot \mathrm{Col}_{\mathrm{h}} \cdot 120\left({ }^{\circ} \mathrm{C}\right)$ (microscopic observation until $120^{\circ} \mathrm{C}$; dec. at ca. $200^{\circ} \mathrm{C}$ )

459/LiNTf $2(50: 50)+5$ wt.\% * propylene carbonate: $\mathrm{Cub}_{\mathrm{V}}(\operatorname{Ia} \overline{3} d) \cdot 81-92 \cdot \mathrm{I}\left({ }^{\circ} \mathrm{C}\right)$

459/LiNTf $2(50: 50)+10$ wt.\% * propylene carbonate: $\mathrm{Cub}_{\mathrm{V}}(\operatorname{Ia} \overline{3} d) \cdot 74-86 \cdot \mathrm{I}\left({ }^{\circ} \mathrm{C}\right)$ 
459/LiNTf $2+\geq 15$ wt.\% $\%$ propylene carbonate: biphasic

* with respect to the mass of the zwitterion

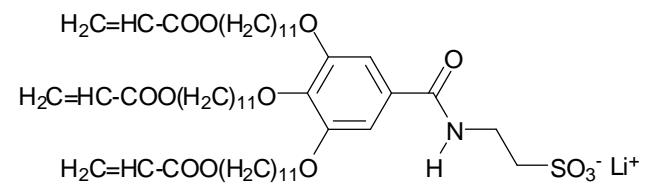

460

460 + 5-25 wt.\% propylene carbonate: $\mathrm{Cub}_{\mathrm{V}}(\operatorname{Ia} \overline{3} d / P n \overline{3} m)$ at r.t.

$460+5-20$ wt. $\% \mathrm{LiClO}_{4}(0.245 \mathrm{M})$ in propylene carbonate: $\mathrm{Cub}_{\mathrm{V}}(\mathrm{Ia} \overline{3} d / \mathrm{Pn} \overline{3} m)$ at r.t.

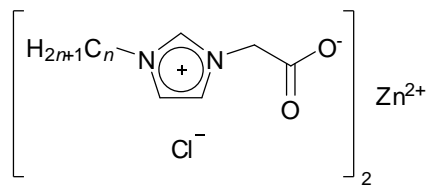

461-n

461-1: $\mathrm{Cr} \cdot 110 \cdot \mathrm{I}\left({ }^{\circ} \mathrm{C}\right)$

461-16: $\mathrm{Cr} \cdot 113 \cdot \mathrm{SmX}($ ?) $)$ ca. $250 \cdot \operatorname{dec} .\left({ }^{\circ} \mathrm{C}\right)$ 


\section{References}

(1) Atkins, P.; Jones, L. Chemical Principles: The Quest for Insight; 2nd ed.; W. H. Freeman \& Co.: New York, 2002.

(2) Atkins, P.; de Paula, J. Atkins' Physical Chemistry; 7th ed.; Oxford University Press: Oxford, 2002.

(3) Israelachvili, J. N. Intermolecular and Surface Forces; 3rd ed.; Academic Press: London, 2010.

(4) Hunter, C. A. Quantifying Intermolecular Interactions: Guidelines for the Molecular Recognition Toolbox. Angew. Chem., Int. Ed. 2004, 43, 5310-5324.

(5) Meot-Ner, M. The Ionic Hydrogen Bond. Chem. Rev. 2005, 105, 213-284.

(6) Hosseini, M. W. Molecular Tectonics: From Simple Tectons to Complex Molecular Networks. Acc. Chem. Res. 2005, 38, 313-323.

Meot-Ner (Mautner), M. Update 1 of: Strong Ionic Hydrogen Bonds. Chem. Rev. 2012, 112, PR22-PR103.

(8) Steiner, T. The Hydrogen Bond in the Solid State. Angew. Chem., Int. Ed. 2002, 41, 48-76.

(9) Steiner, T. Unrolling the Hydrogen Bond Properties of C-H $\cdots$ O Interactions. Chem. Commun. 1997, 727734.

(10) Skarmoutsos, I.; Dellis, D.; Matthews, R. P.; Welton, T.; Hunt, P. A. Hydrogen Bonding in 1-Butyl- and 1Ethyl-3-Methylimidazolium Chloride Ionic Liquids. J. Phys. Chem. B 2012, 116, 4921-4933.

(11) Lee, C. H.; Su, F. Y.; Lin, Y. H.; Chou, C. H.; Lee, K. M. Anion-Controlled Assemblies of C-H $\cdots O$ Hydrogen Bonded Grid, Stair or Bilayer Structures by L-Shaped Pyridinium Salts. CrystEngComm 2011, 13, 2318-2323.

(12) Lee, K. M.; Chen, J. C.; Huang, C. J.; Lin, I. J. B. Anion-Controlled Assemble of C-H $\cdots$ X Hydrogen Bonded Helical Tubes or Catemers by Crescent Imidazolium Salts. CrystEngComm 2009, 11, 2804-2809.

(13) Lee, K. M.; Lee, Y. T.; Lin, I. J. B. Supramolecular Liquid Crystals of Amide Functionalized Imidazolium Salts. J. Mater. Chem. 2003, 13, 1079-1084.

(14) Kuduva, S. S.; Craig, D. C.; Nangia, A.; Desiraju, G. R. Cubanecarboxylic Acids. Crystal Engineering Considerations and the Role of C- $\mathrm{H}^{\cdots} \mathrm{O}$ Hydrogen Bonds in Determining O-H $\cdots \mathrm{O}$ Networks. J. Am. Chem. Soc. 1999, 121, 1936-1944.

(15) Kölle, P.; Dronskowski, R. Hydrogen Bonding in the Crystal Structures of the Ionic Liquid Compounds Butyldimethylimidazolium Hydrogen Sulfate, Chloride, and Chloroferrate(II,III). Inorg. Chem. 2004, 43, 2803-2809.

(16) Kempter, V.; Kirchner, B. The Role of Hydrogen Atoms in Interactions Involving Imidazolium-Based Ionic Liquids. J. Mol. Struct. 2010, 972, 22-34.

(17) Holbrey, J. D.; Reichert, W. M.; Rogers, R. D. Crystal Structures of Imidazolium Bis(Trifluoromethanesulfonyl)Imide 'Ionic Liquid' Salts: The First Organic Salt With a Cis-TFSI Anion Conformation. Dalton Trans. 2004, 2267-2271. 
(18) Elaiwi, A.; Hitchcock, P. B.; Seddon, K. R.; Srinivasan, N.; Tan, Y. M.; Welton, T.; Zora, J. A. HydrogenBonding in Imidazolium Salts and Its Implications for Ambient-Temperature Halogenoaluminate(III) Ionic Liquids. J. Chem. Soc., Dalton Trans. 1995, 3467-3472.

(19) Dymek, C. J.; Grossie, D. A.; Fratini, A. V.; Adams, W. W. Evidence for the Presence of Hydrogen-Bonded Ion-Ion Interactions in the Molten-Salt Precursor, 1-Methyl-3-Ethylimidazolium Chloride. J. Mol. Struct. 1989, 213, 25-34.

(20) Desiraju, G. R. C-H $\cdots \mathrm{O}$ and Other Weak Hydrogen Bonds. From Crystal Engineering to Virtual Screening. Chem. Commun. 2005, 2995-3001.

(21) Desiraju, G. R. Hydrogen Bridges in Crystal Engineering: Interactions Without Borders. Acc. Chem. Res. 2002, 35, 565-573.

(22) Desiraju, G. R. The C-H $\cdots$ O Hydrogen Bond: Structural Implications and Supramolecular Design. Acc. Chem. Res. 1996, 29, 441-449.

(23) Chang, H. C.; Lee, K. M.; Jiang, J. C.; Lin, M. S.; Chen, J. S.; Lin, I. J. B.; Lin, S. H. Charge-Enhanced C$\mathrm{H} \cdots \mathrm{O}$ Interactions of a Self-Assembled Triple Helical Spine Probed by High-Pressure. J. Chem. Phys. 2002, 117, 1723-1728.

(24) Abdul-Sada, A. K.; Al-Juaid, S.; Greenway, A. M.; Hitchcock, P. B.; Howells, M. J.; Seddon, K. R.; Welton, T. Upon the Hydrogen-Bonding Ability of the H4 and H5 Protons of the Imidazolium Cation. Struct. Chem. 1990, 1, 391-394.

(25) Abdul-Sada, A. K.; Greenway, A. M.; Hitchcock, P. B.; Mohammed, T. J.; Seddon, K. R.; Zora, J. A. Upon the Structure of Room-Temperature Halogenoaluminate Ionic Liquids. J. Chem. Soc., Chem. Commun. 1986, 1753-1754.

(26) Desiraju, G. R.; Steiner, T. The Weak Hydrogen Bond in Structural Chemistry and Biology; Oxford University Press: Oxford, 1999.

(27) Dupont, J. On the Solid, Liquid and Solution Structural Organization of Imidazolium Ionic Liquids. J. Braz. Chem. Soc. 2004, 15, 341-350.

(28) Fumino, K.; Wulf, A.; Ludwig, R. Strong, Localized, and Directional Hydrogen Bonds Fluidize Ionic Liquids. Angew. Chem., Int. Ed. 2008, 47, 8731-8734.

(29) Fumino, K.; Wulf, A.; Ludwig, R. The Potential Role of Hydrogen Bonding in Aprotic and Protic Ionic Liquids. Phys. Chem. Chem. Phys. 2009, 11, 8790-8794.

Roth, C.; Peppel, T.; Fumino, K.; Koeckerling, M.; Ludwig, R. The Importance of Hydrogen Bonds for the Structure of Ionic Liquids: Single-Crystal X-Ray Diffraction and Transmission and Attenuated Total Reflection Spectroscopy in the Terahertz Region. Angew. Chem., Int. Ed. 2010, 49, 10221-10224.

(31) Wulf, A.; Fumino, K.; Ludwig, R. Spectroscopic Evidence for an Enhanced Anion-Cation Interaction From Hydrogen Bonding in Pure Imidazolium Ionic Liquids. Angew. Chem., Int. Ed. 2010, 49, 449-453.

(32) Fumino, K.; Peppel, T.; Geppert-Rybczynska, M.; Zaitsau, D. H.; Lehmann, J. K.; Verevkin, S. P.; Koeckerling, M.; Ludwig, R. The Influence of Hydrogen Bonding on the Physical Properties of Ionic Liquids. Phys. Chem. Chem. Phys. 2011, 13, 14064-14075. 
(33) Peppel, T.; Roth, C.; Fumino, K.; Paschek, D.; Koeckerling, M.; Ludwig, R. The Influence of HydrogenBond Defects on the Properties of Ionic Liquids. Angew. Chem., Int. Ed. 2011, 50, 6661-6665.

(34) Avent, A. G.; Chaloner, P. A.; Day, M. P.; Seddon, K. R.; Welton, T. Evidence for Hydrogen-Bonding in Solutions of 1-Ethyl-3-Methylimidazolium Halides, and Its Implications for Room-Temperature Halogenoaluminate(III) Ionic Liquids. J. Chem. Soc., Dalton Trans. 1994, 3405-3413.

(35) Steiner, T. Hydrogen-Bond Distances to Halide Ions in Organic and Organometallic Crystal Structures: UpTo-Date Database Study. Acta Cryst. B 1998, 54, 456-463.

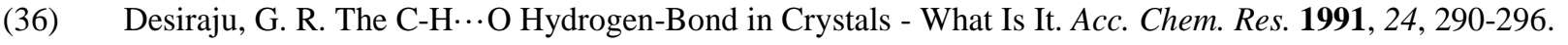

(37) Steiner, T.; Desiraju, G. R. Distinction Between the Weak Hydrogen Bond and the Van Der Waals Interaction. Chem. Commun. 1998, 891-892.

(38) Mele, A.; Tran, C. D.; Lacerda, S. H. D. The Structure of a Room-Temperature Ionic Liquid With and Without Trace Amounts of Water: The Role of $\mathrm{C}-\mathrm{H} \cdots \mathrm{O}$ and $\mathrm{C}-\mathrm{H} \cdots \mathrm{F}$ Interactions in 1-n-Butyl-3Methylimidazolium Tetrafluoroborate. Angew. Chem., Int. Ed. 2003, 42, 4364-4366.

(39) Suarez, P. A. Z.; Einloft, S.; Dullius, J. E. L.; De Souza, R. F.; Dupont, J. Synthesis and Physical-Chemical Properties of Ionic Liquids Based on 1-n-Butyl-3-Methylimidazolium Cation. J. Chim. Phys. 1998, 95, 1626-1639.

(40) Getsis, A.; Mudring, A. V. Imidazolium Based Ionic Liquid Crystals: Structure, Photophysical and Thermal Behaviour of $\left[\mathrm{C}_{n} \operatorname{mim}\right] \mathrm{Br} \cdot x \mathrm{H}_{2} \mathrm{O}(n=12,14 ; x=0,1)$. Cryst. Res. Technol. 2008, 43, 1187-1196.

(41) Yang, M.; Mallick, B.; Mudring, A. V. On the Mesophase Formation of 1,3-Dialkylimidazolium Ionic Liquids. Cryst. Growth Des. 2013, 13, 3068-3077.

(44) Wang, M.; Pan, X.; Dai, S.; Chen, J. Influence of Intermolecular Interactions on the Mesogenic Properties of Imidazolium Salts. Acta Phys. Chim. Sin. 2015, 31, 653-659.

(45) Yang, M.; Mallick, B.; Mudring, A. V. A Systematic Study on the Mesomorphic Behavior of Asymmetrical 1-Alkyl-3-Dodecylimidazolium Bromides. Cryst. Growth Des. 2014, 14, 1561-1571.

(46) Bradley, A. E.; Hardacre, C.; Holbrey, J. D.; Johnston, S.; Mcmath, S. E. J.; Nieuwenhuyzen, M. SmallAngle X-Ray Scattering Studies of Liquid Crystalline 1-Alkyl-3-Methylimidazolium Salts. Chem. Mater. 2002, 14, 629-635.

(47) Yang, M.; Campbell, P. S.; Santini, C. C.; Mudring, A. V. Small Nickel Nanoparticle Arrays From Long Chain Imidazolium Ionic Liquids. Nanoscale 2014, 6, 3367-3375.

(48) Li, T.; Xu, F.; Shi, W. Ionic Liquid Crystals Based on 1-Alkyl-3-Methylimidazolium Cations and Perfluorinated Sulfonylimide Anions. Chem. Phys. Lett. 2015, 628, 9-15.

(49) Yang, J.; Li, F. F.; Zhang, J. A.; Li, J.; Wang, W. X. Hydroformylation of Oct-1-Ene in Novel Ionic Liquid Crystals. Helv. Chim. Acta 2010, 93, 1653-1660. 
(50) Godinho, M.; Cruz, C.; Teixeira, P., I; Ferreira, A.; Costa, C.; Kulkarni, P.; Afonso, C. Shear-Induced Lamellar Phase of an Ionic Liquid Crystal at Room Temperature. Liq. Cryst. 2008, 35, 103-107.

(51) Luo, S. C.; Sun, S.; Deorukhkar, A. R.; Lu, J. T.; Bhattacharyya, A.; Lin, I. J. B. Ionic Liquids and Ionic Liquid Crystals of Vinyl Functionalized Imidazolium Salts. J. Mater. Chem. 2011, 21, 1866-1873.

(52) Campbell, P. S.; Yang, M.; Pitz, D.; Cybinska, J.; Mudring, A. V. Highly Luminescent and Color-Tunable Salicylate Ionic Liquids. Chem. Eur. J. 2014, 20, 4704-4712.

(53) Chiou, J. Y. Z.; Chen, J. N.; Lei, J. S.; Lin, I. J. B. Ionic Liquid Crystals of Imidazolium Salts With a Pendant Hydroxyl Group. J. Mater. Chem. 2006, 16, 2972-2977.

(54) Wang, X. J.; Sternberg, M.; Kohler, F. T. U.; Melcher, B. U.; Wasserscheid, P.; Meyer, K. Long-AlkylChain-Derivatized Imidazolium Salts and Ionic Liquid Crystals With Tailor-Made Properties. RSC Adv. 2014, 4, 12476-12481.

(55) Wang, X.; Vogel, C. S.; Heinemann, F. W.; Wasserscheid, P.; Meyer, K. Solid-State Structures of DoubleLong-Chain Imidazolium Ionic Liquids: Influence of Anion Shape on Cation Geometry and Crystal Packing. Cryst. Growth Des. 2011, 11, 1974-1988.

(56) Kato, T.; Mizoshita, N.; Kishimoto, K. Functional Liquid-Crystalline Assemblies: Self-Organized Soft Materials. Angew. Chem., Int. Ed. 2006, 45, 38-68.

(57) Cheng, X. H.; Bai, X. Q.; Jing, S.; Ebert, H.; Prehm, M.; Tschierske, C. Self-Assembly of ImidazoliumBased Rodlike Ionic Liquid Crystals: Transition From Lamellar to Micellar Organization. Chem. Eur. J. 2010, 16, 4588-4601.

(58) Ichikawa, T.; Kato, T.; Ohno, H. 3D Continuous Water Nanosheet As a Gyroid Minimal Surface Formed by Bicontinuous Cubic Liquid-Crystalline Zwitterions. J. Am. Chem. Soc. 2012, 134, 11354-11357.

(59) Cook, A. G.; Baumeister, U.; Tschierske, C. Supramolecular Dendrimers: Unusual Mesophases of Ionic Liquid Crystals Derived From Protonation of DAB Dendrimers With Facial Amphiphilic Carboxylic Acids. J. Mater. Chem. 2005, 15, 1708-1721.

(60) Chen, B.; Zeng, X. B.; Baumeister, U.; Diele, S.; Ungar, G.; Tschierske, C. Liquid Crystals With Complex Superstructures. Angew. Chem., Int. Ed. 2004, 43, 4621-4625.

(61) Saielli, G.; Voth, G. A.; Wang, Y. Diffusion Mechanisms in Smectic Ionic Liquid Crystals: Insights From Coarse-Grained MD Simulations. Soft Matter 2013, 9, 5716-5725.

(62) Ji, Y.; Shi, R.; Wang, Y.; Saielli, G. Effect of the Chain Length on the Structure of Ionic Liquids: From Spatial Heterogeneity to Ionic Liquid Crystals. J. Phys. Chem. B 2013, 117, 1104-1109.

(63) Leclercq, L.; Noujeim, N.; Schmitzer, A. R. Development of $N, N$-Diaromatic Diimidazolium Cations: Arene Interactions for Highly Organized Crystalline Materials. Cryst. Growth Des. 2009, 9, 4784-4792.

(64) Kohmoto, S.; Okuyama, S.; Nakai, T.; Takahashi, M.; Kishikawa, K.; Masu, H.; Azumaya, I. Crystal Structure of Hydrates of Imidazolium Salts. J. Mol. Struct. 2011, 998, 192-197.

(65) Ichikawa, T.; Yoshio, M.; Taguchi, S.; Kagimoto, J.; Ohno, H.; Kato, T. Co-Organisation of Ionic Liquids With Amphiphilic Diethanolamines: Construction of 3D Continuous Ionic Nanochannels Through the Induction of Liquid-Crystalline Bicontinuous Cubic Phases. Chem. Sci. 2012, 3, 2001-2008. 
(66) Yamashita, A.; Yoshio, M.; Shimizu, S.; Ichikawa, T.; Ohno, H.; Kato, T. Columnar Nanostructured Polymer Films Containing Ionic Liquids in Supramolecular One-Dimensional Nanochannels. J. Polym. Sci., Part A: Polym. Chem. 2015, 53, 366-371.

Soberats, B.; Uchida, E.; Yoshio, M.; Kagimoto, J.; Ohno, H.; Kato, T. Macroscopic Photocontrol of IonTransporting Pathways of a Nanostructured Imidazolium-Based Photoresponsive Liquid Crystal. J. Am. Chem. Soc. 2014, 136, 9552-9555.

(68) He, C.; He, Q.; Zhang, S.; Tan, X.; Gao, H.; Cheng, X. Ionic Bent Shape Ternary Facial Amphiphiles. Chin. J. Chem. 2013, 31, 839-844.

(69) Wu, B. P.; Pang, M. L.; Tan, T. F.; Meng, J. B. The T ' Phase and Its 'Sandwich-Like Layer' Structure As Shown by Ionic Liquid Crystals Containing a Biphenyl Ester-Based Rigid-Core Modified by 3Alkylimidazolium Salts. Liq. Cryst. 2012, 39, 579-594.

(70) Sepelj, M.; Lesac, A.; Baumeister, U.; Diele, S.; Nguyen, H.; Bruce, D. W. Intercalated Liquid-Crystalline Phases Formed by Symmetric Dimers With an $\alpha, \omega$-Diiminoalkylene Spacer. J. Mater. Chem. 2007, 17, 1154-1165.

(71) Ichikawa, T.; Yoshio, M.; Hamasaki, A.; Taguchi, S.; Liu, F.; Zeng, X.-b.; Ungar, G.; Ohno, H.; Kato, T. Induction of Thermotropic Bicontinuous Cubic Phases in Liquid-Crystalline Ammonium and Phosphonium Salts. J. Am. Chem. Soc. 2012, 134, 2634-2643.

Ma, K.; Shahkhatuni, A. A.; Somashekhar, B. S.; Gowda, G. A. N.; Tong, Y.; Khetrapal, C. L.; Weiss, R. G. Room-Temperature and Low-Ordered, Amphotropic-Lyotropic Ionic Liquid Crystal Phases Induced by Alcohols in Phosphonium Halides. Langmuir 2008, 24, 9843-9854.

Taguchi, S.; Ichikawa, T.; Kato, T.; Ohno, H. Nano-Biphasic Ionic Liquid Systems Composed of Hydrophobic Phosphonium Salts and a Hydrophilic Ammonium Salt. Chem. Commun. 2012, 48, 52715273.

(74) Lava, K.; Evrard, Y.; Van Hecke, K.; Van Meervelt, L.; Binnemans, K. Quinolinium and Isoquinolinium Ionic Liquid Crystals. RSC Adv. 2012, 2, 8061-8070.

Asaftei, S.; Ciobanu, M.; Lepadatu, A. M.; Song, E.; Beginn, U. Thermotropic Ionic Liquid Crystals by Molecular Assembly and Ion Pairing of 4,4'-Bipyridinium Derivatives and Tris(Dodecyloxy)Benzenesulfonates in a Non-Polar Solvent. J. Mater. Chem. 2012, 22, 14426-14437.

Goossens, K.; Lava, K.; Nockemann, P.; Van Hecke, K.; Van Meervelt, L.; Driesen, K.; Görller-Walrand, C.; Binnemans, K.; Cardinaels, T. Pyrrolidinium Ionic Liquid Crystals. Chem. Eur. J. 2009, 15, 656-674.

Mathevet, F.; Masson, P.; Nicoud, J.-F.; Skoulios, A. Smectic Liquid Crystals From Supramolecular Guanidinium Alkanesulfonates. J. Am. Chem. Soc. 2005, 127, 9053-9061.

Sauer, S.; Steinke, N.; Baro, A.; Laschat, S.; Giesselmann, F.; Kantlehner, W. Guanidinium Chlorides With Triphenylene Moieties Displaying Columnar Mesophases. Chem. Mater. 2008, 20, 1909-1915.

Maeda, H.; Chigusa, K.; Sakurai, T.; Ohta, K.; Uemura, S.; Seki, S. Ion-Pair-Based Assemblies Comprising Pyrrole-Pyrazole Hybrids. Chem. Eur. J. 2013, 19, 9224-9233.

Haketa, Y.; Honsho, Y.; Seki, S.; Maeda, H. Ion Materials Comprising Planar Charged Species. Chem. Eur. J. 2012, 18, 7016-7020. 
(81) Huang, Y.; Yan, Y.; Smarsly, B. M.; Wei, Z.; Faul, C. F. J. Helical Supramolecular Aggregates, Mesoscopic Organisation and Nanofibers of a Perylenebisimide-Chiral Surfactant Complex Via Ionic SelfAssembly. J. Mater. Chem. 2009, 19, 2356-2362.

(82) Faul, C. F. J.; Krattiger, P.; Smarsly, B. M.; Wennemers, H. Ionic Self-Assembled Molecular ReceptorBased Liquid Crystals With Tripeptide Recognition Capabilities. J. Mater. Chem. 2008, 18, 2962-2967.

(83) Li, W.; Yin, S. Y.; Wang, J. F.; Wu, L. Tuning Mesophase of Ammonium Amphiphile-Encapsulated Polyoxometalate Complexes Through Changing Component Structure. Chem. Mater. 2008, 20, 514-522.

(84) Floquet, S.; Terazzi, E.; Hijazi, A.; Guénée, L.; Piguet, C.; Cadot, E. Evidence of Ionic Liquid Crystal Properties for a DODA ${ }^{+}$Salt of the Keplerate $\left[\mathrm{Mo}_{132} \mathrm{O}_{372}\left(\mathrm{CH}_{3} \mathrm{COO}\right)_{30}\left(\mathrm{H}_{2} \mathrm{O}\right)_{72}\right]^{42-}$. New J. Chem. 2012, 36, 865-868.

(85) Cui, L.; Miao, J. J.; Zhu, L. Spacer Length Controlled Oblique-Columnar to Lamello-Columnar Mesophase Transition in Liquid Crystalline DNA-Discotic Cationic Lipid Complexes. Macromolecules 2006, 39, 25362545.

(86) Ishida, Y.; Kai, Y.; Kato, S.-y.; Misawa, A.; Amano, S.; Matsuoka, Y.; Saigo, K. Two-Component Liquid Crystals As Chiral Reaction Media: Highly Enantioselective Photodimerization of an Anthracene Derivative Driven by the Ordered Microenvironment. Angew. Chem., Int. Ed. 2008, 47, 8241-8245.

(87) Downard, A.; Earle, M. J.; Hardacre, C.; Mcmath, S. E. J.; Nieuwenhuyzen, M.; Teat, S. J. Structural Studies of Crystalline 1-Alkyl-3-Methylimidazolium Chloride Salts. Chem. Mater. 2004, 16, 43-48.

(88) Zhang, S.; Liu, S.; Zhang, Y.; Deng, Y. Photoinduced Isothermal Phase Transition of Ionic Liquid Crystals. Chem. Asian J. 2012, 7, 2004-2007.

(89) Wang, M.; Pan, X.; Xiao, S.; Zhang, C.; Li, W.; Dai, S. Regulating Mesogenic Properties of Ionic Liquid Crystals by Preparing Binary or Multi-Component Systems. J. Mater. Chem. 2012, 22, 2299-2305.

(90) Holbrey, J. D.; Seddon, K. R. The Phase Behaviour of 1-Alkyl-3-Methylimidazolium Tetrafluoroborates; Ionic Liquids and Ionic Liquid Crystals. J. Chem. Soc., Dalton Trans. 1999, 2133-2139.

(91) Biswas, M.; Dule, M.; Samanta, P. N.; Ghosh, S.; Mandal, T. K. Imidazolium-Based Ionic Liquids With Different Fatty Acid Anions: Phase Behavior, Electronic Structure and Ionic Conductivity Investigation. Phys. Chem. Chem. Phys. 2014, 16, 16255-16263.

(92) Xu, F.; Matsumoto, K.; Hagiwara, R. Effects of Alkyl Chain Length and Anion Size on Thermal and Structural Properties for 1-Alkyl-3-Methylimidazolium Hexafluorocomplex Salts $\left(\mathrm{C}_{x} \mathrm{MImAF}_{6}, x=14,16\right.$ and 18; A = P, As, Sb, Nb and Ta). Dalton Trans. 2012, 41, 3494-3502.

(93) Gordon, C. M.; Holbrey, J. D.; Kennedy, A. R.; Seddon, K. R. Ionic Liquid Crystals: Hexafluorophosphate Salts. J. Mater. Chem. 1998, 8, 2627-2636.

(94) Bara, J. E.; Hatakeyama, E. S.; Wiesenauer, B. R.; Zeng, X.; Noble, R. D.; Gin, D. L. Thermotropic Liquid Crystal Behaviour of Gemini Imidazolium-Based Ionic Amphiphiles. Liq. Cryst. 2010, 37, 1587-1599.

(95) Yang, M.; Stappert, K.; Mudring, A. V. Bis-Cationic Ionic Liquid Crystals. J. Mater. Chem. C 2014, 2, 458473.

(96) Gao, Y.; Slattery, J. M.; Bruce, D. W. Columnar Thermotropic Mesophases Formed by Dimeric LiquidCrystalline Ionic Liquids Exhibiting Large Mesophase Ranges. New J. Chem. 2011, 35, 2910-2918. 
Yoshio, M.; Ichikawa, T.; Shimura, H.; Kagata, T.; Hamasaki, A.; Mukai, T.; Ohno, H.; Kato, T. Columnar Liquid-Crystalline Imidazolium Salts. Effects of Anions and Cations on Mesomorphic Properties and Ionic Conductivity. Bull. Chem. Soc. Jpn. 2007, 80, 1836-1841.

(98) Starkulla, G.; Kaller, M.; Frey, W.; Axenov, K. V.; Laschat, S. Liquid Crystalline Imidazolium Salts Bearing 5-Phenylpyrimidine: Dependence of Mesomorphic Properties on Spacer Lengths, Terminal $\mathrm{N}$ Alkyl Group and Counterions. Liq. Cryst. 2011, 38, 1515-1529.

(99) Starkulla, G. F.; Klenk, S.; Butschies, M.; Tussetschläger, S.; Laschat, S. Towards Room Temperature Ionic Liquid Crystals: Linear Versus Bent Imidazolium Phenylpyrimidines. J. Mater. Chem. 2012, 22, 2198721997.

(100) Sakuda, J.; Yoshio, M.; Ichikawa, T.; Ohno, H.; Kato, T. 2D Assemblies of Ionic Liquid Crystals Based on Imidazolium Moieties: Formation of Ion-Conductive Layers. New J. Chem. 2015, 39, 4471-4477.

(101) Alam, M. A.; Motoyanagi, J.; Yamamoto, Y.; Fukushima, T.; Kim, J.; Kato, K.; Takata, M.; Saeki, A.; Seki, S.; Tagawa, S.; et al. "Bicontinuous Cubic" Liquid Crystalline Materials From Discotic Molecules: A Special Effect of Paraffinic Side Chains With Ionic Liquid Pendants. J. Am. Chem. Soc. 2009, 131, 1772217723.

(102) Lee, J. J.; Yamaguchi, A.; Alam, M. A.; Yamamoto, Y.; Fukushima, T.; Kato, K.; Takata, M.; Fujita, N.; Aida, T. Discotic Ionic Liquid Crystals of Triphenylene As Dispersants for Orienting Single-Walled Carbon Nanotubes. Angew. Chem., Int. Ed. 2012, 51, 8490-8494.

(103) Farrand, L. D.; Vaughan-Spickers, J.; Hardacre, C.; Sheppard, O. Ionic Mesogenic Compounds. WO 2004065523 A1, 2004.

(104) Wang, Y. J.; Marques, E. F. Thermotropic Phase Behavior of Cationic Gemini Surfactants and Their Equicharge Mixtures With Sodium Dodecyl Sulfate. J. Phys. Chem. B 2006, 110, 1151-1157.

(105) Manet, S.; Karpichev, Y.; Dedovets, D.; Oda, R. Effect of Hofmeister and Alkylcarboxylate Anionic Counterions on the Krafft Temperature and Melting Temperature of Cationic Gemini Surfactants. Langmuir 2013, 29, 3518-3526.

(106) Mihelj, T.; Popovic, J.; Skoko, Z.; Tomasic, V. Thermotropic Phase Transitions of Catanionic Dodecylsulfates With Multi-Charged and Multi-Tailed Quaternary Ammonium Centers. Thermochim. Acta 2014, 591, 119-129.

(107) Tomasic, V.; Mihelj, T.; Zhang, R.; Liu, F.; Ungar, G. Mesomorphism of a New Series of Catanionic 4-(1Pentylheptyl)Benzenesulfonates. Soft Matter 2014, 10, 7887-7896.

(108) Mihelj, T.; Vojta, D.; Tomasic, V. The Diversity in Thermal Behavior of Novel Catanionic Cholates: The Dominant Effect of Quaternary Ammonium Centers. Thermochim. Acta 2014, 584, 17-30.

(109) Maximo, G. J.; Santos, R. J. B. N.; Lopes-da-Silva, J. A.; Costa, M. C.; Meirelles, A. J. A.; Coutinho, J. A. P. Lipidic Protic Ionic Liquid Crystals. ACS Sustainable Chem. Eng. 2014, 2, 672-682.

(110) Ishida, Y.; Amano, S.; Saigo, K. Template Polymerization of Columnar Architectures Based on the Salts of a Carboxylic Acid and 2-Amino Alcohols: Application to the Molecular Recognition of 2-Amino Alcohols. Chem. Commun. 2003, 2338-2339. 
(111) Amano, S.; Ishida, Y.; Saigo, K. Solid-State Hosts by the Template Polymerization of Columnar Liquid Crystals: Locked Supramolecular Architectures Around Chiral 2-Amino Alcohols. Chem. Eur. J. 2007, 13, 5186-5196.

(112) Ishida, Y.; Achalkumar, A. S.; Kato, S.-y.; Kai, Y.; Misawa, A.; Hayashi, Y.; Yamada, K.; Matsuoka, Y.; Shiro, M.; Saigo, K. Tunable Chiral Reaction Media Based on Two-Component Liquid Crystals: Regio-, Diastereo-, and Enantiocontrolled Photodimerization of Anthracenecarboxylic Acids. J. Am. Chem. Soc. 2010, 132, 17435-17446.

(113) Hernández-Ainsa, S.; Barberá, J.; Marcos, M.; Serrano, J. L. Nanoobjects Coming From Mesomorphic Ionic PAMAM Dendrimers. Soft Matter 2011, 7, 2560-2568.

(114) Hernández-Ainsa, S.; Fedeli, E.; Barberá, J.; Marcos, M.; Sierra, T.; Serrano, J. L. Self-Assembly Modulation in Ionic PAMAM Derivatives. Soft Matter 2014, 10, 281-289.

(115) Chen, H.; Kwait, D. C.; Gonen, Z. S.; Weslowski, B. T.; Abdallah, D. J.; Weiss, R. G. Phase Characterization and Properties of Completely Saturated Quaternary Phosphonium Salts. Ordered, RoomTemperature Ionic Liquids. Chem. Mater. 2002, 14, 4063-4072.

(116) Ma, K.; Somashekhar, B. S.; Gowda, G. A. N.; Khetrapal, C. L.; Weiss, R. G. Induced Amphotropic and Thermotropic Ionic Liquid Crystallinity in Phosphonium Halides: "Lubrication" by Hydroxyl Groups. Langmuir 2008, 24, 2746-2758.

(117) Abdallah, D. J.; Robertson, A.; Hsu, H. F.; Weiss, R. G. Smectic Liquid-Crystalline Phases of Quaternary Group VA (Especially Phosphonium) Salts With Three Equivalent Long $n$-Alkyl Chains. How Do Layered Assemblies Form in Liquid-Crystalline and Crystalline Phases? J. Am. Chem. Soc. 2000, 122, 3053-3062.

(118) Shahkhatuni, A. A.; Ma, K. F.; Weiss, R. G. Designing Amphotropic Smectic Liquid Crystals Based on Phosphonium Salts for Partial Ordering of Solutes As Monitored by NMR Spectroscopy. J. Phys. Chem. B 2009, 113, 4209-4217.

(119) Matsumoto, T.; Ichikawa, T.; Sakuda, J.; Kato, T.; Ohno, H. Design of Amphiphilic Zwitterions Forming Liquid-Crystalline Phases and Effects of Lithium Salt Addition on Their Phase Behavior. Bull. Chem. Soc. Jpn. 2014, 87, 792-796.

(120) Bhowmik, P. K.; Han, H. S.; Nedeltchev, I. K.; Cebe, J. J. Room-Temperature Thermotropic Ionic Liquid Crystals: Viologenbis(Triflimide) Salts. Mol. Cryst. Liq. Cryst. 2004, 419, 27-46.

(121) Safavi, A.; Tohidi, M. Design and Characterization of Liquid Crystal-Graphite Composite Electrodes. J. Phys. Chem. C 2010, 114, 6132-6140.

(122) Casella, G.; Causin, V.; Rastrelli, F.; Saielli, G. Viologen-Based Ionic Liquid Crystals: Induction of a Smectic A Phase by Dimerisation. Phys. Chem. Chem. Phys. 2014, 16, 5048-5051.

(123) Sauer, S.; Saliba, S.; Tussetschläger, S.; Baro, A.; Frey, W.; Giesselmann, F.; Laschat, S.; Kantlehner, W. pAlkoxybiphenyls With Guanidinium Head Groups Displaying Smectic Mesophases. Liq. Cryst. 2009, 36, 275-299.

(124) Butschies, M.; Sauer, S.; Kessler, E.; Siehl, H. U.; Claasen, B.; Fischer, P.; Frey, W.; Laschat, S. Influence of $N$-Alkyl Substituents and Counterions on the Structural and Mesomorphic Properties of Guanidinium Salts: Experiment and Quantum Chemical Calculations. ChemPhysChem 2010, 11, 3752-3765. 
(125) Tan, B. H.; Yoshio, M.; Ichikawa, T.; Mukai, T.; Ohno, H.; Kato, T. Spiropyran-Based Liquid Crystals: the Formation of Columnar Phases Via Acid-Induced Spiro-Merocyanine Isomerisation. Chem. Commun. 2006, 4703-4705.

(126) Maeda, H.; Chigusa, K.; Yamakado, R.; Sakurai, T.; Seki, S. Carboxylate-Driven Supramolecular Assemblies of Protonated meso-Aryl-Substituted Dipyrrolylpyrazoles. Chem. Eur. J. 2015, 21, 9520-9527.

(127) Liu, K.; Pesce, D.; Ma, C.; Tuchband, M.; Shuai, M.; Chen, D.; Su, J.; Liu, Q.; Gerasimov, J. Y.; Kolbe, A.; et al. Solvent-Free Liquid Crystals and Liquids Based on Genetically Engineered Supercharged Polypeptides With High Elasticity. Adv. Mater. 2015, 27, 2459-2465. 